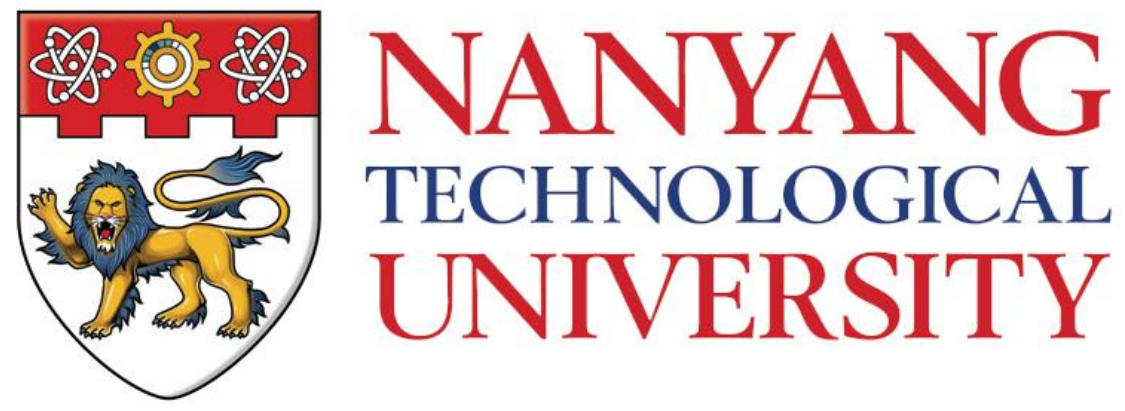

RESPONSES OF REINFORCED CONCRETE STRUCTURES UNDER PROGRESSIVE COLLAPSE SUBJECTED TO DIFFERENT LOADING METHODS AND LOADING RATES

PHAM ANH TUAN

SCHOOL OF CIVIL AND ENVIRONMENTAL ENGINEERING 


\section{RESPONSES OF REINFORCED CONCRETE STRUCTURES UNDER PROGRESSIVE COLLAPSE SUBJECTED TO DIFFERENT LOADING METHODS AND LOADING RATES}

\section{PHAM ANH TUAN}

School of Civil and Environmental Engineering

A thesis submitted to the Nanyang Technological University in partial fulfilment of the requirement for the degree of

Doctor of Philosophy 


\section{ACKNOWLEDGEMENT}

I would like to express my gratitude and appreciation to my supervisor, Professor Tan Kang Hai, for his guidance, support, and encouragement throughout the Ph.D. study. His enthusiasm and passion for research are incredible and admirable. I am so fortunate and grateful to work with him.

I would like to thank to Nanyang Technological University (NTU) for providing me the research position, as well as to Ministry of Home Affairs (MHA), Singapore, for the financial support.

I also wish to thank all of my seniors and friends in NTU, particularly Dr. Nguyen Minh Phuong, Dr. Nguyen Tuan Trung, Dr. Nguyen Truong Thang, Dr. Yu Jun, Mr. Vu Ngoc Son, Ms. Nguyen Thi Nguyet Hang, for their valuable discussions and supports in research and life, as well as Dr. Namyo Salim Lim for his help on testing procedure and for his sharing of knowledge.

I am thankful to the technical staff at Protective Engineering Laboratory, Mr. Jee Kim Tian and Mr. Ho Yaow Chan, for their assistance and support during the experimental work. Thanks are also attended to Dr. Tassilo Rinder and Mr. Christian Brenneis at Fraunhofer Institute for High-Speed Dynamics EMI (Germany) for their work and contribution during the blast tests.

Finally, I am deeply indebted to my parents, my wife and children, who have loved and supported me unconditionally. 


\section{TABLE OF CONTENTS}

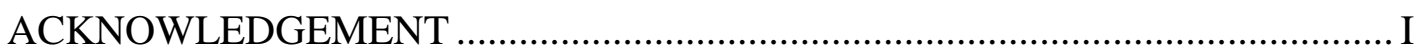

TABLE OF CONTENTS ................................................................................ III

SUMMARY

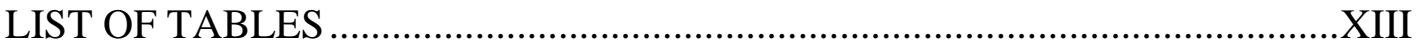

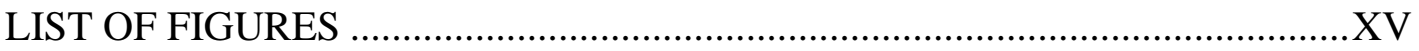

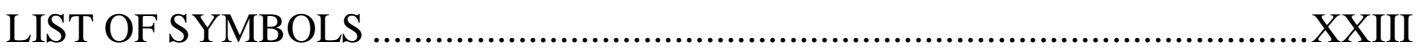

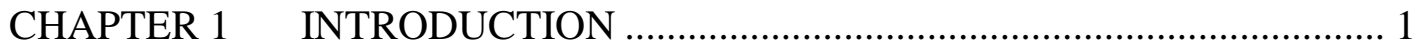

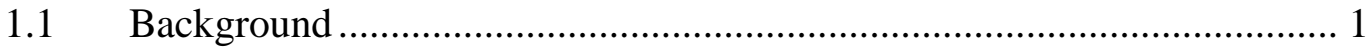

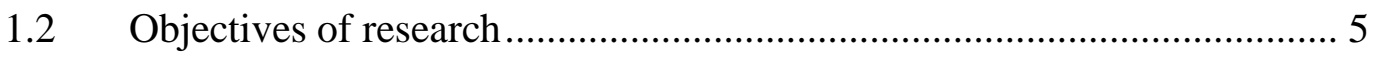

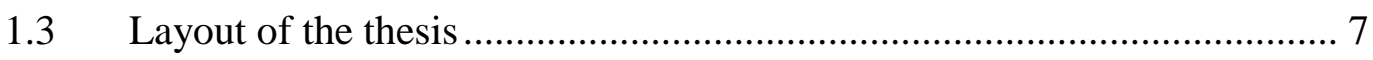

CHAPTER 2 LITERATURE REVIEW ….................................................. 9

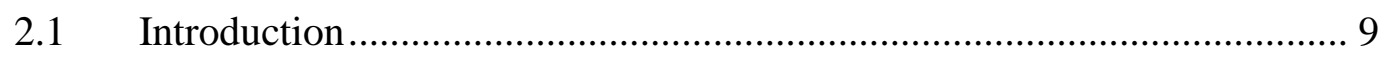

2.2 Design methods for mitigating progressive collapse ............................. 9

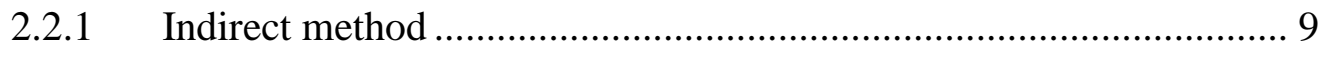

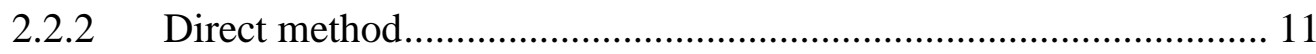

2.3 Studies on Alternate Load Paths of beam-column structures ................. 13

2.3.1 Quasi-static analyses of beam-column structures ........................... 14

2.3.2 Dynamic analyses of beam-column structures ............................ 23

2.3.3 Blast-induced dynamic behaviour of beam-column structures........ 30

2.3.4 Summary of experimental studies on beam-column structures ....... 33

2.4 Alternate load paths of beam-slab structures ....................................... 35

2.4.1 Analytical models for TMA in slab-only structures ...................... 35

2.4.2 Studies on TMA in RC beam-slab systems under progressive

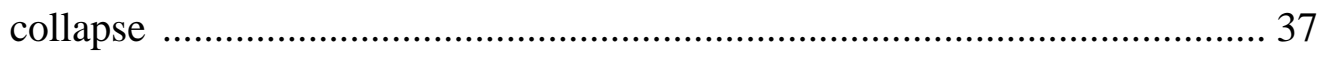

2.5 Summary of literature review and research scope of work.................... 40 


\section{CHAPTER 3 NUMERICAL INVESTIGATION ON BEAM-COLUMN SUBSTRUCTURES UNDER DYNAMIC RESPONSES ....................................45}

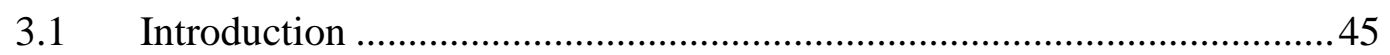

3.2 Numerical validations on beam-column substructures.............................45

3.2.1 Quasi-static and contact-detonation tests on RC sub-assemblages

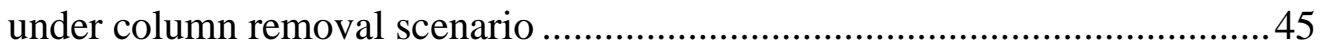

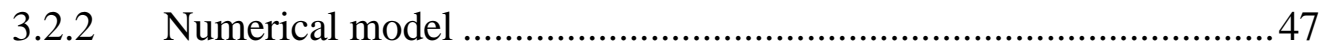

3.2.3 Model validations by pull-out tests ................................................56

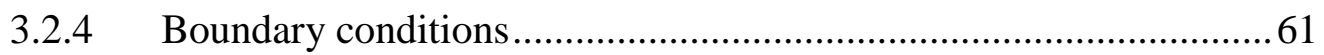

3.2.5 Analysis procedure and validation results for quasi-static tests .......63

3.2.6 Strain rate effect on the simulation of the blast series ..................... 70

3.2.7 Simulation of blast pressure in LS-Dyna...................................... 71

3.2.8 Analysis procedure and validation results for blast tests.................74

3.3 Parametric studies on RC substructures under progressive collapse.........77

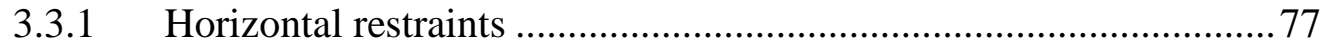

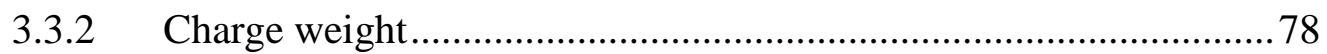

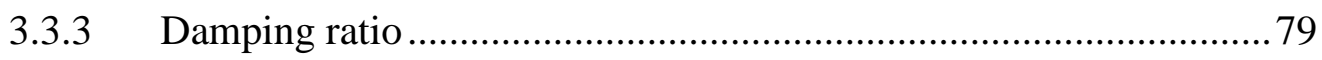

3.3.4 Comparison between dynamic analysis and static analysis associated with the energy approach for dynamic evaluation ...................................... 80

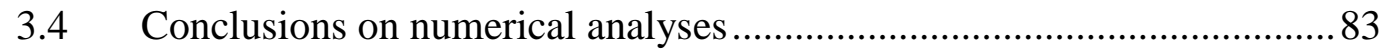

\section{CHAPTER 4 A SIMPLIFIED MODEL OF CATENARY ACTION IN RC} FRAMES UNDER AXIALLY RESTRAINED CONDITIONS ...........................85

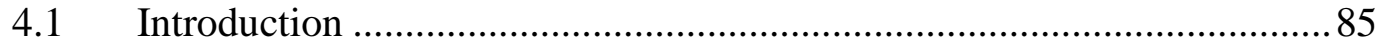

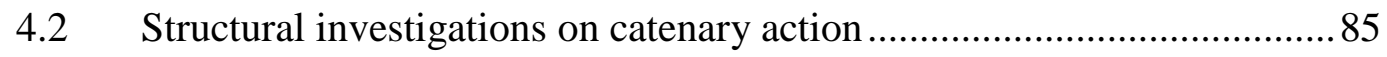

4.2.1 Proposed simplified structural response under supporting column

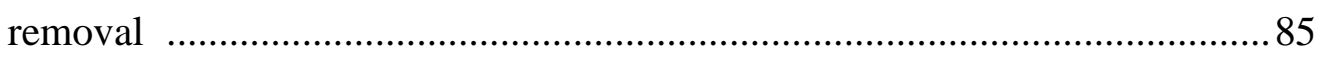

4.2.2 Proposed normalised factors for sub-assemblage behaviour............87

4.2.3 Investigations on structural behaviour.......................................... 89

4.3 Simplified semi-analytical model on catenary action............................92

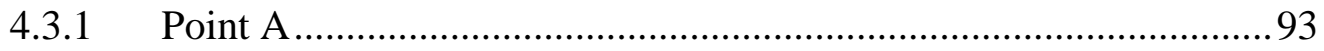

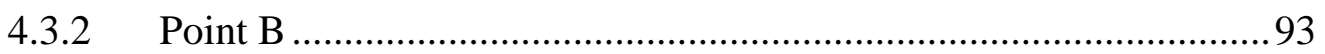




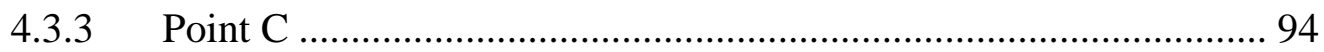

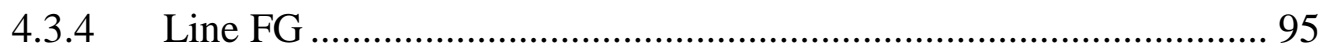

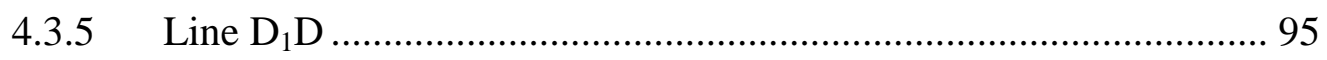

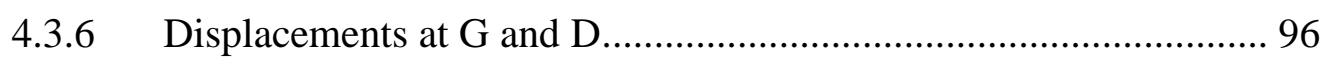

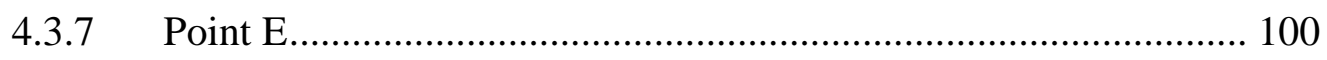

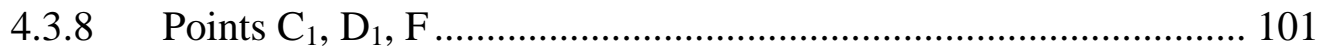

4.3.9 Minimum value for horizontal stiffness ratio $k_{\text {hor } \ldots \ldots \ldots \ldots \ldots \ldots \ldots \ldots . . . . . . . . . . . .102}$

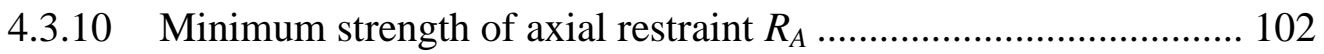

4.3.11 Comparisons between the simplified model and the FEM results. 103

4.3.12 Dynamic assessment of RC sub-assemblages under single column

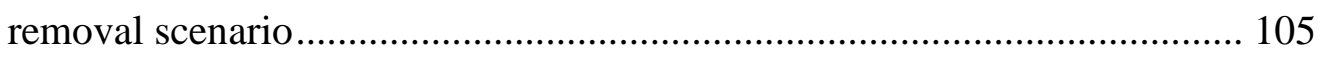

4.4 Test validations using the semi-analytical model .............................. 107

4.5 Discussions on the semi-analytical model for sub-assemblages........... 112

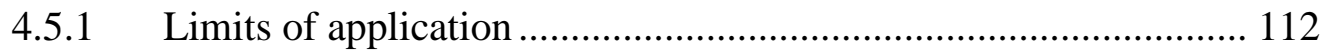

4.5.2 Discussions on symmetric conditions ......................................... 112

4.6 Conclusions on the simplified model.................................................. 116

CHAPTER 5 EXPERIMENTAL PROGRAMME ON RC SUBSTRUCTURES.

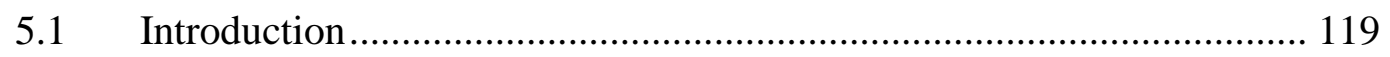

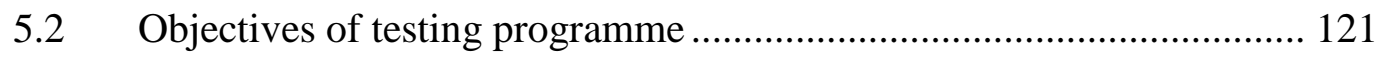

5.2.1 Free-fall dynamic tests on RC frames under CL condition (Series I).. 121

5.2.2 Static and dynamic tests on RC frames under UDL condition (Series

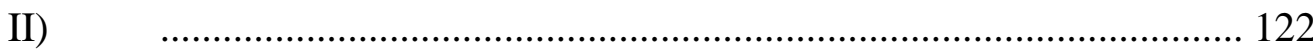

5.2.3 Blast-induced tests on RC frames under CL condition (Series III) 122 $5.3 \quad$ Specimen design and test setup.................................................... 123

5.3.1 Prototype building and quasi-static specimens under CL condition....

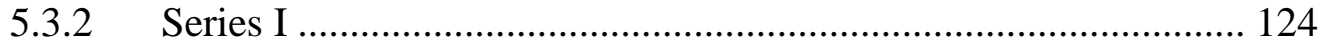

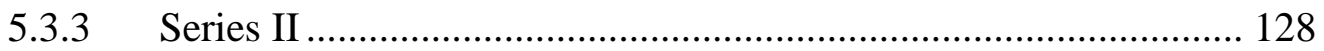

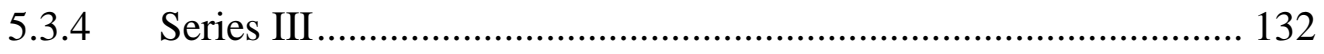

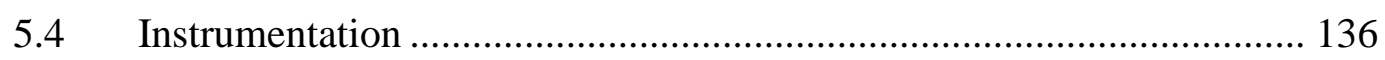




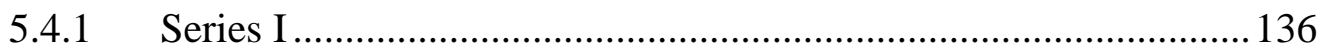

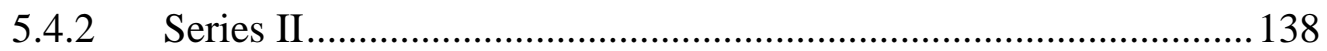

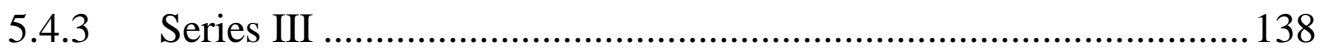

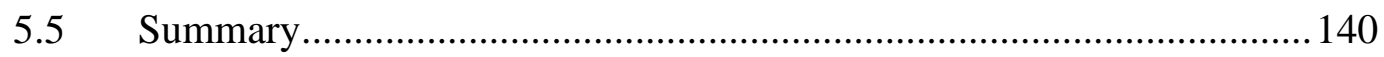

CHAPTER 6 EXPERIMENTAL AND NUMERICAL RESULTS OF RC SUBSTRUCTURES UNDER CL FREE-FALL DYNAMIC CONDITION (SERIES I)

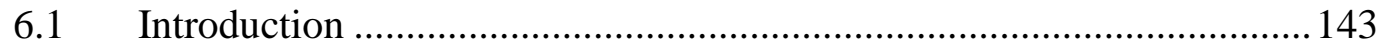

6.2 Test results of Series I ..................................................................... 143

6.2.1 Testing procedure and overall behaviour ................................... 143

6.2.2 Damage patterns of RC frames under applied loads of 20 and $25 \mathrm{kN}$. 148

6.2.3 Damage patterns of specimens under $29 \mathrm{kN}$ load level and above 150

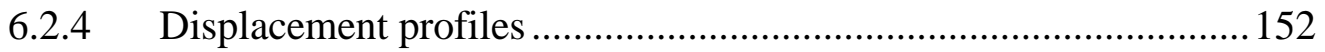

6.2.5 Strain rate effects on material properties ..................................... 155

6.2.6 Dynamic load increase factors..................................................157

6.3 Comparisons between static and dynamic responses at structural and

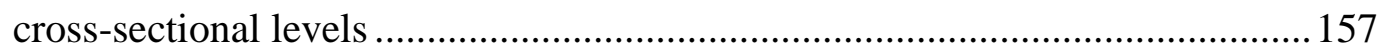

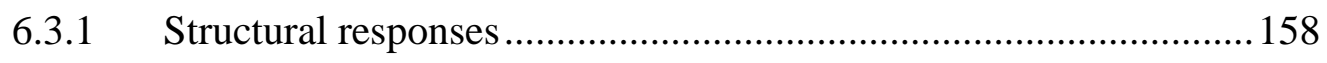

6.3.2 Stiffness of boundary restraints .................................................. 161

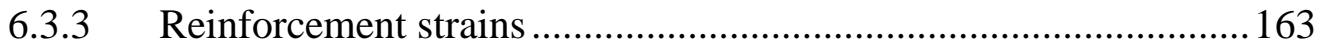

6.3.4 Comparisons of dynamic loading capacity between actual tests and

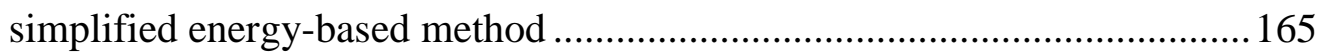

6.4 Numerical studies on 2D frames under CL condition ...........................166

6.4.1 Validations and investigations of static responses using 3D FEM

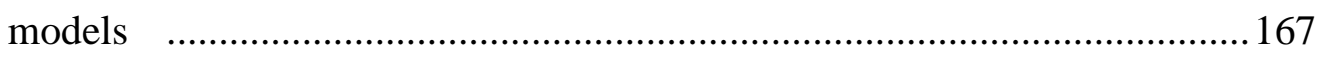

6.4.2 Validations and investigations of dynamic responses using 3D FEM

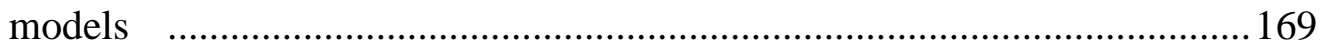

6.4.3 Simplified analysis using equivalent SDOF system ...................... 172

6.5 Conclusions on experimental and numerical results of Series I............. 175

Appendix 6A - Details of test results from Series I........................................ 178 


\section{CHAPTER 7 EXPERIMENTAL RESULTS OF FRAME STRUCTURES}

UNDER UDL STATIC AND DYNAMIC CONDITIONS (SERIES II)

7.1 Introduction 181

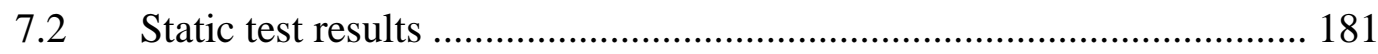

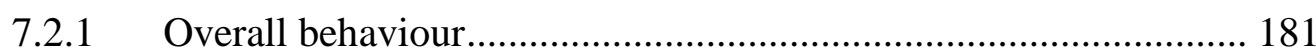

7.2.2 Damage patterns and failure modes .......................................... 186

7.2.3 Beam-end rotation and horizontal reaction at top rebar fracture ... 193

7.3 Analytical relationship of structural behaviours between UDL and CL

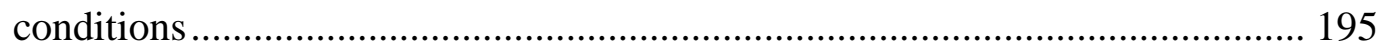

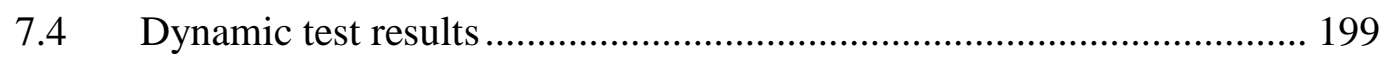

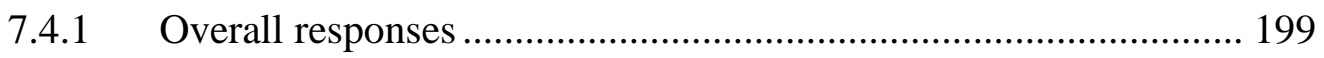

7.4.2 Structural damages and failure modes ...................................... 202

7.4.3 Strain rate effects on material strength ....................................... 203

7.5 Comparisons of structural responses between static and dynamic tests

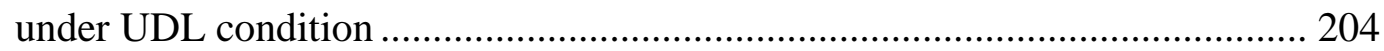

7.5.1 Structural force-based and displacement-based DIFs.................. 204

7.5.2 Reaction forces........................................................................ 204

7.6 Comparisons of load-carrying capacity between actual dynamic tests and

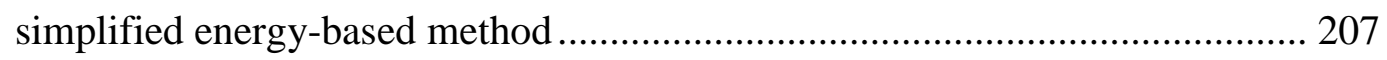

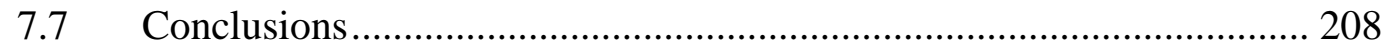

Appendix 7A - Details of test results from Series II ....................................... 211

CHAPTER 8 BLAST-INDUCED RESPONCES OF RC FRAMES UNDER CL

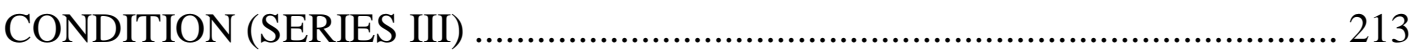

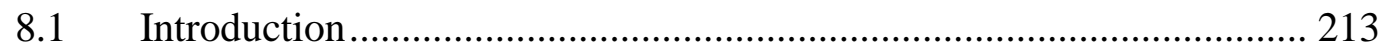

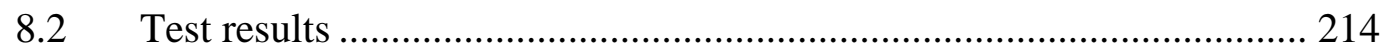

8.2.1 CD1-P/25/M..................................................................... 214

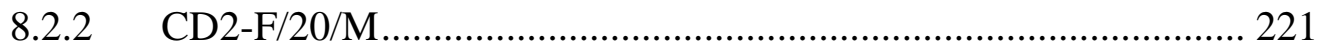

8.3 Comparisons between free-fall and blast-induced dynamic tests ......... 225

$8.4 \quad$ Numerical validations for the blast tests .............................................. 226

8.4.1 Verification of blast pressure using CONWEP .......................... 226

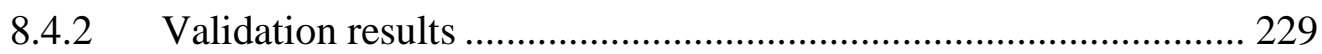


8.5 Conclusions on experimental and numerical results of Series III .230

CHAPTER 9 EVALUATION OF TENSILE MEMBRANE ACTION IN RC BEAM-SLAB STRUCTURES UNDER COLUMN REMOVAL SCENARIOS .233

9.1 Introduction .233

9.2 Numerical models of beam-only and beam-slab structures under progressive collapse. .234

9.2.1 Quasi-static tests on RC structures under internal-column removal scenario .234

9.2.2 Numerical model 235

9.2.3 Boundary condition 236

9.2.4 Validation results for beam-only tests .237

9.2.5 Validation results for beam-slab test 239

9.3 Investigations of structural responses comparing between CL and UDL conditions 240

9.3.1 Proposed loading method representing UDL condition 240

9.3.2 Slab-only structure. 242

9.3.3 Beam-slab structure .245

9.3.4 Discussions on the combined effects of beams and slabs under both

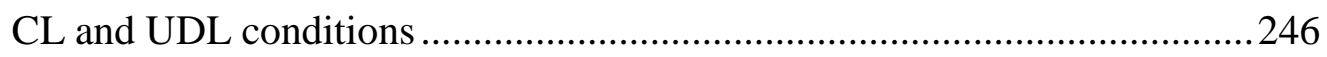

9.4 Effect of boundary stiffness on mobilisation of TMA .........................247

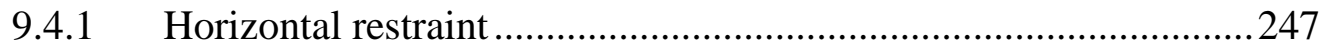

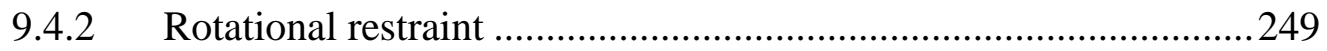

9.5 Effect of location of removed column on structural response ...............249

9.5.1 Corner-column removal..........................................................252

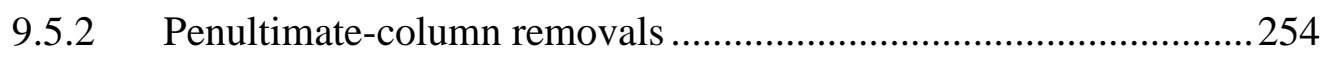

9.6 Simplified analytical model for structural response of RC beam-slab

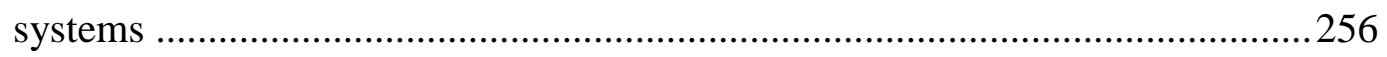

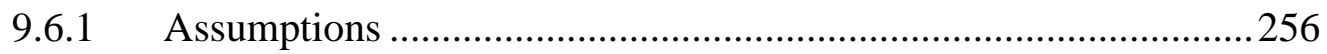

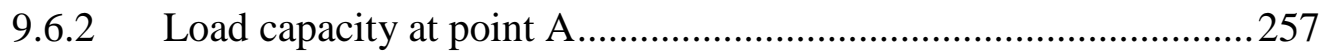

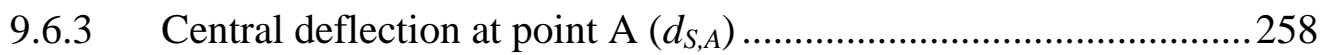

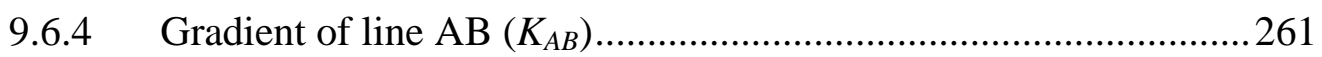

9.6.5 Maximum deflection at point B of the beam-slab model ..............267 
9.6.6 Validations of the simplified model for beam-slab structures ....... 269

9.7 Effect of contact detonation on 3D beam-slab structures ..................... 271

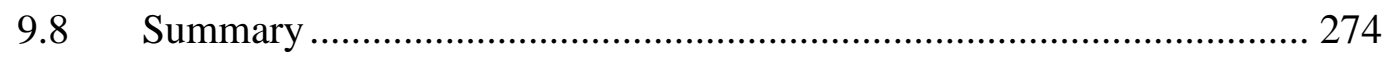

CHAPTER 10 CONCLUSIONS AND FUTURE WORK .............................. 277

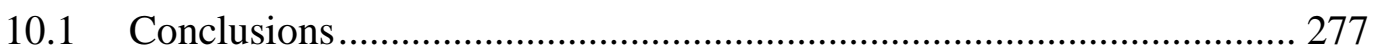

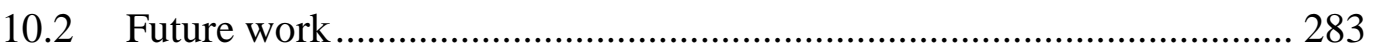

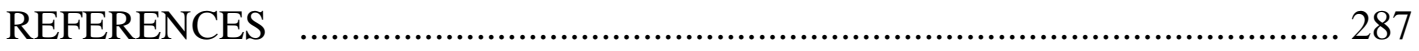




\section{SUMMARY}

Nowadays, the risks of progressive collapse of governmental and public buildings have been substantially increased due to elevated danger in terrorist attacks. Several methods and design guidelines have been released to mitigate such threats. Among them, Alternate Load Path approach applying the single column removal assumption is an effective solution to investigate structural resistance to progressive collapse. In this study, a research programme on static and dynamic behaviour of reinforced concrete $(\mathrm{RC})$ substructures against progressive collapse threats subjected to different loading rates (from quasi-static to actual blast environment), loading configurations (concentrated and distributed loads), as well as structural types (beam-column and beam-slab), was conducted. Numerical analyses of twodimensional beam-column sub-assemblages based on finite element method (FEM) were developed and validated by test results for both quasi-static and ultra-fast dynamic loading regimes. Based on the numerical results, a semi-analytical approach was proposed to predict static response of $\mathrm{RC}$ sub-assemblages under concentrated loading (CL) configuration, taking into account the mobilisation of catenary action (CA) before and after the fracture of bottom rebars at the middle joint.

Besides the development of the semi-analytical model for static response, three sets of static/free-fall dynamic/blast-induced tests (Series I, II, and III) were conducted on two-dimensional RC beam-column frames. First, the investigation on free-fall dynamic behaviour under CL condition (Series I) confirmed structural responses and failure modes observed in previous quasi-static studies. It also highlighted dynamic effects of the sudden column loss event such as dynamic load increase factors or strain rate enhancement on material strength. Secondly, results of quasistatic and dynamic tests under uniformly distributed loading (UDL) condition (Series II) were used to develop analytical relationship of structural behaviours between the two different loading configurations, viz. CL and UDL. Based on dynamic test data from Series I and II, the use of a simplified method for dynamic assessment based on energy criterion was discussed and verified. For Series III 
which included contact detonation tests under CL condition, blast effects from an explosive charge placed at the middle column were observed via initial structural uplift as well as incident blast pressure measured at the beam and column locations. Structural capacities in the blast tests were noticeably weaker than those in the freefall dynamic tests with similar applied loads.

Last but not least, a numerical investigation was carried out to study the development of tensile membrane action (TMA) in slabs for RC beam-slab systems under different loading methods (CL and UDL), boundary stiffness (horizontal and rotational restraints), as well as column loss locations. It was shown that TMA was less influenced by horizontal restraint, but was noticeably affected by rotational restraint stiffness of the slab edges if the structure was subjected to UDL condition. Moreover, under UDL configuration, the most critical scenario of progressive collapse was the removal of penultimate column, rather than corner column. Finally, a simplified analytical approach was proposed to predict structural response of beam-slab systems under internal-column loss scenario taking into account the contribution of TMA from tensile yielding forces of slab top rebars in the vicinity of negative-moment yield lines. 


\section{LIST OF TABLES}

Table 2.1 Summary of current experimental studies on beam-column structures... 34

Table 2.2 Summary of current experimental studies on beam-slab structures ........ 38

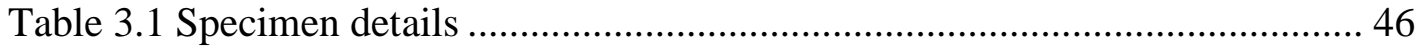

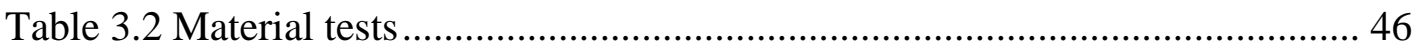

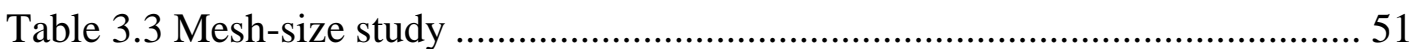

Table 3.4 Properties of rebars in the pull-out tests ............................................... 58

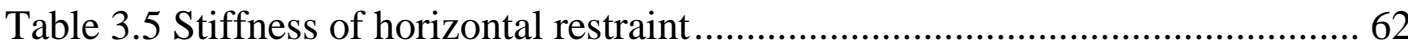

Table 3.6 Comparisons between FEM and test results (1) .................................. 66

Table 3.7 Comparisons between FEM and test results (2) .................................. 67

Table 4.1 Numerical parametric studies ............................................................. 92

Table 4.2 Test data and proposed model parameters ........................................... 109

Table 4.3 Controlling points from the proposed model ........................................ 110

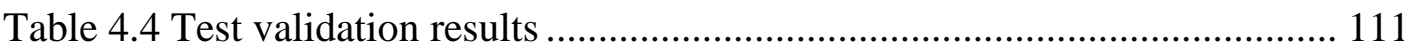

Table 5.1 Specimen properties of Series I ....................................................... 128

Table 5.2 Material properties for the dynamic tests ......................................... 128

Table 5.3 Specimen properties of Series II ...................................................... 132

Table 5.4 Test plan for contact detonation experiments (Series III) ...................... 136

Table 6.1 Summary of dynamic test results (Series I) ........................................ 146

Table 6.2 Summary of material strength enhancements ..................................... 156

Table 6.3 Boundary conditions of the dynamic tests .......................................... 163

Table 6.4 Comparisons of average restraint stiffness between static and dynamic

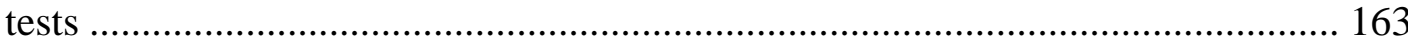

Table 7.1 Summary of static test results (Series II) ............................................ 185

Table 7.2 Summary of dynamic test results (Series II) ...................................... 201

Table 7.3 Summary of strain rates and DIF for rebars (Series II) ........................ 204

Table 7.4 Equivalent restraint stiffness of end joints from static and dynamic tests

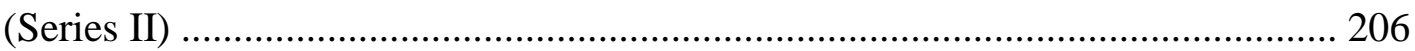

Table 7.5 Predictions of dynamic capacities based on static tests ........................ 208

Table $9.1 \alpha$ factor (Timoshenko et al. 1959) .................................................... 261 


\section{LIST OF FIGURES}

Fig. 1.1 Disproportionate collapse of Ronan Point Apartment (1968).................... 1

Fig. 1.2 Iconic events of progressive collapse ........................................................ 2

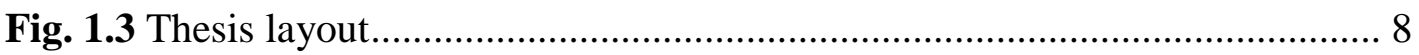

Fig. 2.1 Tie requirement for building structures (DOD 2013) .............................. 11

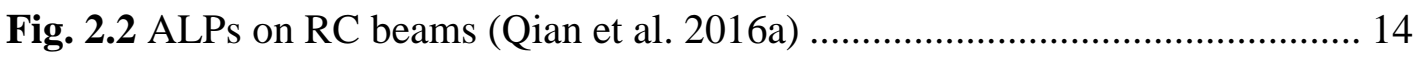

Fig. 2.3 Specimen design and test setup (Yi et al. 2008)...................................... 16

Fig. 2.4 Test setup and instrumentation layout (Sadek et al. 2011)........................ 16

Fig. 2.5 Test results for IMF specimen (Sadek et al. 2011).................................. 17

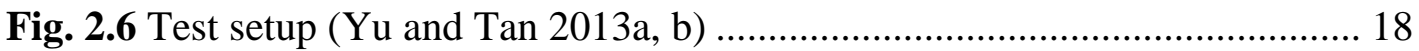

Fig. 2.7 Test results for S1 and S2 (Yu and Tan 2013a) .................................... 18

Fig. 2.8 Configuration for beam-column tests (Lim et al. 2015)............................ 19

Fig. 2.9 Quasi-static test results for 2D frames (Lim et al. 2015) .......................... 20

Fig. 2.10 Catenary action model from Li et al. (2014) ........................................ 23

Fig. 2.11 Simplified dynamic approach (Izzuddin et al. 2008) ............................. 25

Fig. 2.12 Characteristic response and DIF value for different systems (Izzuddin and

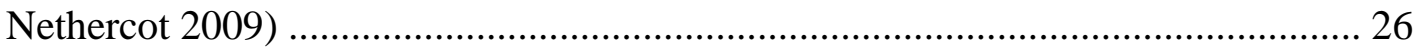

Fig. 2.13 Test results of F5 and DF5 (Qian and Li 2012c, b) .............................. 27

Fig. 2.14 Test results of F6 and DF6 (Qian and Li 2012c, b) .............................. 27

Fig. 2.15 Different loading configurations for static and dynamic tests (Sagiroglu

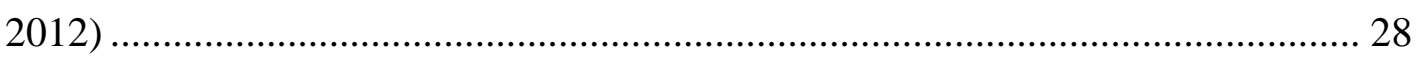

Fig. 2.16 Test setup for sudden column removal scenario (Liu et al. 2015) ........... 29

Fig. 2.17 Release time from the mechanism........................................................ 29

Fig. 2.18 Position of removed column B5 (Sasani et al. 2007) .............................. 31

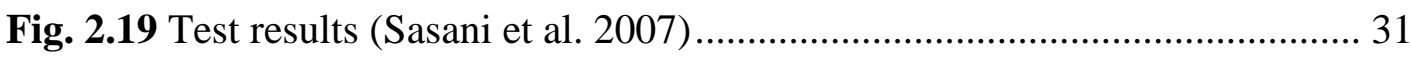

Fig. 2.20 Specimen design (Yu et al. 2014) ........................................................ 32

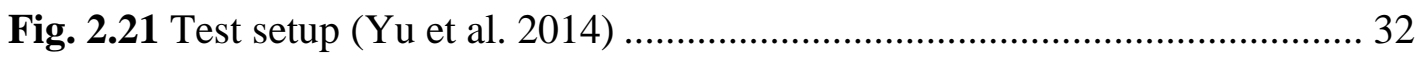

Fig. 2.22 Upper bound mechanisms for ALPs of RC structures ........................... 35

Fig. 2.23 Response of fully fixed slabs under UDL (Park 1964) ........................... 36

Fig. 2.24 Slab failure mode under large deformations (Bailey 2001) .................... 37

Fig. 2.25 In-plane stress distribution (Bailey 2001) ............................................. 37 
Fig. 2.26 Different scenarios of column removal (Stevens et al. 2009) .39

Fig. 2.27 Formation of yield lines and plastic hinges in beam-slab system (Pham et al. 2015) .39

Fig. 2.28 Testing programme on 2D beam-column frames .43

Fig. 3.1 Symmetrical model in LS-Dyna: a) Specimen modelling; b) Reinforcement modelling..... .48

Fig. 3.2 Schematic of yield surface .50

Fig. 3.3 Stress-strain relationship of a single element with $f^{\prime}=30 \mathrm{MPa}$ (Murray 2007) .50

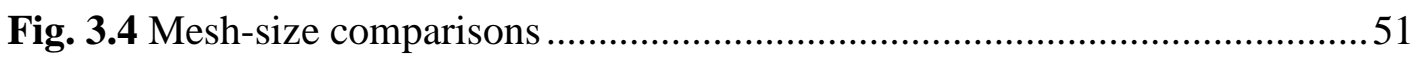

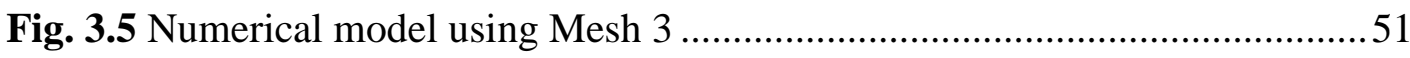

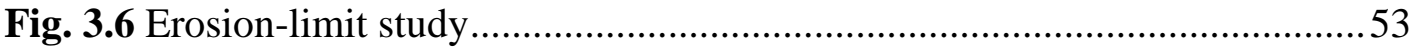

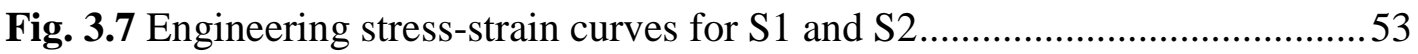

Fig. 3.8 Bond stress-slip relationship based on CEB model and simplified model for

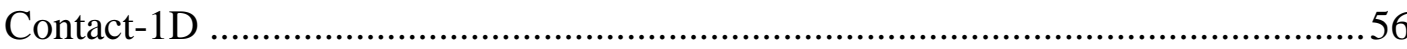

Fig. 3.9 Typical pull-out specimen with joint block and grooved bar ....................57

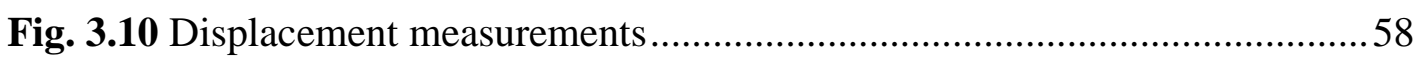

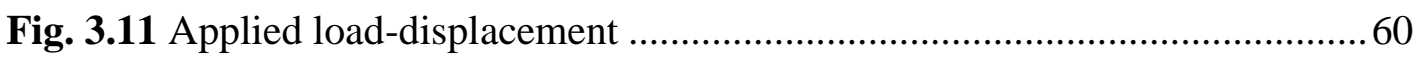

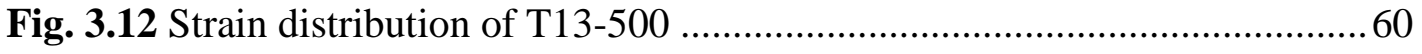

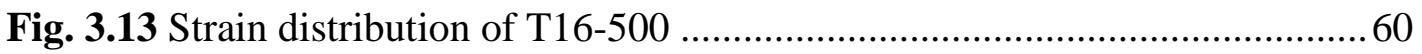

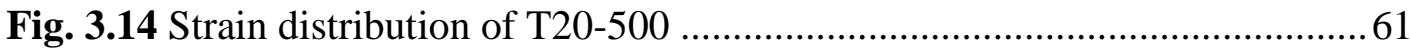

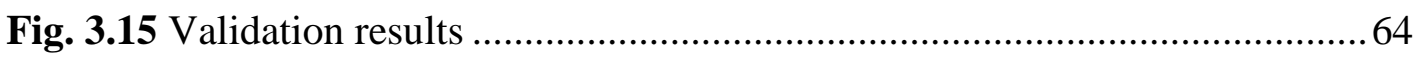

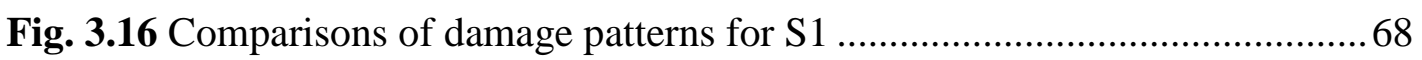

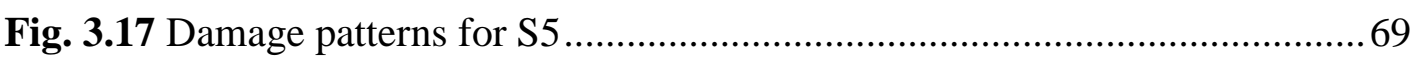

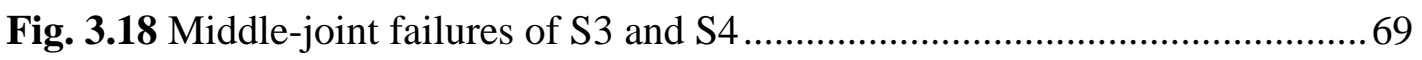

Fig. 3.19 Failures observed by FEM for S6 ..................................................... 70

Fig. 3.20 Time-history of a typical blast pressure ................................................ 72

Fig. 3.21 Different types of free-field explosion (UFC 3-340-02).........................73

Fig. 3.22 The exponentially narrowing envelop of displacement response with

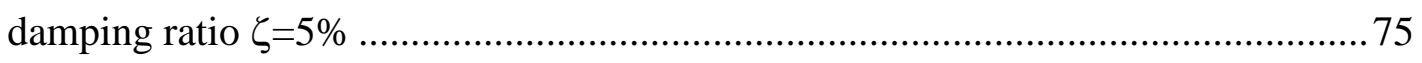

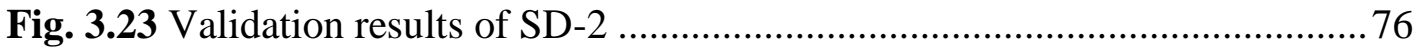

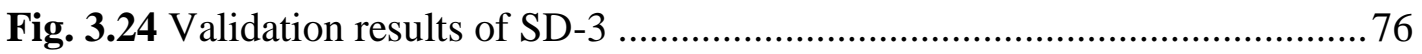


Fig. 3.25 Comparison of damage patterns of SD-3 …....................................... 77

Fig. 3.26 Damage patterns of middle-column ...................................................... 77

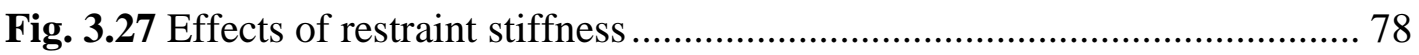

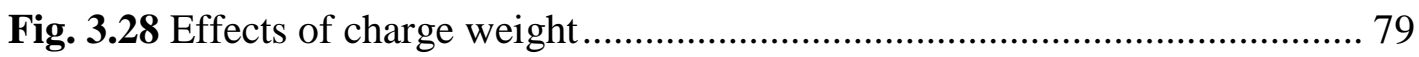

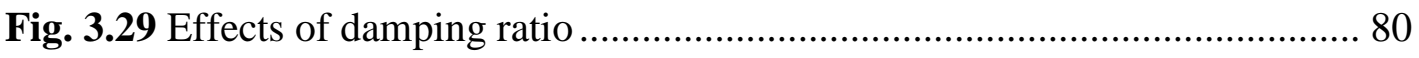

Fig. 3.30 Comparison of different analysis procedures ..................................... 81

Fig. 4.1 Simplified response of beam-column structures .................................... 86

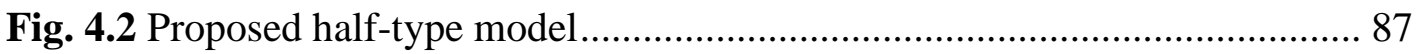

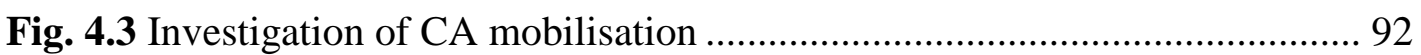

Fig. 4.4 Analytical model for compressive arch action (Yu and Tan 2014) ........... 94

Fig. 4.5 Proposed response and curvature distribution at point D......................... 96

Fig. 4.6 Proposed response and curvature distribution at point G......................... 98

Fig. 4.7 Special cases of the simplified model .................................................. 101

Fig. 4.8 Comparisons between simplified and FEM models ................................. 105

Fig. 4.9 Dynamic assessment for the simplified response.................................. 106

Fig. 4.10 Procedure of the simplified model ...................................................... 106

Fig. 4.11 Test validation using the proposed model ........................................... 108

Fig. 4.12 Combined response from two sides of the sub-assemblage .................. 114

Fig. 4.13 Example of sub-assemblage with two different sides ......................... 115

Fig. 4.14 Comparisons between FEM and simplified method for sub-assemblage

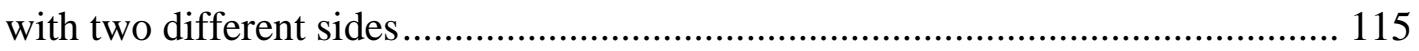

Fig. 5.1 Relationships between test series ....................................................... 121

Fig. 5.2 Prototype elevation view and locations of test specimens (Lim 2017) .... 123

Fig. 5.3 Specimen design of Series I .............................................................. 125

Fig. 5.4 Bending moment diagram of a 2D frame after column removal (Yu 2012)

Fig. 5.5 Test setup of full-restraint specimens from Series I............................. 125

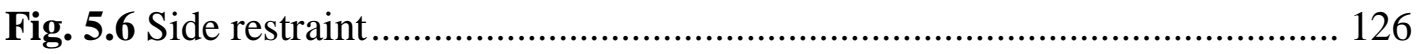

Fig. 5.7 Applied load and release mechanism from Series I ............................. 127

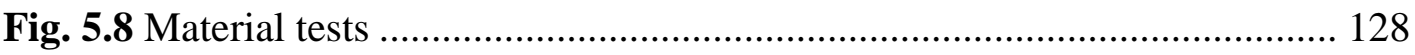

Fig. 5.9 Specimen design of Series II ......................................................... 129

Fig. 5.10 Comparisons between actual UDL and 4-point loading methods .......... 130 


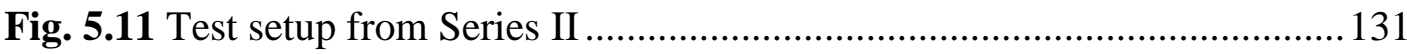

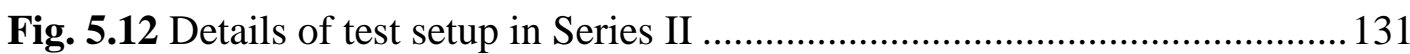

Fig. 5.13 Specimen design for full-restraint setup (Series III) ............................. 133

Fig. 5.14 Test setup of full-restraint specimen (Series III) .................................. 134

Fig. 5.15 Photo of partial-restraint specimen (Series III) .................................... 134

Fig. 5.16 Explosive charge and temporary support (Series III) ........................... 135

Fig. 5.17 Displacement transducers from Series I ............................................. 137

Fig. 5.18 Strain gauges from Series I ............................................................ 137

Fig. 5.19 Instrumentation of static tests from Series II ....................................... 138

Fig. 5.20 Displacement and reaction measurement from Series III ...................... 139

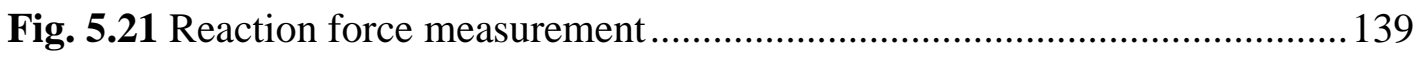

Fig. 5.22 Measurements of blast pressure ….................................................... 140

Fig. 6.1 Displacement time-history responses................................................. 144

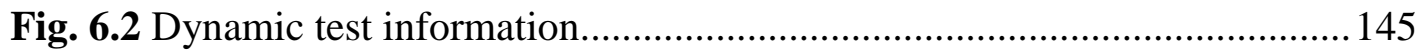

Fig. 6.3 Total vertical reaction time-history responses ....................................... 147

Fig. 6.4 Total horizontal reaction time-history responses (left side)...................... 147

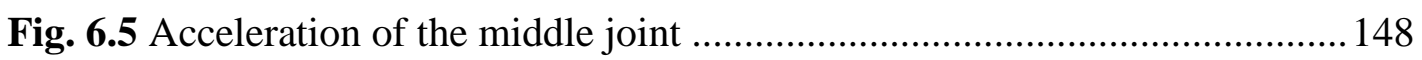

Fig. 6.6 Damage patterns and failure modes of the static and the dynamic tests... 149

Fig. 6.7 Reinforcement strains at end joint and middle joint .............................. 150

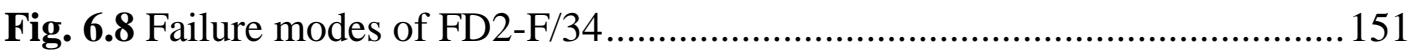

Fig. 6.9 Behaviour of partially restrained column in FD4-P/30.5 _....................... 152

Fig. 6.10 Comparisons between transducer readings and displacements from

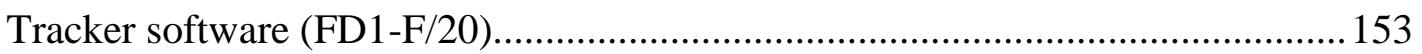

Fig. 6.11 Comparisons of displacement profiles for full-restraint specimens ....... 154

Fig. 6.12 Displacement profile of partially restrained side columns...................... 154

Fig. 6.13 Concrete strains and strain rates at middle and end joints of FD2-F/34.156

Fig. 6.14 Rebar strains and strain rates at Sections 1 and 4 of FD2-F/34 ............. 157

Fig. 6.15 Structural responses within flexure/CAA ............................................. 158

Fig. 6.16 Structural responses within CA …....................................................... 160

Fig. 6.17 Simplified model for RC beam-column frames .................................... 162

Fig. 6.18 Comparisons of beam reinforcement strains between static and dynamic

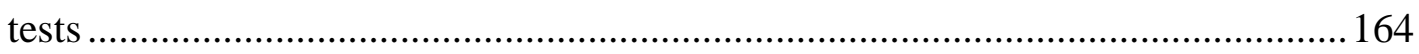


Fig. 6.19 Comparisons of column reinforcement strains between static and dynamic tests

Fig. 6.20 Comparisons between dynamic tests and pseudo-static responses from Izzuddin's method 166

Fig. 6.21 FEM models for 2D frame specimens ................................................ 167

Fig. 6.22 Validation results of FEM models with quasi-static tests .................... 168

Fig. 6.23 Failure modes in quasi-static models ................................................ 168

Fig. 6.24 Effects of column compression force .................................................. 169

Fig. 6.25 Validation results for FD1-F/20 .................................................... 170

Fig. 6.26 Validation results for FD1-F/29 ..................................................... 171

Fig. 6.27 Validation results for FD2-F/34 and FD4/P-30.5 ............................. 171

Fig. 6.28 Failure modes in FD4-P/30.5 model ................................................. 171

Fig. 6.29 Parametric studies for FD1-F/20 model .............................................. 172

Fig. 6.30 Parametric studies for FD1-F/29 model ............................................ 172

Fig. 6.31 The simplified model for dynamic response ....................................... 174

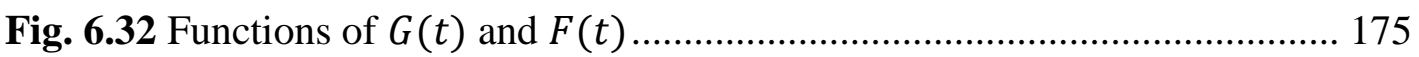

Fig. 6.33 Prediction from equivalent SDOF models ...................................... 175

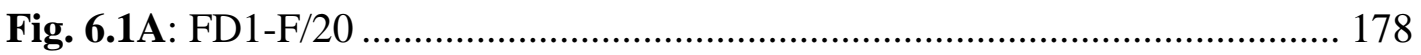

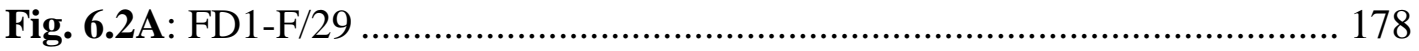

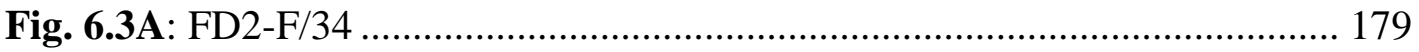

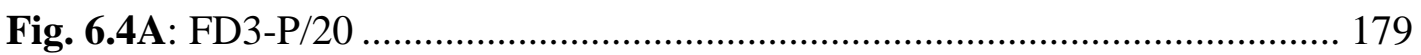

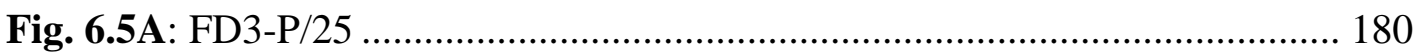

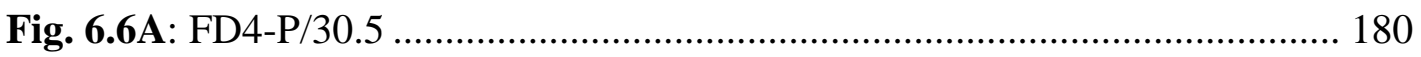

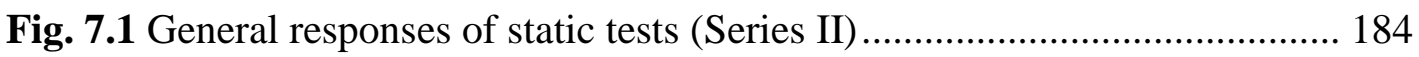

Fig. 7.2 Crack patterns during flexure and CAA stage ...................................... 187

Fig. 7.3 Tension zone development in static tests ............................................ 188

Fig. 7.4 Calculation of sectional bending moment $(\mathrm{kN}, \mathrm{m})$.............................. 189

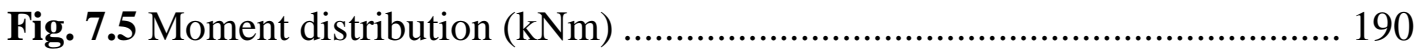

Fig. 7.6 Final failure modes of static tests ....................................................... 191

Fig. 7.7 Comparisons of rebar strains between CL and UDL static tests ............. 193

Fig. 7.8 Comparison of beam-end rotations at " 1 st top rebar fracture" ................. 194 
Fig. 7.9 Total horizontal reaction and rebar forces at the end joint at " 1 st top rebar fracture" state.

Fig. 7.10 Moment distribution at yielding point 195

Fig. 7.11 Force equilibrium at large deformations 196

Fig. 7.12 Displacement profiles under UDL 197

Fig. 7.13 Simplified CL and UDL responses of a sub-assemblage. 198

Fig. 7.14 Simplified UDL prediction of FR-U. 199

Fig. 7.15 General responses of UDL dynamic tests .200

Fig. 7.16 Structural reactions from UDL dynamic tests 201

Fig. 7.17 Damage patterns and failure modes of dynamic tests .203

Fig. 7.18 Structural responses of dynamic tests with load levels of 42 and 55 kN 205

Fig. 7.19 Structural responses of dynamic tests with $61 \mathrm{kN}$ .205

Fig. 7.20 Comparisons between pseudo-static predictions and actual dynamic tests

Fig. 7.1A: FR-U .211

Fig. 7.2A PR-U. .211

Fig. 7.3A FDU1-P/42 211

Fig. 7.4A FDU1-P/61 212

Fig. 7.5A FDU2-F/55 212

Fig. 7.6A FDU3-P/61 212

Fig. 8.1 Blast test procedure of CD1-P/25/M. .215

Fig. 8.2 Response of the middle joint (CD1-P/25/M) 215

Fig. 8.3 Damages and failure modes of CD1-P/25/M .216

Fig. 8.4 Damages at the bottom of partially-restrained column. 217

Fig. 8.5 Blast pressures on beam and column 218

Fig. 8.6 Horizontal reaction (upper right side) 219

Fig. 8.7 Horizontal displacement (left-side end joint). 219

Fig. 8.8 Bottom reinforcement strains (CD1-P/25/M) 220

Fig. 8.9 Reinforcement strains and strain rates 221

Fig. 8.10 Response of the middle joint (CD2-F/20/M) .....................................222

Fig. 8.11 Damages and failure modes of CD2-F/20/M......................................222

Fig. 8.12 Damages of middle column 223 
Fig. 8.13 Horizontal reaction 224

Fig. 8.14 Concrete and reinforcement strains 224

Fig. 8.15 Validations of Izzuddin's method with blast tests. .226

Fig. 8.16 FEM model for blast test validation (full-restraint) 227

Fig. 8.17 Validation of blast incident pressure using CONWEP data (Pc for column pressure and $\mathrm{Pb}$ for beam pressure) 228

Fig. 8.18 Distribution of incident pressure $\left(\mathrm{N} / \mathrm{m}^{2}\right)$ 228

Fig. 8.19 Validation results for blast tests 229

Fig. 8.20 Damages and failure modes of CD1 model. 230

Fig. 8.21 Damage patterns of CD2 model 230

Fig. 9.1 Test setup (Qian et al. 2014) 235

Fig. 9.2 Horizontal support of the system 237

Fig. 9.3 FEM models 237

Fig. 9.4 Test validation results of beam-only structures 238

Fig. 9.5 Damage patterns of beam-only structures 238

Fig. 9.6 Test validation results of $\mathrm{S} 2$ 239

Fig. 9.7 Damage patterns of S2 239

Fig. 9.8 Equivalent UDL by multiple-point loading systems 241

Fig. 9.9 Comparisons between true UDL force-controlled and equivalent multiplepoint displacement-controlled methods 241

Fig. 9.10 Responses of slab-only structures 243

Fig. 9.11 Yield-line patterns of slab-only structures 244

Fig. 9.12 Failure modes of slab-only structures 244

Fig. 9.13 Yielding of slab-only rebars at $55 \mathrm{~mm}$ deflection (1.0dslab) 245

Fig. 9.14 Responses of beam-slab structures .................................................. 246

Fig. 9.15 Failure modes of beam-slab structures .............................................. 246

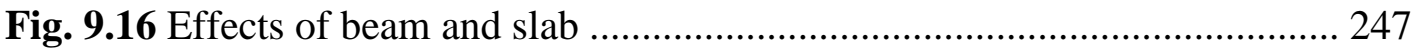

Fig. 9.17 S2 models for restraint stiffness studies ............................................ 248

Fig. 9.18 Effects of horizontal restraint stiffness on beam-slab structures ........... 248

Fig. 9.19 Effects of rotational restraint on beam-slab structures ......................... 249

Fig. 9.20 Progressive collapse scenarios of multi-storey buildings..................... 251

Fig. 9.21 Structural responses from different column loss scenarios ................... 252 
Fig. 9.22 Rebar yielding at $55 \mathrm{~mm}$ deflection of internal- and corner-column cases 253

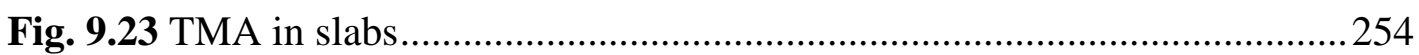

Fig. 9.24 Rebar yielding at $55 \mathrm{~mm}$ deflection of PI- and PE-column cases ..........255

Fig. 9.25 Simplified response of RC beam-slab systems ...................................257

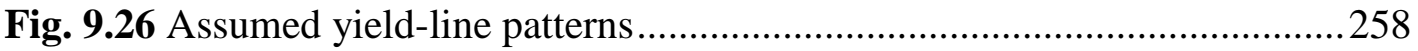

Fig. 9.27 Distribution of load to internal beams under UDL case .......................260

Fig. 9.28 Yielding forces of top rebars at negative yield lines............................263

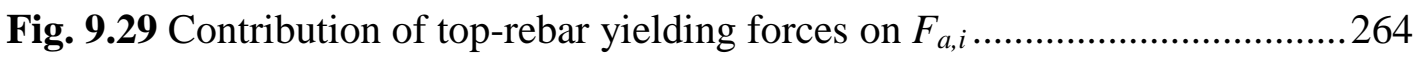

Fig. 9.30 Ideal scenario of isotropically-reinforced square slab .........................265

Fig. 9.31 Conversion of actual orthotropic slab into isotropic slab ......................266

Fig. 9.32 Distribution of slab top-rebar yielding force (UDL).............................267

Fig. 9.33 Dynamic capacity based on the simplified model ...............................269

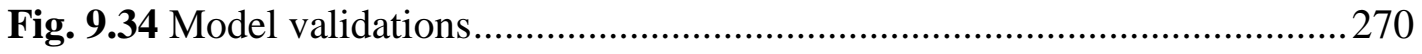

Fig. 9.35 Dynamic responses of the slab structure .............................................273

Fig. 9.36 Effects of blast loading at maximum uplift deformation ........................2274 


\section{LIST OF SYMBOLS}

\section{CHAPTER 4}

$A_{c} \quad$ sectional area of concrete

$A_{s} \quad$ sectional area of total longitudinal rebars at the end joint

$A_{s}^{\text {top }} \quad$ sectional area of top longitudinal rebars in the beam near the end joint

$A \quad$ area of beam section, $A=A_{c}+A_{s}$

$d_{A}, d_{B}, d_{C_{1}}, d_{C}, d_{D_{1}}, d_{D}, d_{E}, d_{F}, d_{G} \quad$ displacements at points A, B, C 1, C, D $, ~ D, E$, F, G

$d_{\text {beam }} \quad$ concrete beam depth

$d \quad$ distance from extreme compression fibre of concrete to tension reinforcement

$d^{\prime} \quad$ distance from extreme compression fibre of concrete to compression reinforcement

$d_{\text {mid }}, d_{\text {end }} \quad$ diameters of tensile rebars at the middle and the end joints

$E_{c} \quad$ elastic modulus of concrete, $E_{c}=4.7 \sqrt{f_{c k}} \mathrm{GPa}$

$E_{S} \quad$ elastic modulus of longitudinal rebars

$F_{\text {top }}^{y} \quad$ yield force of the top reinforcement, $F_{\text {top }}^{y}=f_{y} A_{s}^{\text {top }}$

$F_{t o p}^{u} \quad$ ultimate tensile force of the top reinforcement, $F_{t o p}^{u}=f_{u} A_{s}^{\text {top }}$

$f_{c k} \quad$ concrete compressive strength

$f_{y} \quad$ yield strength of longitudinal rebars

$f_{u} \quad$ ultimate strength of longitudinal rebars

$H, H_{\max } \quad$ horizontal reaction and maximum horizontal reaction of the subassemblage 
$I_{g} \quad$ moment of inertial of the gross section area

$K_{A} \quad$ horizontal spring of the end-joint restraint

$K_{R} \quad$ rotational spring of the end-joint restraint

$k_{\text {span }} \quad$ span-depth ratio

$k_{\text {hor }} \quad$ normalised horizontal stiffness

$k_{\text {flex }} \quad$ relative bending stiffness

$k_{\text {topbar }} \quad$ steel-to-concrete stiffness ratio

$k_{\text {rot }} \quad$ normalised rotational stiffness

$L_{n} \quad$ clear span of the single beam

$L_{n}^{\text {mid }}, L_{n}^{\text {end }} \quad$ equivalent cantilever spans

$L_{s p} \quad$ plastic hinge length at the end joint (for calculation of $d_{G}$ )

$L_{s p}^{\text {mid }}, L_{s p}^{e n d} \quad$ plastic hinge lengths at the middle and the end joints (for calculation of $\left.d_{D}\right)$

$M_{\text {mid }}, M_{\text {end }} \quad$ sagging moment capacities of beam section near the middle joint and hogging moment capacity of beam section near the end joint

$P_{A}, P_{B}, P_{C_{1}}, P_{C}, P_{D_{1}}, P_{D}, P_{E}, P_{F}, P_{G} \quad$ applied loads at points $\mathrm{A}, \mathrm{B}, \mathrm{C}_{1}, \mathrm{C}, \mathrm{D}_{1}, \mathrm{D}, \mathrm{E}$, F, G

$P_{B}^{d}, P_{D}^{d}, P_{G}^{d} \quad$ equivalent dynamic loads at displacements $d_{B}, d_{D}, d_{G}$

$P_{\text {left }}, P_{\text {right }} \quad$ simplified responses of the "left" and the "right" systems

$P_{\text {total }} \quad$ simplified response of the total system

$R_{A} \quad$ capacity of the horizontal restraint

$\alpha_{1}, \alpha_{2}, \beta_{1}, \beta_{2}$ variables of the proposed model for sub-assemblages

$\Delta_{X Y}, \Delta_{Y Z} \quad$ contributions of beam deflection within segments $X Y$ and $Y Z$.

$\Delta_{u}^{m i d} \quad$ beam deformation caused by ultimate curvature at plastic hinge (segment $X Y$ ) 
$\Delta_{y}^{\text {mid }} \quad$ beam deformation caused by linear distribution of curvature along segment $X Y$

$\Delta_{c} \quad$ beam deformation caused by constant yield curvature at curtailment region

$\Delta_{u} \quad$ beam deformation caused by ultimate curvature at plastic hinge

$\Delta_{y} \quad$ beam deformation caused by linear distribution of curvature along the beam

$\phi_{y}, \phi_{u} \quad$ yield and ultimate curvatures of concrete beam section at the end joint under hogging moment

$\phi_{y}^{\prime}, \phi_{u}^{\prime} \quad$ yield and ultimate curvatures of concrete beam section at the middle joint under sagging moment

$\phi_{y}^{c} \quad$ yield curvature of concrete beam section at the curtailment region under hogging moment

\section{CHAPTER 7}

$d_{D^{\prime}} \quad$ displacement at point $D^{\prime}$

$F a_{\text {top }}, F a_{\text {bot }}$ resultant forces from top and bottom reinforcement at Section 1

$F_{c} \quad$ resultant force of compressive concrete region at beam bottom surface (Section 1)

$H_{C L}, H_{U D L} \quad$ horizontal reactions of beam-column structures subjected to CL and UDL

$P_{C L}, P_{U D L} \quad$ total applied loads of beam-column structures subjected to CL and UDL

$P_{A \prime}, P_{B^{\prime}}, P_{D^{\prime}} \quad$ applied loads at points $\mathrm{A}^{\prime}, \mathrm{B}^{\prime}, \mathrm{D}^{\prime}$

$\theta_{\max } \quad$ beam-end rotation at the state of " 1 st top rebar fracture"

$\delta_{C L}, \delta_{U D L} \quad$ middle-joint displacements of beam-column structures subjected to CL and UDL 


\section{CHAPTER 9}

$A_{S} \quad$ reinforcement area per unit length of slab

$m_{s}, m_{s}^{\prime} \quad$ positive and negative moment capacities per unit width of slab

$d_{S, A}, d_{S, B} \quad$ displacements at points A, B

$d_{s} \quad$ effective depth of slab

D slab stiffness coefficient

E elastic modulus of concrete

$F_{a, i} \quad$ tensile force per unit width along $\delta i$

$F_{a}^{x}, F_{a}^{y} \quad$ yield forces per unit width of slab top reinforcement along x- and ydirections

$I_{x}, I_{y} \quad$ moments of inertial of concrete sections from Beam X and Beam Y

$K_{\text {beam }}^{x}, K_{\text {beam }}^{y}$ structural stiffness of Beam X and Beam Y

$K_{\text {slab }} \quad$ structural stiffness of slab-only part

$K_{O A}, K_{A B} \quad$ beam-slab structural stiffness at parts $\mathrm{OA}$ and $\mathrm{AB}$

$L_{x}, L_{y} \quad$ clear spans in $\mathrm{x}$ - and y-directions of the beam-slab structure

$L_{n}^{x}, L_{n}^{y} \quad$ clear spans of original internal beams in $\mathrm{x}$ - and y-directions

$M_{b, x}, M_{b, x}^{\prime}$ positive and negative bending capacities of internal beams in $\mathrm{x}$ direction $($ Beam $\mathrm{X})$

$M_{b, y}, M_{b, y}^{\prime} \quad$ positive and negative bending capacities of internal beams in $y$ direction (Beam Y)

$m_{s x}, m_{s x}^{\prime} \quad$ positive and negative bending capacities per unit width of slab in $\mathrm{x}-$ direction

$m_{s y}, m_{s y}^{\prime} \quad$ positive and negative bending capacities per unit width of slab in ydirection

$n \quad$ slab aspect ratio 
$P_{c l}^{s l a b}, P_{u d l}^{\text {slab }}$ total applied loads of slab under CL and UDL (yield-line method)

$P_{S, A}, P_{S, B} \quad$ applied loads at points A, B

$P_{x}, P_{y} \quad$ total applied loads on Beam $\mathrm{X}$ and Beam Y (UDL case)

$P_{d y n} \quad$ maximum dynamic load of beam-slab system

$Q_{x}, Q_{y} \quad$ applied loads per unit length on Beam X and Beam Y (UDL case)

$t_{s} \quad$ slab thickness

$\alpha \quad$ dimensionless factor of slab

$\delta i \quad$ arbitrary length along the negative-moment yield line of slab

$\delta d \quad$ deflection increment of beam-slab system

$\delta P \quad$ load increment of beam-slab system

$\delta P_{i} \quad$ increment of vertical applied load caused by tensile forces along $\delta i$

$v \quad$ Poisson's factor of concrete

$\mu \quad$ slab converting factor 


\section{CHAPTER 1 INTRODUCTION}

\subsection{Background}

With increasing risks from terrorist attacks to governmental and public facilities nowadays, there is growing importance to protect structures from such fatal incidents, including progressive collapse. In this connection, the American Society of Civil Engineering (ASCE) Standard ASCE 7-10 (2010) defines progressive collapse as "the spread of local damage, from an initiating event, from element to element resulting, eventually, in the collapse of an entire structure or a disproportionately large part of it". It was first coined after the collapse of Ronan Point Apartment in May 1968, East London (Fig. 1.1). The incident was triggered by a gas explosion in a corner flat on the $18^{\text {th }}$ floor, causing the sudden collapse of an external bearing wall which was the main supporting element for the flats above. It was the first time when progressive collapse was assessed as a critical issue for structural design under abnormal loads such as impact or explosion. In the following decades, provisions and requirements have been implemented into the codes of practice for structural design to prevent occurrences of such fatal incidents, firstly in the British Standards and then in other international standards such as EN 1991-1-7 (2006) and ACI 318-11.

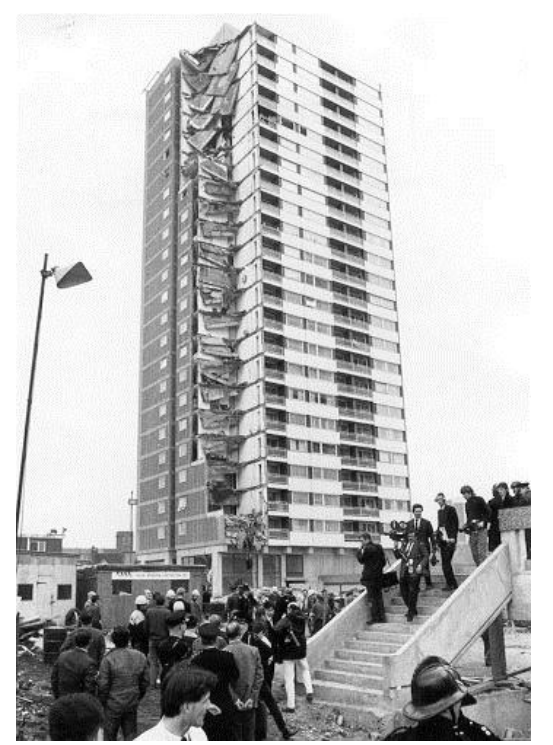

Fig. 1.1 Disproportionate collapse of Ronan Point Apartment (1968) 
In the early 1990s, the threat from terrorist attacks using deadly weapons and explosives started raising serious concerns on the design approach to protect buildings from progressive collapse initiated by localised damages due to explosives. The partial failure of Murrah Federal Building in April 1995 due to a car bomb and the tragic collapse of the twin towers of World Trade Center in September 2001 were classic examples of terrorist threats (Fig. 1.2).

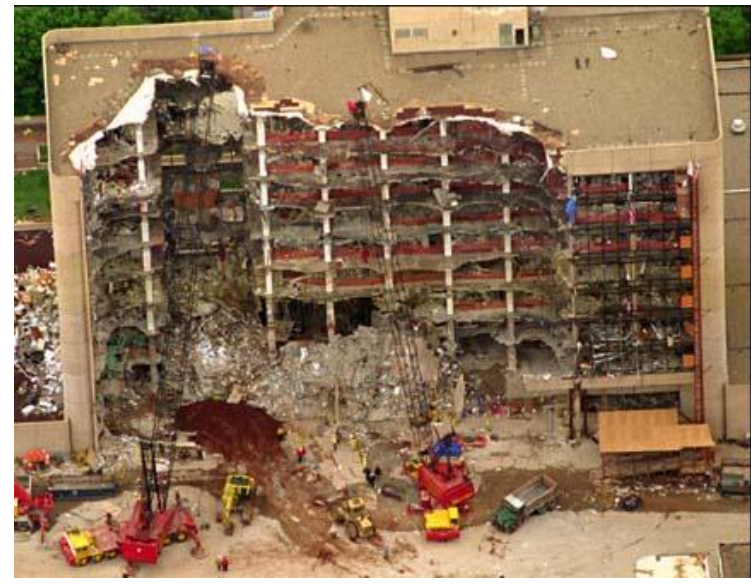

a) Murray Federal Building collapse (1995)

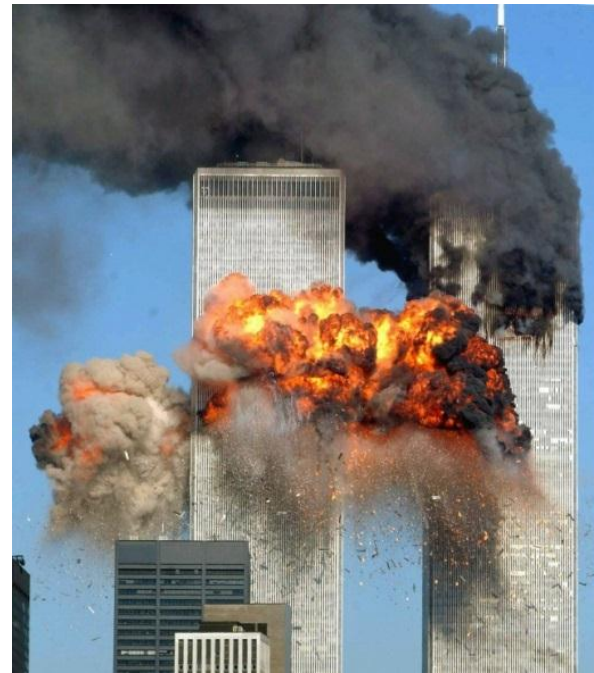

b) World Trade Center second hit (2001)

Fig. 1.2 Iconic events of progressive collapse

To date, public awareness on progressive collapse of buildings has been raised. The U.S Government has issued several guidelines to counter this threat. Among them, the General Service Administration GSA (2003) and the Department of Defence UFC 4-023-03 (DOD 2013) were drafted for governmental buildings and important facilities to resist disproportionate collapse. These guidelines include the requirements for different levels of importance, as well as to provide some methods for analysis and design of building structures against extreme loading conditions. Within the current standards and guidelines, the most commonly-used approaches are the indirect and the direct methods (Ellingwood and Leyendecker 1978). The indirect method requires a certain level of tie forces between all members of the structure to ensure mobilisation of catenary or tensile membrane action, as well as to enhance structural continuity and ductility. It was first implemented into the 
British codes after the collapse of Ronan Point Apartment and has been employed in EN 1991-1-7 and DOD (2013). However, no structural analysis is required for this method. On the other hand, the direct method analyses structural response under abnormal loading events. This approach includes two types of procedure, namely, the Alternate Load Path (ALP) and the Specific Local Resistance (SLR) design methods. While the former allows a localised damage and analyses the remaining structure to prevent this failure from propagating, the latter focuses on the design of key members in the structure to survive a specific threat.

The ALP method is based on the assumption of a sudden column loss, in which a load-bearing column or a load-bearing wall is forcibly removed due to an extreme event like blast or impact, and the remaining structure is analysed if it can develop double-span bridging behaviour and if there is adequate horizontal restraint for that part of the affected structure. However, in ALP method, the origin of the threat causing the column removal is not required. Therefore, this threat-independent design approach is widely accepted in both practical design and scientific studies for assessing the progressive collapse resistance of a structure. As the main members to resist disproportionate collapse in reinforced concrete (RC) buildings, beam-column substructures have been extensively investigated both experimentally and numerically (Izzuddin and Elghazouli 2004, Sasani and Kropelnicki 2008, Yi et al. 2008, Su et al. 2009, Valipour and Foster 2010, Bao et al. 2014). It was shown that, besides flexural behaviour, catenary action (CA) is a promising alternate mechanism to withstand collapse under larger deformations. It is normally activated when the central displacement exceeds one beam depth. Quasi-static tests show that after the fracture of bottom rebars at the middle joint, structural response undergoes a sudden drop. As the double-span structure keeps moving downwards, the loadcarrying capacity may increase again until fracture of the remaining top rebars occurs (Sasani and Kropelnicki 2008, Yu and Tan 2013a, Lim et al. 2015). However, most experimental studies focused on structural behaviour under quasistatic condition applying a concentrated loading (CL) method due to simplicity and expediency in the test setup. In this method, a quasi-static force is applied onto the middle joint of the double-span structure, causing this joint to move downwards 
under a displacement-controlled manner. Therefore, behaviour of beam-column structures under uniformly distributed loading (UDL) condition, which is closer to reality, has yet to be investigated. Moreover, as progressive collapse is a dynamic event, experimental programmes of structural behaviour under dynamic conditions, especially the mobilisation of CA before and after the fracture of bottom rebars occurs, are of great importance. To date, there are very few such studies.

When applying ALP approach to mitigate progressive collapse, structural behaviour is usually analysed using linear or nonlinear static methods. Dynamic effects are considered by adopting simplified approaches such as Dynamic Increase Factor in DOD (2013), or the energy method proposed by Izzuddin et al. (2008). Although these approaches are computationally efficient, their validity has yet to be demonstrated through experimental tests, in particular, when CA is mobilised. Hence, actual dynamic tests are required to confirm the validity of the simplified methods using nonlinear static procedure.

On the other hand, although it is convenient to apply column removal design scenarios, the shortcoming is that the approach does not consider the initial state of damage of remaining structural elements (Krauthammer 2003). It is assumed that the load-bearing capacities of such members remain unchanged after the supporting column has been removed. Nevertheless, realistic events of explosive attacks from man-made hazards clearly indicate high potential of disproportionate collapse after several structural members are severely damaged by a blast incident. Hence, this column removal scenario may lead to an unsafe design of structural members. Indeed, the damage state of surviving structures should be evaluated when assessing progressive collapse potential triggered by explosion. Therefore, measuring the blast effect on remaining structures during a blast-induced column loss event is of practical importance.

Similar to CA in beams, tensile membrane action (TMA) in RC slabs is shown to increase significantly the overall structural capacity under large deformations (Park 1964, Bailey et al. 2000, Foster et al. 2004). Mobilisation of this mechanism is 
denoted by the formation of a compressive ring at the slab edges and a tensile net at the middle area. Experimental studies on beam-slab structures under single column removal scenarios usually showed that the load-bearing capacity was larger than the predictions based on plastic-hinge and yield-line theories (Pham and Tan 2013, Qian et al. 2014, Lu et al. 2016). However, such studies did not distinguish the contribution of TMA in slabs from CA in beams. As a result, mobilisation and capacity of TMA have not yet been investigated quantitatively. The influence of translational and rotational restraint stiffness on TMA in the beam-slab system has not yet been investigated. Besides, previous studies on beam-slab structures considered corner-column loss scenario as the most critical situation since both CA and TMA are believed to be limited. However, due to the complexity and cost constraint of beam-slab structural tests, there is no existing experimental data considering all the possible locations of column loss to verify this finding.

\subsection{Objectives of research}

From the shortcomings of previous studies on column removal scenario and the simplified dynamic methods, as well as the need to quantify the contribution of upper-bound tensile mechanisms such as CA or TMA, a research programme is designed and presented in this study. The scope is mostly confined to the behaviour of two-dimensional (2D) RC beam-column structures under progressive collapse event initiated by sudden column removal from accidental/terrorist attacks. Three series of experiments on 2D RC frames will be carried out, together with the development of numerical and simplified analyses. Moreover, three-dimensional (3D) beam-slab systems are also studied. The objectives are listed as follows:

(1) To gain an overall understanding of RC behaviour under the single column removal event, physics-based numerical analyses will be conducted for $2 \mathrm{D}$ subassemblages and validated under both quasi-static and dynamic blast-induced conditions. Based on numerical predictions and published test data, a semianalytical model will be proposed focusing on the development of CA before and after the fracture of bottom rebars at middle-joint interfaces. The simplified 
model will provide a quick tool to designers for preliminary sizing against progressive collapse.

(2) To compare and contrast the behaviour and failure modes of 2D beam-column structures subject to CL quasi-static condition, a series of six free-fall dynamic tests on 2D beam-column frames will be conducted applying CL condition (Series I). Results will be compared with the quasi-static tests from a related study (Lim et al. 2015) and dynamic effects will also be investigated. Actual test results are also compared with predictions from the simplified method for dynamic assessment proposed by Izzuddin et al. (2008) to evaluate the usefulness and conservatism of this approach.

(3) To fill the gap of knowledge on beam-column structural behaviour under progressive collapse subjected to distributed loads which is more common in reality, a series of two quasi-static and four free-fall dynamic tests (Series II) will be conducted. Based on experimental static results, an analytical relationship of behaviours between CL and UDL conditions will then be developed. Besides, the dynamic tests will confirm the use of nonlinear static analysis for beam-column frames subjected to UDL.

(4) To evaluate effects of initial blast on structural responses under contact detonation, two close-in blast tests on RC beam-column frames applying CL method (Series III) will be conducted. By comparing the actual blast test results to the free-fall dynamic tests (Series I), and to analytical predictions based on quasi-static responses, limitations of the single column removal assumption and Izzuddin's method on dynamic assessment will be addressed.

(5) Besides the studies on 2D sub-assemblages and 2D beam-column frames, an investigation on $3 \mathrm{D}$ beam-slab systems will be carried out focusing on the contribution of TMA in slabs to the overall capacity of the substructure. A simplified analytical model will also be proposed for RC beam-slab structures under internal-column loss scenario, taking into account TMA. 


\subsection{Layout of the thesis}

The layout of this thesis, including the objectives labelled from (1) to (5), is presented in Fig. 1.3. The thesis includes ten chapters. This first chapter introduces the background of research as well as the motivations to conduct realistic dynamic studies on progressive collapse initiated by single column loss events. The objectives are presented.

In Chapter 2, an extensive literature review of progressive collapse studies on RC structures is carried out. The direct method for progressive collapse resistance, based on ALP approach, is discussed. An overview of experimental and analytical studies on RC beam-column structures against single column removal, from quasistatic to free-fall dynamic and actual blast conditions, is presented to point out technical gaps in research. Finally, studies on beam-slab structures under progressive collapse are also reviewed.

In Chapter 3, numerical analyses using general-purpose finite element software, LSDyna, are conducted and validated by some reported progressive collapse experiments on RC structures. Modelling issues such as material constitutive model, erosion criteria, bond-slip behaviour between concrete and reinforcement, blast loading simulation, are addressed. Parametric studies are then performed in Chapter 4 to investigate the effects of significant factors on CA development. Thereafter, a semi-analytical approach is proposed to predict static behaviour of sub-assemblages under CL condition taking into account CA.

Chapter 5 presents an experimental programme for 2D RC frames against progressive collapse. Three test series, namely, Series I, II and III, are proposed. Objectives, specimen design, test setup, as well as instrumentation system, are introduced. The specimens are inspired by a related quasi-static test programme on RC frame structures from Lim et al. (2015). Results of Series I on free-fall dynamic tests applying CL condition is discussed in Chapter 6 at both structural and crosssectional levels. 
Chapter 7 presents the investigation of quasi-static and free-fall dynamic tests under UDL condition from Series II. Chapter 8 discusses the blast-test results of Series III, which employ similar boundary condition and applied loads with the free-fall tests of Series I.

In Chapter 9, a numerical investigation is carried out to study the effect of TMA in a combined RC beam-slab system. A simplified analytical approach is also proposed to predict structural response of beam-slab structures under internalcolumn removal.

Finally, conclusions of the research work as well as recommendations for future work are presented in Chapter 10.

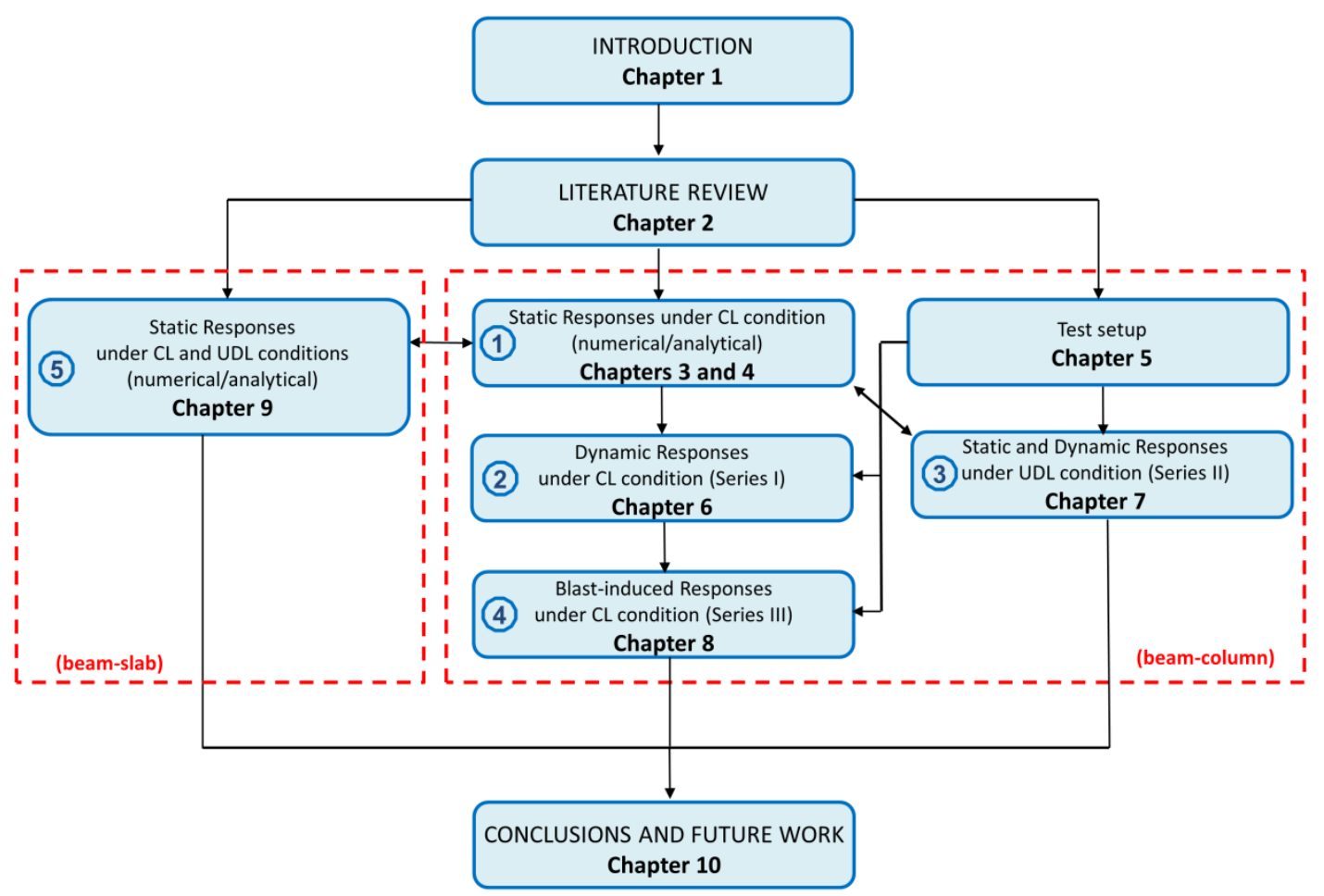

Fig. 1.3 Thesis layout 


\section{CHAPTER 2 LITERATURE REVIEW}

\subsection{Introduction}

In the first chapter, design procedures on mitigating progressive collapse are briefly presented. In this chapter, provisions and methodologies on structural analysis and design to resist this extreme event are illustrated more clearly by reviewing current standards and design guides for abnormal loading. The review focuses on the Alternate Load Path method which employs the assumption of single column removal to simulate a progressive collapse incident. Research work related to RC beam-column structures under quasi-static, free-fall dynamic, as well as actual blast environments, is considered. Thereafter, current studies on beam-slab systems under progressive collapse are also mentioned. The purpose of the literature review is to identify technical gaps in the subject area and to develop the scope of work for the current study.

\subsection{Design methods for mitigating progressive collapse}

To date, most standards or design guidelines related to progressive collapse resistance are based on two methods, namely, indirect and direct methods proposed by Ellingwood and Leyendecker (1978). The former is a prescriptive approach which requires a minimum level of connectivity between different structural members, whereas the latter is a performance-based method analysing structural response under a specific threat. The provisions on these two approaches can be found in DOD (2013) design guide as well as ASCE 7-10 (2010) standard.

\subsubsection{Indirect method}

Basically, the indirect method requires a certain level of continuity and integrity within structural members. This approach was developed after the event of Ronan Point Apartment collapse, in which the connections between precast concrete members were damaged after supporting precast wall panels were blown away by a gas explosion. Provisions of this tie force method according to some popular standards/guidelines are reviewed below: 
ASCE 7-10 provides a general provision (part 1.4) which requires all structures and buildings to have sufficient integrity to resist local damages and prevent disproportionate collapse. However, it does not give any quantitative clauses on how to achieve such level of continuity and integrity.

BS 8110:1997, on the other hand, requires reinforcement design for notional tie forces to satisfy the integrity demand of RC structures. The tying reinforcement has to be calculated for all peripheral, internal, horizontal and vertical ties. The value of notional tie forces is clearly defined. Such requirements are also specified in EN 1991-1-7 (2006).

In DOD (2013), the indirect method is explicitly prescribed through provisions of tie forces. The application of this approach is based on different categories of occupancy which are linked to the importance of buildings and the effects of progressive collapse to potential loss of human life. DOD (2013) describes a minimum requirement of tie force that structural members need to satisfy so that sufficient alternate paths for load redistribution can be created in case of supporting structures damaged by sudden overloading situations. Suffice to say, the tie force method relies on the mobilisation of tensile mechanisms to redistribute the applied loads after some structural members are damaged by accidental/terrorist events. Adequate internal, peripheral and vertical ties shall be provided for all load-bearing elements, as shown in Fig. 2.1. For internal ties, the tie strength $F_{1}$ in the longitudinal or transverse direction is equal to $3 w_{F} L_{1}$ where $w_{F}$ is the floor load and $L_{1}$ is the larger span of the slab. For peripheral tie $F_{P}$, it is computed as $6 w_{F} L_{1} L_{p}+$ $3 W_{c}$ with $L_{p}$ equal to $1.0 \mathrm{~m}$ and $W_{c}$ equal to 1.2 times of cladding dead loads. Regarding vertical ties, they must have tensile strength equal to the largest vertical load applied on the column from any storey based on the floor load $w_{F}$.

The purpose of the tie force requirements from the indirect method is to ensure reinforcement continuity between members inside a structure, confirming that all of them are tied together both vertically and horizontally. By providing tie force members in this manner, it helps to increase redundancy, ductility, capacity and 
improve structural robustness. So long as the integrity of the building is ensured, threats from localised damages can be reduced.

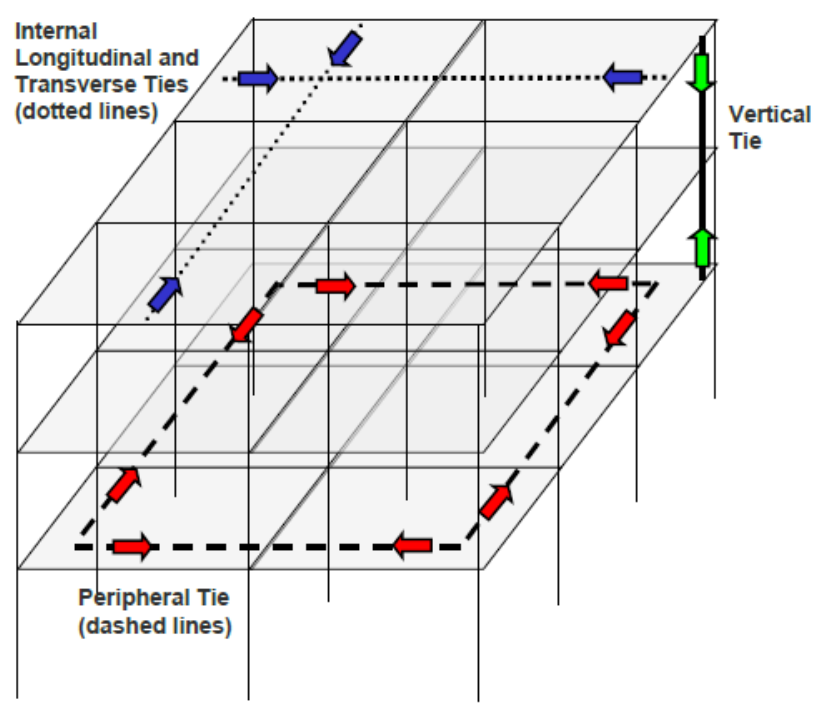

Fig. 2.1 Tie requirement for building structures (DOD 2013)

\subsubsection{Direct method}

By satisfying some notional values of tie force with little effort of calculation and without any structural analysis, applying the indirect method is deemed to have met the requirements of structural integrity. However, the actual effect of this prescriptive approach has not yet been verified in a realistic collapse scenario. On the other hand, the direct method, which is performance-based, requires extensive calculations for structural strength and capacity under specific cases of abnormal loads. In this method, the progressive collapse potential is evaluated by analysing structural members or systems under an initiating threat or damage. According to ASCE 7-10 and DOD (2013), two forms of direct method shall be applied for progressive collapse resistance, viz. Alternate Load Path (ALP) and Specific Load Resistance (SLR) methods.

\section{ALP method}

DOD (2013) specifies that the ALP method is used when a structural design cannot satisfy the requirements of vertical tie forces, or when the occupancy category of the building is type III or IV. This method is based on an assumption, called the 
column removal scenario, in which a vertical load-bearing component is notionally removed from the system. The remaining structure must be able to bridge over this item to prevent collapse propagation. Both the ALP and the Tie force methods can be considered as threat-independent because it is not necessary to define the origin of the incident causing the removal of vertical bearing components. The damage caused by an initial event to the remaining structural components is therefore neglected. Besides, both methods provide robustness and resistance to structures against some localised damages. In DOD guideline, ALP is the most important method for mitigating progressive collapse. Analysis procedures, as well as acceptance criteria of the ALP method, are included in this document. Three types of analysis procedure are mentioned, including linear static, nonlinear static and nonlinear dynamic. The idea of ALP is also mentioned in BS 8110:1997 and EN 1991-1-7, which state the requirement for design of bridging elements under the column removal scenario.

\section{SLR method}

Compared to the ALP method which requires multiple analyses of the global structure through the removal of key elements one at a time, the SLR method necessitates the key members to resist a specific blast loading condition. The main idea is to eliminate the cause of progressive collapse by providing a sufficient level of safety to structural members under the specific scenarios analysed. If the initial cause of extreme loads is a blast event, then relevant manuals on structural member design against explosion can be applied. The U.S Army handbook TM5-1300 (1990) and the new version UFC 3-340-02 (2008) have been widely used for component design to resist blast effects. These guidelines are based on semiempirical approaches as well as test data collected from blast trials throughout several decades.

Since SLR is a threat-dependent approach, it requires the engineer to define a specific loading scenario to have a cost-efficient design, but such threat information can be used by terrorists to plan an attack on the structure. Therefore, this kind of information should be kept classified and confidential. However, in DOD (2013) 
(and also in BS 8110), the SLR is still considered as threat-independent, in which a notional value of extreme load, or a notional level of safety factor for flexure or shear resistance, is proposed. In BS 8110:1997, both the key member and its connections must withstand an ultimate load of $34 \mathrm{kN} / \mathrm{m}^{2}$. In DOD (2013), SLR analysis for Occupancy category IV requires a safety factor of 2 for flexural resistance of columns and 1.5 for load-bearing walls. Indeed, SLR is aimed to improve load resistance of perimeter columns and walls on the first and second storeys of a building.

Compared to SLR, ALP employing the single column removal assumption is more convenient for assessing progressive collapse potential of RC structures because it can be applied to any type of events without characterising the original threat. In the following sections, experimental and analytical work regarding the two most important RC substructures, i.e. the beam-column and the beam-slab systems, is reviewed to find out technical gaps of existing research.

\subsection{Studies on Alternate Load Paths of beam-column structures}

In an RC building with conventional design, the most important members to support gravity loads are the beams and columns. The beam system must be able to carry vertical loads from slabs and other non-structural features and to contribute to the continuity and stability of the building. Likewise, the columns have to transfer both vertical and lateral loads to foundations. Together with the shear wall system, the columns ensure horizontal stiffness and stability of the structure. In the event of extreme loads, the beams and columns play a significant role in maintaining the integrity of the entire structure and preventing disproportionate collapse. Hence, most of the studies on the ALP method for concrete structures focus on the behaviour of beam-column sub-assemblages and beam-column frames.

Basically, when an RC beam-column joint undergoes downward movements after the supporting column is removed, flexural mechanism will first be mobilised in beams at small deformations. If the beams are provided with adequate lateral restraint, compressive arch action (CAA) can enhance the ultimate moment capacity 
by mobilising axial compression (Fig. 2.2(a)). After which, if the displacement of the joint (at the location with the column removed) keeps increasing to a value equal to one beam depth, then catenary action (CA) may take place (Fig. 2.2(b)). This mechanism is considered as the last line of defence to prevent a progressive collapse event (Sasani and Kropelnicki 2008, Yi et al. 2008, Sadek et al. 2011). Since progressive collapse is a nonlinear dynamic phenomenon which involves complicated structural responses, most previous studies on ALP method try to consider the dynamic effects within the static analysis procedure. In the following part, experimental and analytical studies on $\mathrm{RC}$ beam-column substructures subjected to single column removal scenarios will be reviewed, from quasi-static, to free-fall dynamic, and finally actual blast conditions.

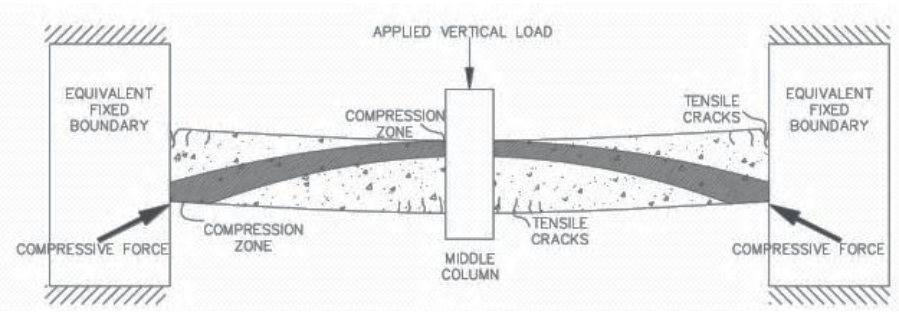

a) CAA

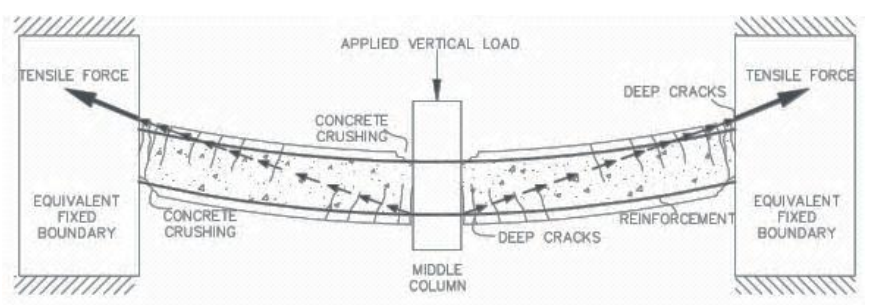

b) $\mathrm{CA}$

Fig. 2.2 ALPs on RC beams (Qian et al. 2016a)

\subsubsection{Quasi-static analyses of beam-column structures}

Despite the dynamic characteristics of progressive collapse, quasi-static response provides a lot of information on the development of different structural mechanisms, especially the response of joint displacement versus applied load. From such results, insight on structural behaviour can be obtained and investigated before performing any dynamic study. 


\subsubsection{Quasi-static tests}

Recently, there have been extensive quasi-static experimental studies on ALP approach of beam-column structures (Sasani and Kropelnicki 2008, Yi et al. 2008, Su et al. 2009, Choi and Kim 2011, Sadek et al. 2011, Stinger and Orton 2013, Valipour et al. 2013, Yu and Tan 2013b, a, Lim et al. 2015, Lu et al. 2016). Most of them applied concentrated loading (CL) condition at the middle joint of the doublespan beam to investigate structural responses against progressive collapse situations under quasi-static conditions. Mobilisation and development of both CAA and CA, which strongly depend on adjacent axial restraint conditions, were clearly observed. Behaviours of relevant experimental studies investigating mobilisation of CA are presented below.

\section{Yi's test on a multiple-bay 2D frame (Yi et al. 2008)}

A quasi-static test on a 2D RC frame with multiple bays and storeys was conducted at Hunan University by $\mathrm{Yi}$ et al. (2008) to study the static behaviour and load redistribution under the scenario of middle column removal. Fig. 2.3 shows the test setup and overall dimensions of the frame specimen. The test results clearly indicated three different mechanisms. At first, elastic behaviour was evident at small displacement. Subsequently, plastic response was observed when longitudinal rebars started yielding and plastic hinges formed at two ends of the beams on the first floor. Finally, CA took place when the structural response increased noticeably progressing to large deformation state. The results of this study show that CA in beams, which depends strongly on surrounding axial restraints, plays an important role to protect the structure from collapse under single-column removal scenarios. The test was terminated when the bottom rebars at the middle joint of the first floor fractured, which was considered as the collapse of the specimen. It should be noted that there was still residual catenary strength from the top rebars at the section. 


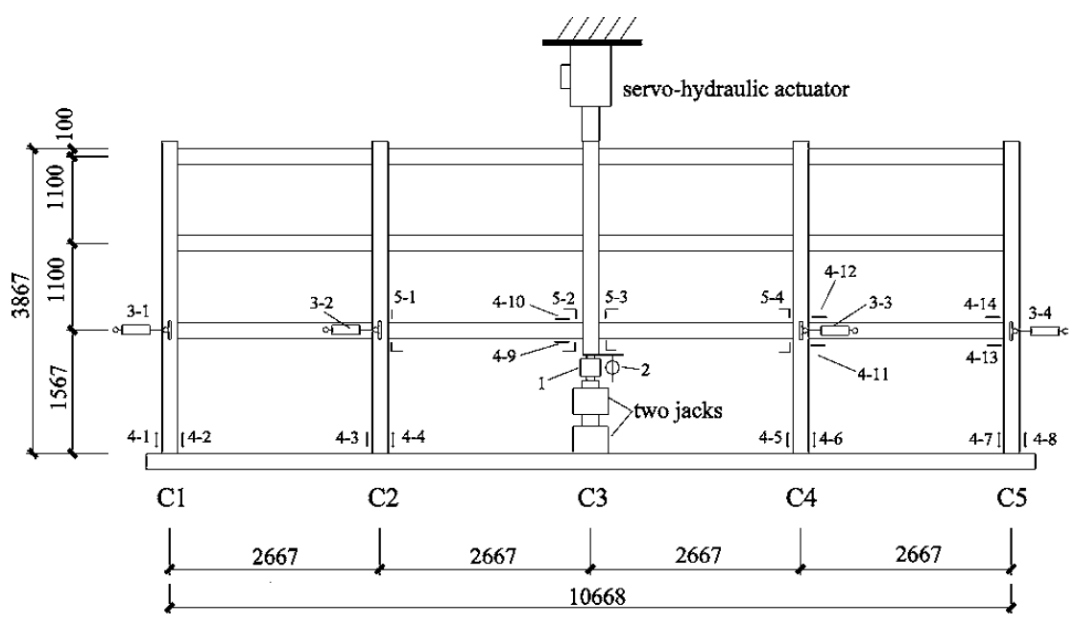

Fig. 2.3 Specimen design and test setup (Yi et al. 2008)

\section{Sadek's test on concrete beam-frame assemblies (Sadek et al. 2011)}

In this study, two RC beam-column assemblies were cast and tested under vertical displacement of the centre beam-column joint to simulate the column loss scenario. One specimen was designed as an intermediate moment frame - IMF (conventional design for moderate seismic resistance) while the other was a special moment frame - SMF (highly seismic-resistant design). Column removal was simulated by applying a vertical load to the middle joint through four steel rods which were anchored to the strong floor as shown in Fig. 2.4.

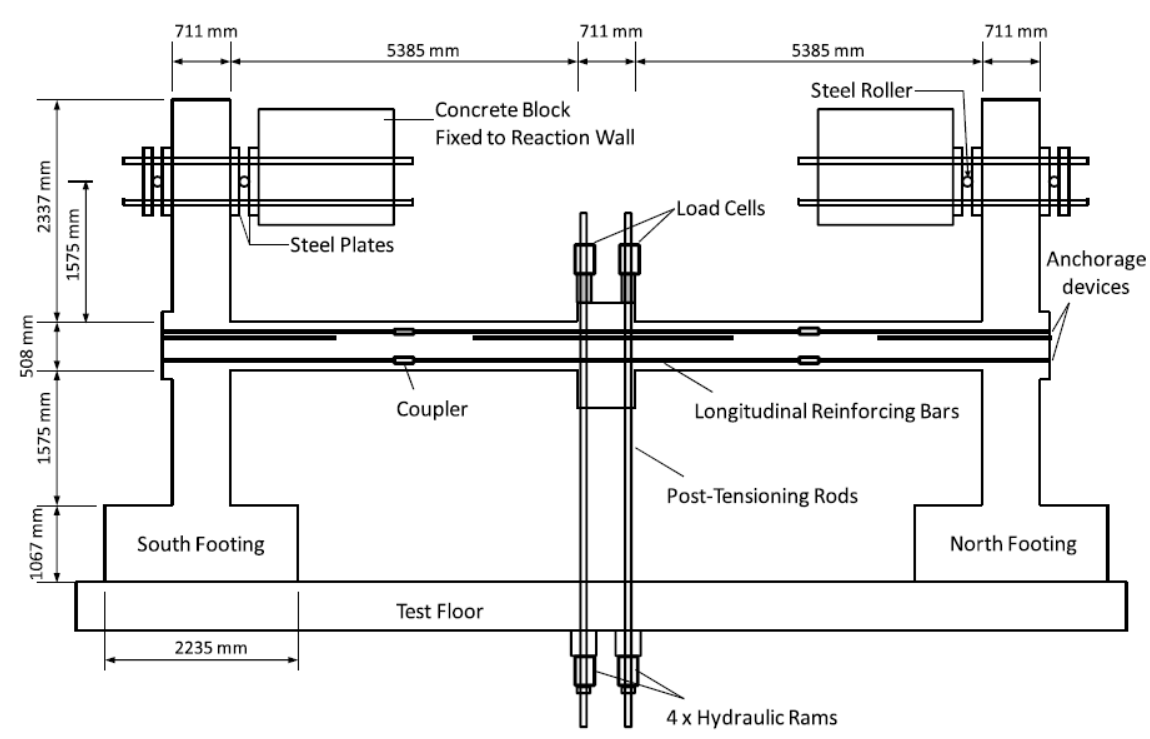

Fig. 2.4 Test setup and instrumentation layout (Sadek et al. 2011) 
Results of specimen IMF are presented in Fig. 2.5. As the middle joint was gradually moving downwards, the specimen sustained elastic behaviour and reached its maximum flexural capacity at the first peak of $267 \mathrm{kN}$ for the vertical load. After which, there was a decrease in load capacity caused by concrete crushing in the compressive zone. This decreasing period was not clearly observed in the test of Yi et al. (2008). With an increase of downward displacement, the vertical load increased again as CA was mobilised and reached the second peak at a value of 547 $\mathrm{kN}$. Afterwards, the vertical load reduced when the bottom longitudinal rebars fractured, considered as termination of the test. Test results from specimen SMF showed a similar phenomenon with a greater value of vertical force due to strengthening of both longitudinal and stirrup reinforcement.

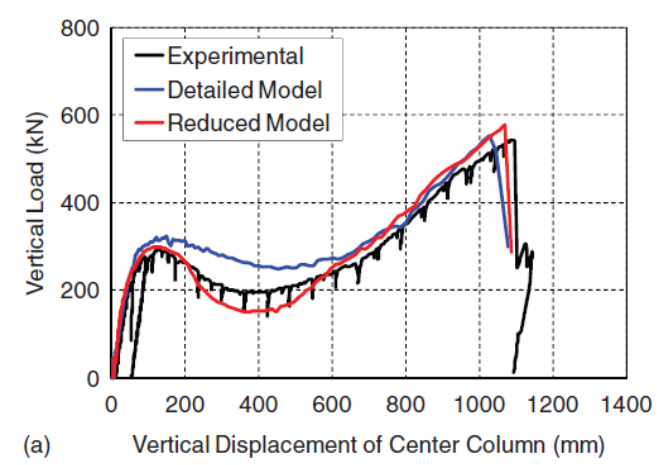

Fig. 2.5 Test results for IMF specimen (Sadek et al. 2011)

In both series of tests from Yi et al. (2008) and Sadek et al. (2011), collapse was defined at the moment when the bottom longitudinal rebars in the beams near the middle joint fractured. This evaluation criterion seems rather conservative because the remaining top rebars could still contribute to CA with an even higher value of vertical applied load, as witnessed by Sasani and Kropelnicki (2008), Choi and Kim (2011), Yu and Tan (2013a, b), and Lim et al. (2015).

\section{Yu's tests on RC sub-assemblages (Yu and Tan 2013a, b)}

An experimental study on progressive collapse resistance of beam-column subassemblages with the scenario of middle column removal was conducted at Nanyang Technological University. The test series included eight specimens from 
S1 to S8. The first six specimens had the same geometry and different reinforcement ratios and arrangements, while the last two (S7 and S8) had different beam spans. The structure consisted of a two-span beam with a middle joint and two column stubs as shown in Fig. 2.6. To represent translational and rotational restraints of the end joints, one roller support and two horizontal restraints were placed at each column stub. Removal of the supporting column was simulated by slowly increasing the displacement of the middle joint using a vertical actuator.

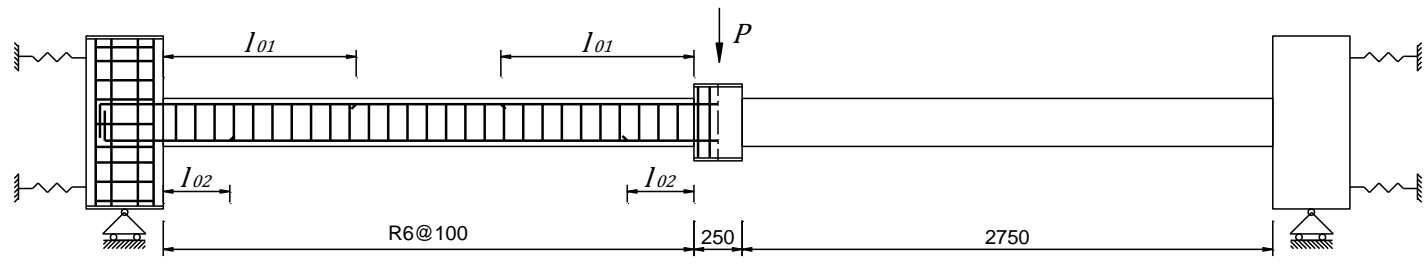

Fig. 2.6 Test setup (Yu and Tan 2013a, b)

The test results clearly showed three phases of behaviour including purely flexural action at the beginning, CAA and CA (Fig. 2.7) in this sequence. CA was indicated by a change of horizontal reactions from compression to tension. After the first fracture of bottom rebars at a load level of $41 \mathrm{kN}$ for $\mathrm{S} 1$ and $39 \mathrm{kN}$ for $\mathrm{S} 2$, the specimens could still withstand additional displacement and the vertical load could still increase. Total collapse took place when the top rebars near the end joints fractured with the applied load reaching $69 \mathrm{kN}$ for $\mathrm{S} 1$ and $66 \mathrm{kN}$ for S2. This phenomenon was not observed in the tests of Yi et al. (2008) and Sadek et al. (2011) due to early termination of the tests after bottom rebars had fractured at the middle joint.

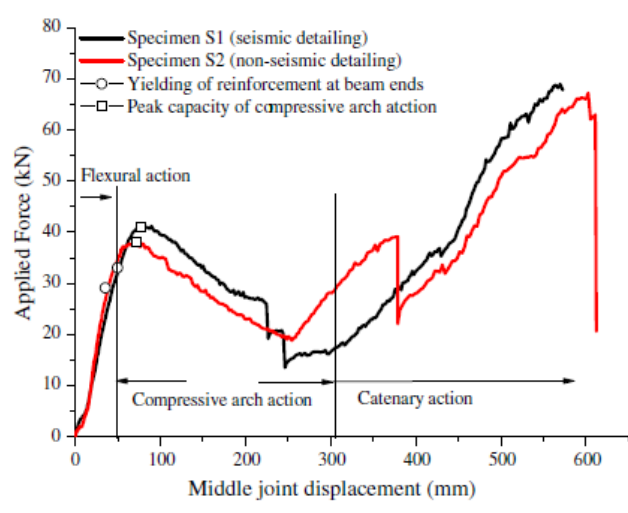

a) Vertical applied load

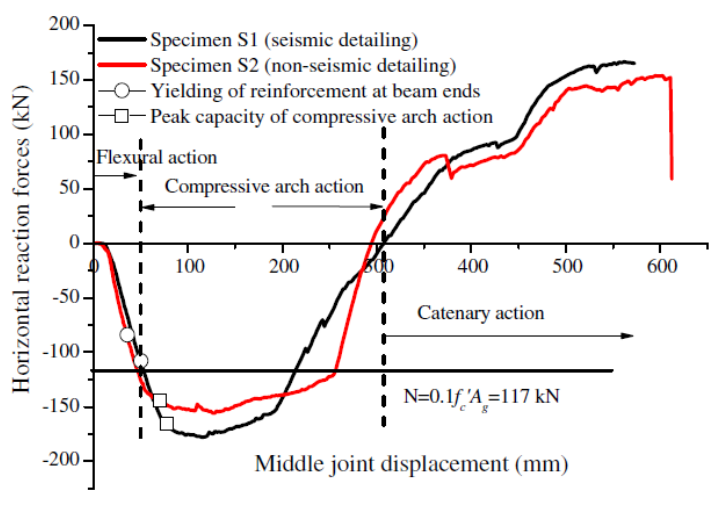

b) Horizontal reaction

Fig. 2.7 Test results for S1 and S2 (Yu and Tan 2013a) 


\section{Lim's tests on 2D beam-column frames (Lim et al. 2015)}

To study the mobilisation of CA in beam-column substructures under different conditions of adjacent restraints, two frame tests were conducted by Lim et al. (2015). The two specimens, named as FR and PR, shared the same geometry and reinforcement design, except that the boundary conditions at two sides of the specimens were different. While FR (Fig. 2.8(a)) represented an RC frame with both sides fully restrained (interior column loss), the frame in PR test (Fig. 2.8(b)) was fully restrained at the left side but partially restrained at the right side (representing next-to-outermost column loss). Test results elucidated the differences of structural behaviour between the two specimens regarding CA, which was significantly mobilised under the full-restraint condition (FR) even after the fracture of bottom rebars at the middle joint (Fig. 2.9(a)). Considering the static test of PR, after the bottom rebars in the beam had fractured, the partially-restrained side column started moving inward excessively with negligible development of CA. Hence, CA did not provide significant enhancement to structural load-carrying capacity (Fig. 2.9(b)). To protect laboratory equipment from being damaged by the abrupt collapse of the side column, PR specimen was stopped at a MJD of $396 \mathrm{~mm}$ before any top rebar fracture had occurred.

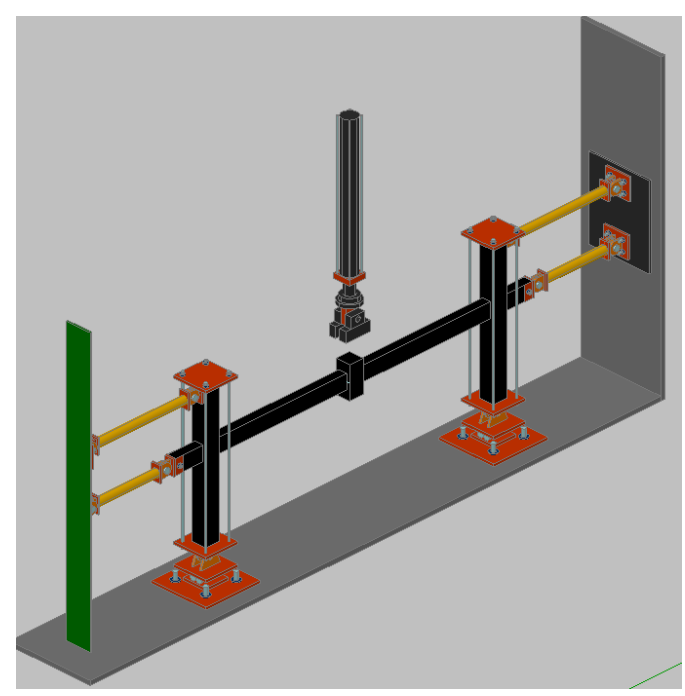

a) Full-restraint setup (FR)

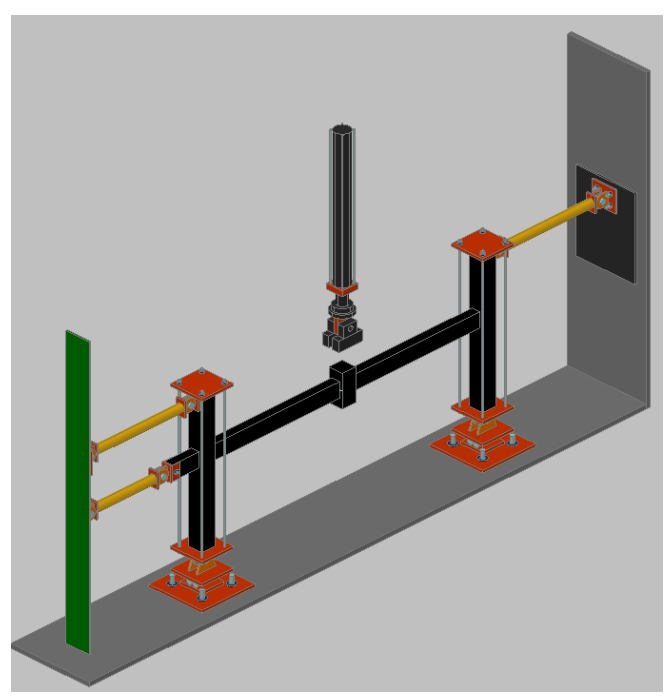

b) Partial-restraint setup (PR)

Fig. 2.8 Configuration for beam-column tests (Lim et al. 2015) 


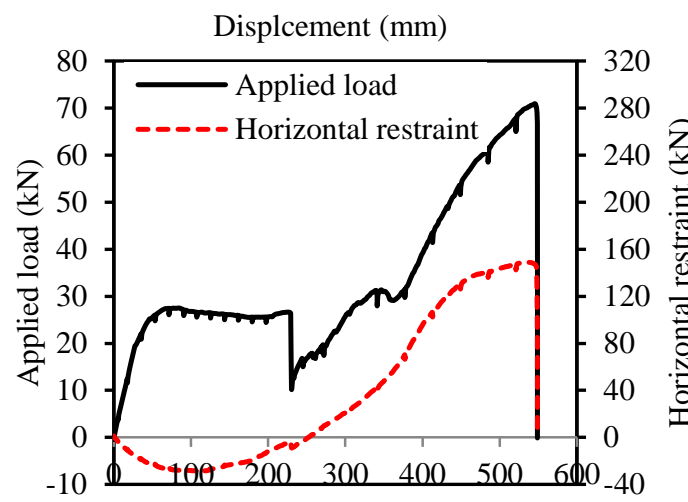

a) FR specimen

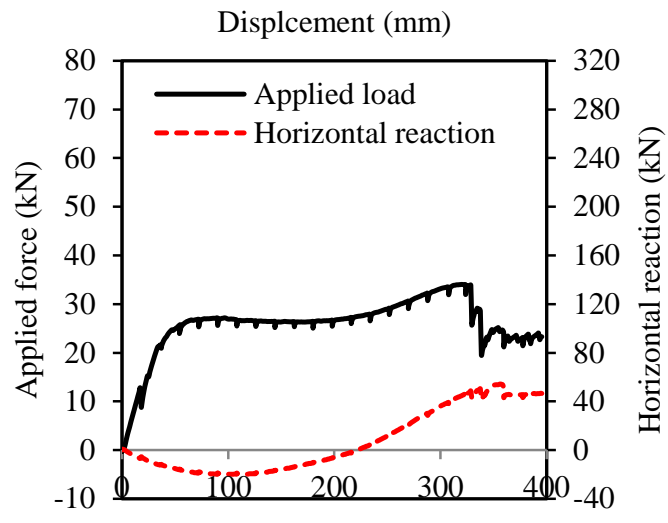

b) PR specimen

Fig. 2.9 Quasi-static test results for 2D frames (Lim et al. 2015)

To summarise, from previous quasi-static tests on $\mathrm{RC}$ beam-column structures under single column removal scenario, mobilisation and development of both CAA and CA, which depend strongly on the lateral restraint conditions, were clearly observed. CA was observed to start kicking in when the middle-joint displacement (MJD) of a two-span beam had reached around one beam depth. Subsequently, as the deflection kept increasing, beam bottom rebars near the middle joint started fracturing, leading to a sudden drop in load capacity. The displacement corresponding to this failure was about $1 / 8$ to $1 / 11$ of the single clear span in the tests of Yu and Tan (2013a, b) and Lim et al. (2015), whereas in Yi et al. (2008) and Sadek et al. (2011), that ratio was $1 / 6$ and $1 / 5$, respectively. Suffice to say, the fracture of bottom rebars is significantly influenced by span-depth ratio, rebar arrangement, and ultimate strain of longitudinal reinforcement. If the downward displacement was kept increasing after the fracture of bottom rebars (Sasani and Kropelnicki 2008, Yu and Tan 2013a, b, Lim et al. 2015), the load capacity would increase again and the final load resistance might be greater than both the peak strength provided by CAA and CA stages before the fracture of bottom rebars. Nonetheless, most of the quasi-static tests on beam-column structures applied CL condition due to expediency in testing. In actual buildings, the beam-column substructure experiencing column loss scenarios can be subjected to either CL or uniformly distributed loading (UDL) condition. The important question is whether $\mathrm{CA}$ in RC frames can be fully mobilised under UDL case. However, to date, there has not been any quasi-static test on 2D beam-column structures subjected to UDL 
condition, possibly due to complexity in loading configuration. In this thesis, static tests on 2D frames under UDL method will be proposed to study if the loading condition may affect the mobilisation of CAA or CA.

\subsubsection{Numerical and analytical studies on quasi-static behaviour}

Besides experimental work, several numerical and analytical studies have been conducted on the static behaviour of beam-column structures which involves various nonlinear phenomena such as changing of geometry under large displacements, crushing of concrete at large strains, yielding and post-yielding behaviour of reinforcement. The use of sophisticated finite-element-method (FEM) modelling, either with physics-based solid elements or with simplified fibre elements incorporating a component-based joint model (Valipour and Foster 2010, Yu and Tan 2013a, Bao et al. 2014, Pham et al. 2016), can provide reasonable predictions compared to actual responses. In terms of static condition, numerical simulations using simplified components such as fibre beam elements or beamcolumn joint models can be applied to reduce computational cost (Bao et al. 2008, Yu and Tan 2013a). Nonetheless, users are required to possess a high level of modelling skill, knowledge of FEM and vast computing resources when resorting to numerical simulations.

In the conceptual design stage where engineers need to explore various options to arrive at reasonable and economical solutions, a well-validated and simple approach is preferred instead of detailed finite element simulations. In this regard, Park proposed a model to calculate the compressive membrane action (CMA) capacity and peak reaction force of horizontally restrained RC slabs (Park and Gamble 2000). Similarly, this model can also be used to estimate the CAA capacity of RC beam-column structures. The CAA capacity of a sub-assemblage subjected to a concentrated load at the middle joint is determined by Eq. (2.1) (Yu and Tan 2010). Based on this model, Yu and Tan (2014) developed an analytical model to predict CAA of sub-assemblages considering imperfect boundary conditions in the static tests such as connection gaps, and rotational restraint stiffness. 


$$
\begin{aligned}
& P=\frac{2}{L_{n}}\left(0 . 8 5 f ^ { \prime } { } _ { c } \beta _ { 1 } b h \left[\frac{h}{2}\left(1-\frac{\beta_{1}}{2}\right)+\frac{\delta}{4}\left(\beta_{1}-3\right)+\frac{L_{n}^{2}}{2 \delta}\left(\beta_{1}-1\right) \varepsilon_{t}+\right.\right. \\
& \left.\frac{L_{n}^{2}}{2 h}\left(1-\frac{\beta_{1}}{2}\right) \varepsilon_{t}+\frac{\delta^{2}}{8 h}\left(2-\frac{\beta_{1}}{2}\right)-\frac{\beta_{1} L_{n}^{4}}{4 \delta^{2} h} \varepsilon_{t}^{2}\right]-\frac{\left(T^{\prime}-T-C_{s}^{\prime}+C_{s}\right)}{3.4{f^{\prime}}^{\prime}{ }^{b}}+\left(C_{s}^{\prime}+C_{s}\right)\left(\frac{h}{2}-\right. \\
& \left.\left.d^{\prime}-\frac{\delta}{2}\right)+\left(T^{\prime}+T\right)\left(d-\frac{h}{2}+\frac{\delta}{2}\right)\right)
\end{aligned}
$$

where $L_{n}$ is the single clear span of beams; $b$ and $h$ are the beam width and depth, respectively; $f^{\prime}{ }_{c}$ is the cylinder compressive strength of concrete; $\beta_{1}$ is the ratio of the depth of concrete equivalent stress block to the depth of section neutral axis; $T$ and $T^{\prime}$ are tensile resultant forces of rebars of sections near the middle joints and near the end joints, respectively; $C_{s}$ and $C_{s}^{\prime}$ are compressive resultant forces of rebars of sections near the middle joints and near the end joint, respectively; $d$ and $d^{\prime}$ are the distances from tensile and compressive rebars to the extreme compressive concrete fibre, respectively; $\delta$ is the middle joint displacement; and $\varepsilon_{t}$ is the total strain due to beam axial deformation and movement of beam end supports.

Regarding CA, Li et al. (2014) proposed equations for curve-type and straight-type catenary mechanisms under UDL and point loading, respectively, defining the relationship between applied load $R_{N}$ and corresponding deformation $\Delta$ (Fig. 2.10). Structural capacity after yielding of a sub-assemblage system is computed based on Eqs. (2.2) and (2.3) for UDL and point-loading conditions, respectively. However, Li's model assumes perfectly-rigid horizontal restraints at both sides of the twospan beam, which is difficult to achieve in both the reported tests and actual building structures. Besides, this mechanism does not distinguish the behaviour of CA before and after the fracture of the bottom rebars in the double-span beam. 


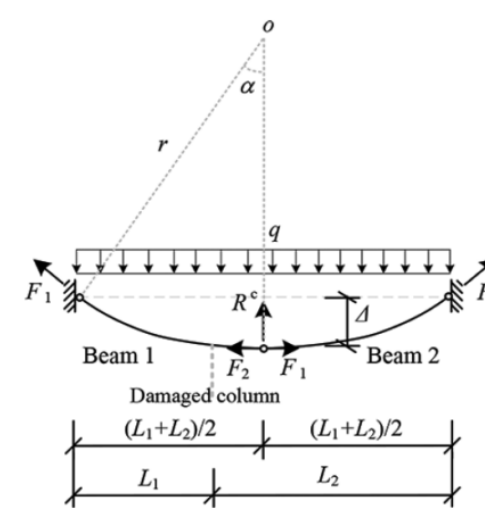

(a) Curve-type catenary mechanism

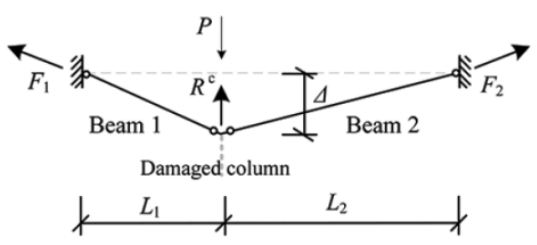

(b) Straight-type catenary mechanism

Fig. 2.10 Catenary action model from Li et al. (2014)

$R_{N}=\frac{8 F_{1 y}}{\left(L_{1}+L_{2}\right)} \Delta$

$R_{N}=\frac{\left(L_{1}+L_{2}\right) F_{1 y}}{L_{1} L_{2}} \Delta$

where $L_{1}$ and $L_{2}$ are the two spans of the structure (Fig. 2.10); $F_{1 y}$ is the yield tensile force of the beam.

In summary, although numerical studies have been well established to investigate the beam-column quasi-static behaviour under progressive collapse, it is timeconsuming and not suitable for normal design. Thus, there is a need to develop a simplified analysis to predict the structural response of RC sub-assemblages taking into account development of CA as well as actual stiffness of boundary conditions. This is one of the objectives of the current study.

\subsubsection{Dynamic analyses of beam-column structures}

As progressive collapse is a dynamic phenomenon, a number of analytical studies have been made to evaluate its dynamic effects on structures based on nonlinear static responses. Besides the analytical work, some free-fall dynamic tests have also been conducted to simulate actual missing column scenarios within laboratory conditions. 


\subsubsection{Dynamic assessments based on nonlinear static responses}

Both DOD and GSA documents propose simplified methods to take account of dynamic effect using static analysis. A simplified approach proposed by GSA (2003) incorporates the dynamic effects on static analysis by a factor of 2 on applied loads. This amplification, however, is only valid for linear elastic behaviour and cannot realistically represent the highly nonlinear behaviour of progressive collapse phenomenon. It does not simulate the sequence of collapse mechanisms correctly. On the other hand, DOD (2013) guideline applies Dynamic Increase Factor (DIF) as a coefficient to consider the dynamic effects based on nonlinear static analysis. The increased gravity load used for the static analysis is defined as follows:

$G_{N}=\Omega_{N}[1.2 D+0.5(L$ or $0.2 S)]$

The terms $D, L$ and $S$ denote the dead load, live load and snow load acting on the area of removed column, while $\Omega_{N}$ is the DIF value incorporating the dynamic behaviour of the structure. $\Omega_{N}$ is calculated for steel and concrete structures based on Eqs. (2.5) and (2.6), respectively:

For steel frames: $\Omega_{N}=1.08+0.76 /\left(\frac{\theta_{p r a}}{\theta_{y}}+0.83\right)$

For concrete frames: $\Omega_{N}=1.04+0.45 /\left(\frac{\theta_{p r a}}{\theta_{y}}+0.48\right)$

where $\theta_{y}$ and $\theta_{\text {pra }}$ are the yield and the plastic rotation angles of flexural members, respectively.

The DIF method from DOD (2013) presented by equation (2.4) only considers flexural mechanism to the capacity of structures under column removal scenarios. This method may underestimate the contribution from other upper-bound mechanisms such as CAA or CA.

With the aim of considering all the complex structural responses, a simplified frame-work was proposed by Izzuddin et al. (2008) to evaluate the maximum dynamic response based on nonlinear static analyses. The method includes three 
separate stages, namely: (1) specifying the nonlinear static response of the structure under column removal scenario, (2) assessing the dynamic behaviour, and (3) assessing the ductility of the connections. The novel contribution of this approach is the use of energy method in converting dynamic response to pseudo-static resistance, and in considering the column sudden removal as a sudden application of additional loading on the structure. At the moment when the structure attains its maximum response, the velocity reduces to zero and the work done by the external load is equal to the strain energy of the structure. The maximum dynamic response can be calculated by multiplying the static behaviour of a similar loading value with an amplification factor $\lambda_{b}$. For a given static response, at each displacement-point $u_{d, 1}$ (Fig. 2.11(a)), the corresponding dynamic load-level $P_{1}$ which produces the same maximum displacement $u_{d, 1}$ as the static load can be determined as follows (Izzuddin et al. 2008):

$P_{1}=\frac{1}{u_{d, 1}} \int_{0}^{u_{d, 1}} P d U_{S}$

By applying the above integral equation for every point in the static response curve, a pseudo-static response can be easily constructed (Fig. 2.11(b)). The dynamic amplification factor can then be calculated with regard to specific levels of gravity load. Compared to the DIF method from DOD (2013) which only considers the ductility capacity of sections, Izzuddin's method incorporates the overall nonlinear response of the member. Hence, it can consider hardening or snap-through phenomena shown in Fig. 2.12. Although Izzuddin's method is simple and computationally efficient, the application of this approach for CA stage needs to be validated by actual tests conducted under both quasi-static and free-fall dynamic environments.

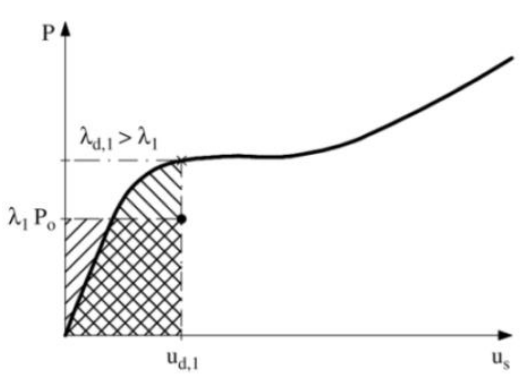

a) Nonlinear static response

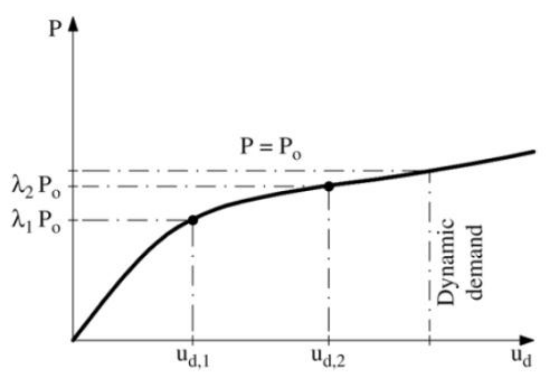

b) Pseudo-static response

Fig. 2.11 Simplified dynamic approach (Izzuddin et al. 2008) 


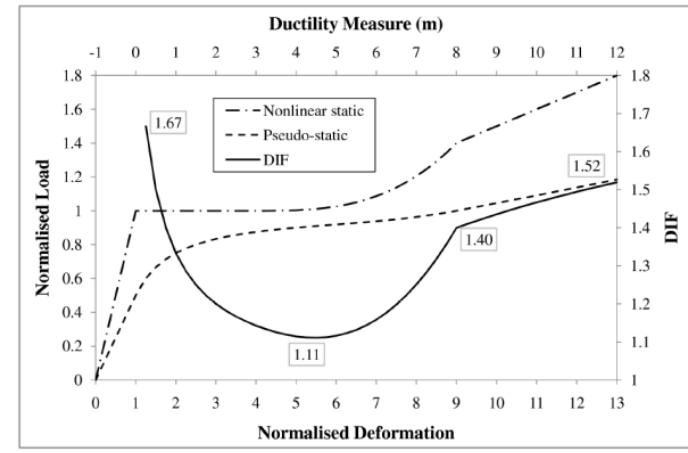

a) Elastic-plastic with hardening

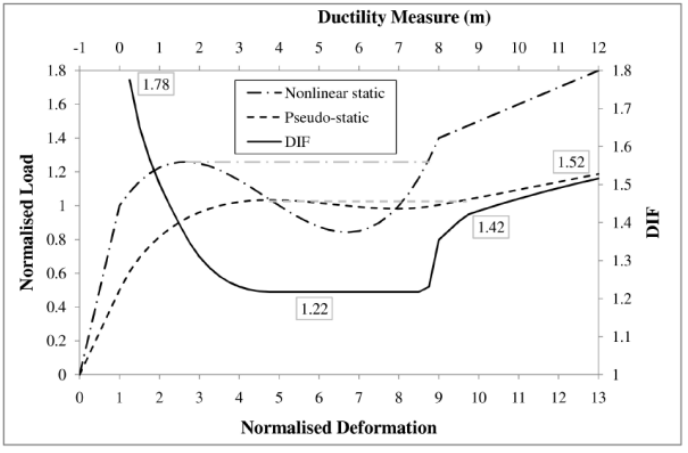

b) Elastic-plastic with snap-through

Fig. 2.12 Characteristic response and DIF value for different systems (Izzuddin and Nethercot 2009)

\subsubsection{Free-fall dynamic tests}

Despite the difficulties in simulating actual dynamic behaviour within laboratory environment, some experimental work on free-fall dynamic tests of beam-column structures has been conducted. In such tests, the column-missing scenario was simulated by a sudden removal of the support while the specimen was loaded with self-weight and additional loads representing imposed actions. Immediately after the release, the double-span specimen started falling freely under gravitational forces. The dynamic tests normally followed some quasi-static experiments conducted earlier in terms of structural design and boundary condition to have direct comparisons on dynamic effects.

\section{Study on corner-column removal by Qian}

Based on quasi-static results of RC substructures under the missing scenario of a corner column (Qian and Li 2012c), a series of dynamic tests was conducted to verify the response under free-fall condition (Qian and Li 2012b). Before testing, the specimen was temporarily supported by a special rotatable column and was loaded with steel plates to simulate the additional self-weight from the slab system as well as variable actions. The specially-designed supporting column was then suddenly struck off by a hammer, allowing the specimen to deflect freely under gravity. One interesting observation from these dynamic tests compared to the relevant static tests was the change of failure mode in some cases. The final failure 
stage of specimens F5 and F6 in the quasi-static tests included opening of vertical cracks in the beam near the end-joints together with fracture of longitudinal rebars as shown in Fig. 2.13(a) and Fig. 2.14(a) (flexural failure). In the corresponding dynamic tests, i.e. DF5 and DF6, the dominant failure was due to severe diagonal cracks of the beam near the end-joint, and the longitudinal steel had not yet fractured as shown in Fig. 2.13(b) and Fig. 2.14(b) (shear failure). This change in failure mode came from the differences in loading configuration between the two test series. While the static tests applied CL configuration at the corner joint, the dynamic tests employed multiple-point loading configuration along the longitudinal and the transverse beams. Therefore, results from the two test series could not be directly compared.

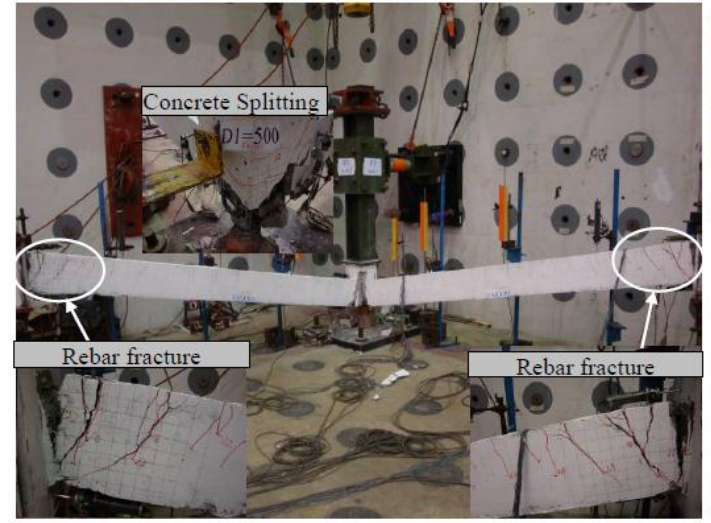

a) Quasi-static (F5)

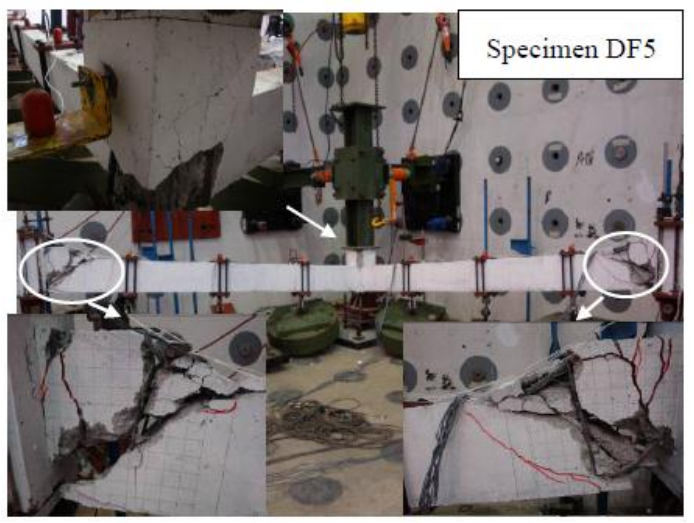

b) Dynamic (DF5)

Fig. 2.13 Test results of F5 and DF5 (Qian and Li 2012c, b)

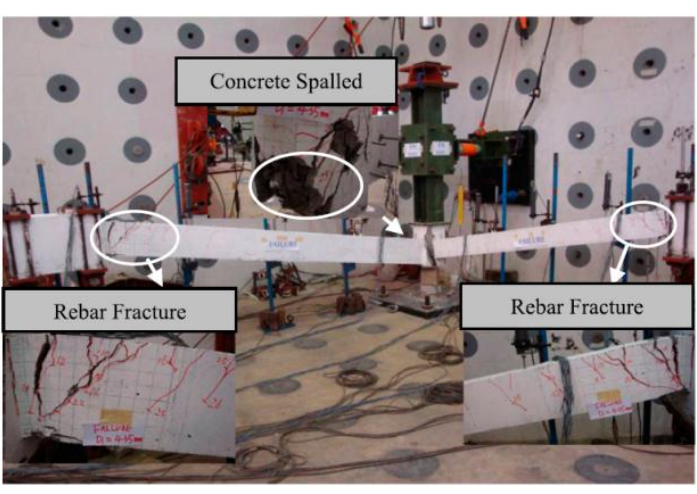

a) Quasi-static (F6)

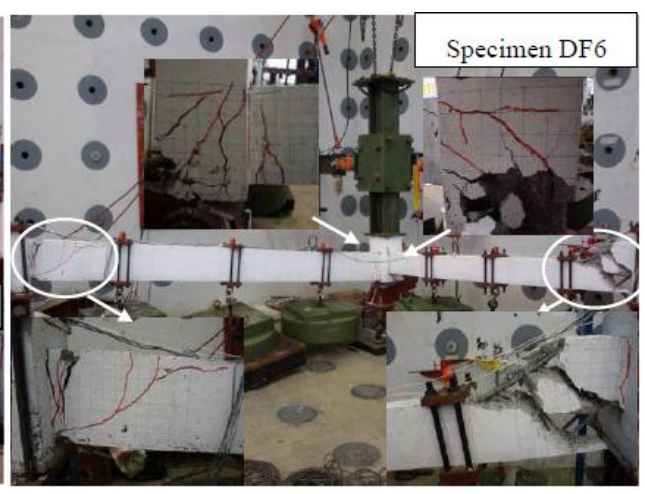

b) Dynamic (DF6)

Fig. 2.14 Test results of F6 and DF6 (Qian and Li 2012c, b) 
Some similar pairs of "static and dynamic" test series were also conducted, including: Su et al. (2009) and Tian and Su (2011), Sagiroglu (2012) (with both static and dynamic tests), Stinger and Orton (2013) and Orton and Kirby (2013). In those studies, although the specimens from both testing environments shared the same design and boundary condition, they were subjected to different loadings (Fig. 2.15). While the static tests had CL configuration above the removed column, the dynamic tests adopted a multiple-point loading method by fixing several weights along the double-span structure. Such a difference in loading configurations could lead to different structural behaviours, load-carrying capacities, and failure modes. Therefore, these tests cannot be used to validate the accuracy of the frame-work for dynamic assessment as proposed by Izzuddin et al. (2008).

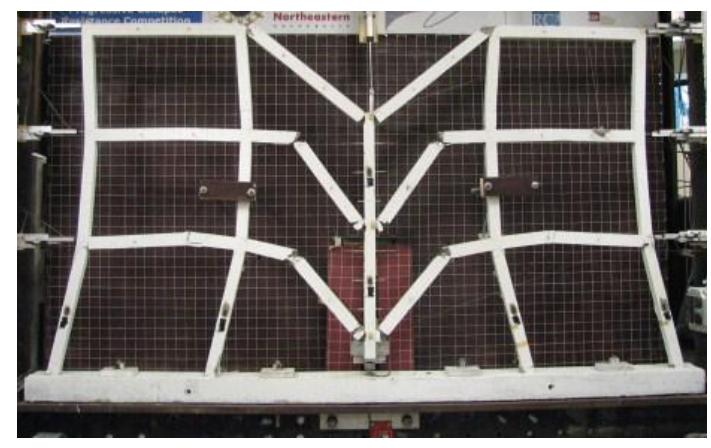

a) CL static test

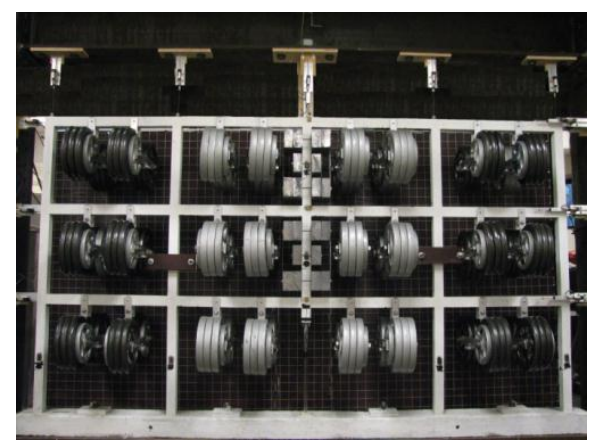

b) UDL dynamic test

Fig. 2.15 Different loading configurations for static and dynamic tests (Sagiroglu 2012)

\section{Liu's dynamic tests of steel connections under middle column removal (Liu et al.} 2015)

An experimental programme (Liu et al. 2015) was carried out to study the dynamic response of steel connections in comparison with the quasi-static tests conducted by Tan and Yang (2012). The configuration of the test setup is shown in Fig. 2.16. Only one-half of each span beam was constructed and connected to the horizontal reactions by a pin connection. The middle beam-column joint was hung from a reaction frame by a quick-release mechanism. This special hanging system could allow a sudden release of the middle joint and simulate free-fall behaviour. 
Based on the test results (Fig. 2.17), the release time of the mechanism was fairly consistent for all the tests at around $30 \mathrm{~ms}$. This value was smaller than one-fourth of the natural period, which satisfies the requirement to create a real dynamic response to a structural system according to Biggs (1964). This study also concluded that the DIF value recommended by DOD overestimated the dynamic behaviour within the range of CAA, but underestimated realistic response when the specimen underwent CA stage. That is to say, using DIF method proposed in DOD guideline for designing structures may lead to unsafe predictions of CA capacity. As a result, additional dynamic tests need to be conducted to verify this finding in the context of RC beam-column structures.

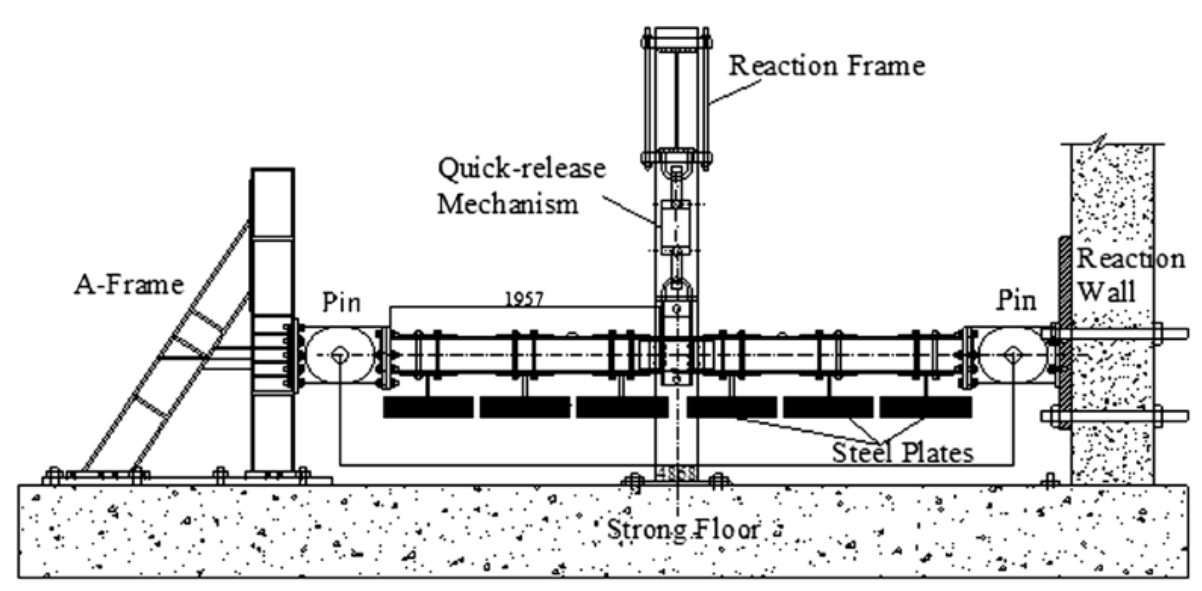

Fig. 2.16 Test setup for sudden column removal scenario (Liu et al. 2015)
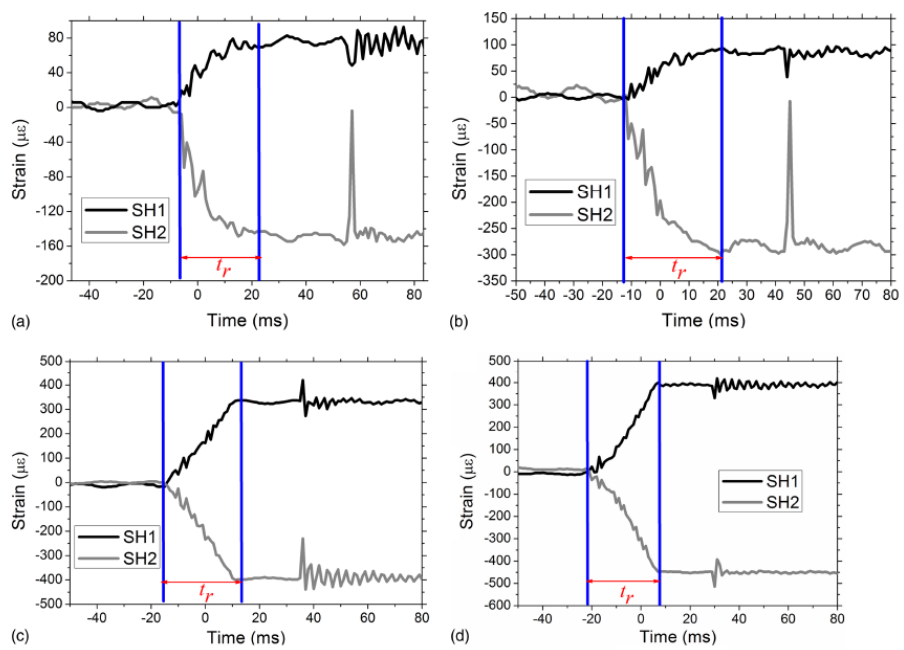

Fig. 2.17 Release time from the mechanism 
From the review of current analytical and experimental work on dynamic responses of RC structures under progressive collapse, there is a need to conduct systematic free-fall dynamic experiments based on quasi-static tests, with the same loading configuration and boundary restraint conditions. This testing programme will not only be used to study the behaviour observed in the nonlinear static response, but also verify the usefulness of the simplified methods for dynamic assessment. Both loading conditions, viz. CL and UDL, shall be considered in the testing programme discussed in Chapter 5.

\subsubsection{Blast-induced dynamic behaviour of beam-column structures}

Although some experimental and analytical studies on dynamic behaviour of RC structures under single column removal scenario have been conducted, the shortcoming of this assumption, however, is that it does not consider initial damages of remaining structural elements. As mentioned in Chapter 1, most of the iconic events resulting in progressive collapse incidents were triggered by explosions, whether it was a gas explosion or a bomb attack. Therefore, a study of progressive collapse taking into account actual damages from the initial explosive event is necessary. Nevertheless, the conduct of such experiments involving explosives is constrained by cost and safety requirements. If the charge weight is too small, the structure is hardly damaged and the targeted column is even not yet totally removed; but if the charge weight is too much, the structure is probably blasted into chunks and pieces. Currently, there is very limited number of tests associated with detonation. The aim was to apply sufficient charge weight to force the structure into large deformation state. In this section, an explosion experiment on existing building and another detonation test series on RC specimens are reviewed.

Sasani's blast test on existing building subjected to the removal of one edge column (Sasani et al. 2007)

A ten-storey RC building was tested under the implosion of one edge column on the ground floor to trigger progressive collapse and to study the behaviour of the remaining structure (Fig. 2.18). Before the test was conducted, all non-structural 
features and partition walls were removed, and the building only resisted its selfweight. Data received from the beam system above the removed column indicated that flexural behaviour (Vierendeel action in frames) was the main mechanism for load redistribution (ALP) after the ground column was removed. The measured vertical displacement remained relatively small, only $6.4 \mathrm{~mm}$ (Fig. 2.19(a)). The structure responded within elastic behaviour and no evidence of CA was observed. The test results also indicated that the time for the axial force of the above column on the second floor to reduce to a comparatively small value was less than $12 \mathrm{~ms}$ (Fig. 2.19(b)). This duration was defined as the time for the ground column to be totally removed by the detonation. Based on this experiment, similar blast tests on existing buildings were also conducted including Sasani and Sagiroglu (2010) and Sasani et al. (2011). However, in such tests, effects of the initial blast on surrounding structural members were not measured.

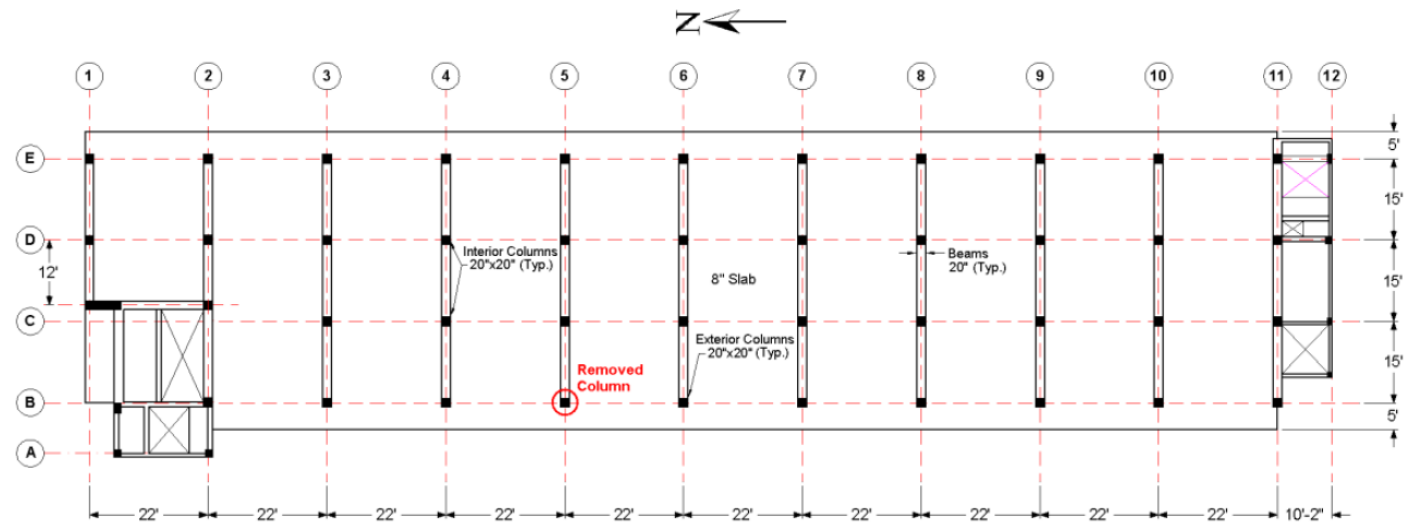

Fig. 2.18 Position of removed column B5 (Sasani et al. 2007)
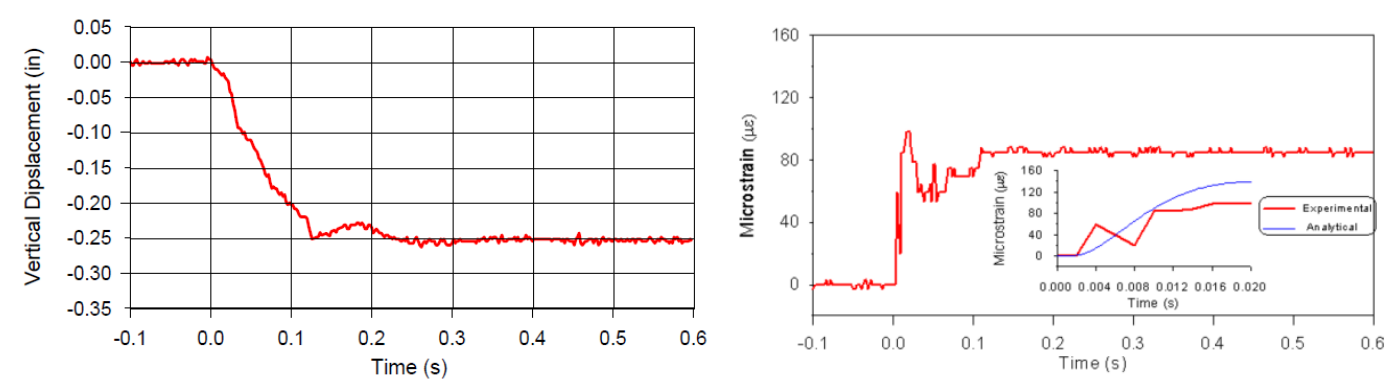

a) Displacement time-history of joint B5 in second-floor

b) Variation of axial strain in column B5 at fifth-storey

Fig. 2.19 Test results (Sasani et al. 2007) 


\section{Yu's blast tests on sub-assemblages under contact detonation (Yu et al. 2014)}

To investigate the effects of ultra-fast dynamics compared to quasi-static loading regimes, a series of progressive collapse tests induced by contact detonation was conducted at Fraunhofer EMI, Germany (Yu et al. 2014). The specimen design and boundary conditions were similar to specimen S2 from the static tests of $\mathrm{Yu}$ and Tan (2013a). The test series included three specimens from SD-1 to SD-3. Column removal was simulated by detonating a C-4 charge placed at the middle column of the specimen. Right after the detonation, the blast pressure immediately blew away the end part of the column, triggering collapse. The specimen design and general configuration of the contact detonation tests are shown in Fig. 2.20 and Fig. 2.21, respectively.

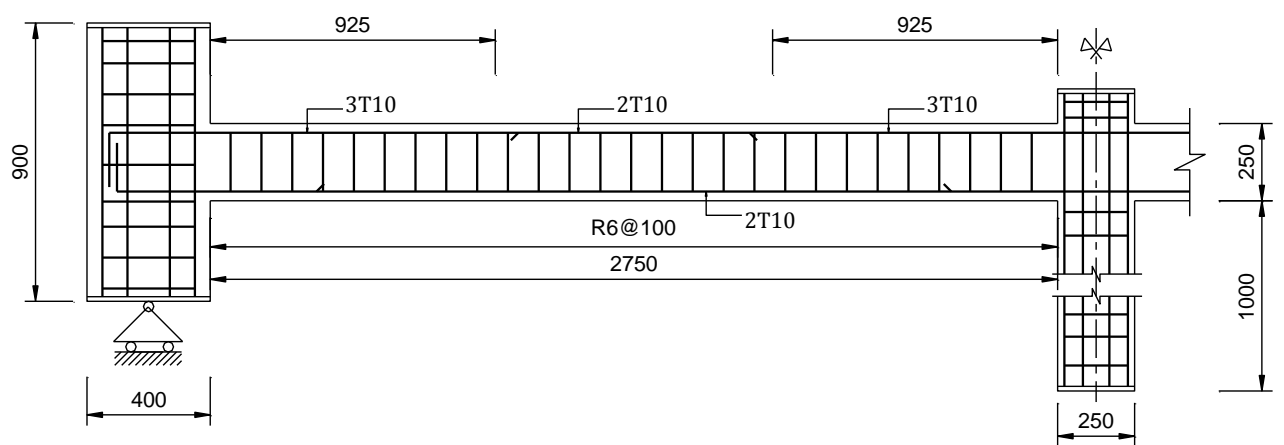

Fig. 2.20 Specimen design (Yu et al. 2014)

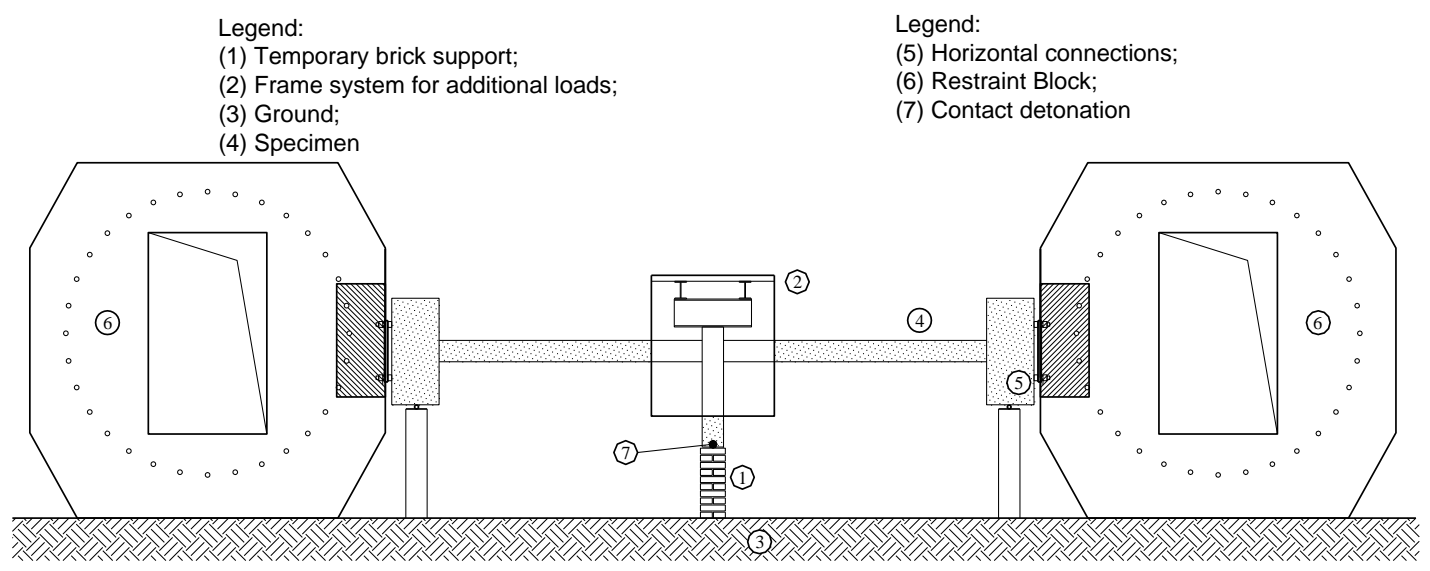

Fig. 2.21 Test setup (Yu et al. 2014) 
For specimen SD-1, the massive blocks at two sides of the specimen were not properly fixed to the ground. As a result, under the blast pressure from detonation, these restraints were shifted. For the remaining tests, the blocks were fixed to the ground by bolts. For specimen SD-2, after the middle support was removed by blast pressure, the middle joint experienced an uplift within the first $100 \mathrm{~ms}$ before falling freely under gravity load and finally sustaining a residual deflection of 50.2 $\mathrm{mm}$. With an imposed load of $27 \mathrm{kN}, \mathrm{SD}-2$ showed flexural deformation with CAA evident by the mobilisation of horizontal forces at the two end supports. Compared to SD-2, SD-3 with a larger imposed load of $47 \mathrm{kN}$ responded beyond the maximum CAA capacity. Displacement of the middle joint kept increasing until the specimen hit the ground at $471.7 \mathrm{~mm}$. The horizontal reaction of SD-3 was compression at the beginning but switched to tension when the middle joint deflected by more than one beam depth, indicating the commencement of CA before the specimen hit the ground. No top rebar fracture occurred after the test had finished. Therefore, it was not so clear whether SD-3 had indeed failed or could have sustained the applied load of $47 \mathrm{kN}$ if there had been ample headroom for deflection. An important finding from the study is that the dynamic increase effect was larger than the prediction obtained from the simplified frame-work proposed by Izzuddin et al. (2008). It shows clearly a significant initial reduction in the strength of the sub-assemblages caused by blast pressure. However, the stiffness of horizontal restraints which significantly affects structural behaviour of subassemblage systems (Yu and Tan 2013a) was not measured in this research work. Moreover, similar to the structural test by Sasani et al. (2007), the blast effects were not quantitatively measured in this study.

\subsubsection{Summary of experimental studies on beam-column structures}

The review of experimental work on beam-column structures against progressive collapse under different loading rates and loading configurations is presented in Table 2.1. It clearly shows a lack of 2D quasi-static structural tests under UDL condition, as well as the need to study mobilisation of CA under dynamic regime. Besides, measurements of initial blast effects on surrounding structural members in close-in blast tests are essential for accurate numerical modelling. 
Table 2.1 Summary of current experimental studies on beam-column structures

\begin{tabular}{|c|c|c|c|c|c|c|c|}
\hline \multirow[b]{2}{*}{ Research } & \multicolumn{3}{|c|}{ Loading rate } & \multicolumn{2}{|c|}{$\begin{array}{c}\text { Loading } \\
\text { configuration }\end{array}$} & \multirow{2}{*}{$\begin{array}{c}\text { Mobilisation } \\
\text { of CA after } \\
\text { bottom rebar } \\
\text { fracture }\end{array}$} & \multirow{2}{*}{$\begin{array}{c}\text { Measurement } \\
\text { of initial blast } \\
\text { effect }\end{array}$} \\
\hline & $\begin{array}{l}\text { Quasi- } \\
\text { static }\end{array}$ & $\begin{array}{l}\text { Free-fall } \\
\text { dynamic }\end{array}$ & $\begin{array}{c}\text { Close- } \\
\text { in } \\
\text { blast }\end{array}$ & CL & UDL & & \\
\hline $\begin{array}{l}\text { Sasani and } \\
\text { Kropelnicki } \\
\text { (2008) }\end{array}$ & $\mathrm{x}$ & & & $\mathrm{x}$ & & $\mathrm{x}$ & \multirow{17}{*}{ N/A } \\
\hline $\begin{array}{l}\text { Yi et al. } \\
\text { (2008) }\end{array}$ & $\mathrm{x}$ & & & $\mathrm{x}$ & & & \\
\hline $\begin{array}{l}\text { Orton et al. } \\
\text { (2009) }\end{array}$ & $\mathrm{x}$ & & & $\mathrm{x}$ & & & \\
\hline $\begin{array}{l}\text { Su et al. } \\
\text { (2009) }\end{array}$ & $\mathrm{x}$ & & & $\mathrm{x}$ & & & \\
\hline $\begin{array}{l}\text { Sadek et al. } \\
\text { (2011) }\end{array}$ & $\mathrm{x}$ & & & $\mathrm{x}$ & & & \\
\hline $\begin{array}{c}\text { Choi and } \\
\text { Kim (2011) }\end{array}$ & $\mathrm{x}$ & & & $\mathrm{x}$ & & $\mathrm{x}$ & \\
\hline $\begin{array}{l}\text { Qian and Li } \\
(2012 \mathrm{c})\end{array}$ & $\mathrm{x}$ & & & $\mathrm{x}$ & & & \\
\hline $\begin{array}{l}\text { Stinger and } \\
\text { Orton } \\
(2013) \\
\end{array}$ & $\mathrm{x}$ & & & $\mathrm{x}$ & & & \\
\hline $\begin{array}{l}\text { Valipour et } \\
\text { al. (2013) }\end{array}$ & $\mathrm{x}$ & & & $\mathrm{x}$ & & & \\
\hline $\begin{array}{c}\text { Yu and Tan } \\
\text { (2013a) }\end{array}$ & $\mathrm{x}$ & & & $\mathrm{x}$ & & $\mathrm{x}$ & \\
\hline $\begin{array}{c}\text { Yu and Tan } \\
\text { (2013b) }\end{array}$ & $\mathrm{x}$ & & & $\mathrm{x}$ & & $\mathrm{x}$ & \\
\hline $\begin{array}{l}\text { Lim et al. } \\
\text { (2015) }\end{array}$ & $\mathrm{x}$ & & & $\mathrm{x}$ & & $\mathrm{x}$ & \\
\hline $\begin{array}{l}\text { Lu et al. } \\
(2016)\end{array}$ & $\mathrm{x}$ & & & $\mathrm{x}$ & & & \\
\hline $\begin{array}{l}\text { Tian and Su } \\
\text { (2011) }\end{array}$ & & $\mathrm{x}$ & & & $\mathrm{x}$ & & \\
\hline $\begin{array}{l}\text { Qian and Li } \\
\text { (2012b) }\end{array}$ & & $\mathrm{x}$ & & & $\mathrm{x}$ & & \\
\hline $\begin{array}{c}\text { Sagiroglu } \\
\text { (2012) }\end{array}$ & $\mathrm{x}$ & $\mathrm{x}$ & & $\begin{array}{l}\mathrm{x} \text { (for } \\
\text { static) }\end{array}$ & $\begin{array}{c}\mathrm{x} \text { (for } \\
\text { dynamic) }\end{array}$ & & \\
\hline $\begin{array}{c}\text { Orton and } \\
\text { Kirby } \\
(2013)\end{array}$ & & $\mathrm{x}$ & & & $\mathrm{x}$ & & \\
\hline $\begin{array}{c}\text { Sasani et al. } \\
(2007)\end{array}$ & & & $\mathrm{x}$ & & $\begin{array}{c}\text { self- } \\
\text { weight }\end{array}$ & & No \\
\hline $\begin{array}{l}\text { Sasani and } \\
\text { Sagiroglu } \\
(2010)\end{array}$ & & & $\mathrm{x}$ & & $\begin{array}{l}\text { self- } \\
\text { weight }\end{array}$ & & No \\
\hline $\begin{array}{l}\text { Sasani et al. } \\
\text { (2011) }\end{array}$ & & & $x$ & & $\begin{array}{c}\text { self- } \\
\text { weight }\end{array}$ & & No \\
\hline $\begin{array}{l}\text { Yu et al. } \\
\text { (2014) }\end{array}$ & & & $\mathrm{x}$ & $\mathrm{x}$ & & $x^{1}$ & No \\
\hline
\end{tabular}

Note: ${ }^{1}$ specimens hit the ground before any top rebar fracture had occurred. 


\subsection{Alternate load paths of beam-slab structures}

In an RC building, although the beam-column frames have significant importance in resisting the sudden column loss event, contribution of the slab system to overall structural resistance is substantial. The neglect of slab behaviour when assessing progressive collapse potential can lead to uneconomical design of beam and column sections. Moreover, besides CA in beams, tensile membrane action (TMA) in slabs is generally believed as an upper-bound mechanism to provide an alternate path for load redistribution to mitigate collapse. While the development of CA is normally denoted by the change of axial force in the beam from compression to tension (Fig. 2.22(a)), mobilisation of TMA is indicated by the formation of a tensile net at the middle of the slab and a compressive ring near the edges (Fig. 2.22(b)). In this section, some analytical models developed for TMA in slabs are presented, together with a review of current studies on combined beam-slab systems under progressive collapse.

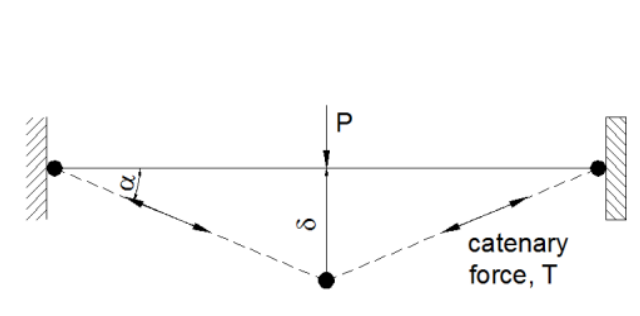

a) CA in axially-restrained beams

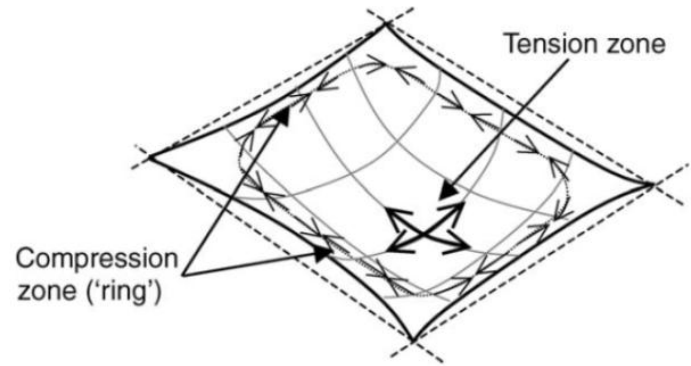

b) TMA in slabs

Fig. 2.22 Upper bound mechanisms for ALPs of RC structures

\subsubsection{Analytical models for TMA in slab-only structures}

\section{Park's model for fully fixed slabs under UDL}

Park (1964) conducted a test series on slab panels under UDL condition applying full-restraint boundary condition. Reinforcement ratio of the slabs varied from $0.16 \%$ to $2.39 \%$. Typical structural response and damage patterns are presented in

Fig. 2.23. Compressive membrane action, which occurred at about one-half of the slab thickness, was followed by TMA until the slabs failed by fracture of 
reinforcement parallel to the short span. Park suggested using a defection ratio of $1 / 10$ of the short span for a conservative estimation of ultimate load capacity for TMA. Park also proposed a conservative linear equation for TMA neglecting the contribution of flexural mechanism:

$$
\frac{w L_{x}{ }^{2}}{T_{x} \Delta}=\frac{\pi^{3}}{\sum_{\substack{n, 3,5, . . \\ n^{3}}}^{\infty}(-1)^{\frac{n-1}{2}}\left(1-\frac{1}{\cosh \left(\frac{n \pi L_{y}}{2 L_{x}} \sqrt{\frac{T_{x}}{T_{y}}}\right)}\right)}
$$

where $w$ and $\Delta$ are the applied load and the displacement of the slab, respectively; $L_{x}$ and $L_{y}$ are the shorter and the longer span, respectively; $T_{x}$ and $T_{y}$ are the yield forces per unit width in the $\mathrm{x}$ and $\mathrm{y}$ directions, respectively.

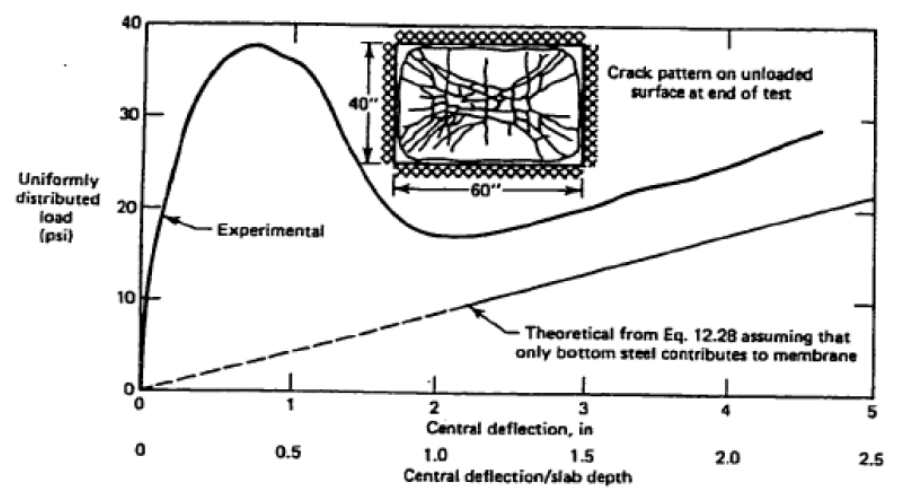

Fig. 2.23 Response of fully fixed slabs under UDL (Park 1964)

\section{Bailey's model for simply supported slabs under UDL}

Based on large-scale tests on composite slabs, Bailey (2001) developed a theoretical approach to predict TMA capacity of unrestrained RC slabs based on equilibrium approach. Two modes of failure are considered as shown in Fig. 2.24. By assuming rigid-plastic behaviour, the distribution of flexural and membrane actions can be separated into different in-plane elements (Fig. 2.25). Bailey's model also provides a simple equation to predict maximum deformation due to slab reinforcement fracture. 


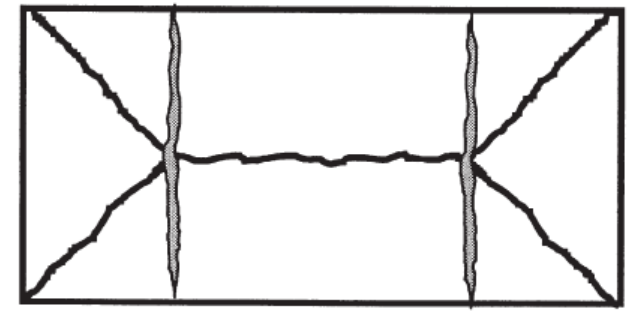

a) Mode of failure (i) - cracks forming at intersection of yield lines

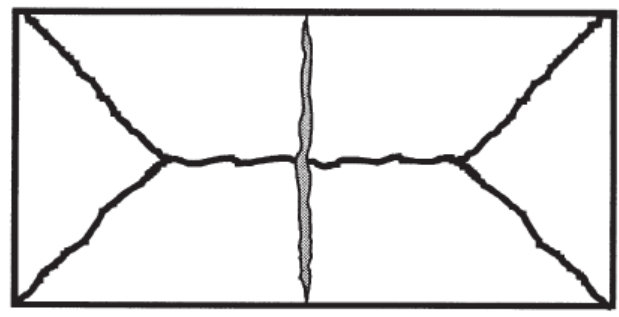

b) Mode of failure (ii) - cracks forming at centre of slab

Fig. 2.24 Slab failure mode under large deformations (Bailey 2001)

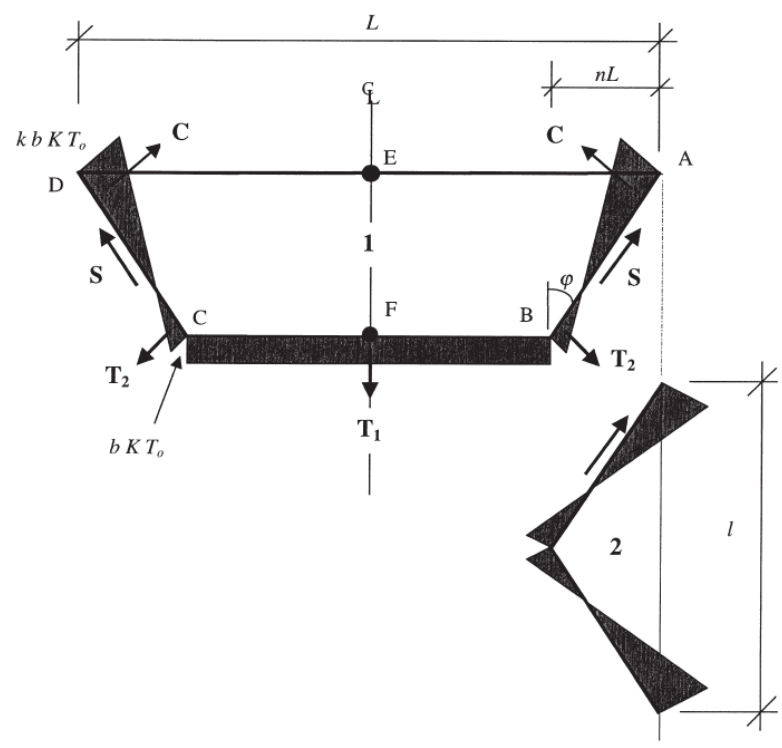

Fig. 2.25 In-plane stress distribution (Bailey 2001)

\subsubsection{Studies on TMA in RC beam-slab systems under progressive collapse}

To investigate the behaviour of the slab in a combined beam-slab system against column loss scenarios under quasi-static condition, laboratory scaled tests applying both CL (Qian and Li 2012a, Qian et al. 2014, Lu et al. 2016) and UDL (Pham and Tan 2013, 2015, Qian et al. 2016b) configurations were conducted. Table 2.2 categorises the published tests in terms of loading configuration, location of the removed column, as well as development of TMA as observed in the tests. In the CL tests, a point load was applied directly onto the joint above the removed column, while in the UDL tests, distributed loads on the slabs were applied by a 12-point 
loading system (Foster et al. 2004). The static tests on beam-slab structures, whether conducted under UDL or CL condition, showed enhancement in loadcarrying capacity beyond the predicted yield-line load for the slab. However, such studies did not clearly account for the respective contribution of CA and TMA to actual structural resistance. Moreover, experimental investigations on the sensitivity of TMA to external restraint conditions of slabs are constrained by costs and laboratory facility, and therefore have not been comprehensively studied. Besides, when assessing the vulnerability of a structure under progressive collapse threats, the engineer is required to consider various scenarios of column removal (GSA 2003, DOD 2013), as shown in Fig. 2.26. Among all the cases, the loss of a corner column is generally believed to be the most critical scenario due to a lack of restraint from two adjacent sides of the corner slab, as shown in the CL static tests of Qian and Li (2012a). In their work, CA and TMA are conservatively neglected for corner column loss and only flexural mechanism is considered for both beam and slab members. Nonetheless, this assumption may not be true if the system is under UDL condition.

Table 2.2 Summary of current experimental studies on beam-slab structures

\begin{tabular}{|c|c|c|c|c|c|c|c|}
\hline \multirow{2}{*}{ Research } & \multicolumn{2}{|c|}{$\begin{array}{c}\text { Loading } \\
\text { configuration }\end{array}$} & \multicolumn{4}{|c|}{ Column loss location } & \multirow{2}{*}{$\begin{array}{c}\text { Mobilisation } \\
\text { of TMA }\end{array}$} \\
\hline & CL & UDL & Internal & Edge & Penultimate & Corner & \\
\hline $\begin{array}{l}\text { Qian and Li } \\
\text { (2012a) }\end{array}$ & $\mathrm{X}$ & & & & & $\mathrm{x}$ & \\
\hline $\begin{array}{l}\text { Pham and Tan } \\
\quad(2013)\end{array}$ & & $\mathrm{X}$ & & & $\mathrm{x}$ & & x (limited) \\
\hline $\begin{array}{l}\text { Pham and Tan } \\
\text { (2015) }\end{array}$ & & $\mathrm{x}$ & & & $\mathrm{x}$ & & \\
\hline $\begin{array}{c}\text { Qian et al. } \\
\text { (2014) }\end{array}$ & $\mathrm{X}$ & & $\mathrm{x}$ & & & & $\mathrm{x}$ \\
\hline $\begin{array}{l}\text { Qian et al. } \\
\text { (2016b) }\end{array}$ & & $\mathrm{x}$ & & & $\mathrm{x}$ & $\mathrm{x}$ & x (limited) \\
\hline $\begin{array}{c}\text { Lu et al. } \\
\text { (2016) }\end{array}$ & $\mathrm{x}$ & & & $\mathrm{x}$ & & & $\mathrm{x}$ \\
\hline $\begin{array}{l}\text { Ren et al. } \\
(2016)\end{array}$ & $\mathrm{x}$ & & $\mathrm{x}$ & & & & $\mathrm{x}$ \\
\hline
\end{tabular}




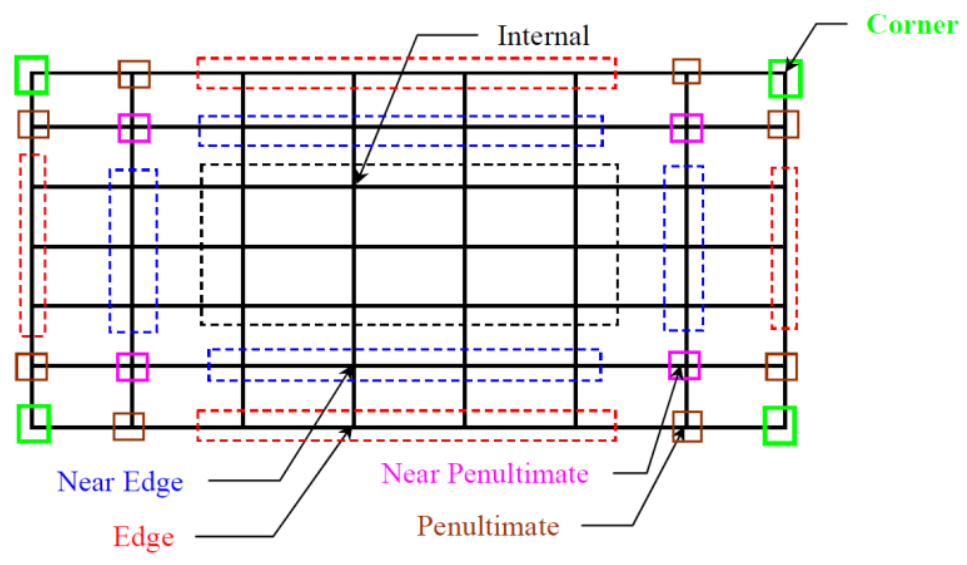

Fig. 2.26 Different scenarios of column removal (Stevens et al. 2009)

In terms of practical approaches for RC beam-slab systems under progressive collapse, Pham et al. (2015) proposed a simplified model to calculate structural capacity for a penultimate column removal scenario under UDL incorporating the contribution of both internal beams and slabs. Four-segment yield-line pattern is assumed to form in the slab whereas positive-moment plastic hinges in beams are assumed to form at intersections with the slab yield-lines (Fig. 2.27). However, only flexural mechanism is considered and no upper-bound action (TMA) is taken into account. To date, there is no practical/engineering method considering TMA in the combined beam-slab system, which has great inherent structural resistance to collapse.

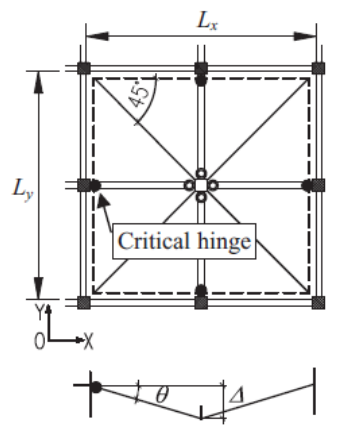

(a) $L_{x}=L_{y}$ (PI scenario)

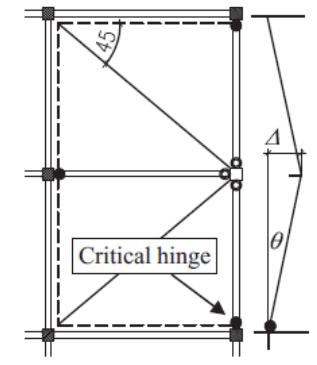

(b) $L_{y}=2 L_{x}(\mathrm{PE}$ scenario)

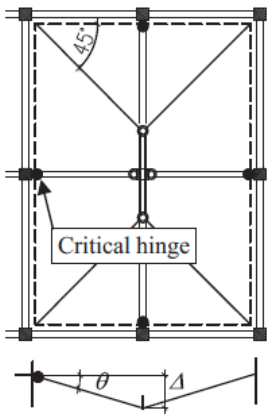

(c) $L_{x}<L_{y}$ (PI scenario)

Fig. 2.27 Formation of yield lines and plastic hinges in beam-slab system (Pham et al. 2015) 
In summary, the review of ALPs in slabs under progressive collapse scenarios highlights the need to investigate contribution of TMA to the overall structural capacity of the beam-slab system. Besides, sensitivity of this mechanism to factors such as boundary condition, location of the removed column, is of great importance. Moreover, there is a lack of simplified engineering methods to quantify the enhancement of TMA in the context of combined beam-slab structures. This work will be discussed in Chapter 9 of the thesis.

\subsection{Summary of literature review and research scope of work}

Based on an extensive review of progressive collapse studies on RC structures, the following shortcomings and technical gaps have been identified to form the scope of work of research:

(1) The studies on 2D beam-column substructures under missing column scenario with quasi-static conditions showed that both CAA and CA can significantly enhance overall behaviour for resisting progressive collapse. The mobilisation and development of these two mechanisms strongly depend on the boundary conditions and ductility/continuity level of structures. Numerical simulations using either simplified fibre elements or physics-based solid elements successfully captured the static behaviour of beam-column structures under progressive collapse. However, such methods are time-consuming and require extensive knowledge of finite elements which is not applicable for practical design. Therefore, in this study, a simplified engineering method taking into account actual boundary stiffness and mobilisation of CA is proposed. The 2D sub-assemblage includes a double-span beam with a middle joint and a column stub at each end to represent boundary restraints from actual buildings. Due to symmetry in boundary and loading conditions, only half of the structure is modelled. Several parameters affecting CA mobilisation such as span-depth ratio, horizontal restraint stiffness, and reinforcement ratio are considered. Actual strength of the horizontal support is also taken into account.

(2) CA, which was observed in quasi-static tests, is a promising alternate path for 
load redistribution. However, corresponding dynamic tests conducted to compare with quasi-static tests either did not share the same loading configuration or did not have sufficient headroom resulting in the specimen hitting the ground before CA could be fully mobilised. As a result, structural response of RC beam-column frames under dynamic condition was not fully investigated. Hence, dynamic experiments in Series I of this research are conducted for this reason. Compared to the sub-assemblage structures in the quasi-static study, more realistic boundary conditions including side columns and extended beams are employed at two sides of the frame specimens to simulate two scenarios of column loss, namely, interior column and next-tooutermost column.

(3) Most existing quasi-static tests on beam-column members applied CL condition whereas there was hardly any beam-column test subjected to UDL. As a result, structural behaviour, failure modes, as well as CA capacity under this loading configuration, have not been investigated. To plug this gap of knowledge, Series II programme including both quasi-static and free-fall dynamic tests under UDL condition are proposed. It employs similar specimens with Series I, but applies UDL condition via an equivalent 4-point loading method. While the UDL static tests can be directly compared with the previous CL static tests from a related study (Lim et al. 2015) to identify differences in structural responses between the two loading configurations, the UDL free-fall tests will highlight the dynamic effects of sudden column removal event. Innovative methods incorporating the dynamic behaviour of structures based on quasistatic response are convenient tools to assessing progressive collapse resistance. One such method is the energy approach by Izzuddin et al. (2008). In this study, dynamic tests from Series I and II are used to verify applicability and accuracy of Izzuddin's approach.

(4) Despite the efforts on simulating the realistic situation of progressive collapse triggered by detonation, most previous studies were focused on structural behaviour under missing column scenario. Researchers did not measure 
quantitatively the initial damages caused by blast effects to adjacent members of the structure (Sasani et al. 2007, Yu et al. 2014). Therefore, in the contact detonation tests of Series III, blast effects on structures are carefully measured compared with structural responses from the pristine state of the free-fall dynamic tests in Series I. Blast effects of the explosion event will be highlighted by studying initial uplift and blast pressure. Due to limitations on funding as well as safety concern, only a limited number of detonations can be carried out. Therefore, test results will also be used to validate finite element models in terms of blast loading, structural response, and damage patterns. The validated numerical modelling technique is then used to study the behaviour of 3D beam-slab structures subjected to a charge weight presented in Chapter 9.

(5) TMA in RC slabs under large deformations has been extensively studied in previous research work. However, contribution of this mechanism in the context of beam-slab systems under column removal scenarios has not been quantitatively investigated. Due to constraint by cost and facilities, the sensitivity of TMA to boundary stiffness, as well as to the location of the removed column, has not been comprehensively considered. Besides, simplified methods for quantifying TMA in combined beam-slab structures are needed. The investigation on beam-slab structures conducted in Chapter 9 will address this topic by numerically investigating a 2-by-2-span panel with internal and edge beams as well as slab extensions. Various conditions of loading methods (CL and UDL), boundary stiffness (translational and rotational restraints), and column loss scenarios (internal/edge/penultimate/corner column) will be considered. Analytical model for TMA of beam-slab structures will also be proposed.

In the following chapters, numerical and experimental studies on behaviour of beam-column and beam-slab substructures under realistic loading configurations and loading rates are conducted and presented. Numerical models using FEM software LS-Dyna are first developed and validated by published test results of RC sub-assemblages. Parametric studies are then conducted to develop a semi- 
analytical approach for beam-column structures taking into account CA. Experimental work on 2D RC beam-column frames under different loading configurations and different loading rates is carried out (Series I to III), including actual blast tests. Relationships between the three test series are shown in Fig. 2.28. Last but not least, TMA in beam-slab systems is investigated numerically and analytically. The objective of the research programme is to fill in technical gaps in practical engineering for the design of $\mathrm{RC}$ structures under progressive collapse condition.

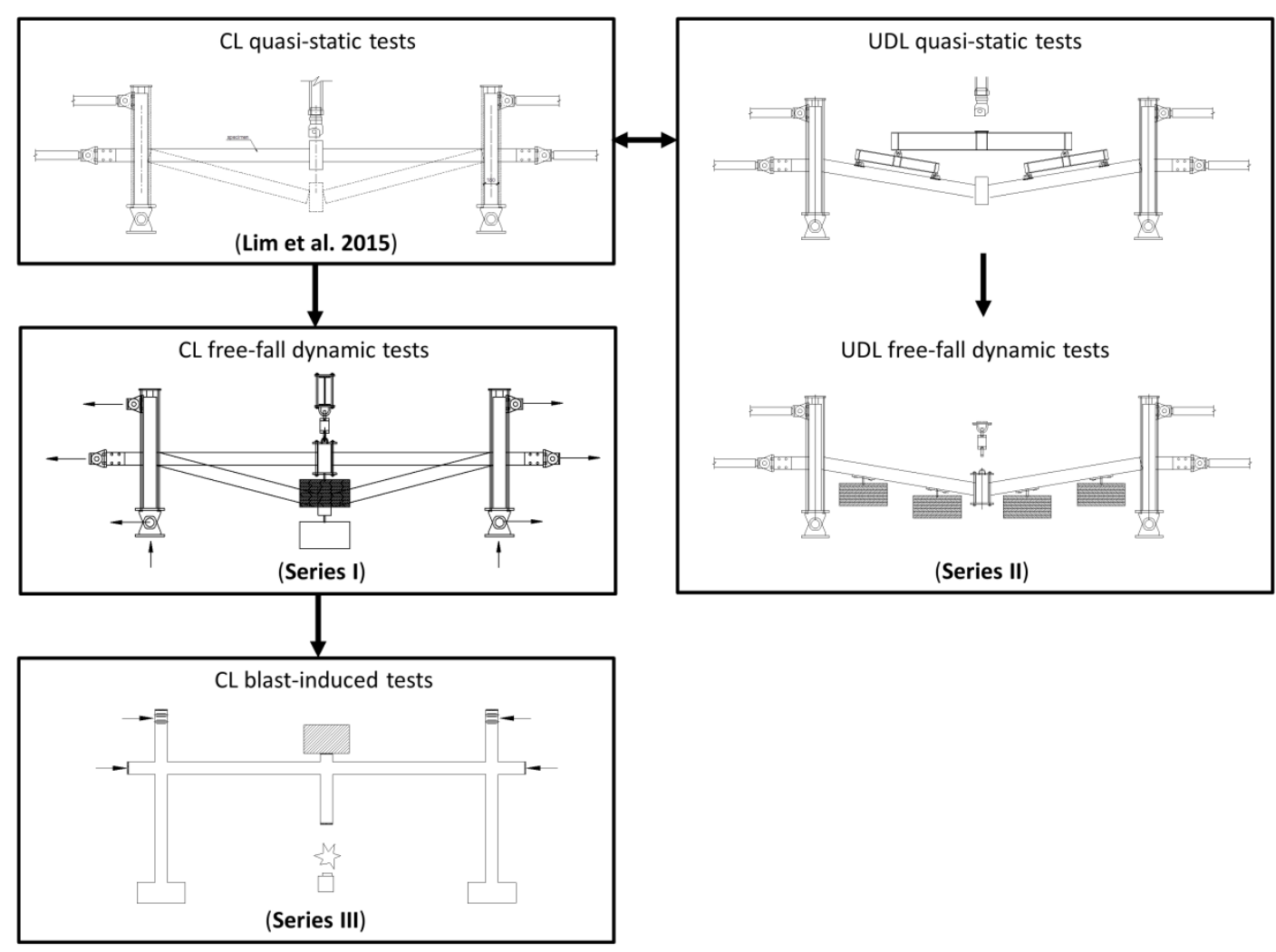

Fig. 2.28 Testing programme on 2D beam-column frames 



\section{CHAPTER 3 NUMERICAL INVESTIGATION ON BEAM- COLUMN SUBSTRUCTURES UNDER DYNAMIC RESPONSES}

\subsection{Introduction}

Due to the complexity in structural response under progressive collapse, especially when explosive conditions are involved, detailed numerical simulation using FEM is highly recommended for the design of protective structures (Krauthammer 2008). This advanced analytical method considers several nonlinear behaviours including material plasticity and damage, stress concentration and local failure, strain rate effects, etc. In this chapter, numerical models are developed to study the behaviour of RC beam-column sub-assemblages under the scenario of supporting column loss (Pham et al. 2016). An explicit finite element software LS-Dyna (Hallquist 2007) is used to simulate the sub-assemblages due to a wide variety of available constitutive models in the programme as well as its abilities in modelling the blast pressure on structures. Two sets of published test results are used for the validation, namely, the quasi-static tests on sub-assemblages under progressive collapse from $\mathrm{Yu}$ and Tan (2013a, b) with detailed data from Yu (2012), and the dynamic progressive collapse tests on RC assemblages under contact detonation from $\mathrm{Yu}$ et al. (2014). Subsequently, parametric studies are carried out to investigate the effect of some significant variables on structural dynamic behaviour such as boundary stiffness, damping effect, charge weight, load level, etc. The simplified method proposed by Izzuddin et al. (2008) is also verified numerically. The modelling procedure presented in this chapter is also applied in some other chapters of the thesis to investigate static and dynamic responses of different RC structures.

\subsection{Numerical validations on beam-column substructures}

\subsubsection{Quasi-static and contact-detonation tests on RC sub-assemblages under column removal scenario}

As reviewed in Section 2.3.1.1, a quasi-static experimental programme on progressive collapse resistance of beam-column sub-assemblages with the middle 
column "removed" was conducted by Yu and Tan (2013a, b). The test results are used to validate numerical models for quasi-static condition developed in this chapter. Test setup of this series is generally described in Fig. 2.6. Among the eight specimens, results from the first seven tests showed significant improvement of CA on structural behaviours. Whereas in the last specimen, S8, CA had almost no effect on structural capacity due to its small span-depth ratio and the specimen was severely damaged under shear failure. Rebar detailing and material test of all specimens are presented in Table 3.1 and Table 3.2, respectively.

Table 3.1 Specimen details

\begin{tabular}{|c|c|c|c|c|c|c|c|c|}
\hline \multirow{3}{*}{ Test } & \multirow{3}{*}{$\begin{array}{c}L_{n} \\
(\mathrm{~mm})\end{array}$} & \multirow{3}{*}{$\begin{array}{l}\text { Beam } \\
\text { section } \\
(\mathrm{mm})\end{array}$} & \multirow{2}{*}{\multicolumn{2}{|c|}{$\begin{array}{c}\text { Rebar } \\
\text { curtailment } \\
(\mathrm{mm})\end{array}$}} & \multicolumn{4}{|c|}{ Longitudinal reinforcement } \\
\hline & & & & & \multicolumn{2}{|c|}{ End span } & \multicolumn{2}{|c|}{ Mid span } \\
\hline & & & $l_{01}$ & $l_{02}$ & Top & Bottom & Top & Bottom \\
\hline S1 & 2750 & $150 \times 250$ & 1000 & NA & $1 \mathrm{~T} 13+2 \mathrm{~T} 10$ & 2T10 & $2 \mathrm{~T} 10$ & $2 \mathrm{~T} 10$ \\
\hline $\mathrm{S} 2$ & 2750 & $150 \times 250$ & 925 & NA & $3 \mathrm{~T} 10$ & $2 \mathrm{~T} 10$ & $2 \mathrm{~T} 10$ & $2 \mathrm{~T} 10$ \\
\hline S3 & 2750 & $150 \times 250$ & 1000 & 345 & $3 \mathrm{~T} 13$ & $2 \mathrm{~T} 10$ & $2 \mathrm{~T} 13$ & T10+1T13 \\
\hline S4 & 2750 & $150 \times 250$ & 1000 & $\mathrm{NA}$ & $3 \mathrm{~T} 13$ & $2 \mathrm{~T} 13$ & $2 \mathrm{~T} 13$ & $2 \mathrm{~T} 13$ \\
\hline S5 & 2750 & $150 \times 250$ & 1000 & NA & $3 \mathrm{~T} 13$ & $3 \mathrm{~T} 13$ & $2 \mathrm{~T} 13$ & $3 \mathrm{~T} 13$ \\
\hline S6 & 2750 & $150 \times 250$ & 1000 & 345 & $3 \mathrm{~T} 16$ & $2 \mathrm{~T} 13$ & $2 \mathrm{~T} 16$ & $3 \mathrm{~T} 13$ \\
\hline $\mathrm{S} 7$ & 2150 & $150 \times 250$ & 780 & NA & $3 \mathrm{~T} 13$ & 2T13 & $2 \mathrm{~T} 13$ & $2 \mathrm{~T} 13$ \\
\hline
\end{tabular}

Table 3.2 Material tests

\begin{tabular}{|c|c|c|c|c|c|c|}
\hline Test & Reinforcement & $\begin{array}{c}\text { Elastic } \\
\text { modulus } \\
(\mathrm{MPa})\end{array}$ & $\begin{array}{c}\text { Yield } \\
\text { strength } \\
(\mathrm{MPa})\end{array}$ & $\begin{array}{c}\text { Ultimate } \\
\text { strength } \\
(\mathrm{MPa})\end{array}$ & $\begin{array}{c}\text { Fracture } \\
\text { strain } \\
(\%)\end{array}$ & $\begin{array}{c}\text { Concrete } \\
\text { strength } \\
(\mathrm{MPa})\end{array}$ \\
\hline \multirow{2}{*}{ S1, } & $\mathrm{R} 6$ & 178500 & 310 & 422 & 14.00 & \multirow{3}{*}{31.24} \\
\cline { 2 - 6 } S2 & $\mathrm{T} 10$ & 182611 & 511 & 731 & 12.32 & \\
\cline { 2 - 6 } & $\mathrm{T} 13$ & 185763 & 527 & 640 & 10.76 & \\
\cline { 2 - 6 } S3 to & $\mathrm{R} 6$ & 199177 & 349 & 459 & - & \multirow{3}{*}{38.15} \\
\cline { 2 - 6 } S8 & $\mathrm{T} 10$ & 211020 & 511 & 622 & 11.00 & \\
\cline { 2 - 6 } & $\mathrm{T} 13$ & 185873 & 494 & 593 & 10.92 & \\
\cline { 2 - 6 } & $\mathrm{T} 16$ & 184423 & 513 & 612 & 13.43 & \\
\hline
\end{tabular}

Based on the results from specimen S2 (Yu and Tan 2013a), dynamic blast-induced tests were conducted (Yu et al. 2014) as mentioned in Section 2.3.3 to investigate dynamic responses of the actual progressive collapse event. Two test results, i.e. SD-2 and SD-3, are used in this chapter to validate proposed FEM models for dynamic condition. These two specimens had similar design, test setup and charge weight placed at the bottom of the middle column. The vertical applied loads on the 
middle column were $27 \mathrm{kN}$ and $47 \mathrm{kN}$ for SD-2 and SD-3, respectively. The results from the first specimen, SD-1, were not so reliable due to inappropriate ground fixing of the two massive restraints (Fig. 2.21) which led to a shift of these buttresses during the blast process. Hence, SD-1 is not considered in the present study.

\subsubsection{Numerical model}

\subsubsection{Geometric properties}

From Yu and Tan (2013a), component-based models using fibre-based beam elements have been employed to simulate the structural response, which was shown to be computationally efficient compared to $3 \mathrm{D}$ solid elements used in the current study. This simplified numerical analysis is helpful for engineers to quickly evaluate the overall static response of sub-assemblages under column removal scenarios. On the other hand, the 3D physics-based models developed in this chapter is more complicated to employ and more time-consuming, but can provide a more detailed structural response, e.g. graphical damage patterns of concrete or physical fracture of rebars. Most importantly, it can simulate dynamic responses under blast effects, which cannot be performed using the simplified beam models.

The explicit finite element software LS-Dyna (Hallquist 2007) is used to simulate the sub-assemblage tests due to its numerical stability, as well as a wide variety of available constitutive models. Based on the assumption of symmetry for loading, geometry, boundary conditions and material properties from the test, only one-half of the specimen is modelled as shown in Fig. 3.1. Concrete is simulated using 8node solid elements with reduced integration scheme. Reinforcing bars are explicitly modelled by 2-node Hughes-Liu beam elements with 2x2 Gauss quadrature integration. This beam formulation is based on degenerated 8-node solid elements. Therefore, the beam element is fully compatible with solid elements, and can still provide computational efficiency and robustness. The Hughes-Liu beam formulation can consider the behaviour of axial force, bi-axial bending, and finite transverse shear strains. 


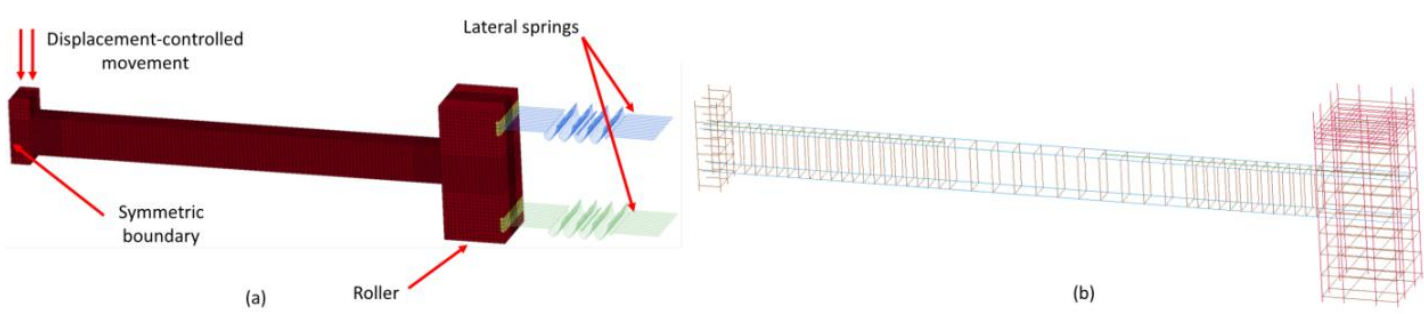

Fig. 3.1 Symmetrical model in LS-Dyna: a) Specimen modelling; b) Reinforcement modelling

\subsubsection{Material models and mesh sizes}

Since numerical results are very sensitive to material properties, choosing the appropriate constitutive model is very important in FEM analyses. To date, many efforts have been made to accurately simulate the behaviour of concrete materials which involves fully nonlinear phenomena such as softening, cracking, and strain rate hardening. Within the material library of LS-Dyna, there are a number of constitutive models which can be used for modelling concrete behaviour such as Mat_Johnson_Holmquist_Concrete (MAT_111), continuous surface cap model MAT_159 (CSCM), Karagozian and Case Concrete Damage REL3 (K\&C), Mat_Winfrith_Concrete (MAT_084), and Riedel-Hiermaier-Thoma model (RHT) (Hallquist 2007). Among them, the three most popularly used models for RC simulations under extreme conditions such as blast or impact loads are K\&C, RHT and CSCM. All of them can effectively capture post-peak softening, shear dilation, confinement effect, and strain rate hardening (Brannon and Leelavanichkul 2009, $\mathrm{Wu}$ et al. 2012). All three of them possess isotropic plasticity and rate-dependence which employ three shear strength surfaces in the field of stress space.

For these three models, the ultimate strength of concrete is dependent on pressure and shear stress. Under uniaxial loading condition, deformation is initially linear within the elastic range and concrete is assumed to be isotropic. But when deformation further increases and a peak stress is reached, degradation in stiffness and strength of material will occur. Concrete will start yielding or failing when the material properties attain the yield surface. During the stress increment, if the trial elastic stress $\sigma^{T}$ is outside the yield surface, plastic-update equations will be 
activated to re-calculate the stress state of the element. When damage occurs, concrete strength is reduced by collapsing the strength surface in the stress space. On the other hand, incorporation of loading rate is accompanied by expanding the yield surface and higher strength levels. Besides the yield surface, all the three models apply two other surfaces to simulate the maximum stress limit and the residual strength of the material, so-called the limit and the residual surfaces (Fig. 3.2(a)). If a stress state attains the limit surface, irreversible damage will occur and cause the boundary of the stress state to collapse until it reaches the residual surface, representing total damage of the material. In RHT and CSCM models, a cap is introduced into the yield surface to consider an elastic limit in pure hydrostatic compression and to allow porosity to affect shear strength.

All three constitutive models have the advantage of simple keyword input, based on unconfined axial compressive strength. This value can be obtained from uniaxial compression tests. Other relevant parameters can be automatically generated by the model itself. Such functions are very useful when there is limited information on the concrete used. In this study, the CSCM model is chosen to simulate the concrete solid elements for both static and dynamic cases due to its accuracy, stability and user-friendly features.

The CSCM model uses a complex formulation that combines shear failure surface with hardening compaction surface. Fig. 3.2(b) shows the general shape of the yield surface of CSCM. The yield surface is formed in terms of three stress invariants, namely, $J_{1}, J^{\prime}{ }_{2}$ and $J^{\prime}{ }_{3}$. The default parameters for inputting concrete materials of CSCM are based on three input data: unconfined compressive strength, aggregate size, and the units used in the simulation. Automatic-generated parameters are most suitable for concrete with the compressive strength between 28 and $48 \mathrm{MPa}$. The best-fit method is primarily based on CEB-FIP Concrete Model Code (2010). Fig. 3.3 shows the stress-strain results from automatic generation of MAT_159 for a single element using concrete with a compressive strength of $30 \mathrm{MPa}$. 


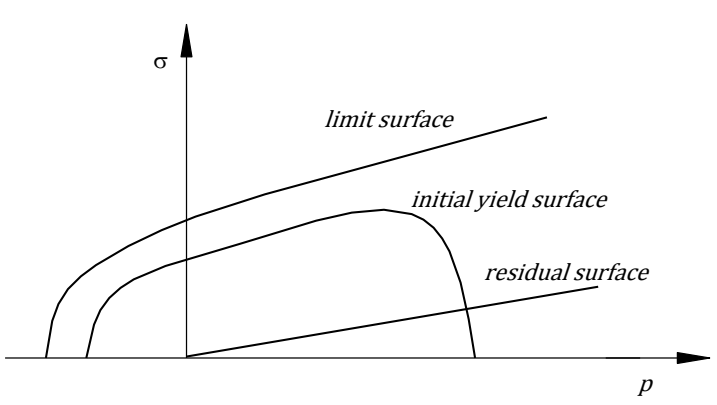

a) For general material models

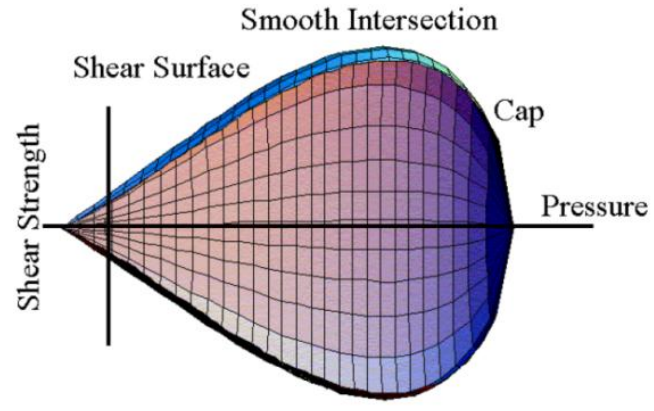

b) For CSCM

Fig. 3.2 Schematic of yield surface

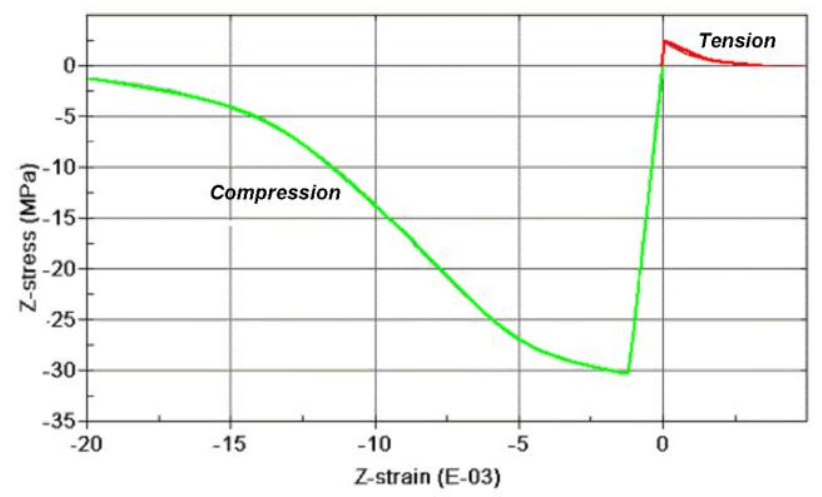

Fig. 3.3 Stress-strain relationship of a single element with $f^{\prime}{ }^{\prime}=30 \mathrm{MPa}$ (Murray 2007)

An isotropic elastic-plastic material model Mat_Piecewise_Linear_Plasticity (MAT_024) is used for steel reinforcement assuming the material behaviour in tension is identical to that in compression. A convergence study is carried out employing three mesh sizes (Table 3.3). Geometry and material properties, as well as the boundary conditions (restraint stiffness and connection gaps) from specimen S1 (Yu 2012) are used for the convergence study. As a result, the mesh size of concrete elements is chosen as $12.5 \mathrm{~mm}$ for the joint regions and $25 \mathrm{~mm}$ for the other regions. Further efforts on refining the mesh do not lead to any remarkable convergence but instead take much longer time and more resources. A comparison study of load-displacement relationship for different mesh sizes is shown in Fig. 3.4 while Fig. 3.5 gives detailed information on the model using Mesh 3. The length of beam element is $25 \mathrm{~mm}$. 
Table 3.3 Mesh-size study

\begin{tabular}{|c|c|c|c|}
\hline Mesh type & Mesh 1 & Mesh 2 & Mesh 3 \\
\hline Mesh size at joint locations (mm) & $25 \times 25 \times 25$ & $12.5 \times 12.5 \times 25$ & $12.5 \times 12.5 \times 12.5$ \\
\hline Mesh size at normal locations (mm) & $25 \times 25 \times 25$ & $25 \times 25 \times 25$ & $12.5 \times 12.5 \times 25$ \\
\hline Total number of solid elements & 16,666 & 19,726 & 48,832 \\
\hline Total number of beam elements & 2,836 & 3,298 & 4,232 \\
\hline Running time (s) & 3,247 & 4,862 & 16,724 \\
\hline Peak compressive arch action (kN) & 53.5 & 51.6 & 50.7 \\
\hline Displacement at the peak (mm) & 80 & 82.5 & 87.5 \\
\hline
\end{tabular}

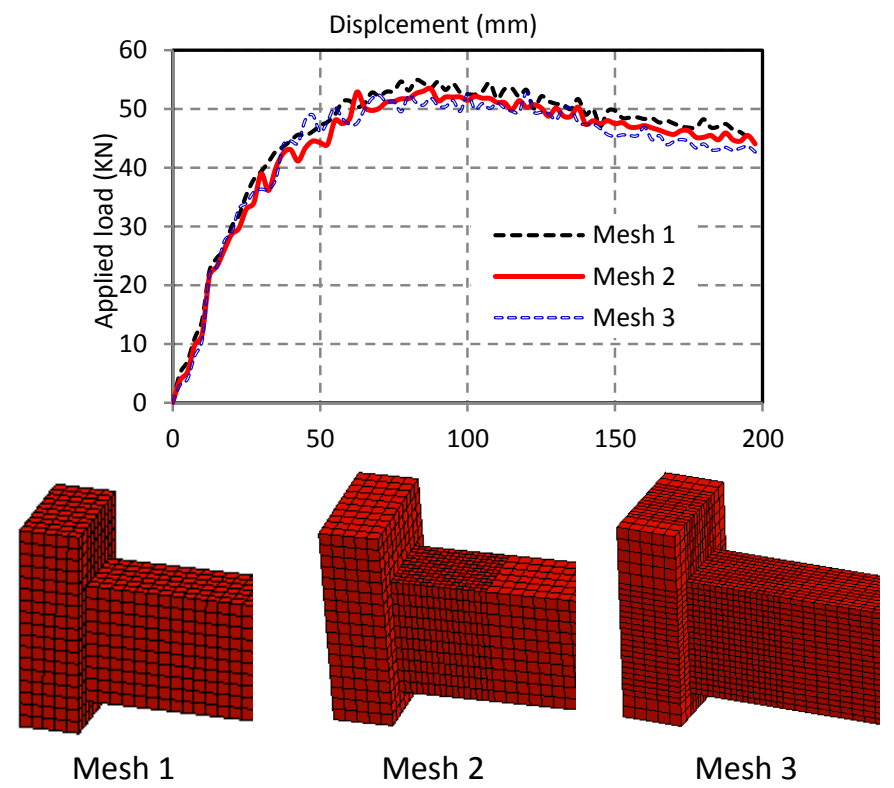

Fig. 3.4 Mesh-size comparisons

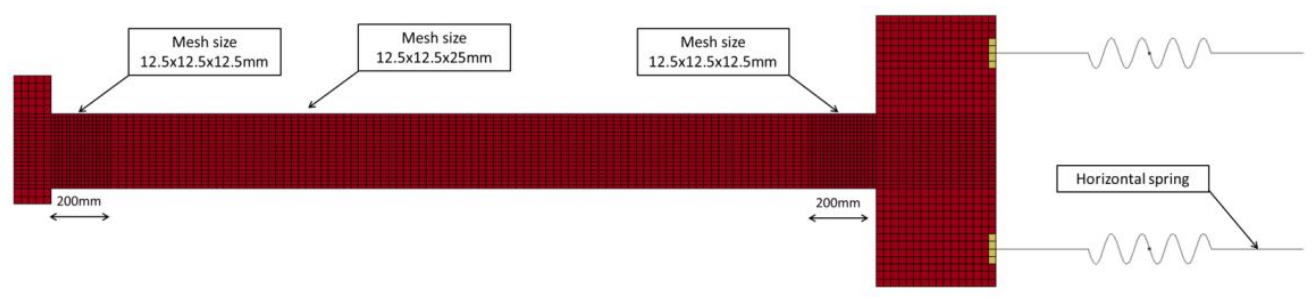

Fig. 3.5 Numerical model using Mesh 3

Although element erosion is not a physical phenomenon for material behaviour, it helps to overcome computational difficulties when large mesh-distortion occurs 
within Lagrangian grids. In the context of concrete material, this attribute allows graphical representation of spalling and separation of concrete under high tensile forces. Among numerical simulations, many erosion criteria are available such as strain-based approaches (maximum principal strain, shear strain), or stress-based approaches (pressure, principal stress, etc.). Their applications, however, require calibrations and validations with actual tests. Previous studies show that erosion criteria and limits are dependent on mesh size (Luccioni and Aráoz 2011). Nonetheless, failure criteria vary significantly between different structural problems as well as among different users.

For RC structures subject to impulsive loads such as impact or blast, the strainbased criterion for erosion using maximum principal strain is preferred (Shi et al. 2010, Tang and Hao 2010, Wu et al. 2011). For sub-assemblages under the column removal scenario, test results showed that the governing mechanisms are flexural and tensile actions, primarily denoted by opening of vertical cracks at the beam ends and development of cracks along the double-span beam. Therefore, the maximum principal strain is also a suitable criterion for erosion algorithm to represent the development of concrete separations during testing. When applying this method, a solid element will be deleted if $\varepsilon_{1} \geq \varepsilon_{\max }$ where $\varepsilon_{1}$ is the maximum principal strain and $\varepsilon_{\max }$ is the principal strain at failure. Since the erosion limits are dependent on mesh size, the appropriate value should be carefully selected based on a specific mesh size to reasonably match with actual damage patterns from actual experiments. In this study, several values of maximum principal strain are tried and the numerical predictions are compared to the sub-assemblage test results (Yu and Tan 2013a, b). It is shown that the maximum strain value of 0.1 gives the best fit with test results in terms of concrete cracking and spalling in tension (Fig. 3.6). Element erosion is also applied for reinforcement material as it reaches the maximum tensile strain to represent fracture of rebars. Engineering stress-strain curves from rebar tensile tests (Fig. 3.7) are converted into true stress-strain curves based on actual gauge length of the material tests and beam-element mesh size in the FEM model. The maximum value of tensile strain is defined when tensile stress reaches the maximum value (before necking part). 
The CSCM model for concrete, incorporating element erosion, does not detect the occurrence and development of cracks in the RC structure at tensile region. Instead, the damage index in the model indicates the level of material failure (from 0 to 1 , i.e. complete failure). On the other hand, element erosion technique helps to illustrate the severe separation of concrete under large deformations (catenary action stage).

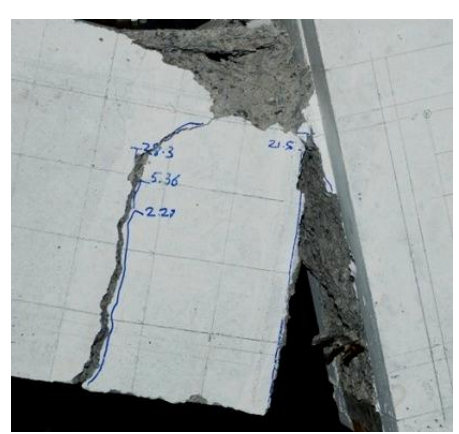

a) Actual test

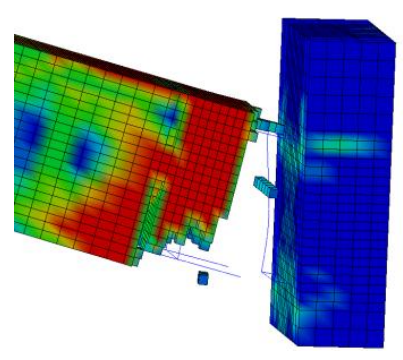

d) $\varepsilon_{\max }=0.05$

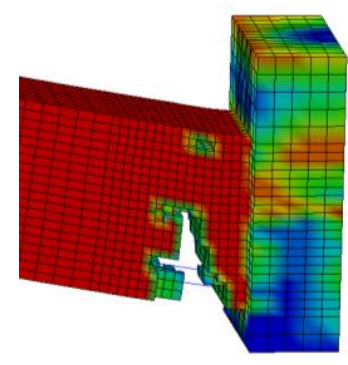

b) $\varepsilon_{\max }=0.22$

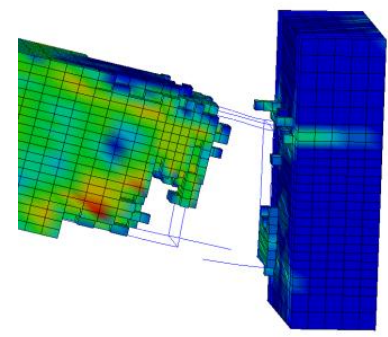

e) $\varepsilon_{\max }=0.01$

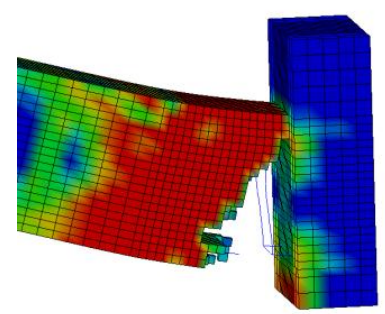

c) $\varepsilon_{\max }=0.1$

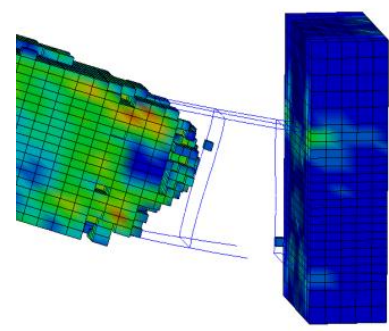

f) $\varepsilon_{\max }=0.003$

Fig. 3.6 Erosion-limit study

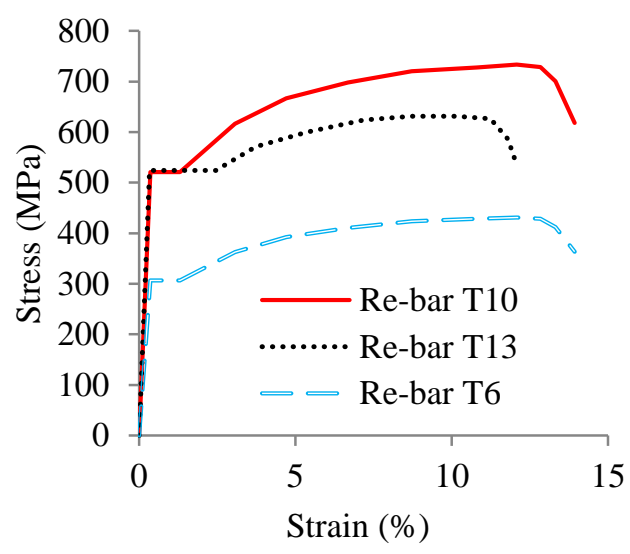

Fig. 3.7 Engineering stress-strain curves for S1 and S2 


\subsubsection{Bond-slip behaviour}

Basically, composite action between concrete and reinforcement is mobilised through bond stress-slip relationship. The assumption of perfect bonding may overestimate the bearing capacity, and leads to premature fracture of rebars (Bao et al. 2014). In this numerical analysis, the bond-slip behaviour is modelled by applying a one-dimensional contact function (Contact_1D) between the concrete solid elements and the rebar beam elements. The slave nodes from the beam elements are mapped onto the master nodes from the solid elements through virtual springs. The value of the spring force depends on relative displacements between the slave nodes and the master nodes (Hallquist 2007).

The bond-slip relationship proposed in CEB-FIB Model Code (2010) is applied in this study. The bond stress $\tau_{b}$ between concrete and rebars for pull-out failure can be defined as follows:

$$
\begin{aligned}
& \tau_{b}=\tau_{\text {bmax }}\left(s / s_{1}\right)^{\alpha} \text { for } 0 \leq s \leq s_{1} \\
& \tau_{b}=\tau_{b \max } \text { for } s_{1} \leq s \leq s_{2} \\
& \tau_{b}=\tau_{b \max }-\left(\tau_{b \max }-\tau_{b f}\right)\left(s-s_{2}\right) /\left(s_{3}-s_{2}\right) \text { for } s_{2} \leq s \leq s_{3} \\
& \tau_{b}=\tau_{b f} \text { for } s_{3} \leq s
\end{aligned}
$$

The parameters $\tau_{\text {bmax }}, \tau_{b f}, s_{1}, s_{2}, s_{3}$ are defined according to Concrete Model Code (2010) and depend on the compressive strength of concrete as well as the bonding condition. On the other hand, the bonding relationship within Contact_1D (LSDyna) is assumed to be elastic-perfectly-plastic. After attaining the maximum shear stress, the bond stress will decay following an exponential damage curve. The constitutive law of bond stress $\tau$ with respect to slip $s$ can be represented by:

$$
\begin{aligned}
& \tau=G_{s} s \text { for } s \leq s_{\max } \\
& \tau=\tau_{\max } e^{-h_{d m g} D} \text { for } s \geq s_{\max }
\end{aligned}
$$

where $G_{s}$ is the bond shear modulus, $s_{\max }$ is the maximum elastic slip, $h_{d m g}$ is the damage curve exponential coefficient and $D$ is the damage parameter. 
To incorporate the bond-slip model from Concrete Model Code (2010) into the onedimensional contact function of LS-Dyna, a simplified relationship proposed by Shi et al. (2009) is adopted. First, $G_{S}$ is defined as the slope of the actual stress-slip curve for the initial elastic range, and then $s_{\max }$ is defined as:

$s_{\max }=\tau_{\max } / G_{S}$

The final parameter, damage curve exponential factor $h_{d m g}$, is chosen to have the best-fit result with the CEB model curve. This method is based on the assumption that the bond stress will be uniformly distributed along the interface between the concrete and the reinforcement, and the average bond stress is used instead of local bond stress. Hence, this Contact_1D function may not give accurate descriptions of local bond-slip behaviour, but can simulate the overall effect of bond-slip on the response of a sub-assemblage subject to CA.

The parameters of the bond-slip model from Concrete Model Code (2010) are defined with regard to concrete cylinder strength $f_{c k}=30 \mathrm{MPa}$, with the assumption of good bonding condition: $\tau_{\max }=13.7 \mathrm{MPa}, s_{1}=1 \mathrm{~mm}, s_{2}=2 \mathrm{~mm}, s_{3}=10 \mathrm{~mm}$, $\tau_{b f}=5.48 \mathrm{MPa}, \alpha=0.4$. The simplified bond-slip model for Contact-1D is defined with the following parameters: $G_{s}=27.4 \mathrm{MPa} / \mathrm{mm}, s_{\max }=0.5 \mathrm{~mm}, h_{d m g}=0.1$. The results for stress-slip relationship of both models are described in Fig. 3.8(a). Test results (Yu and Tan 2013a, b) showed that the bond-slip behaviour and pullout phenomena occurred mostly within the joint regions. To save computational cost, the one-dimensional contact function is applied for longitudinal reinforcement in the joint regions only (Fig. 3.8(b)). For longitudinal rebars in other parts of the beams, perfect bonding between concrete and reinforcement is assumed. The LSDyna keyword Constrained_Lagrange_In_Solid is used to embed the rebar beam elements into the concrete solid elements at these regions. 


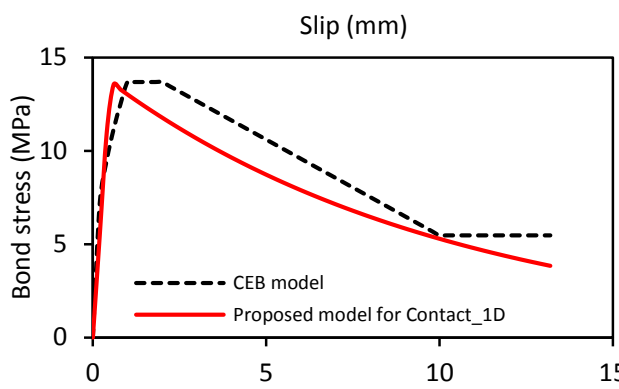

a) CEB vs. Contact_1D

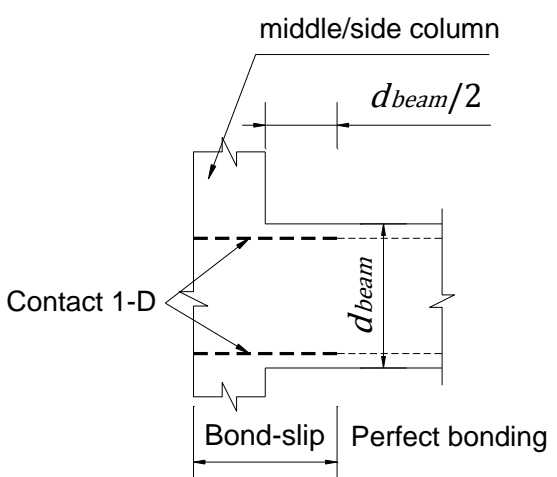

b) Joint region for bond-slip modelling

Fig. 3.8 Bond stress-slip relationship based on CEB model and simplified model for Contact-1D

Comparing numerical models with and without bond-slip simulation (i.e. perfect bonding condition), the one with the bond-slip model captures better rebar fracturing states, whereas in the model with perfect bonding condition, rebars tend to fracture prematurely.

\subsubsection{Model validations by pull-out tests}

In seismic engineering, the bar slip is a critical component affecting the hysteresis behaviour and the deformation capacity of RC structures. Therefore, numerous experimental studies have been conducted to determine bond-slip behaviour. For example, systematic pull-out tests of reinforcing bars were used to investigate local bond-slip behaviour (Viawanthanatepa et al. 1979, Hawkins et al. 1982, Eligehausen et al. 1983, Ueda et al. 1986, Shima et al. 1987). In these tests, large slips of bars occurred when they were subjected to small strains. Compared with bar slips in seismic research, the bond conditions for the bars in progressive collapse are different in the sense that the bars experience large post-yield tension up to fracture. This fact is typically neglected in seismic research. This is because the tensile forcedisplacement relationship determines the slip of the embedded bar at the joint interface, further affecting the capacity of the fixed-end rotation at the beam ends. In this current study, a series of pull-out tests was conducted (Pham et al. 2016) focusing more on the inelastic bond behaviour of continuous bars under wellconfined conditions, and the plastic strain could reach more than 5\%. Typically in previous pull-out tests, inelastic bond behaviour is studied at very limited plastic 
range. It is known that bar yielding weakens the bond, and the current bond-slip tests push this weakening effect to a more extreme condition. The test results are used to ascertain the validity of critical parameters for concrete elements (mesh size, erosion limit, etc.) as well as the proposed bond-slip relationship applied in the numerical models.

The specimens included a reinforcing bar with the middle part embedded in a concrete joint block with the dimensions of $250 \times 250 \times 500 \mathrm{~mm}^{3}$ (Fig. 3.9). Two ends of the rebar extend out from the joint block and were pulled by an Instron testing machine. To investigate the strains of rebar inside the embedment length of 500 $\mathrm{mm}$, three tests were conducted with different diameters: 13, 16 and $20 \mathrm{~mm}$. The mean cylinder strength of concrete was $30 \mathrm{MPa}$. Post-yield strain gauges were arranged within the embedded part of the reinforcing bar at a spacing of 2.5 times the bar diameter. To ensure uniformity of bond stress along the rebar, the bars were grooved and strain gauges were installed inside the groove to prevent them from affecting the actual bond stress distribution. The grooved bar was fabricated by machining two channels along the two longitudinal ribs. Due to this prefabrication, actual perimeter and area of the bar were reduced compared to nominal values. Fig. 3.9 shows an overview of the specimen and the details of strain gauges installed inside the groove, while material properties of reinforcement as well as actual perimeter and area of the grooved bars are included in Table 3.4.
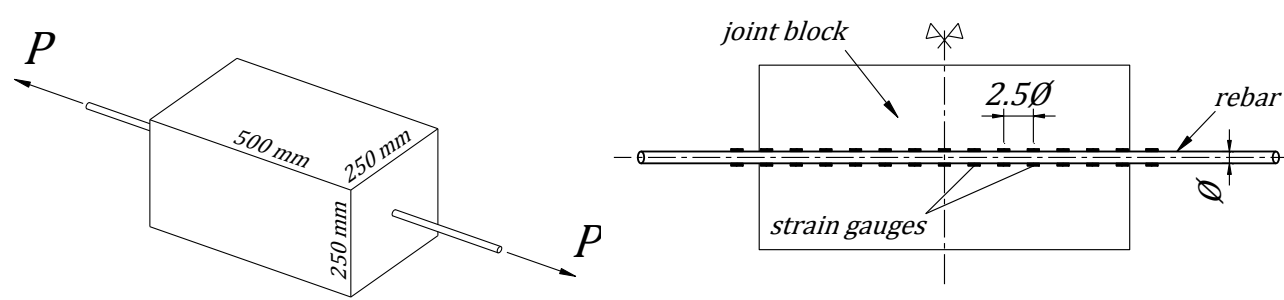

a) Joint block design

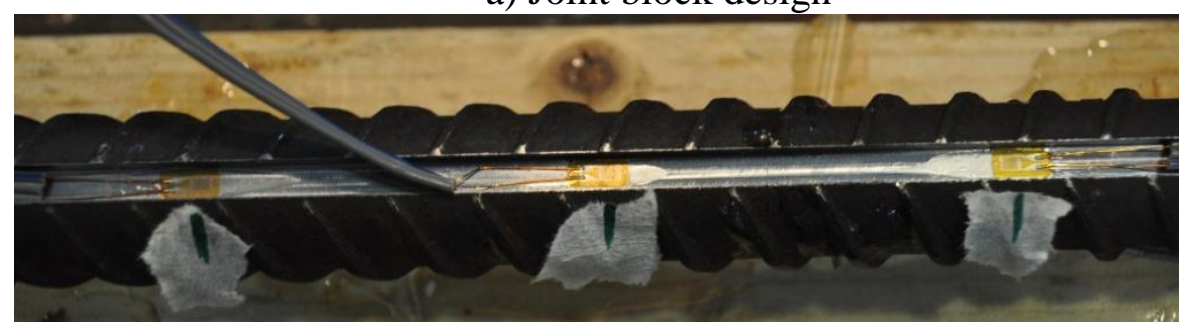

b) Strain gauges installed inside the groove

Fig. 3.9 Typical pull-out specimen with joint block and grooved bar 
Table 3.4 Properties of rebars in the pull-out tests

\begin{tabular}{|c|c|c|c|c|c|c|}
\hline \multirow{2}{*}{ Test } & $\begin{array}{c}\text { Yield } \\
\text { strength }\end{array}$ & $\begin{array}{c}\text { Tensile } \\
\text { strength }\end{array}$ & $\begin{array}{c}\text { Elastic } \\
\text { Modulus }\end{array}$ & $\begin{array}{c}\text { Maximum } \\
\text { strain }\end{array}$ & \multicolumn{2}{|c|}{ Grooved bar } \\
\cline { 2 - 7 } & $\begin{array}{c}f_{y} \\
(\mathrm{MPa})\end{array}$ & $\begin{array}{c}f_{u} \\
(\mathrm{MPa})\end{array}$ & $\begin{array}{c}E_{s} \\
(\mathrm{Mpa})\end{array}$ & $\varepsilon_{u}(\%)$ & $\begin{array}{c}\text { Perimeter } \\
(\mathrm{mm})\end{array}$ & $\begin{array}{c}\text { Area } \\
(\mathrm{mm} 2)\end{array}$ \\
\hline $\mathrm{T} 13-500$ & 550 & 656 & 197443 & 10.3 & 32.2 & 103.19 \\
\hline $\mathrm{T} 16-500$ & 573 & 672 & 218080 & 9.7 & 41.27 & 170.5 \\
\hline $\mathrm{T} 20-500$ & 561 & 689 & 204497 & 13.46 & 55.13 & 279.82 \\
\hline
\end{tabular}

The two ends of the rebar were rigidly gripped by the Instron testing machine. The specimen was fixed at the bottom end and was pulled at the top end following a displacement-controlled manner. The load was measured directly from the machine while deformations were captured by seven linear variable displacement transducers (LVDTs) named D1 to D7, as shown in Fig. 3.10. The LVDTs were divided into three types: (1) overall deformation of the bar (D1), (2) displacements at certain points near the top end (D2 and D3) and near the bottom end (D6 and D7) of the bar, and (3) movement of the joint block (D4 and D5). Due to symmetry, strain values from both sides of the joint block were identical.
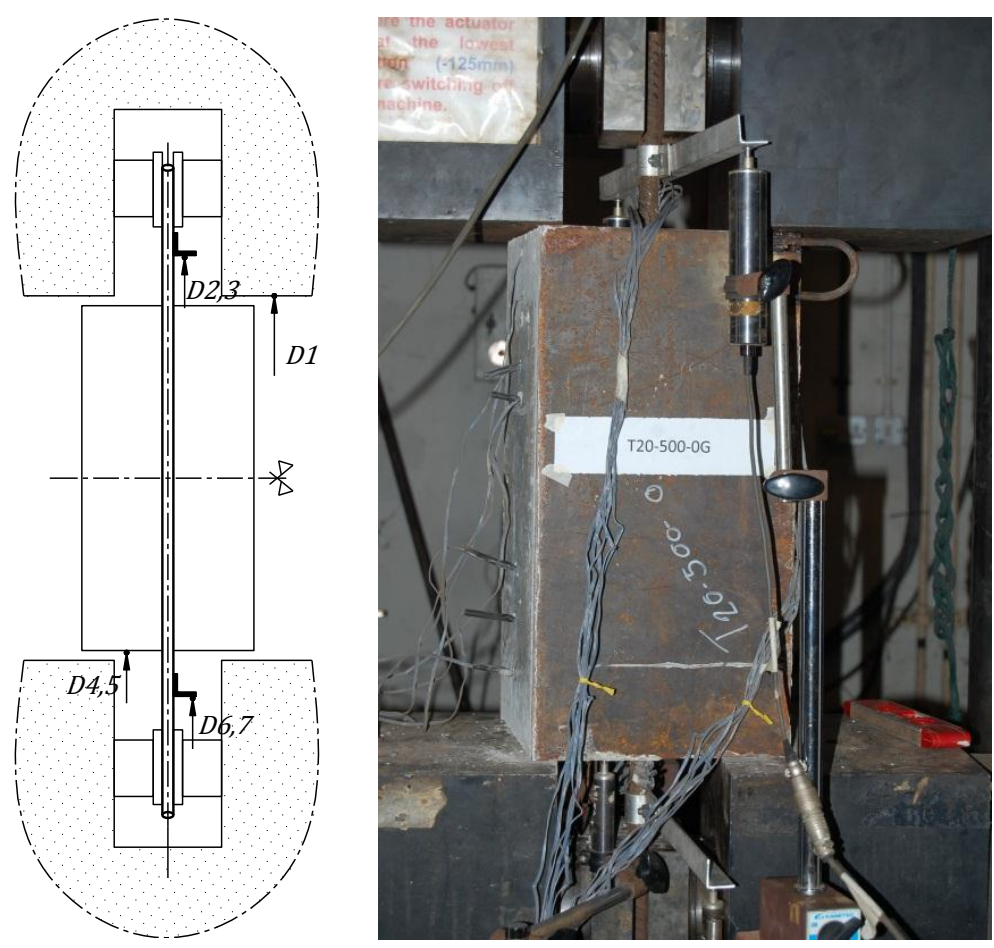

Fig. 3.10 Displacement measurements 
The numerical models applied for the pull-out tests employ similar parameters with the FEM models which simulate the sub-assemblage tests. These variables include material models, mesh size, erosion limit, and bond-slip relationship. The pull-out test data for the validations consist of relationship between the applied load and the displacement of the rebar at the interface of the joint block and the strain distribution in the reinforcement within the embedment length. The FEM models give very good agreement in terms of load-displacement relationship with all the three tests (Fig. 3.11). With regard to strain distribution, the numerical models have good prediction of the strain values along the embedment length if the rebar is still within the elastic range. When applied load is relatively large and reinforcement starts yielding, the strain values from FEM do not always agree well with test results. Nonetheless, the FEM simulations can still provide some qualitative information on the extent of yielding of the embedded reinforcing bar. In other words, the number of strain gauges which have yielded is similar between the numerical and the test results. Fig. 3.12 to Fig. 3.14 present comparisons of strain distribution between the numerical predictions and the tests for all three specimens. Due to symmetry, only the strains from half of the embedment length are shown. With the validation results from the pull-out tests, the proposed parameters of the FEM models are shown to be reliable. These models and the associated parameters are then applied to simulate sub-assemblage tests in Section 3.2.1.

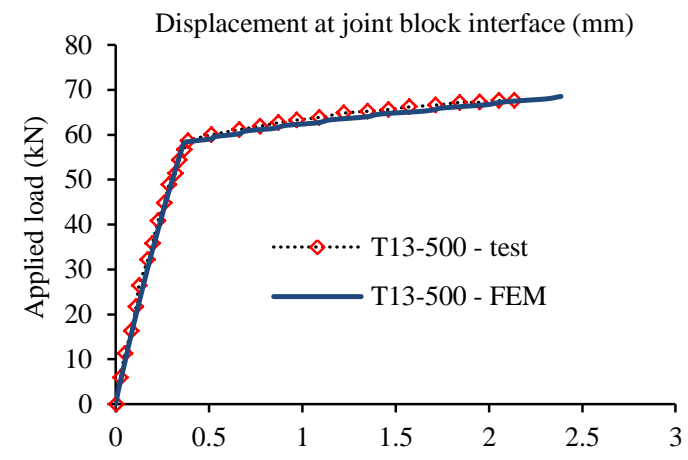

a) $\mathrm{T} 13-500$

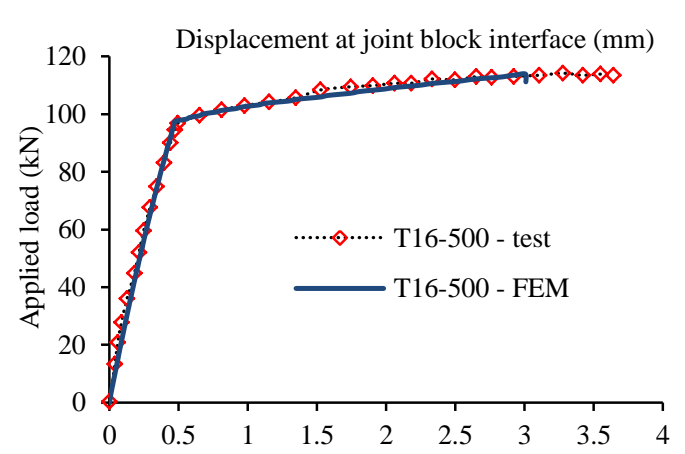

b) $\mathrm{T} 16-500$ 


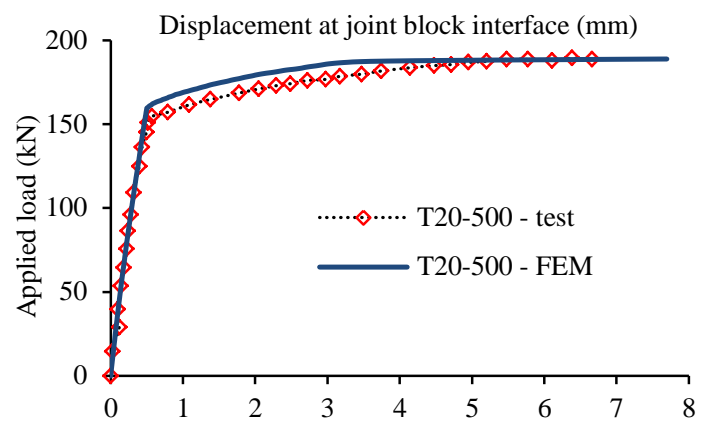

c) $\mathrm{T} 20-500$

Fig. 3.11 Applied load-displacement

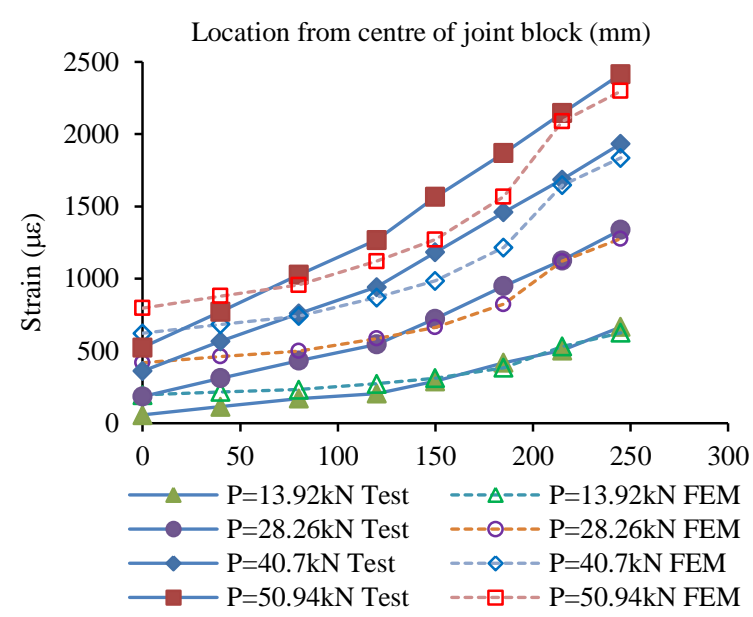

a) Elastic range

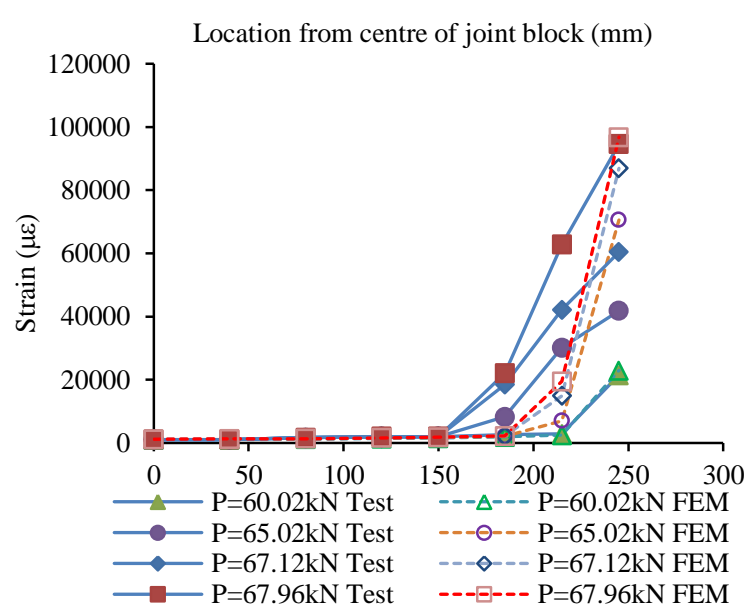

b) Post-yield range

Fig. 3.12 Strain distribution of $\mathrm{T} 13-500$

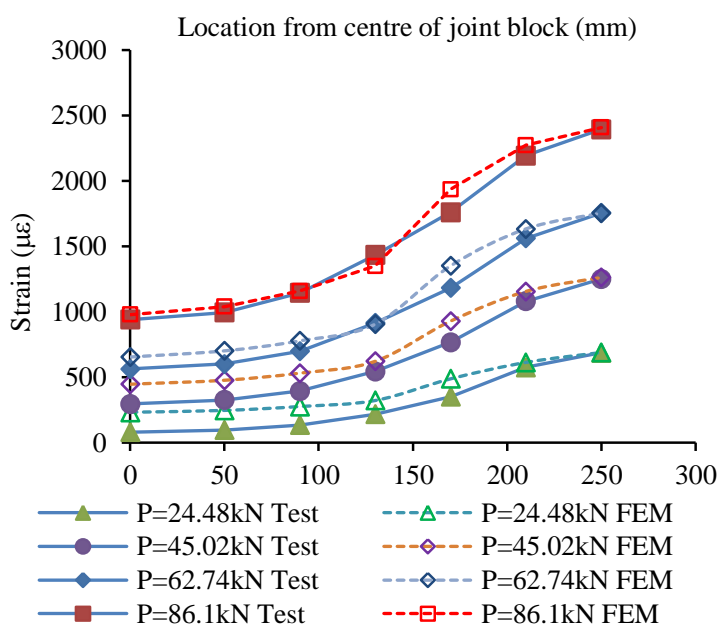

a) Elastic range

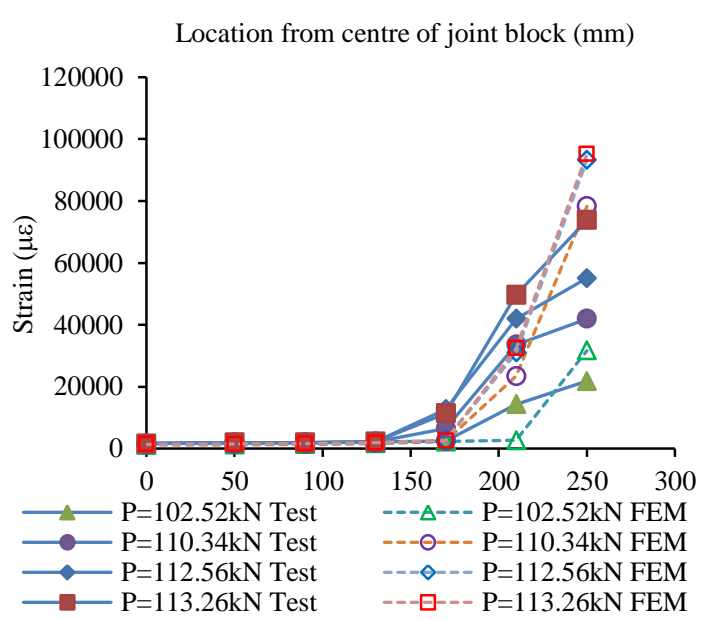

b) Post-yield range

Fig. 3.13 Strain distribution of T16-500 


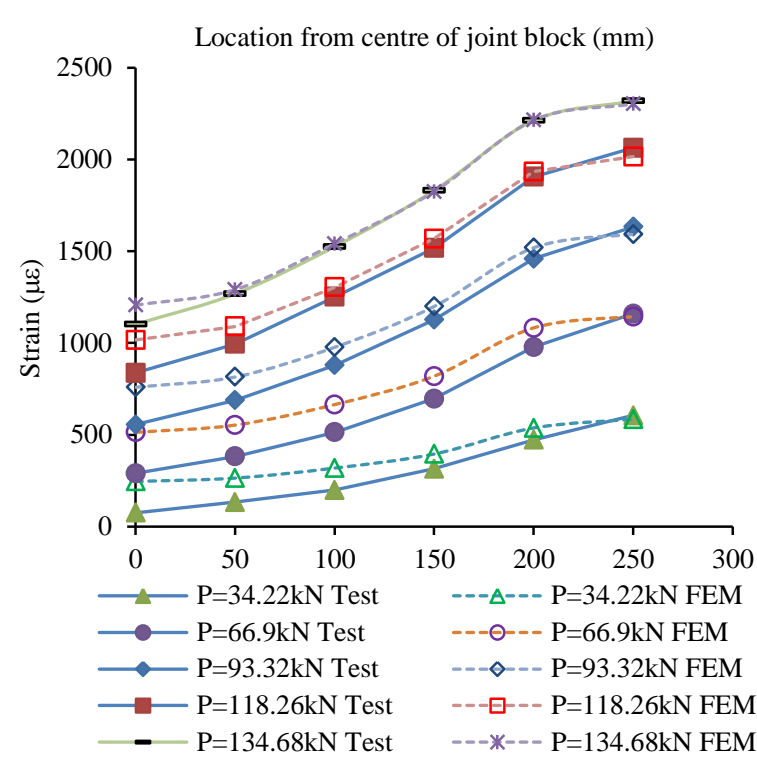

a) Elastic range

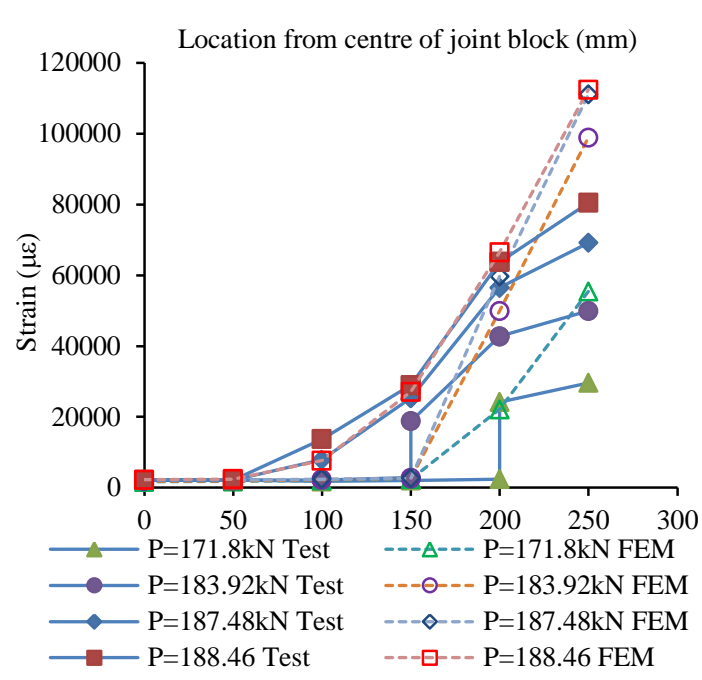

b) Post-yield range

Fig. 3.14 Strain distribution of T20-500

\subsubsection{Boundary conditions}

Imperfect boundary conditions have significant effects on structural behaviour, especially on the development of CAA and CA. Static test results (Yu 2012) showed different stiffness values of horizontal restraints for the sub-assemblage tests. Besides, connection gaps in the supports were also recorded in the tests through horizontal load cell readings and horizontal displacement-transducer measurements. In this study, the boundary conditions are considered by a series of translational springs with nonlinear elastic behaviour, assuming that the spring stiffness values from both sides of the specimens are similar (Table 3.5). However, in the blast tests (Yu et al. 2014), the reaction stiffness value of horizontal restraints was not measured. Hence, it is treated as a variable to be determined by trial and error from actual blast test measurements. From structural dynamics, the fundamental period of an SDOF system depends on the mass and stiffness by the following equation:

$w_{D}=w_{n} \sqrt{\left(1-\zeta^{2}\right)}=\sqrt{\frac{k}{m\left(1-\zeta^{2}\right)}}$ 
where $w_{D}$ and $w_{n}$ are the damped circular frequency and natural circular frequency of the system, $k$ and $m$ are the stiffness and the mass of the system, respectively, and $\zeta$ is the equivalent damping ratio.

Assuming the fundamental mode of the sub-assemblage system is the vertical vibration of the middle joint, the fundamental period can be determined using Eq. (3.8). From test results of SD-2, the fundamental period of vibration within the gravity-load phase was $310 \mathrm{~ms}$. For an RC member under dynamic vibration, the damping ratio of the global system can be generally specified as 5\% and it usually does not significantly affect the value of the frequency. Moreover, the mass of the sub-assemblage system is already known. Therefore, only the k-stiffness is a variable which depends on lateral spring stiffness. The stiffness values of top and bottom springs are assumed to be equal. From trial and error, total lateral stiffness of each spring series within the range of $3.5 \times 10^{4}$ to $5 \times 10^{4} \mathrm{kN} / \mathrm{m}$ gives reasonable predictions of the fundamental period. In this study, the stiffness value of $3.5 \times 10^{4}$ $\mathrm{kN} / \mathrm{m}$ is chosen because it gives the best fit with test data in terms of deflections of the middle joint.

Table 3.5 Stiffness of horizontal restraint

\begin{tabular}{|c|c|c|c|c|c|}
\hline Specimen & $\begin{array}{c}\text { Horizontal } \\
\text { restraints }\end{array}$ & $\begin{array}{c}\text { Tension stiffness } \\
(\mathrm{kN} / \mathrm{m})\end{array}$ & $\begin{array}{c}\text { Compression } \\
\text { stiffness }(\mathrm{kN} / \mathrm{m})\end{array}$ & $\begin{array}{c}\text { Tension } \\
\text { Gap (mm) }\end{array}$ & $\begin{array}{c}\text { Compression } \\
\text { Gap (mm) }\end{array}$ \\
\hline \multirow{2}{*}{ S1 } & Top & 43234 & & 1.8 & \\
\hline & Bot & 42589 & 122602 & 1.2 & 4.6 \\
\hline \multirow{2}{*}{ S2 } & Top & 55957 & & 0 & \\
\hline & Bot & 63942 & 102327 & 1.7 & 3.9 \\
\hline \multirow{2}{*}{ S3 } & Top & 103079 & & 2.5 & \\
\hline & Bot & 40786 & 159871 & 4.4 & 1 \\
\hline \multirow{2}{*}{ S4 } & Top & 130483 & & 1.6 & \\
\hline & Bot & 65952 & 214886 & 3.8 & 3.2 \\
\hline \multirow{2}{*}{ S5 } & Top & 132227 & & 2.1 & \\
\hline & Bot & 82650 & 195344 & 3.8 & 1.95 \\
\hline \multirow{2}{*}{ S6 } & Top & 116470 & & 2.9 & \\
\hline & Bot & 73976 & 189707 & 2 & 4.9 \\
\hline \multirow{2}{*}{ S7 } & Top & 91500 & & 2 & \\
\hline & Bot & 72000 & 234000 & 2.5 & 4.2 \\
\hline \multirow{2}{*}{ S8 } & Top & 88000 & & 3.5 & \\
\hline & Bot & 120000 & 195000 & 3.5 & 3.4 \\
\hline
\end{tabular}




\subsubsection{Analysis procedure and validation results for quasi-static tests}

For quasi-static models, initially gravity load is gradually applied, and then the middle joint is forcibly moved downwards through a displacement-controlled regime until both the bottom and the top reinforcing bars of the beam-column joint fracture. Explicit analysis procedure is employed to ensure stability and convergence of numerical solutions. The numerical predictions are compared to the test results. Generally, the FEM models show reasonably good agreement in terms of overall response, horizontal reactions, as well as failure modes of both the concrete and the reinforcement near the joint regions. Fig. 3.15 shows a comparison between the test and the numerical results for eight specimens (viz. S1 to S8) with regard to MJD versus applied load and horizontal reaction. Numerical models give very close predictions of the first peak from CAA and the final phase due to residual capacity from CA. From the first peak to the instant when the bottom rebars fracture, numerical models give reasonable results for S3, S4, S6 and S7. However, numerical results of S1, S2 and S5 have some divergence compared to the test behaviour. During the quasi-static tests of Yu and Tan (2013a), S1 and S2 did not have rotational restraint at the middle joint. Therefore, when the MJD became remarkable, the middle joint started rotating. On the other hand, in the simulation, perfect rotational restraint is presumed. As a result, there are differences between the test and the modelling results for S1 and S2. For S5, bottom rebars at two sides of the middle joint fractured at different times, which was not the case for the FEM analysis. Therefore, the actual response of S5 showed higher capacity compared to the FEM prediction.

When comparing among the test results, it is observed that the vertical deflection corresponding to the fracture of bottom reinforcement was not consistent from test to test. Experimental observations also revealed that rebars from two sides of the middle joint did not fracture at the same time. Compared to applied load, model predictions of horizontal reactions match better with test results. In other words, the structural stiffness of adjacent restraints from the tests is well captured in all the numerical validations. Results from both test and numerical model show very limited enhancement of CA in specimen S8. 

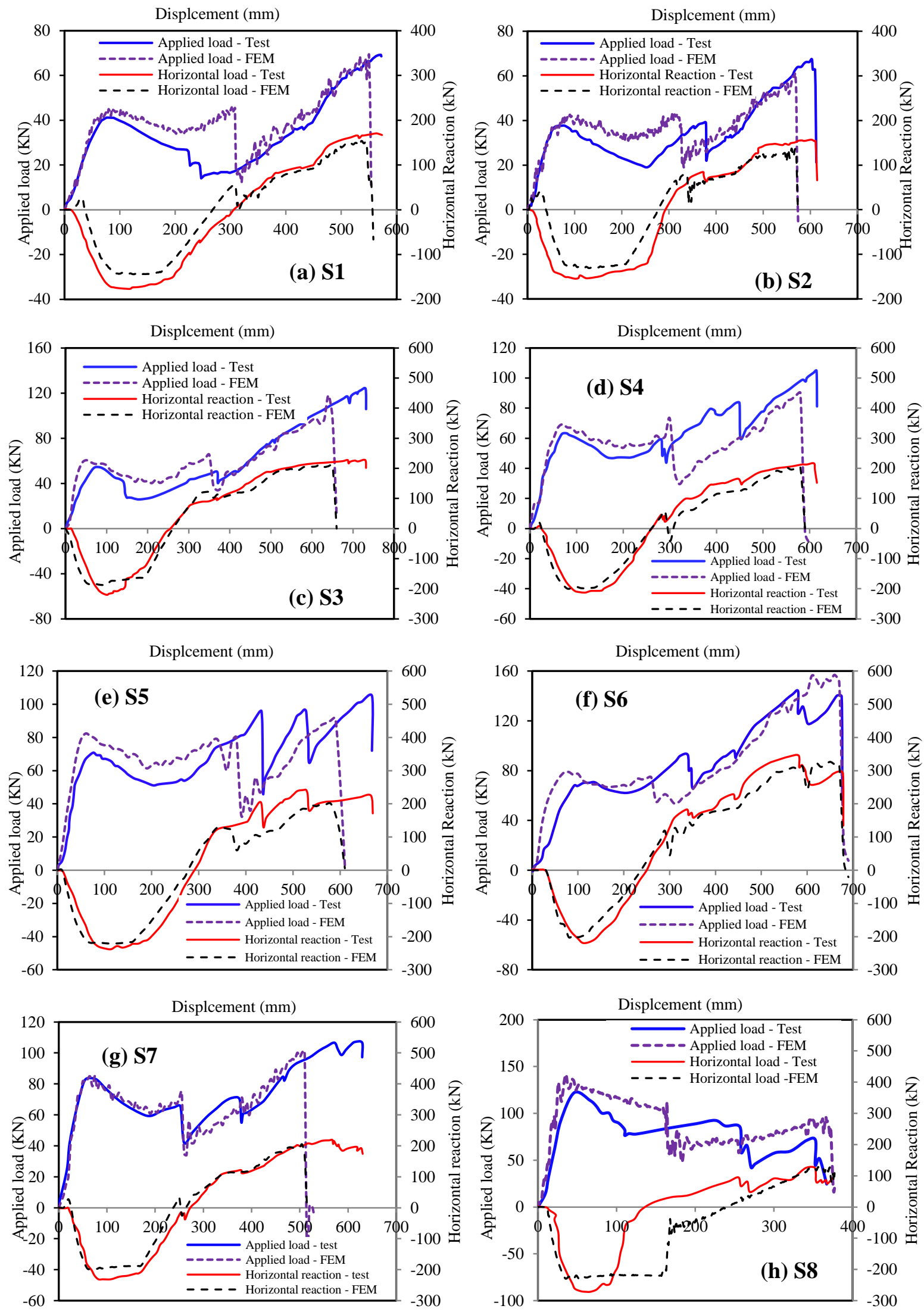

Fig. 3.15 Validation results 
To quantitatively evaluate the accuracy of the simulations, an error analysis is conducted comparing critical values between FEM predictions and test results. They include the applied load at (1) the peak of CAA, the instants of (2) bottom rebar fracture and (3) top rebars fracture. The minimum and maximum horizontal reactions are also used for comparison. Besides, the Izzuddin method is employed to calculate the maximum dynamic loads (based on strain energy) for both the actual tests and the FEM results. For each comparison, Mean and COV (coefficient of variation) values are calculated. The results are shown in Table 3.6 and Table 3.7. In this error analysis, a "close" or "good" agreement is defined when the mean value is in the range of 0.9 to 1.1 and the $\mathrm{COV}$ is not larger than 0.15 . As such, it is shown that most of the values used in the comparison except the applied load corresponding to fracture of bottom rebar satisfy this criterion. 


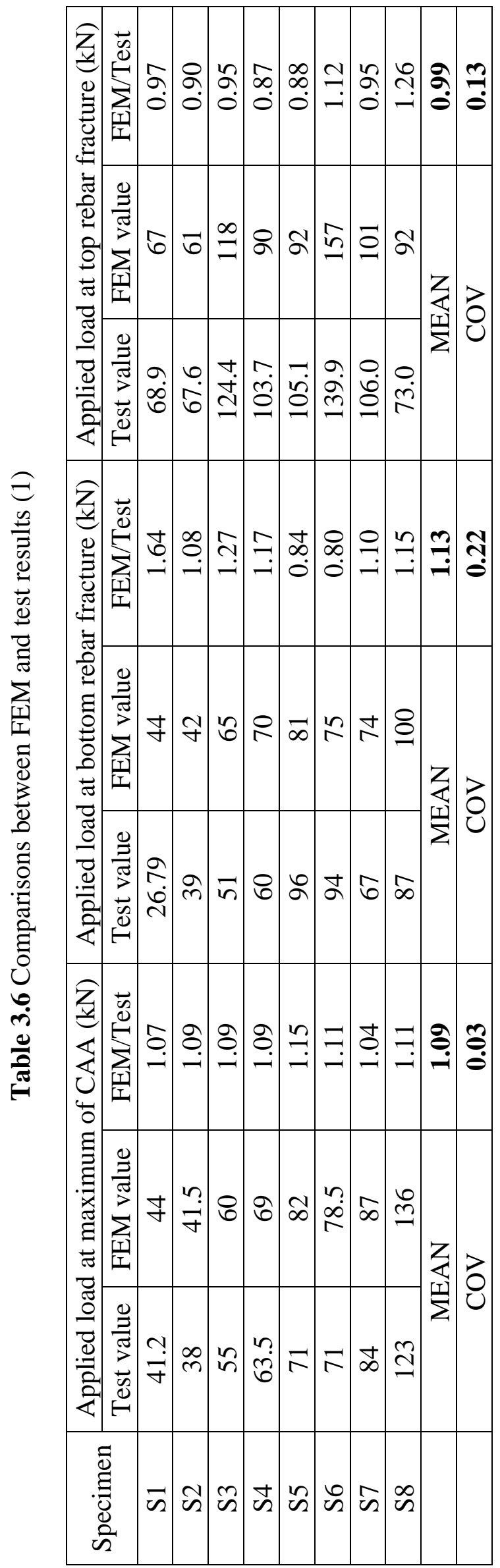




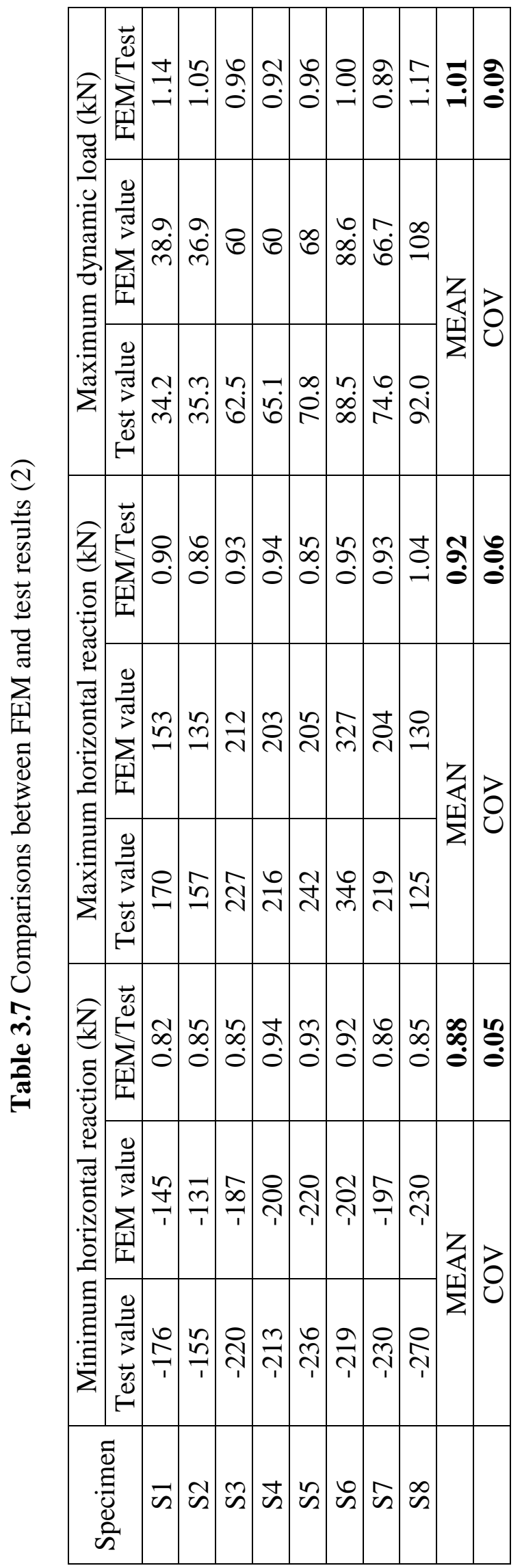


In terms of damage patterns, numerical models agree fairly well with tests for both concrete and longitudinal reinforcement. Fig. 3.16 shows a comparison of the final stage of S1 between the test and the simulation. In the FEM results, concrete failure is represented by the value of Damage index and the erosion of concrete element. An index of 0 denotes no failure and 1 indicates that concrete material is completely damaged. It is clear that both the concrete damage by cracking (at the beam surface near the end joint and at the middle span) and by spalling (at the beam end near the middle joint) are successfully simulated in the model. Besides, the fracture of bottom rebars at the middle joint and top rebars at the end joint are simulated in the models. Fig. 3.17 shows another comparison of damage patterns between the test and the numerical results for specimen S5. The damage level in the joint regions is also portrayed through the FEM analyses. Fig. 3.18 and Fig. 3.19 describe the failure modes of joint regions for specimens S3, S4 and S6. In summary, spalling and damaging of concrete as well as fracturing of reinforcement are clearly captured in FEM analyses and they show good resemblance with experimental results.

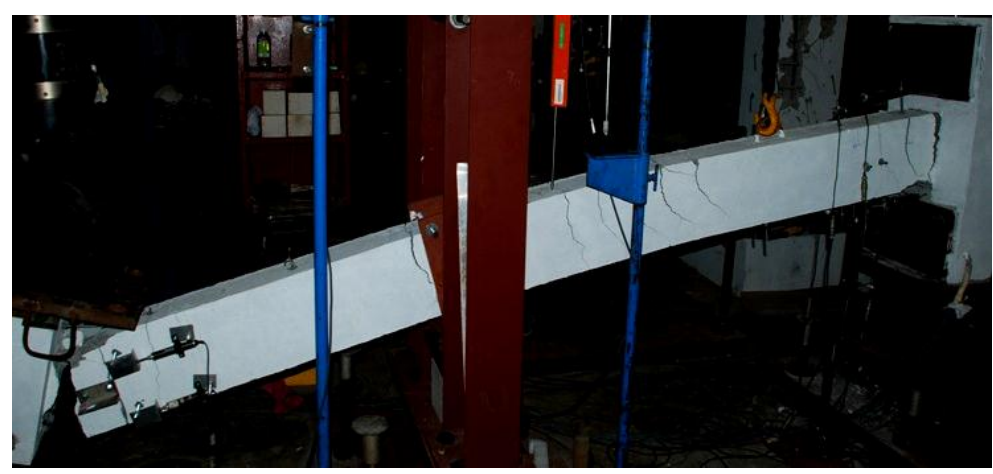

a) Photographs of final stage (Yu and Tan 2013a)

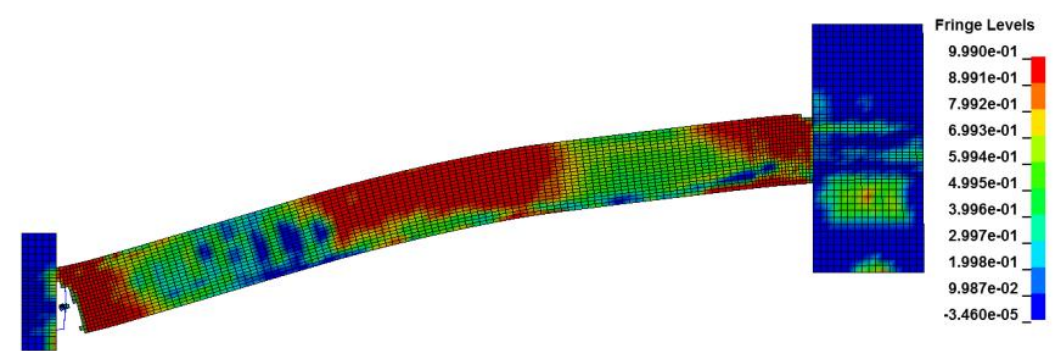

b) Damage index and concrete erosion from FEM model

Fig. 3.16 Comparisons of damage patterns for $\mathrm{S} 1$ 


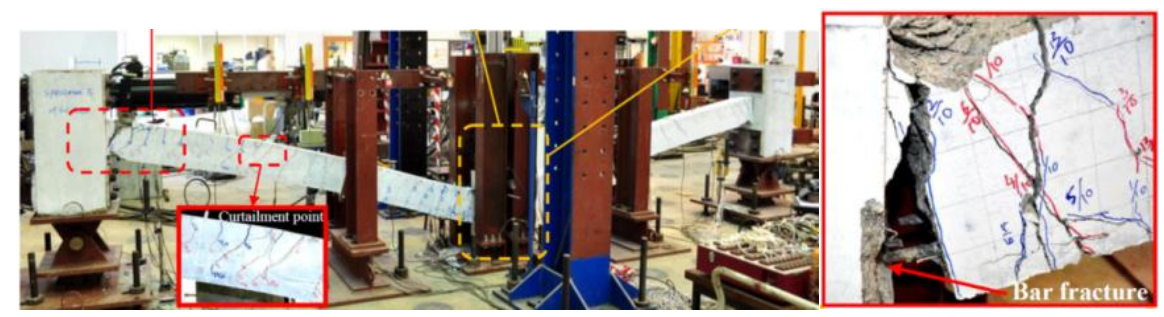

a) Failures observed during test ( $\mathrm{Yu}$ and Tan 2013b)
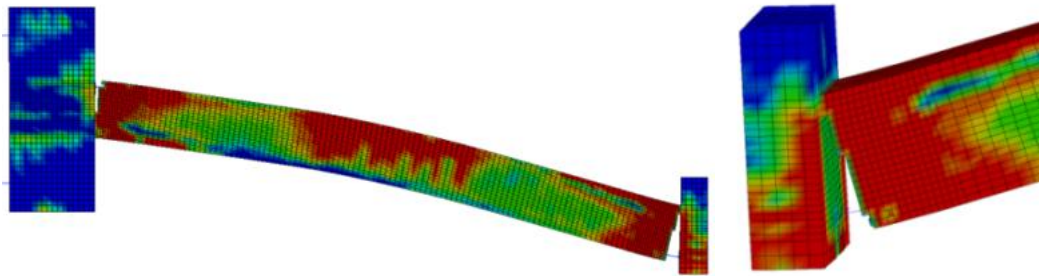

b) Failures observed during FEM

Fig. 3.17 Damage patterns for S5
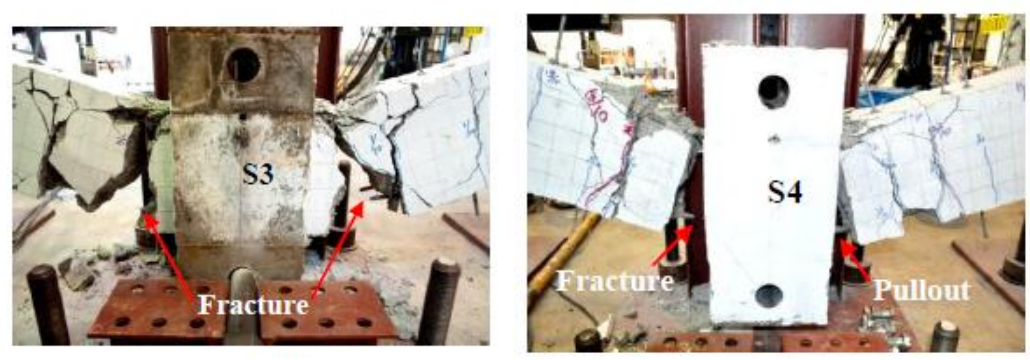

a) Test observations (Yu and Tan 2013b)
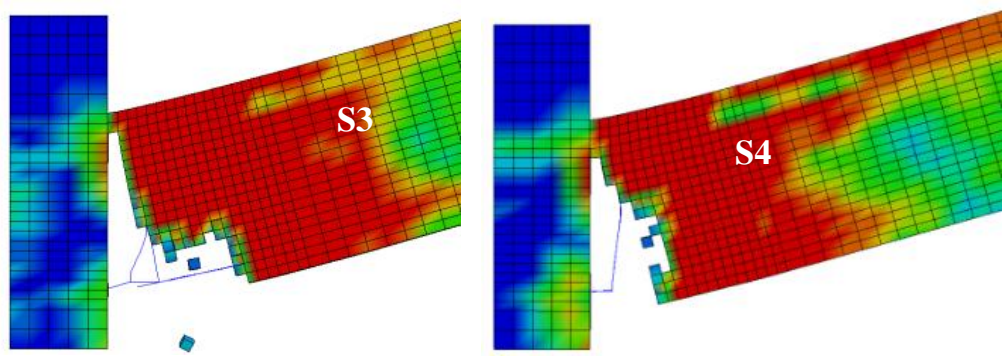

b) FEM

Fig. 3.18 Middle-joint failures of S3 and S4 


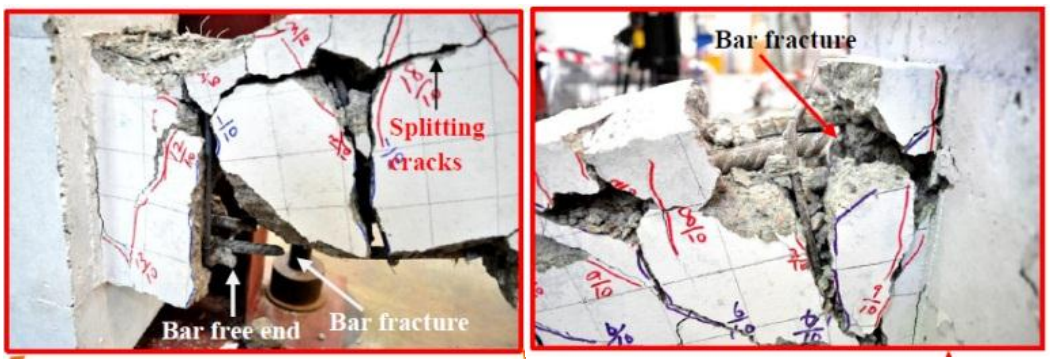

a) Failures observed by test (Yu and Tan 2013b)

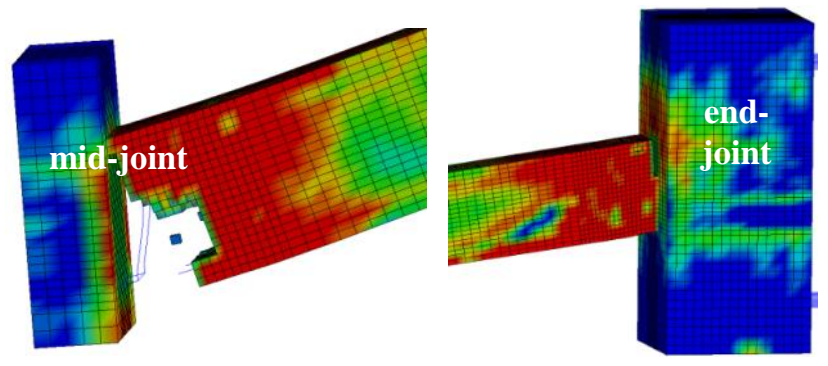

b) Failures observed by FEM

Fig. 3.19 Failures observed by FEM for S6

\subsubsection{Strain rate effect on the simulation of the blast series}

Strain rate effects on material behaviour are usually represented by a Dynamic Increase Factor (DIF) which is the ratio of dynamic strength to static strength with regard to a specific strain rate. The CEB-FIB Model Code (2010) proposes Eqs. (3.9) to (3.12) to determine the DIF values for compression and tension strengths of concrete with the assumption that they only depend on strain rate. These equations are applied in this study to consider the effect of strain rate on concrete material. For reinforcing steel, the empirical Eqs. (3.13) to (3.15) on strain rate enhancement factors for steel proposed by Malvar (1998) is utilised and the DIF is determined for both yield strength and ultimate strength.

\section{Concrete}

For concrete in compression:

$$
\begin{aligned}
& C D I F=\frac{f_{c d}}{f_{c s}}=\left(\frac{\dot{\varepsilon}_{c}}{\dot{\varepsilon}_{c 0}}\right)^{0.014} \text { for } \dot{\varepsilon}_{c} \leq 30 s^{-1} \\
& C D I F=\frac{f_{c d}}{f_{c s}}=0.012\left(\frac{\dot{\varepsilon}_{c}}{\dot{\varepsilon}_{c 0}}\right)^{1 / 3} \text { for } \dot{\varepsilon}_{c} \geq 30 s^{-1}
\end{aligned}
$$


For concrete in tension:

$$
\begin{aligned}
& \text { TDIF }=\frac{f_{t d}}{f_{t s}}=\left(\frac{\dot{\varepsilon}_{c t}}{\dot{\varepsilon}_{c t 0}}\right)^{0.018} \text { for } \dot{\varepsilon}_{c t} \leq 10 s^{-1} \\
& T D I F=\frac{f_{t d}}{f_{t s}}=0.0062\left(\frac{\dot{\varepsilon}_{c t}}{\dot{\varepsilon}_{c t 0}}\right)^{1 / 3} \text { for } \dot{\varepsilon}_{c t} \geq 10 s^{-1}
\end{aligned}
$$

where $f_{c s}$ and $f_{t s}$ are the static compressive strength and static tensile strength of concrete, $f_{c d}$ and $f_{t d}$ are the dynamic compressive strength and dynamic tensile strength of concrete, respectively. $\dot{\varepsilon}_{c}$ and $\dot{\varepsilon}_{c t}$ are strain rates in $\mathrm{s}^{-1}$ for concrete in compression and tension, respectively. $\dot{\varepsilon}_{c 0}$ is equal to $30 \times 10^{-6} \mathrm{~s}^{-1}$ and $\dot{\varepsilon}_{c t 0}$ is equal to $1 \times 10^{-6} \mathrm{~s}^{-1}$.

\section{Reinforcing steel}

$\mathrm{DIF}=\left(\frac{\dot{\varepsilon}}{10^{-4}}\right)^{\alpha}$

For yield strength: $\alpha=\alpha_{f y}=0.074-0.040 \frac{f_{y}}{414}$

For ultimate strength: $\alpha=\alpha_{f u}=0.019-0.009 \frac{f_{y}}{414}$

where $\dot{\varepsilon}$ is the strain rate in $\mathrm{s}^{-1}$ and $f_{y}$ is the steel yield strength in MPa.

\subsubsection{Simulation of blast pressure in LS-Dyna}

An explosion is a physical-chemical phenomenon which causes a shock wave, or a so-called blast pressure, propagating away from the detonation point. The velocity and magnitude of the shock wave depend not only on the properties of the explosive but also on the medium that it travels through. Basically, the time-history of a blast pressure includes two phases, i.e. the positive phase and the negative phase. The latter is normally neglected in structural analysis. The positive phase is usually described by two important parameters, namely, peak pressure $p_{\max }$ and duration time $t_{d}$. For a free-field air-burst explosion, the blast pressure can be predicted by using the modified Friedlander equation (Baker 1973): 


$$
p(t)=p_{0}+p_{\max }\left(1-\frac{t^{\prime}}{t_{d}}\right) \exp \left(-\frac{b t^{\prime}}{t_{d}}\right)
$$

where $t^{\prime}=t-t_{a}$ in which $t_{a}$ is the arrival time of the blast (see Fig. 3.20).

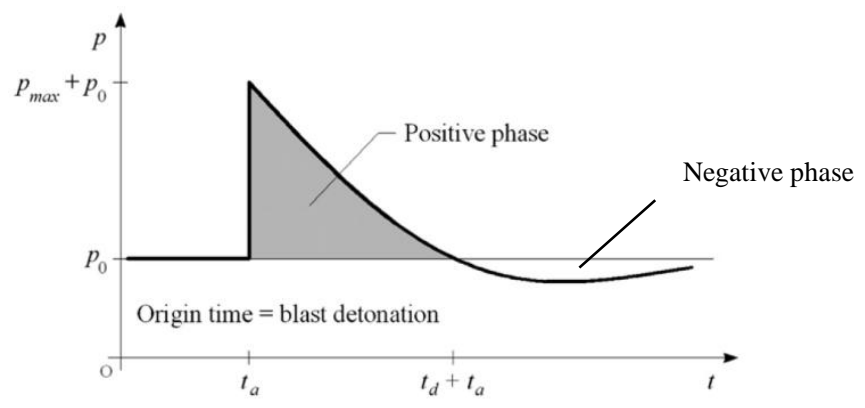

Fig. 3.20 Time-history of a typical blast pressure

The total kinetic energy of a blast pressure, called the blast impulse, is equal to the area under the positive phase curve and can be calculated by the equation below:

$i_{s}=\int_{t_{a}}^{t_{a}+t_{d}} p(t) d t$

To normalise the effect of both explosive weight and distance to the target, blast scaling laws have been proposed based on the weight of an equivalent TNT explosive. The most commonly-used scaling law, called the "cube root scaling", states that if two charges with similar explosive and geometry are being detonated in the same environmental condition, the generated blast waves are similar with the same scaled distance $Z$. The following equation defines the value of the scaled distance $Z$ :

$Z=\frac{R}{\sqrt[3]{W}}$

where $W$ is the weight of the explosive and $R$ is the distance from the explosion.

During the propagation period, if the shock wave encounters a target or a surface, a reflected pressure will be generated. The reflected pressure has a similar overall shape compared to the incident pressure but with a greater peak value. The parameters of the reflected pressure depend on the incident wave as well as the 
contact angle with the surface. Different types of free-field explosion can create different values of blast pressure consisting of free air-burst, air-burst and groundburst. Fig. 3.21 describes the differences in blast location with regard to the ground surface and target position for these three scenarios.

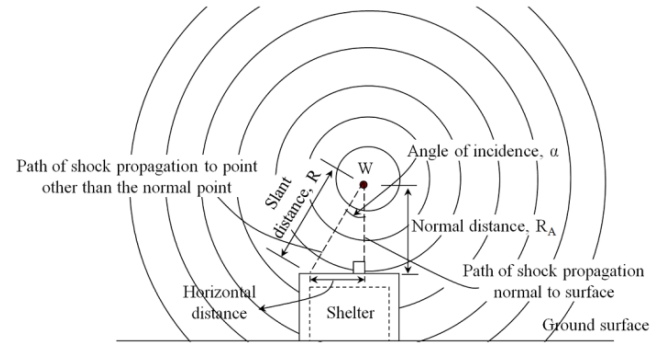

(a) Free air-burst

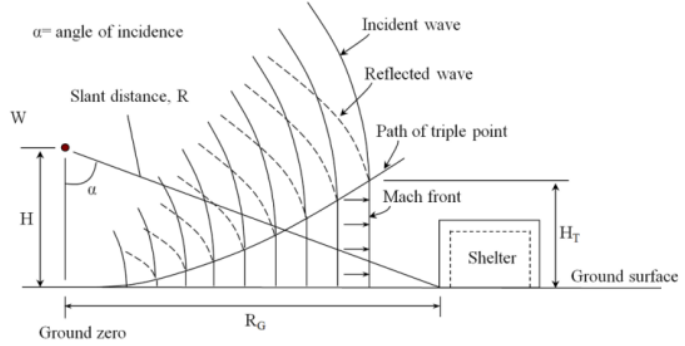

(b) Air-burst with ground reflection

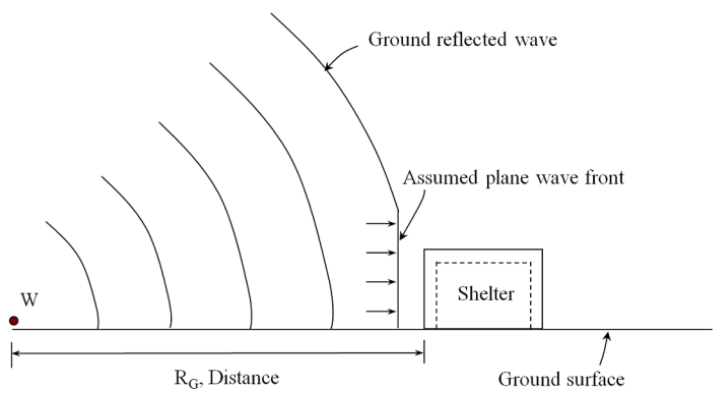

(c) Ground-burst

Fig. 3.21 Different types of free-field explosion (UFC 3-340-02)

The evaluation of blast loads on structures based on the scaled distance of equivalent TNT weight can be found in U.S. Army manuals such as TM5-855-1 (1986) or UFC 3-340-02 (2008) for these three air-blast cases. An analytical programme developed in the early 1990's, CONWEP (Hyde 1991), has been widely used by researchers and engineers to calculate the pressure and effect from air blast for different explosive situations. This programme is based on semi-empirical equations and tabulated data from TM5-855-1 (1986) manual. It can be used to calculate blast pressure on structural members.

To calculate the blast loads acting on the assemblage, a blast load function integrated with LS-Dyna, Load_blast_enhanced keyword, is implemented in this study. This function employs analytical formulae and blast data available in the 
CONWEP. It allows users to define a specific weight of equivalent TNT explosion at a certain position and a detonation time of the blast. Three blast scenarios are available, namely, spherical air-burst, hemispherical ground-burst, and spherical airburst with ground reflection (Hallquist 2007). The time-history of blast loads will then be calculated automatically and applied to the targeted surfaces of structural models. The value of both incident and reflected pressure can be computed for a specific position. The applicable range of this function is for the scaled distance from 0.147 to $40 \mathrm{~m} / \mathrm{kg}^{1 / 3}$ for spherical air-burst, and from 0.178 to $40 \mathrm{~m} / \mathrm{kg}^{1 / 3}$ for hemispherical surface-burst. In the blast tests, the same amount of composite explosive C-4 was used for both SD-2 and SD-3. Due to confidentiality of the test, the value of charge weight could not be revealed in the technical paper for experimental programme (Yu et al. 2014). In this current study, therefore, the same value of ideal TNT with the tests, so-called $m \mathrm{~kg}$ of TNT, is chosen for the Load_blast_enhanced function to generate an equivalent blast impulse. The location of blast is selected under the bottom of the middle-column to simulate the blast effect. Blast loads are assigned for the bottom and the side faces of both the beam and the middle-column. It is difficult to validate exactly the blast effect on the substructure since they were not measured in the tests. However, the blast loads on the specimen can still be verified by comparing the general behaviour between the test results and the FEM simulations. Moreover, a qualitative comparison of damage patterns between the tests and the models can be made for the end part of the middle column.

\subsubsection{Analysis procedure and validation results for blast tests}

At the beginning of the analysis, the explosive defined by the Load_blast_enhanced function is activated and only lasts for a few milliseconds. Due to the uplift pressure from blast loading, the assemblage starts deforming upwards. The additional gravity load on top of the middle joint is then applied at $80 \mathrm{~ms}$ when the substructure achieves the maximum upward displacement. Thereafter, the substructure starts oscillating up and down and eventually all movements stop due to inherent damping effects. In this study, the global damping coefficient of the numerical model is set to be $5 \%$ of critical damping as recommended for general RC structures under 
earthquake conditions. Based on logarithmic decrement technique, it is shown that the assumed value of $5 \%$ for damping ratio is reasonable to describe the oscillation of the specimen, as presented in Fig. 3.22.

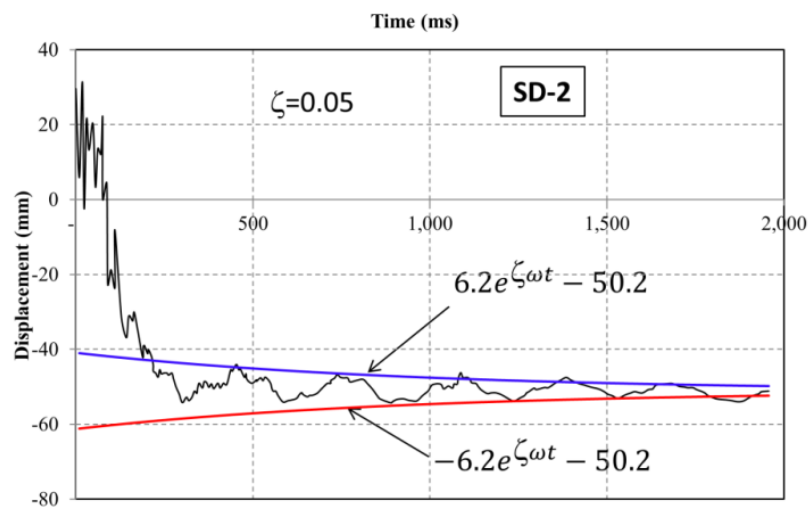

Fig. 3.22 The exponentially narrowing envelop of displacement response with damping ratio $\zeta=5 \%$

The numerical analyses of SD-2 and SD-3 are performed and the results are presented herein. In general, the MJD time-history has been successfully validated in both FEM models. In terms of horizontal reactions, the numerical results agree reasonably with the test data, although some divergence in value is observed (Fig. 3.23, Fig. 3.24). For SD-2, the FEM model overestimates the maximum deformation from the actual test by $10 \%$, which is considered as acceptable. For horizontal reaction, the maximum values of the top and bottom reactions from the FEM model are larger than those from the test results by 8 and $20 \%$, respectively. This difference in the bottom reaction force is due to actual stiffness of the horizontal supports was not directly measured in the tests. Instead, this value was assumed in the FEM model. In the simulation, both the upper and the lower horizontal restraints are assumed to have identical stiffness. However, in actual test, the lower restraint could have smaller stiffness compared to the upper. For SD-3, since the failure of the specimen could not be confirmed from the test, it is difficult to compare the deformations between the FEM model and the actual test. However, in terms of maximum compressive force from horizontal reaction, the FEM result is larger than the test result by only $3 \%$. 
With regard to failure modes, the predicted results from FEM are in good agreement with those from the tests (Fig. 3.25). Some qualitative comparisons for damage patterns at the bottom end of the middle column also confirm the validation of charge weight as well as the method of applying the blast loads (Fig. 3.26).

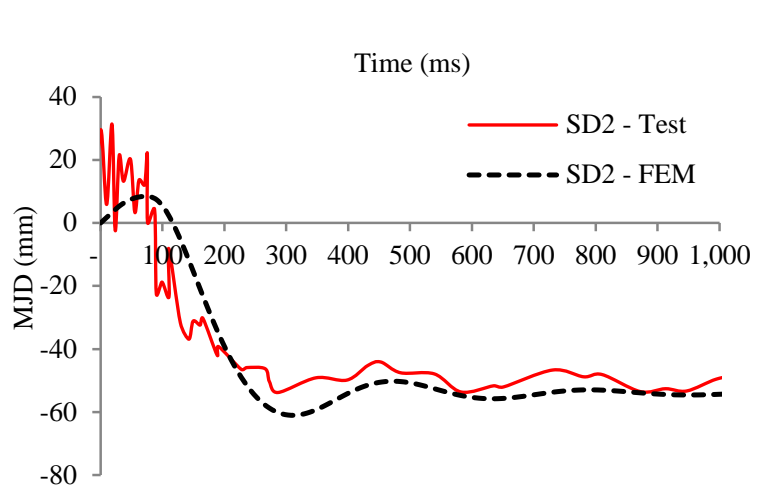

a) Time-history of MJD

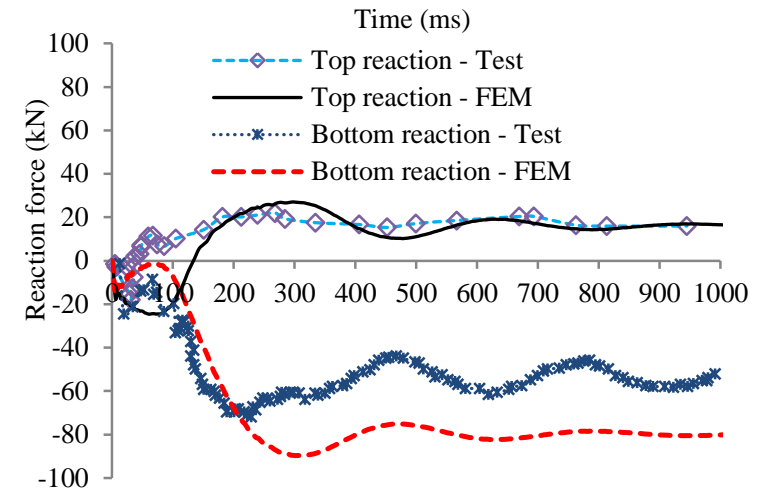

b) Time-history of horizontal reaction

Fig. 3.23 Validation results of SD-2

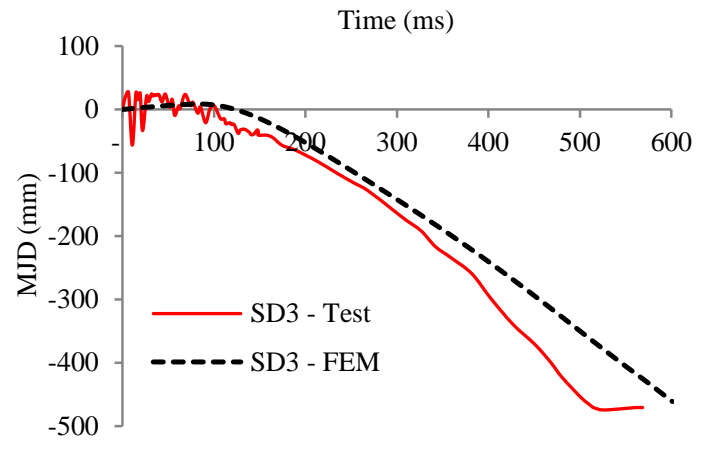

a) Time-history of MJD

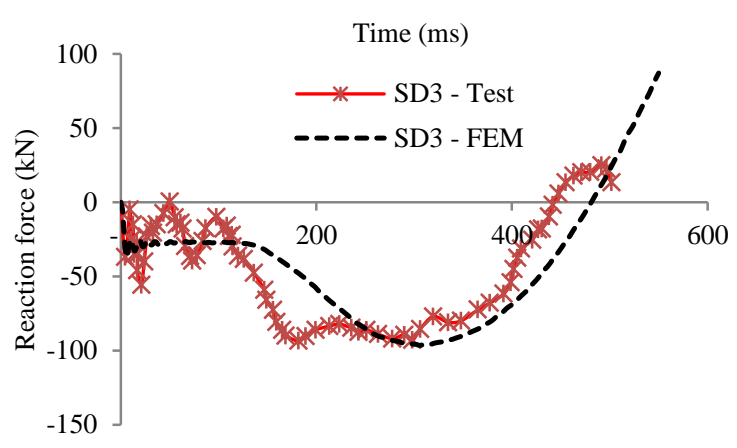

b) Time-history of horizontal reaction

Fig. 3.24 Validation results of SD-3

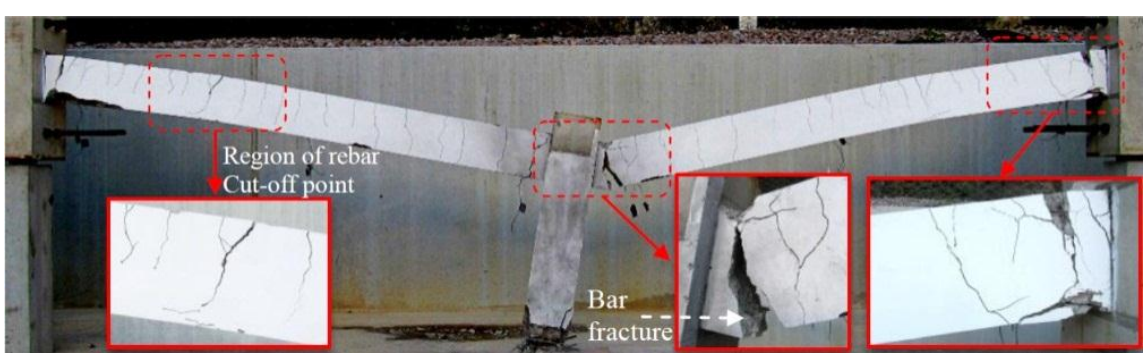

a) Final failures of SD-3 (Yu et al. 2014) 

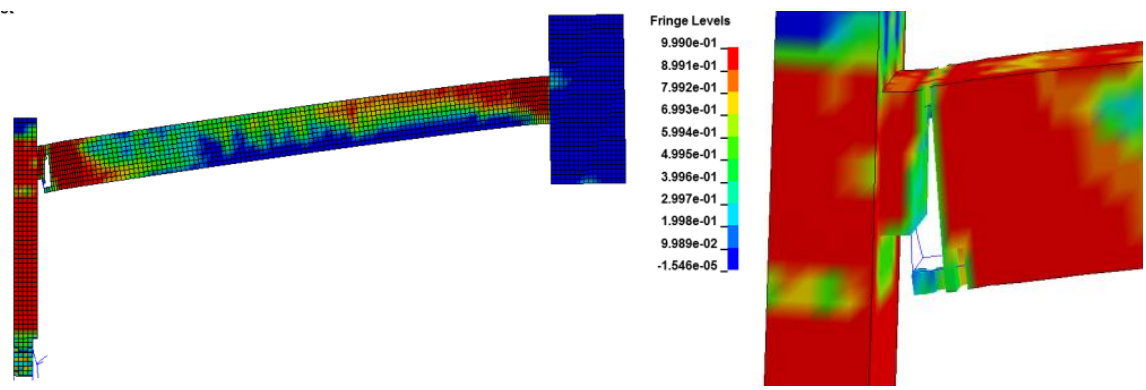

b) Damage patterns of FEM model at $500 \mathrm{~ms}$

Fig. 3.25 Comparison of damage patterns of SD-3

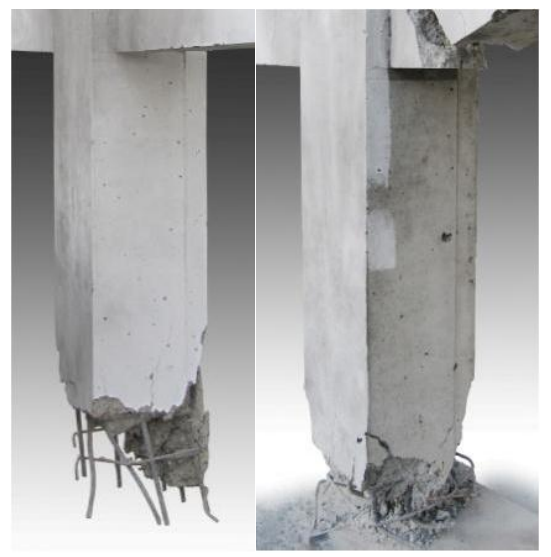

a) Test results of SD-2 and SD-3 (Yu et al. 2014)
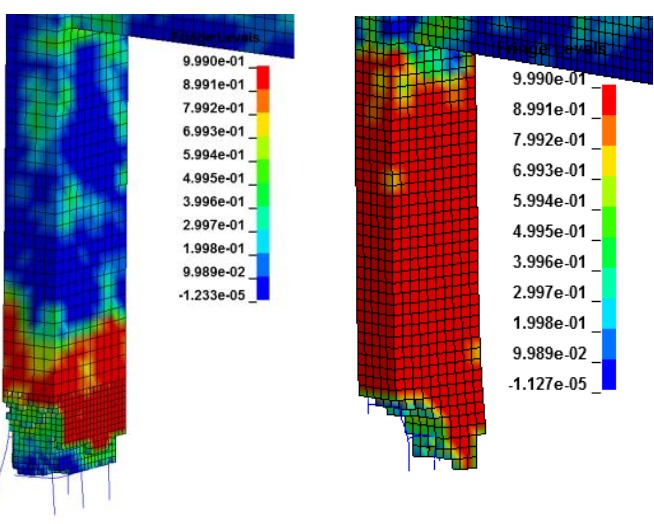

b) FEM results (different meshes)

Fig. 3.26 Damage patterns of middle-column

\subsection{Parametric studies on RC substructures under progressive collapse}

Based on the validated models from both static and contact detonation tests, parametric studies under dynamic condition are conducted and the results are presented here. Factors such as restraint stiffness, load level, damping ratio, and charge weight are considered in the dynamics analyses. Additionally, the energy approach for dynamic assessment (Izzuddin et al. 2008) is also compared with the numerical results with regard to two design situations, viz. free-fall condition and close-in contact detonation.

\subsubsection{Horizontal restraints}

Lateral reaction stiffness plays a vital importance on the overall behaviour of assemblage systems under the blast duration as well as under the free-fall vibration. 
In this section, parametric studies are conducted using several stiffness values for a free-fall dynamic analysis of SD-2 model under a constant applied load of $27 \mathrm{kN}$. Results show that with perfectly rigid constraint, the maximum deflection can be reduced by $64 \%$ compared to the stiffness value of $3.5 \times 10^{4} \mathrm{kN} / \mathrm{m}$ which is chosen for validation of the blast tests (Fig. 3.27). Hence, the monitoring of lateral stiffness is very important when conducting ultra-fast dynamic tests on beam-column structures.

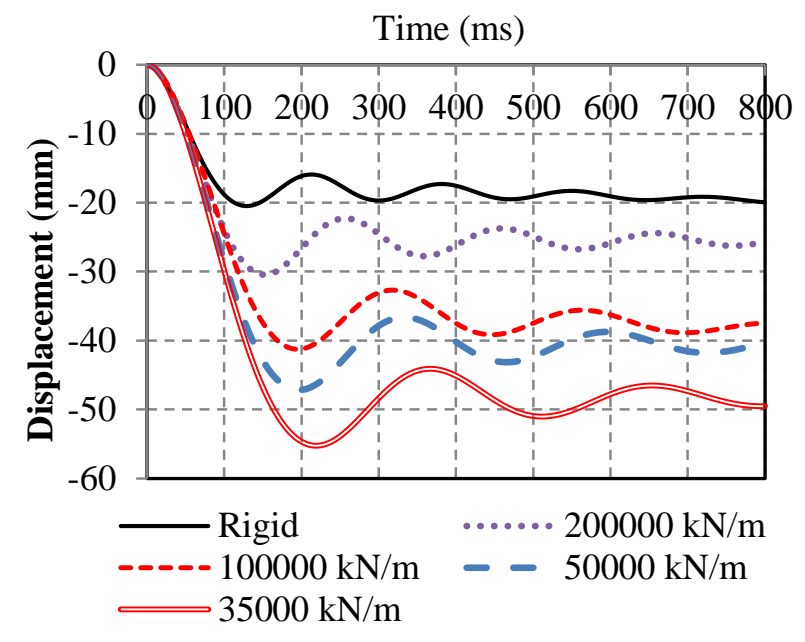

Fig. 3.27 Effects of restraint stiffness

\subsubsection{Charge weight}

Different values of equivalent TNT charge weight are used to study the effect of initial blast pressure on the overall behaviour and resistance of sub-assemblage structures. The charge weight varies from $m \mathrm{~kg}$, similar to the actual test (Yu et al. 2014), to $4 m$ and $8 m \mathrm{~kg}$ of TNT explosive. The additional load is set to $27 \mathrm{kN}$ which is equal to the applied load of SD-2 test. A numerical model using free-fall dynamic analysis with only gravity load is presented as a baseline for comparison. The results clearly show a significant increase in displacement between the free-fall case and the cases with contact detonation as shown in Fig. 3.28. Compared to the free-fall case, the model using $8 \mathrm{~m} \mathrm{~kg}$ of TNT detonation achieves $67 \%$ greater displacement. This shows that the initial effects of blast can cause a significant reduction in load-bearing capacity of structural system under progressive collapse event. This effect is not taken into account when assessing the dynamic effects 
according to DOD (2013) as well as the energy approach by Izzuddin et al. (2008). This numerical observation confirms the significance of blast pressure initiated by close-in detonation, which cannot be considered by the missing column scenario.

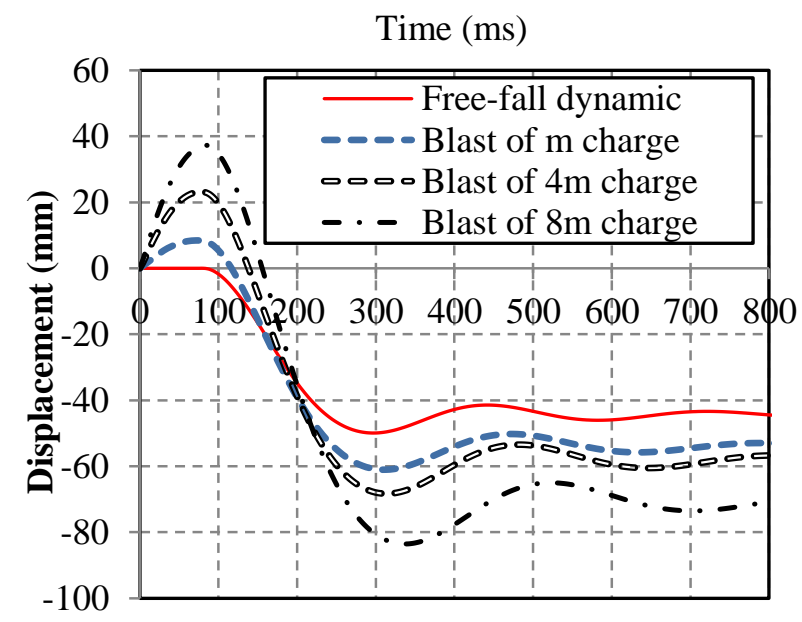

Fig. 3.28 Effects of charge weight

\subsubsection{Damping ratio}

Different damping ratios are used for the FEM model of SD-2 while other parameters such as boundary stiffness, charge weight and load level are kept the same. The damping ratios studied include 3\%, 5\% and $7 \%$ of critical damping, representing three different levels of concrete damage from minor cracks, moderate damage to severe damage. The result from $0 \%$ damping is used as a baseline for comparison. The computational results clearly show that damping effect not only contributes to the decay of vibration, but also reduces the maximum displacement, as well as the magnitude of vibration (Fig. 3.29). Within the first $100 \mathrm{~ms}$ (the blastload phase), damping has almost no effect on the general response of the structure. Energy dissipation from damping is not significant and can be neglected due to extremely short duration of blast loads. Nonetheless, damping ratio has a dominant effect during the gravity-load phase. Hence, it is observed that damping may produce a beneficial effect on mitigating progressive collapse, especially when structures undergo extreme damages such as concrete crushing, rebar yielding and fracturing. 


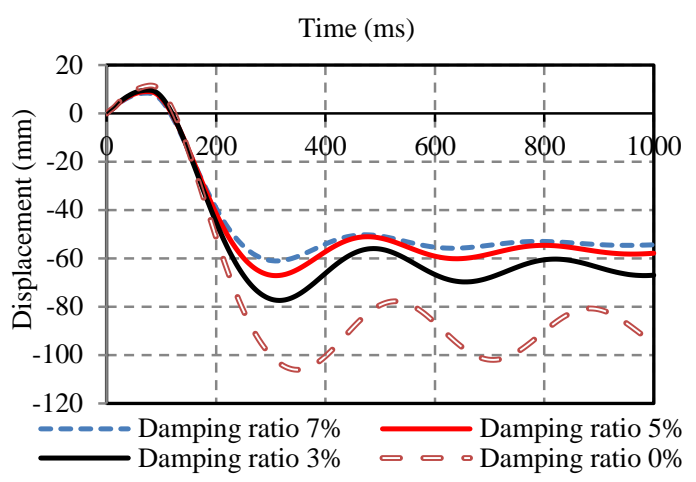

Fig. 3.29 Effects of damping ratio

\subsubsection{Comparison between dynamic analysis and static analysis associated with the energy approach for dynamic evaluation}

The pseudo-static method by Izzuddin et al. (2008) is computationally efficient and can be used in conjunction with the column removal scenario. Based on this method, an example of comparison between two analysis procedures, namely, nonlinear static associated with Izzuddin's method and fully nonlinear dynamic, is made to ascertain if nonlinear static analysis can be used instead of dynamic analysis. A numerical model of SD-2 specimen with similar geometry, material properties and boundary stiffness to the actual test is analysed under quasi-static procedure (push-down analysis) to determine its static response (curve $S$ ). Subsequently, Izzuddin's method is applied to transform the nonlinear static curve of the FEM model to a pseudo-static response incorporating the dynamic effect.

Thereafter, a fully nonlinear dynamic simulation is employed to assess the realistic dynamic behaviour of the model under the sudden-loss scenario of middle column. The support from the middle column is suddenly eliminated with the release time equal to zero to ensure that the loading is dynamic. Other aspects such as damping ratio or blast load are neglected. Several simulations are conducted with the applied load on the middle joint varying from a small value to the greatest value that will cause the structure to fail. At each load level, the maximum displacement of the system is obtained and is used to construct the dynamic capacity curve D1. Comparisons between the pseudo-static curve from Izzuddin's method (curve I) and the one constructed by FEM analyses without damping and blast effects (curve D1) 
as well as the static response (curve S) are presented in Fig. 3.30(a). It is clearly shown that predictions from the pseudo-static response (I) match very well with the results from the dynamic analysis (D1). That is to say, Izzuddin's energy method is suitable for the analyses of structures under a threat-independent scenario of sudden column loss without considering damping and blast effects.

To obtain further understanding of other dynamic effects, two additional series of dynamic analyses are carried out: one series considers the damping ratio of $5 \%$ while the other considers a contact detonation of $m \mathrm{~kg}$ TNT without damping effect. Similar to the first dynamic series (D1), the dynamic capacity curves of these two series are constructed by obtaining the maximum deformations at several load levels and are named as curves D2 and D3, respectively. Fig. 3.30(b) shows a comparison of all dynamic capacity curves from D1 to D3 for all three numerical procedures.

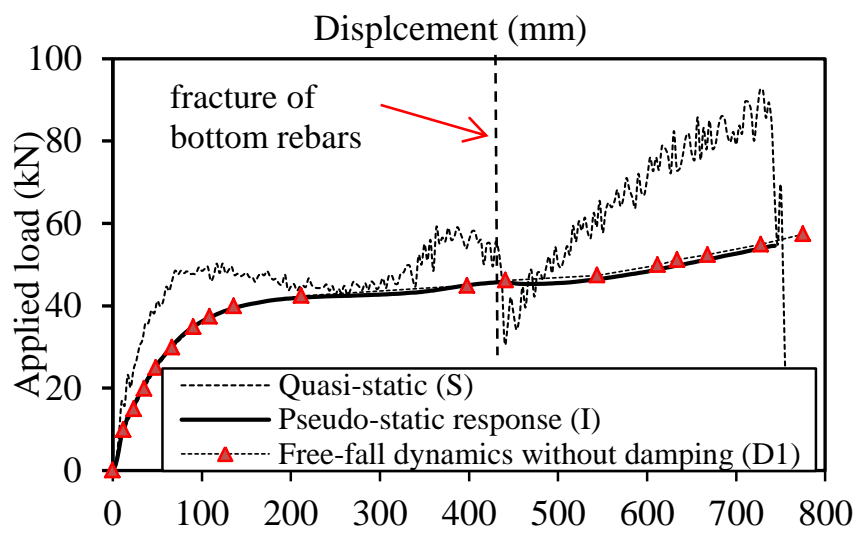

a) Izzuddin vs. free-fall dynamics

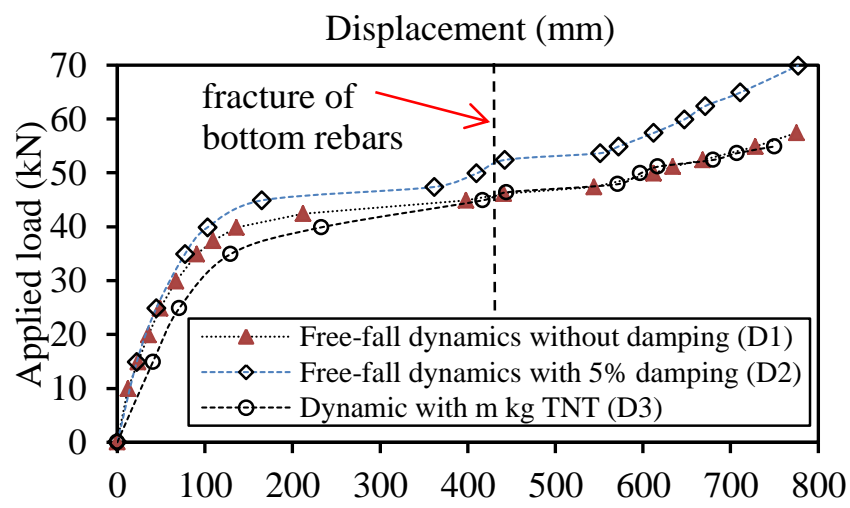

b) Different dynamic analyses

Fig. 3.30 Comparison of different analysis procedures 
For the case of free-fall dynamic analysis with $5 \%$ of critical damping (D2), the difference in the dynamic load-carrying capacity is observed in comparison with the case of zero damping (D1). This difference becomes remarkable when the structure starts yielding, especially after CA starts dominating the overall behaviour of the system. The maximum dynamic load from D2 is $69.9 \mathrm{kN}$, which is $22 \%$ larger than the maximum value from D1 $(57.4 \mathrm{kN})$. This phenomenon is due to energy dissipation from viscous damping which can significantly reduce the effect of dynamic response. However, the damping effect can only be mobilised when structures have already sustained severe damages after the blast-load phase.

Comparing between the free-fall dynamics without damping (D1) and the blastinduced dynamics without damping (D3), a noticeable decrease in load capacity is observed. However, this reduction is only significant when the applied load is less than $40 \mathrm{kN}$ and the structure is within the range of flexure or CAA. The reduction in terms of dynamic loads between D1 and D3 varies from 10 to 30\%. As the applied load is increased and CA is mobilised, the difference between the cases with and without blast effect is almost unnoticeable. As such, the effect of blast pressure on the maximum displacement depends not only on the charge weight but also on the applied load of the double-span structure. Further investigation on blast effects with a larger charge is necessary to fully understand the relationship between the blast loads and the level of imposed load.

For all the three cases of dynamic analysis, D1 to D3, when the applied load is relatively large, the bottom reinforcing bars will fracture under dynamic response. However, the structure can still withstand the final collapse based on the remaining top rebars. This finding confirms the capacity of CA to mitigate final collapse even when all the bottom rebars have already fractured. Similar observation was also obtained in the static tests by $\mathrm{Yu}$ and Tan $(2013 \mathrm{a}, \mathrm{b})$. It is noteworthy that in the actual blast test of SD-3, the testing procedure terminated when the specimen hit the ground with a corresponding displacement of $471.7 \mathrm{~mm}$ and the bottom rebars had already fractured at one side of the middle joint. Hence the ultimate catenary capacity of the assemblage could not be confirmed. From the numerical results of 
FEM dynamic analyses, all the three curves D1 to D3 have maximum loads larger than the applied load of SD-3, i.e. $47 \mathrm{kN}$. Moreover, the maximum deformations of the three curves are much greater than $471.7 \mathrm{~mm}$. Therefore, it is deduced that SD-3 could still withstand the applied load of $47 \mathrm{kN}$ if there was adequate headroom for vertical deflection.

\subsection{Conclusions on numerical analyses}

To provide solid numerical analyses for predicting nonlinear structural behaviour under single column removal scenario, physics-based FEM models are developed in this chapter and are validated by actual tests on RC sub-assemblages. Some brief summaries on the FEM results are listed as follows:

(1) Numerical analyses using LS-Dyna software can well predict the structural behaviour of beam-column sub-assemblages under both quasi-static and blast conditions. However, FEM simulations should be used with great care as the choice of constitutive models and mesh size will significantly affect the accuracy of results. In this study, the continuous surface cap model incorporated with element erosion using the maximum principal strain criterion can provide relatively good agreement with both RC sub-assemblage and component pull-out tests. The numerical analyses are validated by test data regarding the overall response of $\mathrm{RC}$ structures. Moreover, the models can also predict the failure modes such as reinforcement fracturing, concrete spalling, damages from blast effect, etc. As numerical predictions show remarkable dependence on mesh size and erosion limit, it is crucial for FEM models to be validated by actual tests before they are used for parametric studies.

(2) The stiffness of horizontal restraints is shown to have great effect on the strength and behaviour of structures under dynamic regime. The study also numerically verifies the prediction of dynamic response using the energy method proposed by Izzuddin et al. (2008) for the case of free-fall dynamic analysis without damping effect and blast loads. However, damping effect has 
an indispensable influence on the behaviour of concrete structures. Noticeable reduction in strength due to blast loads are also observed from the models.

(3) Based on the numerical models for quasi-static condition validated in this chapter, a comprehensive study will be carried out and presented in Chapter 4, investigating important factors that influence the mobilisation of CA as well as proposing a semi-analytical approach for predicting structural response of RC sub-assemblages.

(4) From the parametric studies under dynamic environment, CA plays an important role to resist collapse, even after the fracture of bottom rebars at the middle joint. This finding will be verified experimentally in the latter part (Chapter 6) on actual dynamic behaviours under sudden column removal scenarios. These dynamic tests will also be used to confirm the conservatism of Izzuddin's method mentioned in this chapter.

(5) As both boundary condition and effect of initial blast on surviving members are crucial for investigating structural behaviour under a contact-detonation event, they must be carefully specified. However, in the dynamic tests of Yu et al. (2014), these data were not quantitatively measured. As a result, during the validation of the FEM models, some assumptions related to these parameters are made. To avoid such inconveniencies, in the blast-induced tests from Series III of this study (Chapter 8), both restraint stiffness and blast pressure on surrounding members will be carefully considered. 


\section{CHAPTER 4 A SIMPLIFIED MODEL OF CATENARY ACTION IN RC FRAMES UNDER AXIALLY RESTRAINED CONDITIONS}

\subsection{Introduction}

To study the behaviour of RC substructures under single column removal scenario, the physics-based models for quasi-static condition developed in Chapter 3 (Pham et al. 2016) are employed to investigate important variables affecting CA. Based on actual static tests and numerical predictions, a semi-analytical model is proposed to provide a practical and yet reliable procedure to predict the overall response of $2 \mathrm{D}$ RC beam-column structures subjected to CL condition (Pham and Tan 2017c). This simplified approach takes into account the effect of partial restraint, as well as structural response before and after the fracture of bottom rebars in the double-span beam. Equivalent dynamic capacity is also considered in the proposed model by using Izzuddin's method (Izzuddin et al. 2008). Published test results (Yu and Tan 2013a, b, Lim et al. 2015) are used to validate the proposed model and to assess its conservatism for design purpose.

\subsection{Structural investigations on catenary action}

\subsubsection{Proposed simplified structural response under supporting column removal}

From the literature test results of RC sub-assemblages, a typical structural response with adequate horizontal restraints can be presented in Fig. 4.1(a) by the curve OABCDEFG. It should be noted that curve OA represents the initial stage in which steel reinforcement behaves elastically and there is little concrete damage. At point A, longitudinal rebars in the beam-column joints start yielding and cracks start developing within the concrete tensile regions if the section is under-reinforced. The first peak at point $\mathrm{B}$ denotes the maximum flexural capacity considering CAA enhancement. Thereafter, vertical load gradually decreases depending on the spandepth ratio and horizontal restraint stiffness of the double-span beam. The 
descending part is due to crushing of concrete at the compressive zones, as well as a reduction of CAA. Point $\mathrm{C}$ in Fig. 4.1(a) marks the onset of $\mathrm{CA}$ and usually coincides with a vertical deflection proportional to one beam depth. Within the ascending phase $\mathrm{CD}$, mobilisation of $\mathrm{CA}$ is identified by a change of axial force in beams from compression to tension. The sudden drop DE marks the fracture of bottom longitudinal rebars in the beams at the middle joint region. It is conservatively assumed that the bottom rebars from both sides of the middle joint fracture simultaneously. In reality, bottom rebars from two sides of the joint can fracture at different times due to imperfections in symmetric condition. After which, if the MJD continues to increase then the top reinforcing bars will contribute to the residual capacity of catenary mechanism until they also fracture.

To provide a simplified response representing the structural capacity of beamcolumn components, the typical response OABCDEFG is represented by a polyline with controlling points from A to $\mathrm{G}$ (Fig. 4.1(b)). For parts $\mathrm{BC}$ and $\mathrm{CD}$, additional intermediate points $C_{1}$ and $D_{1}$ are proposed to make these curves fit better with the actual response in Fig. 4.1(a). For horizontal lines $C_{1} D_{1}$ and EF, it is assumed that the structural capacity remains unchanged while the displacement keeps increasing. Point $\mathrm{C}_{1}$ is defined so that $\mathrm{C}$ is the mid-point of $\mathrm{C}_{1} \mathrm{D}_{1}$. A, B, C, D, E and $\mathrm{G}$ are considered as the primary points of the proposed curve which can be directly obtained from structural analysis. However, $\mathrm{C}_{1}, \mathrm{D}_{1}$ and $\mathrm{F}$ are secondary points which are dependent on the locations of the primary points.

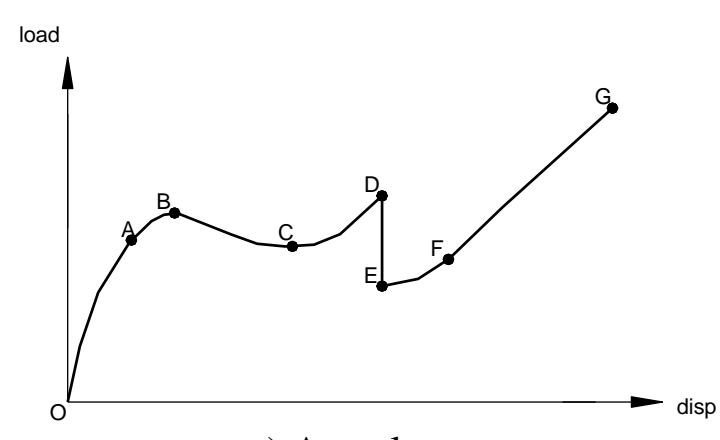

a) Actual response

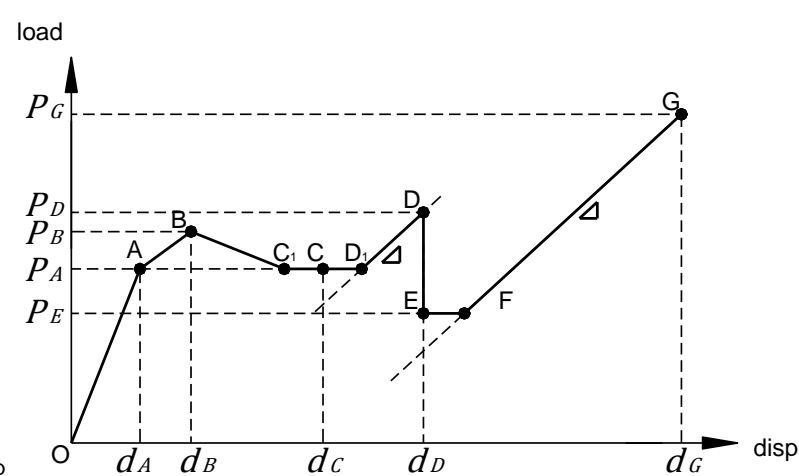

b) Simplified piecewise response

Fig. 4.1 Simplified response of beam-column structures 


\subsubsection{Proposed normalised factors for sub-assemblage behaviour}

A series of normalised parameters is used to investigate the effects of important geometrical, material and external restraint factors on the behaviour of beamcolumn structures. To make the problem tractable, a symmetry in loading, material and boundary conditions is assumed; thus, only one-half of the double-span beam is considered (Fig. 4.2). The middle joint has vertical deflection as the only degree of freedom while its horizontal and rotational degrees of freedom are restrained due to symmetry at the centre. For the end joint, a pin-on-roller support with an axial spring $\left(K_{A}\right)$ and a rotational spring $\left(K_{R}\right)$ are considered as boundary conditions. Horizontal force from the axial spring is denoted as $H$. Such an arrangement allows the consideration of boundary effects (or imperfections) incorporating both horizontal and rotational restraints at the end joint. From tests, it is shown that the governing mechanisms of sub-assemblages under column loss scenario include flexural and compressive/tensile actions in the beam. Therefore, all the parameters studied herein are related to either translational or rotational characteristics. A semianalytical model based on the simplified response shown in Fig. 4.1(b) is developed employing the normalised factors, presented in the following.

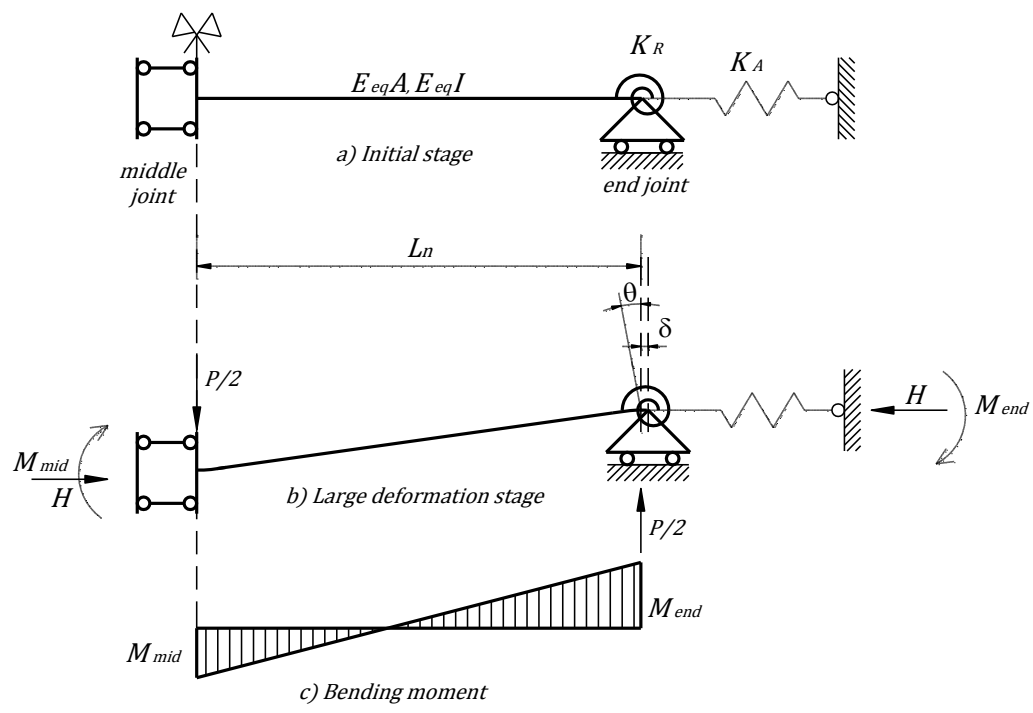

Fig. 4.2 Proposed half-type model 
The first normalised parameter is the span-depth ratio $k_{\text {span }}$ defined by Eq. (4.1). This factor describes the governing behaviour of the structure which is either pure flexure or flexure with shear.

$k_{\text {span }}=\frac{L_{n}}{d_{\text {beam }}}$

where $L_{n}$ is the single clear span before the column is removed and $d_{\text {beam }}$ is the overall beam depth.

The normalised horizontal stiffness, $k_{\text {hor }}$, which describes the axial stiffness of the beam relative to adjacent lateral restraints, is defined by Eq. (4.2). The value of $k_{\text {hor }}$ depends on the axial stiffness of adjacent members to withstand a horizontal force, which is sensitive to the number of bays next to the removed column. A small value of $k_{\text {hor }}$ denotes a weak boundary restraint compared to the beam axial stiffness.

$k_{\text {hor }}=\frac{K_{A} L_{n}}{\left(E_{c} A_{c}+E_{s} A_{s}\right)}$

where $E_{c}, A_{c}$ are respectively the elastic modulus and the total area of uncracked concrete; $E_{S}, A_{s}$ are respectively the elastic modulus and total area of steel reinforcement at the end joint.

The normalised bending stiffness $k_{\text {flex }}$, which represents the flexural capacity of the beam compared to its flexural stiffness, is given by Eq. (4.3). This factor relates the bending moment resistance of the section to the beam elastic stiffness. For example, a high value of $k_{\text {flex }}$ denotes that the beam is highly reinforced.

$k_{\text {flex }}=\frac{M_{\text {mid }}+M_{\text {end }}}{E_{\text {eq }} I_{g} / L_{n}}$

where $I_{g}$ is the moment of inertia of the uncracked concrete cross section; $M_{\text {mid }}$ and $M_{\text {end }}$ are respectively the sagging and the hogging plastic moment capacities (yielding state) of the beam section at the middle joint and the end joint. $M_{\text {mid }}$ and $M_{\text {end }}$ can be obtained from a simplified equation proposed by Paulay and Priestley (1992): 
$M=\left(d-d^{\prime}\right) A_{s}^{t s} f_{y}$

where $d$ and $d^{\prime}$ are the distances from the extreme compression fibre of concrete to the centre of tension and compression reinforcement, respectively; $A_{s}^{t s}$ and $f_{y}$ are the cross-sectional area and yield strength of tension longitudinal rebars, respectively. Eq. (4.4) is applicable for under-reinforced sections in the absence of beam axial forces.

The steel-to-concrete stiffness ratio, $k_{\text {topbar }}$, relates the axial stiffness of the top reinforcement to that of the concrete material in the beam section at the end joint (Eq. (4.5)). A small reinforcement ratio yields a small $k_{\text {topbar }}$ or vice versa.

$k_{\text {topbar }}=\frac{E_{s} A_{s}^{\text {top }}}{E_{c} A_{c}}$

where $A_{s}^{t o p}$ is the area of top reinforcement in the beam near the end joint.

Finally, Eq. (4.6) specifies the normalised rotational stiffness $k_{r o t}$ of the end joint. It is a ratio of the rotational stiffness $K_{R}$ of the boundary condition to the beam elastic stiffness. If the end restraint has low rotational stiffness, it will limit the mobilisation of CAA.

$k_{\text {rot }}=\frac{K_{R}}{E_{e q} I_{g} / L_{n}}$

\subsubsection{Investigations on structural behaviour}

Based on the validated model for quasi-static condition from Chapter 3, parametric studies are conducted for the five factors ranging from $k_{\text {span }}$ to $k_{\text {rot }}$ and for concrete strength; the results are shown in Fig. 4.3. The purpose of the comparison is to find critical parameters which should be incorporated into the simplified model for CA. While direct comparisons are made for $k_{\text {span }}, k_{\text {hor }}$ and $k_{\text {rot }}$, the influences of $k_{\text {flex }}$ and $k_{\text {topbar }}$ are indirectly illustrated by comparing different ratios of top and bottom rebars at the end joint. From both numerical simulations presented in Fig. 4.3(a) and test results from Yu and Tan (2013a, b), it is shown that if $k_{\text {span }}$ is 
smaller than 7, then after the first peak, structural response will decrease quickly. There will be no ascending phase, i.e. flexural and compressive arch mechanisms will govern the response and shear will dominate the failure instead of CA.

In terms of axial restraint, five values of $k_{h o r}$ are compared ranging from 0.01 to 2.27 (Pham et al. 2016). The results presented in Fig. 4.3(b) clearly show that $k_{\text {hor }}$ has significant effect on structural response, as well as development of catenary mechanism in the beams. In this comparison, the value of $k_{\text {hor }} \geq 2$ can be considered as perfectly rigid condition. On the other hand, if the horizontal restraint is too weak, say $k_{\text {hor }}<0.01$ (for $k_{\text {span }}=11$ ), catenary mechanism cannot be efficiently mobilised. This observation agrees with Yu and Tan (2013a) regarding the sensitivity of CA to horizontal restraint stiffness.

Regarding the effect of longitudinal reinforcement on CA before and after the fracture of bottom rebars near the middle joint, Fig. 4.3(c) and (d) show that while the top rebars significantly affect the structural response before and after the fracture of the bottom rebars, the bottom rebars only affect the behaviour prior to their fracture at the middle joint. After the fracture, load-carrying capacity drops to point $\mathrm{E}$ and the bottom rebars can no longer contribute to $\mathrm{CA}$.

The normalised rotational stiffness, $k_{\text {rot }}$, is studied in the range of 0.04 to perfectly rigid condition. Results show that rotational restraint stiffness has less effect on structural response after the first peak (Fig. 4.3(e)). To gain a thorough understanding of the effect of $k_{\text {rot }}$ on CA, a more extensive investigation is made in which the span-depth ratio $k_{\text {span }}$ is also considered. Five values of $k_{\text {span }}(7,9,11$, 12.8 and 15) and five values of $k_{\text {rot }}(0.04,0.41,0.85,4.36$ and 8.9) are taken into account simultaneously. It is found that the effect of rotational stiffness on the curve from $\mathrm{C}_{1}$ to $\mathrm{G}$ (Fig. 4.1(b)) is almost constant if both $k_{\text {span }}$ and $k_{\text {rot }}$ satisfy Eq. (4.7). Moreover, the case of beam-column sub-assemblages with very little rotational restraint at the end supports $\left(k_{\text {rot }}<0.2\right)$ is not realistic for concrete structures. Hence, it is concluded that the development of CA does not depend on $k_{\text {rot }}$. 
$\frac{5}{k_{\text {span }}}+k_{\text {rot }} \geq 1$

Considering concrete strength, it is observed that $f_{c k}$ greatly affects the peak of CAA (point B) but has limited effect on the remaining points from $\mathrm{C}$ to $\mathrm{G}$ (Fig. 4.3(f)). In this phase, CA governs the response and is only dependent on $k_{\text {span }}$, $k_{\text {hor }}$, and $k_{\text {topbar }}$. Connection gaps, which exist in actual tests, can remarkably affect the mobilisation of CAA (Yu and Tan 2014). Nevertheless, it has very little influence on residual response after all the bottom rebars have fractured (part EG). Moreover, this boundary imperfection only occurs in the laboratory tests and has little physical meaning for cast-in-situ concrete structures. In summary, all the four important factors $\left(k_{\text {span }}, k_{\text {hor }}, k_{\text {flex }}, k_{\text {topbar }}\right)$ have been incorporated into the proposed model, while three other less important ones $\left(k_{\text {rot }}\right.$, concrete strength and connection gaps) can be ignored.

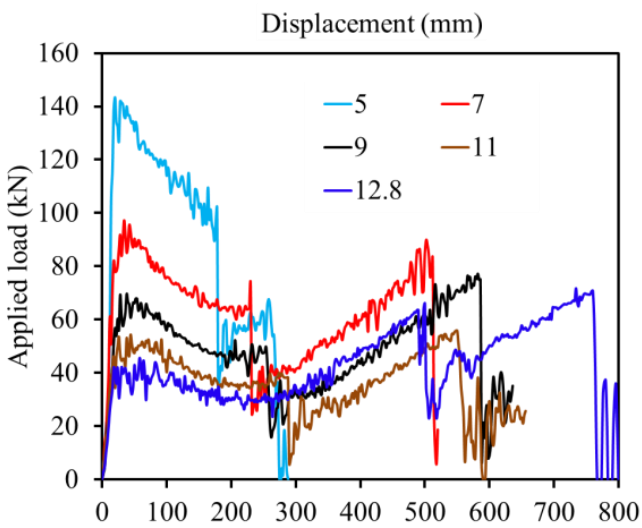

a) Span-depth ratio $k_{\text {span }}$

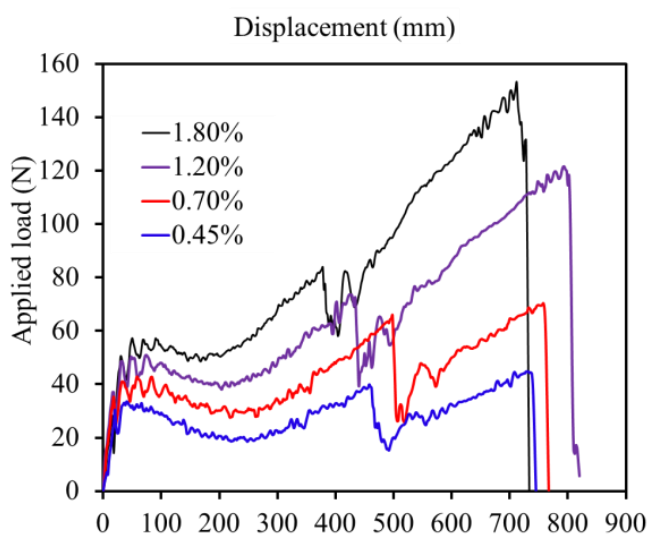

c) Top rebars $(\%)$

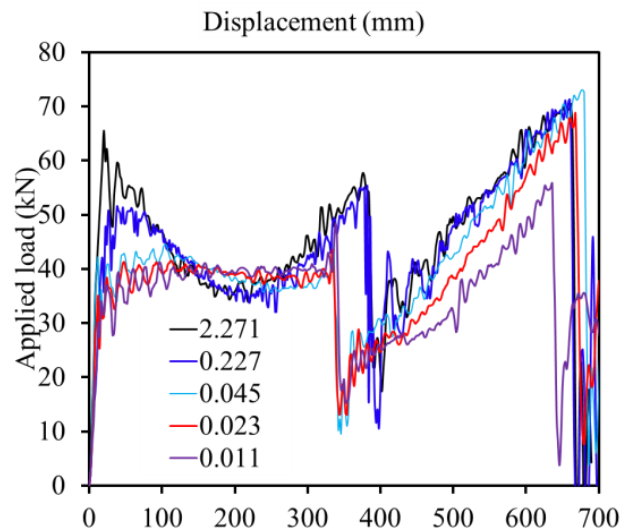

b) Horizontal stiffness $k_{\text {hor }}$

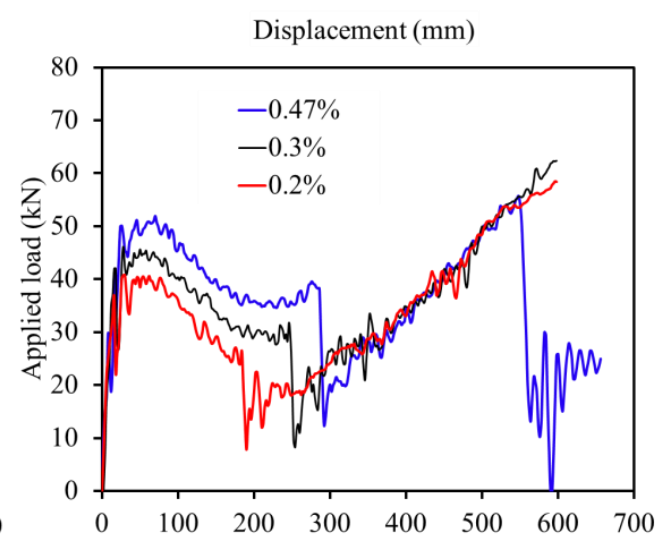

d) Bottom rebars $(\%)$ 


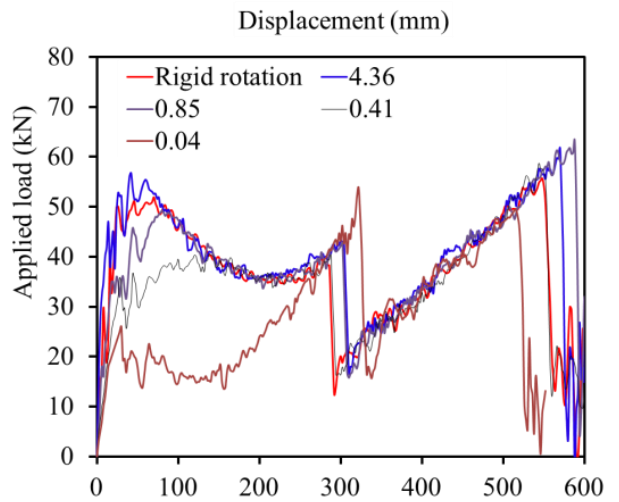

e) Rotational stiffness $k_{\text {rot }}$

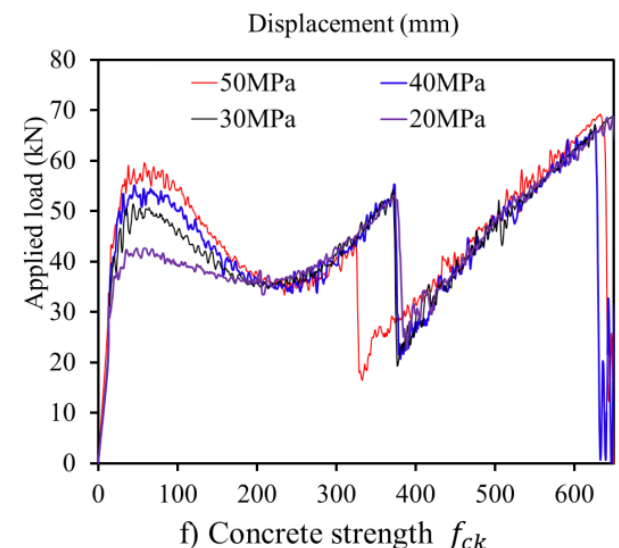

f) Concrete strength $f_{c k}$

Fig. 4.3 Investigation of CA mobilisation

\subsection{Simplified semi-analytical model on catenary action}

Based on the comparison in Section 4.2.3, a comprehensive numerical parametric study on CA is employed to obtain a semi-analytical approach for predicting the simplified polyline response from Fig. 4.1(b). All the investigated parameters are summarised in Table 4.1. A total number of 120 case studies are considered including four types of rebar arrangement, five different spans and six values of horizontal stiffness. The reinforcement contents are chosen within the practical range of beam design. Constant parameters include the beam cross-sectional dimensions, concrete grade and reinforcement strength. Perfect rotational restraint and zero connection gap are assumed since they have little effects on CA. From numerical predictions, the values of controlling points (from A to G of Fig. 4.1(b)) can be defined and semi-analytical equations are proposed as follows.

Table 4.1 Numerical parametric studies

\begin{tabular}{|c|c|c|c|c|c|c|}
\hline Parameters & \multicolumn{6}{|c|}{ Value } \\
\hline Concrete & \multicolumn{6}{|c|}{$f_{c k}=30 \mathrm{MPa}$} \\
\hline Beam dimension & \multicolumn{6}{|c|}{$150 \mathrm{~mm}$ wide by $250 \mathrm{~mm}$ depth } \\
\hline Reinforcement & \multicolumn{6}{|c|}{$f_{y}=520 \mathrm{MPa}, f_{u}=620 \mathrm{MPa}, \varepsilon_{y}=0.27 \%, \varepsilon_{u}=12 \%$} \\
\hline Top rebars & \multicolumn{2}{|c|}{$3 \mathrm{~T} 8$} & $3 \mathrm{~T} 10$ & \multicolumn{2}{|c|}{$3 \mathrm{~T} 13$} & $3 \mathrm{~T} 16$ \\
\hline $\mathrm{mm}^{2} / \%$ & \multicolumn{2}{|c|}{$151(0.45 \%)$} & $236(0.7 \%)$ & \multicolumn{2}{|c|}{$398(1.2 \%)$} & $603(1.8 \%)$ \\
\hline Bottom rebars & \multicolumn{2}{|c|}{$2 \mathrm{~T} 8$} & $2 \mathrm{~T} 10$ & \multicolumn{2}{|c|}{$2 \mathrm{~T} 10$} & $2 \mathrm{~T} 10$ \\
\hline $\mathrm{mm}^{2} / \%$ & \multicolumn{2}{|c|}{$101(0.3 \%)$} & $157(0.47 \%)$ & \multicolumn{2}{|c|}{$157(0.47 \%)$} & $157(0.47 \%)$ \\
\hline Span (mm) & \multicolumn{6}{|c|}{$1750 / 2250 / 2750 / 3200 / 3750$} \\
\hline $\begin{array}{c}\text { Horizontal } \\
\text { stiffness }(\mathrm{kN} / \mathrm{m})\end{array}$ & $5 \times 10^{3}$ & $10^{4}$ & $2 \times 10^{4}$ & $4 \times 10^{4}$ & $10^{5}$ & $10^{6}$ \\
\hline
\end{tabular}




\subsubsection{Point A}

Assuming yielding of both the top rebars near the end joint and the bottom rebars near the middle joint, plastic hinge theory is employed to predict the capacity at point A (Fig. 4.1(b)) as shown in Eq. (4.8). Based on numerical results of displacement when structural capacity reaches the value of $P_{A}$, the displacement at point $\mathrm{A}\left(d_{A}\right)$ is determined by Eq. (4.9). In this equation, $\alpha_{1}$ is a coefficient that considers the effect of horizontal restraint stiffness $\left(k_{\text {hor }}\right)$ defined by Eq. (4.10). Nonetheless, since the proposed model assumes rigid condition for rotational restraint, it tends to overestimate the stiffness at point $\mathrm{A}$ and yields a smaller value of $d_{A}$ compared to test results.

$$
\begin{aligned}
P_{A} & =\frac{2\left(M_{\text {mid }}+M_{\text {end }}\right)}{L_{n}} \\
d_{A} & =\frac{\alpha_{1}\left(M_{\text {mid }}+M_{\text {end }}\right) L_{n}{ }^{2}}{3 E_{C} I_{g}} \\
\alpha_{1} & =\frac{1}{\left(1.25+k_{\text {hor }}\right)^{4}}+0.56
\end{aligned}
$$

\subsubsection{Point B}

In this study, the analytical model proposed by Yu and Tan (2014) is employed to define the load-carrying capacity and the corresponding deformation at B. Yu's model can quickly and accurately predict the CAA capacity of RC sub-assemblages under a column-missing scenario. The model considers all the design parameters in beams and the imperfect boundary conditions of sub-assemblages, including partial axial and rotational restraints, and connection gaps at the beam ends. In this model, vertical resistance $P$ is determined based on moment equilibrium in the one-bay beam as shown in the equation and figure below.

$P=2 V=2\left(M_{u 1}+M_{u}-N \delta-\frac{q l_{n}^{2}}{2}\right) / l_{n}$ 


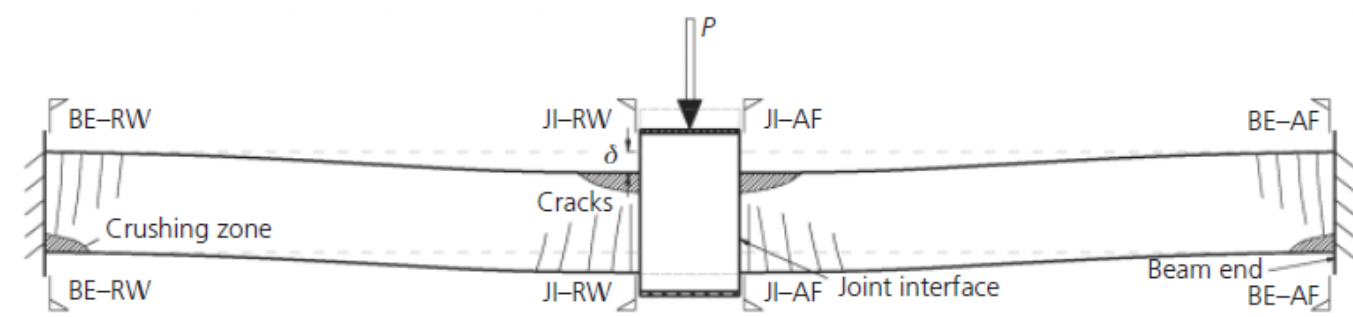

(a)

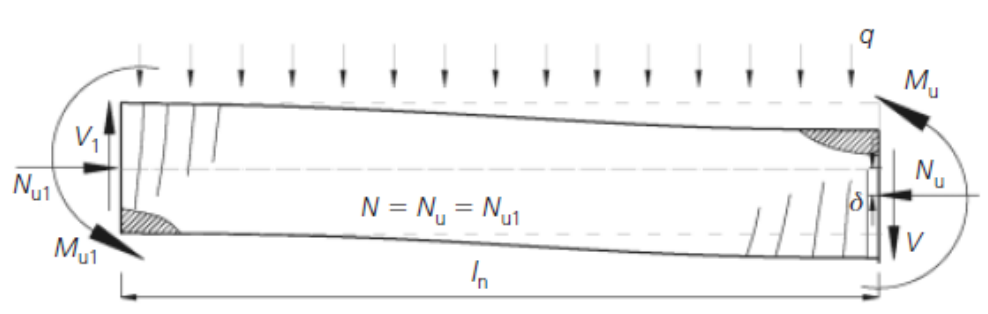

(b)

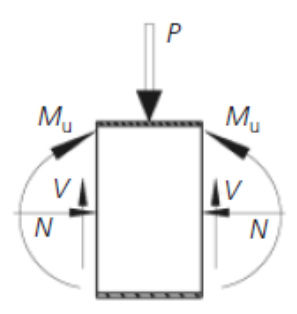

(c)

Fig. 4.4 Analytical model for compressive arch action (Yu and Tan 2014)

To determine the $\mathrm{P}-\delta$ relationship, it is necessary to obtain $\mathrm{M}-\delta$ and $\mathrm{N}-\delta$ relationships. Yu's model applies only after plastic hinges have occurred at several critical sections. The following assumptions are adopted in Yu's model:

(a) A plane section remains plane;

(b) At each critical section, concrete in compression has attained its strength with an idealised equivalent rectangular stress block and an ultimate strain of 0.003 in the extreme compression fibre of concrete; tension reinforcement has yielded and concrete tensile strength is ignored;

(c) The restraints from surrounding structures are converted to equivalent axial and rotational restraints with linear elastic stiffness;

(d) Axial shortening of beams is only induced by elastic axial compressive strains, which are uniform throughout the whole two-bay beam;

(e) The material property of compressive steel reinforcement is elastic-perfectlyplastic, and the unloading slope after yielding equals the initial elastic modulus.

\subsubsection{Point C}

At point $\mathrm{C}$ (Fig. 4.1(b)), the effect of CAA almost ceases and CA is about to be mobilised. The applied load at $\mathrm{C}, P_{C}$, is assumed to be equal to $P_{A}$ (flexural 
strength). The vertical deflection at $\mathrm{C}, d_{C}$, is assumed to be proportional to $d_{\text {beam }}$, and depends on $k_{\text {span }}$ and $k_{\text {hor }}$. A large value of $k_{\text {span }}$ and a small value of $k_{\text {hor }}$ lead to a large value of $d_{C}$. On the other hand, a small $d_{C}$ denotes dominant influence of arching action. Consequently, Eqs. (4.11) and (4.12) are used together to define $d_{C}$.

$d_{C}=\alpha_{2} d_{\text {beam }}$

where $\alpha_{2}$ is defined by Eq. (4.12)

$$
\alpha_{2}=\left(0.4+0.065 k_{\text {span }}-12 k_{\text {flex }}\right)\left(\frac{1}{\left(1.07+k_{\text {hor }}\right)^{15}}+1\right)
$$

\subsubsection{Line FG}

From the results of parametric studies, it is shown that the gradient of line FG greatly depends on the yield force of the top reinforcement $F_{\text {top }}^{y}$ at the end joint and the span-depth ratio $k_{\text {span }}$, while it is less influenced by axial restraint ratio $k_{h o r}$. Nonetheless, the position of FG is dominated by the stiffness of axial restraint. Therefore, a linear function for line FG is proposed herein:

$P_{F G}(d)=\beta_{1} \frac{F_{t o p}^{y}}{L_{n}}\left(d-\beta_{2} d_{\text {beam }}\right)$

$\beta_{1}$ and $\beta_{2}$ are specified by Eqs. (4.14) and (4.15), respectively.

$\beta_{1}=\left(1.2+0.24 k_{\text {span }}-9 k_{\text {topbar }}\right)$

$\beta_{2}=\left(-0.45+0.125 k_{\text {span }}-3.6 k_{\text {topbar }}\right)\left(\frac{1}{\left(1.05+k_{\text {hor }}\right)^{10}}+1\right)$

\subsubsection{Line $\mathrm{D}_{1} \mathrm{D}$}

Observations from both the test and numerical results show that line $\mathrm{D}_{1} \mathrm{D}$ tends to be parallel to FG (Fig. 4.1(b)). Hence, for simplicity it is assumed that they share the same gradient, which mostly depends on the yield strength $F_{\text {top }}^{y}$ of the top reinforcement (Eq. (4.13)). The reduction in terms of load-carrying capacity between $\mathrm{D}_{1} \mathrm{D}$ and $\mathrm{FG}$ is due to positive moment at the middle joint $M_{\text {mid }}$ which 
terminates after all the bottom rebars have fractured at D. Eq. (4.16) shows the load capacity of the structure within $\mathrm{D}_{1} \mathrm{D}$.

$P_{D_{1} D}(d)=P_{F G}(d)+\frac{2 M_{\text {mid }}}{L_{n}}=\beta_{1} \frac{F_{t o p}^{y}}{L_{n}}\left(d-\beta_{2} \cdot d_{\text {beam }}\right)+\frac{2 M_{\text {mid }}}{L_{n}}$

After obtaining the separate functions of $F G$ and $D_{1} D$, the load-carrying capacities at points $\mathrm{D}$ and $\mathrm{G}$ can be defined by specifying corresponding displacements of these points. In the next section, an analytical approach based on plastic hinge length will be used to determine the corresponding deflections $d_{D}$ and $d_{G}$.

\subsubsection{Displacements at $G$ and $D$}

The deformation at point $\mathrm{D}\left(d_{D}\right)$ is calculated based on the deflection caused by segments $\mathrm{XY}\left(\Delta_{\mathrm{XY}}\right)$ and $\mathrm{YZ}\left(\Delta_{\mathrm{YZ}}\right)$ shown in Fig. 4.5(a), in which Y is the contraflexural point. In segment XY, plastic hinge is assumed to have fully developed in the beam section near the middle joint with a plastic hinge length $L_{s p}^{\text {mid }}$ (Fig. 4.5(b)). Beam curvature within the plastic hinge length is assumed to reach the ultimate value $\phi_{u}^{\prime}$ whereas the beam curvature along XY is uniformly distributed from $\phi_{y}^{\prime}$ (yield value) to zero. Hence, $\Delta_{X Y}=\Delta_{u}^{m i d}+\Delta_{y}^{\text {mid }}$ in which $\Delta_{u}^{m i d}$ is due to ultimate curvature $\phi_{u}^{\prime}$ within $L_{s p}^{\text {mid }}$, and $\Delta_{y}^{\text {mid }}$ comes from linear distribution of curvature along XY.

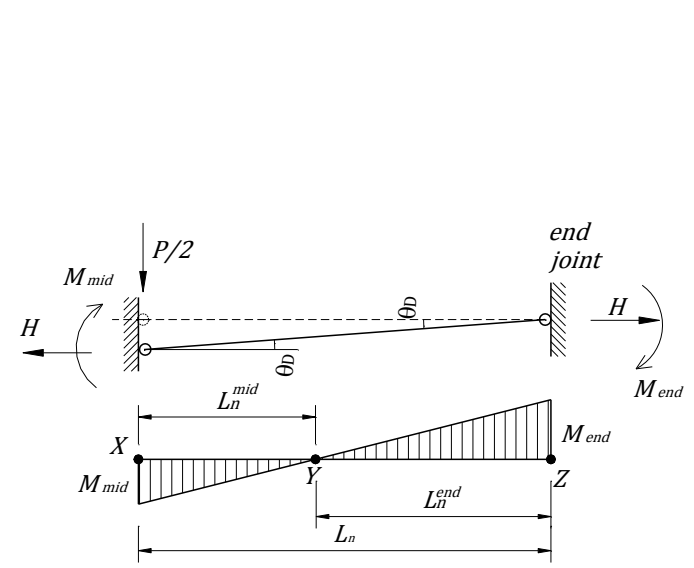

a) Assumption of moment distribution

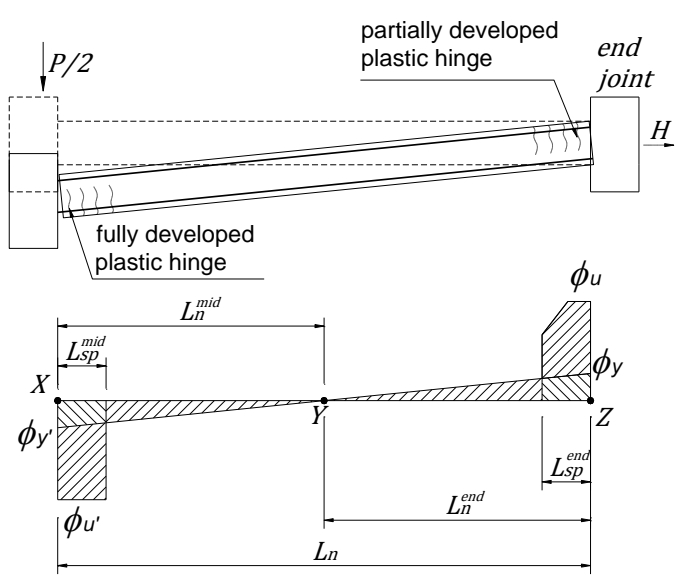

b) Assumption of curvature distribution

Fig. 4.5 Proposed response and curvature distribution at point D 
In segment YZ, plastic hinge is partially developed within a plastic hinge length $L_{s p}^{e n d}\left(\right.$ Fig. 4.5(b)). Along YZ, beam curvature is assumed to be linear from $\phi_{y}$ (yield value) to zero. Since plastic hinge is not yet fully developed, not all of the beam curvature within $L_{s p}^{e n d}$ reaches ultimate value $\phi_{u}$ (Fig. 4.5(b)). Therefore, $\Delta_{Y Z}$ is defined based on $\Delta_{X Y}$ noting that both segments have the same rotational angle $\theta_{D}$ (Fig. 4.5(a)).

When the structure reaches the maximum capacity at $G$ and finally collapses at the two ends, the middle joint can be considered as a pin support (Fig. 4.6). At this stage, severe damages occur along the double-span beam and especially concentrate near the joint regions (Fig. 4.6(a)). Near the end joint, multiple tensile cracks develop and concrete in the compression zone is crushed. Prior to top rebars fracturing, a plastic hinge is assumed to have fully developed at the end joint. Near the middle joint, concrete spalling penetrates through most of the beam section, except at the region surrounding the top rebars. Therefore, the middle joint behaves like a pin support. Within the region of top rebar cut-off points (curtailment regions), several flexural cracks are observed owing to negative bending moment reaching the yield capacity of the beam section. Total displacement $d_{G}$ is divided into three components, i.e. $d_{G}=\Delta_{y}+\Delta_{u}+\Delta_{c}$. The first component $\Delta_{y}$ is due to linear distribution of curvature along the beam: from zero at the middle joint to $\phi_{y}$ at the end joint. The second component $\Delta_{u}$ is the displacement caused by ultimate curvature $\phi_{u}$ within the ideal plastic hinge length $L_{s p}$ near the end joint. The last term $\Delta_{c}$ comes from curvature distribution along the curtailment region $L_{c}$ where some top rebars are cut off. Based on test results and numerical studies, it is assumed that the remaining top rebars along the curtailment region attain the yield strength. Also the curvature reaches the yield value $\phi_{y}^{c}$ and is constant in this part (Fig. 4.6(b)).

To calculate the displacements $d_{D}$ and $d_{G}$, a method proposed by Paulay and Priestley (1992) for cantilever columns under horizontal displacements is applied. The detailed derivations of $d_{D}$ and $d_{G}$ are presented in the following part. 
Afterwards, the load capacities at G and D are obtained from Eqs. (4.13) and (4.16), respectively.
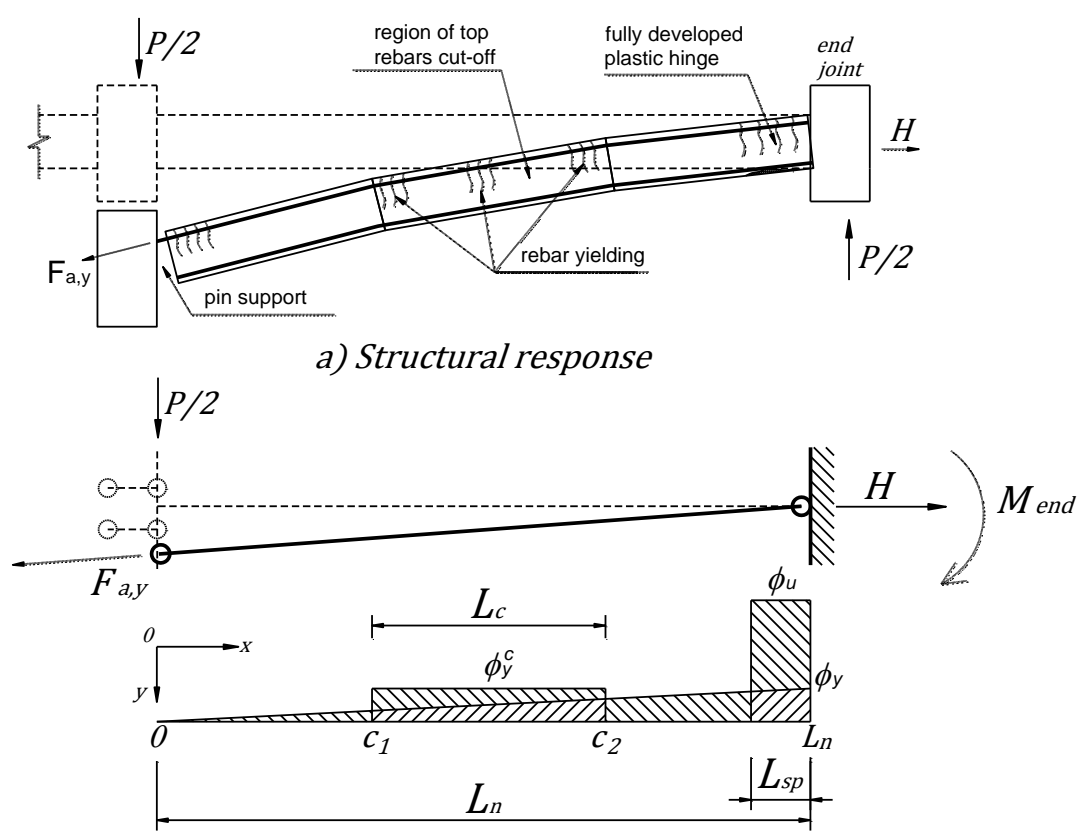

b) Curvature distribution

Fig. 4.6 Proposed response and curvature distribution at point $\mathrm{G}$

\subsubsection{Point D}

At point $\mathrm{D}$, it is assumed that the beam sections at the middle and the end joints remain the maximum plastic moment capacities, $M_{\text {mid }}$ and $M_{\text {end }}$, besides the development of CA (Fig. 4.5(a)). To calculate the plastic hinge lengths near the middle joint $\left(L_{s p}^{\text {mid }}\right)$ and near the end joint $\left(L_{s p}^{e n d}\right)$, Paulay and Priestley (1992) method is applied. However, the equation of plastic hinge length from this method is proposed for a cantilever column subjected to horizontal displacement at the free end. To employ this equation, therefore, equivalent cantilever spans, $L_{n}^{\text {mid }}$ and $L_{n}^{\text {end }}$, are derived which represent the beam spans of segments XY and YZ (Fig. 4.5(a)). As a result, $L_{n}^{\text {mid }}$ and $L_{s p}^{m i d}$ can be computed from Eq. (4.17). Thereafter, $L_{s p}^{m i d}$ and $L_{s p}^{e n d}$ are defined using Eqs. (4.18) and (4.19), respectively. Subsequently, yield and plastic displacements of segment XY can be obtained by Eqs. (4.20) and (4.21), respectively. 
$\frac{L_{n}^{\text {mid }}}{L_{n}^{\text {end }}}=\frac{M_{\text {mid }}}{M_{\text {end }}}$

$L_{s p}^{m i d}=0.08 L_{n}^{\text {mid }}+0.022 d_{\text {mid }} f_{y}$

$L_{s p}^{e n d}=0.08 L_{n}^{\text {mid }}+0.022 d_{\text {end }} f_{y}$

where $d_{\text {mid }}$ and $d_{\text {end }}$ are the diameters of longitudinal tensile bars at the middle and the end joints, respectively.

$\Delta_{y}^{m i d}=\frac{\phi_{y}^{\prime} L_{n}^{m i d^{2}}}{3}$

$\Delta_{u}^{m i d}=\left(\phi_{u}^{\prime}-\phi_{y}^{\prime}\right) L_{s p}^{m i d}\left(L_{n}^{m i d}-0.5 L_{s p}^{m i d}\right)$

where $\phi_{y}^{\prime}$ and $\phi_{u}^{\prime}$ are the yield and ultimate curvatures of the concrete beam section at the middle joint under sagging moment. These values depend on cross-sectional geometry and material properties of concrete and reinforcement stress-strain curve. Park and Paulay (1975) have developed a rational method to calculate these values to construct the moment-curvature response of a flexural section. Nowadays, there are several convenient tools to obtain this response curve. In this study, a crosssectional design tool integrated into the structural analysis software SAP 2000 (Habibullah and Wilson 2005) is employed to determine the yield and ultimate values of curvature at different beam sections based on the material and geometry properties. The input data used in the SAP 2000 are based on concrete cylinder strength, yield strength and ultimate tensile strength of reinforcement with corresponding strains. The simple parametric stress-strain curves of the reinforcement as well as of the unconfined concrete model are automatically generated by SAP2000. After getting $\Delta_{X Y}, \Delta_{Y Z}$ is defined using Eq. (4.22).

$\frac{\Delta_{X Y}}{\Delta_{Y Z}}=\frac{L_{n}^{\text {mid }}}{L_{n}^{\text {end }}}$

\subsubsection{Point $G$}

In terms of vertical loading resistance, the beam at this stage behaves as a cantilever with the span equal to $L_{n}$. Hence, the method from Paulay and Priesley (Eq. (4.23)) can be directly applied to calculate the plastic hinge length at the end joint. All the 
components of $d_{G}\left(\Delta_{y}, \Delta_{u}\right.$ and $\left.\Delta_{c}\right)$ are defined by Eqs. (4.24), (4.25) and (4.26), respectively.

$$
\begin{aligned}
& L_{s p}=0.08 L_{n}+0.022 d_{e n d} f_{y} \\
& \Delta_{y}=\frac{\phi_{y} L_{n}^{2}}{3} \\
& \Delta_{u}=\left(\phi_{u}-\phi_{y}\right) L_{s p}\left(L_{n}-0.5 L_{s p}\right) \\
& \Delta_{c}=\int_{c_{1}}^{c_{2}} \phi_{y}^{c}\left(L_{n}-x\right) d x
\end{aligned}
$$

where $\phi_{u}$ and $\phi_{y}$ are the yield and the ultimate curvatures of the concrete beam section at the end joint under hogging moment. $\phi_{y}^{c}$ is the yield curvature of the concrete beam section at the curtailment region under hogging moment. $c_{1}$ and $c_{2}$ denote locations of the curtailment region (Fig. 4.6(b)). The method for deriving $\phi_{y}$, $\phi_{u}$ and $\phi_{y}^{c}$ is similar to $\phi_{y}^{\prime}$ and $\phi_{u}^{\prime}$.

During the validation of the proposed model by the FEM predictions, it is found that the model-predicted displacements at G have good agreement with FEM values if $k_{\text {span }}>9$. If $k_{\text {span }} \leq 9$, however, the proposed equation provides smaller values compared to FEM results. The reason is that tensile forces are ignored in the beams. Previous studies show that axial tensile force on columns can also help in increasing the ductility of the plastic hinge, especially when the span-depth ratio is small. To overcome this shortcoming, a modification for Eq. (4.23) for determining the plastic hinge length is made when $k_{\text {span }} \leq 9$. In such situations, $L_{s p}$ will be derived by Eq. (4.27).

$L_{s p}=\operatorname{maximum}\left(0.08 L_{n}, d_{\text {beam }}\right)+0.022 d_{\text {end }} f_{y}$

\subsubsection{Point E}

After the fracture of bottom rebars at both sides of the middle joint, the vertical load capacity has a sudden drop from D to E. Results obtained from tests and FEM analyses show that the applied loads at D and E are proportional to the ratio of plastic moment resistance at end joint and total plastic moment resistance from the 
middle and the end joints. Knowing the value of $P_{D}$, one can compute $P_{E}$ from Eq. (4.28).

$$
\frac{P_{E}}{P_{D}}=\frac{M_{\text {end }}}{M_{\text {mid }}+M_{\text {end }}}
$$

\subsubsection{Points $\mathrm{C}_{1}, \mathrm{D}_{1}, \mathrm{~F}$}

After all the primary points A, B, C, D, E, G and the equations of straight lines $\mathrm{D}_{1} \mathrm{D}$ and FG have been determined, the secondary points $C_{1}, D_{1}, F$ can then be defined based on these data. Point $\mathrm{D}_{1}$ is the intersection of $\mathrm{D}_{1} \mathrm{D}$ and $\mathrm{C}_{1} \mathrm{C}$, i.e. $P_{D_{1}}=P_{C}$. Similarly, Point $\mathrm{F}$ belongs to the line $\mathrm{FG}$ and $P_{F}=P_{E}$. Lastly, $\mathrm{C}_{1}$ is determined from $\mathrm{C}_{1} \mathrm{D}_{1}$ in which $\mathrm{C}$ is the mid-point. However, if the results show that $P_{D}$ is smaller than $P_{C}$ then point $\mathrm{D}_{1}$ does not exist. In such case, $P_{D}$ will be equal to $P_{C}$ while $d_{D}$ remains unchanged and $\mathrm{C}_{1}$ is chosen so that $\mathrm{C}_{1} \mathrm{C}=\mathrm{CD}$ (Fig. 4.7(a)). This scenario represents the case when bottom rebar fracture occurs early before CA is efficiently mobilised. Moreover, if $\mathrm{E}$ lies below line FG then $\mathrm{F}$ is determined as the intersection of lines DE and FG and point $\mathrm{E}$ is no longer needed (Fig. 4.7(b)). It demonstrates the response of a sub-assemblage with relatively large horizontal restraint stiffness.

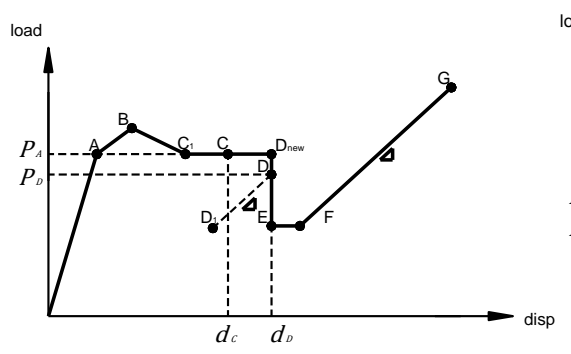

a) $P_{D}<P_{C}$

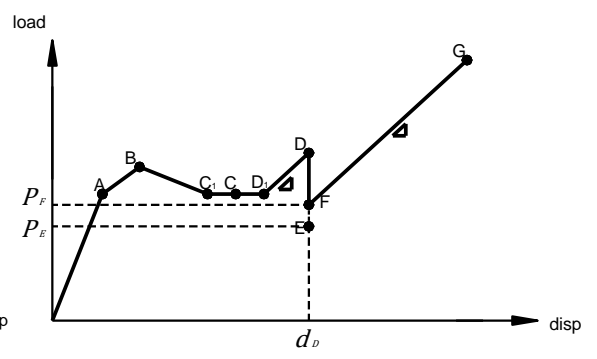

b) Point E lies below FG

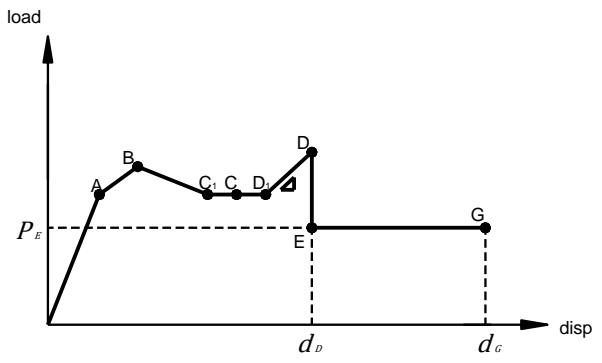

c) $k_{\text {hor }}$ too small

Fig. 4.7 Special cases of the simplified model 


\subsubsection{Minimum value for horizontal stiffness ratio $\boldsymbol{k}_{h o r}$}

Observations from different span-depth ratios show that after the fracture of bottom rebars at the middle joint, CA can be efficiently mobilised at the latter part (FG) only if $k_{\text {hor }}$ is not less than a certain value defined by Eq. (4.29). Therefore, in the proposed response, polyline EFG will be considered as a horizontal line EG if $k_{\text {hor }}$ does not satisfy this stiffness requirement. In this case, $P_{G}$ is equal to $P_{E}$ and point $\mathrm{F}$ is no longer needed (Fig. 4.7(c)).

$$
k_{\text {hor }} \geq \quad \begin{aligned}
& 0.011 \text { if } k_{\text {span }} \geq 11 \\
& 0.019 \text { if } 11 \geq k_{\text {span }} \geq 9 \\
& 0.029 \text { if } 9 \geq \mathrm{k}_{\text {span }} \geq 7
\end{aligned}
$$

\subsubsection{Minimum strength of axial restraint $R_{A}$}

In the numerical simulations, the horizontal restraint stiffness $K_{A}$ is assumed to behave elastically. Nonetheless, in actual buildings, boundary restraints may respond beyond the elastic range and may be subject to large inward deflections if ductile failure governs, or may suddenly fail in a brittle manner. In this approach, the horizontal restraint is only allowed to behave elastically with the maximum elastic strength $R_{A}$. If the horizontal reaction force exceeds $R_{A}$, CA cannot be developed in FG part. To fully mobilise CA in the beam-column structure, the capacity of horizontal restraint $R_{A}$ must be greater than the maximum horizontal reaction $H_{\max }$ which can be fully developed in the sub-assemblage before the top rebars fracture. At point $\mathrm{G}$, it is assumed that the beam top rebars near the end joint section reaches its ultimate strength $f_{u}$. Through numerical predictions and neglecting the tensile force from the bottom rebars as well as the tensile strength of concrete, $H_{\max }$ is related to ultimate tensile force of top reinforcement $F_{\text {top }}^{u}$ by Eq.

$R_{A} \geq H_{\text {max }}=F_{\text {top }}^{u}\left(1-\frac{1}{\left(k_{\text {span }}-4.5\right)^{1.6}}\right)\left(1-\frac{1}{\left(k_{\text {hor }}+1.05\right)^{20}}\right)$

when both $k_{\text {span }}$ and $k_{\text {hor }}$ are large $\left(k_{\text {span }} \geq 11\right.$ and $\left.k_{\text {hor }} \geq 0.12\right), \mathrm{H}_{\max }$ is equal to $F_{\text {top }}^{u}$. But when $k_{\text {span }}$ is small or $k_{\text {hor }}$ is weak, $H_{\max }$ can be reduced to $50 \%$ of $F_{\text {top }}^{u}$. 
For the single column loss scenario, $K_{A}$ and $R_{A}$ can be computed based on structural properties of adjacent members next to the double-span beam. If $R_{A} \geq H_{\max }$, then CA can be fully developed following all the equations of the proposed model. But if $R_{A}<H_{\text {max }}, \mathrm{CA}$ is neglected after all the bottom rebars have fractured and EFG will be replaced by a horizontal line EG, similar to the case of relatively small $k_{\text {hor }}$ presented in Fig. 4.7(c).

\subsubsection{Comparisons between the simplified model and the FEM results}

Parameters derived from the proposed model such as displacements and loads at controlling points are compared to FEM predictions of 120 cases (Table 4.1) and the results are shown in Fig. 4.8. For each comparison, error statistics is carried out calculating the mean value and the COV (coefficient of variation) value. If the mean value is in the range of $0.9 \sim 1.1$ and the COV value is not larger than 0.15 , the simplified model is considered to be acceptable. If the mean value or the COV are outside this range, they must be on the conservative side (i.e. underestimating the structural capacity provided by FEM) to be considered as acceptable. Basically, the values from the proposed model are either close to or more conservative than the numerical predictions.

For point A: A comparison of $d_{A}$ between the proposed model and the corresponding displacement from FEM which has the applied load equal to $P_{A}$ is shown in Fig. 4.8(a).

For point C: Results of $P_{C}$ and $d_{C}$ are compared between FEM analyses and the proposed equations. The comparisons show relatively good agreement. Values from the simplified model are more conservative than FEM models in most cases (Fig. 4.8(b) and (c)).

For line FG: In Eq. (4.13), $\beta_{1} F_{a}^{y} / L_{n}$ represents the gradient of FG while $\beta_{2} . d_{\text {beam }}$ is the root of the equation $P_{F G}(d)=0$ which represents the location of FG. Comparisons of the gradient and the root from the function of line FG between numerical and simplified methods are carried out and illustrated in Fig. 4.8(d) and 
(e). The predicted values for the gradient are relatively close to the FEM results. On the other hand, the proposed model gives more conservative values of the root compared to FEM when $k_{\text {hor }}$ is relatively small. Thus, the use of this model will provide safe results for the case of weak axial restraints.

For point E: Fig. 4.8(f) describes a comparison of $P_{E} / P_{D}$ between the numerical predictions and the results from Eq. (4.28). The errors are within an acceptable range.

Displacement at D and G: Comparisons are made between FEM and analytical results for the displacements $d_{D}$ and $d_{G}$. The results are shown in Fig. 4.8(g) and (h). Basically, the simplified method has good agreement with numerical results for $d_{D}$ and gives more conservative results for $d_{G}$. Maximum tensile reaction $H_{\text {max }}$ : The analytical values of $H_{\text {max }}$ show relatively good agreement with those computed by FEM (Fig. 4.8(i)).

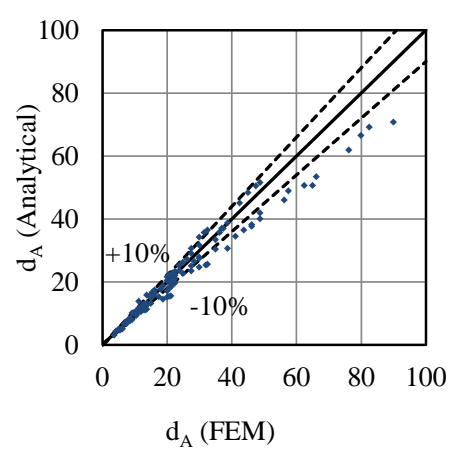

a) $d_{A}, \mathrm{~mm}$

(Mean $=0.94, \mathrm{COV}=0.11$ )

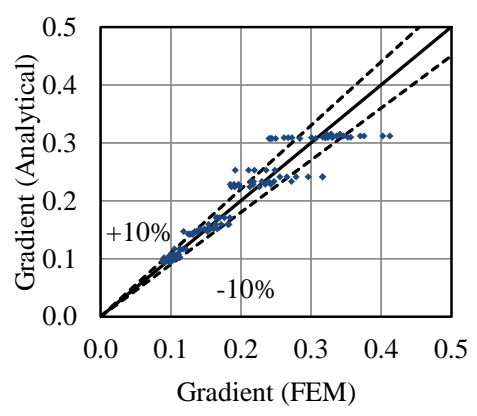

d) Gradient of line FG, kN/mm

(Mean=1.02, $\mathrm{COV}=0.11$ )

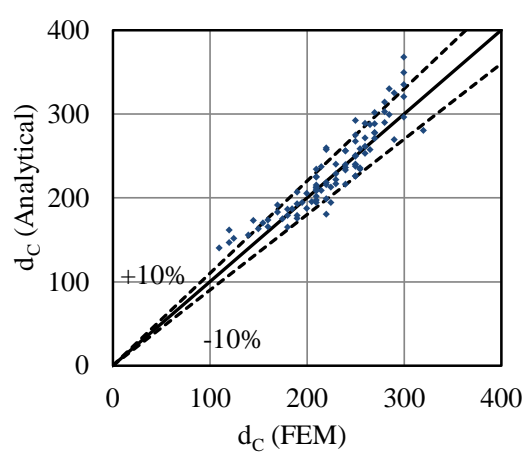

b) $d_{C}, \mathrm{~mm}$

(Mean=1.03, $\mathrm{COV}=0.09)$

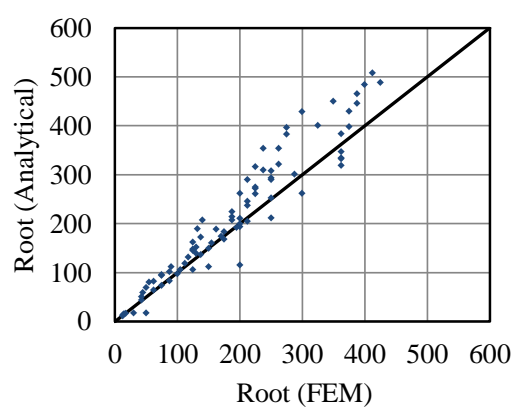

e) Root of line FG, mm

(Mean=1.12, $\mathrm{COV}=0.18$ )

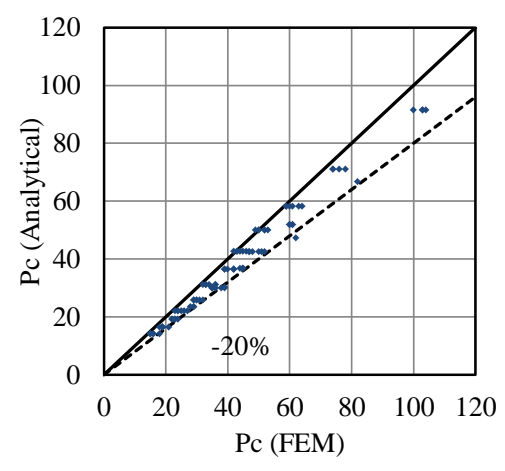

c) $P_{C}, \mathrm{kN}$

(Mean $=0.89, \mathrm{COV}=0.07)$

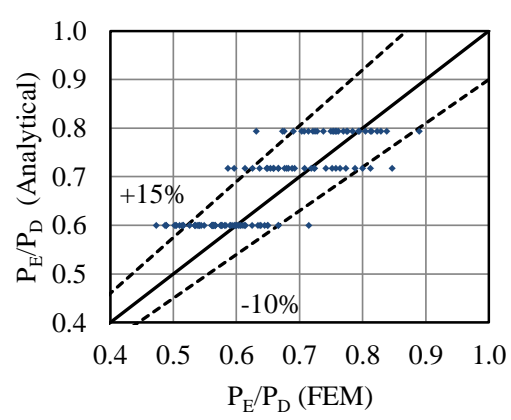

f) $P_{E} / P_{D}$

(Mean=1.05, $\mathrm{COV}=0.10$ ) 


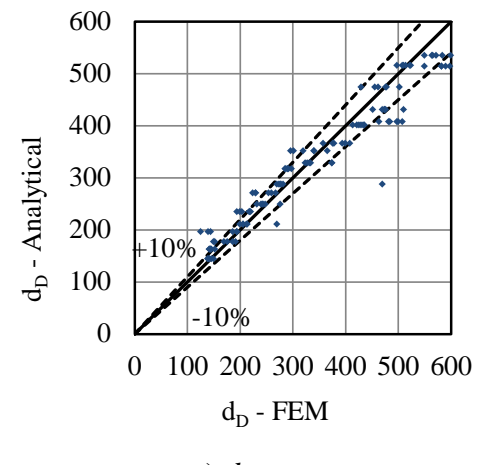

g) $d_{D}, \mathrm{~mm}$

(Mean=1.01, COV=0.12)

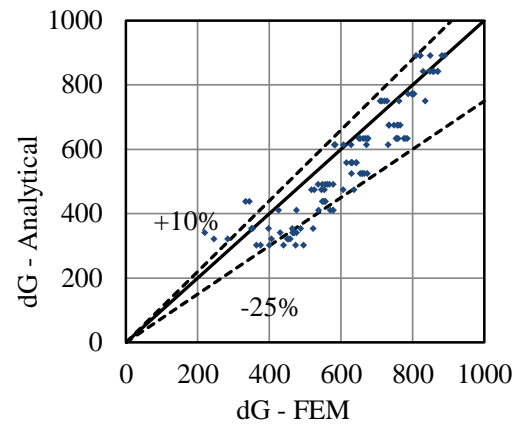

h) $d_{G}, \mathrm{~mm}$

$($ Mean $=0.92 \mathrm{COV}=0.17)$

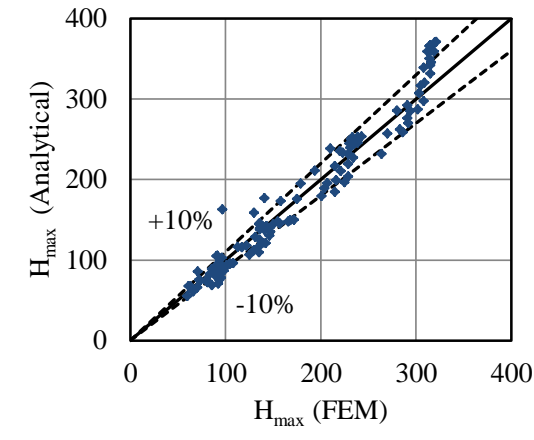

i) $H_{\max }, \mathrm{kN}$

(Mean=1.01, $\mathrm{COV}=0.11)$

Fig. 4.8 Comparisons between simplified and FEM models

\subsubsection{Dynamic assessment of RC sub-assemblages under single column removal scenario}

Once the piecewise static behaviour of the sub-assemblage is already defined, the dynamic effect can be easily achieved by employing Izzuddin's method. Applying this approach to the proposed simplified static polyline, there are three possible peaks of dynamic response at displacements $d_{B}, d_{D}$ and $d_{G}$ (Fig. 4.9). The equivalent dynamic loads at these deflections, i.e. $P_{B}^{d}, P_{D}^{d}$ and $P_{G}^{d}$, are derived by Eqs. (4.31) to (4.33). The largest value from these three dynamic loads is the ultimate dynamic capacity of the sub-assemblage.

$$
\begin{aligned}
P_{B}^{d}=\left(P_{A} d_{A}+\right. & \left.\left(P_{B}+P_{A}\right)\left(d_{B}-d_{A}\right)\right) / 2 d_{B} \\
P_{D}^{d}=\left(P_{A} d_{A}+\right. & \left(P_{B}+P_{A}\right)\left(d_{B}-d_{A}\right)+\left(P_{C}+P_{B}\right)\left(d_{C 1}-d_{B}\right) \\
& \left.+2 P_{C}\left(d_{D 1}-d_{C 1}\right)+\left(P_{D}+P_{D 1}\right)\left(d_{D}-d_{D 1}\right)\right) / 2 d_{D} \\
P_{G}^{d}=\left(P_{A} d_{A}+\right. & \left(P_{B}+P_{A}\right)\left(d_{B}-d_{A}\right)+\left(P_{C}+P_{B}\right)\left(d_{C 1}-d_{B}\right) \\
& +2 P_{C}\left(d_{D 1}-d_{C 1}\right)+\left(P_{D}+P_{D 1}\right)\left(d_{D}-d_{D 1}\right) \\
& \left.+P_{E}\left(d_{F}-d_{E}\right)+\left(P_{G}+P_{F}\right)\left(d_{G}-d_{F}\right)\right) / 2 d_{G}
\end{aligned}
$$




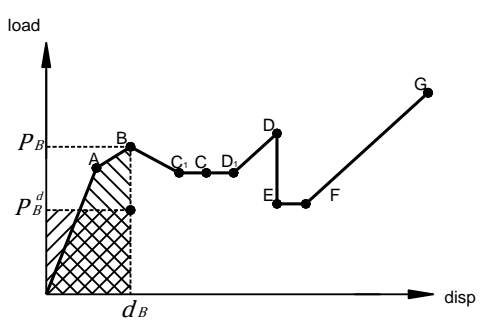

a) Dynamic load $P_{B}^{d}$ at $d_{B}$

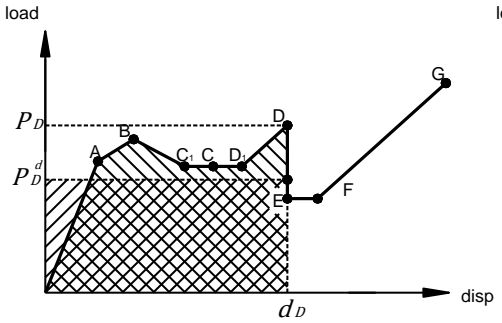

b) Dynamic load $P_{D}^{d}$ at $d_{D}$

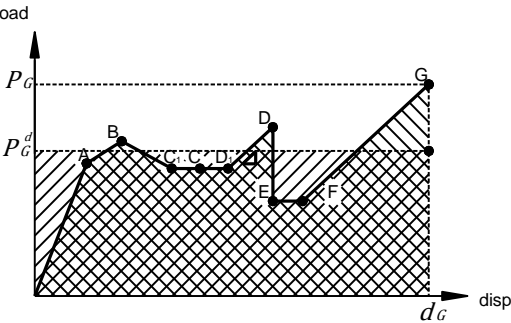

c) Dynamic load $P_{G}^{d}$ at $d_{G}$

Fig. 4.9 Dynamic assessment for the simplified response

In summary, the solution procedure to obtain the simplified semi-analytical method is presented in Fig. 4.10.

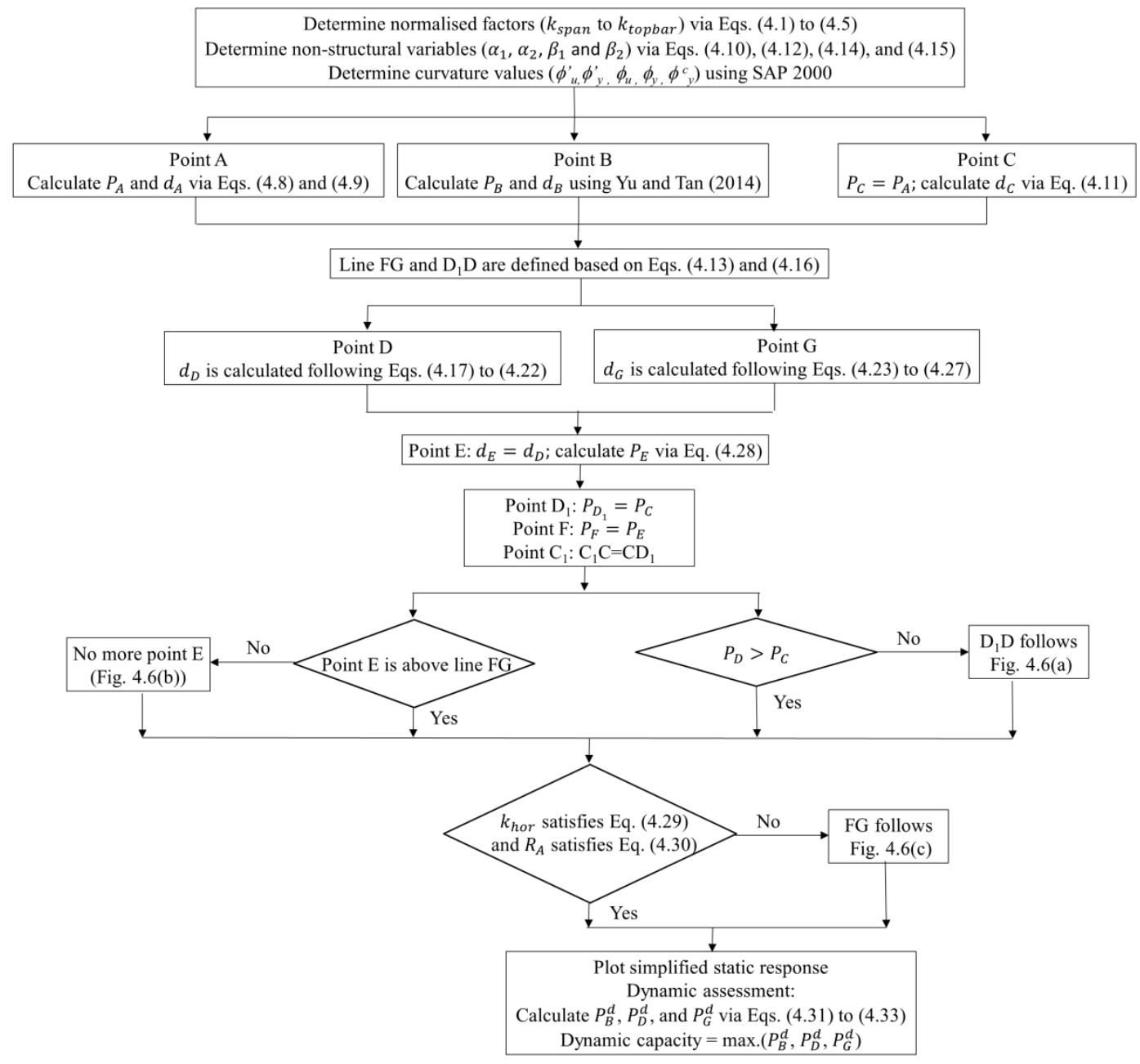

Fig. 4.10 Procedure of the simplified model 


\subsection{Test validations using the semi-analytical model}

To verify the application of the proposed model for CA and structural response, experimental results including seven tests from Yu and Tan (2013a, b) and two tests from Lim et al. (2015) are used to construct the piecewise curve of response using the equations and solution procedure described in Section 4.3. Other tests on 2D beam-column structures (Yi et al. 2008, Su et al. 2009, Sadek et al. 2011) are not used for the validation since the stiffness of horizontal restraints was not directly measured in these tests. Input information as well as all necessary variables for deriving the model are presented in Table 4.2 and Table 4.3. Comparisons between semi-analytical and test results are presented in Fig. 4.11. The proposed model provides reasonably good predictions of structural response, and yet requires much less effort compared to detailed FEM simulations. For specimen PR from Lim et al. (2015), since the test was prematurely terminated at a deflection of $396 \mathrm{~mm}$ before any fracture of top longitudinal rebars occurred, the response is defined up to this displacement only. In most of the tests, lateral boundary conditions for both sides were well restrained by either the strong wall or the A-frame. Hence, the ultimate strength $\boldsymbol{R}_{\boldsymbol{A}}$ of these restraints is considered to be relatively large. However, for specimen PR, one side of the frame was partially restrained while the other was fully restrained. The actual test results showed no enhancement from CA after the fracture of rebars at the middle joint. During the test, the horizontal reaction of the weak side gradually increased to $54 \mathrm{kN}$ before the adjacent column was pulled inwards, indicating the yielding state of the restraint. Thus, in the simplified model, the strength of horizontal restraint $\boldsymbol{R}_{\boldsymbol{A}}$ is limited to $54 \mathrm{kN}$ which is smaller than $\boldsymbol{H}_{\boldsymbol{m a x}}$ calculated from Eq. (4.30) (equal to $107.9 \mathrm{kN}$ ). As a result, the simplified response of specimen PR after the fracture of the bottom rebars is a horizontal line EG (Fig. 4.7(c)), which shows good agreement with the actual test.

By assuming perfectly-rigid rotational restraint, the proposed model neglects the rotational deformation at the end joint, and therefore tends to overestimate the stiffness of the substructure at the initial stage (part OA). As a result, the values of $d_{A}$ calculated from the model are smaller than those from actual tests. Nonetheless, 
this assumption has little effect on the total strain energy of the static response which is important to dynamic assessment of progressive collapse. In terms of maximum displacement and corresponding ultimate load capacity (point G), the simplified model provides relatively good matching with all the test results (Table 4.4). A comparison study between the model predictions and test results regarding maximum reaction force $H_{\max }$ at point $\mathrm{G}$ and maximum equivalent dynamic load using Izzuddin's method shows very good agreement (Table 4.4).

As the semi-analytical model is aimed for hand calculations with its equations well presented, one can easily use Excel spreadsheet to automate the calculation process in order to produce the simplified responses from several structural options with ease. On the other hand, if detailed models (LS-Dyna) are used, it would easily take 4 to 8 hours for one model to run, not to mention the time taken to generate the model itself. Therefore, the effectiveness of the simplified method in terms of time consumption is obvious.
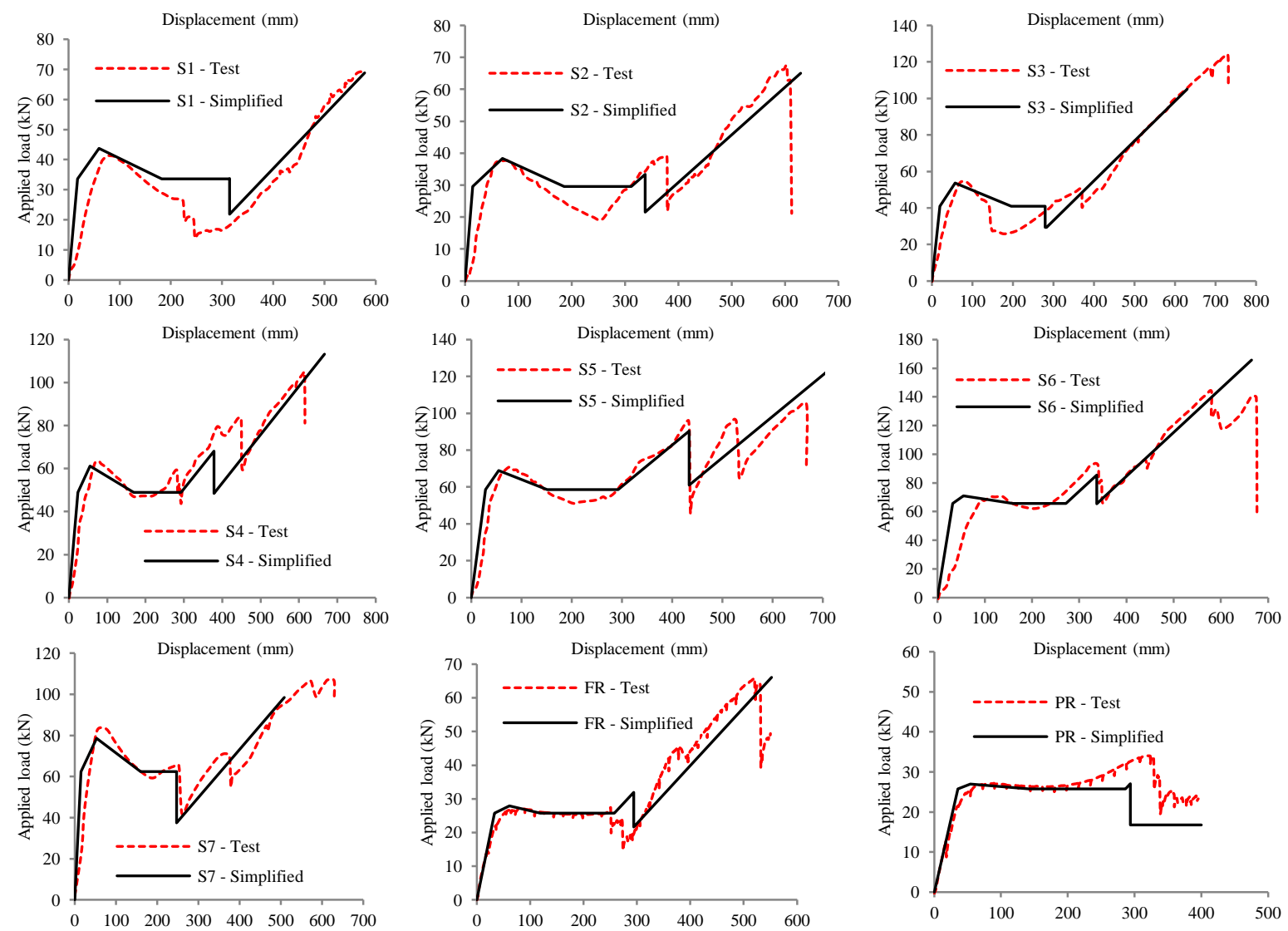

Fig. 4.11 Test validation using the proposed model 


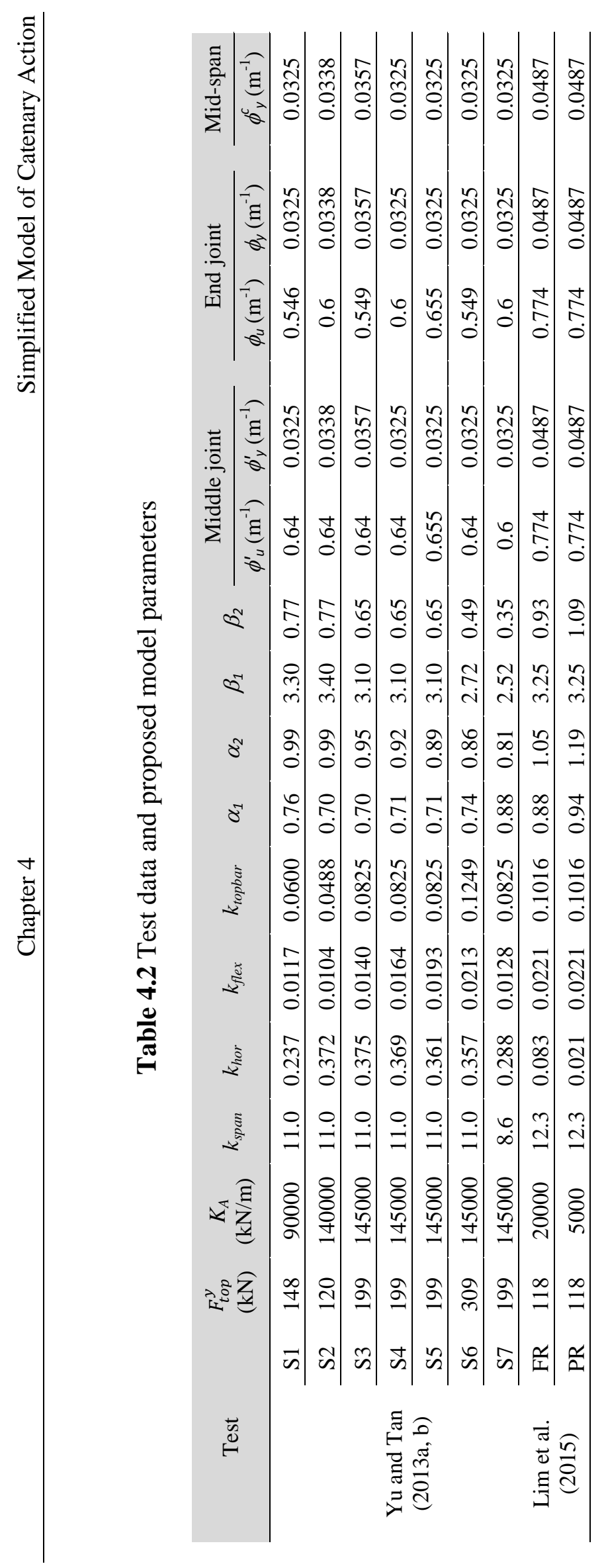




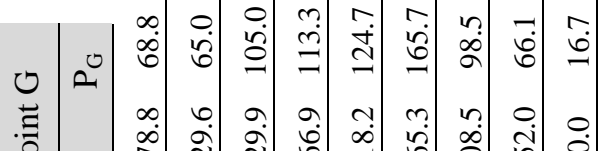

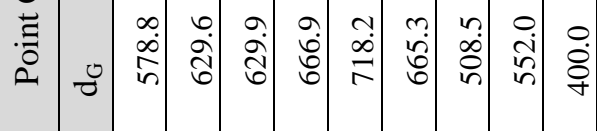

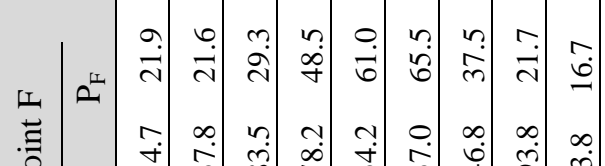

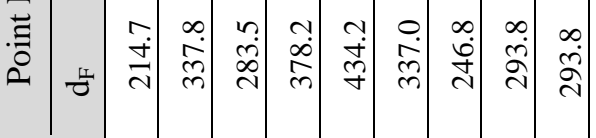

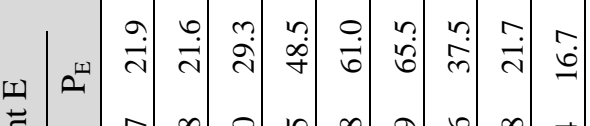

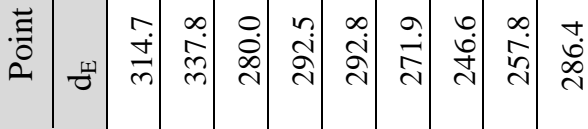

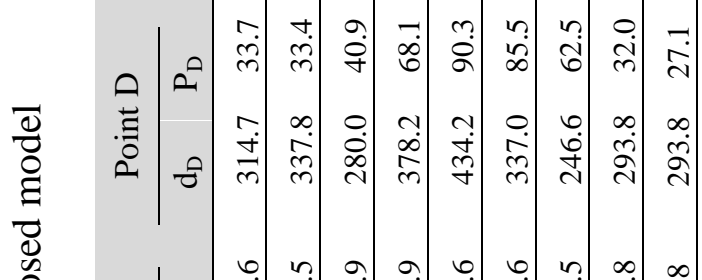

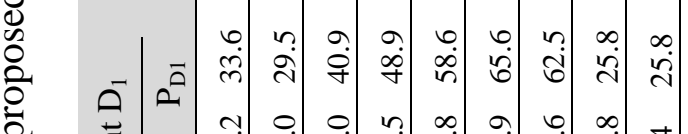

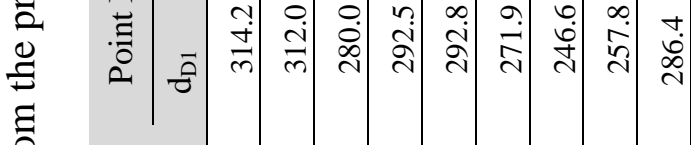

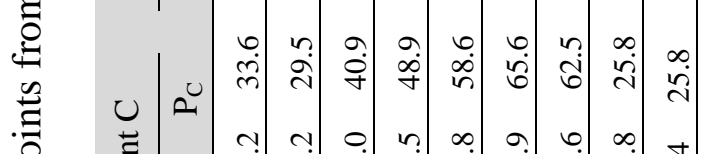

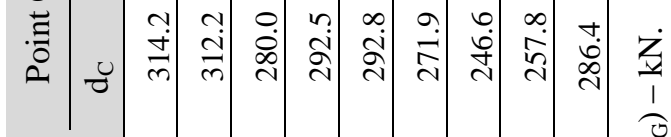

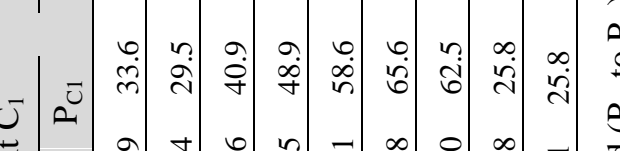

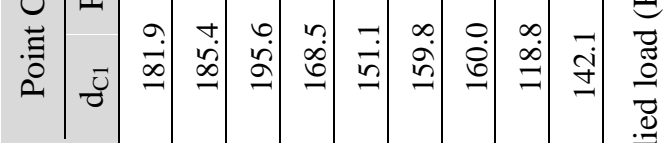

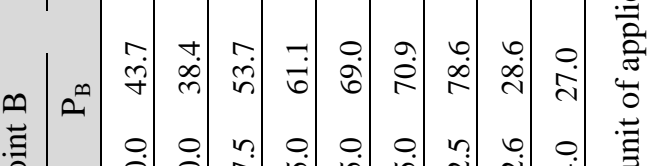

言

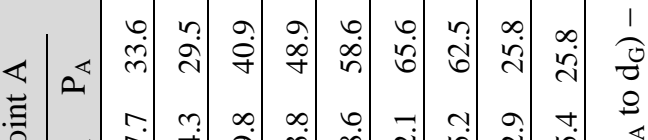

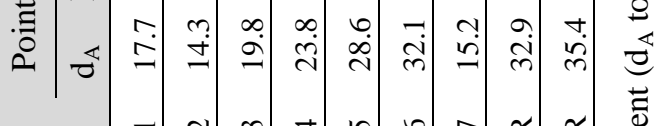
च|

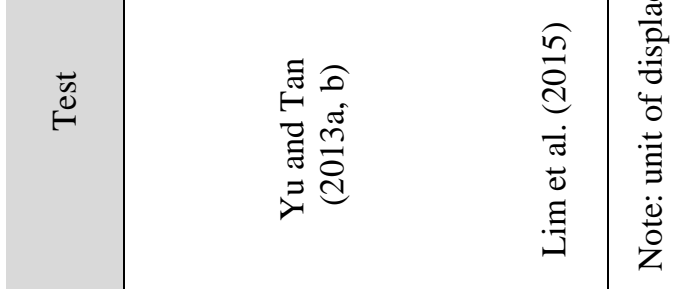




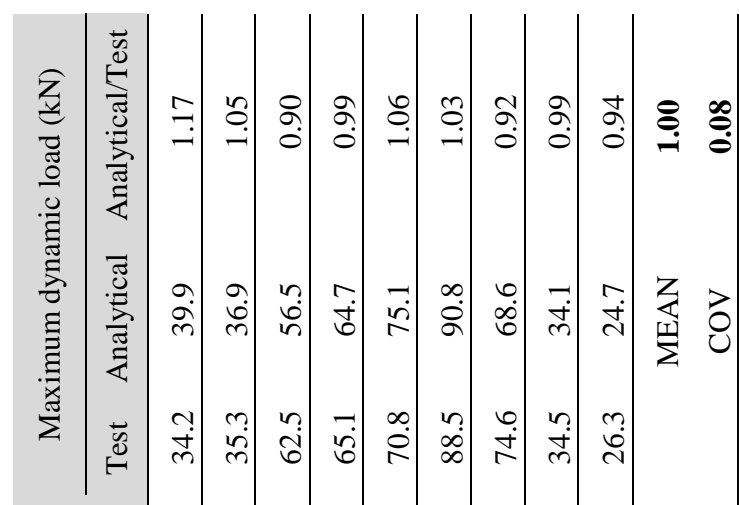




\subsection{Discussions on the semi-analytical model for sub-assemblages}

\subsubsection{Limits of application}

Since the simplified model is developed based on FEM analyses, there are some assumptions that should be clearly stated when applying this approach. First, the model can only be used for structures with concrete cylinder strength ranging from 20 to $50 \mathrm{MPa}$. Structures using high strength or fibre concrete are outside the scope of this research. Secondly, longitudinal reinforcement consists of deformed bars with yield strength up to $600 \mathrm{MPa}$. It is assumed that at the beam sections near the end joint and the middle joint, the ratio of top bars is larger than bottom bars, which is generally satisfied in normal RC design. The anchorage length of reinforcement into the joint regions follows structural requirements, e.g. EN 1992-1-1 (2004), to ensure good bonding between concrete and reinforcement. In terms of structural geometry, the model is only valid when $k_{\text {span }} \geq 7$ where flexure dominates behaviour instead of shear. With regard to CA developed in the double-span beam after the fracture of the bottom reinforcement, it is shown that CA is only significant when the requirements for horizontal stiffness (Eq. (4.29)) and strength (Eq. (4.30)) are satisfied. Concerning rotational stiffness, $k_{\text {rot }}$ has to satisfy Eq. (4.7) to ensure rotational resistance at the end joint will not affect CA. This requirement was easily fulfilled in the tests for both sub-assemblages ( $\mathrm{Yu}$ and Tan 2013a, b) and beam-column frames (Lim et al. 2015). The proposed model neglects connection gaps in the restraint boundaries. Such gaps have little effect on the final CA part (FG); they only affect the first peak of CAA (point B).

\subsubsection{Discussions on symmetric conditions}

The simplified model is based on half-specimen simulations, assuming symmetry for geometry, material and boundary conditions. In practice, it is likely that restraint stiffness values $\left(K_{A}\right.$ and $\left.R_{A}\right)$ from two sides of the two-span beam structure are not the same (e.g. number of adjacent bays on each side is different). In this case, the smaller horizontal stiffness value should be applied. 
In circumstances where geometry and material properties from two sides of the double-span beam are not identical, or the joint may not be located at the middle of the double span, the total behaviour of the sub-assemblage $P_{\text {total }}$ is calculated as the average of the responses from two symmetric systems which are similar to the single-span structures on the left and the right sides of the removed column (Fig. 4.12(a)), so-called the "left" and the "right" systems, corresponding to the same displacement $d$ as shown in Eq. (4.34).

$P_{\text {total }}(d)=\frac{P_{\text {left }}(d)+P_{\text {right }}(d)}{2}$

$P_{\text {left }}(d)$ and $P_{\text {right }}(d)$ can be determined using the simplified model (Fig. 4.12(b)) based on their geometrical and material properties noting that horizontal restraints $\left(K_{A}\right.$ and $R_{A}$ ) of the two systems are identical. However, these two simplified responses are not totally independent. They are coupled with each other at specific displacements when rebars fracture. Due to differences in structural responses, displacements corresponding to top and bottom rebar fractures of the "left" and the "right" systems may not be the same. Fig. 4.12(b) shows an example of the two simplified responses in which bottom rebars of the "left" system fracture earlier compared to those of the "right" one $\left(d_{D, L}<d_{D, R}\right)$, and vice versa regarding the fracture of top rebars $\left(d_{G, L}>d_{G, R}\right)$.

To simply combine the responses of the two systems into the total response $P_{\text {total }}$, it is assumed that after the fracture of bottom rebars from the "left" system (at $\left.d=d_{D, L}\right)$, bottom rebars from the "right" system are also considered as fracture. Therefore, the behaviour of the "right" system will change from $D_{1, R} D_{R} E_{R} F_{R}$ to $\mathrm{D}_{1, \mathrm{R}} \mathrm{D}{ }_{\mathrm{R}} \mathrm{E}_{\mathrm{R}} \mathrm{F}_{\mathrm{R}}$ as shown in Fig. 4.12(b). As a result, $P_{\text {total }}$ at points $\mathrm{D}$ and $\mathrm{E}$ $\left(d=d_{D, L}\right)$ shall be calculated as follows:

$P_{\text {total }}\left(D_{T T}\right)=\frac{P_{\text {left }}\left(D_{L}\right)+P_{\text {right }}\left(D^{\prime}{ }_{R}\right)}{2}$
$P_{\text {total }}\left(E_{T T}\right)=\frac{P_{\text {left }}\left(E_{L}\right)+P_{\text {right }}\left(E^{\prime}{ }_{R}\right)}{2}$ 
Similarly, after the top rebars from the "right" system have fractured (at $d=d_{G, R}$ ) causing a total failure to this structure, behaviour of the "left" system is also considered to have terminated and segment $F_{L} G_{L}$ is replaced by $F_{L} G^{\prime}{ }_{L}$ as presented in Fig. 4.12(b). The corresponding $P_{\text {total }}$ is then calculated by Eq. (4.37).

$$
P_{\text {total }}\left(G_{T T}\right)=\frac{P_{\text {left }}\left(G_{L}^{\prime}\right)+P_{\text {right }}\left(G_{R}\right)}{2}
$$

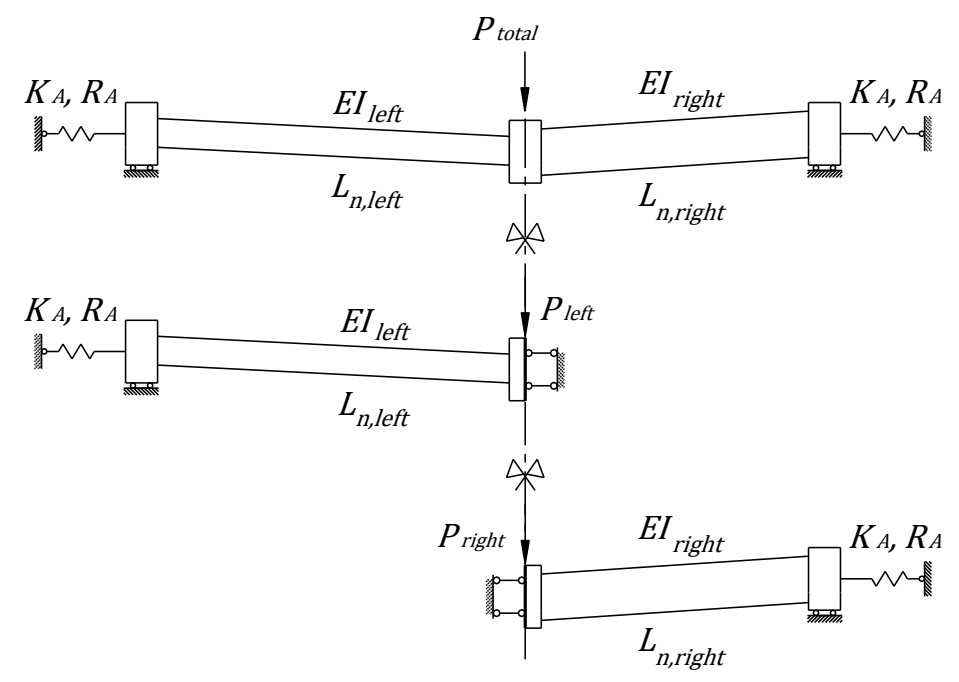

a) The "left" and the "right" systems

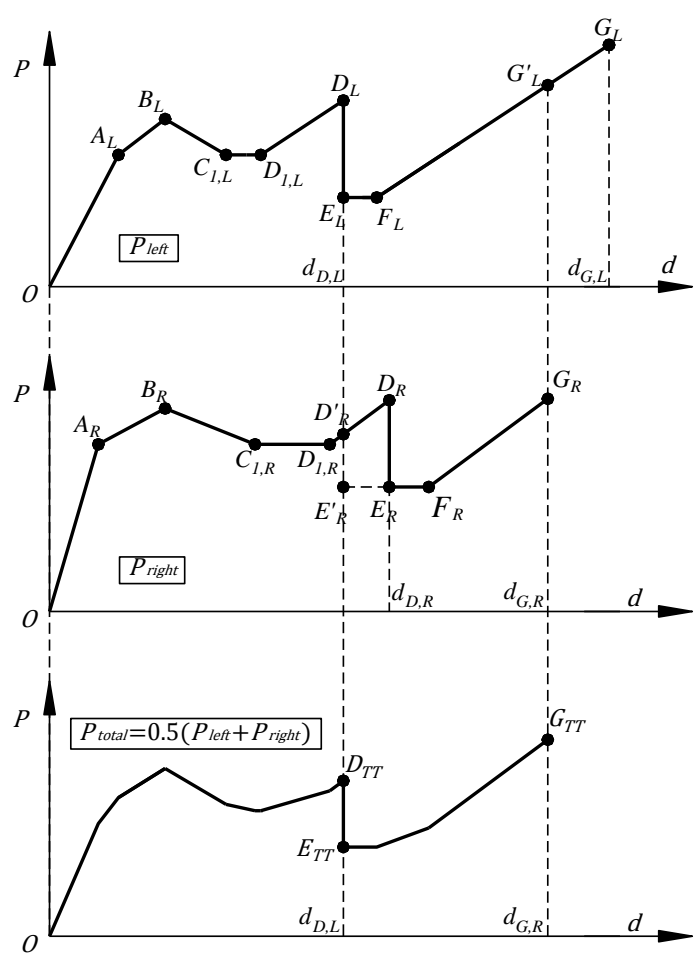

b) Simplified responses of the "left", the "right" and the total systems

Fig. 4.12 Combined response from two sides of the sub-assemblage 
To verify this coupling method for double-span beams with different geometry/material properties from the two sides, FEM analyses are conducted for an example shown in Fig. 4.13. Three simulations are performed representing responses from the "left", the "right", and the "total" systems. The results are compared with predictions from the semi-analytical approach using the coupling of rebar fracture from the "left" and the "right" structures (Fig. 4.14). It is shown that the simplified method gives conservative result compared to FEM prediction.

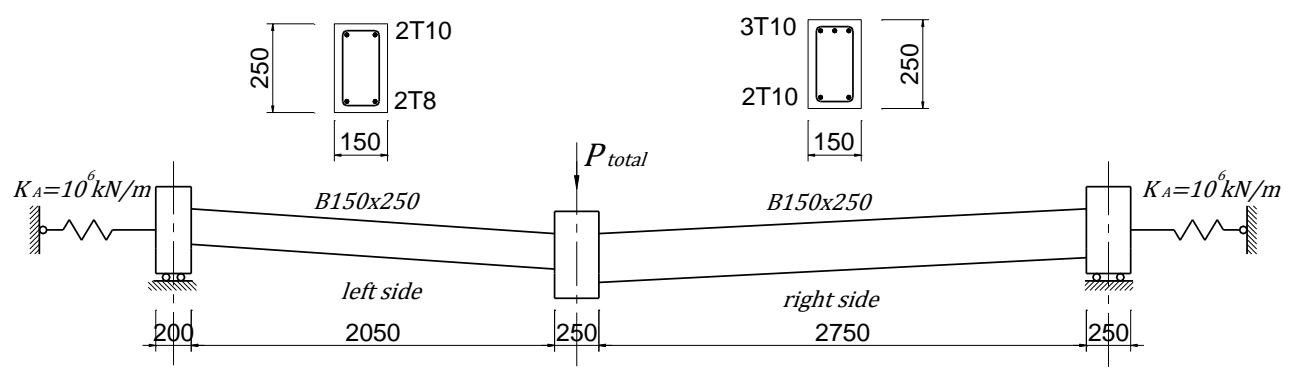

Fig. 4.13 Example of sub-assemblage with two different sides

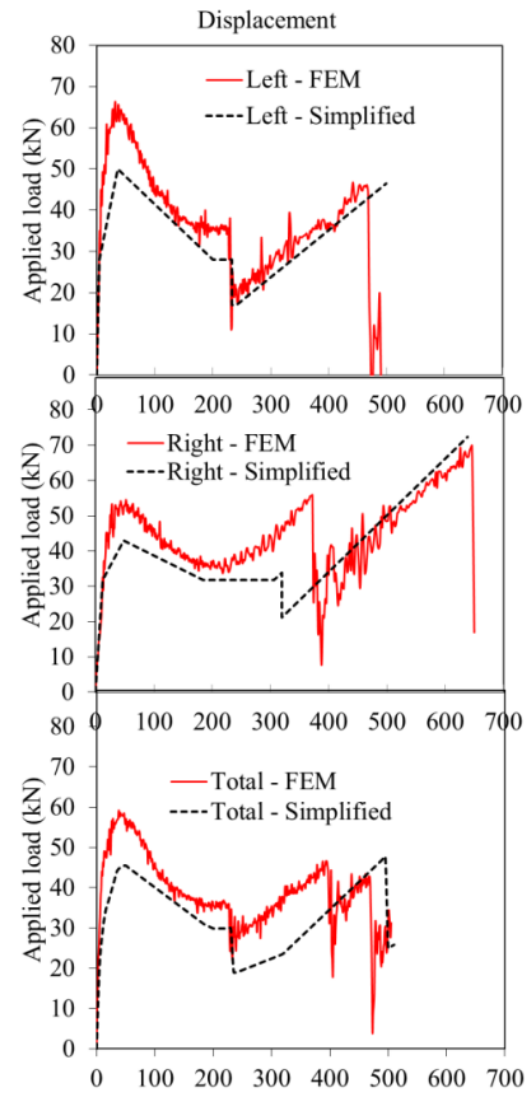

Fig. 4.14 Comparisons between FEM and simplified method for sub-assemblage with two different sides 


\subsection{Conclusions on the simplified model}

In this chapter, parametric studies based on numerical analyses are carried out to gain a better understanding of sub-assemblage static responses, especially the development and contribution of CA before and after the fracture of bottom rebars at the middle joint. The importance of span-depth ratio and horizontal restraint to the mobilisation of $\mathrm{CA}$ in $\mathrm{RC}$ sub-assemblages is quantified. CA can only be considered to be significant if the span-depth ratio is not less than 7. Similarly, CA cannot produce any enhancement in structural capacity after the fracture of bottom rebars if the normalised horizontal stiffness is too small (Eq. (4.29)). It is also shown that rotational stiffness only affects CAA and has less influence on the remaining response. Other factors such as concrete strength, connection gap or bottom reinforcement in beams have little effect on subsequent CA phase. The assumption of elastic stiffness for horizontal restraint simplifies the analysis but is difficult to achieve in practice. Instead, if the strength of horizontal restraint is smaller than the maximum horizontal reaction required by the sub-assemblage, then CA will not be fully mobilised after the fracture of bottom rebars.

The proposed semi-analytical model provides a quick means for estimating the overall behaviour of beam-column sub-assemblages under $\mathrm{CL}$ condition. It simplifies the nonlinear response into a piecewise multi-linear curve with some controlling points. The assumptions of relatively good rotational restraint and bonding condition yield reasonable predictions as the model has been validated by test results. Strain energy as well as the maximum dynamic load can then be easily obtained from the simplified static response. Although the model is developed based on symmetric assumptions of structural and boundary conditions, it can be conservatively applied for non-symmetric sub-assemblages.

After conducting extensive investigations on static response of $\mathrm{RC}$ beam-column structures subjected to CL condition, an experimental programme is proposed and presented in Chapter 5 including three test series. While Series I is aimed to investigate dynamic responses of $2 \mathrm{D}$ frames under CL condition, compared with the related quasi-static tests from Lim et al. (2015), Series II studies quasi-static and 
dynamic responses of 2D structures subjected to UDL, which is one of the technical gaps mentioned in Chapter 2. The last series, Series III, focuses on the blast effect on structural behaviour in a sudden column removal event triggered by contact detonation. 



\section{CHAPTER 5 EXPERIMENTAL PROGRAMME ON RC SUBSTRUCTURES}

\subsection{Introduction}

To simulate the loss of a supporting column, most quasi-static tests applied a displacement-controlled point-load method, in which the beam-column joint above the removed column was pushed downwards by either an actuator or a hydraulic jack (Yi et al. 2008, Su et al. 2009, FarhangVesali et al. 2013, Yu and Tan 2013a, b). Structural response of such CL quasi-static tests, including the mobilisation of CA before and after the fracture of bottom rebars near the middle joint, has been discussed in Chapter 4. To compare the structural behaviours between quasi-static and dynamic regimes on the same specimen design, free-fall dynamic tests were conducted in some previous studies simulating the column loss scenario by suddenly eliminating the supporting mechanism of the middle joint (Tian and $\mathrm{Su}$ 2011, Qian and Li 2012b, Sagiroglu 2012, Orton and Kirby 2013). However, those dynamic studies applied UDL configuration which was different with the corresponding static studies (under CL condition).

Regarding quasi-static environment, as previously mentioned in Section 2.3.1.1, static tests on 2D beam-column structures applying UDL condition have not yet been investigated in any reported experimental work. In terms of free-fall dynamic environment, if CL is employed, a massive weight equal to the total applied load will need to be attached to the middle joint of the structure, which may pose safety concerns. Moreover, the weight attached to the middle joint can affect the head room underneath the specimen, reducing the deformation capacity of the test setup. In the CL dynamic blast-induced tests from Yu et al. (2014), due to insufficient head room for deflection, specimens under high load level $(47 \mathrm{kN})$ hit the ground before any final failure occurred. As a result, the development of CA after the fracture of bottom rebars, which had been confirmed in the corresponding static tests (Yu and Tan 2013a), was not yet demonstrated in the dynamic tests. 
To date, the simplified method proposed by Izzuddin et al. (2008) has been widely used to assess dynamic capacity of a structural system under progressive collapse based on its nonlinear static response. Nonetheless, it is important to establish an experimental programme to verify if this assumption is indeed valid in dynamic free-fall and actual blast environments. This will be critical to establishing a reliable structural model, especially within CA stage.

Although progressive collapse resistance for RC structures has been widely studied in recent years, there are very limited tests that involve explosives or blast load regime. Besides, the effects of blast pressure on structural response were not sufficiently considered in the reported literature (Sasani et al. 2007, Sasani 2008, Yu et al. 2014). In the contact detonation tests by Yu et al. (2014), which has been used to validate the FEM model in Chapter 3, some important data were not measured, i.e. stiffness of horizontal restraints and blast effects on the subassemblages. Thus, some assumptions for these parameters are made leading to difficulties in validating the FEM model. Hence, there is a need to conduct blast tests for RC frames with the objective of measuring all structural responses as well as boundary and loading characteristics. In addition, this experimental work would supplement valuable data on the behaviour of beam-column structures under different loading rates ranging from static, dynamic to blast load regime.

With the aim to expand the study on ALPs in RC structures from quasi-static to free-fall dynamic, and to actual blast condition, as well as to investigate effects of different loading configurations (CL versus UDL), an experimental programme was conducted in this study. There were three test series on 2D RC frames including (Series I) free-fall dynamic tests applying CL condition, (Series II) static and freefall dynamic tests applying UDL condition, and (Series III) contact detonation tests applying CL condition. The experimental programme employed similar geometry, reinforcing arrangement, material properties and boundary restraints from a previous CL quasi-static test series by Lim et al. (2015) which focused on the effects of horizontal restraint conditions on the mobilisation of CA. Compared to the sub-assemblages studied in Chapters 3 and 4 , the beam-column structures 
proposed for the experimental programme involved more realistic boundary conditions, including side columns (instead of column stubs) and extended beams at both sides. Such design represented closer-to-reality scenarios, which were the removal of either an interior column (two sides appropriately restrained) or a nextto-outermost column (restraint from one side was significantly weaker than the other). Fig. 5.1 presents linkages between the proposed testing programme in this study and the previous CL tests (Lim et al. 2015), as well as the relationship among the three test series. Objectives of each series are then presented in the following part.

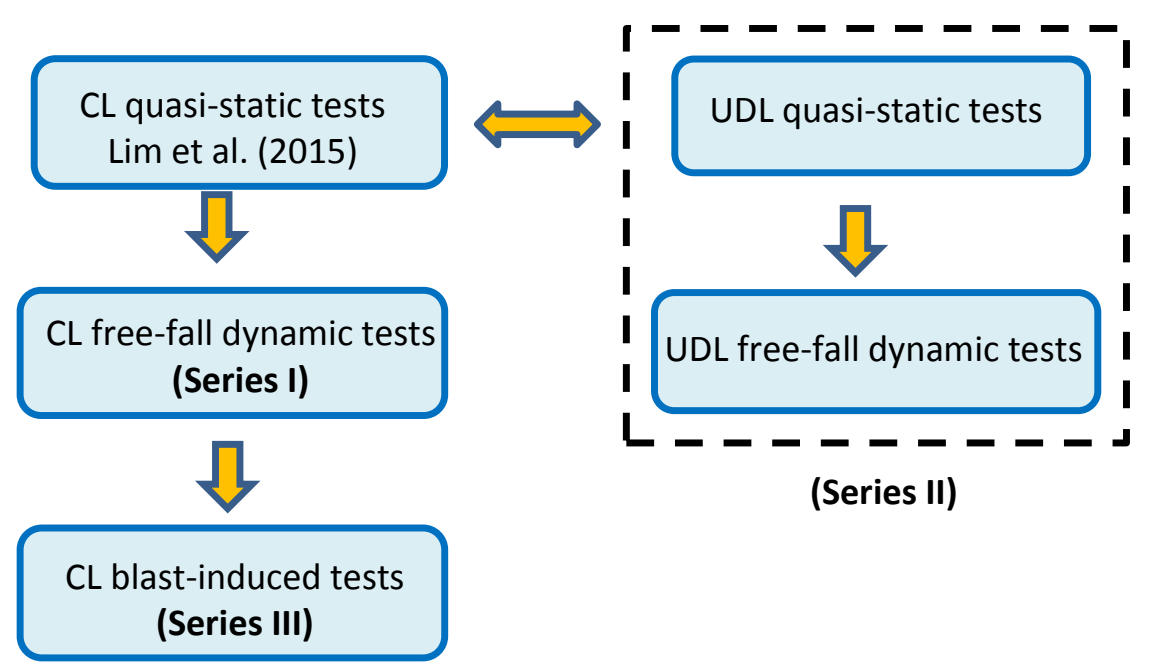

Fig. 5.1 Relationships between test series

\subsection{Objectives of testing programme}

\subsubsection{Free-fall dynamic tests on RC frames under $\mathrm{CL}$ condition (Series I)}

A series of four specimens was cast and tested under the sudden column loss scenario to extend the previous CL quasi-static study (Lim et al. 2015) into the dynamic regime. The objectives of Series I were to (a) investigate dynamic effects caused by the sudden removal of a supporting column; (b) ascertain if the failure modes were different from those of static tests under the same loading configuration; (c) study if CA could be mobilised after the fracture of bottom rebars at the middle joint; and (d) verify Izzuddin's method with actual dynamic 
responses. Test parameters included boundary conditions (full-restraint and partialrestraint) and applied loads at the middle joint. Unlike the displacement-controlled static tests in which the entire displacement-versus-load relationship could be obtained, in each dynamic test a specimen could only be subjected to a fixed imposed load and therefore could only provide one corresponding maximum deflection. Hence, to clearly understand the dynamic behaviour of the frame structures, three load levels were applied for each type of boundary condition. Extensive comparisons between static and dynamic tests were conducted in terms of displacement profiles, damages and failure modes, as well as behaviours at crosssectional and structural levels.

\subsubsection{Static and dynamic tests on $\mathrm{RC}$ frames under UDL condition (Series II)}

To study the resistance of RC beam-column structures against single column removal scenario under UDL condition, both static and dynamic tests were proposed in Series II. The static tests included two specimens under displacementcontrolled manner, while the dynamic tests included three specimens under loadcontrolled free-fall condition. The total loads applied onto the specimens were equally distributed into four points along the double-span beam. The objectives of Series II were to (a) investigate differences in structural responses and failure modes between the two loading configurations, viz. CL and UDL, as well as to (b) elucidate dynamic effects between the two loading rates (quasi-static and free-fall dynamic). Finally, (c) Izzuddin's method was also verified for the UDL condition.

\subsubsection{Blast-induced tests on RC frames under CL condition (Series III)}

The objective of Series III was to evaluate the limitation of the single column removal scenario which is widely accepted in guidelines for progressive collapse, as well as to verify the numerical modelling procedure for blast-induced environment conducted in Chapter 3. Therefore, interested factors of Series III included boundary condition, applied load, and charge weight placed at the middle support. Results from the blast tests were directly compared with those from Series I, and 
would provide valuable data for future numerical studies on blast-induced progressive collapse.

\subsection{Specimen design and test setup}

\subsubsection{Prototype building and quasi-static specimens under CL condition}

Design of the 2D frames under quasi-static tests (Lim et al. 2015) was based on a typical 5-storey prototype building, which was designed and detailed in accordance with EN 1992-1-1 (2004). The prototype building consisted of 4x6 bays with an equal span of $6 \mathrm{~m}$ in two directions. Two column-missing scenarios were chosen for the 2D frame tests, including (1) one interior and (2) one next-to-outermost column. Locations of the 2D frames are shown in Fig. 5.2(a) and (b), respectively. Details of structural design for the prototype building can be found in Lim (2017).

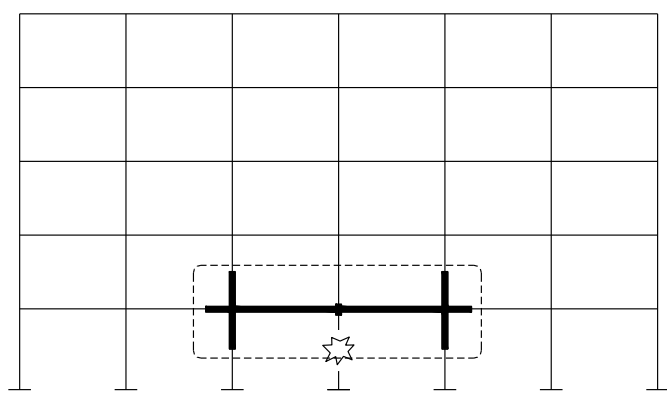

a) interior column removal

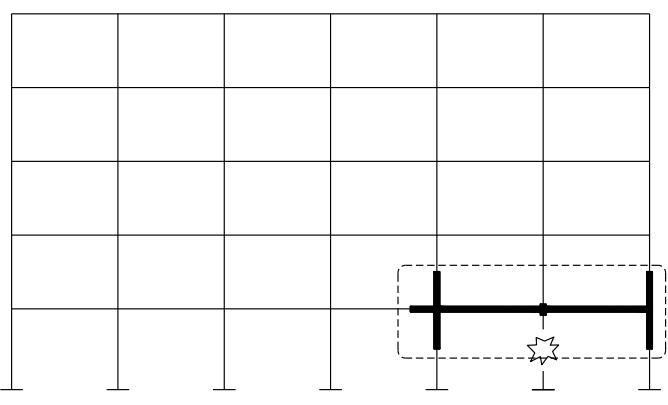

b) next-to-outermost column removal

Fig. 5.2 Prototype elevation view and locations of test specimens (Lim 2017)

In the quasi-static tests of Lim et al. (2015), the 2D frame specimens were scaled down to one-third due to laboratory physical constraints. All the geometries were reduced, but the reinforcement ratio was kept constant for both the columns and the beams. Test setup and test results of this quasi-static series are briefly introduced in Section 2.3.1.1. To have direct comparisons with the CL quasi-static tests, the specimens proposed in this research were similar in terms of structural design and boundary conditions. Only one adjustment was made for the contact detonation tests (Series III), considering the entire storey height of $1.5 \mathrm{~m}$ compared to a reduced height of $0.8 \mathrm{~m}$ in the quasi-static and the free-fall dynamic tests. In the blast tests, a 
concrete footing was provided at each side column to ensure fixed end boundary condition. This modification was made to ensure the behaviour of side columns under blast pressure.

\subsubsection{Series I}

Specimen design in Series I consisted of a double-span beam with a middle joint, two side columns and beam extensions as shown in Fig. 5.3. High strength deformed bars were used for beam longitudinal reinforcement while mild steel round bars were used as stirrups. Three deformed bars T10 with a diameter of 10 $\mathrm{mm}$ were arranged as top reinforcement at each beam end. They were reduced to two T10 at curtailment points which were $650 \mathrm{~mm}$ away from the beam-column interfaces. Only two T10 bars were used for beam bottom reinforcement and were continuous along the double-span beam. The lengths of side columns and beam extensions were chosen to coincide with contra-flexural points of the $2 \mathrm{D}$ frames in the real structure (Fig. 5.4), i.e. half-length for columns and 0.21 span for beam extensions. Therefore, only pin and horizontal supports were needed for such a test setup. Two types of boundary condition, i.e. full-restraint and partial-restraint, were applied. For the full-restraint specimens, beam extensions were arranged at both sides of the double-span beam and were horizontally restrained by either an Aframe or a strong wall. Fig. 5.5 illustrates the test setup for a typical fully restrained specimen in which pinned supports and horizontal restraints were symmetrically arranged at both sides while Fig. 5.6 shows the arrangement of restraints from one side. On the other hand, for the partial-restraint specimens, the beam extension was only designed at the left side. As a result, the beam extension and the horizontal reaction $\mathrm{RH} 2$ at the right side of the specimen (as shown in Fig. 5.5) were omitted and the right-side column was only horizontally restrained at RH1 and RH3. To simulate the axial forces on the side columns generated by gravity loads from the above floors in the actual frame, before conducting the dynamic free-fall tests, preloaded forces were applied onto the side columns using four steel rods and a hydraulic jack seated on top of each column (Fig. 5.6). 


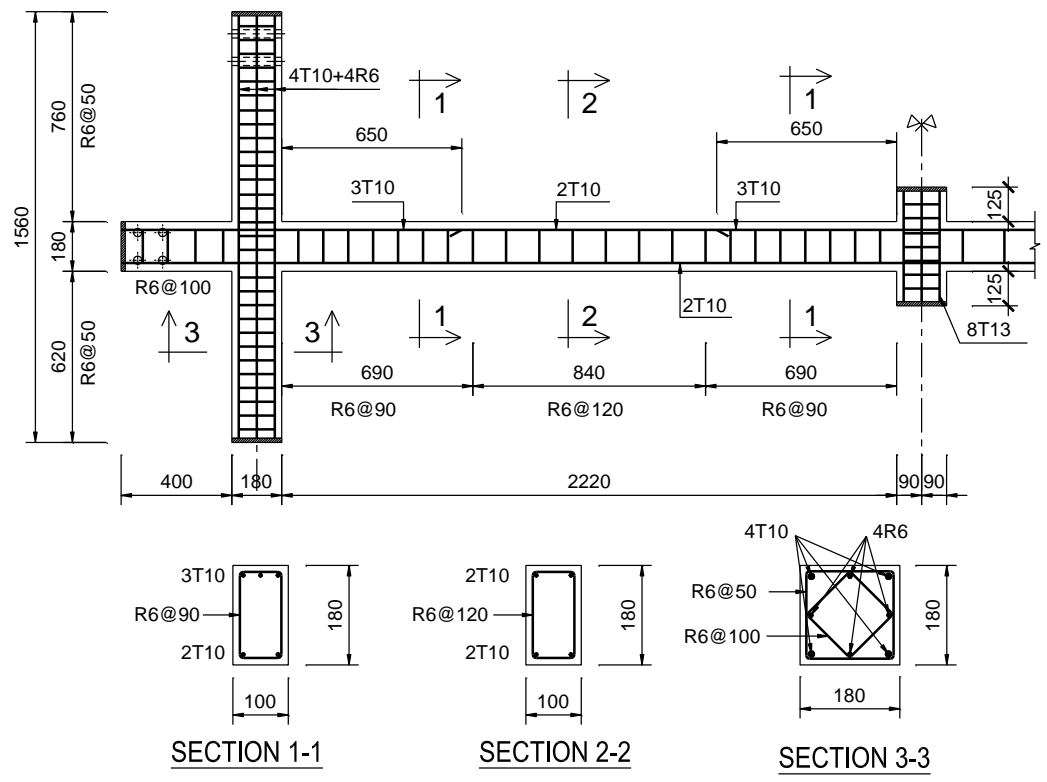

Fig. 5.3 Specimen design of Series I

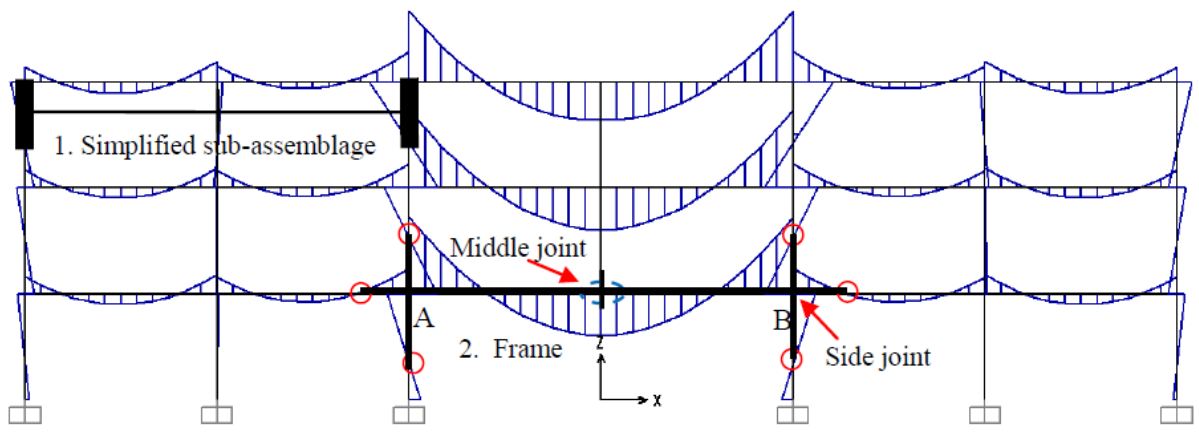

Fig. 5.4 Bending moment diagram of a 2D frame after column removal (Yu 2012)

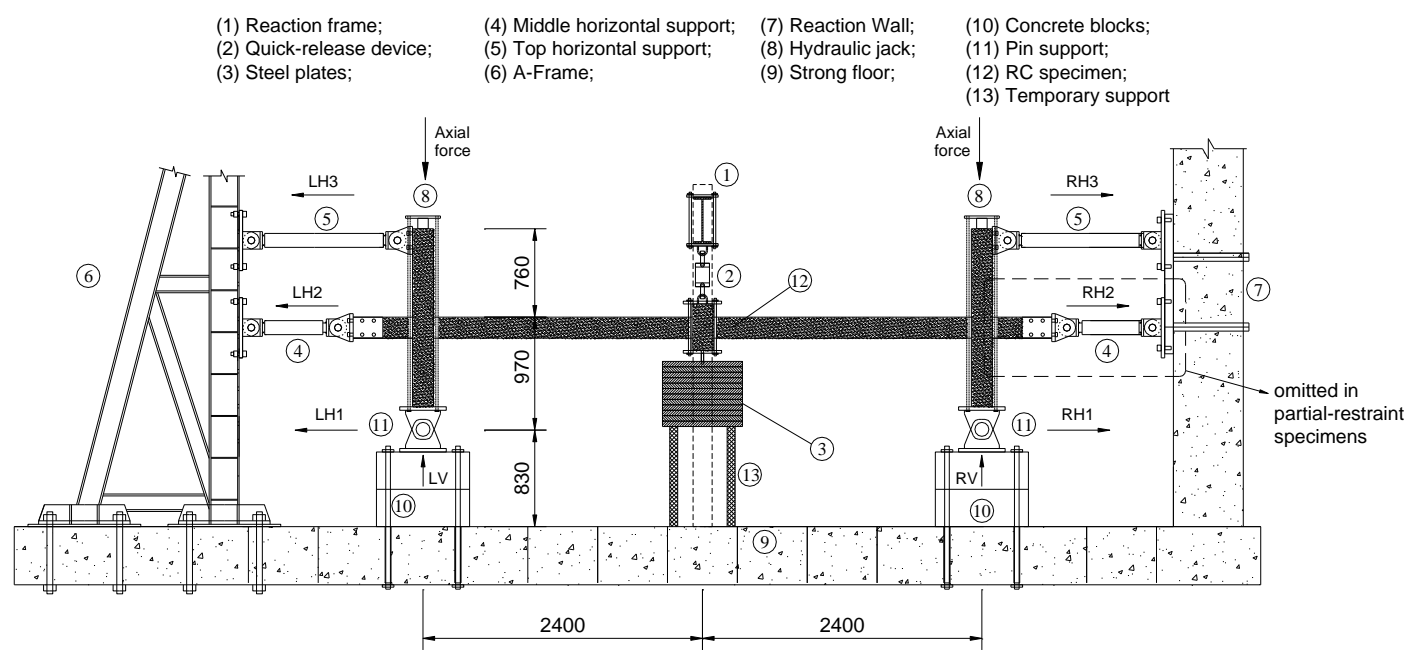

Fig. 5.5 Test setup of full-restraint specimens from Series I 


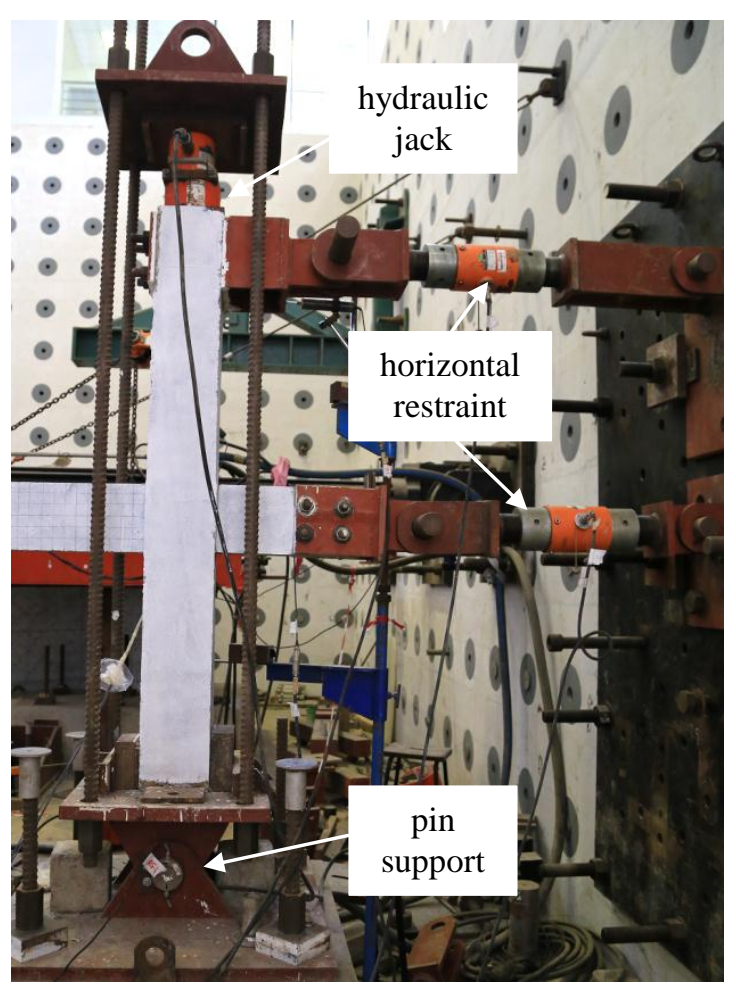

Fig. 5.6 Side restraint

Applied load at the middle joint was imitated using a set of steel plates hung underneath this joint with the total weight varying from test to test (Fig. 5.7(a)). The middle joint was suspended from a supporting $\mathrm{H}$-frame by a quick-release device (Fig. 5.7(b)), which could be suddenly released by yanking a rope connected to it. Such a mechanism for progressive collapse dynamic tests had been successfully applied in a previous study on steel joints by Liu et al. (2015). There was sufficient clearance height below the steel-plate system at the middle joint to ensure that the specimen could only hit the strong floor after final failure had occurred.

Four specimens, named as FD1 to FD4, were fabricated and tested in Series I. Table 5.1 summarises the parameters of the dynamic free-fall tests, as well as the information on the two static tests conducted by Lim et al. (2015). In terms of concrete grade, the dynamic tests had higher cylinder strengths (tested from 150x300mm specimens) compared to the static tests, in particular FD3 and FD4. Concerning reinforcement material, the specimens in the dynamic (Table 5.2) and the static (Lim et al. 2015) tests had fairly similar properties. Fig. 5.8(a) and (b) 
present typical material tests for concrete and reinforcement, respectively. Among the four specimens, FD1 and FD2 represented full-restraint frames and were tested under applied load levels of 20, 29, and $34 \mathrm{kN}$. On the other hand, FD3 and FD4 were designed for partial-restraint frames and were tested with three load levels of 20, 25, and $30.5 \mathrm{kN}$. Static test results from Lim et al. (2015) gave the maximum applied loads of 71 and $34 \mathrm{kN}$ for the full- and the partial-restraint specimens, respectively (Fig. 2.9). It is noteworthy that FD1 and FD3 were tested twice. The first release with only $20 \mathrm{kN}$ of load showed limited damages and deformations to these two specimens. Therefore, after the first test, the double-span beam was pulled back up to the original position and was tested again under higher applied loads of 29 and $25 \mathrm{kN}$ for FD1 and FD3, respectively. All the tests had the same pre-loaded compression of $200 \mathrm{kN}$ on the side columns, similar to that applied for the static tests FR and PR. This compression was equal to $24 \%$ of the stockycolumn resistance based on cylinder concrete strength of $35 \mathrm{MPa}$.

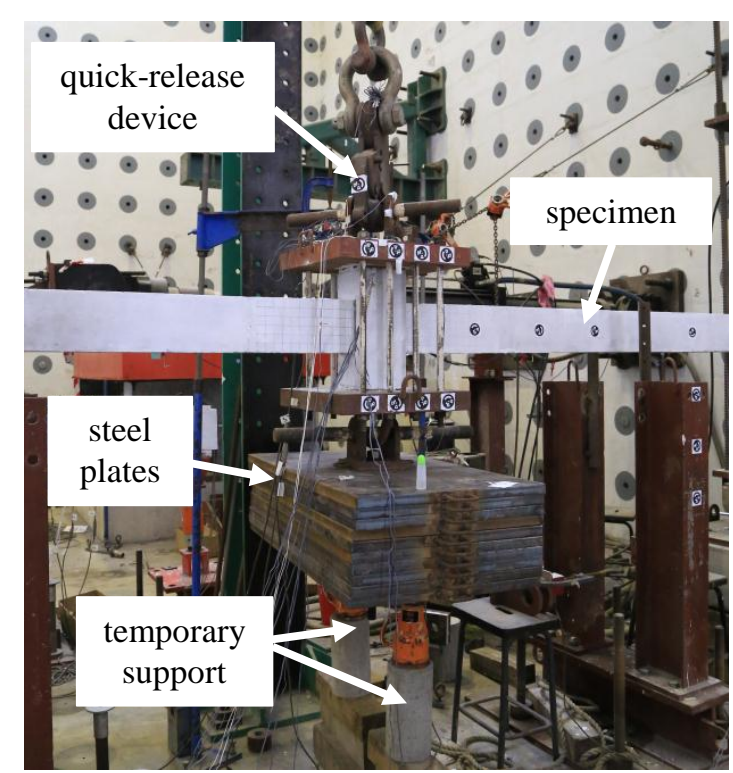

a) Applied load system
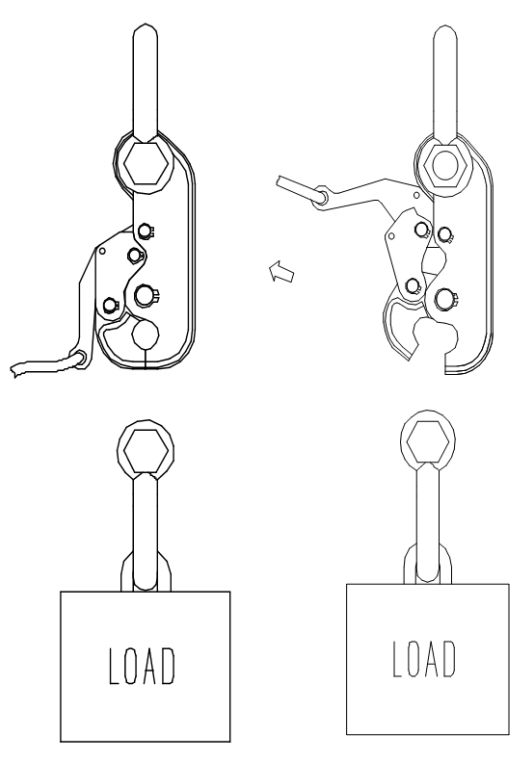

b) Quick-release device

Fig. 5.7 Applied load and release mechanism from Series I 
Table 5.1 Specimen properties of Series I

\begin{tabular}{|c|c|c|c|c|}
\hline Specimen & $\begin{array}{c}\text { Concrete } \\
\text { cylinder } \\
\text { strength (MPa) }\end{array}$ & $\begin{array}{c}\text { Restraint } \\
\text { condition }\end{array}$ & Dynamic test & $\begin{array}{c}\text { Applied } \\
\text { load (kN) }\end{array}$ \\
\hline FR & 32 & Full restraint & \multicolumn{2}{|c|}{ Static (Lim et al. 2015) } \\
\hline PR & 32 & Partial restraint & \multicolumn{2}{|c|}{ Static (Lim et al. 2015) } \\
\hline FD1 & 35 & Full restraint & FD1-F/20 & 20 \\
\cline { 4 - 5 } & 35 & Full restraint & FD1-F/29 & 29 \\
\hline FD2 & 42 & Partial restraint & FD3-P/20 & 20 \\
\cline { 4 - 5 } & & & FD3-P/25 & 25 \\
\hline FD3 & 51 & Partial restraint & FD4-P/30.5 & 30.5 \\
\hline
\end{tabular}

Note: Specimens FD1 and FD3 were tested two times with two applied loads

Table 5.2 Material properties for the dynamic tests

\begin{tabular}{|c|c|c|c|c|c|c|}
\hline Rebar & $\begin{array}{c}\text { Diameter } \\
(\mathrm{mm})\end{array}$ & $\begin{array}{c}\text { Yield } \\
\text { strength } \\
(\mathrm{MPa})\end{array}$ & $\begin{array}{c}\text { Elastic } \\
\text { Modulus } \\
(\mathrm{GPa})\end{array}$ & $\begin{array}{c}\text { Yield } \\
\text { strain } \varepsilon_{\mathrm{y}} \\
(\%)\end{array}$ & $\begin{array}{c}\text { Tensile } \\
\text { strength } \mathrm{f}_{\mathrm{u}} \\
(\mathrm{MPa})\end{array}$ & $\begin{array}{c}\text { Ultimate } \\
\text { strain } \varepsilon_{\mathrm{u}}(\%)\end{array}$ \\
\hline R6 & 6 & 352 & 220 & 0.162 & 539 & 19.2 \\
\hline T10 & 10 & 554 & 196 & 0.288 & 653 & 11.5 \\
\hline T13 & 13 & 535 & 188 & 0.285 & 615 & 8.5 \\
\hline
\end{tabular}

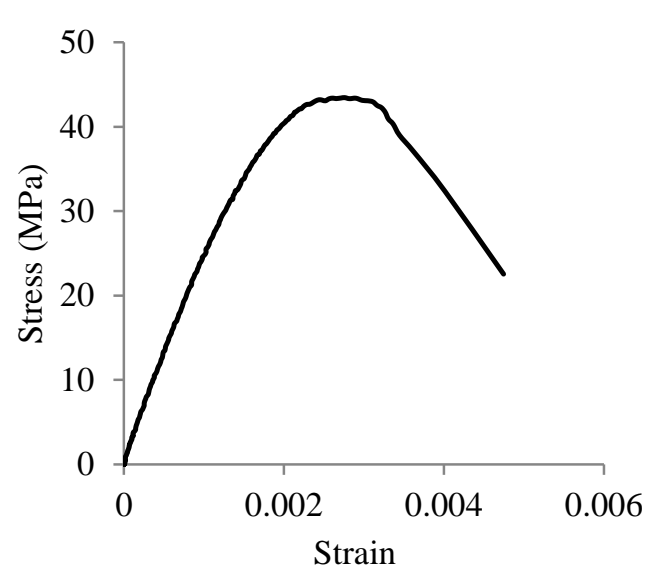

a) Concrete

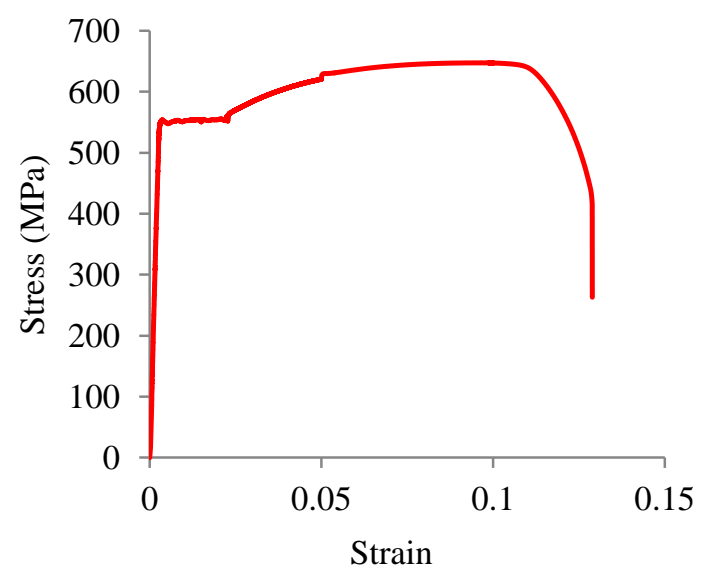

b) Reinforcement T10

Fig. 5.8 Material tests

\subsubsection{Series II}

Compared to Series I, Series II had similar specimen design, boundary condition (both full- and partial-restraint setups), quick-release mechanism (for the dynamic tests), and the applied compression on side columns. However, to avoid shear 
failure, stirrup spacing near the joint interfaces was reduced from 90 to $50 \mathrm{~mm}$ (Fig. 5.9).

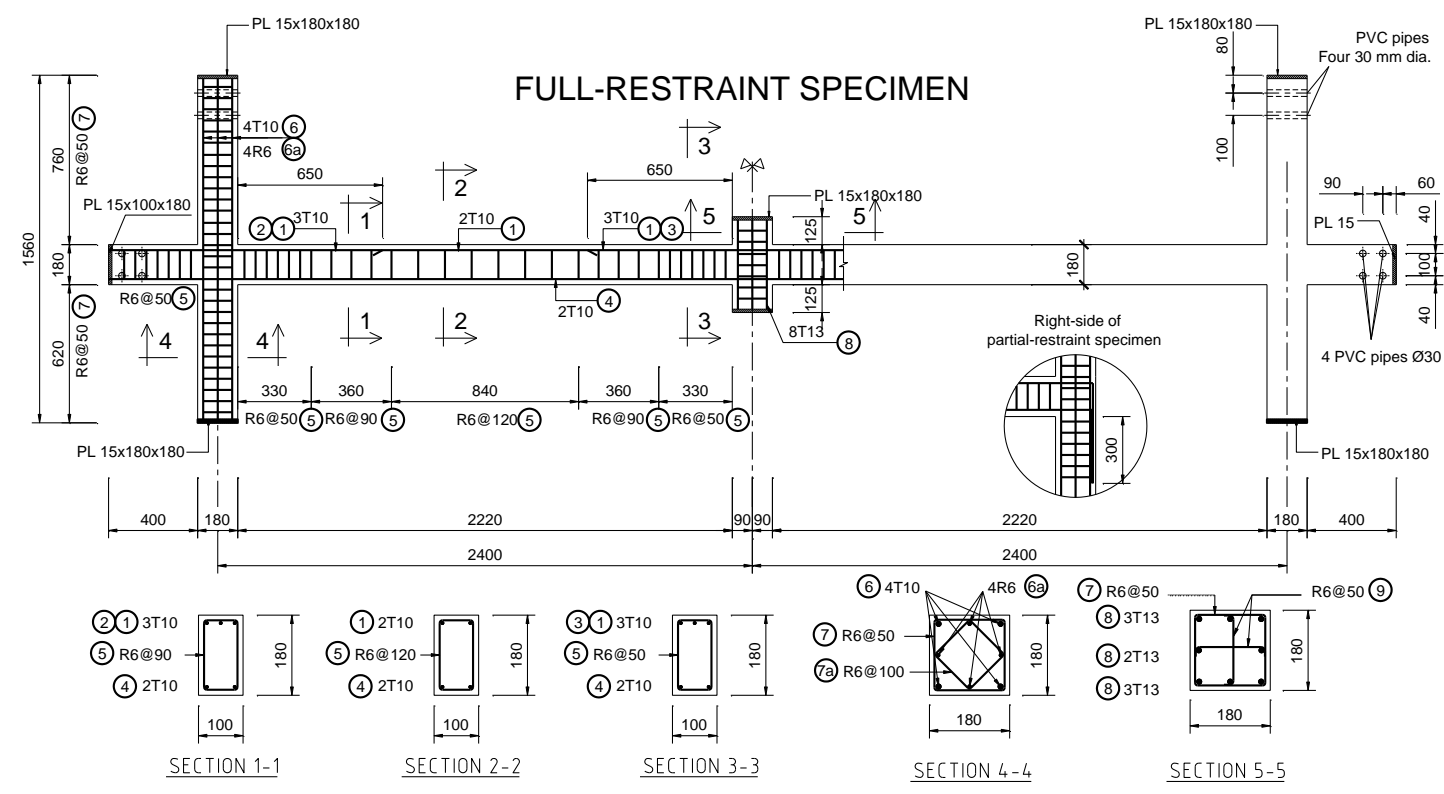

Fig. 5.9 Specimen design of Series II

To simulate distributed loads acting on the structure, a 4-point loading method was employed in both the static and the dynamic tests of Series II. Distances between the four applied loads were chosen to be equal along the double-span beam, i.e. half of the original single span. An elastic analysis comparing deformation shape and moment distribution of a structure subject to UDL and 4-point loading was carried out (Fig. 5.10). It shows that the equivalent loading system produces an almost similar displacement profile to that by UDL, and that both loading methods are in reasonable agreement in terms of bending moments at the end and the middle joints, as well as locations of contra-flexural point.

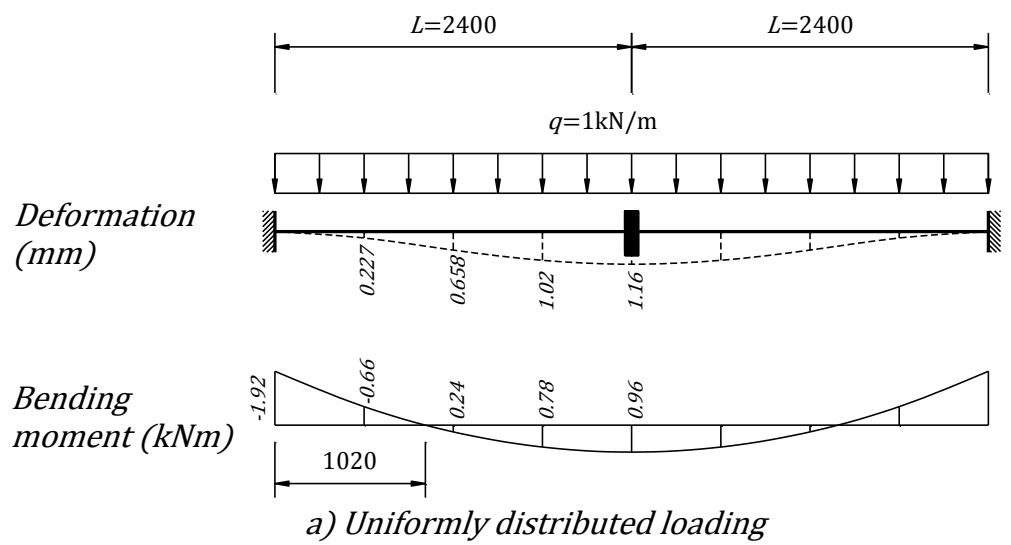




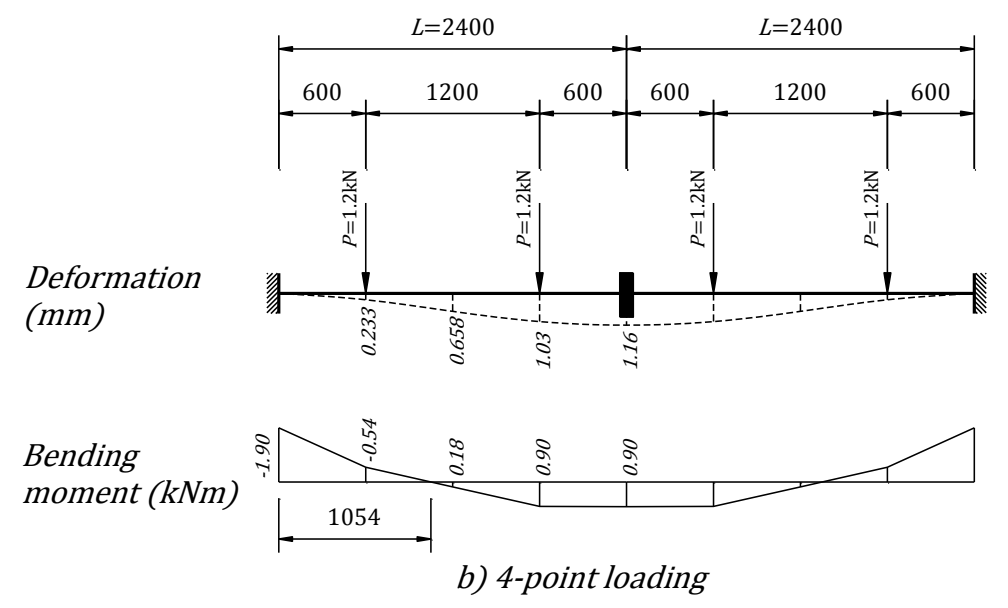

Fig. 5.10 Comparisons between actual UDL and 4-point loading methods

Both types of boundary condition, i.e. full-restraint and partial-restraint, were applied for Series II. Fig. 5.11(a) illustrates a typical test setup for a static fullrestraint specimen with a symmetric arrangement of load pins and horizontal load cells, whereas Fig. 5.11(b) presents a typical partial-restraint specimen from a dynamic test of Series II. For the quasi-static tests, a steel-beam load-transfer system was fabricated as shown in Fig. 5.11(a) to equally divide the load from an actuator above to four roller points on the specimen. Such a test setup ensured that the steel beams could translate and rotate without affecting the deformation shape of the frame specimen.

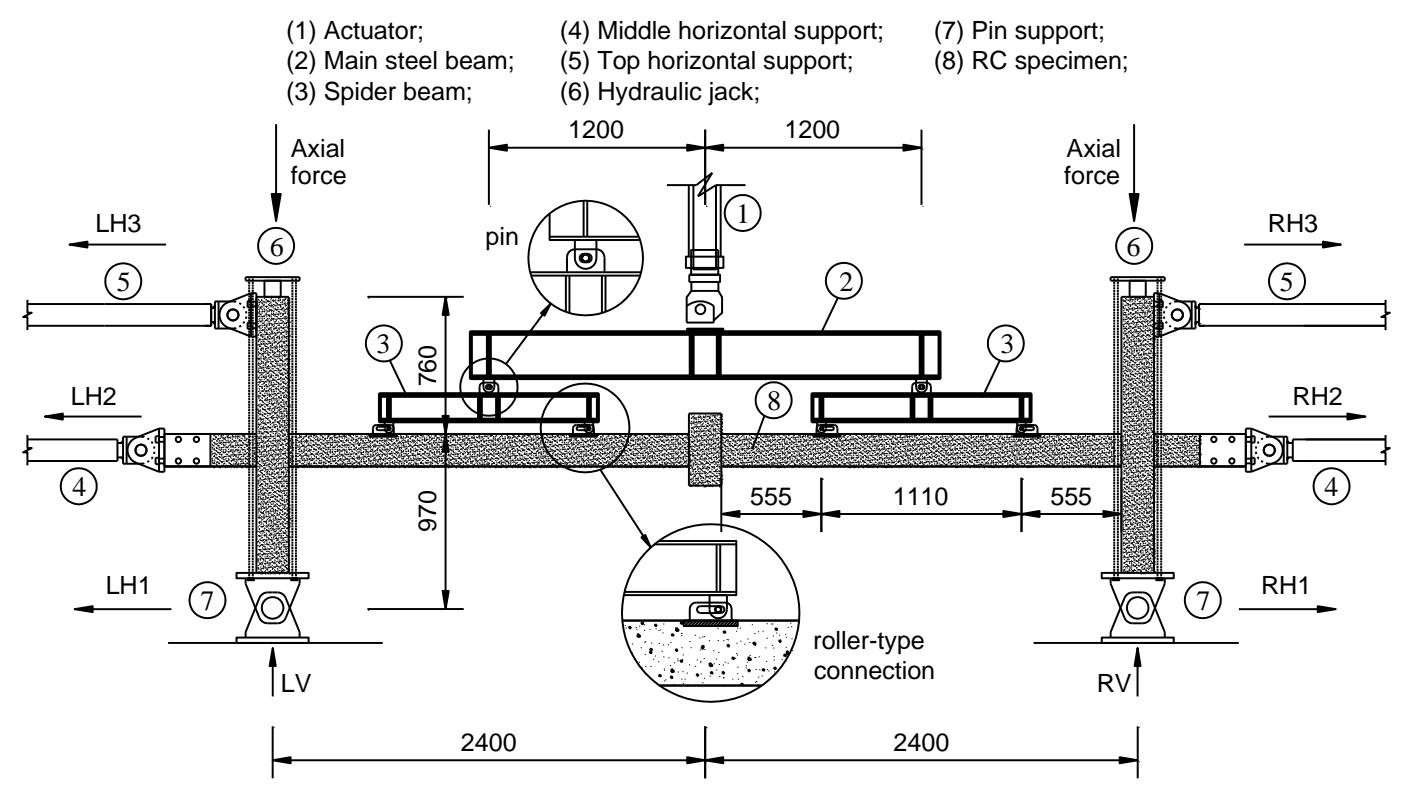

a) Static test (full-restraint specimen) 


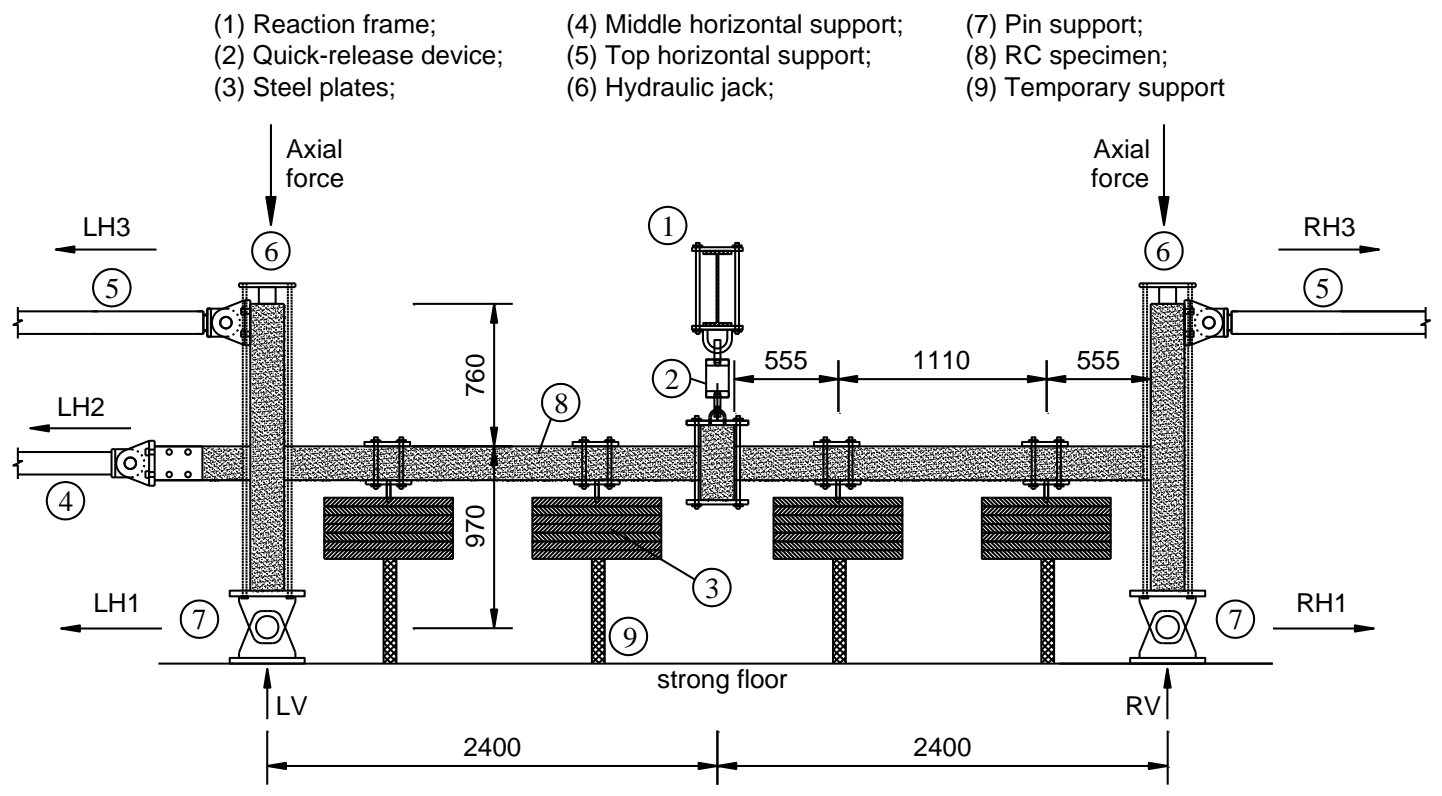

b) Free-fall dynamic test (partial-restraint specimen)

Fig. 5.11 Test setup from Series II

Due to the slenderness of both the specimen and the load-transfer system, lateral restraints were provided at mid-span positions of the single-span beams to prevent out-of-plane movement during the static testing (Fig. 5.12(a)). In the dynamic tests, steel plates with fixed equal weights were hung to the specimens at four locations in accordance with the static tests as shown in Fig. 5.11(b) and Fig. 5.12(b).

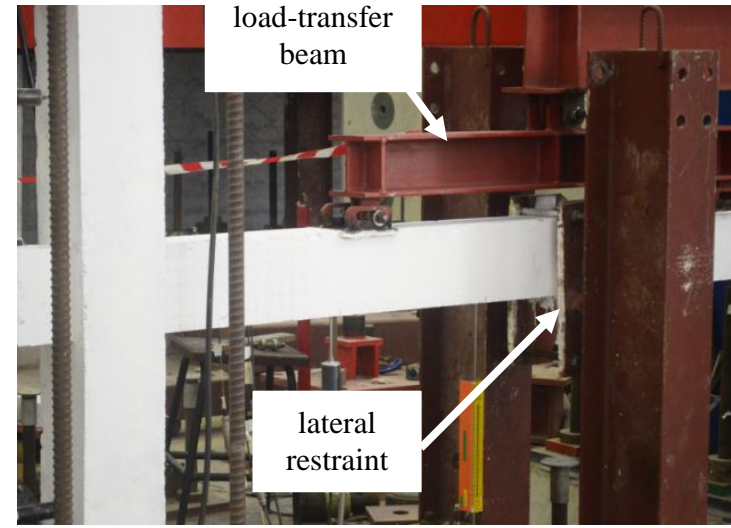

a) Lateral restraint in the static tests

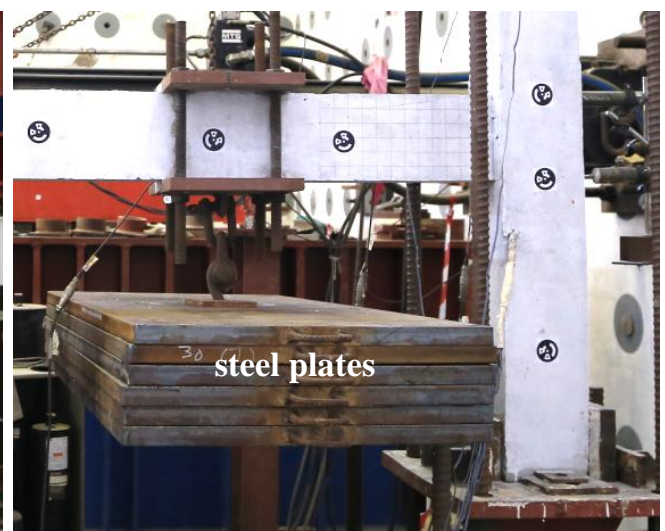

b) Distributed loads in the dynamic tests

Fig. 5.12 Details of test setup in Series II 
For the UDL static tests of Series II, two tests named as FR-U (full-restraint) and PR-U (partial-restraint) were conducted. They corresponded with the two CL static tests of FR and PR from Lim et al. (2015). For the UDL dynamic tests, three specimens, named as FDU1 to FDU3, were fabricated and tested. FDU1 and FDU3 represented partial-restraint frames and were tested under total applied loads of 42 and $61 \mathrm{kN}$, respectively. On the other hand, FDU2 was designed as a full-restraint specimen and was tested with a total load of $55 \mathrm{kN}$. Among the three specimens, FDU1 was tested twice with two different loads whereas the other two were only tested once. After the first release of FDU1 with an applied load of $42 \mathrm{kN}$ (FDU1$\mathrm{P} / 42$ ), the second release (FDU1-P/61) caused a complete collapse to this specimen. Hence, FDU3-P/61 was conducted with the same boundary condition (partialrestraint) and applied load (61 kN) with FDU1-P/61 to study the dynamic behaviour again under pristine condition of the specimen. Table 5.3 summarises the parameters of both the static and the dynamic tests in this series. Similar to Series I, the tests in Series II had higher cylinder strengths compared to the previous tests from Lim et al. (2015). Regarding reinforcement, both Series I and Series II had the same material properties (Table 5.2).

Table 5.3 Specimen properties of Series II

\begin{tabular}{|c|c|c|c|c|}
\hline Specimen & $\begin{array}{c}\text { Concrete cylinder } \\
\text { strength (MPa) }\end{array}$ & $\begin{array}{c}\text { Restraint } \\
\text { condition }\end{array}$ & $\begin{array}{c}\text { Dynamic } \\
\text { test }\end{array}$ & $\begin{array}{c}\text { Total dynamic } \\
\text { load }(\mathrm{kN})\end{array}$ \\
\hline FR-U & 46.6 & Full restraint & \multicolumn{2}{|c|}{ Static test } \\
\hline PR-U & 45 & Partial restraint & \multicolumn{2}{|c|}{ Static test } \\
\hline FDU1 & 44.5 & Partial restraint & FDU1-P/42 & 42 \\
\cline { 2 - 5 } & & Partial restraint & FDU1-P/61 & 61 \\
\hline FDU2 & 44.5 & Full restraint & FDU2-F/55 & 55 \\
\hline FDU3 & 51 & Partial restraint & FDU3-P/61 & 61 \\
\hline
\end{tabular}

Note:* Specimen FDU1 was tested twice with two different applied loads

\subsubsection{Series III}

Basically, the specimens for contact detonation tests in Series III and for free-fall dynamic tests in Series I used similar structural design, loading configuration, as well as boundary conditions except for the side-column design. The side columns in the blast tests were extended and connected to concrete footings at the bottom end 
to simulate a fixed restraint. Due to this modification, compression forces on the side columns, which were used in Series I and II, were not applied in Series III. Both full-restraint and partial-restraint setups were adopted in Series III. Specimen design of this test series is presented in Fig. 5.13.

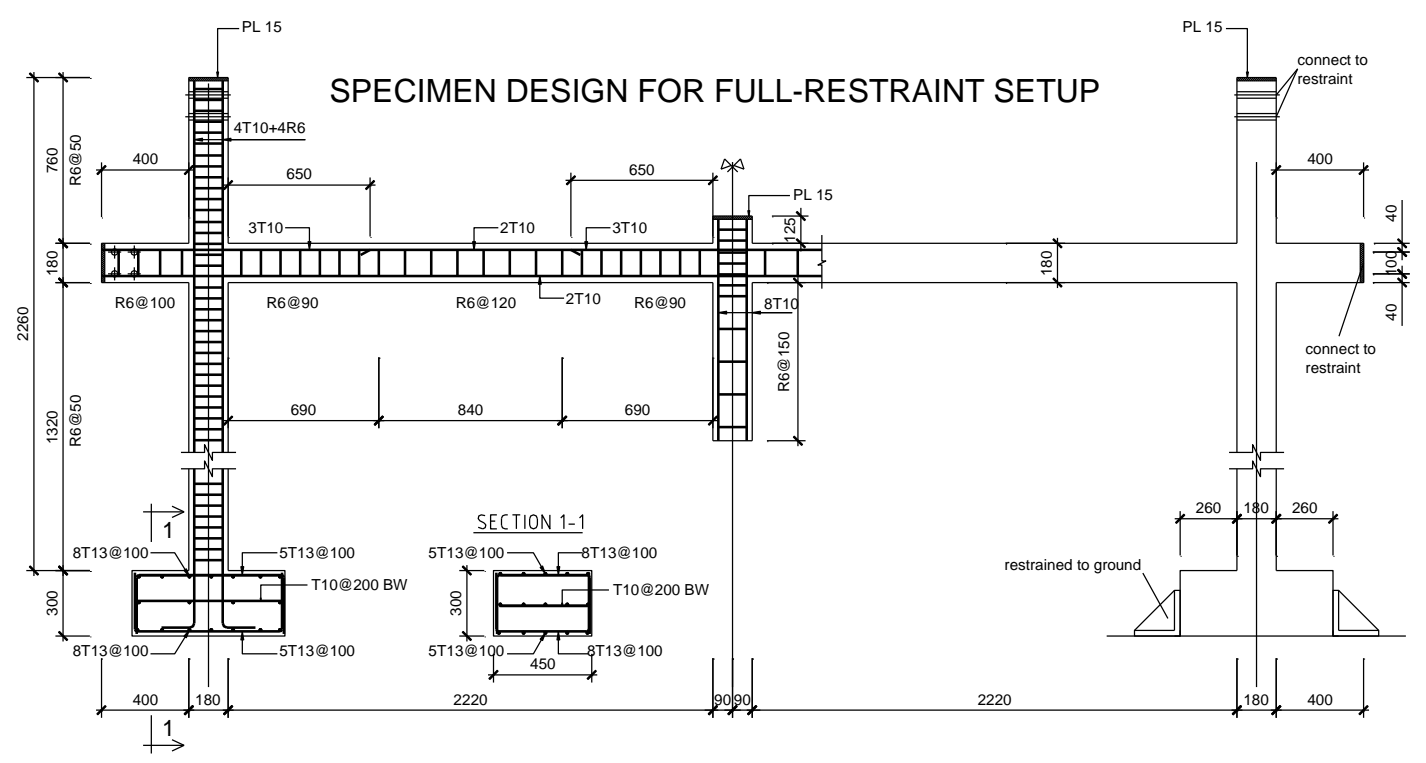

Fig. 5.13 Specimen design for full-restraint setup (Series III)

It was impossible to conduct structural tests under blast loading condition in the laboratory due to safety concern. Therefore, Series III was conducted on a test site at Fraunhofer Institute for High-Speed Dynamics EMI, Germany. The specimens were directly placed on the ground with the column footings fixed to the underneath concrete slab to provide fully fixed supports. Horizontal restraints at the two sides were provided and connected to massive blocks which were also fixed to the ground. Additional imposed load on the substructure was simulated by a concretesteel frame system placed on top of the middle joint. This system was appropriately fixed to the specimen so that it could apply its mass to the specimen after the middle support was removed by an explosive. Such horizontal restraint and loading systems were similar to those from previous tests of Yu et al. (2014). Fig. 5.14 presents the setup of a fully restrained specimen, while the photo of a partially restrained specimen is provided in Fig. 5.15. 


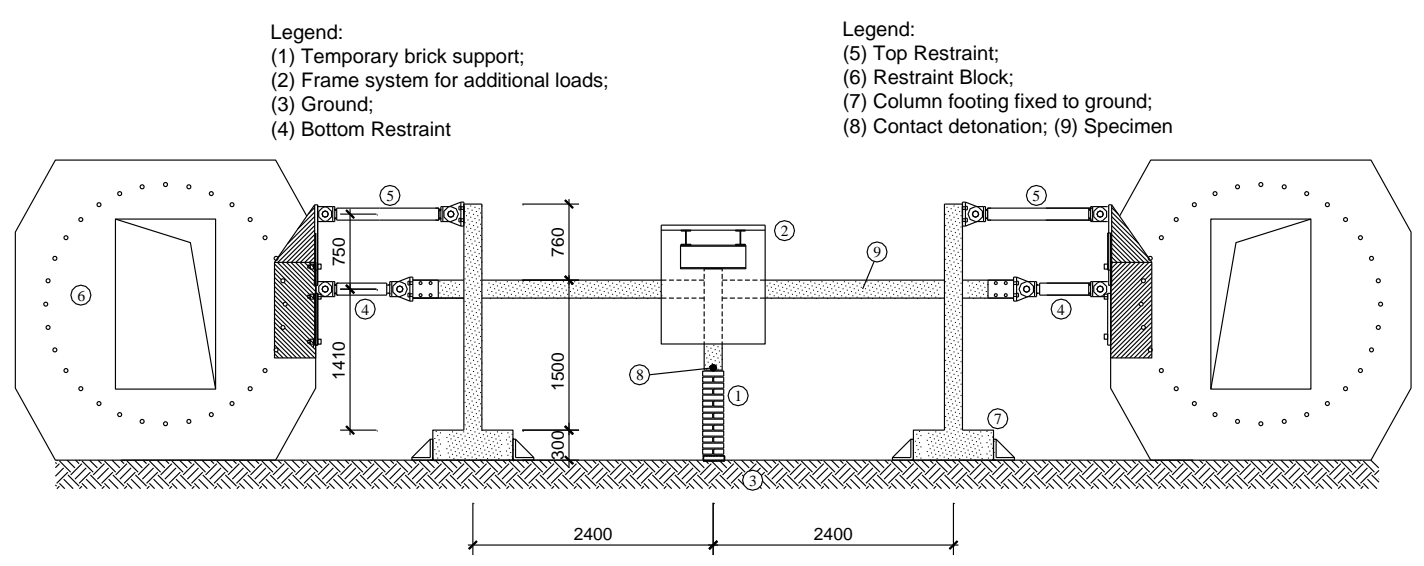

Fig. 5.14 Test setup of full-restraint specimen (Series III)

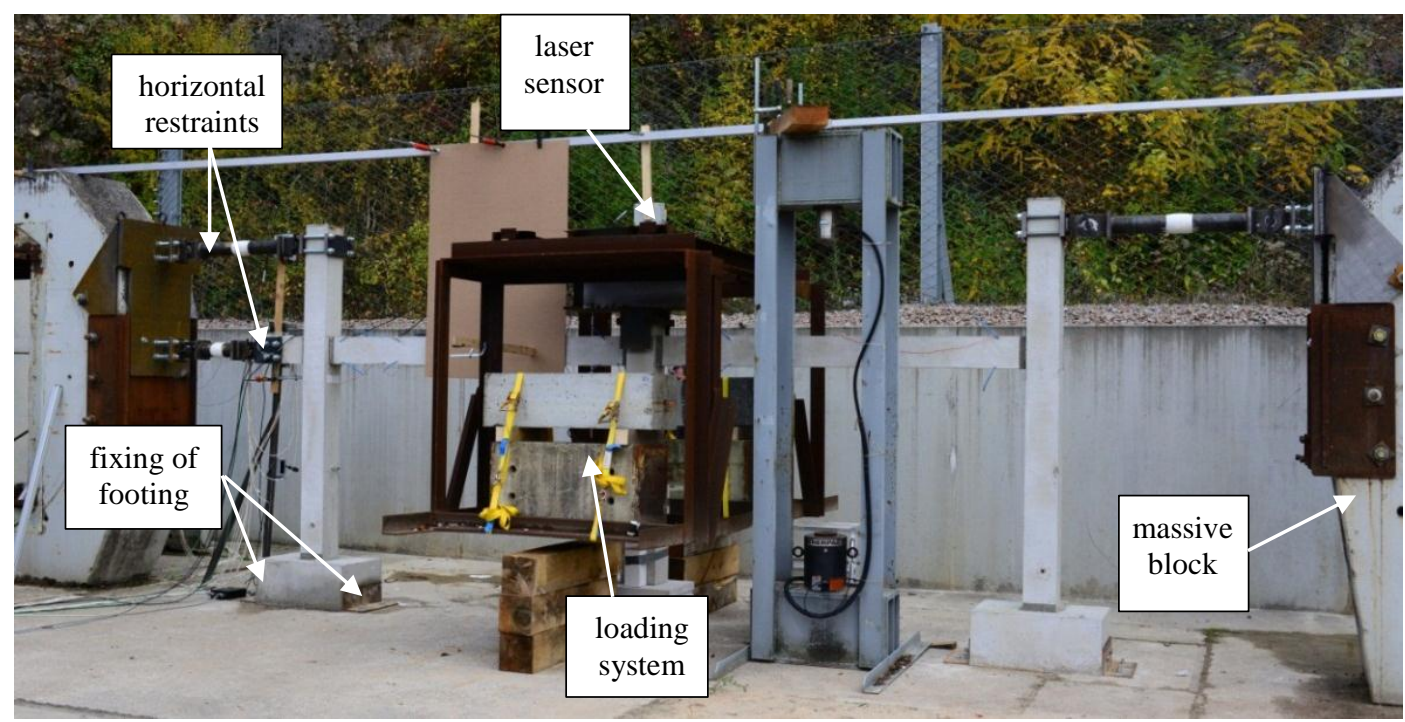

Fig. 5.15 Photo of partial-restraint specimen (Series III)

The middle column in Series III was extended and was placed on a temporary brick support (Fig. 5.16). Spherical PETN explosive was attached to the bottom part of the middle column. When the explosive was detonated, the blast pressure would blow away the temporary support and severely damage a large part of the middle column, triggering a column removal by blast event. Such a design did not fully represent actual situations when a column is attacked by contact detonation because the middle column did not have a proper fixed restraint to the ground. This came from the difficulty of choosing an appropriate charge weight of detonation to clearly destroy the column. If the charge was too small, the column would not be totally removed and could still provide some vertical support to the specimen, violating the 
single column removal assumption. On the other hand, if the charge was too big, it would severely damage the instrumentation system of the test, including displacement, acceleration, and pressure gauges. To avoid this difficulty, it was decided that the middle column would only sit on some temporary support which could be easily removed by the blast.

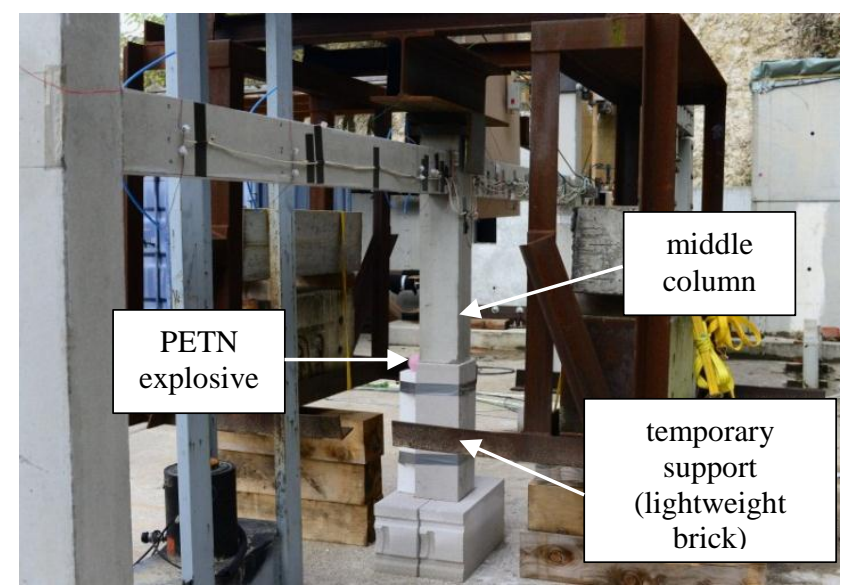

Fig. 5.16 Explosive charge and temporary support (Series III)

Two contact-detonation tests were proposed and identified as CD1 and CD2. Table 5.4 shows the information on boundary condition, applied load at the middle joint, and charge weight used for each test. The first specimen CD1 was partially restrained while the second was fully restrained. The load levels applied in the blast tests ( $20 \mathrm{kN}$ for full-restraint, $25 \mathrm{kN}$ for partial-restraint) were similar to those in the free-fall dynamic tests of Series I to provide direct comparisons between the two loading environments viz. free-fall and ultra-fast dynamic regimes. For both blast tests, one value of charge weight was used, i.e. 0.4 (denoted as M) $\mathrm{kg}$ of PETN explosive. The charge weight used in the tests was chosen to maximise the blast effects on the specimens but still within a safety margin to protect instrumentation from damages. 
Table 5.4 Test plan for contact detonation experiments (Series III)

\begin{tabular}{|l|c|c|c|c|}
\hline Specimen ID & $\begin{array}{c}\text { Type of horizontal } \\
\text { restraint }\end{array}$ & $\begin{array}{c}\text { Applied } \\
\text { load }\end{array}$ & $\begin{array}{c}\text { Charge weight } \\
\text { (PETN explosive) }\end{array}$ & $\begin{array}{c}\text { Corresponding } \\
\text { tests from Series I }\end{array}$ \\
\hline $\mathrm{CD} 1-\mathrm{P} / 25 / \mathrm{M}$ & Partial restraint & $25 \mathrm{kN}$ & $\mathrm{M}=0.4 \mathrm{~kg}$ & $\mathrm{FD} 3-\mathrm{P} / 25$ \\
\hline $\mathrm{CD} 2-\mathrm{F} / 20 / \mathrm{M}$ & Full restraint & $20 \mathrm{kN}$ & $\mathrm{M}=0.4 \mathrm{~kg}$ & $\mathrm{FD} 1-\mathrm{F} / 20$ \\
\hline
\end{tabular}

\subsection{Instrumentation}

\subsubsection{Series I}

Reaction forces from the dynamic tests were measured using load pins and horizontal load cells as shown in Fig. 5.5 and Fig. 5.6, which were similar to the static tests (Lim et al. 2015). Total horizontal reaction force from one side of the specimen was calculated by summing all the horizontal forces on that side. It was verified in each test that the total horizontal reactions from the left and the right sides were relatively close in magnitude, regardless of whether the specimen was fully restrained on both sides or partially restrained on one side. LVDTs and line transducers were arranged along the double-span beam as well as at the end joint region to measure vertical and horizontal deformations of the specimen (denoted as L1 to L7 in Fig. 5.17). One accelerometer (A0) with a capacity of 50g was placed on top of the middle joint. To protect the accelerometer from being damaged when the specimen hit the ground (complete failure), it was only employed for tests with a small applied load, i.e. $20 \mathrm{kN}$. Dynamic data acquisitions at a sampling rate of $1000 \mathrm{~Hz}$ were used for capturing data from the arranged channels. However, due to limited channel number in the free-fall dynamic tests, the measuring system could only provide deflections from fewer locations compared to the static tests. To overcome this issue, two slow-motion cameras at a rate of 240 frames per second were used to capture the in-plane deflections using marked points on the specimens as described in Fig. 5.17. One camera was used to record vertical movements of the right-side beam, while the other was used for horizontal displacements of the rightside column. 


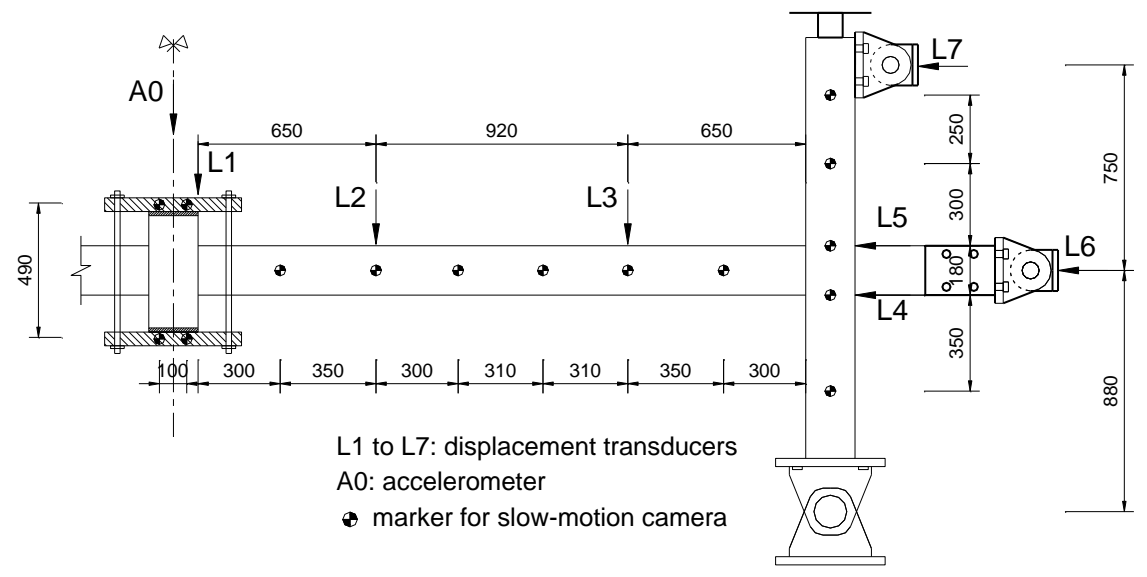

Fig. 5.17 Displacement transducers from Series I

The arrangement of steel and concrete strain gauges is illustrated in Fig. 5.18. Strain gauges were placed along beam longitudinal bars at four critical sections, i.e. endjoint interface (Section 1), rebar curtailment point near the end joint (Section 2), rebar curtailment point near the middle joint (Section 3), and middle-joint interface (Section 4). Strain gauges were also arranged for column longitudinal bars within the end-joint region. To measure the release time, i.e. the time during which the supporting force totally reduced to zero, two strain gauges (HS1 and HS2) were attached to the hooks on top of the middle joint. Two concrete strain gauges (CS1 and CS2) were also placed at expected compression regions near to the middle and the end joints as shown in Fig. 5.18.

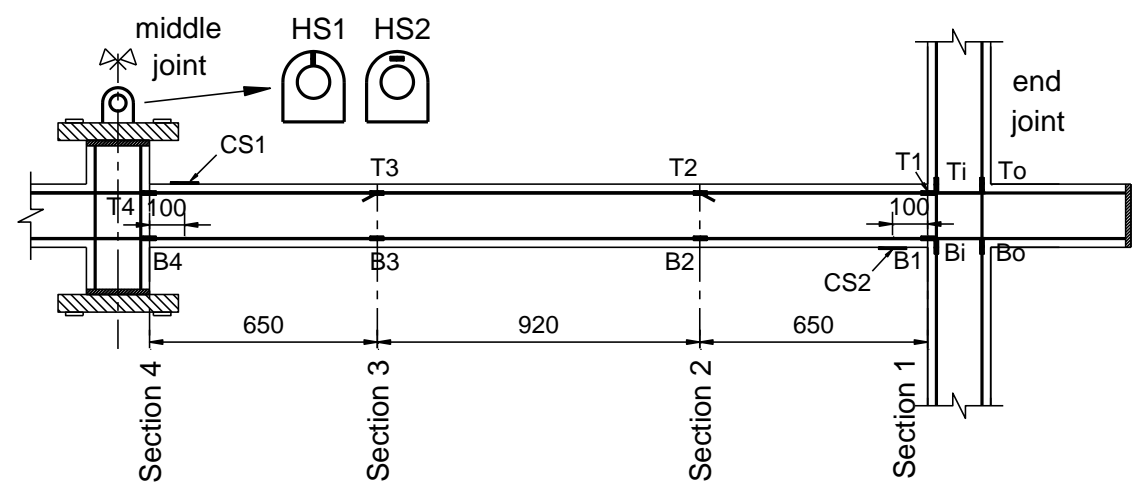

Fig. 5.18 Strain gauges from Series I 


\subsubsection{Series II}

For the UDL static tests, displacement transducers were arranged along the doublespan beam as well as along the side column to measure vertical and horizontal deformations of the structure, showing in Fig. 5.19(a). Strain gauges were placed along the beam longitudinal bars as well as at the beam-column joint (Fig. 5.19(b)), similar to the static tests (Lim et al. 2015). For the UDL dynamic tests, the arrangement of displacement transducers and steel strain gauges followed that of Series I (Fig. 5.17 and Fig. 5.18).
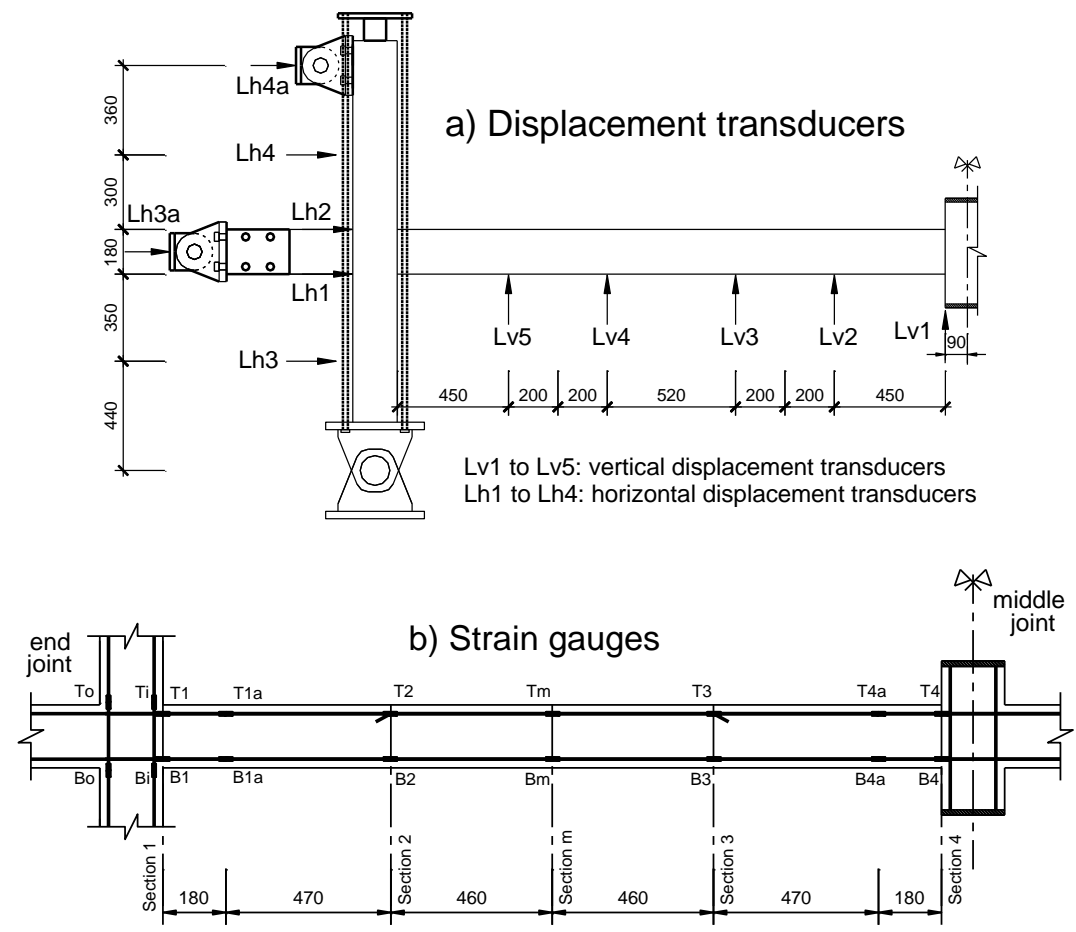

Fig. 5.19 Instrumentation of static tests from Series II

\subsubsection{Series III}

Compared to Series I, the substructure tests under blast condition from Series III had a relatively similar configuration of instrumentation for displacements, reactions, strains of reinforcement and concrete. Data acquisition systems at sampling rates of 2.5 and $5 \mathrm{MHz}$ were employed in the blast tests to appropriately monitor ultra-fast dynamic responses. In addition, a high-speed camera at a rate of 1000 frames per second was used to capture the overall response of the specimens. 
Displacement (D1 to D5) and acceleration (A0) transducers were arranged on top of the double-span beam and along the side column (Fig. 5.20). To measure reaction forces of the restraints, strain gauges were attached onto the horizontal steel-tube supports (Fig. 5.21). Post-yield strain gauges were arranged in longitudinal reinforcement of the beams at four sections similar to Series I (Fig. 5.18). Besides, two concrete strain gauges were employed near the middle and the end joint (CS1, CS2). One of the most important objectives to conduct the blast tests was to directly measure the effect of blast pressure on the sub-assemblages. Due to the complexity in geometry of the specimen, the distribution of blast loads along the beams and the columns would not be uniform. In this study, only a limited number of piezoelectric pressure gauges were used at critical locations due to high cost. One pressure gauge was attached at the beam soffit and one at the side surface of the column to measure incident pressure at these locations (Fig. 5.22).

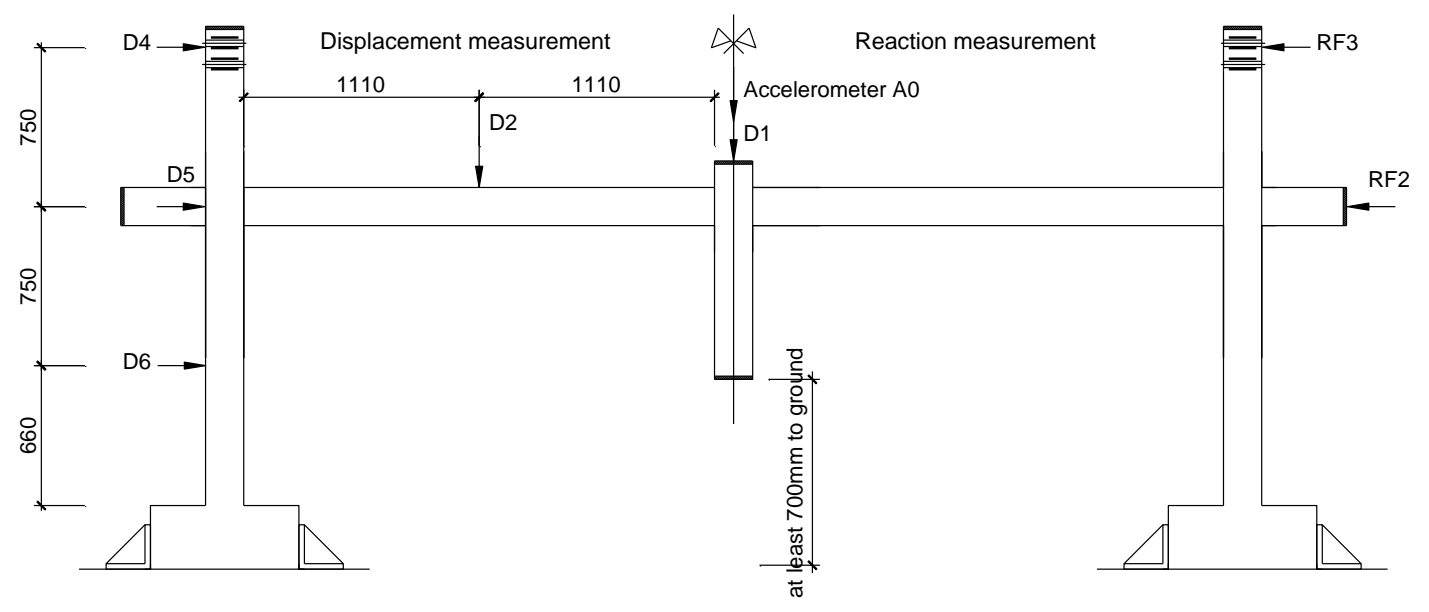

Fig. 5.20 Displacement and reaction measurement from Series III

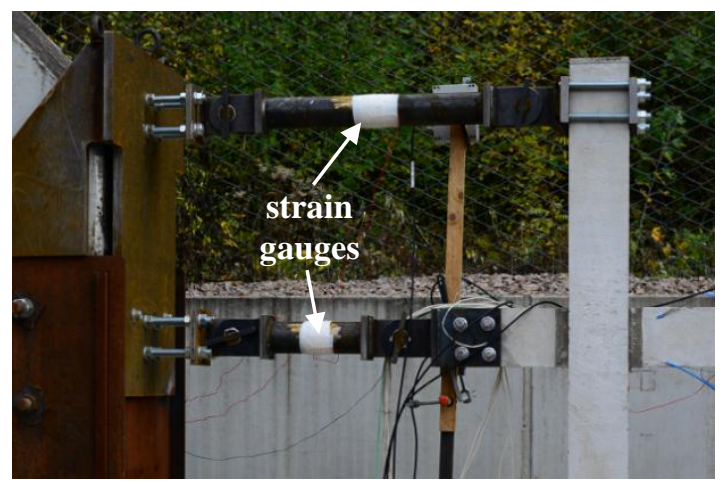

Fig. 5.21 Reaction force measurement 


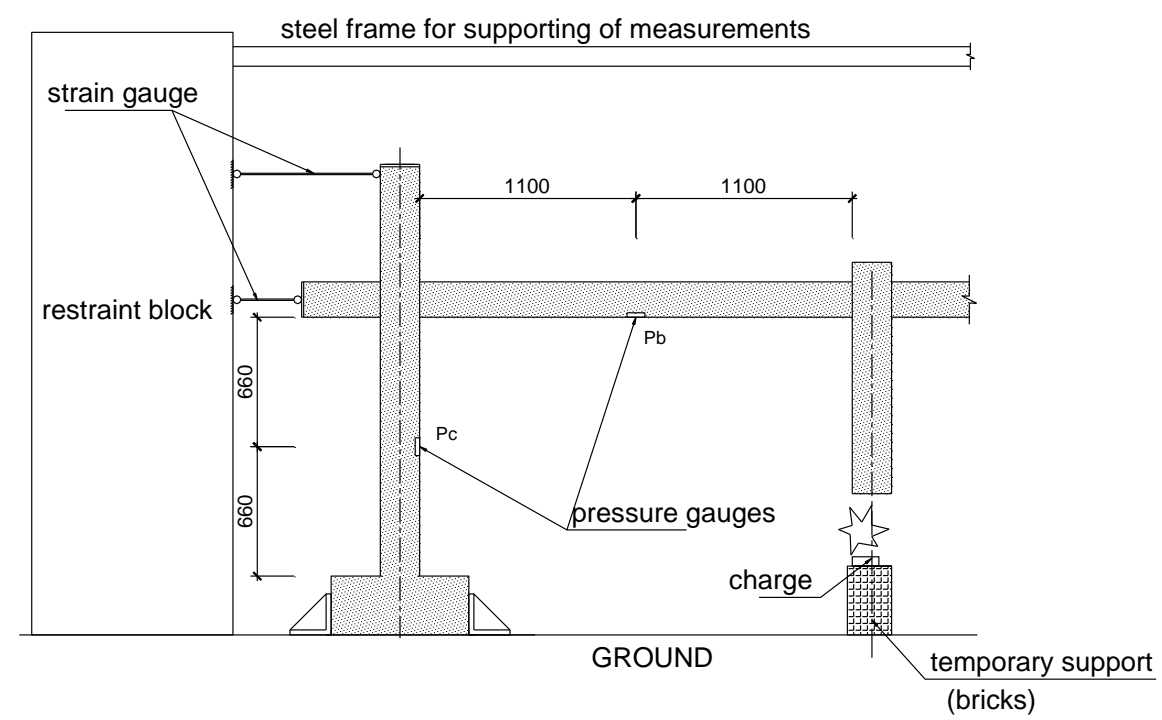

a) Pressure gauge arrangement

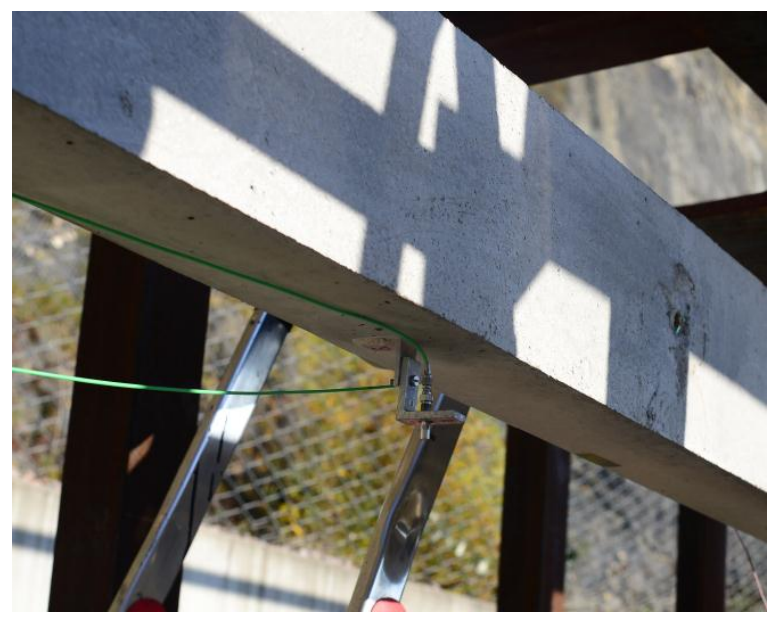

b) Pressure gauge at beam soffit $(\mathrm{Pb})$

Fig. 5.22 Measurements of blast pressure

\subsection{Summary}

In this chapter, an experimental programme on beam-column structures under single column removal scenario was proposed. The specimen design and the boundary conditions of the proposed tests followed the quasi-static tests from Lim et al. (2015). Three test series were conducted including the CL free-fall dynamic tests, the UDL static and dynamic tests, and the CL contact detonation (ultra-fast dynamic) tests. For Series I, the sudden loss of the middle column was simulated by the quick-release mechanism while the specimen was pre-loaded with steel plates at 
the middle joint. For Series II, the equivalent 4-point loading system was proposed to simulate UDL for both static and free-fall dynamic regimes. On the other hand, for Series III, detonation charge would trigger collapse by destroying the middle column and its temporary support. The imposed load in Series III was implemented by a weight-frame system fixed on top of the middle joint. The investigated parameters in Series I included load level and boundary restraint. Compared to Series I, Series II studied the effect of loading method, boundary condition, and load level (dynamic tests). In Series III, blast effect and boundary restraint were the main two parameters studied. Results from the three test series are presented and discussed in the following chapters. 



\section{CHAPTER 6 EXPERIMENTAL AND NUMERICAL RESULTS OF RC SUBSTRUCTURES UNDER CL FREE-FALL DYNAMIC CONDITION (SERIES I)}

\subsection{Introduction}

Following the objectives, specimen design, test setup, and instrumentation introduced in Chapter 5, experimental results of RC beam-column frames under a sudden column removal scenario applying CL condition (Series I) is presented in this chapter (Pham and Tan 2017a). Dynamic effects of the event are investigated and compared with responses from static tests of Lim et al. (2015). Izzuddin's method for dynamic assessment is also verified for the case of CL configuration. Thereafter, numerical analyses using both sophisticated and simplified SDOF models are employed to further study the frame structural behaviour under free-fall dynamic regime.

\subsection{Test results of Series I}

\subsubsection{Testing procedure and overall behaviour}

Shortly before conducting each free-fall dynamic test, axial forces were slowly applied onto the side columns. The temporary support under the steel-plate system (item (13) from Fig. 5.5) was also removed. Due to the applied weight and imperfection of the connection between the release device and the middle joint, the specimen slightly sagged and initial non-zero values were also captured from the reaction forces as shown in Table 6.1. After all the readings from data channels were stable to ensure that the specimen was totally at rest, the rope from the release device was yanked suddenly, generating a free-fall event to the specimen. Under the gravity effect of applied load, the specimen immediately moved downwards. If progressive collapse did not occur, the double-span beam would oscillate within its final deformed position and come to rest under damping effect. Otherwise, a complete collapse would take place through the fracture of both top and bottom rebars in the double-span beam at the middle and the end joint regions, causing the 
specimen to hit the strong floor. Among the six tests (Table 5.1), all of them survived the complete collapse, except FD2-F/34 which was loaded up to $34 \mathrm{kN}$, the highest load used. Time histories of the MJD for all the tests are shown in Fig. 6.1. For the full-restraint specimens (FD1, FD2), large deformations were observed at load levels greater than $20 \mathrm{kN}$. For the partial-restraint specimen FD3, small deflections were observed at load levels of 20 and $25 \mathrm{kN}$. Thereafter, at an applied load of $30.5 \mathrm{kN}$ for FD4, large MJD of $517 \mathrm{~mm}$ was recorded and yet the specimen did not collapse.

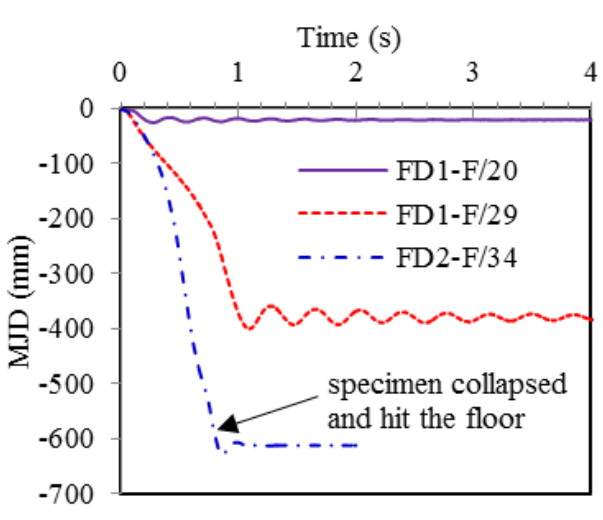

a) Full restraint

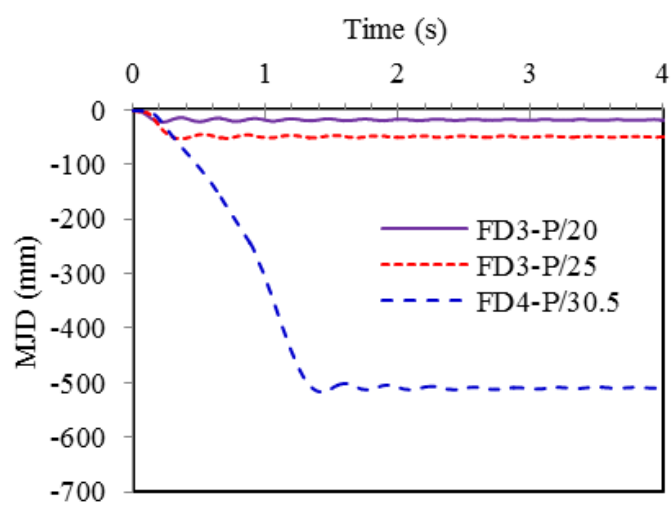

b) Partial restraint

Fig. 6.1 Displacement time-history responses

According to Biggs (1964), when a single-degree-of-freedom system is subjected to a constant force with finite rise time $\left(t_{r}\right)$, if $t_{r}$ is relatively large compared to the natural period $T_{d}$, then the response will follow the applied load and the dynamic effect is negligible. On the other hand, if $t_{r} / T_{d} \leq 1 / 4$, the effect is essentially identical to a suddenly applied load and the influence of $t_{r}$ can be omitted. In the free-fall dynamic tests simulating sudden column removal scenario, the constant force is the gravitational action from the weight applied at the middle joint, and the rise time is represented by the release time $t_{r}$ of the mechanism during which the supporting force of the connection totally reduces to zero. Therefore, the requirement of $t_{r} / T_{d} \leq 1 / 4$ proposed by Biggs (1964) can be suitably applied to the free-fall tests to evaluate the dynamic effect of the release mechanism. For the surviving specimens, the measured vibrations were used to provide information on natural vibration periods $\left(T_{d}\right)$ of the RC frames, which could then be compared with 
the release time $\left(t_{r}\right)$ measured by the strain gauges attached to the middle-joint hook. It was shown that most of the tests (except FD3-P/20) satisfied the requirement of $t_{r} / T_{d} \leq 1 / 4$ to ensure the dynamic effect of free fall was significant. Damping ratios $(\zeta)$ of the vibration stage was also determined based on logarithmic decrement method, ranging from 3.0 to $4.0 \%$. Detailed information on $t_{r}, T_{d}$, and $\zeta$ are presented in Table 6.1, while Fig. 6.2(a) and (b) respectively show the release time and damping ratio from typical free-fall tests.

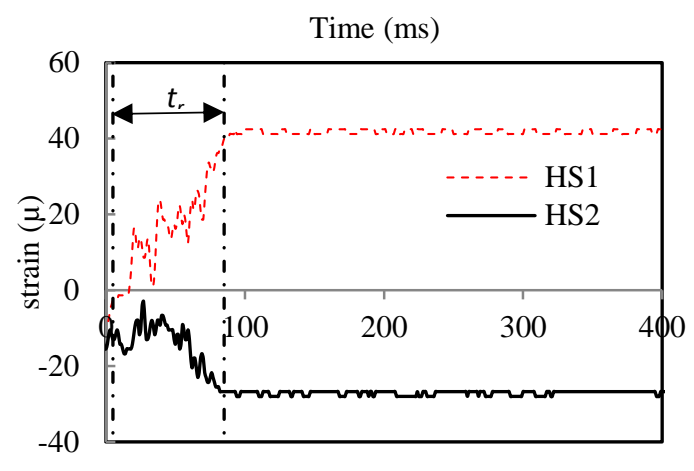

a) Release time (FD3-P/20)

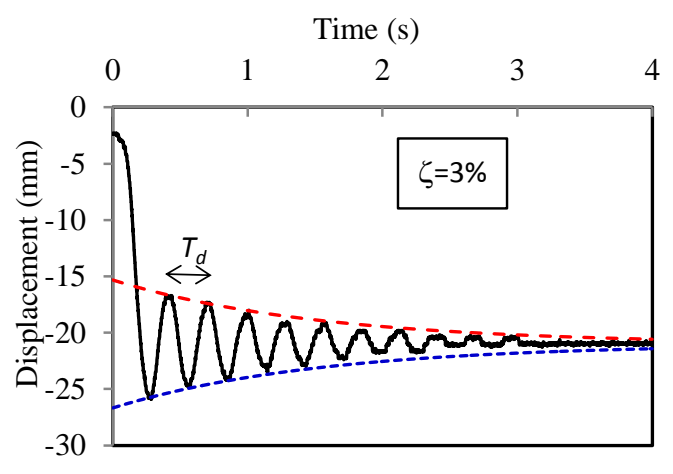

b) Damping ratio (FD1-F/20)

Fig. 6.2 Dynamic test information 


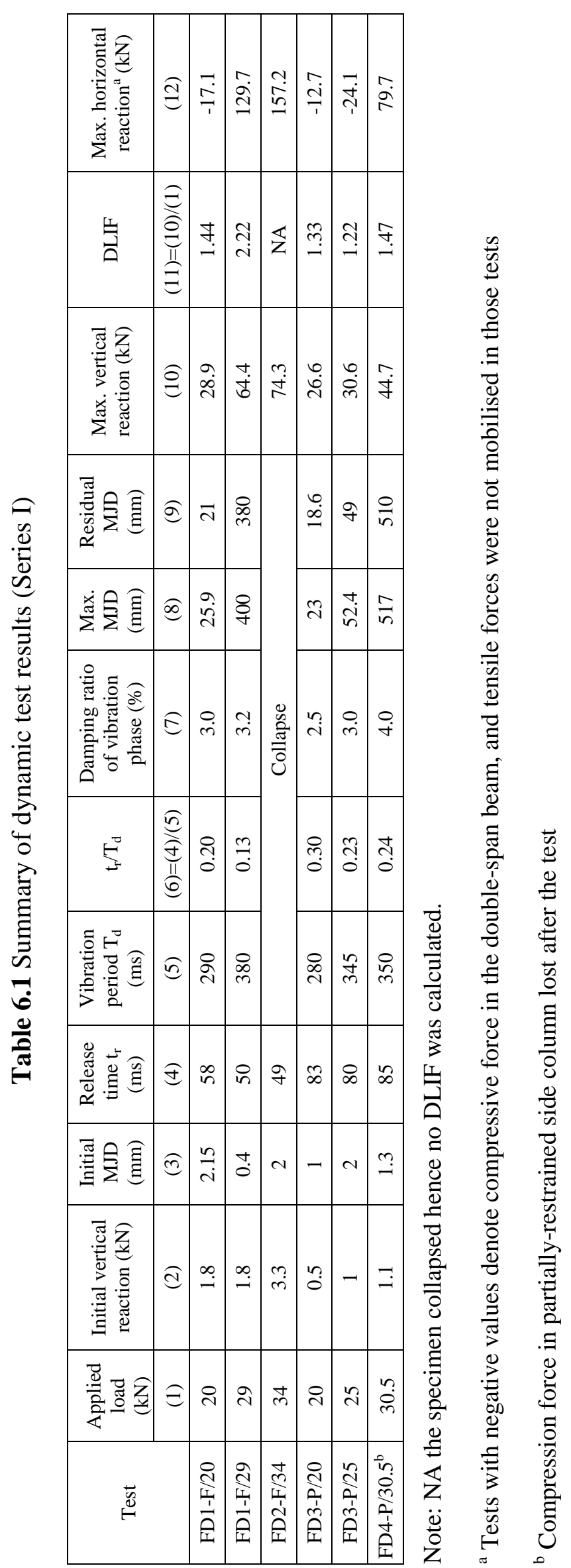


Time histories of total vertical reaction (two sides) and horizontal reaction (left side) of all specimens are presented in Fig. 6.3 and Fig. 6.4, respectively. Fig. 6.4 shows that $\mathrm{CA}$, marked by a change of horizontal reaction from compression to tension, was developed in the full-restraint tests with load levels of 29 and $34 \mathrm{kN}$ and in the partial-restraint test with $30.5 \mathrm{kN}$ load level. Evidence of complete collapse of FD2-F/34 was shown in the time histories of both the horizontal and the vertical reactions as they suddenly dropped to zero at $0.75 \mathrm{~s}$ (Fig. 6.3(a) and Fig. 6.4(a)). Shortly after, the double-span beam of FD2 hit the strong floor at $0.85 \mathrm{~s}$, causing distortions to the reaction forces. The maximum vertical and horizontal reactions of the dynamic tests are summarised in Table 6.1. Fig. 6.5 shows responses of vertical acceleration recorded at the middle joint in the dynamic tests with $20 \mathrm{kN}$ applied load. The maximum acceleration, attained when the MJD reached its maximum value, was $3.7 \mathrm{~g}$ and $14.6 \mathrm{~g}$ for FD1-F/20 and FD3-F/20, respectively.

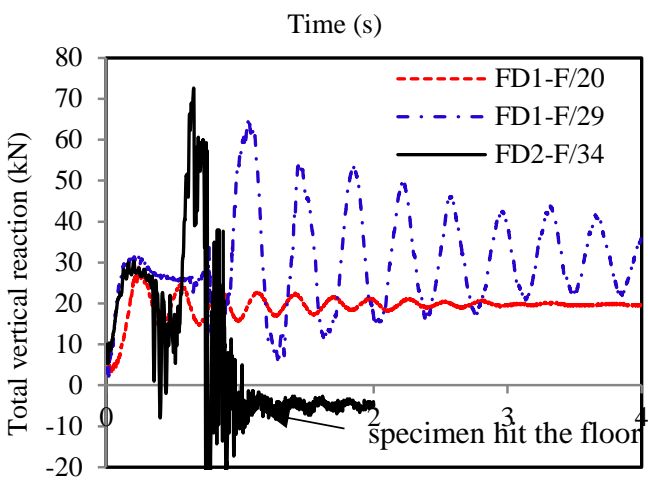

a) Full restraint

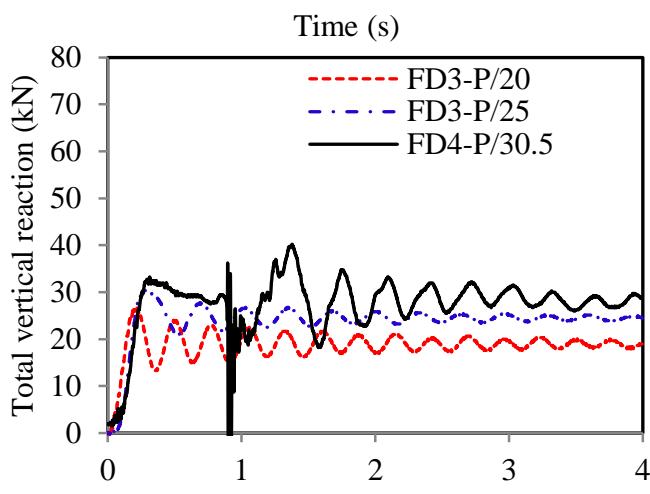

b) Partial restraint

Fig. 6.3 Total vertical reaction time-history responses

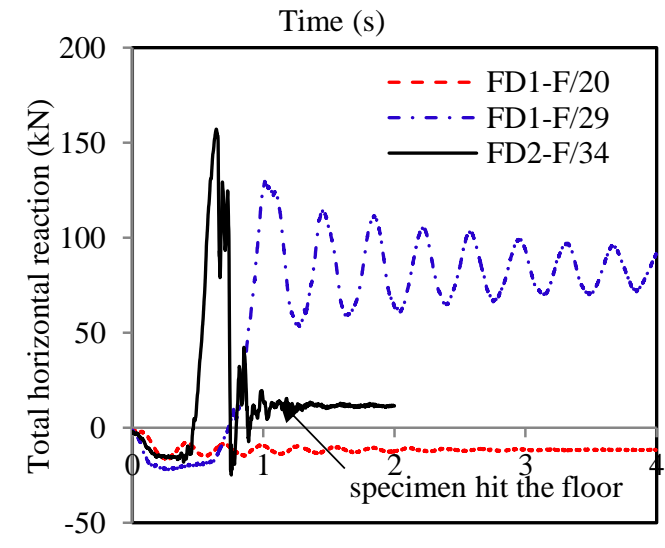

a) Full restraint

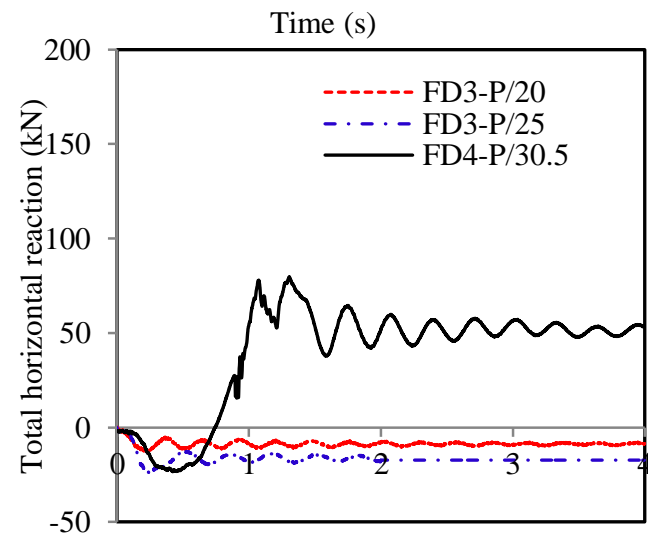

b) Partial restraint

Fig. 6.4 Total horizontal reaction time-history responses (left side) 


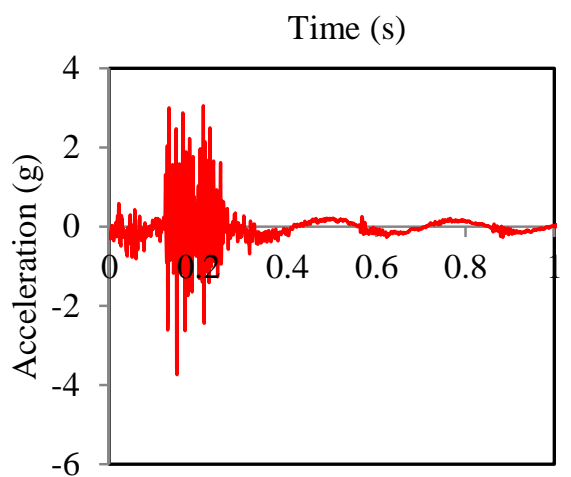

a) FD1-F/20

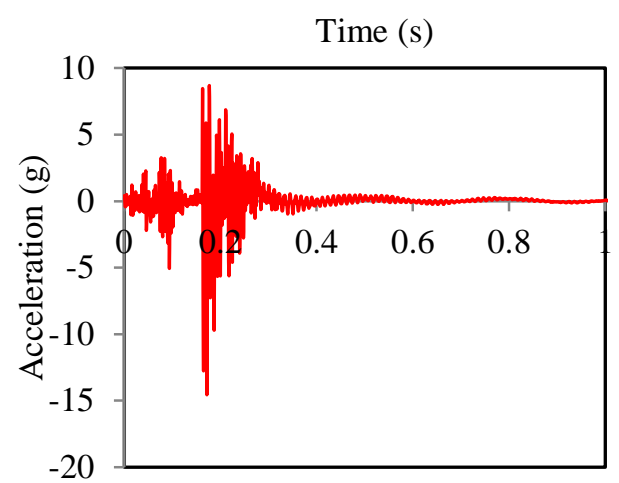

b) FD3-F/20

Fig. 6.5 Acceleration of the middle joint

\subsubsection{Damage patterns of $\mathrm{RC}$ frames under applied loads of 20 and 25 $\mathbf{k N}$}

Fig. 6.6(a) and (e) respectively illustrate the crack patterns of specimens FD1 and FD3 after the first release with $20 \mathrm{kN}$ applied load, whereas Fig. 6.7 shows the time histories of strain gauge readings on longitudinal reinforcement at Sections 1 and 4 (Fig. 5.18) from these tests. It was shown that only some minor cracks were found near the middle and the end joints, which agreed well with strain gauge readings showing yielding of the bottom rebar in Section 4 adjacent to the middle joint (B4 from Fig. 5.18). Suffice to say, FD1 and FD3 only sustained little damages after the first release, and thus, the results of these specimens under the second release of higher loads could still be useful. Subsequently, FD3 was tested again with a $25 \mathrm{kN}$ applied load (FD3-P/25). Despite its non-symmetric partial-restraint boundary condition, crack pattern of this test was symmetrically distributed about the middle joint, as shown in Fig. 6.6(f). Vertical flexural cracks uniformly distributed from the end-joint and middle-joint interfaces towards the rebar cut-off (curtailment) points. In addition, there was no sign of critical damages such as concrete crushing, occurrence of plastic hinges or rebar fracturing. In short, although FD3 was tested twice with two different loads, it still behaved within flexure/CAA phase (Fig. 6.4(b)) with moderate cracks (Fig. 6.6(f)) and deformations (Fig. 6.1(b)). 


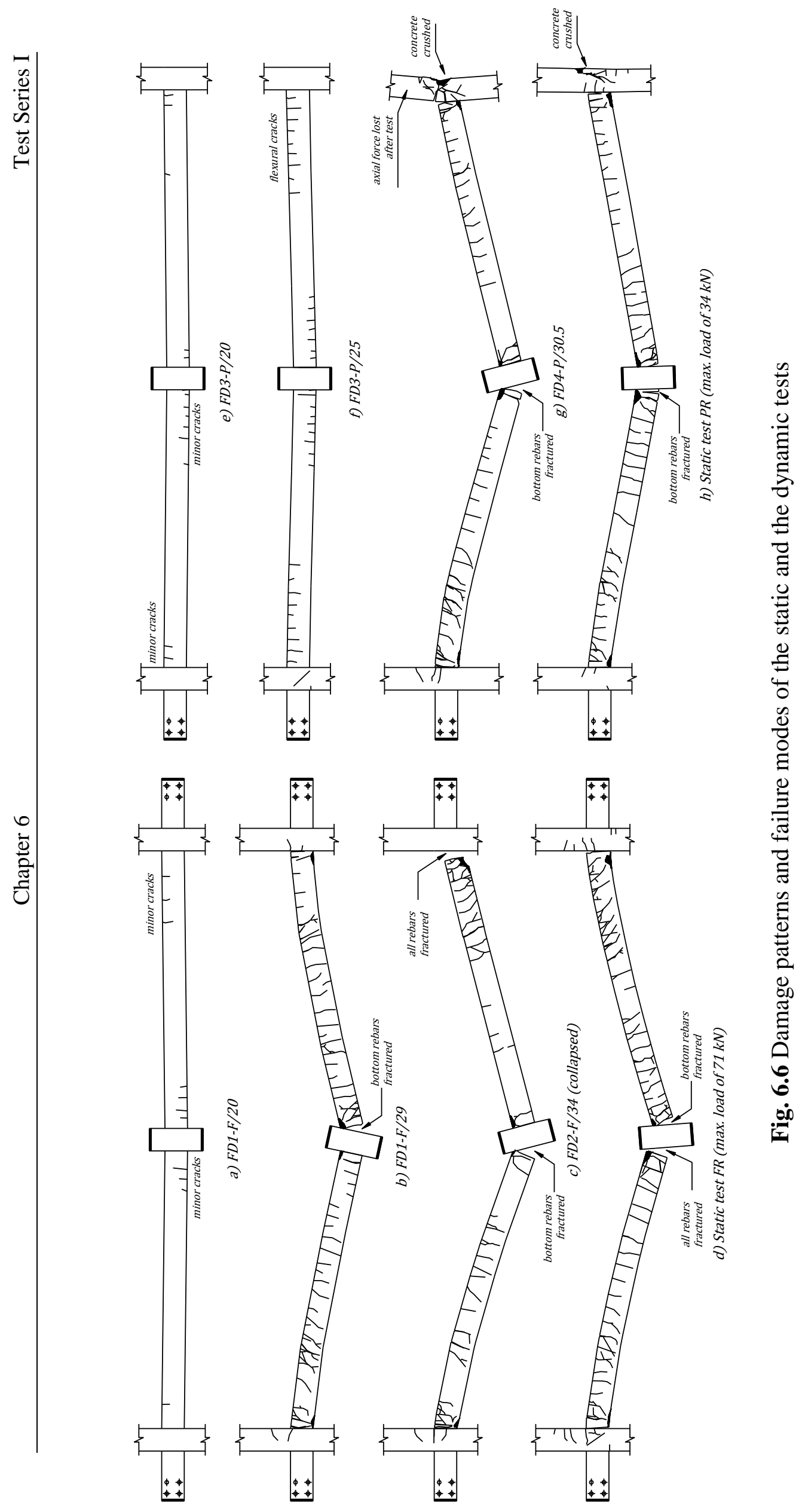

g 


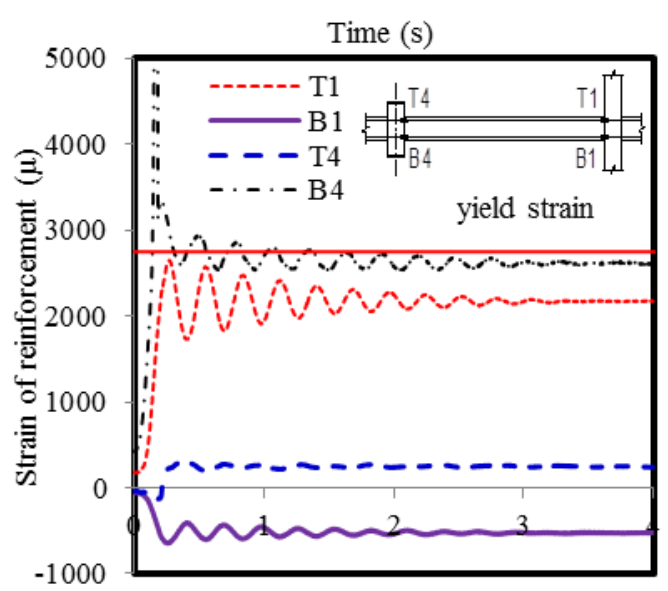

a) FD1-F/20

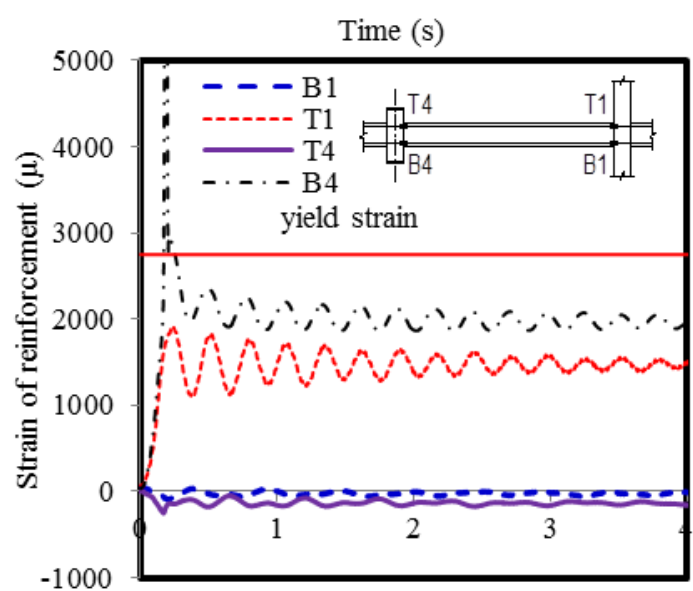

b) FD3-P/20

Fig. 6.7 Reinforcement strains at end joint and middle joint

\subsubsection{Damage patterns of specimens under $29 \mathrm{kN}$ load level and above}

As shown in Fig. 6.1(a) and Fig. 6.4(a), full-restraint specimens under load levels of 29 and $34 \mathrm{kN}$ underwent large vertical deflections and development of CA. Failure modes of FD1 and FD2 specimens are illustrated in Fig. 6.6(b) and (c), respectively. FD1-F/29 (second test of FD1) showed severe cracks in the vicinity of the middle and the end joints, as well as along the double-span beams. In addition, clearly, bottom rebars had fractured on the right side of the middle joint. However, FD1 did not collapse yet. FD2-F/34 with a higher applied load of $34 \mathrm{kN}$ sustained more critical damages as both the bottom rebars at the middle joint and all the reinforcing bars at the right-side end joint had fractured, causing the specimen to collapse completely. The failure mode and damages at the joint regions of this test are shown more clearly in Fig. 6.8. Suffice to say, the dynamic tests on full-restraint specimens demonstrated the mobilisation of CA after the fracture of bottom rebars in the middle joint from FD1-F/29 and yet the specimen survived from collapse. It shows that under dynamic free-fall environment, the remaining top rebars could sustain the applied load and prevent progressive collapse, which was not evident in the reported blast tests by Yu et al. (2014). 


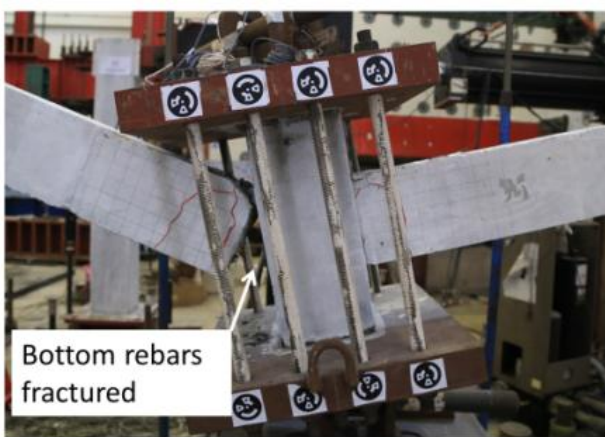

a) Middle joint

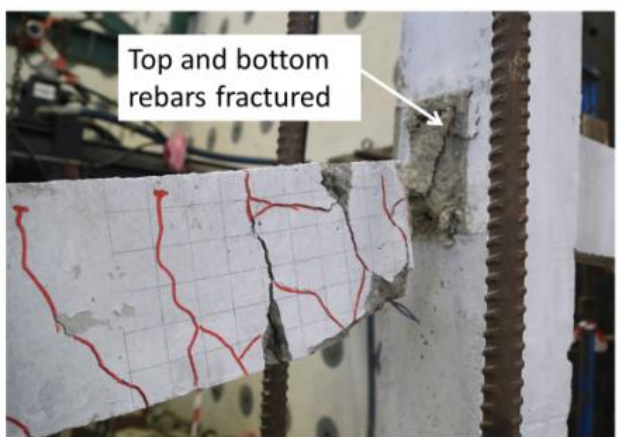

b) End joint

Fig. 6.8 Failure modes of FD2-F/34

With applied load of $30.5 \mathrm{kN}$, FD4-P/30.5 underwent a large deformation with several major failures including fracture of bottom rebars from one side of the middle joint and excessive inward movement of the partially restrained side column causing severe damages to the right-side end joint (Fig. 6.6(g) and Fig. 6.9(a)). Besides the formation of a plastic hinge at the beam end, an additional hinge was formed at the side column with partial restraint. However, beam top rebar fracture had not occurred and the specimen did not collapse yet. This observation, together with the response of FD1-F/29, showed that CA under dynamic condition could still be mobilised in the beam after the fracture of bottom rebars. It should also be noted that after the test of FD4-P/30.5, the axial force on the partially-restrained side column dropped to $28 \%$ of its original value (Fig. 6.9(b)). This was due to a widening gap between the hydraulic jack and the steel plate seated on top of the column. This gap appeared when the end joint moved inwards too much, affecting the alignment of axial-force loading system in the partially-restrained side column. That is to say, this side column would have failed under buckling if its axial force had remained.

Compared to the damages observed in the static tests for both the full- (FR) and the partial-restraint (PR) cases (Fig. 6.6(d) and (h)), the crack patterns from the dynamic tests were fairly similar. Under large deformations, top-surface flexural cracks propagated from the plastic hinges at the end joint to the curtailment points (Sections 2 and 3 of Fig. 5.18). Full-depth tension cracks also occurred in FD1-F/29 and FD2-F/34, denoting that CA had fully developed across some beam sections. 
Besides, concrete crushing extensively occurred at compressive zones of the middle joint (top surface) and the end joint (bottom surface). However, the number of cracks distributing from Sections 3 to 4 in the dynamic tests was fewer than that in the static tests as there was no rotational restraint of the middle joint in the dynamic tests. In terms of fracture of reinforcement, consistency was found between the static and the dynamic tests as the bottom rebars at the middle joint fractured first followed by the fracture of top rebars. In addition, no shear failure was found in either the static or the dynamic tests. Effect of weak restraint on the partial-restraint specimens was also consistent between the static (PR) and the dynamic (FD4$\mathrm{P} / 30.5)$ tests since in both tests the partially-restrained columns were excessively pulled in when bottom rebars at the middle joint had already fractured and CA started developing. Detailed photos of all dynamic tests from Series I can be found in Appendix 6A.

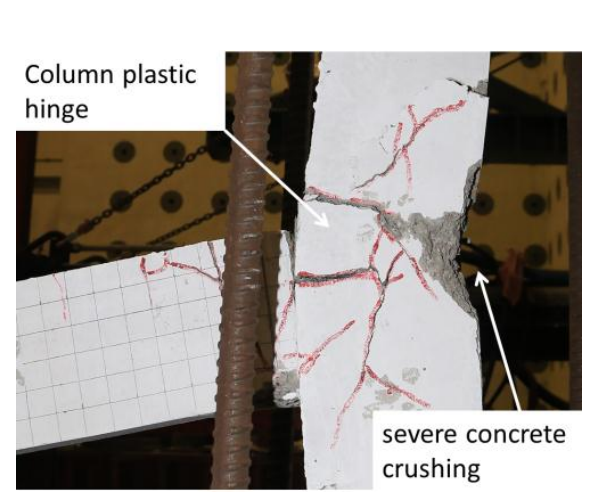

a) End-joint damages

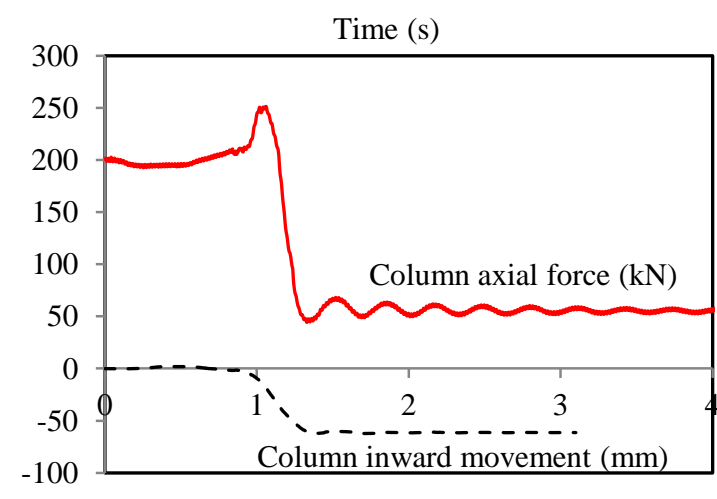

b) Time histories of column axial force and inward movement

Fig. 6.9 Behaviour of partially restrained column in FD4-P/30.5

\subsubsection{Displacement profiles}

Displacement profiles of the dynamic tests were constructed based on displacementtransducer readings and slow-motion camera recordings and were compared to the corresponding static tests by Lim et al. (2015). Deflection measurements were obtained from recorded videos by tracking the movement of marked points using an image analysis software named Tracker (Brown 2012). Comparing the deflection profile obtained from the slow-motion videos to those from the displacement transducers (L1, L2 and L3 from Fig. 5.17), it was found that the former agreed 
well with the latter provided that the maximum deflection was greater than $10 \mathrm{~mm}$ and the magnitude of free vibration was greater than $2 \mathrm{~mm}$. For such conditions, the tolerance of the tracking method for the peak deflection was well within 5\%. Fig. 6.10 presents the comparisons of vertical displacements for FD1-F/20 between the two methods at locations L2 and L3 (Fig. 5.17), respectively. While the software accurately captured larger deflections at L2, it gave less matching results for smaller deflections at L3.
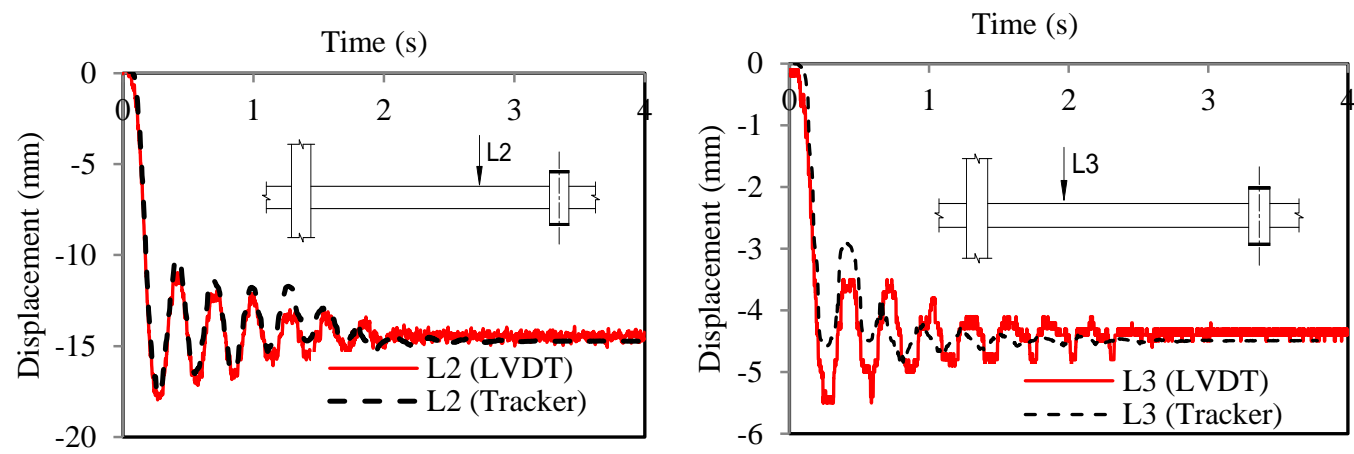

Fig. 6.10 Comparisons between transducer readings and displacements from Tracker software (FD1-F/20)

Beam displacement profiles of FR and FD2-F/34 corresponding to MJDs equal to one, two and three times the beam depth $\left(d_{\text {beam }}\right)$ are plotted in Fig. 6.11. These displacements represented the respective stages of behaviour regarding (a) the end of CAA, (b) CA after the fracture of bottom rebars at the middle joint, and (c) CA at the fracture of top rebars. It is shown that the displacement profiles of both the static and the dynamic tests at one and two $d_{\text {beam }}$ deformations were fairly similar and were almost straight lines. However, at the MJD of three $d_{\text {beam }}$, the displacement profile of FD2 was still linear, whereas in FR, plastic hinges were formed at the locations of top rebar cut-off (L2 and L3), making its profile as a polyline. This is because during the static test of FR, the middle joint was rotationally restrained while there was no such restraint in the dynamic test. Consequently, after the fracture of bottom rebars at the middle joint, plastic hinges at curtailment points were readily formed in the static tests compared to the dynamic tests.

Due to early termination of PR test, its maximum deformation (396 mm) was smaller than that of FD4 under the applied load of $30.5 \mathrm{kN}(517 \mathrm{~mm})$. The same 
observations were obtained when comparing the column horizontal displacement profile of the static to that of the dynamic tests (Fig. 6.12). The maximum inward movement of the PR side column was $3.5 \mathrm{~mm}$ at the applied load of $30.5 \mathrm{kN}$ and was $17.5 \mathrm{~mm}$ when the test stopped. On the other hand, in the corresponding dynamic test of FD4-P/30.5, the side column was pulled inward up to $66 \mathrm{~mm}$ and yet no obvious failure had occurred. Although FD4 achieved a greater displacement profile compared to the static test, it could have failed since it continued losing the column axial force during the excessive inward movement of the end joint (Fig. 6.9(b)).

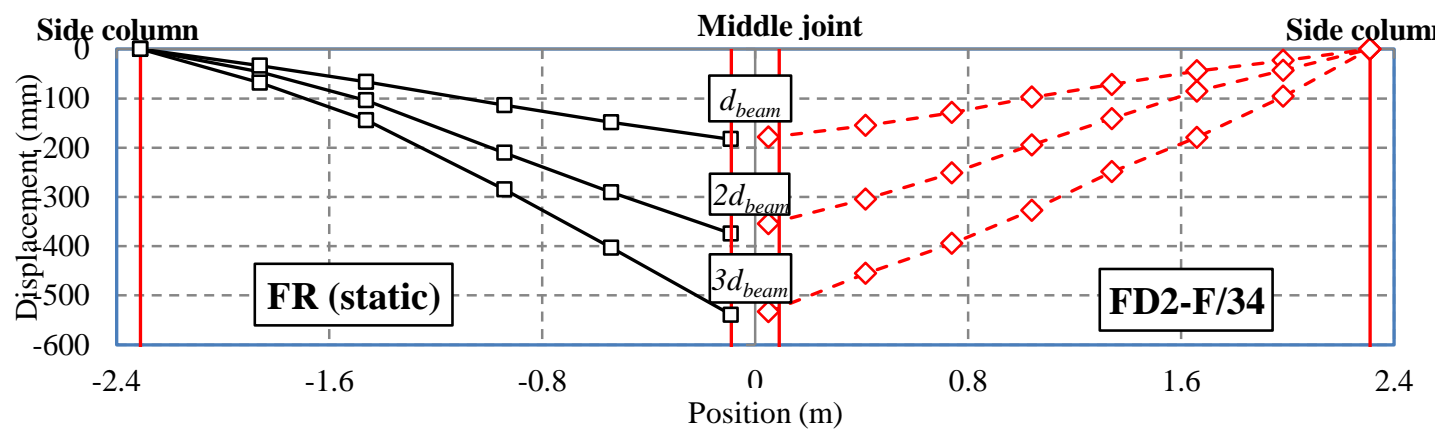

Fig. 6.11 Comparisons of displacement profiles for full-restraint specimens

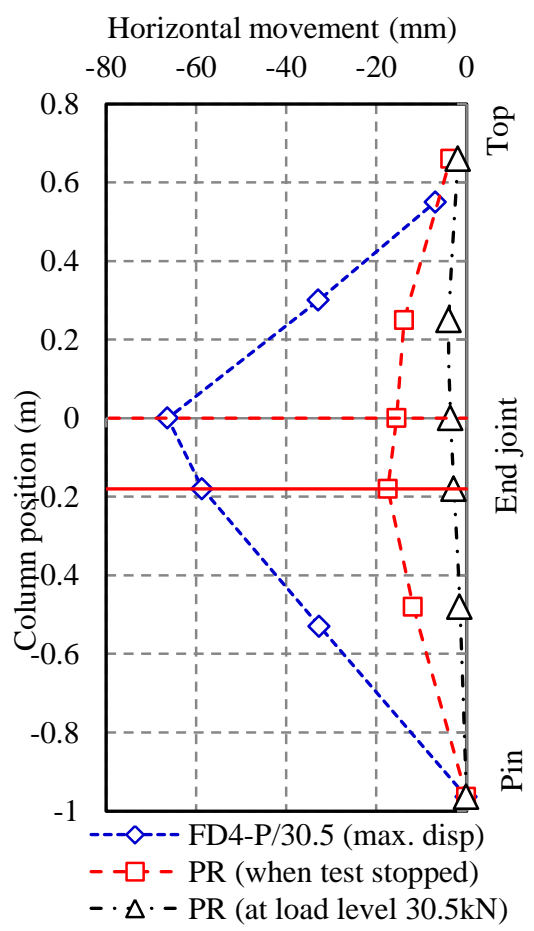

Fig. 6.12 Displacement profile of partially restrained side columns 


\subsubsection{Strain rate effects on material properties}

Based on the rebar strain gauges at Sections 1 and 4 as well as the concrete strain gauges near the joints (CS1 and CS2 in Fig. 5.18), maximum strain rate values from all the dynamic tests were calculated to determine material strength and stiffness enhancements. The CEB model for Concrete Structures (2010) was applied to calculate Dynamic Increase Factors for compression and tension strength (Eqs. (3.9) to (3.12)) and for modulus of elasticity (Eq. (6.1)).

Modulus-of-elasticity dynamic increase factor $\left(\mathrm{DIF}_{\mathrm{E}}\right)$ :

$\operatorname{DIF}_{\mathrm{E}}=\left(\frac{\dot{\varepsilon}_{\mathrm{c}}}{\dot{\varepsilon}_{\mathrm{c} 0}}\right)^{0.026}$

where $\dot{\varepsilon}_{C}$ is the respective strain rates in $\mathrm{s}^{-1}$ for concrete in compression. $\dot{\varepsilon}_{c 0}$ is equal to $30 \times 10^{-6} \mathrm{~s}^{-1}$.

Normal rebar strain gauges are reliable up to the yield point. Since they were used for longitudinal reinforcement, strain rates were calculated for beam tensile rebars at Sections 1 and 4 up to the yield strain value. To calculate the strain rate effect on steel, the empirical Eqs. (3.13) to (3.15) proposed by Malvar (1998) were utilised to calculate the Dynamic Increase Factor (DIF) for yield strength.

Table 6.2 summarises the material increase factors for both concrete and tensile rebar at the end joint and the middle joint of the dynamic tests. Fig. 6.13 presents the strains and the corresponding strain rates at two positions near the middle (CS1) and the end (CS2) joints from FD2-F/34. It is noteworthy that the dynamic increase values for materials near the end and the middle joints were fairly similar. For concrete, the maximum CDIF and TDIF were 1.110 and 1.190, respectively, whereas the maximum $\mathrm{DIF}_{\mathrm{E}}$ was 1.215 for concrete in compression. Regarding reinforcement (Fig. 6.14), the maximum DIFs for yield strength was 1.189 (Table 6.2). The rebar strain rates recorded in the dynamic tests was much larger compared to the static tests (with the equivalent strain rate of about $10^{-4} / \mathrm{s}$ ). If plastic moment capacities of the end and the middle joints were simply calculated based on Eq. (4.4) proposed by Paulay and Priestley (1992), then structural capacity of the 
specimen based on plastic hinge theory (Eq. (4.8)) could be increased up to $18.9 \%$ due to strain rate effect on steel bars. In addition, if the maximum strain rates used for yield strength were applied to calculate DIFs for rebar ultimate strength following Eq. (3.15), the enhancement was not significant (Table 6.2), with the largest DIF of 1.060. In summary, the strain rate enhancement in terms of strength and stiffness of concrete and rebar yield strength, which are beneficial to the development of flexure/CAA, was moderate in the tests. On the other hand, enhancement of rebar ultimate strength which significantly affects the CA capacity was negligible.

Table 6.2 Summary of material strength enhancements

\begin{tabular}{|c|c|c|c|c|c|c|c|c|}
\hline \multirow{3}{*}{ Test } & \multicolumn{3}{|c|}{ Tensile rebars at Sections 1 and 4} & \multicolumn{5}{|c|}{ Concrete at CS1 and CS2 } \\
\hline & \multirow{2}{*}{$\begin{array}{l}\text { Max. strain } \\
\text { rate (1/s) up } \\
\text { to yielding }\end{array}$} & \multicolumn{2}{|c|}{ DIF } & \multicolumn{3}{|c|}{ In compression } & \multicolumn{2}{|c|}{ In tension } \\
\hline & & $\begin{array}{l}\text { Yield } \\
\text { strength }\end{array}$ & $\begin{array}{l}\text { Ultimate } \\
\text { strength }\end{array}$ & $\begin{array}{l}\text { Max. strain } \\
\text { rate }(1 / s)\end{array}$ & CDIF & $\mathrm{DIF}_{\mathrm{E}}$ & $\begin{array}{l}\text { Max. strain } \\
\text { rate }(1 / s)\end{array}$ & TDIF \\
\hline FD1-F/20 & 0.099 & 1.155 & 1.050 & 0.013 & 1.089 & 1.172 & \multicolumn{2}{|c|}{ NA } \\
\hline FD1-F/29 & 0.253 & 1.178 & 1.057 & 0.053 & 1.110 & 1.215 & 0.133 & 1.186 \\
\hline FD2-F/34 & 0.394 & 1.189 & 1.060 & 0.049 & 1.109 & 1.212 & 0.155 & 1.190 \\
\hline FD3-P/20 & 0.212 & 1.173 & 1.055 & \multirow{3}{*}{\multicolumn{5}{|c|}{ not measured }} \\
\hline FD3-P/25 & 0.117 & 1.159 & 1.051 & & & & & \\
\hline FD4-P/30.5 & 0.315 & 1.183 & 1.058 & & & & & \\
\hline
\end{tabular}

Note: NA concrete in tension was not mobilised

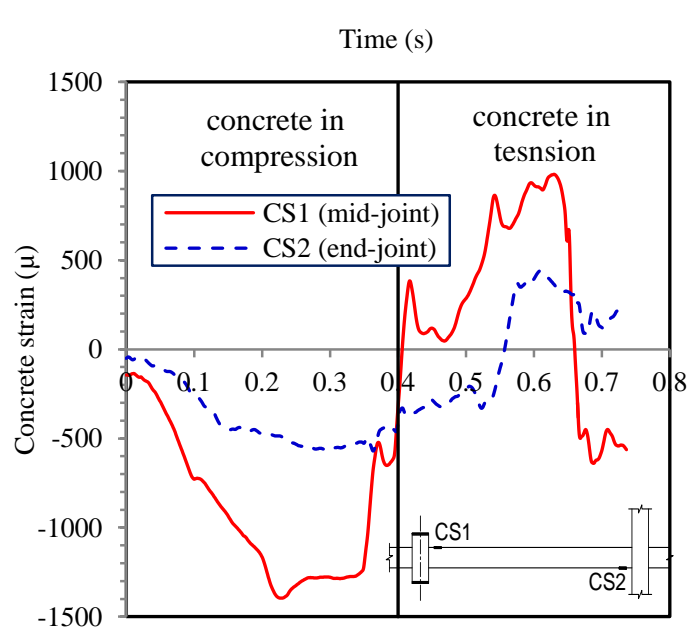

a) Concrete strain

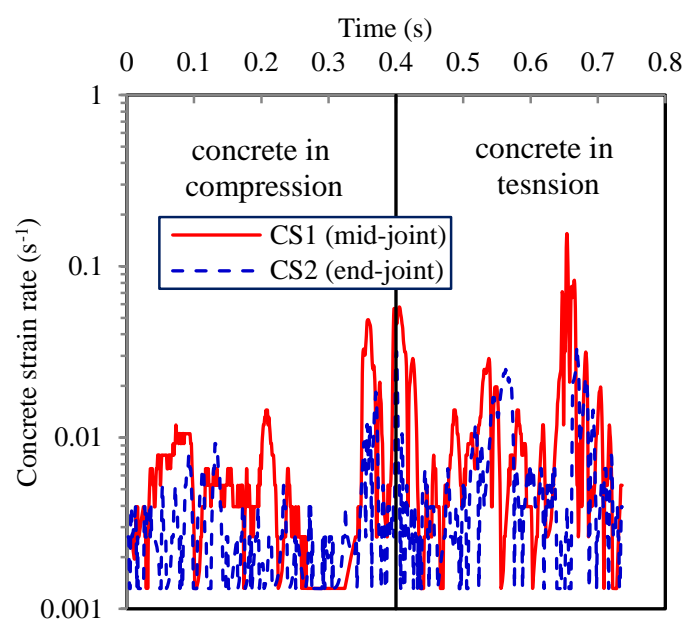

b) Concrete strain rate

Fig. 6.13 Concrete strains and strain rates at middle and end joints of FD2-F/34 


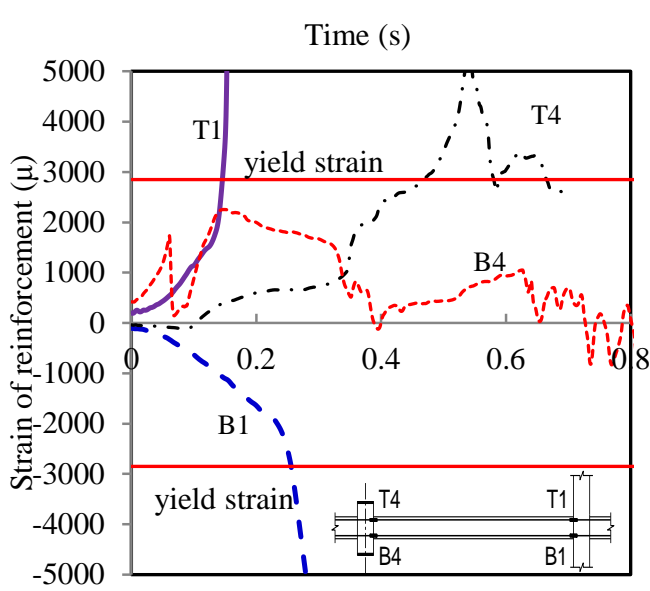

a) Rebar strain

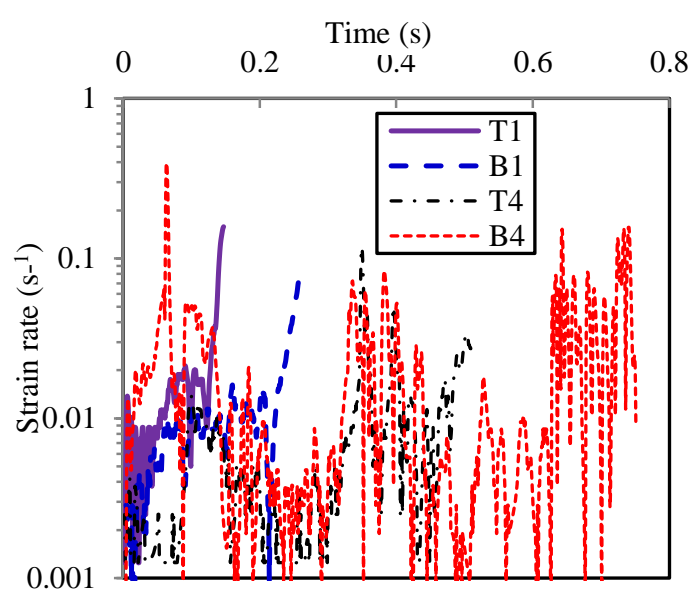

b) Rebar strain rate

Fig. 6.14 Rebar strains and strain rates at Sections 1 and 4 of FD2-F/34

\subsubsection{Dynamic load increase factors}

The maximum value of vertical reaction force from each test, if the specimen had not collapsed yet, was used to calculate its ratio with the corresponding applied load from steel weights of the test to define the dynamic load increase factor (DLIF) presented in Table 6.1. This factor highlighted the inertial effect of the steel weights on the frame structure, ranging from 1.22 to 2.22. According to Tsai (2010), for a typical elasto-plastic behaviour under progressive collapse, the DLIF normally decreases from 2 (elastic stage) towards 1 (plastic stage). In this experimental study, this observation agrees with test results under load levels of 20 and $25 \mathrm{kN}$ in which only flexure/CAA was mobilised (Table 6.1). Nonetheless, when the applied load was higher leading to mobilisation of CA, DLIF increased and showed larger values compared to the CAA stage (2.22 for FD2-F/29 and 1.47 for FD4-P/30.5). Hence, although CA can significantly increase structural capacity in quasi-static condition, the mobilisation of this mechanism under dynamic regime leads to higher dynamic increase factors. As a result, engineers should pay particular attention to the design of frame structures if CA is mobilised.

\subsection{Comparisons between static and dynamic responses at structural and cross-sectional levels}




\subsubsection{Structural responses}

In the static tests, all the collected data corresponded to MJD, whereas in the dynamic tests they were recorded with respect to time. Therefore, to compare the structural behaviour of the static tests with the corresponding dynamic tests at structural level, data from the dynamic tests such as vertical and horizontal reactions were plotted with reference to MJD instead of time. Fig. 6.15 illustrates the comparisons between the static tests (FR and PR) during CAA and flexure phase and corresponding dynamic tests at 20 and $25 \mathrm{kN}$ applied loads. It is shown that structural responses of specimens FD1 and FD3 under the applied load of 20 $\mathrm{kN}$ were almost straight lines for both vertical and horizontal reactions, demonstrating that these frames mostly behaved within elastic range. This observation agrees well with Section 6.2.2 which concludes that the second release of FD1 and FD3 with higher loads could still provide reliable test results. Compared to FR and PR, all the dynamic tests at load levels of 20 and $25 \mathrm{kN}$ showed higher structural stiffness during their flexure/CAA behaviours, although both test series shared the same reinforcement design and boundary condition. The reason for this deviation arises from the difference of concrete strength shown in Table 5.1 since this factor remarkably affects the mobilisation of CAA. FD1 and FD3 respectively had concrete cylinder strengths of $9 \%$ and $31 \%$ greater than FR and PR. Moreover, there was a noticeable enhancement on concrete elastic modulus due to strain rate effects (up to $21.5 \%$ for concrete in compression as shown in Table 6.2) which also helped to increase the overall structural stiffness.

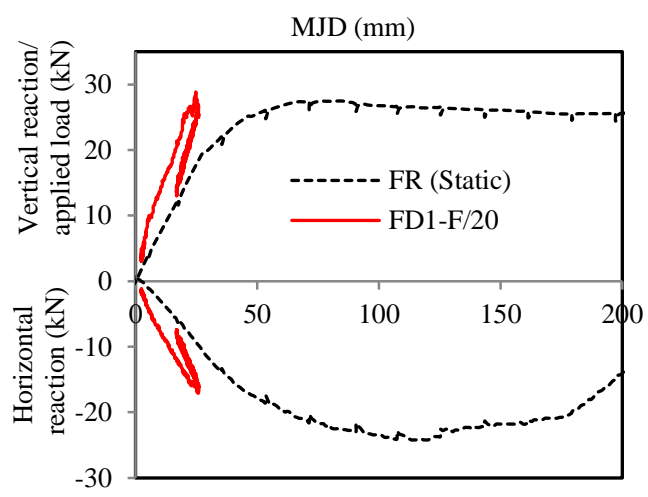

a) Full-restraint specimens

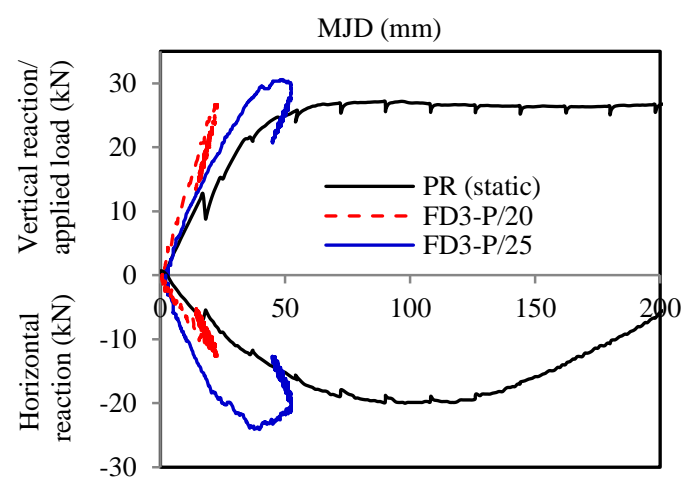

b) Partial-restraint specimens

Fig. 6.15 Structural responses within flexure/CAA 
Fig. 6.16(a) presents similar comparisons between the static test FR and the fullrestraint dynamic tests (FD1-F/29 and FD2-F/34) in which CA was mobilised. Both the vertical and the horizontal reactions from the dynamic tests followed well with those from the static tests during the CAA stage when the beam axial force was still under compression. Thereafter, when CA started developing together with the fracture of bottom rebars, structural responses of the dynamic tests showed larger stiffness than the static tests. Nonetheless, the maximum vertical and horizontal reactions of the dynamic tests did not exceed the maximum values from the static test. Regarding FD1-F/29, although its maximum MJD was only $74 \%$ of the ultimate MJD from FR, its reactions reached $91 \%$ and $89 \%$ of FR vertical and horizontal reactions, respectively. Similarly for FD2-F/34 which experienced a complete collapse, its maximum reactions at the MJD of $452 \mathrm{~mm}$ (83\% of the ultimate MJD from FR) were almost the same as those of the static test. After which, at a MJD of $530 \mathrm{~mm}$, both reactions dropped to zero denoting final failure. The similarity in maximum forces for both vertical and horizontal reactions between the two test series showed a smaller influence of strain rate effect on CA capacity.

For the case of partial-restraint specimens shown in Fig. 6.16(b), the static (PR) and the dynamic (FD4-P/30.5) tests had more or less similar structural behaviour up to the MJD when PR test was stopped $(396 \mathrm{~mm})$. The horizontal reaction of FD4 attained a peak value of $78 \mathrm{kN}$ at $357 \mathrm{~mm}$ MJD before gradually reducing. This reduction was due to inward movement of the partially restrained column which became excessive. In summary, although each dynamic test with one applied load value (forced-controlled) only provided one maximum MJD, the relationship between reaction forces and MJD could be plotted out. From Fig. 6.15 and Fig. 6.16, these derived curves were relatively similar to the applied load/reactiondisplacement curves obtained from the static tests. 

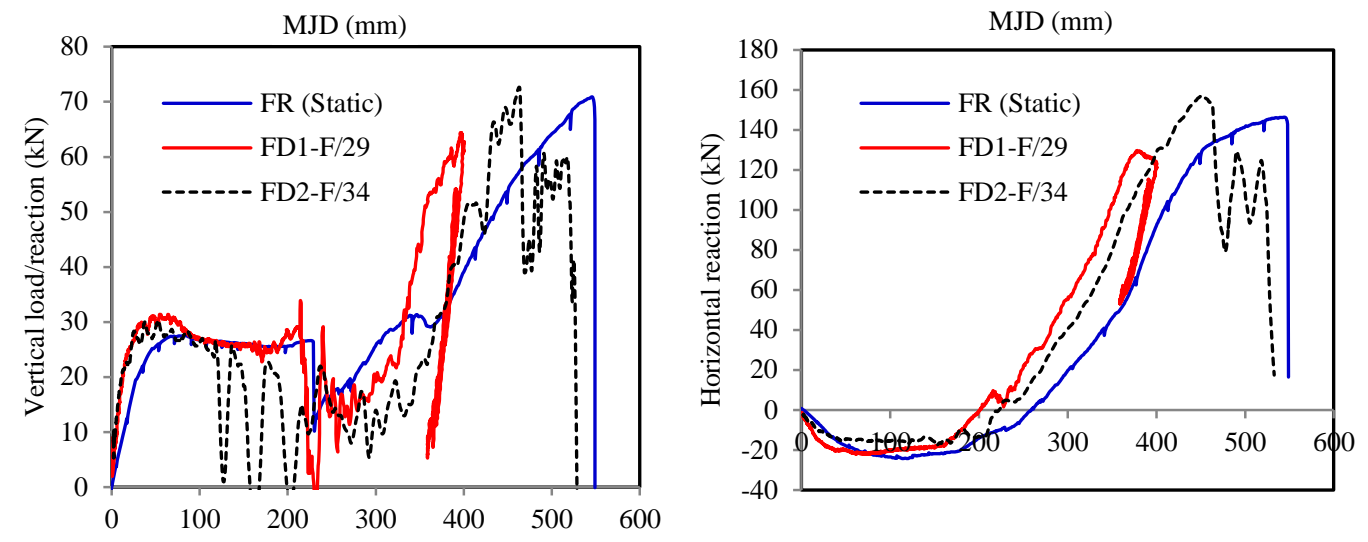

a) Full-restraint specimens
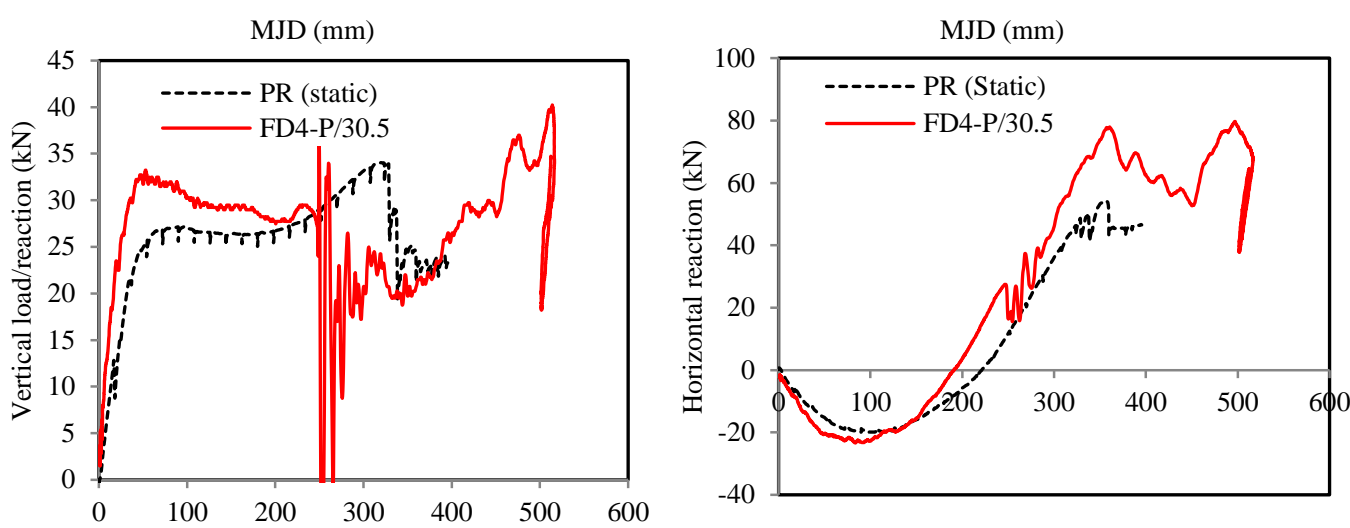

b) Partial-restraint specimens

Fig. 6.16 Structural responses within CA

Concerning the influence of different boundary conditions, at a lower applied load of $20 \mathrm{kN}$, there was hardly any difference in terms of structural behaviour and crack patterns between full-restraint (FD1-F/20) and partial-restraint (FD3-F/20) tests (Fig. 6.15). For low applied loads, the reaction forces at the extended beam level (LH2 or RH2 in Fig. 5.5) were not yet activated during flexure/CAA regime due to connection gaps in the restraint system. At this stage, the horizontal reaction was mostly contributed by the load pin underneath (LH1 or RH1) and partially by the horizontal reaction on top of the side column (LH3 or RH3). For a higher applied load (say $\geq 29 \mathrm{kN}$ ), the development of CA was activated and the difference in behaviour between full- and partial-restraint setups became more apparent. Compared to the full-restraint test of FD1-F/29 with $29 \mathrm{kN}$ applied load (Fig. 6.16(a)), FD4-P/30.5 had much lower structural response in terms of both vertical 
and horizontal reactions (Fig. 6.16(b)) showing that partial-restraint boundary condition significantly reduced the load-carrying capacity under a high applied load.

Considering the failure modes, the dynamic tests experienced the same ductile failure as the static tests. No brittle shear failure was observed even in FD2-F/34 when complete collapse took place (Fig. 6.8). Moreover, the responses of vertical reaction forces in the dynamic tests, which represented shear force at the end joint, followed those in the static tests (Fig. 6.16). Therefore, it can be inferred that if shear failure does not occur during static tests, it will not occur in corresponding dynamic tests even if the applied load in dynamic tests is relatively large. In other words, the sudden removal of a supporting column will not change the failure mode obtained from static environment.

\subsubsection{Stiffness of boundary restraints}

The structural behaviour of the beam-column frames used in the static and the dynamic tests was simplified into a double-span sub-assemblage structure with lateral $\left(K_{A}\right)$ and rotational $\left(K_{R}\right)$ springs at the two ends as described in Fig. 6.17(a). Horizontal reaction $(H)$ and bending moment $\left(M_{\text {end }}\right)$ at each end joint of the subassemblage were defined based on reaction forces from the tests as shown in Fig. 6.17(b). Translational $(d)$ and rotational $(\theta)$ deformations of the end joint were calculated based on the data from two LVDTs L4 and L5 placed at the end joint, illustrated in Fig. 6.17(c). Subsequently, stiffness values of the lateral $\left(K_{A}\right)$ and the rotational $\left(K_{R}\right)$ springs were determined by Eqs. (6.2) and (6.3), respectively.

$$
\begin{aligned}
& K_{A}=\frac{H}{d} \\
& K_{R}=\frac{M_{\text {end }}}{\theta}
\end{aligned}
$$

For the partially-restrained side of FD4-P/30.5, when the deformation was small, the horizontal force was in compression with no visible damage on the column and no yielding of column reinforcement. After the MJD had exceeded one beam depth, 
the horizontal reaction changed to tension and kept increasing as the MJD increased. After the fracture of bottom rebars and as the pulling-in effect became significant on the side column, the tensile horizontal force reached the maximum value of $79.7 \mathrm{kN}$ (Table 6.1) and started reducing (Fig. 6.16(b)). This reduction was due to the $P-\triangle$ effect on the side column which only had partial horizontal restraint. On the other hand, $K_{A}$ of this test is calculated for the partially-restrained side up to the instant before the side column started moving inwards excessively under pulling-in force. $K_{A}$ in compression is computed based on negative horizontal force during CAA stage, whereas $K_{A}$ in tension is computed from the part of positive horizontal reaction before it reaches the tensile peak of $79.7 \mathrm{kN}$. By using this calculation method, the idealised spring $\left(K_{A}\right)$ is assumed to behave elastically in compression and in tension. Therefore, the $K_{A}$ values do not consider $P-\Delta$ effect.

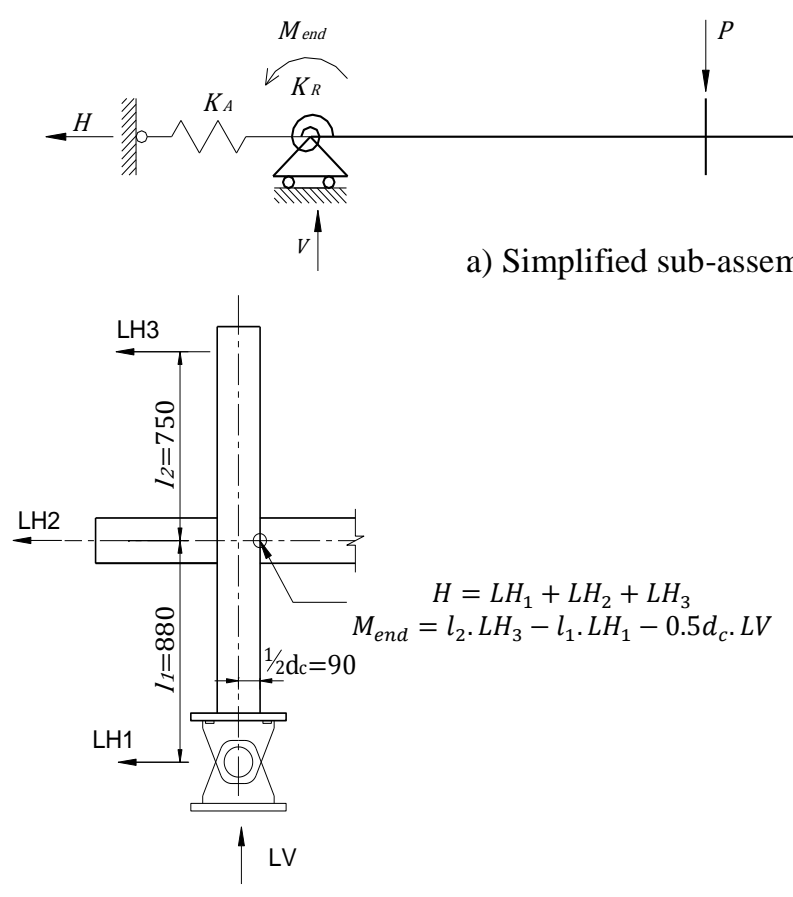

b) Reactions at the end joint

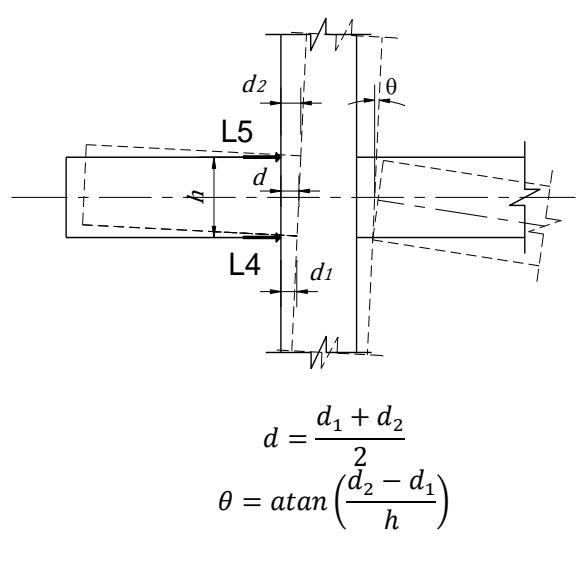

c) Deformations at the end joint

Fig. 6.17 Simplified model for RC beam-column frames

$K_{A}$ and $K_{R}$ were computed for both the left and the right sides of each dynamic test, and the results are shown in Table 6.3. For full-restraint specimens, $K_{A}$ values from the two sides of the double-span beam were reasonably similar. For partial-restraint 
specimens, $K_{A}$ from the right side, which was partially restrained, was noticeably smaller than that from the left side. On the other hand, $K_{R}$ values were quite identical for both sides regardless of the type of boundary condition. Table 6.4 presents the average values of restraint stiffness from the dynamic tests compared with those from the static tests (FR and PR). It is noteworthy that while $K_{A}$ in tension and $K_{R}$ were relatively similar between the static and the dynamic test series, $K_{A}$ in compression from the dynamic tests was remarkably larger than that from the static tests. This result agreed well with the observation from Fig. 6.15 and Fig. 6.16 in which the mobilisation of CAA from the dynamic tests was noticeably larger than that from the static tests whereas both test series showed comparable response of CA.

Table 6.3 Boundary conditions of the dynamic tests

\begin{tabular}{|c|c|c|c|c|c|c|}
\hline \multirow{2}{*}{ Test } & \multicolumn{2}{|c|}{$K_{A}$ of left side $(\mathrm{kN} / \mathrm{m})$} & \multicolumn{2}{c|}{$K_{A}$ of right side $(\mathrm{kN} / \mathrm{m})$} & \multicolumn{2}{c|}{$K_{R}(\mathrm{kNm} / \mathrm{rad})$} \\
\cline { 2 - 7 } & Compression & Tension & Compression & Tension & Left side & Right side \\
\hline FD1-F/20 & 17270 & NA & 23380 & NA & 7690 & 4700 \\
\hline FD1-F/29 & 14290 & 12760 & 16850 & 13570 & 6170 & 7060 \\
\hline FD2-F/34 & 12400 & 12570 & 14000 & 16730 & 3630 & 3060 \\
\hline FD3-P/20 & 39000 & NA & 22000 & NA & 6390 & 5570 \\
\hline FD3-P/25 & 39000 & NA & 28800 & NA & 4950 & 4950 \\
\hline FD4-P/30.5 & 23000 & 19800 & 20000 & 5500 & 4840 & 4280 \\
\hline
\end{tabular}

Note: NA tension force was not developed in the test

Table 6.4 Comparisons of average restraint stiffness between static and dynamic tests

\begin{tabular}{|c|c|c|c|c|c|c|}
\hline \multirow{2}{*}{$\begin{array}{c}\text { Summary of } \\
\text { boundary } \\
\text { condition }\end{array}$} & \multicolumn{3}{|c|}{ Full-restraint sides } & \multicolumn{3}{c|}{ Partial-restraint sides } \\
\cline { 2 - 5 } & \multicolumn{2}{|c|}{$K_{A}(\mathrm{kN} / \mathrm{m})$} & $K_{R}$ & \multicolumn{2}{c|}{$K_{A}(\mathrm{kN} / \mathrm{m})$} & $K_{R}$ \\
\cline { 2 - 3 } & Compression & Tension & $(\mathrm{kNm} / \mathrm{rad})$ & Compression & Tension & $(\mathrm{kNm} / \mathrm{rad})$ \\
\hline Dynamic tests & 22132 & 15086 & 5388 & 20600 & 5500 & 4933 \\
\hline Static tests & 17518 & 14080 & 4258 & 8800 & 5500 & 3250 \\
\hline
\end{tabular}

\subsubsection{Reinforcement strains}

Structural comparisons at cross-sectional level were conducted for the two test series using strain values of reinforcement. Similar to the reaction forces, beam rebar strains from the dynamic tests were plotted with regard to MJD and were compared to related values from the static tests. Fig. $\mathbf{6 . 1 8}$ collates the top- and the 
bottom-rebar strains at the end-joint and the middle-joint interfaces (Fig. 5.18) from FD1-F/29 and compares them with those from FR. It is shown that the strain development had good agreement between the two tests.

To compare the behaviour of the partially-restrained side column between the static (PR) and the dynamic (FD4-P/30.5) tests, strain readings from column rebars (Fig. 5.18) were plotted and presented in Fig. 6.19. Reasonable agreement between static and dynamic tests was observed until the static test was stopped. Initially, inner-face rebars ( $T i$ and $B i)$ were under compression while outer-face rebars ( $T o$ and $B o$ ) were under tension since the side column moved outwards under CAA. During this stage, the column rebars still behaved within elastic range and no obvious column cracks were observed. As the MJD exceeded one beam depth and CA started mobilising, the column-rebar strains changed sign due to inward movement of the column. After the beam bottom rebars had fractured and as the column inward movement had become excessive, the outer face of the side column was under extreme compression force marked by the strain gauge To quickly exceeding compressive yield strain (Fig. 6.19) and the occurrence of severe concrete crushing at this region (Fig. 6.9(a)).

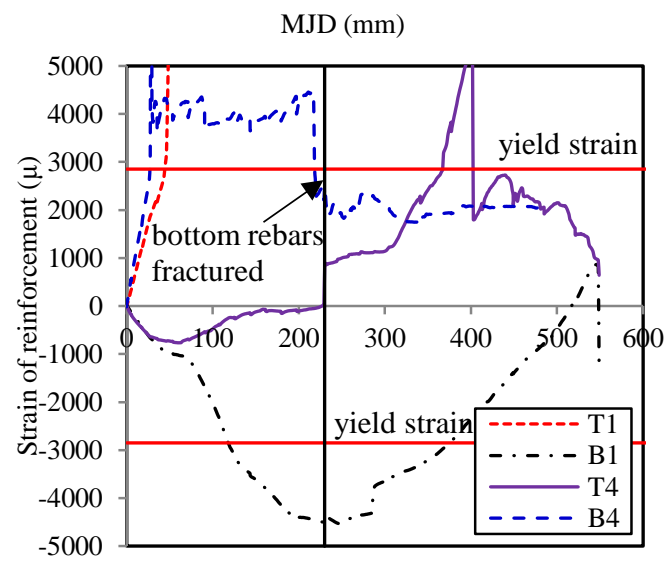

a) FR

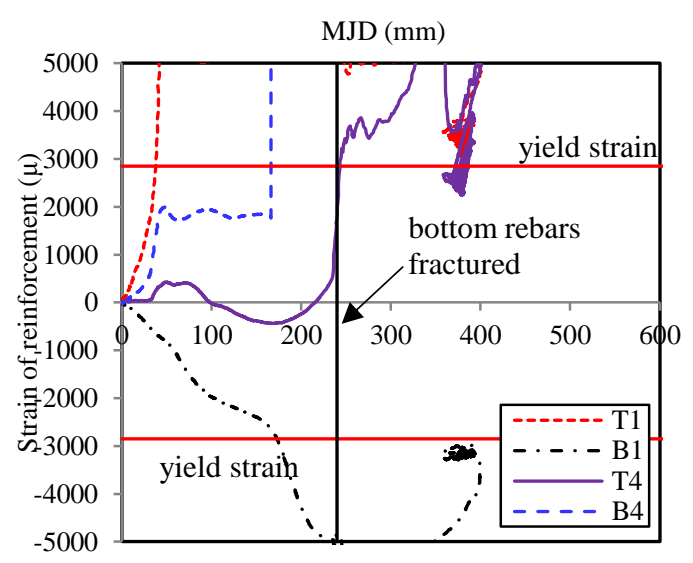

b) Top rebars FD1-F/29

Fig. 6.18 Comparisons of beam reinforcement strains between static and dynamic tests 


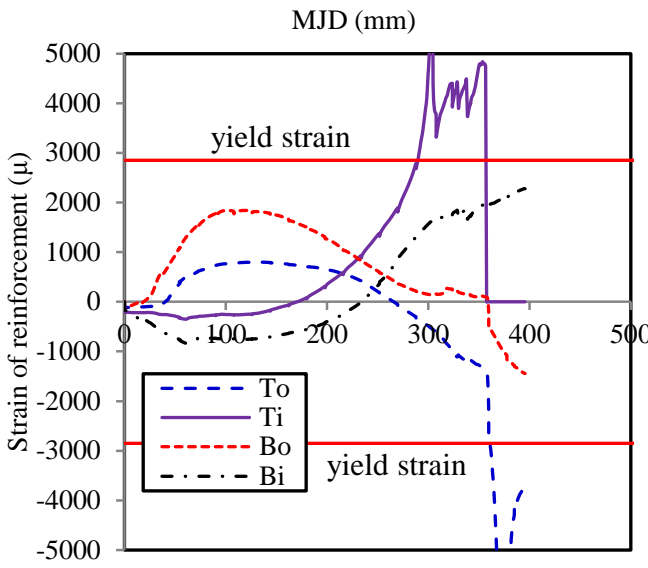

c) Static (PR)

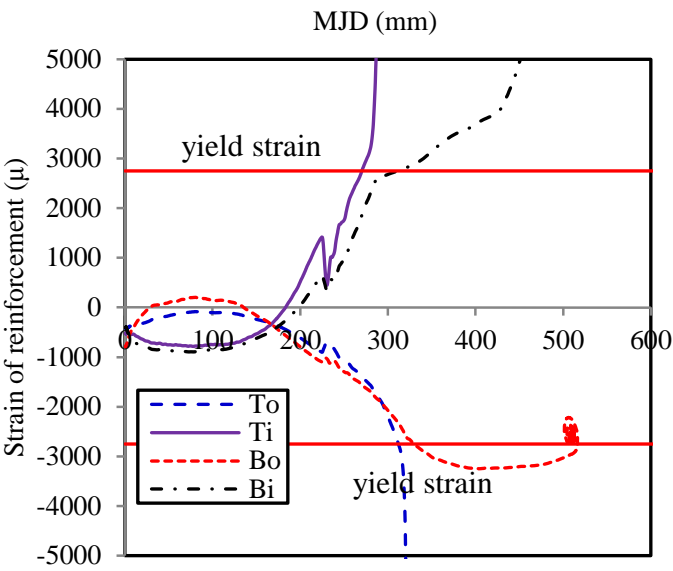

d) Dynamic (FD4-P/30.5)

Fig. 6.19 Comparisons of column reinforcement strains between static and dynamic tests

\subsubsection{Comparisons of dynamic loading capacity between actual tests and simplified energy-based method}

Fig. 6.20 presents the pseudo-static curves generated from the static tests FR and PR using Izzuddin's method together with the MJD-applied load results from all the dynamic tests. For full-restraint case (Fig. 6.20(a)), most dynamic tests showed smaller deformations compared to the pseudo-static curve denoting that such an energy-based framework is conservative as it neglects the effects of damping and strain rate on the material strength. However, in the dynamic test of FD2, the applied load of $34 \mathrm{kN}$ exceeded structural capacity of the specimen leading to a complete collapse while Izzuddin's method also considered this value as the failure load. For the partial-restraint test, due to early termination of PR, its pseudo-static prediction stopped at the MJD of $396 \mathrm{~mm}$ (Fig. 6.20(b)). The simplified method shows smaller structural capacity compared to the actual dynamic tests of specimen FD3 at load levels of 20 and $25 \mathrm{kN}$. On one hand, FD3 had higher concrete strength compared to that of PR which increased the CAA capacity. On the other hand, dynamic strain rate effect could increase the plastic moment capacities at the joints by up to $17.3 \%$ (FD3-P/20) and 15.9\% (FD3-P/25) (see Table 6.2), and could also increase the elastic modulus of concrete. For FD4-P/30.5 which had a higher applied load than the maximum dynamic capacity predicted by Izzuddin's method and yet still survived from collapse, the partially-restrained column would have 
failed if its applied axial force had remained constant during the free-fall event. Therefore, FD4-P/30.5 could be considered as failed. In short, although Izzuddin's method provides conservative predictions of flexure/CAA response based on static tests, its prediction of ultimate dynamic capacity due to CA should not be exceeded during design procedure.

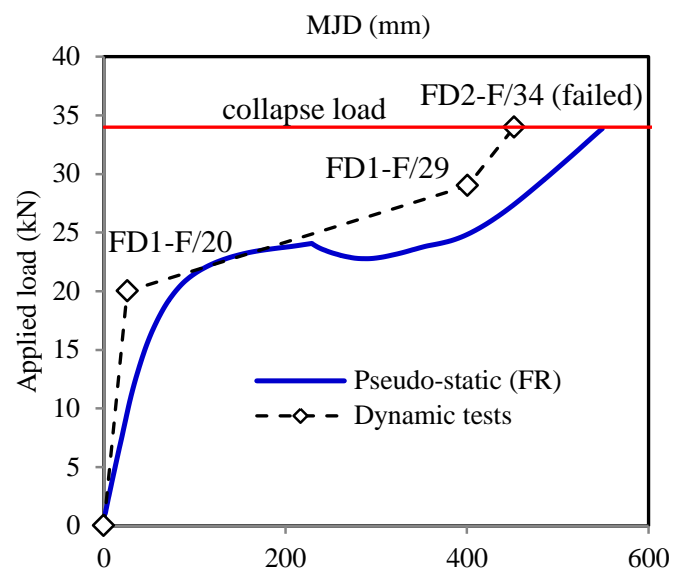

a) Full-restraint cases

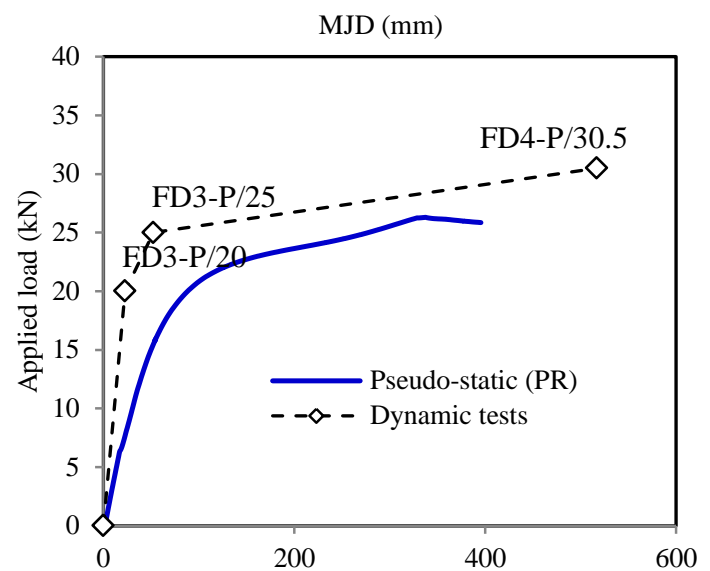

b) Partial-restraint cases

Fig. 6.20 Comparisons between dynamic tests and pseudo-static responses from Izzuddin's method

\subsection{Numerical studies on $2 \mathrm{D}$ frames under $\mathrm{CL}$ condition}

Based on the numerical studies presented in Chapter 3, sophisticated physics-based models are developed for the 2D beam-column frames that were investigated under static tests from Lim et al. (2015) as well as those under CL dynamic tests (Series I). Compared to the half-type models for sub-assemblages in Chapter 3, full-type models of beam-column structures plus horizontal and pinned restraints are employed in this chapter to take into account actual boundary conditions from the tests (Fig. 6.21). Stiffness values of the horizontal restraints are determined from actual test results (Table 6.3 and Table 6.4). Concrete is modelled using 8-node solid elements with two mesh sizes of $10 \mathrm{~mm}$ (joint regions) and $20 \mathrm{~mm}$ (other regions). Reinforcement is modelled using 2-node Hugh-Liu elements with a mesh size of $20 \mathrm{~mm}$. Compared to the simulations in Chapter 3, similar material models are used for concrete and reinforcement. Element erosion for concrete material is also employed with the failure criterion similar to that in Chapter 3. The models are 
validated by both the quasi-static tests from Lim et al. (2015) and the free-fall dynamic tests from Series I. Thereafter, further numerical investigations are conducted to study structural capacities of the 2D frames under sudden column loss scenarios.

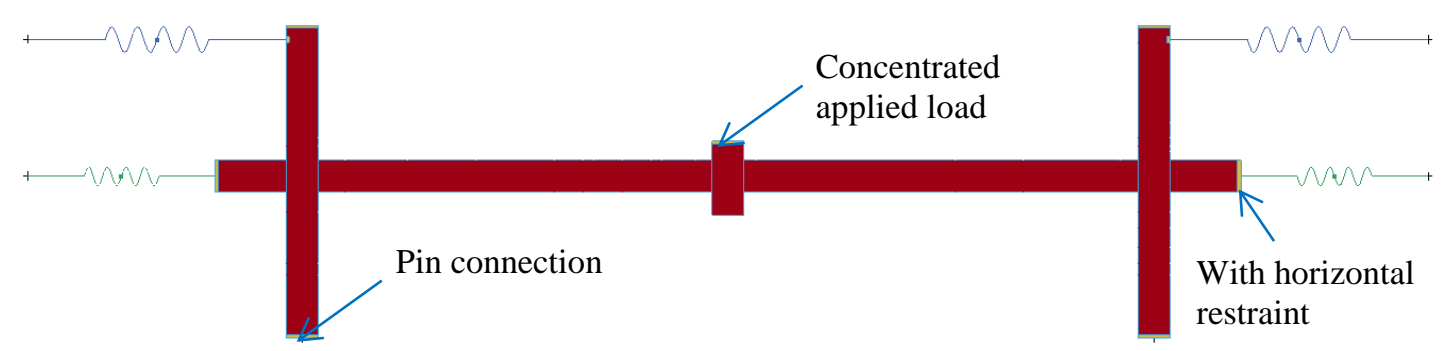

a) Full-restraint setup

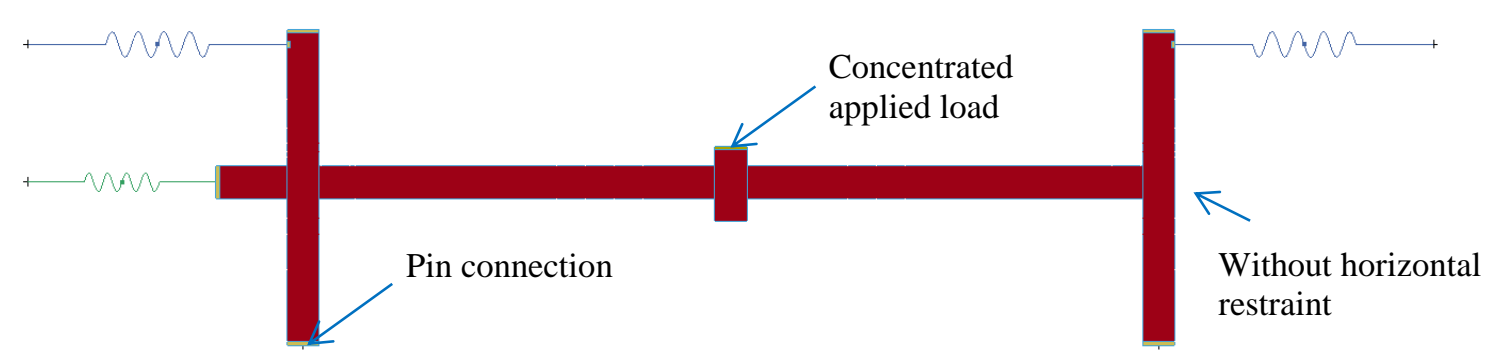

b) Partial-restraint setup

Fig. 6.21 FEM models for 2D frame specimens

\subsubsection{Validations and investigations of static responses using 3D FEM models}

From Fig. 6.22, the models for FR and PR specimens capture well the behaviour of the static tests in terms of both vertical applied load and horizontal reaction versus MJD. Regarding failure mode, the FEM models agree reasonably well with the tests for concrete damage and spalling, rebar fracturing, etc. (Fig. 6.23(a) and (b)). For the partial-restraint model of PR specimen, after the MJD of $396 \mathrm{~mm}$ at which the static test was stopped, the simulation is continued until the structure actually collapses, showing no significant enhancement of structural capacity (Fig. 6.22(b)). When the MJD approaches $600 \mathrm{~mm}$, abrupt failure from the partially-restrained side column occurs, denoting a complete collapse of the beam-column structure (Fig. 
6.23(c)). That is to say, if the MJD had been kept moving downwards and the axial compression force had been kept constant, the side-column failure due to buckling would have dominated the collapse of the partial-restraint specimen PR rather than the fracture of beam top rebars which occurred in the FR test.

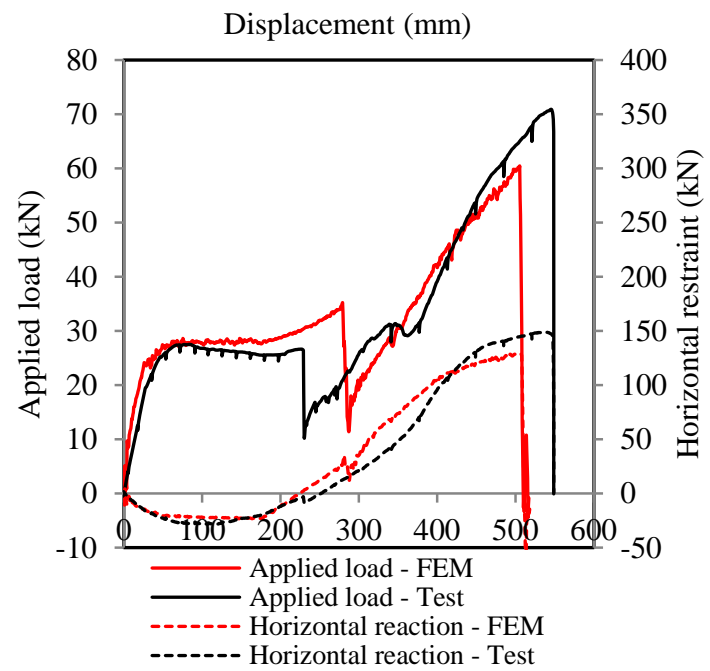

a) FR

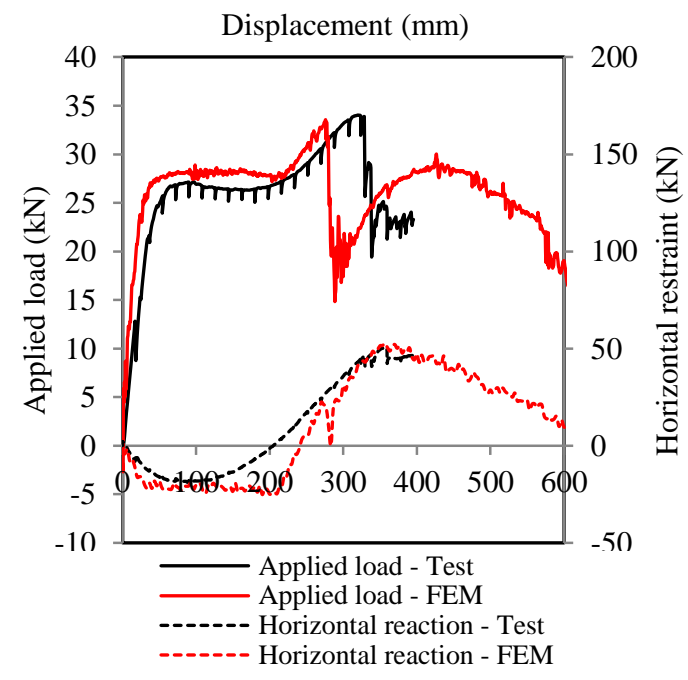

b) PR

Fig. 6.22 Validation results of FEM models with quasi-static tests
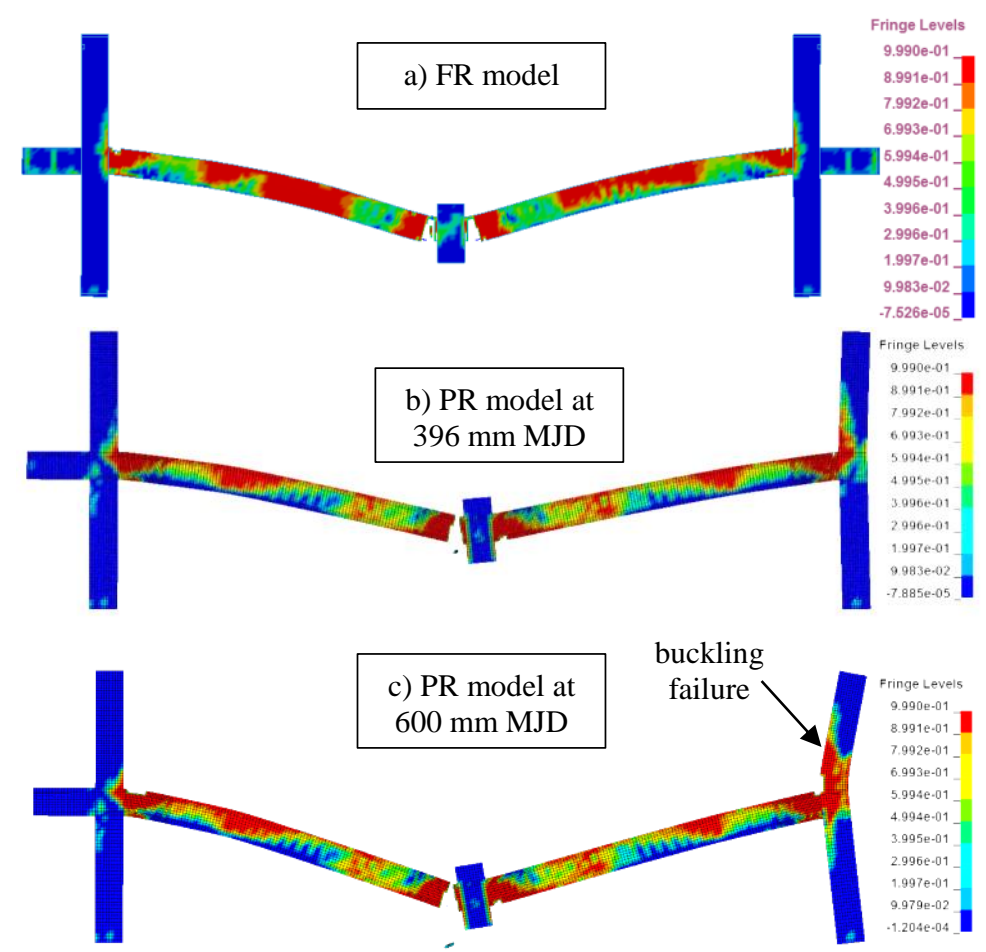

Fig. 6.23 Failure modes in quasi-static models 
To further study the effect of column axial compression on general behaviour of the beam-column frames, a parametric study is conducted involving several values of column force ranging from 200 to $400 \mathrm{kN}$ (equal to 24 to $48 \%$ of the stocky-column capacity). Both the full- and the partial-restraint frames are considered. For the partial-restraint frame, numerical predictions (Fig. 6.24(a)) show that the compression force has limited influence on structural response before the fracture of bottom rebars near the middle joint. After this point, as the compression force increases, the structural response reduces in terms of both load-carrying and displacement capacities. For the full-restraint frame (Fig. 6.24(b)), column axial compression has almost no influence on the structural response, before and after the bottom rebars fracture.

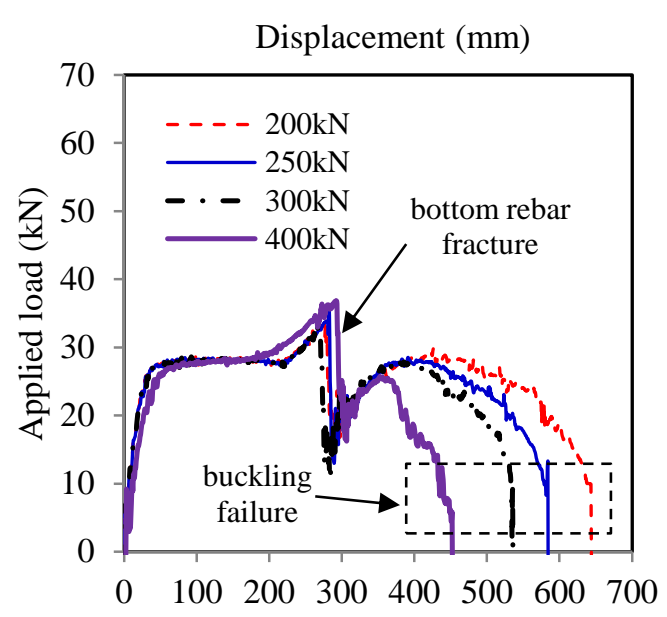

a) Partial-restraint cases

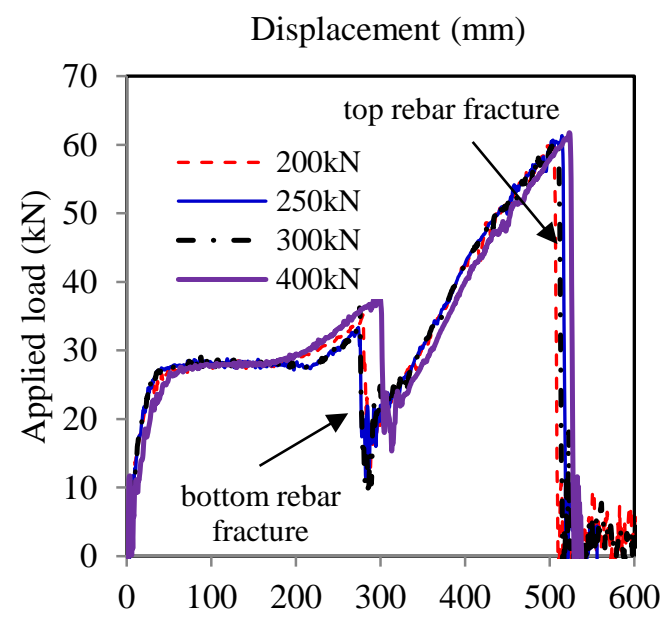

b) Full-restraint cases

Fig. 6.24 Effects of column compression force

\subsubsection{Validations and investigations of dynamic responses using 3D FEM models}

The FR model in section 6.4.1 is employed to simulate the responses of dynamic tests FD1-F/20, FD1-F/29, and FD2-F/34, while the PR model is used to predict the behaviour of FD4-P/30.5. Imposed loads in the dynamic tests are simulated by additional mass attached to the middle joint of the model. All information on the dynamic tests such as release time, initial non-zero reaction force (Table 6.1) is taken into account in the simulation. Strain rate effects are also considered similar to Section 3.2.6. For all the dynamic analyses, global damping ratio is set to $5 \%$ to 
be consistent with that used in Section 3.2.8. For FD1-F/20, the FEM model moderately overestimates the deformation compared to the actual test (Fig. 6.25(a)). Nonetheless, both the numerical and the test results agree well with each other in terms of reaction forces (Fig. 6.25(b)). For FD1-F/29, the FEM model gives good results compared to the actual test for both the displacement time-history and the reaction forces (Fig. 6.26). Numerical predictions for FD2-F/34 and FD4-P/30.5 show complete collapses (Fig. 6.27). While the failure from FD2-F/34 model is due to top and bottom rebar fracture, similar to the actual test, the failure of FD4-P/30.5 model is from the abrupt collapse of the partially-restrained side column after the fracture of bottom rebars (Fig. 6.28). This numerical observation confirms the assumption made in Section 6.3.4 considering the FD4-P/30.5 test to be failed after it had lost most of the column axial force.

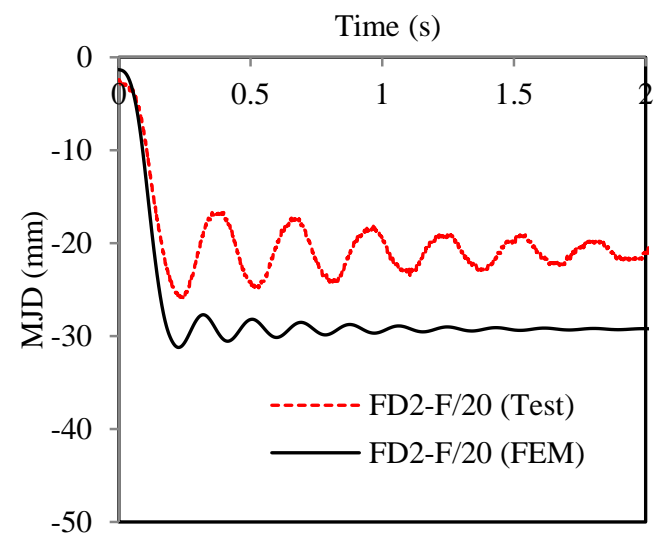

a) Displacement

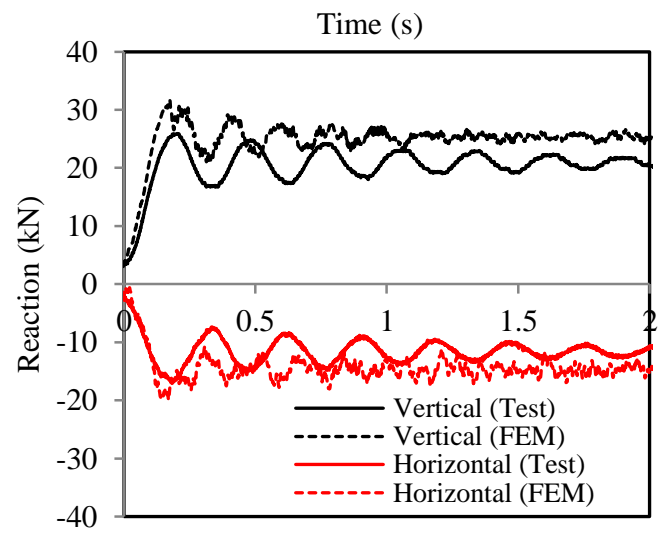

b) Reaction forces

Fig. 6.25 Validation results for FD1-F/20

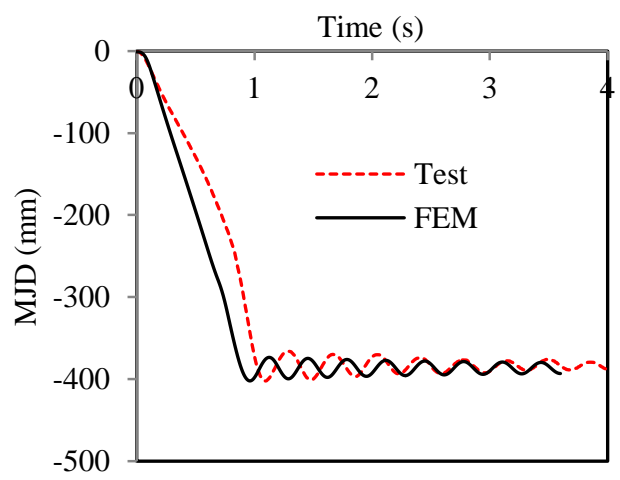

a) Displacement

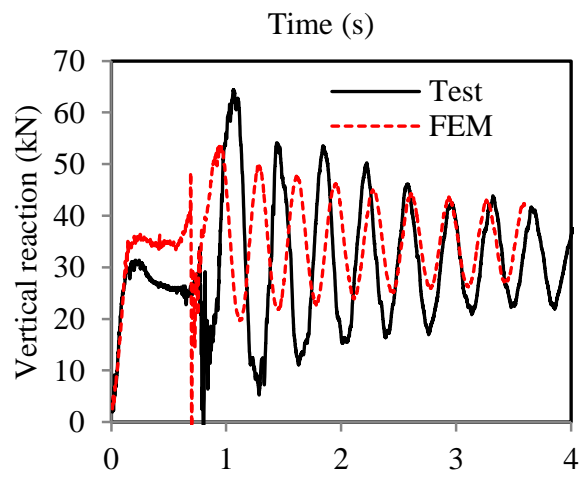

b) Vertical reaction 


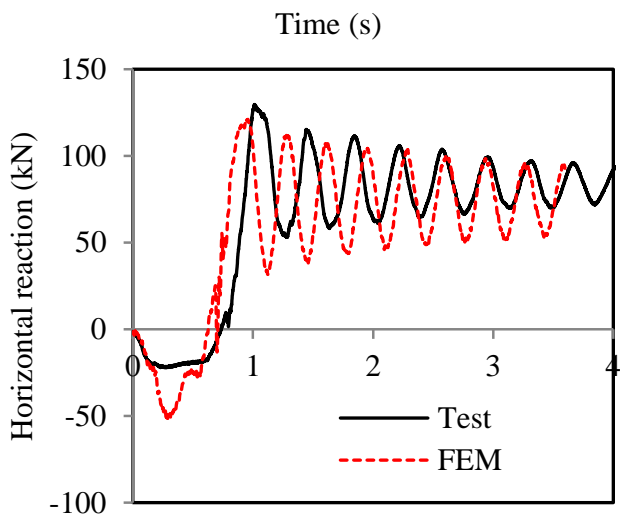

c) Horizontal reaction

Fig. 6.26 Validation results for FD1-F/29

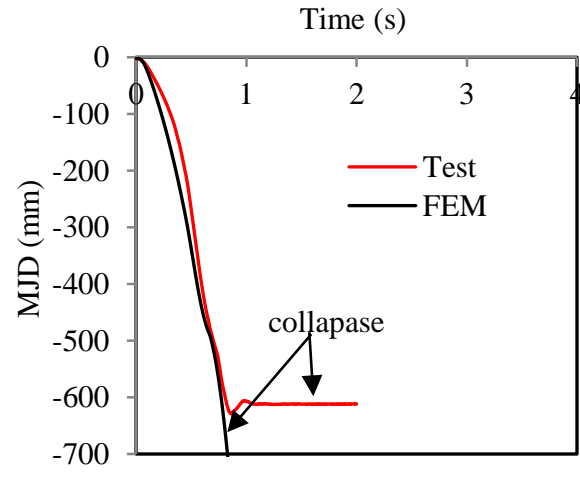

a) FD2-F/34

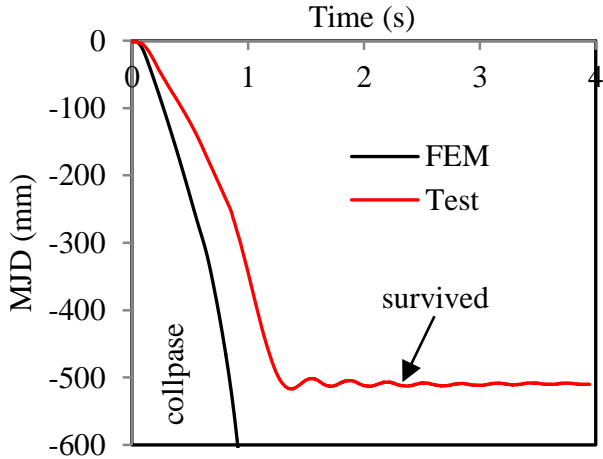

b) FD4-P/30.5

Fig. 6.27 Validation results for FD2-F/34 and FD4/P-30.5

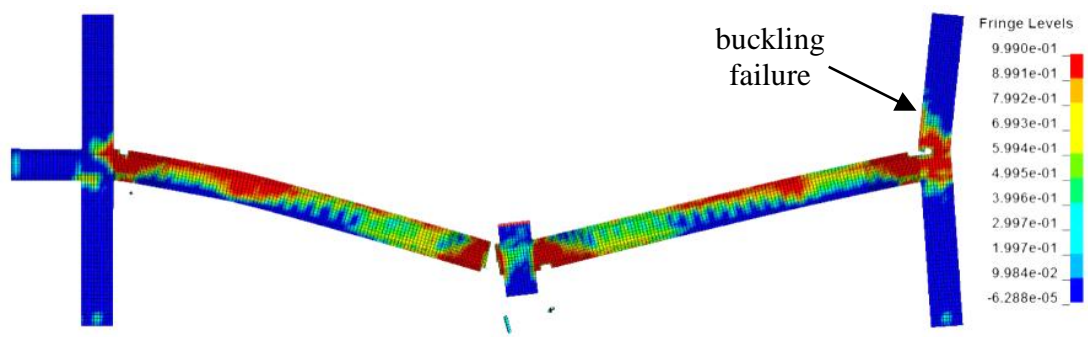

Fig. 6.28 Failure modes in FD4-P/30.5 model

After being validated by actual dynamic tests, the FEM models are used to investigate the influence of some parameters on the dynamic response of the frame specimens. Two FEM models representing the tests of FD1-F/20 and FD1-F/29 are analysed with different release times and concrete grades and the results are shown in Fig. 6.29 and Fig. 6.30. It is found that, when the structure is under CAA stage 
(FD1-F/20), the release time and the concrete grade can notably affect the maximum displacement. However, when the structure goes into CA stage (FD1F/29), these two parameters have insignificant influence on the maximum displacement of the frame.

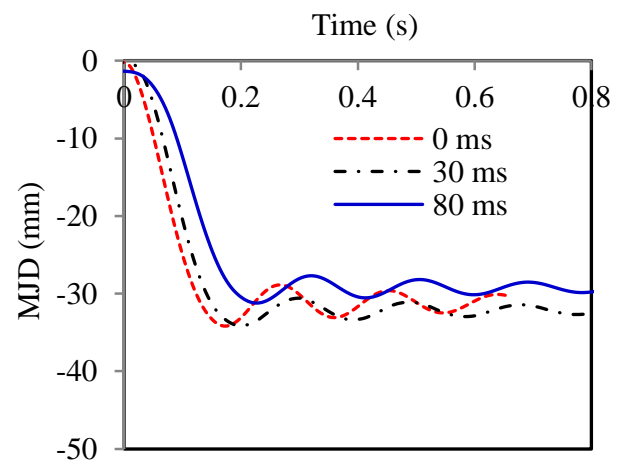

a) Release time

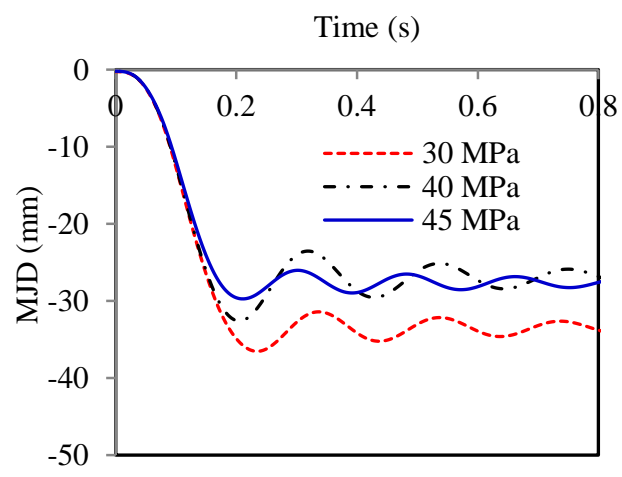

b) Concrete grade

Fig. 6.29 Parametric studies for FD1-F/20 model

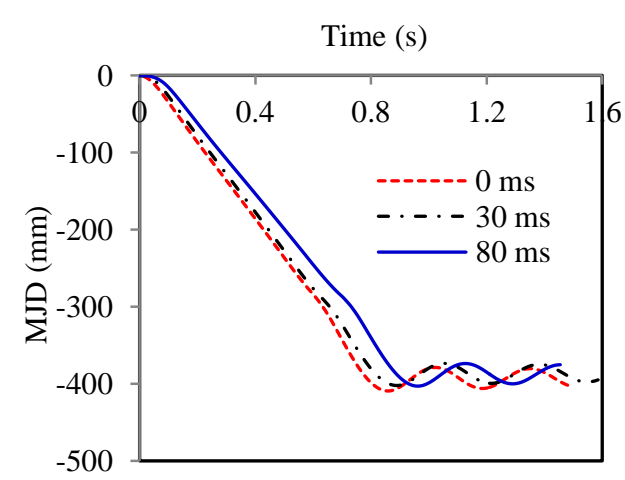

a) Release time

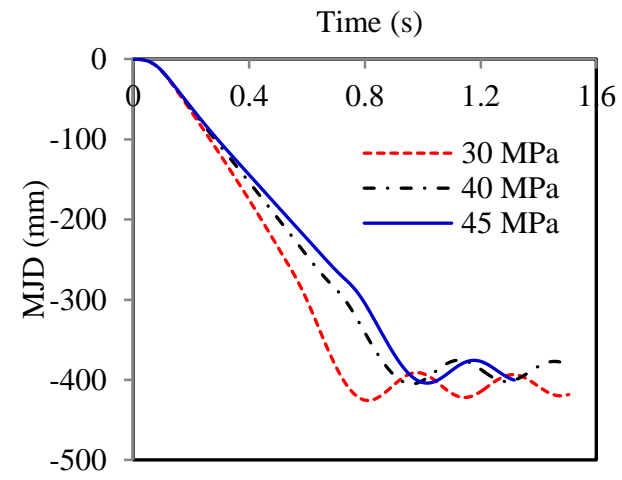

b) Concrete grade

Fig. 6.30 Parametric studies for FD1-F/29 model

\subsubsection{Simplified analysis using equivalent SDOF system}

The use of sophisticated numerical models applying solid elements presented in sections 6.4 .1 and 6.4 .2 is time-consuming especially for analysing dynamic behaviour. To simply predict the structural dynamic response, particularly the maximum displacement of the middle joint, an SDOF system is employed to simulate the dynamic behaviour of the tests in Series I (Fig. 6.31(a)). The governing equation of the system is: 
$m \ddot{u}+c \dot{u}+k u=p(t)=G(t)+F(t)$

where $m, c$, and $k$ are the equivalent mass, viscous damping, and effective stiffness of the system, respectively. $G(t)$ represents gravitational force whereas $F(t)$ is the function of the supporting force at the middle joint. The terms $u, \dot{u}$, and $\ddot{u}$ are displacement, velocity, and acceleration of the mass, respectively. The total equivalent mass $m$ is equal to the sum of the mass from the steel plates hung at the middle joint $m_{s}$ and the mass from the RC double-span beam $m_{b}$ as shown in Fig. 6.31(b). $m_{b}$ can be computed following Eq. (6.5) based on the density function $m(x)$ of continuous mass along the beam (assumed to be uniform) and the shape function $\psi(x)$ of the deformed structure. From dynamic test observations, a linear function $u \frac{x}{L_{n}}$ can be applied for $\psi(x)$. Eq. (6.5) is based on equation (5.1b) from Biggs (1964). However, because the weight from the RC double-span beam was very small compared to the weight of the steel-plate system, the contribution of $m_{b}$ in $m$ is negligible. $c$ is based on damping ratio $\zeta$ and natural frequency $\omega_{n}$, as shown in Eq. (6.6). $\zeta$ is set to $5 \%$ of critical damping.

$m_{b}=\int m(x) \psi^{2}(x) d x \ll m_{s}$

$c=2 \zeta \omega_{n}$

Natural frequency $\omega_{n}$ is derived as $\omega_{n}=\sqrt{k / m}$; while $m$ is independent from time $t$ and displacement $u, k$ is independent from $t$ but dependent on $u$.

The equivalent stiffness $k$ of the SDOF model is obtained from the relationship between the vertical applied load at the middle joint and the corresponding MJD. Assuming that the behaviour and failure mode between static and free-fall dynamic conditions are similar, $k$ is computed based on structural response of the $\mathrm{RC}$ frame during static environment. There are several ways to define this $k$ stiffness, which are based on (1) quasi-static tests, if available (Fig. 2.9), or (2) rigorous analysis employing physics-based models (Fig. 6.22), or (3) semi-analytical approach for sub-assemblages proposed in Section 4.3 (Fig. 4.1(b)). To highlight the simplicity 
of the SDOF model, the $k$ stiffness is determined based on predictions from the semi-analytical model for FR and PR tests (Fig. 4.11).

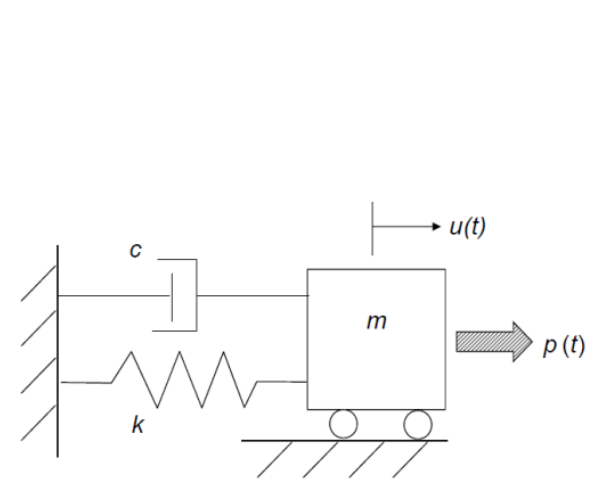

a) Equivalent system

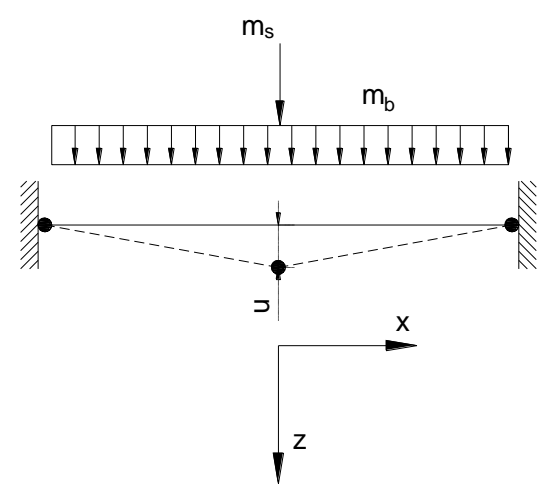

b) Contribution of mass

Fig. 6.31 The simplified model for dynamic response

The functions of $G(t)$ and $F(t)$ are presented in Fig. 6.32. At the beginning $\left(t=t_{s}<0\right)$, both $G(t)$ and $F(t)$ are slowly increased from zero. At $t=0$, both functions gain their maximum magnitude. $G(0)$ is equal to $m g$, i.e. the total weight of the system, whereas $F(0)$ reaches $F_{0}$ which is not necessarily equal to $G(0)$. The subtraction of $G(0)-F(0)$ represents the initial non-zero reaction created in the specimen due to imperfect connection between the quick-release device and the middle joint (Table 6.1). $t_{s}$ is set to be much greater than $T_{d}$ (natural period of the system) to ensure that $G(t)$ and $F(t)$ are statically applied until $t=0$. Thereafter, $F(t)$ is quickly reduced and set to zero at $t=t_{r}$ representing the loss of the supporting mechanism in the actual tests, while $G(t)$ is kept constant. By doing so, the excitation functions of the SDOF model can represent actual conditions from the free-fall tests of Series I, i.e. the removal time and the initial load applied to the specimen before the release (Table 6.1). After all the parameters have been defined, Eq. (6.4) is numerically solved to provide time-history of the displacement $u$ which represents response of the MJD from the actual dynamic tests. 


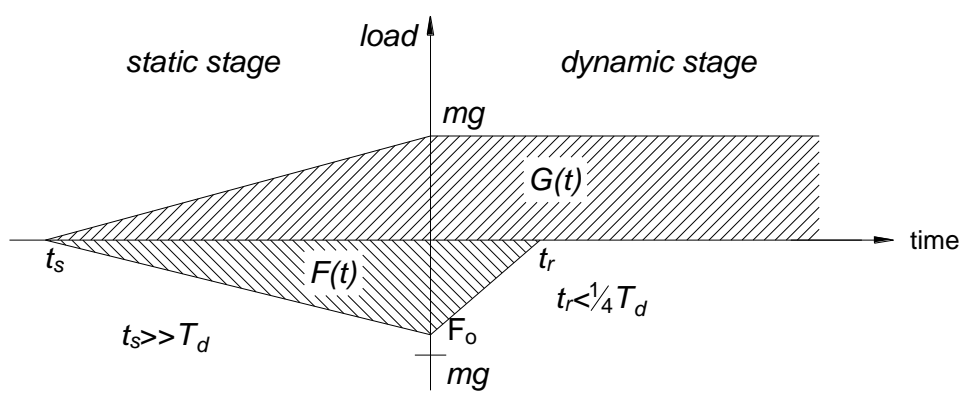

Fig. 6.32 Functions of $G(t)$ and $F(t)$

Compared to the actual test results as well as the detailed FEM models (Fig. 6.33), the simplified SDOF method provides relatively conservative predictions for the maximum displacement of the double-span beam structure. It is because the equivalent stiffness $k$ of the model does not account for material enhancement due to strain rate effects. Nonetheless, compared to the sophisticated 3D model, the equivalent SDOF model is very cost-and-time efficient. Therefore, it is convenient to use such a method to further study the maximum displacement of a system under different free-fall dynamic conditions such as applied load, release time or damping ratio provided that its static response is already well defined.

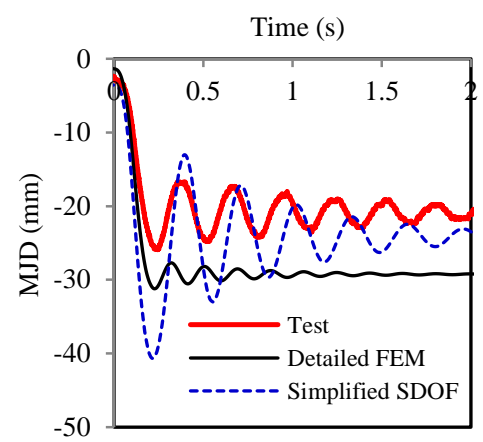

a) FD1-F/20

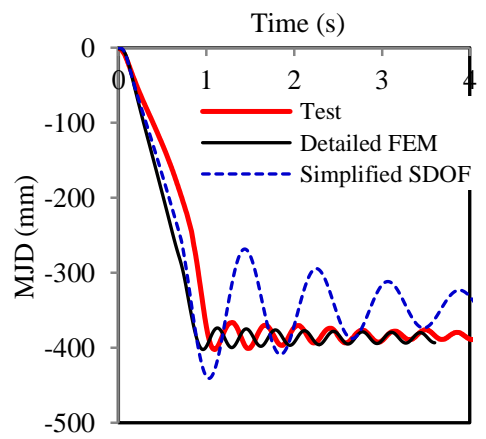

b) FD1-F/29

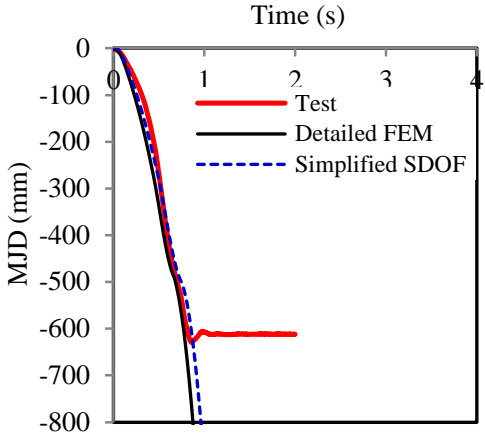

c) $\mathrm{FD} 2-\mathrm{F} / 34$

Fig. 6.33 Prediction from equivalent SDOF models

\subsection{Conclusions on experimental and numerical results of Series I}

Although structural behaviour of 2D beam-column systems has been extensively studied in previous quasi-static experiments, the response under actual dynamic environments was not yet confirmed. To address this problem, free-fall dynamic tests of Series I were conducted based on two previous static tests under full- and 
partial-restraint conditions from Lim et al. (2015). The same CL method was applied at the middle joint for both the static and the dynamic test series. Some conclusions are listed as follows:

(1) The test setup of Series I could simulate the dynamic effects of a column to be suddenly removed since the requirement of release time was satisfied in most of the tests. DIFs for material strength due to strain rate effects were up to 1.189 for rebar yield strength and up to 1.110 and 1.190 for concrete in compression and in tension, respectively. Deformations and damages at a smaller load of $20 \mathrm{kN}$ were negligible and results from the second test of specimens FD1 and FD3 with higher applied loads were useful. The test results showed that CA could still help in preventing collapse even after the fracture of bottom rebars, which has not been confirmed in any previous dynamic tests. However, if CA were utilised, then higher DLIFs associated with CA should be considered for the structure.

(2) Failure modes, deflection profile, and behaviour (at structural and crosssectional levels) of dynamic tests agreed with those observed from corresponding static tests closely. Although each dynamic test had only one applied load, derived curves based on reactions and MJDs could be obtained from the data, which followed reasonably close with the static curves. It is concluded that under the same boundary condition and loading method, the dynamic response caused by a sudden column removal will have similar failure mode with the static response regardless of the applied load level. The study confirms the correctness of nonlinear static analysis for investigating progressive collapse potential of $\mathrm{RC}$ frame structures subjected to $\mathrm{CL}$ condition.

(3) Structural capacities and stiffness within CAA of the dynamic tests were larger than those of the corresponding static tests due to higher concrete strength used, as well as strain rate effect on material strength and stiffness. Nonetheless, the vertical and horizontal reactions under CA from the dynamic tests followed 
those from the static tests showing a weaker influence of strain rate effect on rebar ultimate strength.

(4) Izzuddin's method for dynamic assessment provides conservative predictions of structural responses compared to actual tests since the method ignores damping and strain rate effects. For safety purpose, the dynamic capacity during CA stage predicted by this method should be considered as the maximum load that a structure can resist during a sudden column loss scenario.

(5) Detailed models using LS-Dyna software agree well with both the static and the free-fall dynamic tests not only for overall responses but also for other important aspects such as reaction forces, failure modes. The FEM analyses clearly show the vulnerability of partial-restraint frames against progressive collapse due to buckling failure of the side column, which was not directly observed in both the static test PR and the dynamic test FD4-P/30.5. Besides, the release time and the concrete grade are shown to have noticeable effects on maximum dynamic displacement if the structure is under CAA stage. However, such influences become insignificant when the frame goes into CA regime. Despite good predictions provided by the detailed FEM simulation, the conduct of such complicated analyses is very time-consuming, especially for dynamic regime. On the contrary, the simplified model employing equivalent SDOF system and applying the semi-analytical approach for sub-assemblages (Section 4.3) can conservatively predict the maximum displacement of the system with an absolutely fast run-time (less than one minute). This method is very efficient for studying structural dynamic capacity under different applied loads when the static response is well defined by either actual tests or numerical/simplified predictions. 


\section{Appendix 6A - Details of test results from Series I}

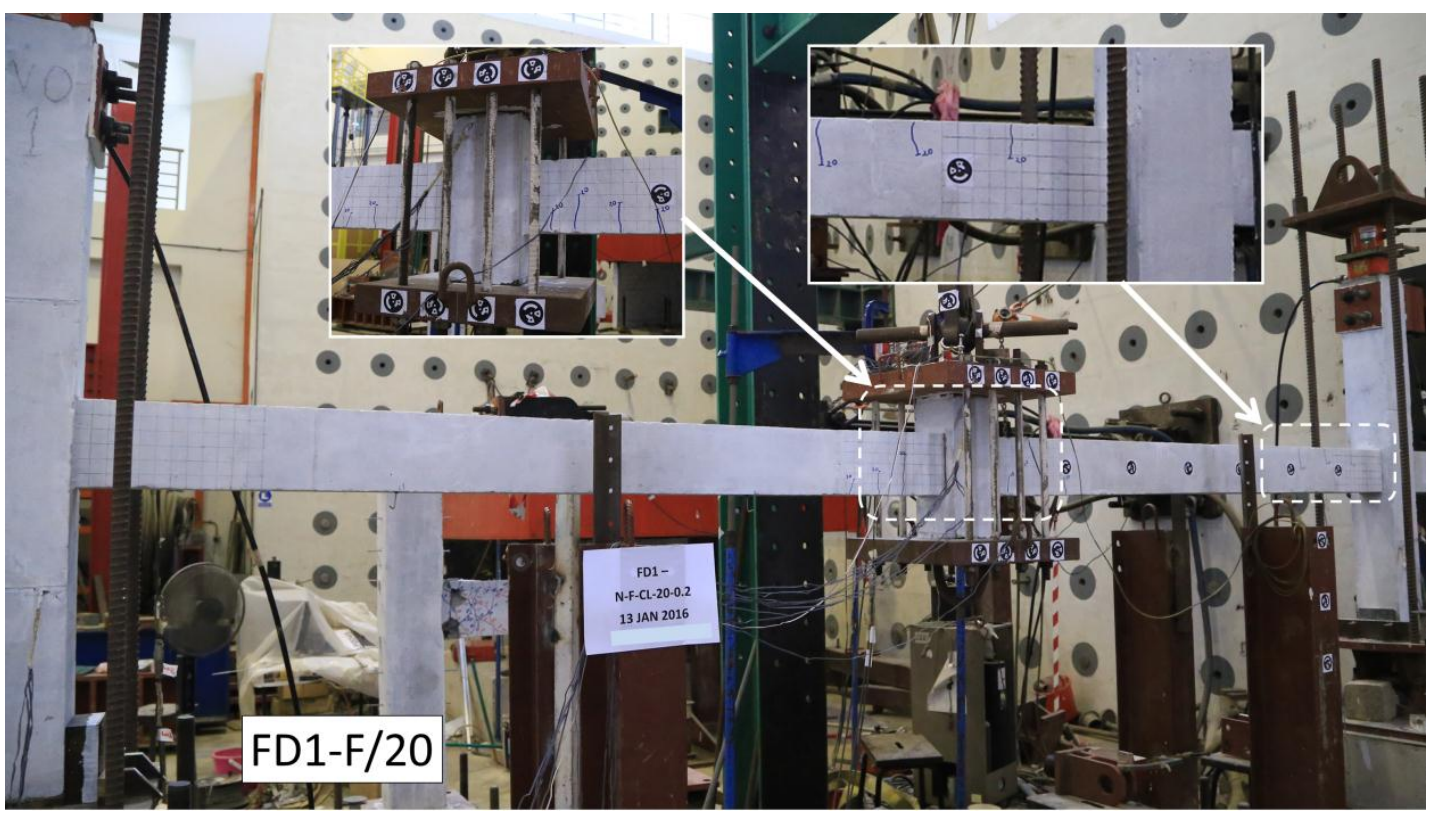

Fig. 6.1A: FD1-F/20

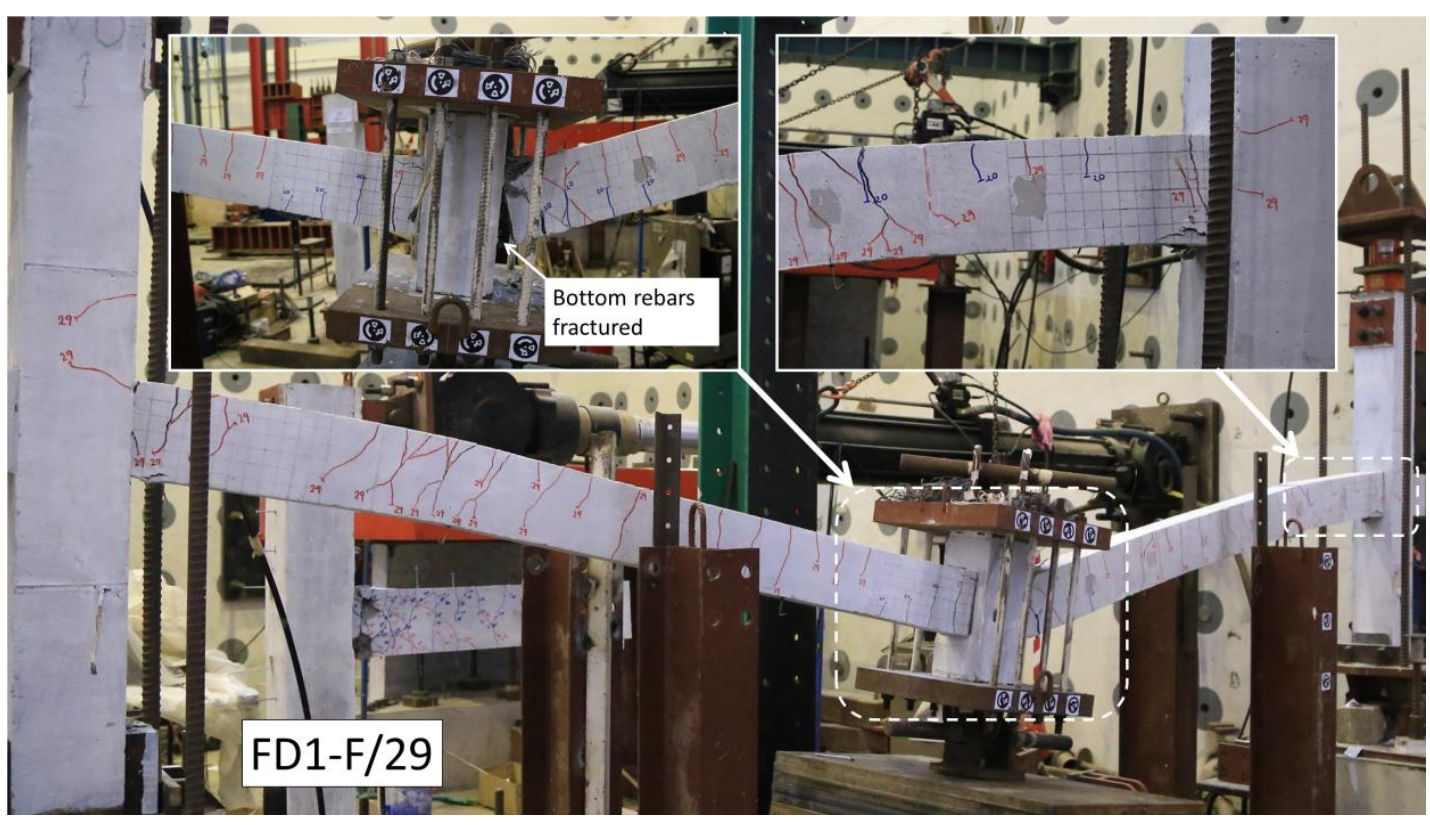

Fig. 6.2A: FD1-F/29 


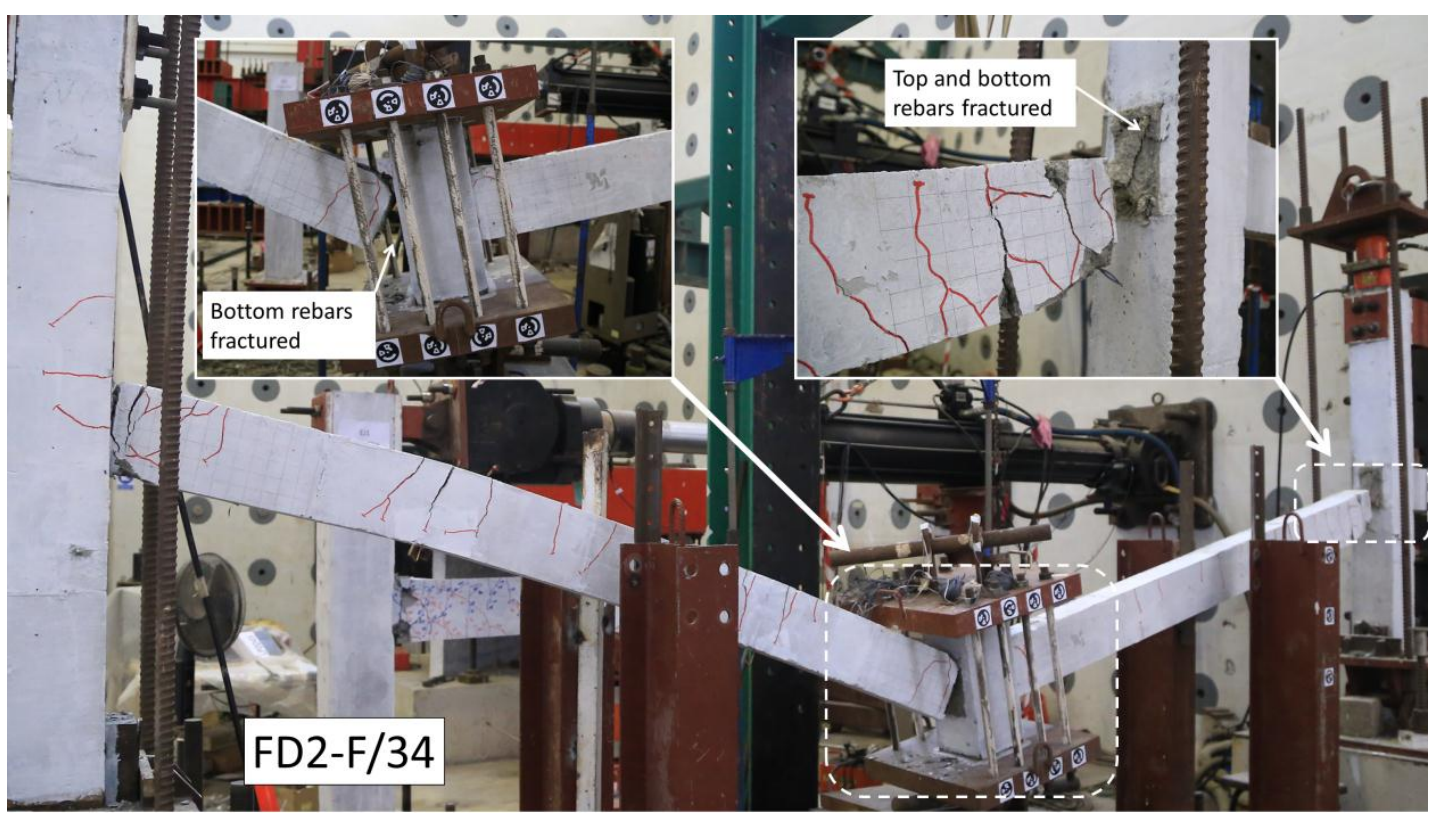

Fig. 6.3A: FD2-F/34

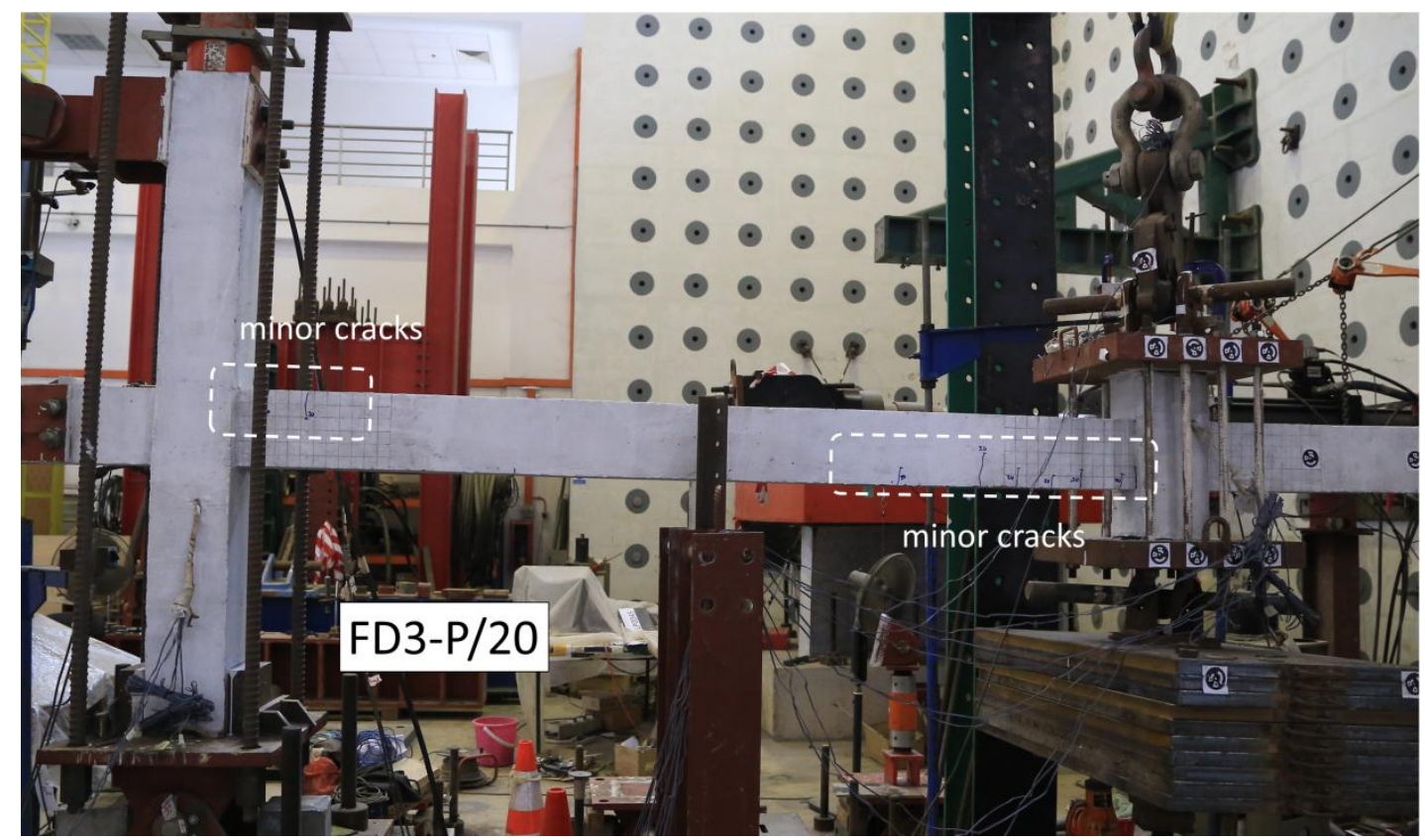

Fig. 6.4A: FD3-P/20 


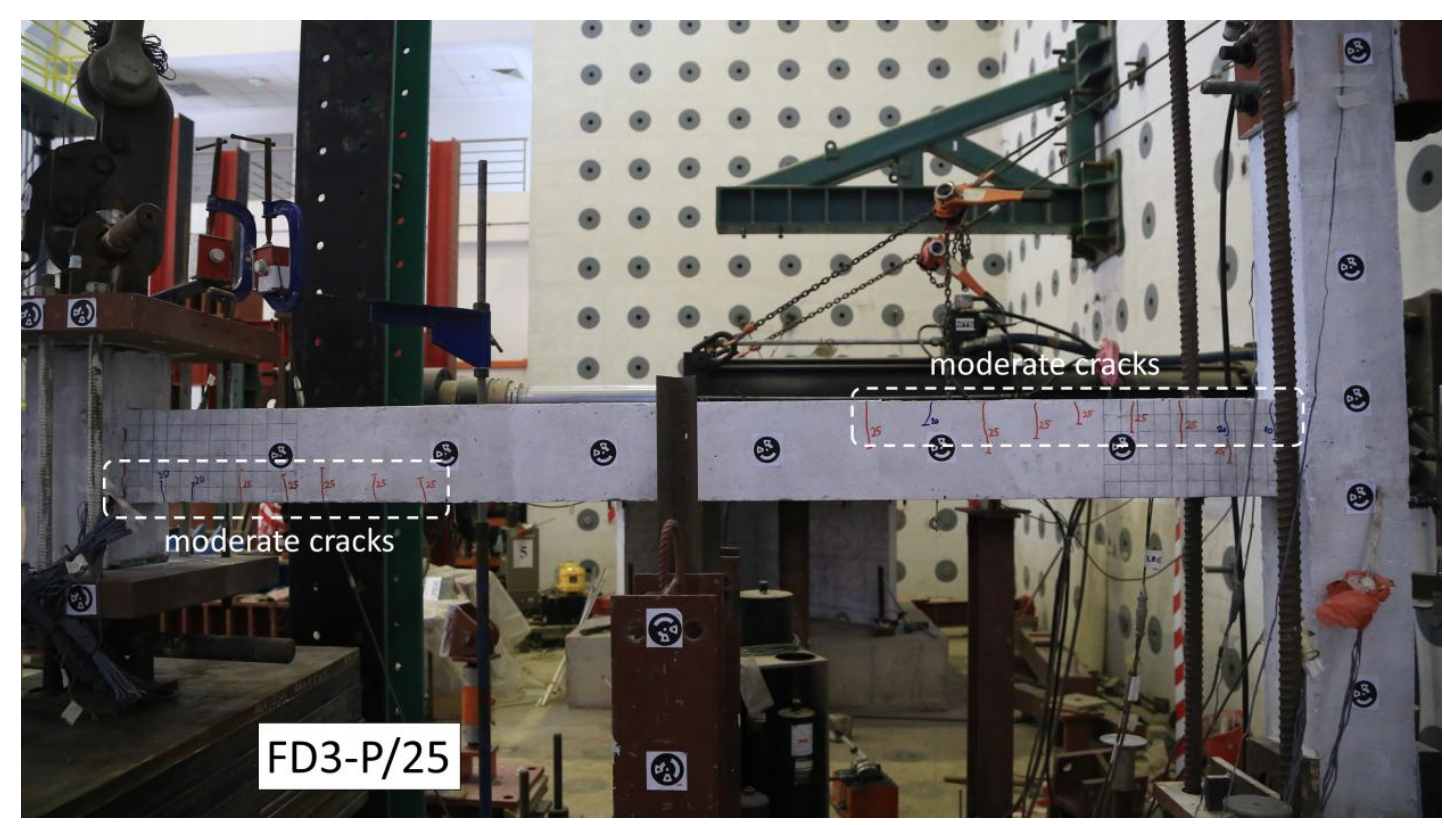

Fig. 6.5A: FD3-P/25

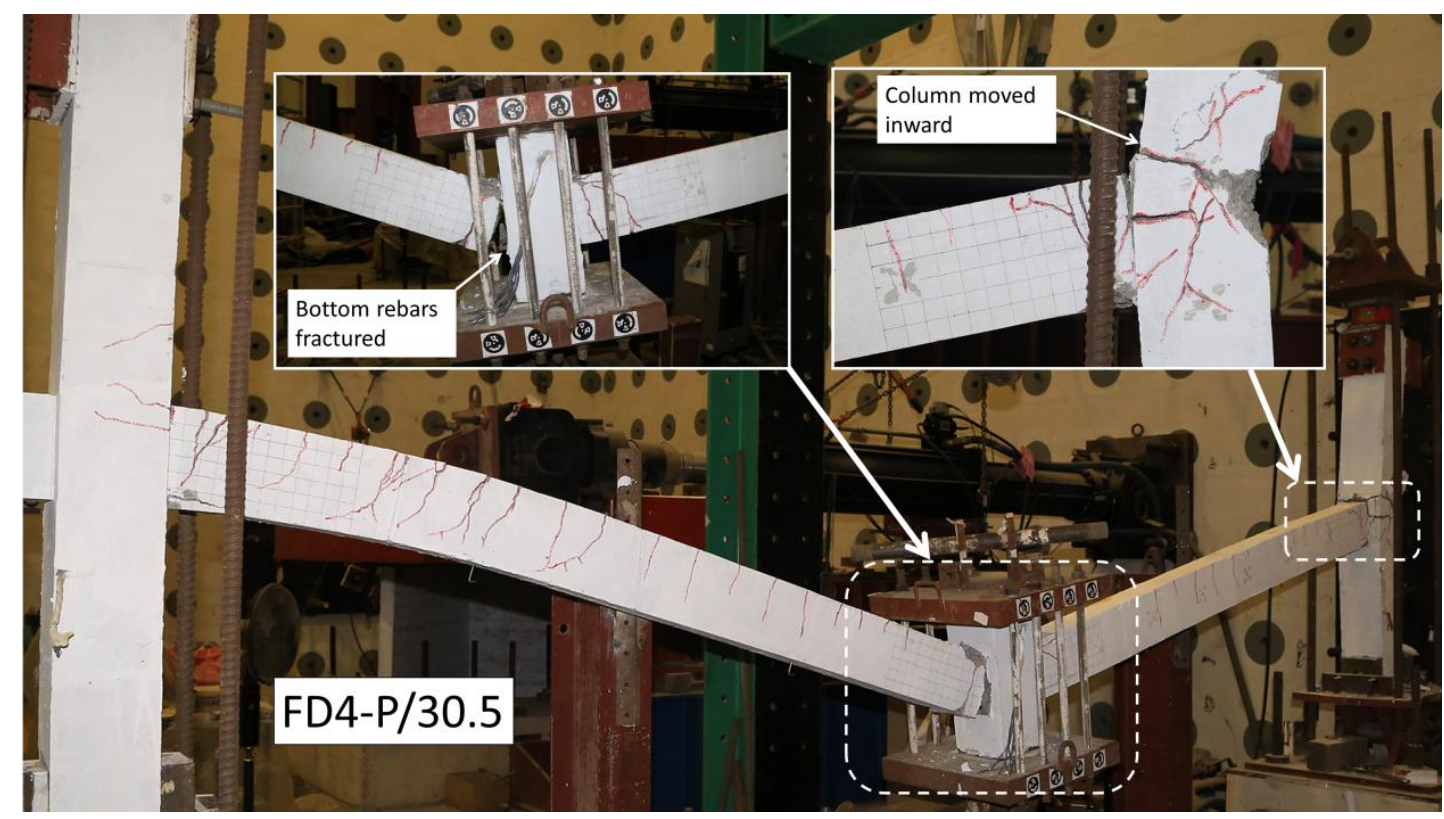

Fig. 6.6A: FD4-P/30.5 


\section{CHAPTER 7 EXPERIMENTAL RESULTS OF FRAME STRUCTURES UNDER UDL STATIC AND DYNAMIC CONDITIONS (SERIES II)}

\subsection{Introduction}

As mentioned in Chapter 2, one important technical issue when investigating structural responses of $2 \mathrm{D}$ beam-column structures under progressive collapse is the lack of experimental studies under UDL condition. For simplicity, most of the previous quasi-static tests employed CL configuration by applying a point load at the middle joint under a displacement-controlled manner. However, load-bearing capacities and failure modes between the two loading methods are not necessarily similar. Evidences for this concern can be found when comparing the quasi-static tests from Qian and $\mathrm{Li}$ (2012c) to the corresponding dynamic tests in Qian and $\mathrm{Li}$ (2012b). To investigate differences in structural responses between the two loading cases, in this chapter, experimental results from UDL static and dynamic tests of Series II are discussed (Pham and Tan 2017b). First, the quasi-static tests on RC frames under UDL are investigated and compared with the related CL static tests by Lim et al. (2015). Based on the comparison, analytical relationship between quasistatic behaviours under different loading configurations is derived. It provides a convenient method to predict the structural response under UDL condition based on that under CL condition which has been studied in Chapter 4. Thereafter, results of the UDL dynamic tests are presented to highlight dynamic effects of the sudden column removal, as well as to confirm structural response and failure modes observed in the UDL static tests. Lastly, the correctness and conservatism of Izzuddin's method for UDL condition are verified.

\subsection{Static test results}

\subsubsection{Overall behaviour}

Total applied loads and total horizontal restraints measured from the two UDL static tests of Series II are presented in Fig. 7.1 together with those from the relevant CL 
tests of FR and PR (Lim et al. 2015) for comparison purpose. Focusing on the static tests FR-U and PR-U, the general frame behaviour subjected to UDL can be summarised into three stages:

Stage I: From the beginning of loading when the structure was elastic under flexure until it gained its first peak due to CAA. In this stage, the structure was about to attain yield at a MJD of $0.25 d_{\text {beam }}$, marked by the yielding of tensile rebars at both Sections 1 and 4 (Fig. 5.19(b)). When the compression force in the beam became larger and significantly enhanced the capacity, the structure reached the first peak at a MJD of about $0.4 d_{\text {beam }}$.

Stage II: After attaining the peak of CAA, concrete crushing started occurring at compressive zones of the end joints, causing structural capacity to gradually reduce. This stage was completed at a MJD of $d_{\text {beam }}$ when structural response started increasing again (for both FR-U and PR-U), indicating a transformation from CAA to CA. In both stages, there were little discernible differences between full- and partial-restraint specimens.

Stage III: At this stage, horizontal reaction changed from compression to tension and the structural capacity kept increasing under the mobilisation of CA. The first failure mode took place when the first top rebar from one end joint fractured, socalled " 1 st top rebar fracture", causing a sudden drop in structural capacity. This failure occurred earlier in PR-U (at $275 \mathrm{~mm}$ MJD) than in FR-U (318 mm). From this point onwards, structural responses of FR-U and PR-U differed significantly. Under increasing displacement, load-carrying capacity of FR-U increased but that of PR-U decreased until all the top rebars at the end joint from one side fractured completely, so-called "one-side top rebar fracture". The increase of MJD after this point led to no further noticeable increase of structural capacity.

All the critical points of response from the UDL static tests are listed in Table 7.1, together with the relevant values from the CL static tests. It was clearly observed that vertical applied loads of the UDL tests under both boundary conditions were 
similar to each other until rebar fracture took place, and were slightly more than twice those of the CL tests (Fig. 7.1(a)). In addition, MJDs corresponding to different stages of response (yielding point, end of Stage I, end of Stage II) were comparable between the two loading methods as shown in Fig. 7.1(a) and in Table 7.1. However, "bottom rebar fracture" at the middle joint which occurred in the CL static tests of Lim et al. (2015), as well as in all reported CL tests in the literature, did not occur in the UDL static tests. Hence, it can be considered as the key difference between the two loading scenarios. In terms of horizontal reaction, the UDL and the CL static tests had relatively similar values (Fig. 7.1(b)), and maximum compression forces during CAA between the two loading methods agreed well with each other (Table 7.1). However, the maximum MJD from FR-U was noticeably smaller than that from FR. As a result, the maximum tension force of the horizontal reaction in FR-U was $45 \%$ smaller than that in FR. Regarding partial-restraint specimens, the MJD and the horizontal reaction of PR-U at the maximum capacity was $16 \%$ and $9 \%$ smaller than those of PR, respectively. However, due to the early termination of PR, the maximum applied load and the horizontal reaction of this test were roughly $48 \%$ and $37 \%$ of the corresponding values from FR. In contrast, the difference between PR-U and FR-U was less (68\% and $60 \%$ for applied load and horizontal reaction, respectively). 


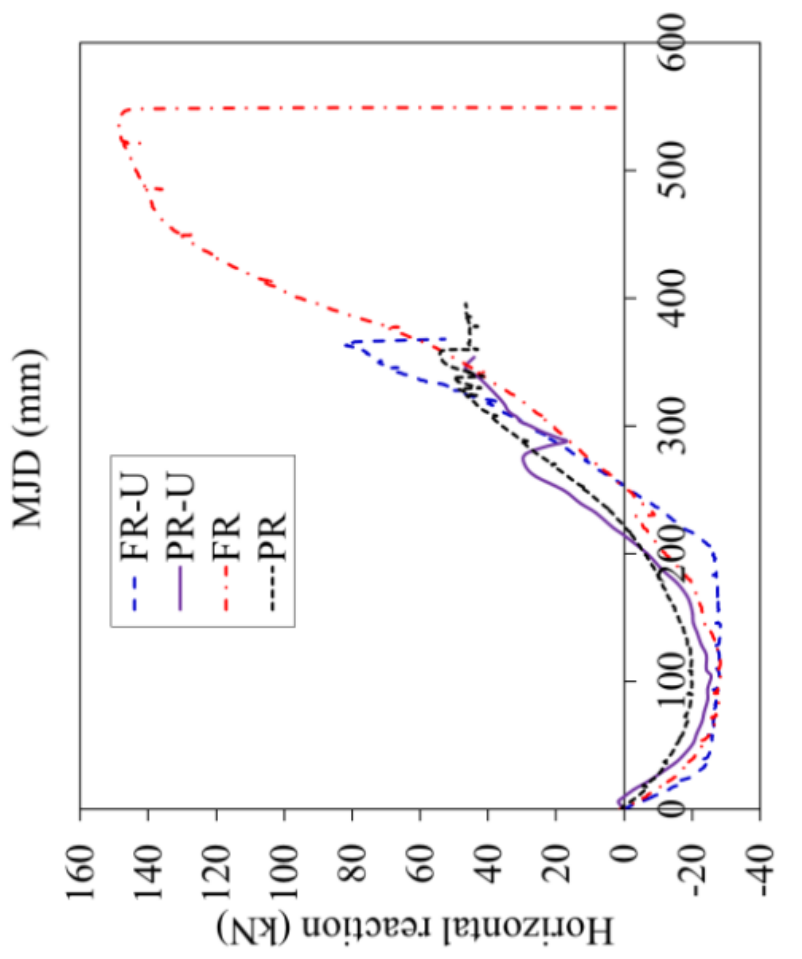

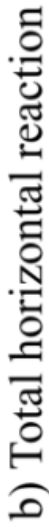

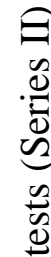

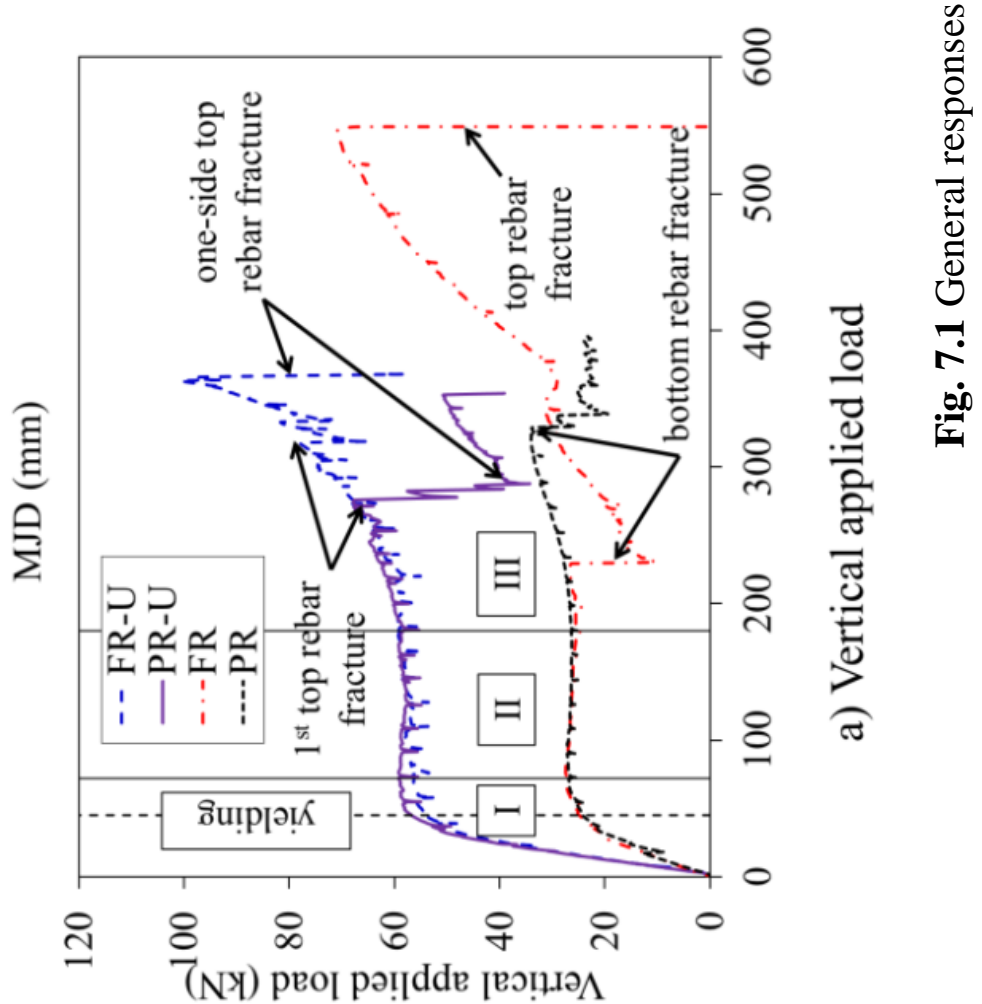




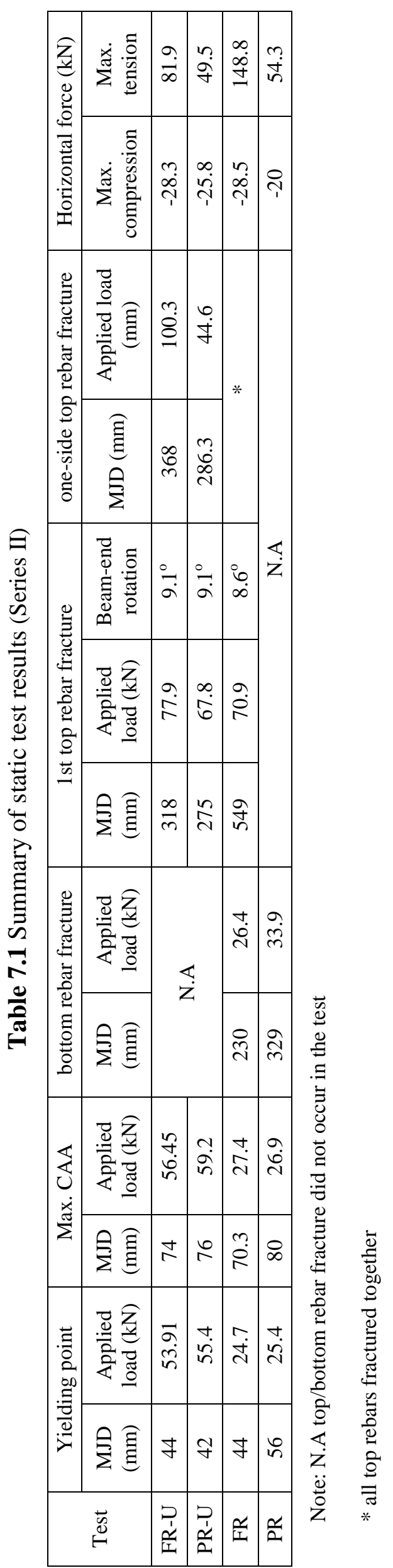




\subsubsection{Damage patterns and failure modes}

Fig. 7.2 presents cracking patterns of the UDL (FR-U) and the CL (FR) static tests at the end of Stages I and II. It was observed that top-surface flexural cracks at the end joint (Section 1) did not significantly propagate towards the curtailment points (Sections 2 and 3) in FR-U. In FR, this top-surface crack propagation was obvious. However, bottom-surface cracks of FR only occurred near the middle joint (Section 4), much fewer than those at the middle joint of FR-U. To further study the development of tension zones in the structure within flexure/CAA stage, compression and tension zones of FR-U and FR were plotted based on strain gauge readings from beam longitudinal bars (Fig. 7.3). It was assumed that at this stage, the beam sections still remained plane and strain distribution along each section was linear. During the elastic range $\left(0.1 d_{\text {beam }}\right)$, tension zones from the two loading cases were relatively similar. As the MJD increased to 0.25 and $0.4 d_{\text {beam }}$, the tension zone near the end joint in FR quickly expanded towards the middle joint. However, in FR-U, the trend was opposite with the tension zone near the middle joint quickly propagating towards the end joint. This difference in the development of tensile region accounted for the difference of crack distribution between the two loading methods during flexure/CAA stage as shown in Fig. 7.2.

At a beam section with a distance $L_{i}$ from the side-column centroid, bending moment $M_{i}$ was calculated based on recorded reaction forces and corresponding vertical deformation $\delta_{i}$ at this section, as shown in Fig. 7.4(a) and (b) for the UDL and the CL tests, respectively. As a result, $M_{i}$ at the end-joint interface as well as at the locations of displacement transducers (Lv1 to Lv5 from Fig. 5.19(a)) was calculated to obtain the bending moment distribution along the beam. Fig. 7.5(a) shows this distribution at a MJD of $0.25 d_{\text {beam }}$ (yielding state), for both FR-U and FR tests. It agrees well with the development of concrete tension zone presented in Fig. 7.3. The bending moment distribution in FR-U followed a parabolic shape, whereas that of FR was linear with a steep curvature at mid-span. Compared to FR, FR-U shows a more uniform distribution of bending moment at the middle joint region. This explains why flexural cracks were more equally spaced out in FR-U (Fig. 7.2(a)). 

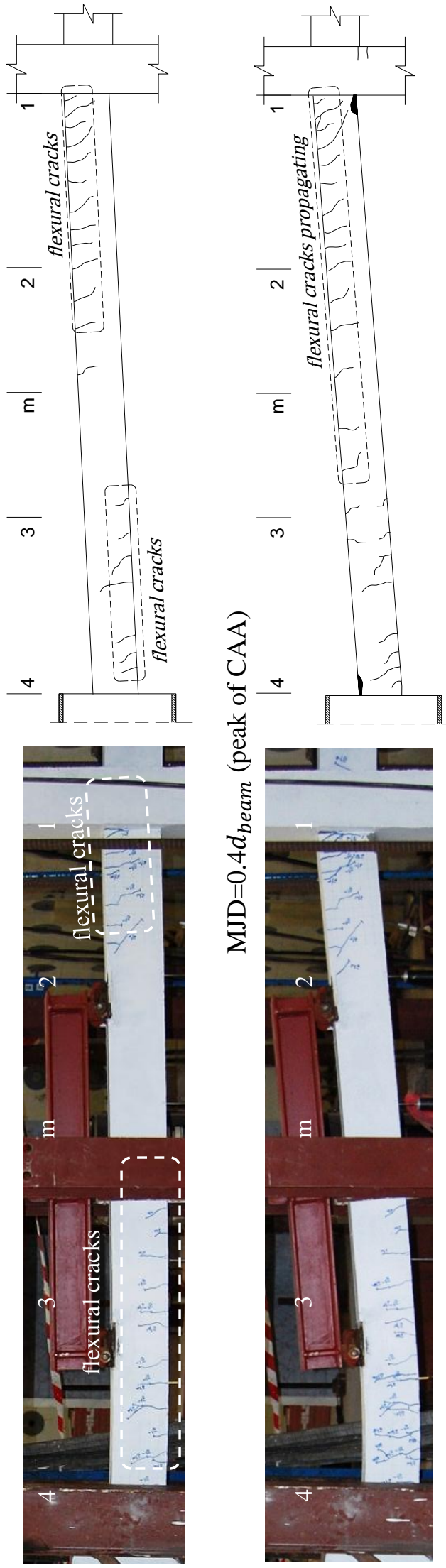

อิ
$\frac{1}{1}$
$\frac{1}{0}$

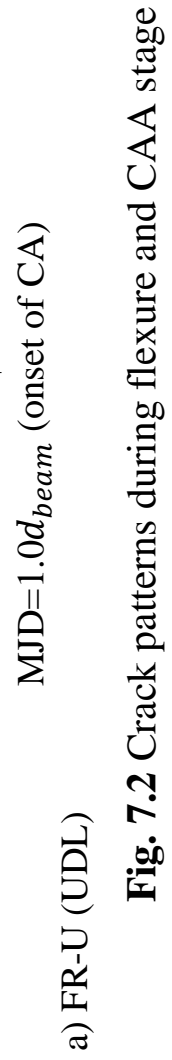



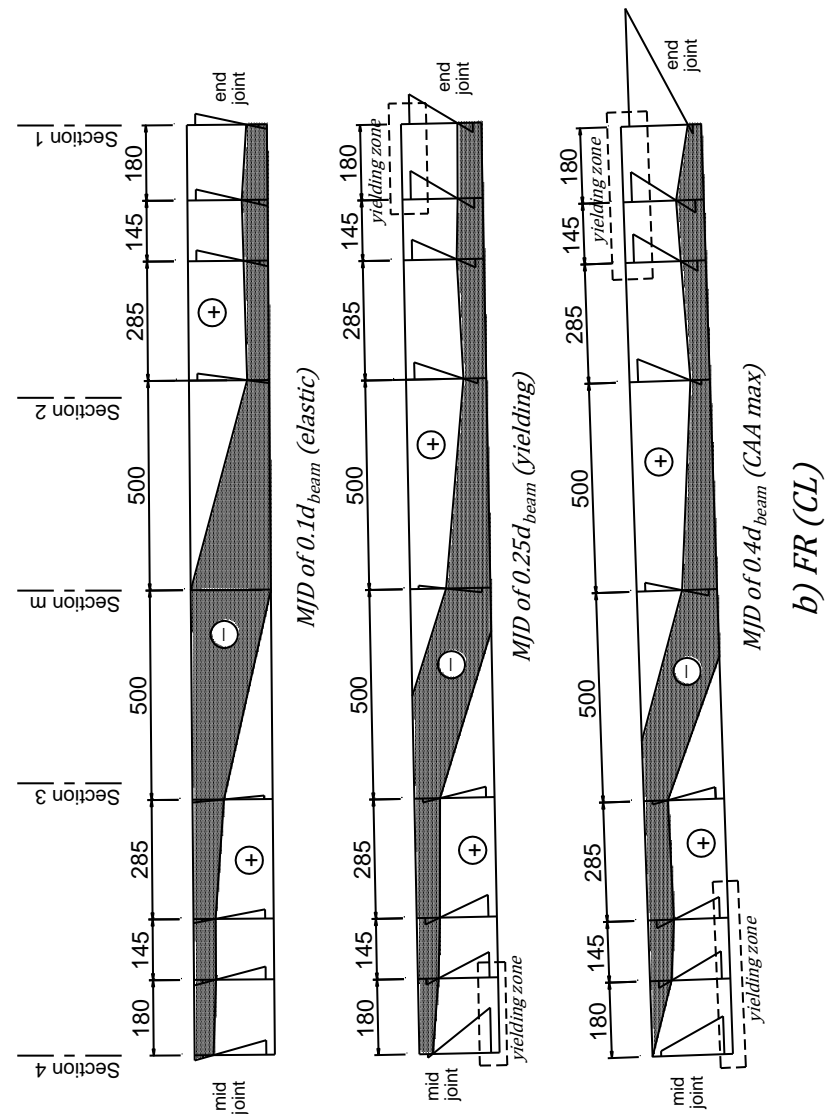

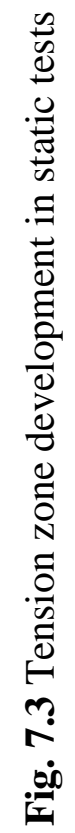
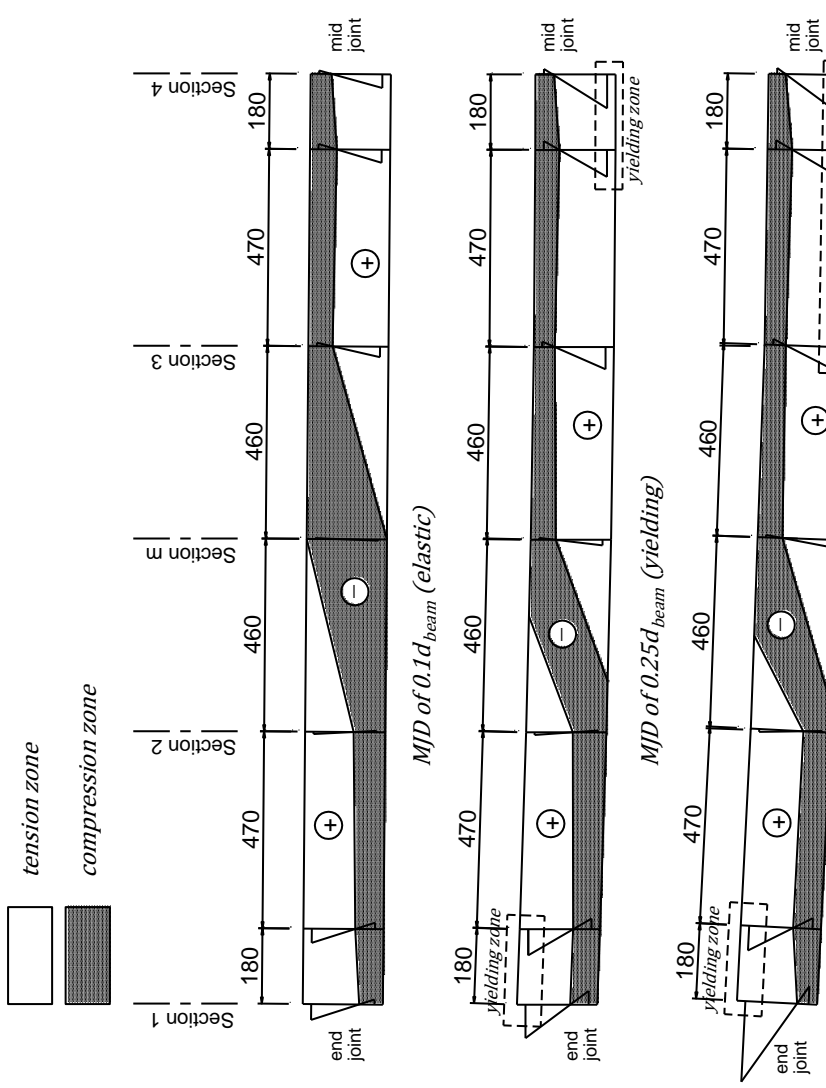

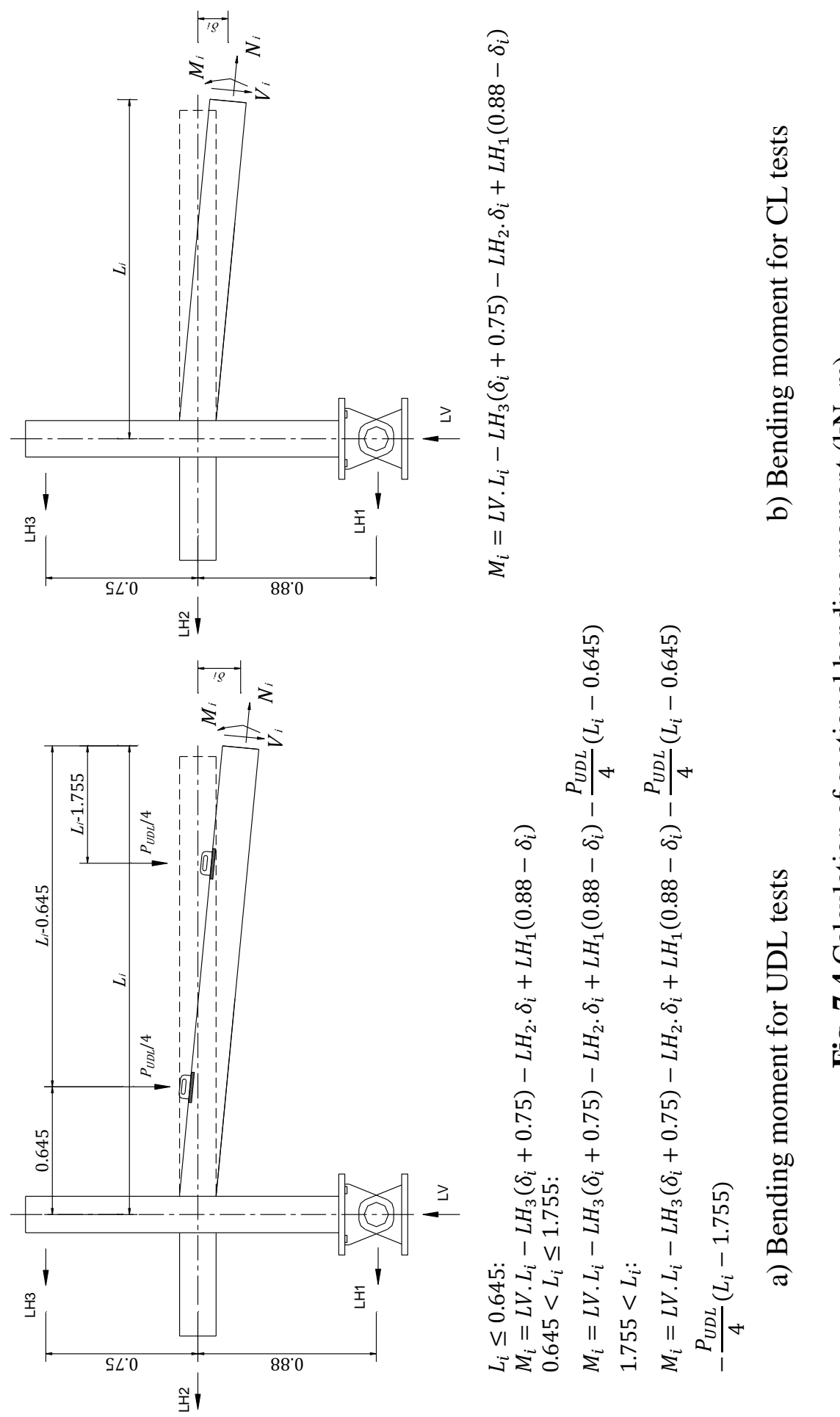

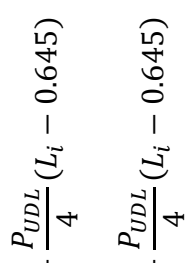
- 11 $\begin{array}{ccc}i 0 & \omega_{0} & \hat{\omega} \\ 1 & 1 & 1\end{array}$ $\begin{array}{lll}\infty & \infty & \infty \\ \infty & \infty & \infty \\ \infty & \infty & \infty \\ 0 & 0 & 0 \\ 0 & 0 & 0\end{array}$

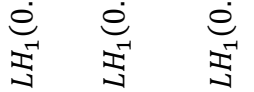

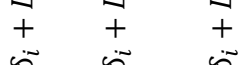
i $\infty \quad 5$ 离 $\begin{array}{llll}1 & 1 & 1\end{array}$

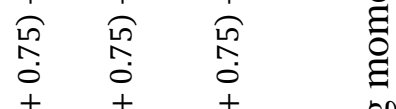
$\dot{\overrightarrow{0}} \ddot{0} \quad \dot{0}$ 包药

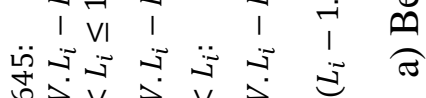

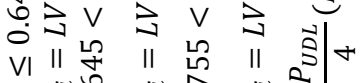

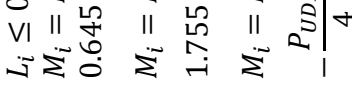

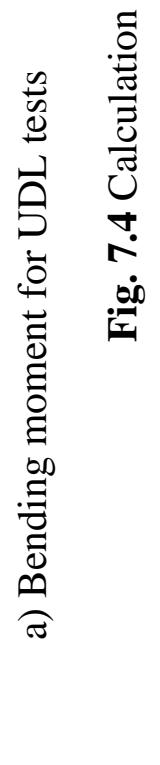




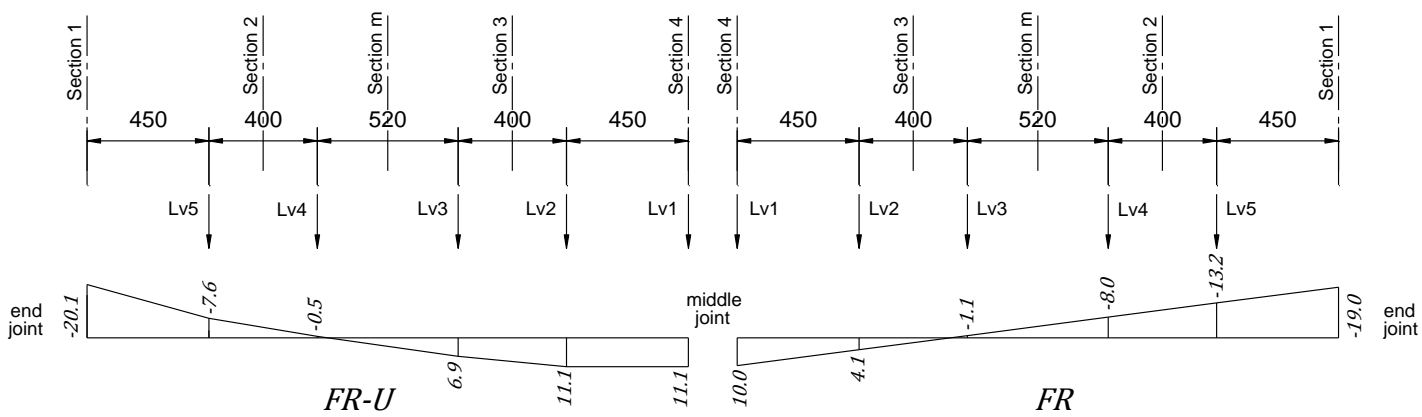

a) at $0.25 d_{\text {beam }}$

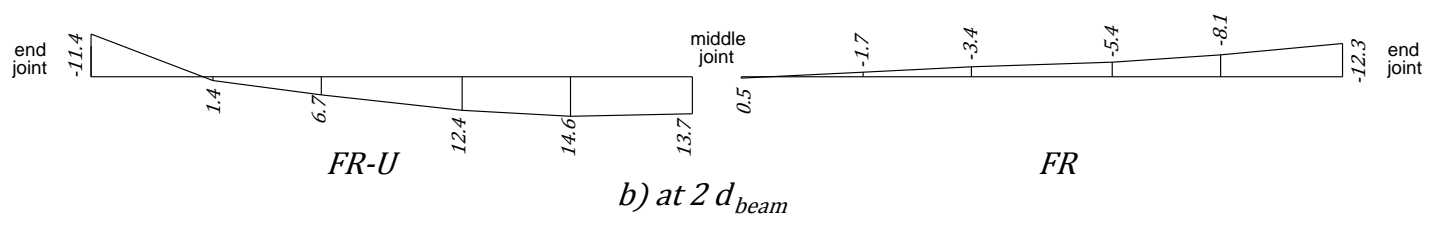

Fig. 7.5 Moment distribution $(\mathrm{kNm})$

After the MJD had exceeded one beam depth and the horizontal reaction had changed to tension, there were only a few new cracks occurring in the UDL tests. Instead, the opening of existing cracks was more dominant. When the top rebars started fracturing at one end joint, bottom-surface cracks near the middle joint propagated towards Section 2 of Fig. 5.19(b). This crack propagation can be explained by the moment distribution of the beam at a MJD of $2 d_{\text {beam }}$, i.e. at large deformation stage (Fig. 7.5(b)). After the " $1^{\text {st }}$ top rebar fracture" of FR-U at 318 $\mathrm{mm}\left(1.8 d_{\text {beam }}\right)$, bending moment at the end joint reduced shifting contra-flexural point towards the end joint, which caused the propagation of bottom-surface cracks. On the other hand, for FR, the bottom rebars had completely fractured at this stage of deformation causing nearly zero bending moment at the middle joint and negative bending moment along the entire beam. As a result, top-surface cracks extensively developed in FR during Stage III. Fig. 7.6 describes the damage patterns and failure modes of UDL static tests at the end of Stage III together with corresponding CL static tests. In FR-U and PR-U, flexural cracks were concentrated at the end joints where fracture of top rebars occurred. They were also equally distributed at two sides of the middle joints due to flatter curvature at mid-span for UDL tests (Fig. 7.5). Besides, there was no major failure (concrete crushing or rebar fracturing) at the middle-joint regions. On the other hand, in the CL tests (FR and PR), top-surface cracks propagated throughout curtailment regions after the 
bottom rebars had fractured. Full-depth cracks also occurred in these tests illustrating that some beam sections were already under pure tension, unlike the behaviour of UDL specimens. There were no full-depth cracks for FR-U and PR-U indicating lower development of tensile force. The middle joints in the CL tests sustained severe damages due to a steep curvature. Regarding displacement profiles, while CL tests showed a rigid-link type of deformation, UDL tests exhibited the usual parabolic shape.
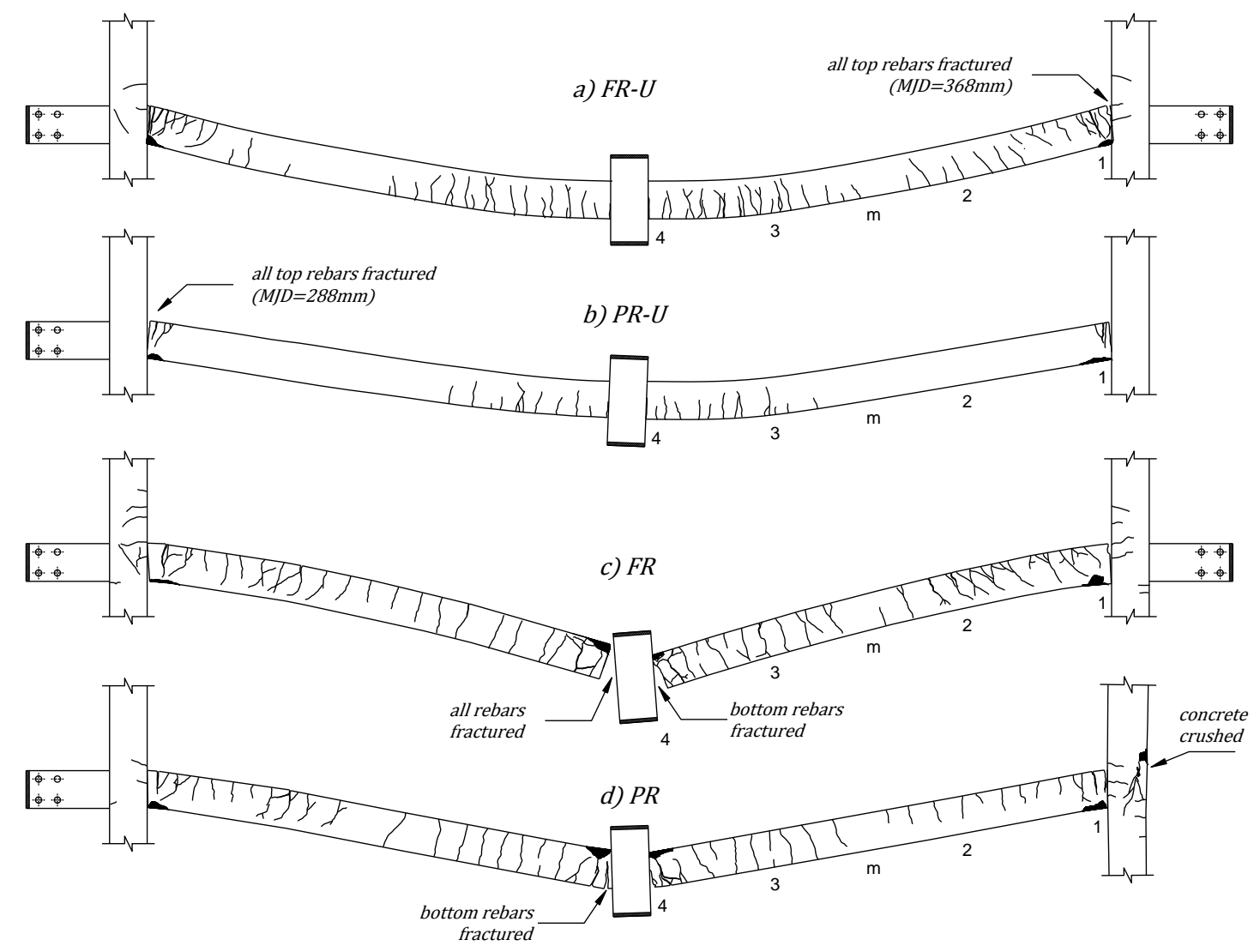

Fig. 7.6 Final failure modes of static tests

When examining the two UDL static tests (Fig. 7.6(a) and (b)), there were fewer flexural cracks at the end joint in PR-U compared to FR-U, suggesting greater strain concentration at the beam-column interface of PR-U. Therefore, the top rebars were fractured earlier in PR-U at $275 \mathrm{~mm}\left(1.5 d_{\text {beam }}\right)$ than in FR-U at $318 \mathrm{~mm}\left(1.8 d_{\text {beam }}\right)$. Moreover, since the collapse of PR-U came from the fracture of top rebars at one end joint, its partially-restrained column underwent little inward movement. This inward column movement was more significant in PR. 
Structural responses of the UDL and the CL static tests were also compared at cross-sectional level by studying the top and bottom reinforcement strains along the double-span beam (Fig. 7.7). In FR, top rebars within curtailment points (T2, Tm and T3) quickly yielded after the fracture of bottom rebars at MJD of $230 \mathrm{~mm}$ (Fig. 7.7(a)), accounting for the occurrence of top-surface cracks at this region (Fig. 7.6(c)). On the other hand, in FR-U, these rebars remained elastic until the end of the test (Fig. 7.7(c)). As a result, negative-moment plastic hinges did not form at the curtailment points in the UDL tests (Fig. 7.6(a) and (b)). Thus, curtailment of top rebars at the middle zone of the single span (Fig. 5.9), which had a significant effect on deflected shape under CL condition, had almost no effect under UDL case. Concerning the beam bottom reinforcement (Fig. 7.7(b) and (d)), the development of tensile strain at Section 3 (B3) during Stages I and II was limited in FR compare to that in FR-U. It agrees well with the bending moment diagram in Fig. 7.5(a) which shows a more uniform distribution of negative moment near the middle joint in FR-U. Prior to top rebar fracture in FR (MJD of $549 \mathrm{~mm}$ ), the bottom rebar at Section 1 (B1) already changed from compression to tension showing that the entire beam section at the end joint was in tension at this stage. However, in FR-U, B1 was still in compression until the end of the test (MJD of $368 \mathrm{~mm}$ ) indicating that tensile force was not yet fully developed throughout the end-joint section.

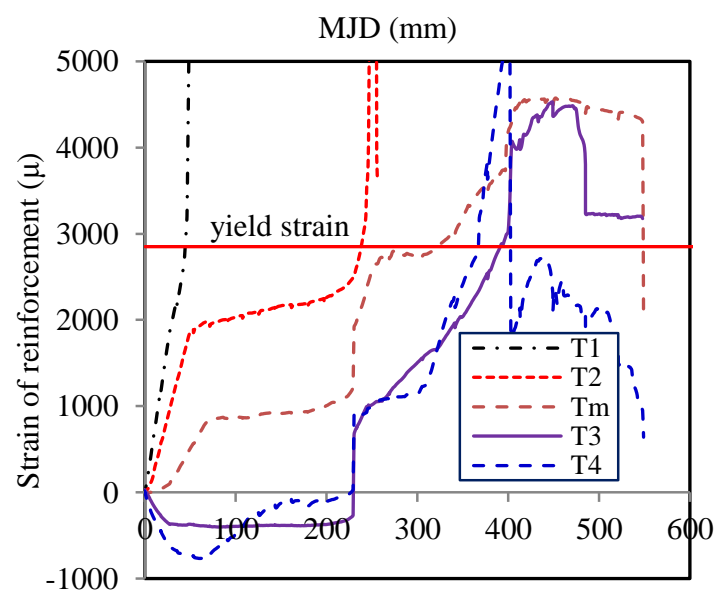

a) Top rebars of FR

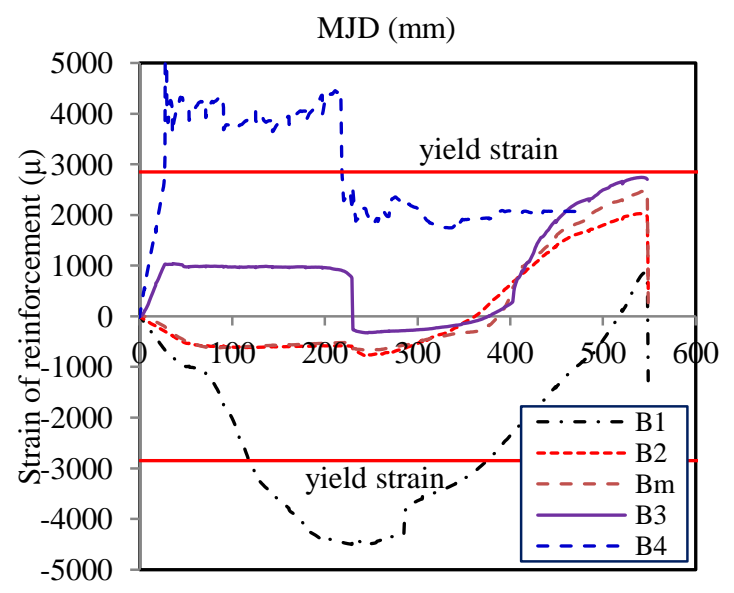

b) Bottom rebars of FR 


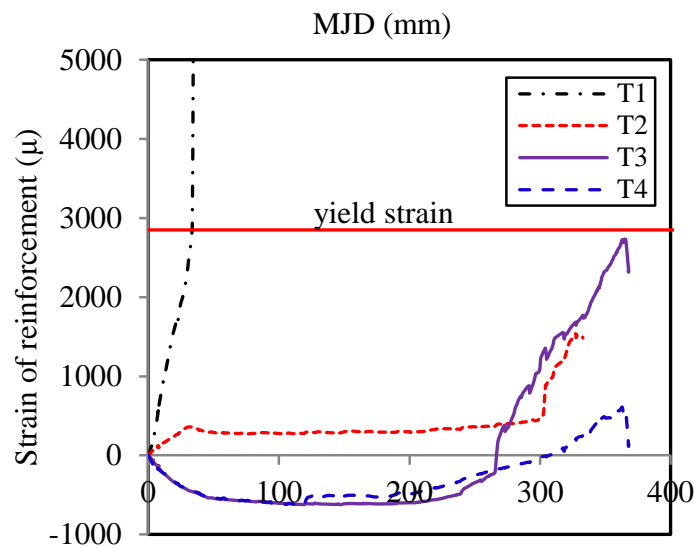

c) Top rebars of FR-U

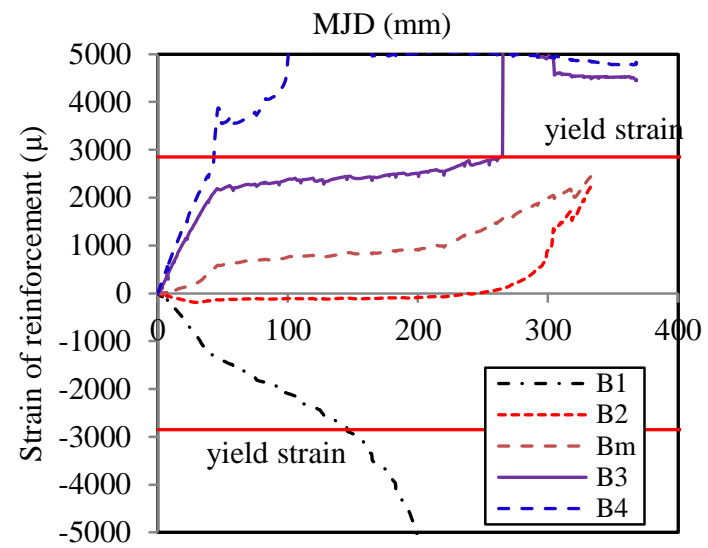

d) Bottom rebars of FR-U

Fig. 7.7 Comparisons of rebar strains between CL and UDL static tests

\subsubsection{Beam-end rotation and horizontal reaction at top rebar fracture}

Behaviours of the UDL and the CL static tests at the state of final collapse, i.e. top rebar fracture, were investigated via relative rotation $\theta$ between the beam end and the end joint. $\theta$ was measured in the tests by two LVDTs Lm1 and Lm2 arranged at this location as shown in Fig. 7.8(a). Development of this rotation with respect to MJD is presented in Fig. 7.8(b) for FR-U, PR-U and FR. Since top rebars did not fracture in PR, rotation result of this test was not considered. It is shown that the beam-end rotations at the state of " $1^{\text {st }}$ top rebar fracture", denoted as $\theta_{\text {max }}$, were relatively similar among all the three tests, roughly equal to $9^{\circ}$ (Table 7.1), although the failure occurred at different MJDs when comparing the UDL and the CL tests. Horizontal reactions of the two loading cases at this state are described in Fig. 7.9 depending on the resultant forces from top $\left(F a_{t o p}\right)$ and bottom $\left(F a_{b o t}\right)$ rebars at Section 1. From Fig. 7.7(b) and (d), $F a_{b o t}$ (represented by strain gauge B1 in Fig. 5.19(b)) was in tension in FR and in compression in FR-U at this state. As a result, horizontal reactions of FR $\left(H_{C L}\right)$ and FR-U $\left(H_{U D L}\right)$ are calculated using Eqs. (7.1) and (7.2), respectively.

$H_{C L}=\cos \left(\theta_{\text {max }}\right)\left(F a_{\text {top }}+F a_{\text {bot }}\right)$

$H_{U D L}=\cos \left(\theta_{\text {max }}\right)\left(F a_{t o p}-F a_{b o t}-F_{c}\right)$

where $F_{c}$ is the resultant force of compressive concrete region at beam bottom surface. 
At the state of top rebar fracture, it is assumed that $F a_{\text {top }}$ had reached the ultimate tensile capacity of reinforcement for both loading cases. As a consequence, if the maximum beam-end rotation $\theta_{\max }$ was assumed to be equal in both cases, $H_{C L}$ would be obviously larger than $H_{U D L}$. In the static tests when the specimens failed, $H_{C L}$ and $H_{U D L}$ were 148.8 and $81.9 \mathrm{kN}$, respectively. Therefore, although having the same beam-end rotation at the final stage of top rebar fracture, CA could not be fully utilised in the UDL tests compared to the CL tests because part of the endjoint section was still under compression.

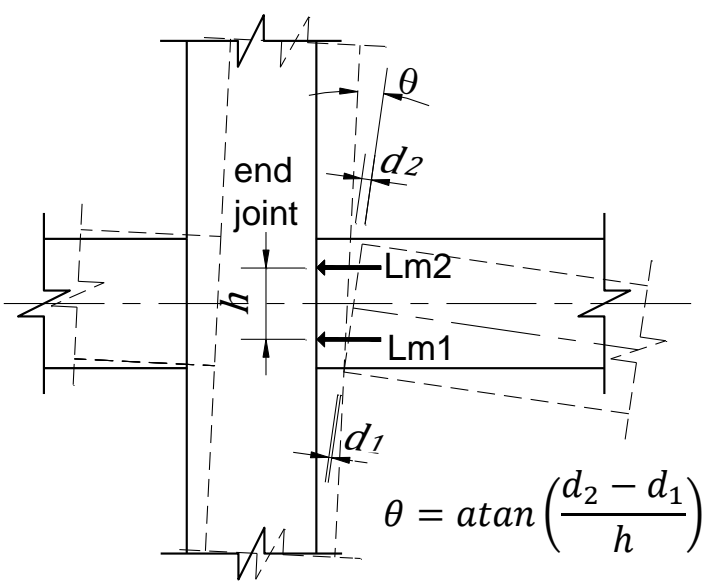

a) Measurement method

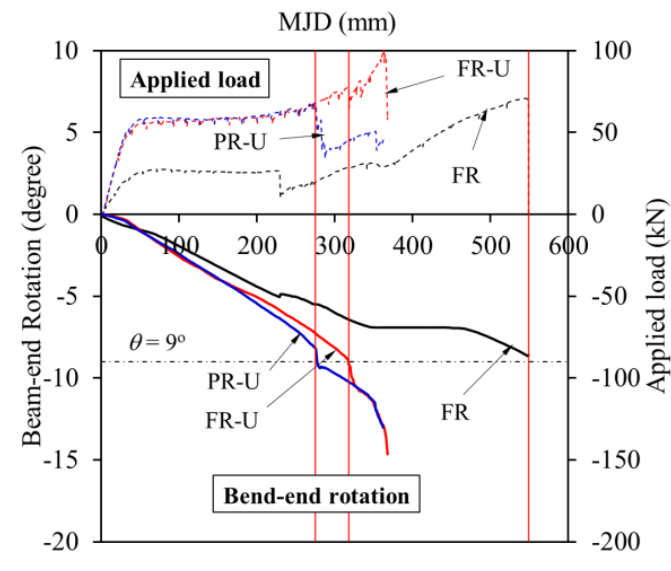

b) Rotation responses

Fig. 7.8 Comparison of beam-end rotations at " 1 st top rebar fracture"

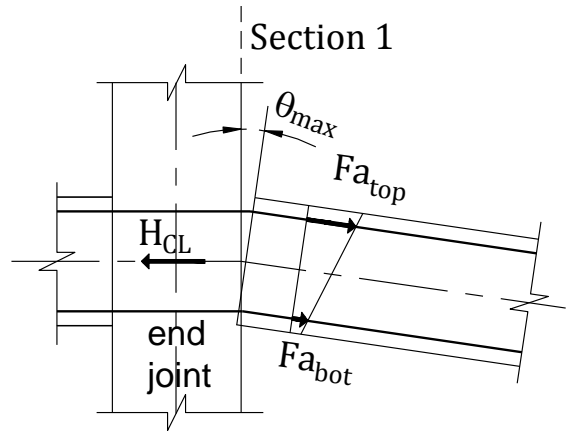

a) CL case

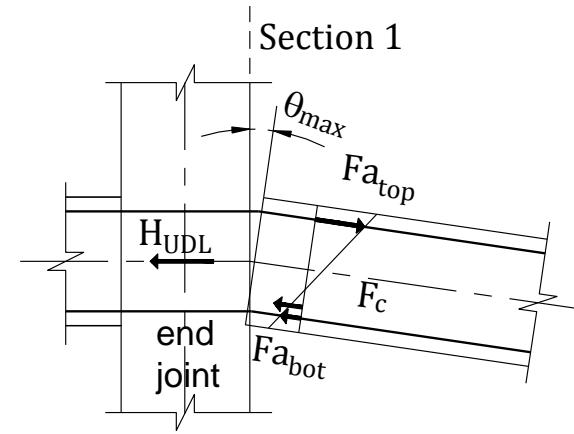

b) UDL case

Fig. 7.9 Total horizontal reaction and rebar forces at the end joint at " 1 st top rebar fracture" state 


\subsection{Analytical relationship of structural behaviours between UDL and CL conditions}

Based on the results of the beam-column tests under UDL (Section 7.2) and CL (Lim et al. 2015) cases, the relationship between behaviours from these two loading configurations is developed. It compares structural responses at the initial yielding stage, the large-deformation stage, as well as at the final failure stage. First, structural capacity of a double-span beam at yielding point under both UDL and CL conditions can be simply calculated by assuming the formation of plastic hinges at both the middle and the end joints as shown in Fig. 7.10. Hence, neglecting the effect of axial compression force and based on analytical relationships between applied loads and moment distributions of the two cases, it is shown from Eqs. (7.3) and (7.4) that total applied load from the UDL case $\left(P_{U D L}\right)$ is twice that from the CL case $\left(P_{C L}\right)$.

$\frac{P_{C L}(2 L)}{4}=M_{m i d}+M_{e n d}$

$\frac{q(2 L)^{2}}{8}=\frac{P_{U D L}(2 L)}{8}=M_{m i d}+M_{e n d}$

where $M_{\text {mid }}$ and $M_{\text {end }}$ are the plastic bending moment capacities at the middle and the end joints, respectively. They can be calculated based on Eq. (4.4) proposed by Paulay and Priestley (1992).

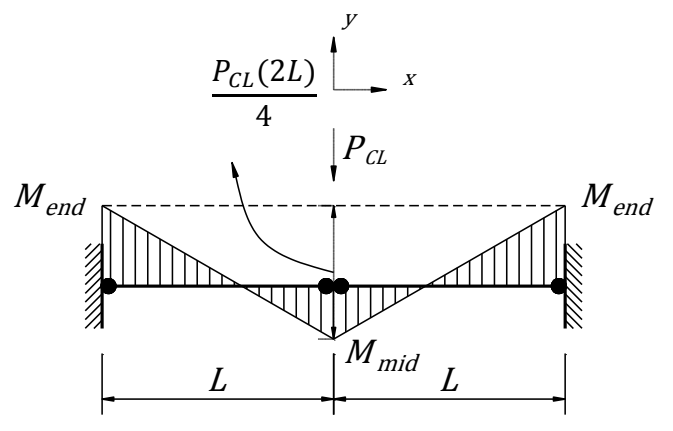

a) $\mathrm{CL}$

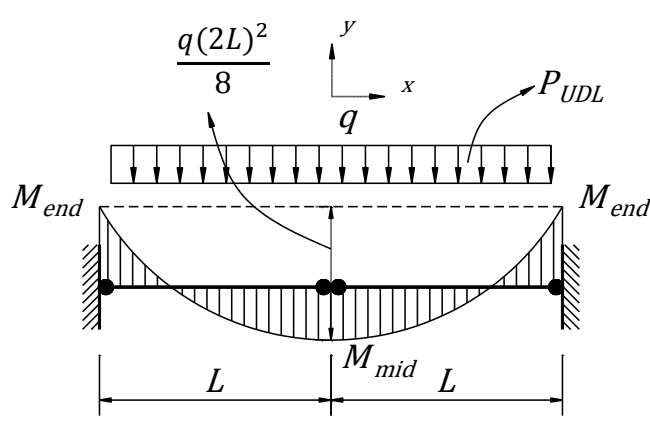

b) UDL

Fig. 7.10 Moment distribution at yielding point 
From Eqs. (7.3) and (7.4), $P_{C L}$ and $P_{U D L}$ at yielding state of the specimens used in this study (Fig. 5.3 and Fig. 5.9) were equal to 28.5 and $57.0 \mathrm{kN}$, respectively, which are relatively similar to test results (Table 7.1).

When the MJD is relatively large and the beam axial force starts affecting structural response significantly, i.e. during CAA and CA stages, based on the equilibrium of moment from half of the structure as illustrated in Fig. 7.11, the relationships between total applied loads and horizontal reactions can be described as follows:

$$
\begin{gathered}
P_{C L}=\frac{2\left(M_{\text {mid }}+M_{\text {end }}+H_{C L} \delta_{C L}\right)}{L} \\
P_{U D L}=\frac{4\left(M_{\text {mid }}+M_{\text {end }}+H_{U D L} \delta_{U D L}\right)}{L}
\end{gathered}
$$

where $H_{C L}$ and $H_{U D L}$ are the horizontal reactions for the CL and UDL cases, respectively. $\delta_{C L}$ and $\delta_{U D L}$ represent MJDs from the CL and UDL cases, respectively.

From the static tests results (Fig. 7.1(b)), $H_{C L}$ and $H_{U D L}$ were relatively similar for the same MJD (i.e. $\delta_{C L}=\delta_{U D L}$ ) before any rebar fracture occurred. Therefore, applying this observation, from Eqs. (7.5) and (7.6) $P_{U D L}$ is equal to $2 P_{C L}$, which was also observed in the static test results for large MJDs, i.e. Stages II and III (Fig. 7.1(a)).

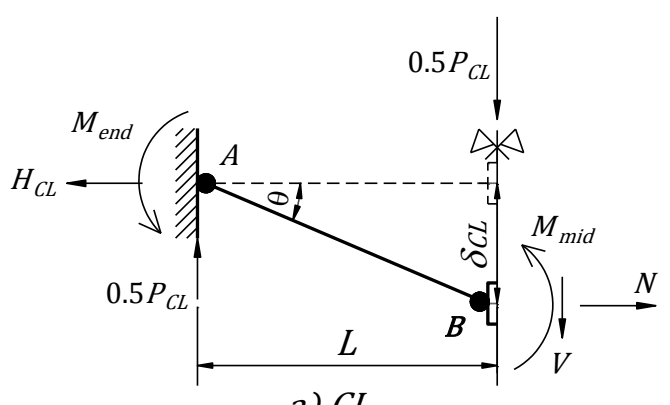

a) $C L$

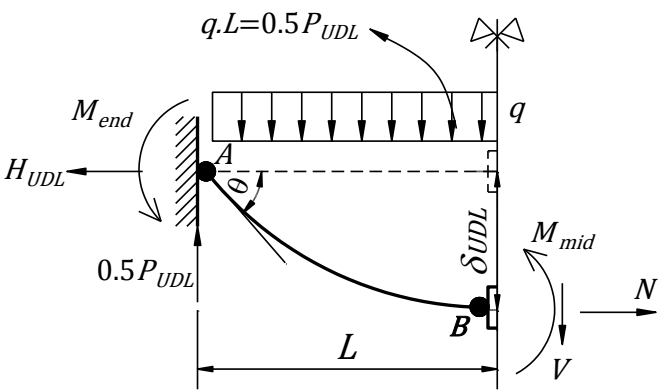

b) $U D L$

Fig. 7.11 Force equilibrium at large deformations

Regarding maximum MJD of the static responses at the failure state of top rebar fracture $\left(\theta=\theta_{\max }\right)$, if the displacement profile of CL case follows a linear function 
defined in Eq. (7.7), then the corresponding MJD of this loading case at $\theta_{\max }$ can be calculated based on Eq. (7.8).

$y=\frac{\delta_{C L}}{L}(x-L)$

$\max \left(\delta_{C L}\right)=\tan \left(\theta_{\max }\right) \cdot L$

To analytically predict the displacement profile of UDL tests, a second order function presented in Eq. (7.9) is used which reasonably well agrees with actual displacement profiles (Fig. 7.12). The corresponding MJD at $\theta_{\max }$ applying the second order function is presented in Eq. (7.10). Hence, if it is assumed that $\theta_{\max }$ is similar for both loading cases at " $1^{\text {st }}$ top rebar fracture" stage, then $\max \left(\delta_{U D L}\right)$ is equal to $0.5 \times \max \left(\delta_{C L}\right)$. Test results from FR-U and FR indicated that this ratio was 0.58 (Table 7.1), which shows that the proposed method to predict the maximum displacement under UDL based on that under CL is conservative.

$y=\frac{\delta_{U D L}}{L^{2}}\left(x^{2}-L^{2}\right)$

$\max \left(\delta_{U D L}\right)=\tan \left(\theta_{\max }\right) \cdot \frac{L}{2}$

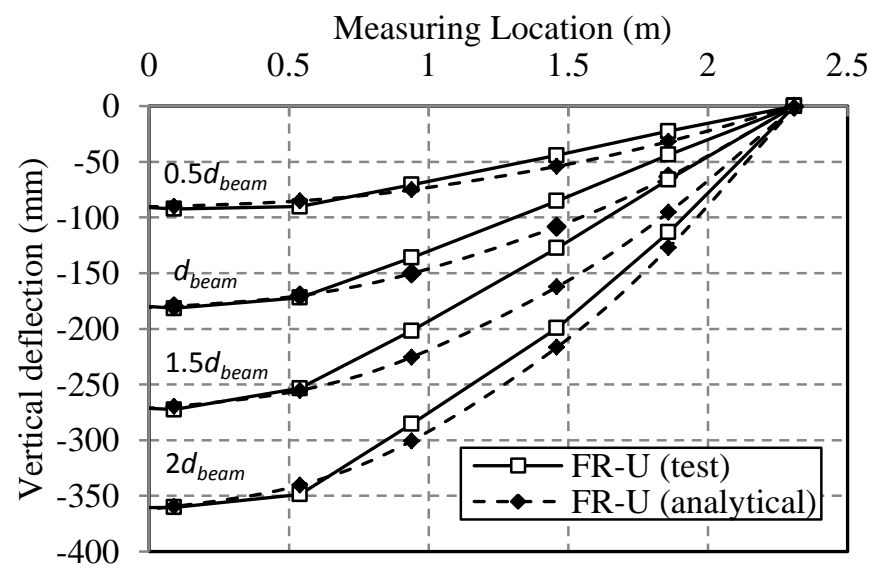

Fig. 7.12 Displacement profiles under UDL 
In summary, at the same MJD, vertical load-carrying capacity of UDL case is twice that of CL case whereas total horizontal reaction is similar between the two loading conditions. This relationship is valid not only at yielding stage but also at the CAA and the CA stages, until fracture starts occurring at either top rebars (UDL) or bottom rebars (CL). At the final stage of CA (top rebar fracture), maximum deformation of UDL case can be conservatively equal to half of that from CL case. The relationship on structural behaviour between the two loading cases can be applied into the semi-analytical model proposed in Section 4.3 to conservatively predict simplified response of sub-assemblages under UDL condition as follows:

For an RC sub-assemblage, if its simplified CL response is presented by polyline $\mathrm{OABC}_{1} \mathrm{CD}_{1} \mathrm{DEFG}$ (defined using the model in Section 4.3), then its simplified UDL response is polyline $\mathrm{OA} \mathrm{B}^{\prime} \mathrm{C}_{1}{ }_{1} \mathrm{C}^{\prime} \mathrm{D}_{1} \mathrm{D}^{\prime}$ as shown in Fig. 7.13. In this polyline, part D ${ }_{1}$ D' represents the mobilisation of CA under UDL case and point D' denotes the moment when top rebar fracture starts occurring. Compared to points $A$ to $D_{1}$ from the CL response, points $A^{\prime}$ to $D^{\prime}{ }_{1}$ from the UDL response have the same displacements but two times the applied loads. The gradient of line $\mathrm{D}_{1}{ }_{1} \mathrm{D}$ ' is also two times that of line $\mathrm{D}_{1} \mathrm{D}$. Displacement at point $\mathrm{D}^{\prime}, d_{D^{\prime}}$, is equal to half of $d_{G}$ from the CL response. However, because rebar cut-off (curtailment) regions do not have any influence on the deflected shape under UDL condition, $\Delta_{c}$ based on Eq. (4.26) shall be omitted when calculating $d_{D^{\prime}}$. Applying this method to predict structural response of specimen FR-U, it gives relatively conservative results compared to the actual test (Fig. 7.14).

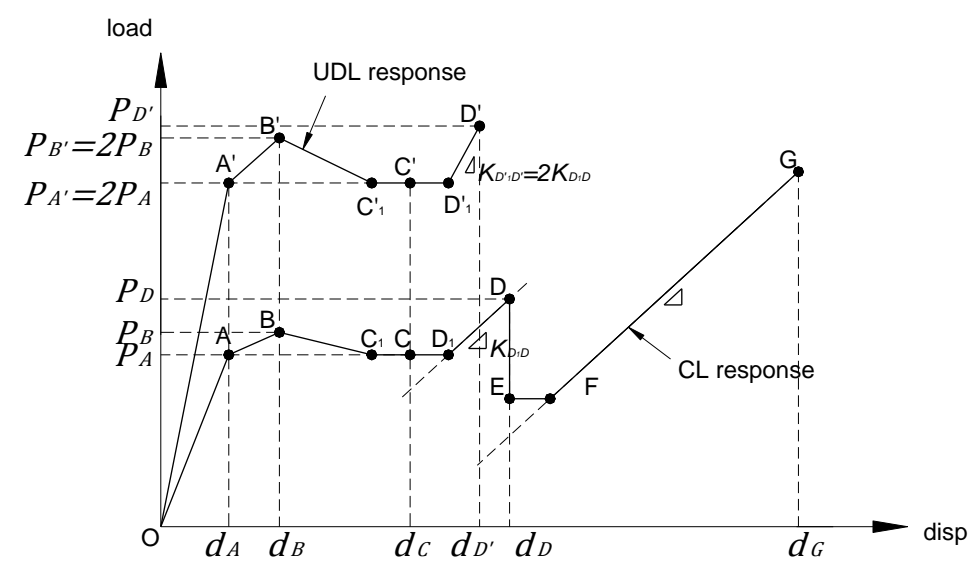

Fig. 7.13 Simplified CL and UDL responses of a sub-assemblage 


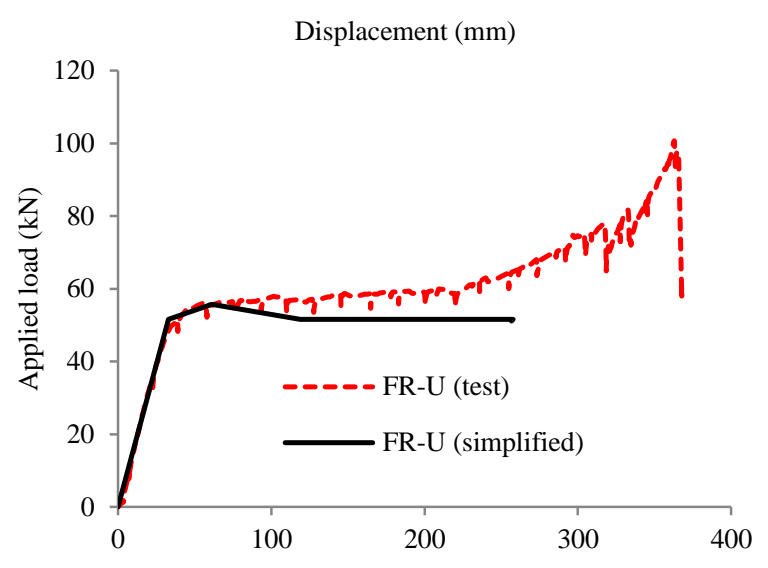

Fig. 7.14 Simplified UDL prediction of FR-U

\subsection{Dynamic test results}

\subsubsection{Overall responses}

Similar to the CL dynamic tests of Series I, before conducting the UDL dynamic tests, axial loads were applied to the side columns. At the same time, temporary supports for the steel-plate systems underneath the specimen (item (9) in Fig. 5.11(b)) were slowly removed. As a result, self-weights from the steel plates were first carried by the single span, causing initial readings in the pin supports as shown in Table 7.2, which summarises important results of the UDL dynamic tests. After readings from all data channels were stable, the rope linking with the quick-release device was suddenly yanked free, releasing the specimen in a free-fall movement. If there was no failure, the specimen would vibrate freely within its final deformation position and slowly came to rest under damping effect. Otherwise, the structure would lose its bearing capacity and drop to the floor with a loud thud. Overall deformations of all the dynamic tests are shown in Fig. 7.15(a). Among the four dynamic tests, only FDU1-P/42 with $42 \mathrm{kN}$ of loads sustained under a small deformation. The others which were under higher loads of 55 and $61 \mathrm{kN}$ attained maximum MJDs larger than one beam depth indicating that the structures had already entered into Stage III of response. Similar to the CL dynamic tests of Series I, the ratio of $t_{r} / T_{d}$ was also used to verify the dynamic characteristic of the sudden column removal event, showing that all the surviving tests from Series II satisfied the requirement of $t_{r} / T_{d} \leq 0.25$ (Table 7.2). Additionally, damping ratio of the 
oscillating phase was shown to be roughly equal to $3.5 \%$ for all specimens (Table 7.2), similar to those from Series I (Table 6.1). Fig. 7.15(b) and (c) present the release time and the damping ratio obtained from the UDL dynamic tests, respectively.

Based on load-pin and load-cell readings, total vertical reaction and total horizontal reaction from the left side of all the UDL dynamic tests were defined and plotted in Fig. 7.16(a) and (b), respectively. While the horizontal reaction of FDU1-P/42 was purely negative showing that the specimen was still under flexure/CAA stage, the horizontal reactions from the other tests changed from negative to positive, illustrating the onset of CA. For FDU1-P/61 which was the second test of specimen FDU1, both the vertical and the horizontal reactions significantly dropped at a time of $0.67 \mathrm{~s}$, denoting a complete failure of the specimen. Thereafter, the specimen hit the strong floor at a time of $1.035 \mathrm{~s}$. For specimens that survived the free-fall event, the DLIF was defined similarly to tests from Series I, ranging from 1.11 to 1.30 as shown in Table 7.2.

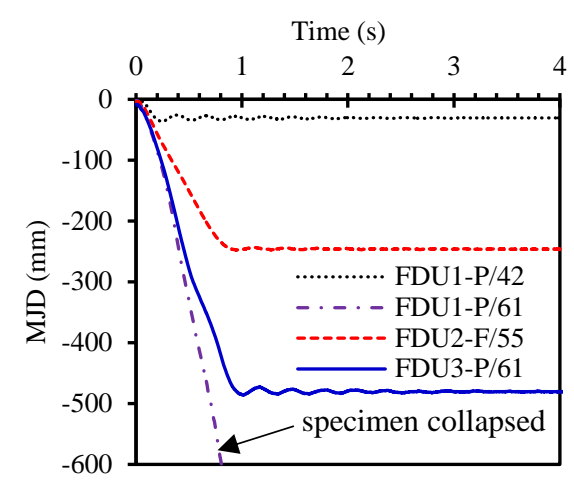

a) MJD time-history

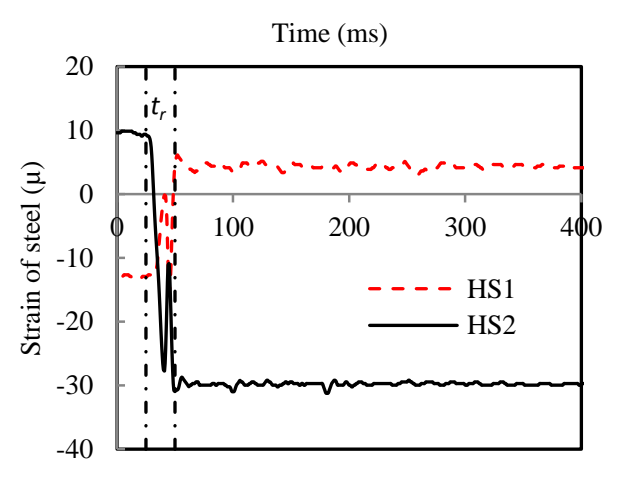

b) Release time (FDU2-F/55)

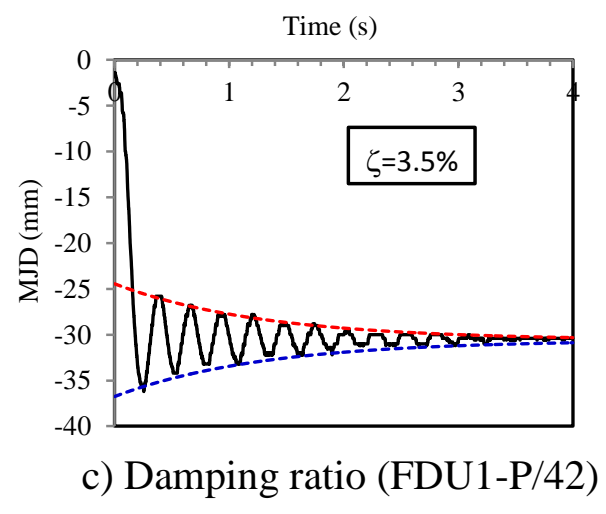

Fig. 7.15 General responses of UDL dynamic tests 


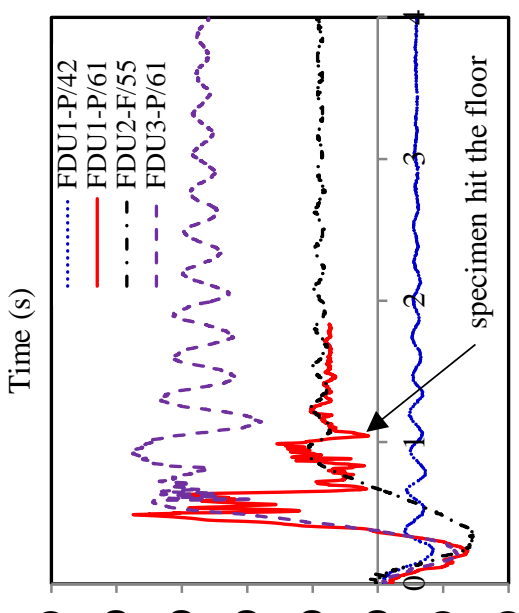

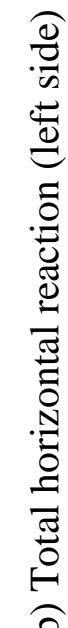

8.8 \%

苟

$\widehat{\infty}$
0
$\vdots$
$\vdots$

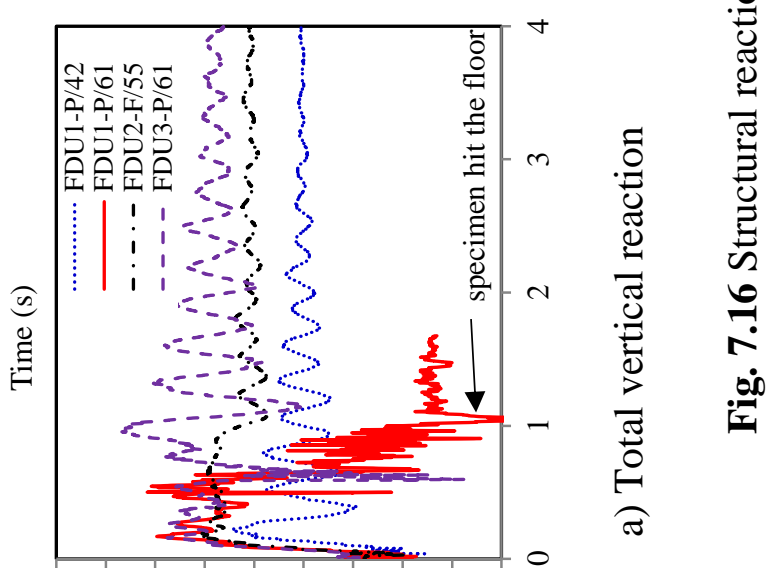

요용유 유응

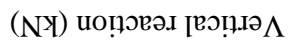




\subsubsection{Structural damages and failure modes}

Structural damages, failure modes, as well as deflected shapes of all the UDL dynamic tests had good resemblance with the corresponding UDL static tests as shown in Fig. 7.17. The first release of the partial-restraint specimen FDU1 under $42 \mathrm{kN}$ of applied load only caused minor flexural cracks to the structure. There was no sign of concrete crushing, indicating that the specimen had not yet reached its maximum capacity under CAA. After the first test, FDU1 was pulled back up to its original position. The second release of this specimen was under $61 \mathrm{kN}$ which led to a complete collapse. The specimen experienced multiple severe damages including the fracture of top rebars at the left-side end joint and the fracture of all sectional rebars at the right-side end joint. However, its partially restrained column experienced less inward movement effect, similar to the corresponding static test PR-U. For FDU2-F/55, multiple cracks occurred at the end joints and within the two sides of the middle joint, together with concrete crushing at bottom surfaces of the end joints. However, no rebar fracture was observed. For FDU3-P/61 which had identical boundary condition and applied load with FDU1-P/61, excessive vertical deflection (up to $485 \mathrm{~mm}$ ) was captured together with severe concrete cracking and crushing. Top rebars at the left-side end joint also fractured after the test. Under large pulling-in effect from the double-span beam, the partially restrained side column underwent an excessive deformation (up to $47 \mathrm{~mm}$ ) and experienced severe concrete crushing at its outer face. However, the specimen sustained the applied load and no collapse took place. Such behaviour of large inward deformation for the side column was not observed in the corresponding UDL static test PR-U (Fig. 7.6(b)), but occurred in the CL static test PR (Fig. 7.6(d)), as well as the CL dynamic test FD4-P/30.5 (Fig. 6.6(g)). Additionally, similar to FD4-P/30.5, the axial force on the partially-restrained side column of FDU3-P/61 after the test had finished was only $35 \%$ of its original value owing to the appearance of a gap between the hydraulic jack and the steel plate on top of the column. As a result, the survival of FDU3 against complete collapse was also questionable. Detailed photos of all static and dynamic tests from Series II are shown in Appendix 7A. 


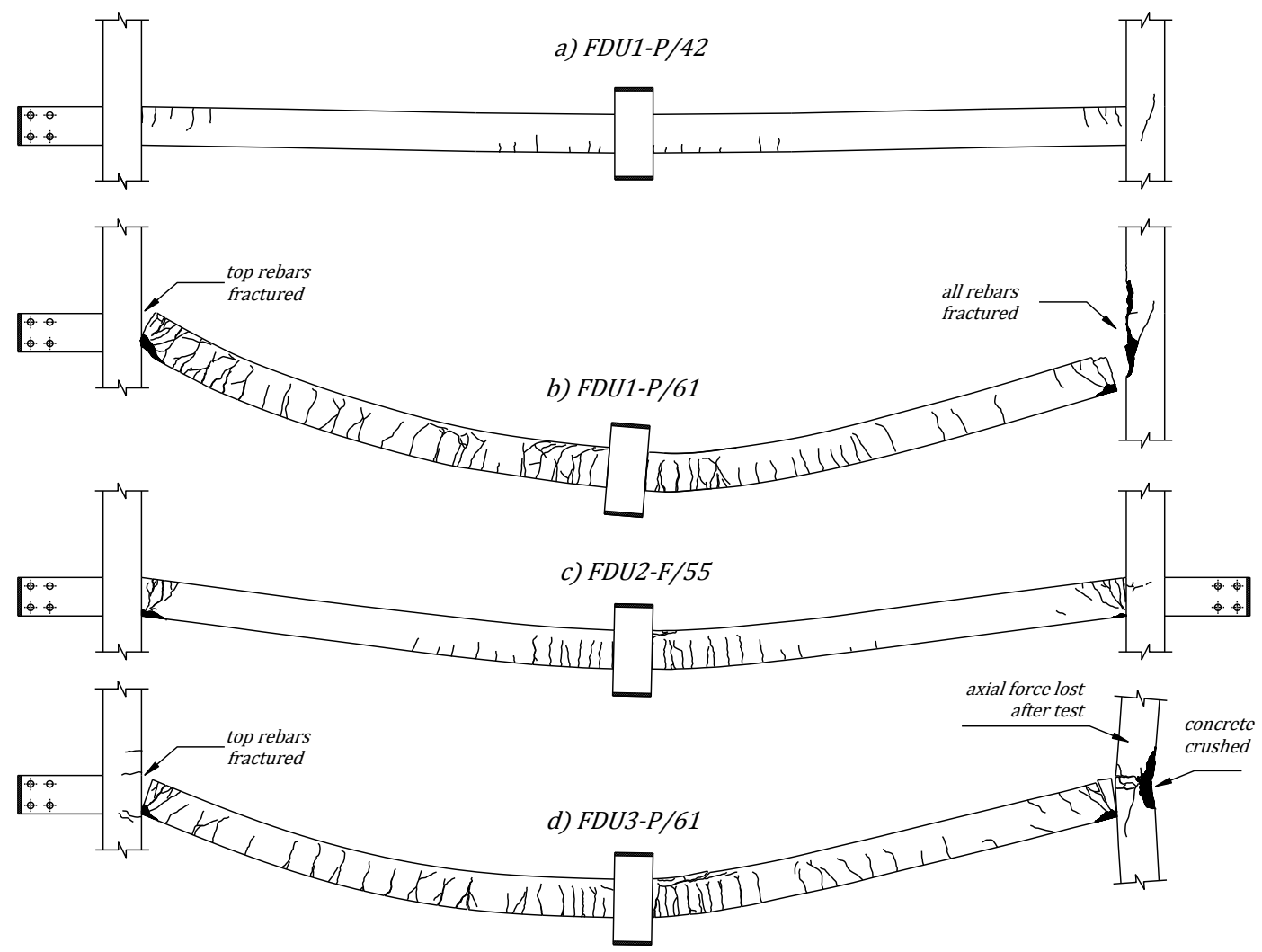

Fig. 7.17 Damage patterns and failure modes of dynamic tests

\subsubsection{Strain rate effects on material strength}

The material DIFs for rebar yield strength and ultimate strength of the UDL dynamic tests were defined following the same procedure from the CL dynamic tests of Series I (Section 6.2.5). Results for maximum strain rates as well as corresponding DIFs are presented in Table 7.3. Unlike the CL dynamic tests (Table 6.2), strain rates of tensile rebars at Section 1 (end joint) from the UDL dynamic tests were greater than those at Section 4 (middle joint). The maximum DIFs for rebar yield strength of Sections 1 and 4 were 1.202 and 1.154 , respectively. It is because, under UDL condition, bending moment was developed faster at the end joint compared to the middle joint. Regarding rebar ultimate strength, the largest DIF was only 1.064, similar to the CL case. In other words, the observation from the CL dynamic tests stating that strain rate effects could remarkably affect the flexure/CAA capacity but could have limited influence on CA capacity was also valid for the UDL dynamic tests. 
Table 7.3 Summary of strain rates and DIF for rebars (Series II)

\begin{tabular}{|c|c|c|c|c|c|c|}
\hline \multirow{3}{*}{ Test } & \multicolumn{3}{|c|}{ Tensile rebars at Section 1} & \multicolumn{3}{|c|}{ Tensile rebars at Section 4} \\
\hline & \multirow{2}{*}{$\begin{array}{l}\text { Max. strain } \\
\text { rate }(1 / \mathrm{s}) \text { up } \\
\text { to yielding }\end{array}$} & \multicolumn{2}{|c|}{ DIF } & \multirow{2}{*}{$\begin{array}{l}\text { Max. strain } \\
\text { rate (1/s) up } \\
\text { to yielding }\end{array}$} & \multicolumn{2}{|c|}{ DIF } \\
\hline & & $\begin{array}{l}\text { Yield } \\
\text { strength }\end{array}$ & $\begin{array}{l}\text { Ultimate } \\
\text { strength }\end{array}$ & & $\begin{array}{l}\text { Yield } \\
\text { strength }\end{array}$ & $\begin{array}{l}\text { Ultimate } \\
\text { strength }\end{array}$ \\
\hline FDU1-P/42 & 0.224 & 1.175 & 1.056 & 0.06 & 1.143 & 1.046 \\
\hline FDU1-P/62 & 0.404 & 1.189 & 1.060 & 0.079 & 1.149 & 1.048 \\
\hline FDU2-F/55 & 0.490 & 1.194 & 1.062 & 0.097 & 1.154 & 1.050 \\
\hline FDU3-P/61 & 0.670 & 1.202 & 1.064 & 0.067 & 1.145 & 1.047 \\
\hline
\end{tabular}

\subsection{Comparisons of structural responses between static and dynamic tests under UDL condition}

\subsubsection{Structural force-based and displacement-based DIFs}

To investigate progressive collapse resistance, structural DIF of a system can be defined in two different ways, which are displacement-based DIF (Biggs 1964) and force-based DIF (Tsai 2010). While the former is the ratio of maximum dynamic MJD to static MJD under the same applied load, the latter is defined by dividing the static applied load to the dynamic applied load under the same displacement. For designing a new structure against progressive collapse, the force-based DIF which provides load capacity is preferred to the displacement-based DIF which only provides displacement capacity. On the other hand, to analyse an existing building for its vulnerability to progressive collapse, the displacement-based DIF can be employed to check the existing rotation capacity.

For a typical elasto-plastic structure, Tsai (2010) showed that as the applied load increases, the force-based DIF decreases from 2 (elastic stage) towards 1 (plastic stage), whereas the displacement-based DIF normally increases from 2 (elastic stage) towards infinity (plastic stage). In general, DIF values calculated from the dynamic tests in this study (Table 7.2) showed a similar trend to Tsai's finding.

\subsubsection{Reaction forces}

Similar to the CL dynamic tests (Series I), reaction forces from the UDL dynamic tests of Series II were also plotted with reference to MJD instead of time, and were used to compared with those from the related UDL static tests. Fig. 7.18 shows the 
comparisons between the dynamic tests under the applied loads of 42 and $55 \mathrm{kN}$ with the static tests of FR-U and PR-U. Due to the effect of applied weights distributing on the single span before the release which was mentioned in Fig. 7.16(a) and Table 7.2, vertical reactions from the UDL dynamic tests had initial non-zero values at zero MJD compared to vertical applied loads from the UDL static tests. Other than that, responses of the vertical and the horizontal reactions from both test series had reasonably good agreement. For the dynamic tests under $61 \mathrm{kN}$ (Fig. 7.19), they also displayed similar trend of response to the relevant static test PR-U. The peaks of CAA from the dynamic tests were larger than those from the static tests by $8 \%$ and $14 \%$ for the full- and the partial-restraint specimens, respectively. It could be due to the enhancement in rebar yield strength which led to an increase of plastic bending moment resistances $M_{\text {mid }}$ and $M_{\text {end }}$ as explained in Section 7.4.3.

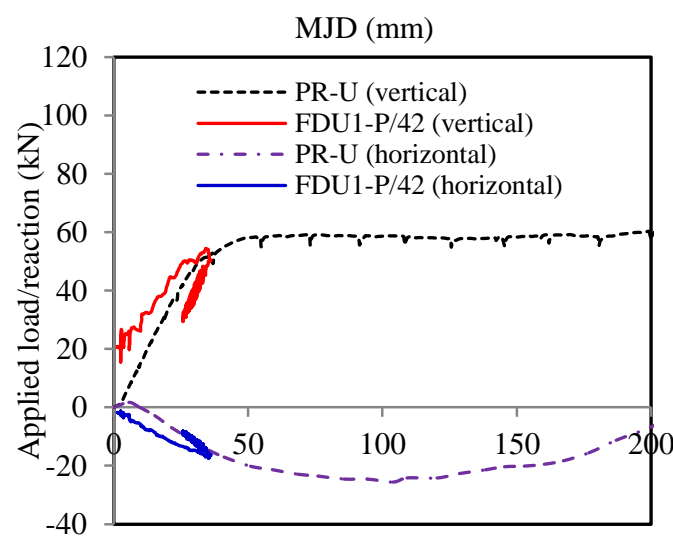

a) Full-restraint specimens

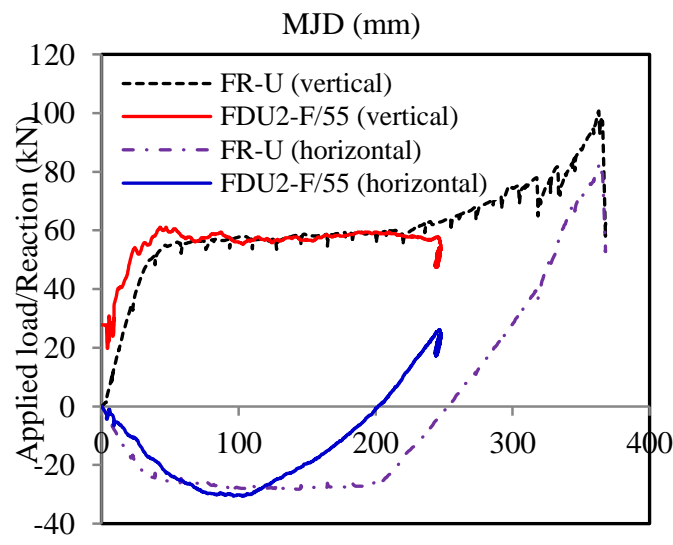

b) Partial-restraint specimens

Fig. 7.18 Structural responses of dynamic tests with load levels of 42 and $55 \mathrm{kN}$

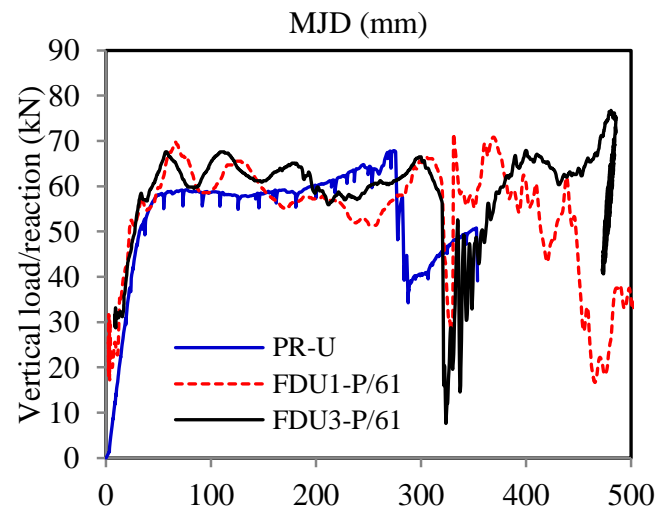

a) Vertical load/reaction

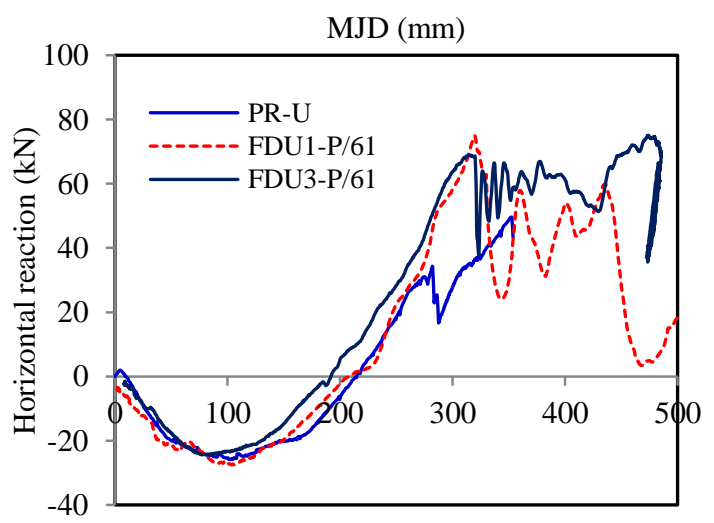

b) Horizontal reaction

Fig. 7.19 Structural responses of dynamic tests with $61 \mathrm{kN}$ 
FDU1-P/61 and FDU3-P/61 had a sudden drop in structural capacity (for both vertical and horizontal reactions) at a MJD of 320 and $324 \mathrm{~mm}$, respectively. It was assumed to be the state when top rebars at the end joints started fracturing during these two dynamic tests, which were comparable with PR-U and FR-U. The beamend rotations at this state were calculated and equal to $9.4^{\circ}$ and $9.6^{\circ}$ for FDU1-P/61 and FDU3-P/61, respectively. It is noteworthy that such rotations had good agreement with those from the UDL and the CL static tests (Table 7.1). After this failure, structural responses of FDU1-P/61 dropped to zero, probably due to initial damages caused by the first release. On the other hand, the structural response of FDU3-P/61 did not drop to zero at the end of the test, denoting that a complete collapse did not occur in FDU3-P/61, although the column axial load dropped to one-third of its original value.

Similar to the CL dynamic tests of Series I (Section 6.3.2), equivalent translational and rotational stiffness of the end-joint restraints from the UDL static and the UDL dynamic tests of Series II were computed and presented in Table 7.4. On average, $K_{A}$ in tension was relatively similar between the two testing environments. Nonetheless, $K_{A}$ in compression from the dynamic tests was noticeably larger than that from the static tests owing to strain rate effects.

Table 7.4 Equivalent restraint stiffness of end joints from static and dynamic tests (Series II)

\begin{tabular}{|c|c|c|c|c|c|c|}
\hline \multirow{2}{*}{ Test } & \multicolumn{2}{|c|}{$K_{A}$ of left side $(\mathrm{kN} / \mathrm{m})$} & \multicolumn{2}{c|}{$K_{A}$ of right side $(\mathrm{kN} / \mathrm{m})$} & \multicolumn{2}{c|}{$K_{R}(\mathrm{kNm} / \mathrm{rad})$} \\
\cline { 2 - 7 } & Compression & Tension & Compression & Tension & Left side & Right side \\
\hline FR-U & 18000 & 15400 & 14500 & 13300 & 3200 & 2800 \\
\hline PR-U & 18000 & 17250 & 11000 & 9000 & 3000 & 5500 \\
\hline FDU1-P/42 & 21000 & NA & 18000 & NA & 4070 & 3160 \\
\hline FDU1-P/61 & 21000 & 21000 & 13650 & 8000 & 6790 & 3190 \\
\hline FDU2-F/55 & 16900 & 19900 & 16900 & 19900 & 5360 & 5860 \\
\hline FDU3-P/61 & 30000 & 9400 & 9600 & 8700 & 4800 & 3800 \\
\hline
\end{tabular}

Note: NA tension force was not developed 


\subsection{Comparisons of load-carrying capacity between actual dynamic tests and simplified energy-based method}

Pseudo-static behaviours for the CL and the UDL static tests based on Izzuddin's method are illustrated in Fig. 7.20. It is shown that FR-U and PR-U had relatively identical dynamic responses with comparable maximum dynamic capacities as shown in Table 7.5. For FR-U, although the static peak of the "one-side top rebar fracture" was 29\% larger than that of the "1st top rebar fracture" (Table 7.1), the difference for the corresponding dynamic peaks was only $5 \%$. Therefore, the " 1 st top rebar fracture" state can be conservatively considered as the final failure mode for both FR-U and PR-U. Comparing the difference of maximum dynamic capacity between the partial- and the full-restraint conditions, FR-U was only $7.5 \%$ larger than PR-U, whereas FR was 28\% larger than PR. Once again, it emphasises the weaker influence of boundary condition on the UDL tests because the maximum MJD from UDL tests was significantly smaller than that from CL tests. Comparing maximum dynamic capacities between the UDL and the CL tests predicted from Izzuddin's method, FR-U was 1.8 times larger than FR and PR-U was 2.1 times larger than PR.

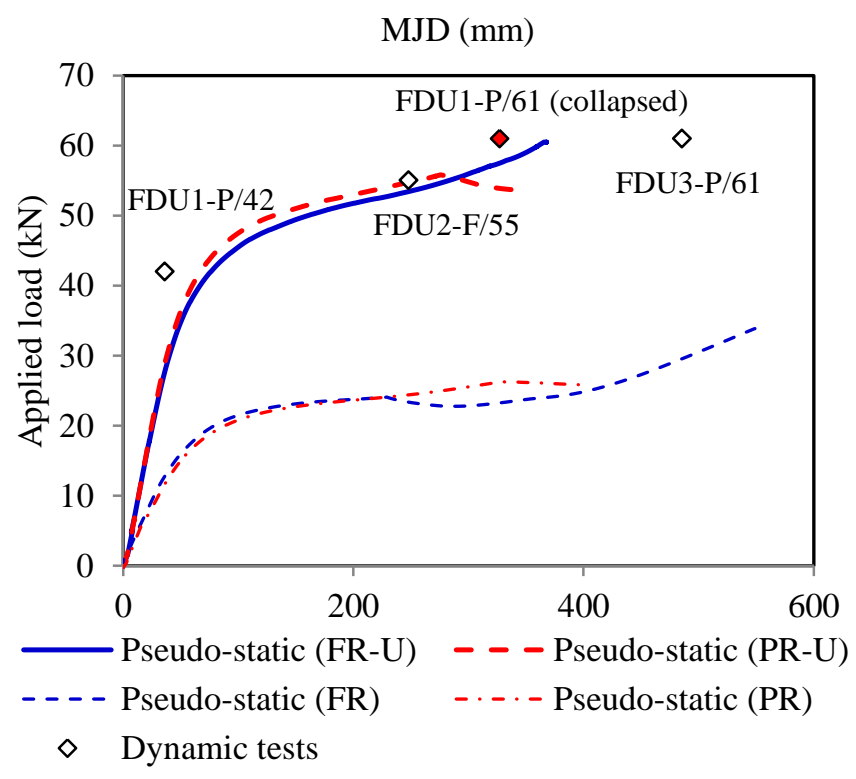

Fig. 7.20 Comparisons between pseudo-static predictions and actual dynamic tests 
Table 7.5 Predictions of dynamic capacities based on static tests

\begin{tabular}{|c|c|c|c|c|}
\hline \multirow{2}{*}{ Test } & \multicolumn{4}{|c|}{ Dynamic capacity (kN) } \\
\cline { 2 - 5 } & $\begin{array}{c}\text { At max. } \\
\text { CAA }\end{array}$ & $\begin{array}{c}\text { At "bottom } \\
\text { rebar fracture" }\end{array}$ & $\begin{array}{c}\text { At "1st top } \\
\text { rebar fracture" }\end{array}$ & $\begin{array}{c}\text { At "one-side top } \\
\text { rebars fracture" }\end{array}$ \\
\hline FR-U & 41.6 & N.A & 57 & 60 \\
\hline PR-U & 44 & N.A & 55.8 & 55.6 \\
\hline FR & 19.1 & 24.1 & \multicolumn{2}{c|}{$33.7 *$} \\
\hline PR & 19.1 & 26.3 & \multicolumn{2}{c|}{ N.A } \\
\hline
\end{tabular}

Note: N.A the top (or bottom) rebar fracture did not occur in the test

* all the top rebars fractured at the same time in FR

The "maximum MJD versus applied load" results from all the UDL dynamic tests were used to compare with dynamic responses predicted by Izzuddin's method as shown in Fig. 7.20. Among the three load levels applied in the UDL dynamic tests, $42 \mathrm{kN}$ was close to the predicted dynamic capacity at the first peak of CAA, while $55 \mathrm{kN}$ was almost equal to the ultimate dynamic capacity of both FR-U and PR-U, whereas $61 \mathrm{kN}$ was slightly larger than these load limits (Table 7.5 and Fig. 7.20). It is shown that the simplified Izzuddin's method provided reasonable and conservative results compared to the actual dynamic capacities at load levels of 42 and $55 \mathrm{kN}$. For the applied load of $61 \mathrm{kN}$, while FDU1-P/61 showed a complete collapse, FDU3-P/61 sustained this load and possessed a higher MJD capacity compared to the prediction from Izzuddin's method. However, similar to FD4$\mathrm{P} / 30.5$, FDU3-P/61 was conservatively considered as failed and the load level of 61 $\mathrm{kN}$ was considered to exceed the actual dynamic capacity of the specimen under UDL condition. Therefore, similar to the CL case, predictions from Izzuddin energy-based method under UDL configuration within CA stage should also be considered as the ultimate capacity that a structure can reach during the sudden column removal scenario.

\subsection{Conclusions}

In this chapter, quasi-static and free-fall dynamic tests under equivalent UDL condition were conducted and presented. Results from the UDL static tests were compared with the previous CL static tests on similar specimens. Besides, dynamic 
response caused by the actual sudden removal of a supporting column was also investigated in the UDL dynamic tests. The following conclusions are highlighted:

(1) The use of 4-point loading method for RC frames reasonably simulated the UDL condition in actual structures.

(2) Differences between behaviours from CL and UDL methods included loadcarrying capacity, sequence of failure (no bottom rebar fracture in UDL tests), deformation profile (linear in CL but parabolic in UDL), as well as the maximum deflection. The analytical calculation on UDL case confirms a $100 \%$ increase for vertical applied load but a 50\% reduction in maximum deflection compared to CL case. However, the two loading methods provided similar horizontal reactions for the same displacement. Moreover, at the state of top rebar fracture, UDL and CL methods had the same beam-end rotation. This can be used as the failure criterion for predictions. As a result, the semi-analytical model proposed in Section 4.3 can be conservatively applied to predict structural response under UDL condition (Fig. 7.13).

(3) CA, which was observed to significantly affect the structural response under CL condition, showed lesser degree of mobilisation under UDL condition, leading to weaker influence of boundary condition on structural response.

(4) Similar to the CL dynamic tests, the release device employed in the UDL dynamic tests could well simulate the sudden loss of a supporting column. Dynamic effects of applied loads were computed in comparison with the UDL static tests through structural displacement-based and force-based DIFs. The enhancement of materials due to strain rate effects was observed to be similar to the CL dynamic tests.

(5) The UDL dynamic tests displayed similar structural response, failure modes, and displacement profile compared to the corresponding UDL static tests, showing that the increase of loading rate from quasi-static $\left(10^{-4} / \mathrm{s}\right)$ to free-fall 
environment (up to $0.67 / \mathrm{s}$ ) did not change the mechanism or the failure mode. The dynamic tests, although following force-controlled manner, had relatively similar trend of reaction responses to those of the static tests. Therefore, it agrees with the conclusion in Chapter 6 for the conservatism of using nonlinear static analysis for designing structural behaviour under progressive collapse of 2D beam-column frames subjected to UDL.

(6) The conservatism of Izzuddin's method was confirmed once again in terms of UDL condition. Nonetheless, for engineering practice, the maximum load during CA stage calculated by this method should be considered as the ultimate capacity of a structure under sudden column loss scenarios. 


\section{Appendix 7A - Details of test results from Series II}

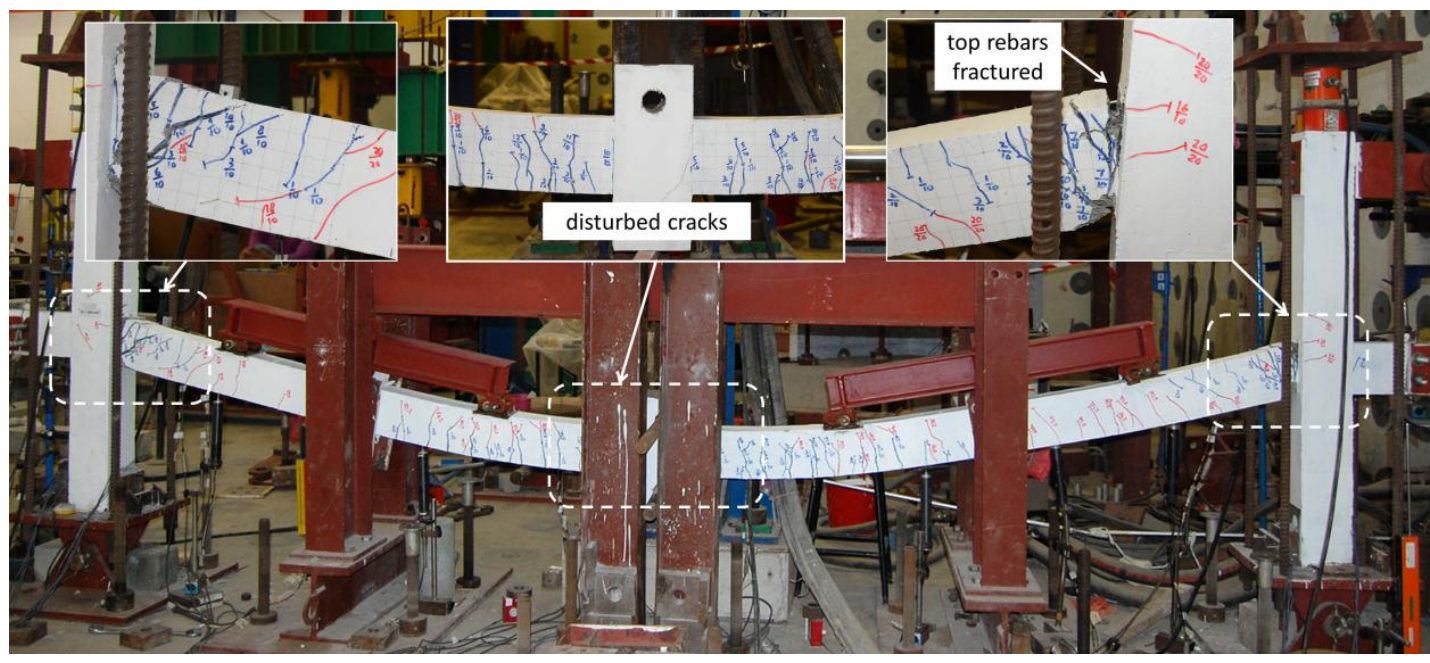

Fig. 7.1A: FR-U

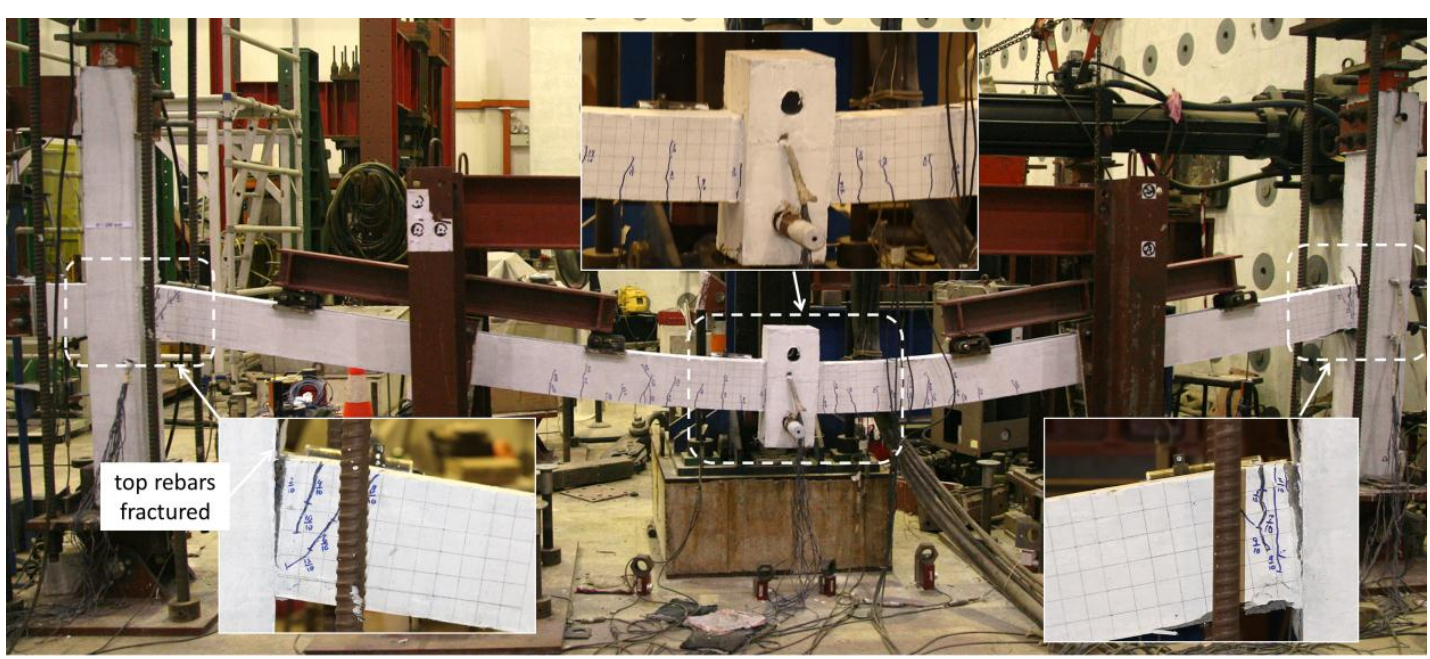

Fig. 7.2A PR-U

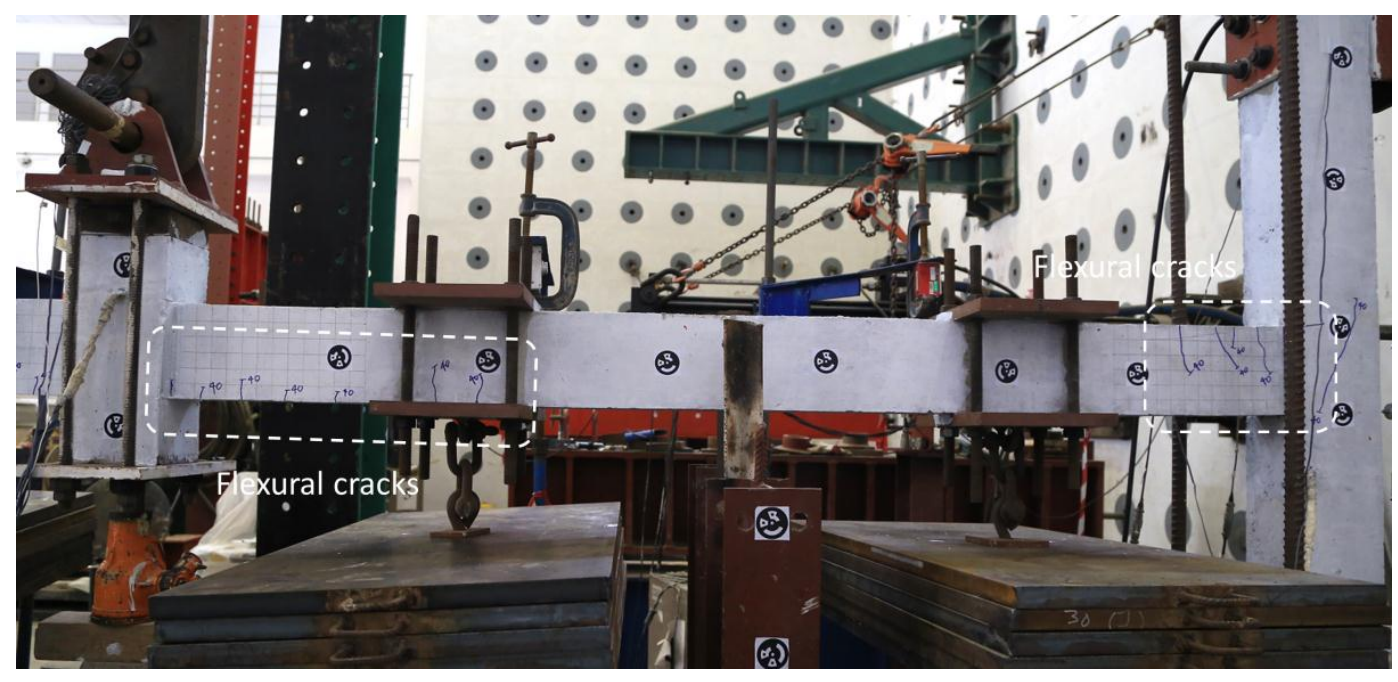

Fig. 7.3A FDU1-P/42 


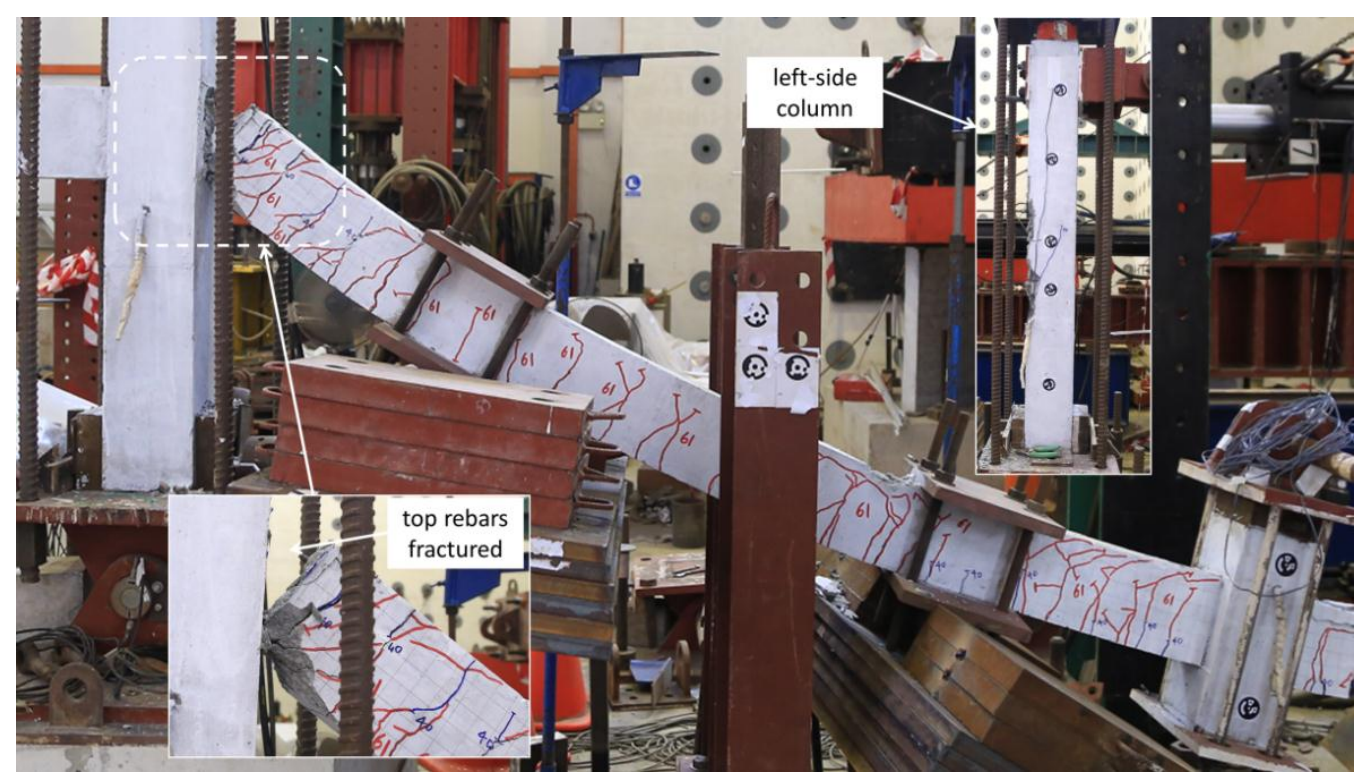

Fig. 7.4A FDU1-P/61

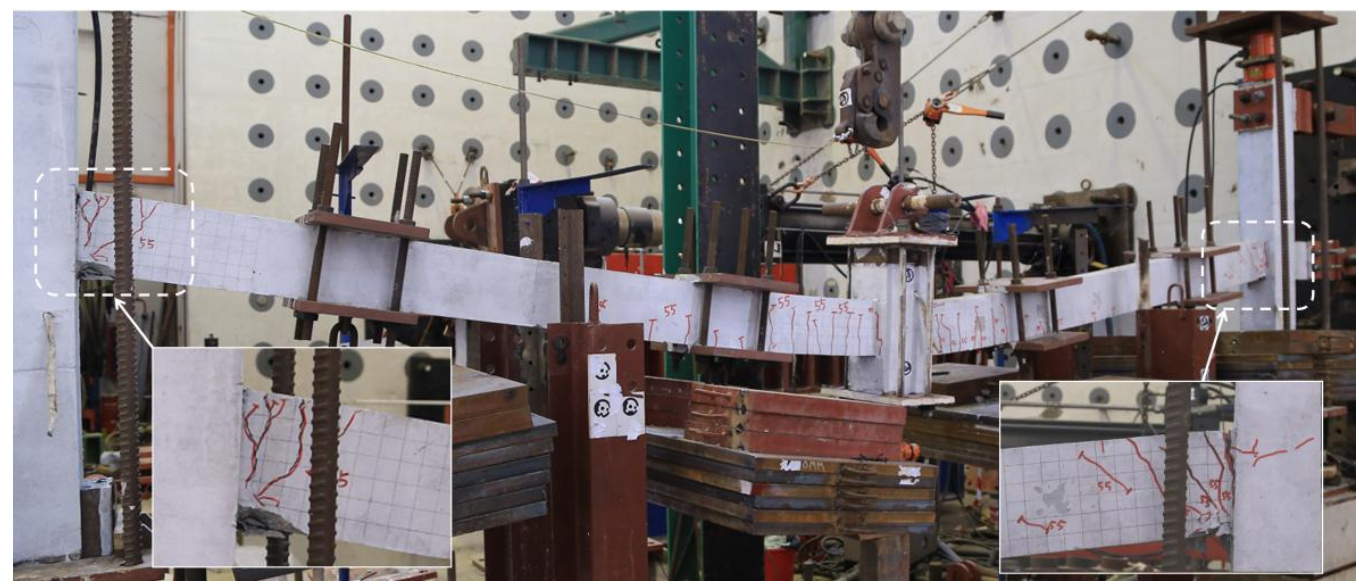

Fig. 7.5A FDU2-F/55

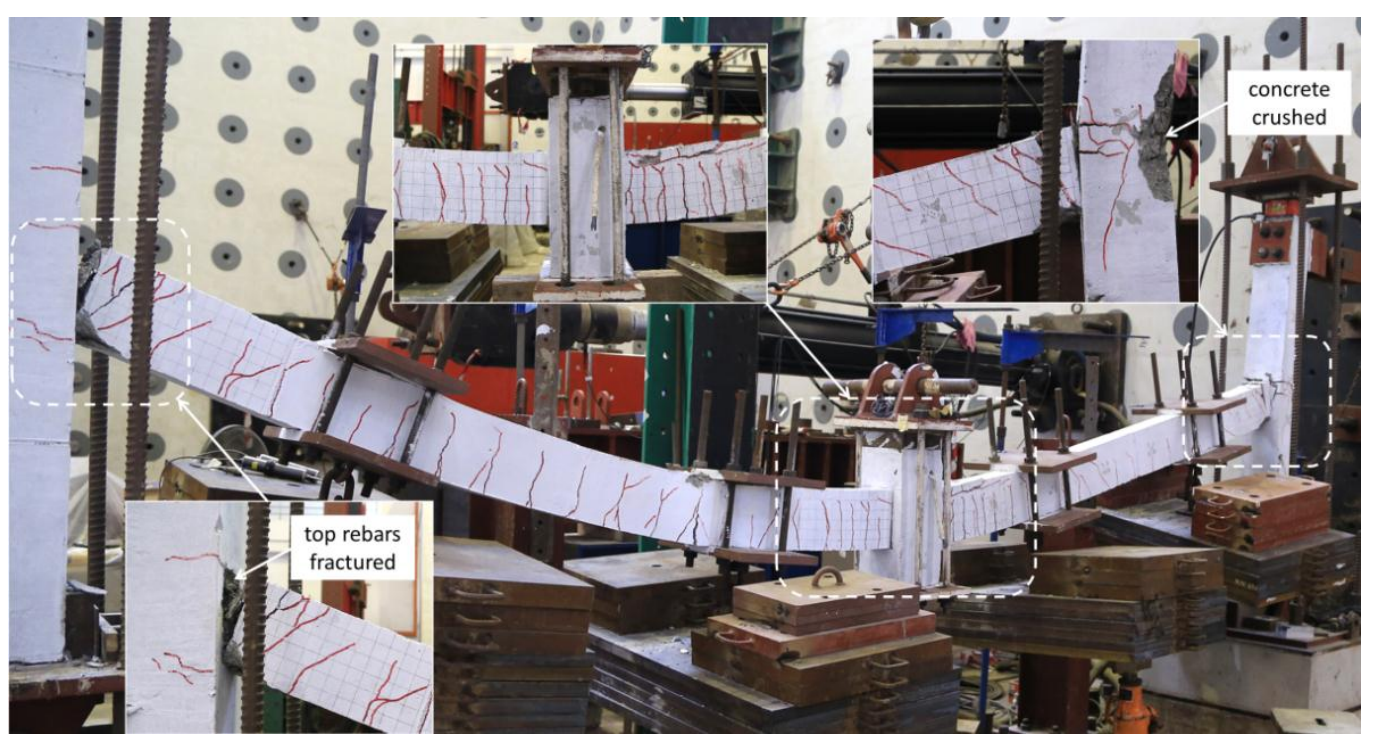

Fig. 7.6A FDU3-P/61 


\section{CHAPTER 8 BLAST-INDUCED RESPONCES OF RC FRAMES UNDER CL CONDITION (SERIES III)}

\subsection{Introduction}

Among the two approaches for direct design method specified in DOD (2013), alternate load path is preferred for assessing progressive collapse potential of structural buildings. It requires direct analyses of structural resistance against a threat-independent localised damage. By using the single column removal assumption, dynamic structural capacity of an RC beam-column system can be straightforwardly computed based on nonlinear static behaviour of a similar structure under the same loading configuration. Results from dynamic tests under both CL (Series I) and UDL (Series II) conditions show that the change of loading rate from quasi-static $\left(10^{-4} / \mathrm{s}\right)$ to free-fall dynamic environment (up to $0.67 / \mathrm{s}$ ) will not change the behaviour and failure modes obtained in static response, regardless of the load level applied in the dynamic regime. In addition, the use of Izzuddin's method for assessing dynamic capacity is shown to be reasonable in the context of notional column removal. Employing this approach, engineers can easily evaluate progressive collapse resistance of an RC beam-column structure without conducting any complicated nonlinear dynamic analysis. Nonetheless, when the initial event is induced by an actual explosive action, such as contact detonation causing a supporting column to be totally destroyed, the correctness and conservatism of this simplified method is questionable.

In this chapter, experimental results of contact detonation tests from Series III are presented and discussed. Two close-in blast tests described in Table 5.4 were conducted. Specimen design and test setup of this series are presented in Chapter 5. The dynamic and blast effects from the tests, including structural response and blast pressure, are addressed. Results from the blast tests are directly compared with those from the corresponding free-fall tests of Series I, as well as with the predictions using Izzuddin's method, to highlight the direct influence of contact detonation in a single column removal event. Thereafter, numerical modelling is 
conducted to simulate the blast tests. CONWEP data integrated into LS-Dyna is employed to compute the blast pressure, compared with actual recorded values. The FEM models for RC frames in Section 6.4.2 are employed incorporating the actual design of the blast tests, viz. column footings and boundary conditions. Blast simulation procedure for sub-assemblages in Section 3.2.7 is also applied in the FEM models.

\subsection{Test results}

\subsubsection{CD1-P/25/M}

\section{Overall responses}

Fig. 8.1, which is extracted from high-speed videos, presents images taken at different times of the blast test CD1-P/25/M. At time $t=0 \mathrm{~ms}$, the explosive attached at the bottom end of the middle column was electrically triggered and immediately blew away the temporary brick support. The fire ball created by the detonation almost covered the specimen within the first $20 \mathrm{~ms}$ and totally ceased at $t=50 \mathrm{~ms}$. After the middle support had been totally destroyed, the double-span beam started deflecting. This downward movement became visible at $t=80 \mathrm{~ms}$. From $t=440 \mathrm{~ms}$ onwards, the middle joint was observed to start rotating significantly, implying that bottom rebars from one side of the joint had fractured. At $t=660 \mathrm{~ms}$, the partially-restrained column on the right side started moving inwards remarkably. Finally, the specimen hit the ground below at a time of $t=789 \mathrm{~ms}$. Fig. 8.2(a) shows the time-history of the MJD with a small uplift within the first $20 \mathrm{~ms}$ due to blast effects. The maximum upward deformation was $2.5 \mathrm{~mm}$. Thereafter, the specimen started moving downwards. At the duration from 0.308 to $0.381 \mathrm{~s}$, some minor distortions were observed in the displacement response, probably due to some dust or debris blocking the laser sensor D1 measuring the MJD. The specimen hit the ground at a MJD of $616 \mathrm{~mm}$. Acceleration time-history of the middle joint is presented in Fig. 8.2(b). Although the acceleration response was not a primary objective of the study, it could provide a quantitative account of the event during the test. There was a sudden peak at the beginning with acceleration values reaching $1000 \mathrm{~g}$ due to the effect of shock wave 
from the blast propagating through the middle column. The duration of this first peak was about $20 \mathrm{~ms}$. Afterwards, the acceleration became very small before rising up again when the specimen hit the ground at around $780 \mathrm{~ms}$. Within these two peaks, at 440 and $560 \mathrm{~ms}$, noticeable variations of the acceleration were observed (up to $50 \mathrm{~g}$ ), probably due to fracture of top/bottom rebars. Compared to the accelerations recorded from the free-fall tests of Series I (Fig. 6.5) as well as from other reported experiments (Qian and Li 2012b, 2014), the acceleration of CD1$\mathrm{P} / 25 / \mathrm{M}$ was much greater in magnitude at the initial stage of the blast event.

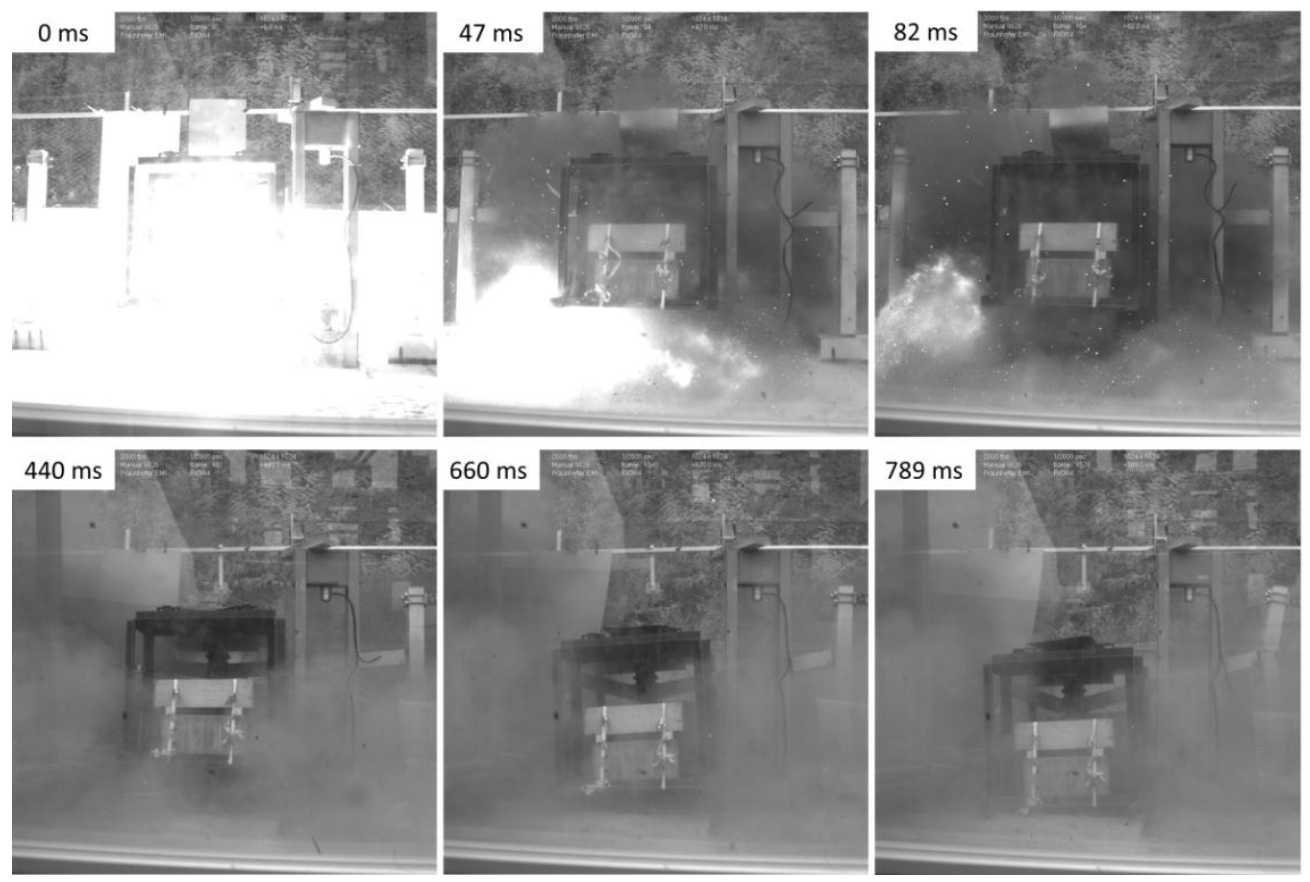

Fig. 8.1 Blast test procedure of CD1-P/25/M

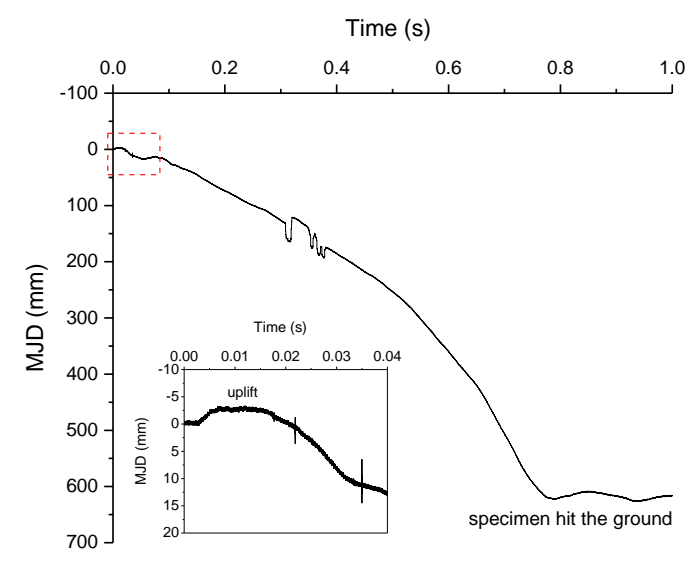

a) Displacement time-history

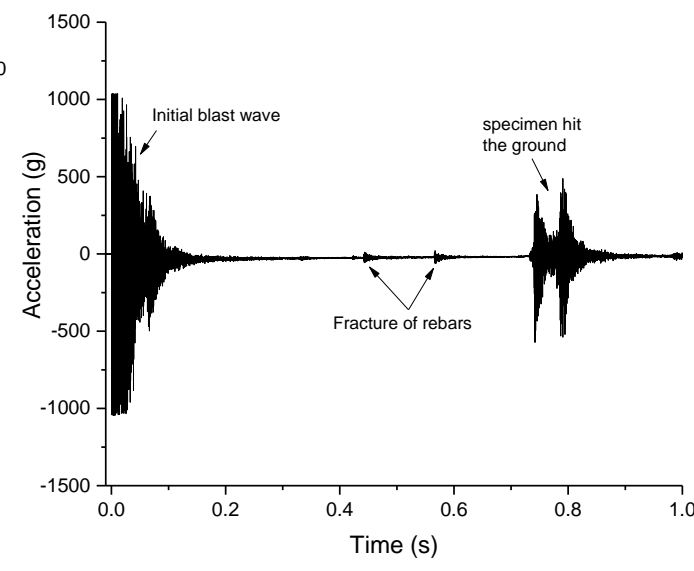

b) Acceleration time-history

Fig. 8.2 Response of the middle joint (CD1-P/25/M) 
Fig. 8.3 presents the failure modes of the specimen after the test, showing that the bottom rebars had fractured at the middle joint whereas the top rebars at the leftside end joint had also fractured. The right-side end joint underwent excessive inward movement, causing concrete crushing at the compression outer face of the column and severe crack openings at the inner face. At the bottom end of the rightside column, flexural cracks were observed denoting that the column bending moment had reached yielding at this location (Fig. 8.4). These damages, which were not observed in the quasi-static and the free-fall dynamic tests, came from the fixed boundary condition of the side column. Unlike SD-3 in Yu et al. (2014) where only bottom rebar fracture had occurred, the damages observed in CD1-P/25/M conclude that the specimen had already failed under the applied load before hitting the ground. Besides, failure modes of CD1-P/25/M agreed well with those from the corresponding static (Lim et al. 2015) and free-fall dynamic (Chapter 6) tests.

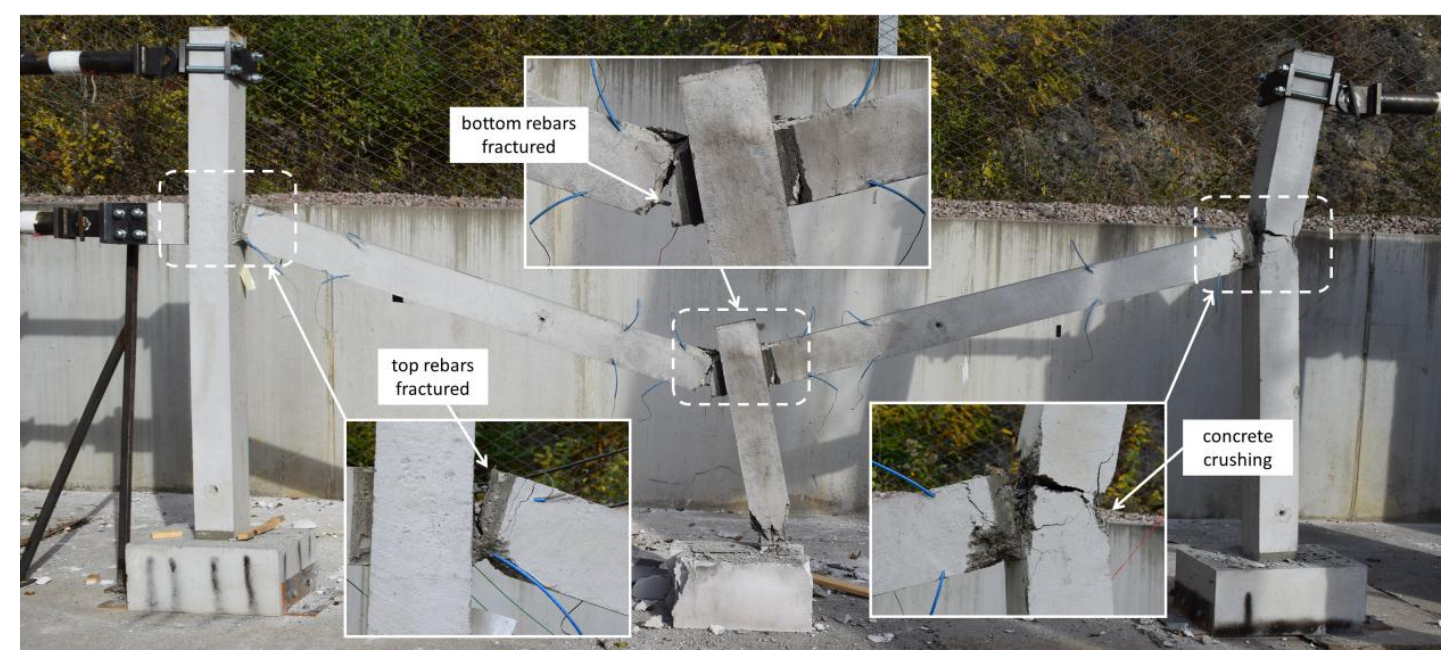

Fig. 8.3 Damages and failure modes of CD1-P/25/M 


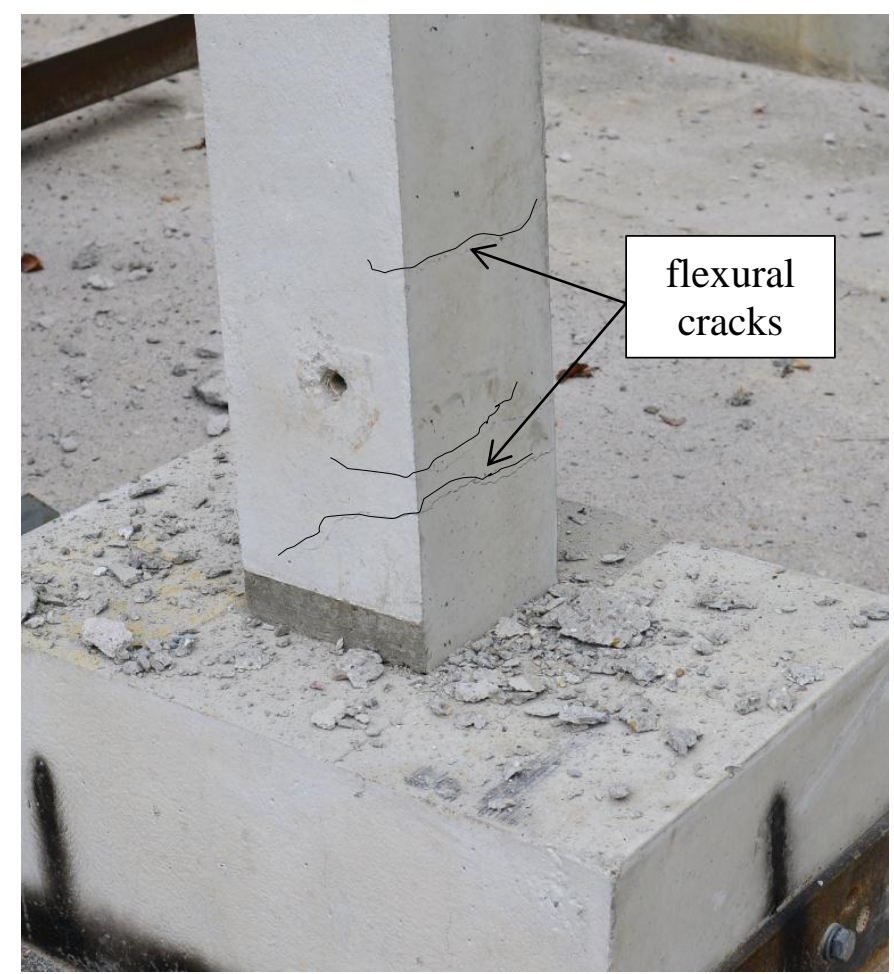

Fig. 8.4 Damages at the bottom of partially-restrained column

Effects of the blast on surviving structural members are investigated via readings from pressure gauges attached to the left-side beam and column, presented in Fig. 8.5. The blast wave arrived at the beam pressure gauge at $t=1.37 \mathrm{~ms}$ with a positive duration of $0.27 \mathrm{~ms}$, earlier than the blast pressure at the side column (arrival time of $t=3.16 \mathrm{~ms}$ and a positive duration of $0.9 \mathrm{~ms}$ ). As the spherical charge was placed about $900 \mathrm{~mm}$ above the ground, there were clearly two peaks of positive pressures for both the beam and the column gauges. The first peak was from free air-burst pressure directly coming from the detonation, while the second was from ground reflection. Due to the position of the charge with respect to the pressure gauges, the peak from the ground reflection was quite obvious compared to the peak from the free air-burst in the column pressure-gauge readings. However, for the beam pressure gauge, this second peak is insignificant compared to the first one. In other words, the free air-burst pressure can be conservatively used to calculate the blast pressure acting on the double-span beam. 


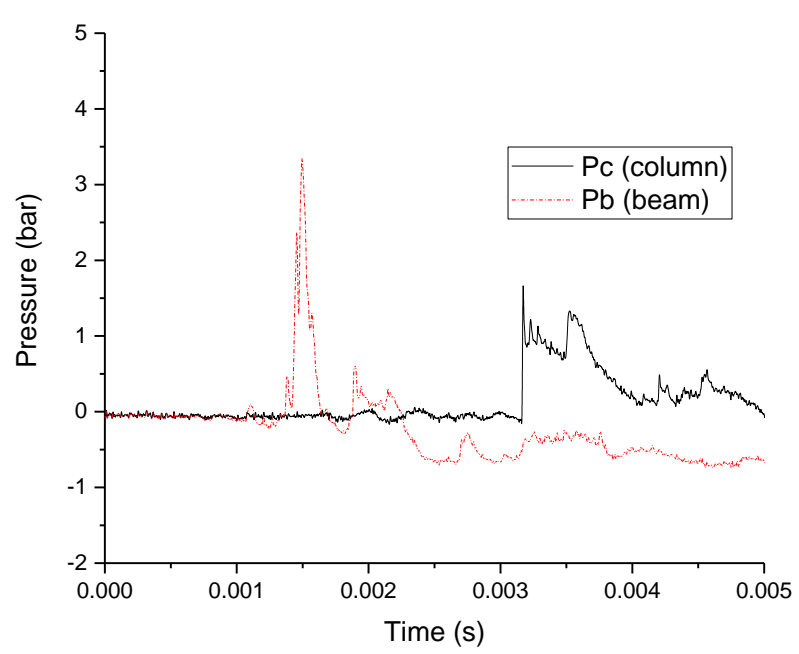

Fig. 8.5 Blast pressures on beam and column

\section{Horizontal reaction and material strain}

Strain gauges attached on the horizontal steel-tube support were used to compute the reaction force of the upper restraint at the right side of the specimen as shown in Fig. 8.6. Within the first few milliseconds, there were some distortions caused by the shock wave propagating from the detonation. After all the blast effects had terminated and as the specimen moved downwards, the horizontal restraint showed negative values denoting the CAA stage of response. Thereafter, it gently changed to tension. At $440 \mathrm{~ms}$, a sudden drop occurred indicating the fracture of bottom rebars at the middle joint. The reaction force reached the final peak of $86 \mathrm{kN}$ at 660 ms and slowly reduced afterwards. This reduction started becoming significant at $720 \mathrm{~ms}$ and the reaction force reduced to almost $30 \%$ of its maximum value at 750 ms demonstrating that the frame specimen had lost most of it load-bearing capacity. The decrease of horizontal reaction force, together with the failure modes observed after the test (Fig. 8.3), clearly show that the specimen had totally failed under the blast-induced collapse event. At $780 \mathrm{~mm}$, distortions occurred in the reading as the specimen hit the ground.

To further investigate the mobilisation of CAA and CA in the double-span beam, readings from LVDT D5 (Fig. 5.20) which showed horizontal movement of the leftside end joint was plotted in accordance with the MJD, as described in Fig. 8.7. 
When the MJD was still small, the end joint was moving outwards with small values of deformation, showing that the mobilisation of CAA in CD1-P/25/M was low. Afterwards, the end joint started moving inwards when the MJD exceeded 180 $\mathrm{mm}$, i.e. one beam depth, denoting the development of CA stage. There was a sudden drop in the reading at a MJD of $260 \mathrm{~mm}$ indicating the fracture of bottom rebars at the middle joint. This rebar-fracture displacement agreed well with both the quasi-static tests (Fig. 2.9(b)) and the free-fall dynamic tests (Fig. 6.16(b)).

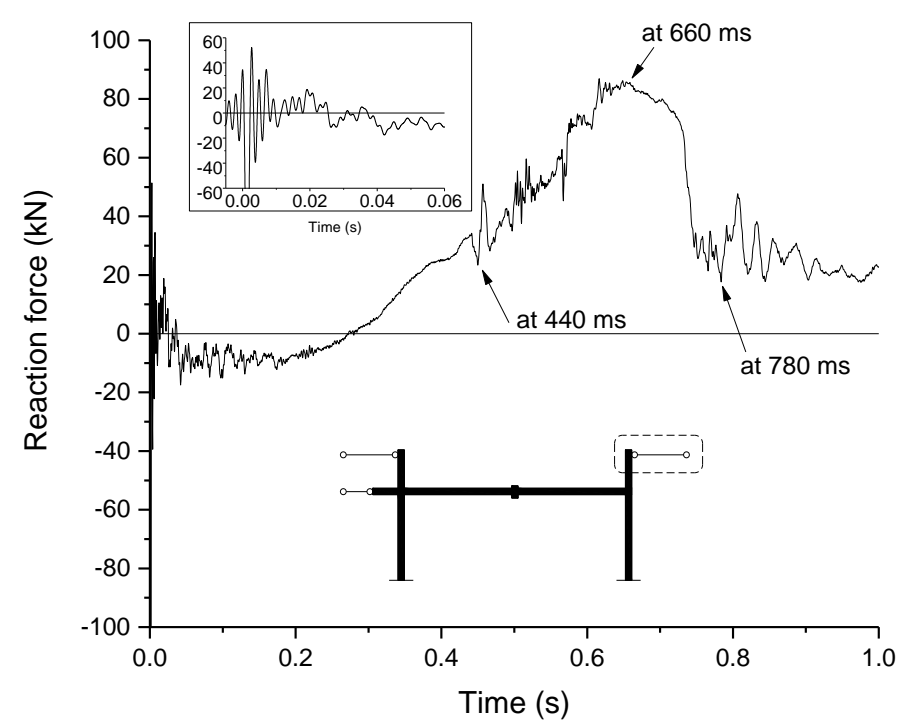

Fig. 8.6 Horizontal reaction (upper right side)

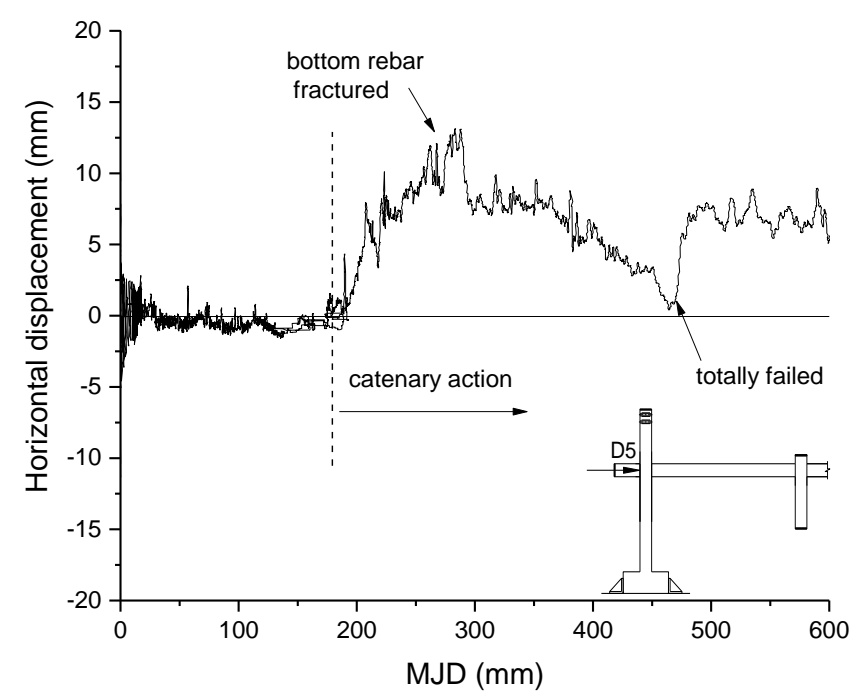

Fig. 8.7 Horizontal displacement (left-side end joint) 
To investigate the response at cross-sectional level, strains of bottom rebars in the double-span beam are plotted in Fig. 8.8. After the blast duration, rebars B1 and B2 were in compression showing a development of negative bending moments at Sections 1 and 2. At the same time, B3 and B4 were in tension showing positive bending moments occurring at Sections 3 and 4 near the middle joint. After the middle support was removed, B4 quickly exceeded the yield value whereas B3 remained elastic until the end of the test. At $440 \mathrm{~ms}$, there was an obvious change in the strain values due to the fracture of bottom rebars near the middle joint.

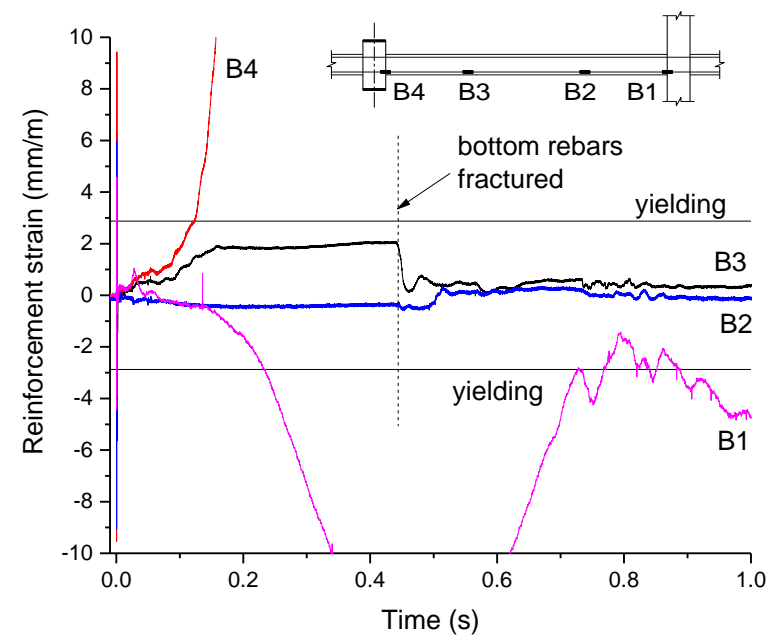

Fig. 8.8 Bottom reinforcement strains (CD1-P/25/M)

Reinforcement strains at the two critical sections, i.e. Sections 1 and 4 , are presented in Fig. 8.9(a) while Fig. 8.9(b) shows the strain rates of tensile rebars T1 and B4 of these two sections. Due to initial damages from the detonation, reading from T4 of Section 4 was unreliable and therefore is not mentioned herein. As the MJD kept moving downwards, strain readings of T1 and B4 quickly exceeded the yield value whereas bottom rebar B1 at Section 1 was always in compression during the whole test. Regarding tensile-rebar strain rates, there was a peak of up to hundred /s during the very early stage of blast duration. The strain-rate peak of B4 $(150 / \mathrm{s})$ was considerably larger than that of T1 (35/s) due to a closer distance of the former to the charge. Nonetheless, this duration was very short (less than $5 \mathrm{~ms}$ ). After the blast event had finished and as the specimen started behaving under the gravitational free-fall stage, the maximum strain rates recorded for the yielding and 
the plastic stages of the rebars were $1 / \mathrm{s}$ and $5 / \mathrm{s}$, respectively. Based on this strain rate values, DIF for the yield and the ultimate strengths of reinforcement were equal to 1.21 and 1.08 , respectively. They were not noticeably larger than those in the free-fall tests of Series I (Table 6.2).

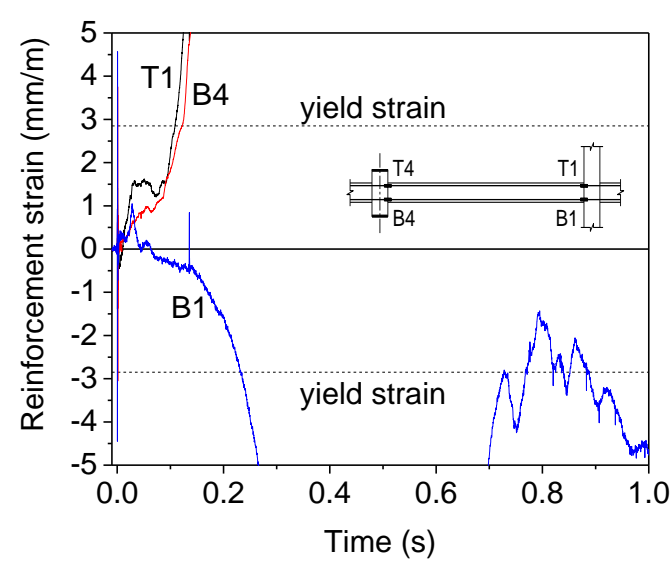

a) Reinforcement strains

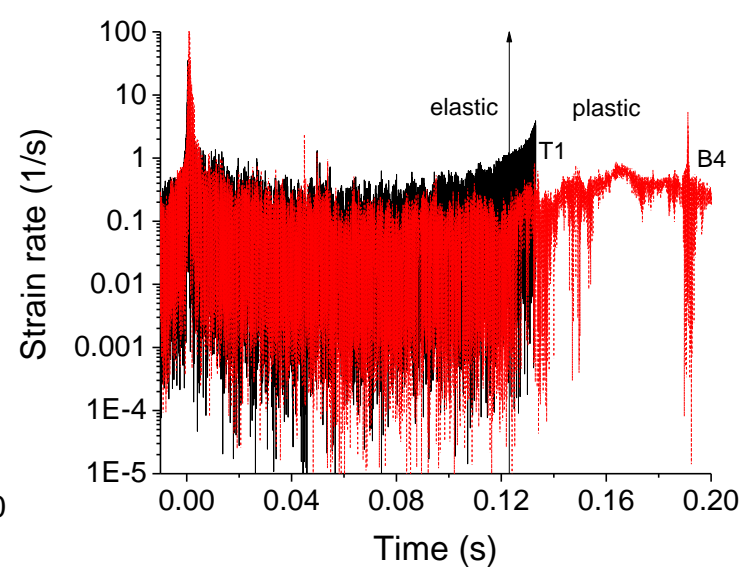

b) Reinforcement strain rates

Fig. 8.9 Reinforcement strains and strain rates

\subsubsection{CD2-F/20/M}

\section{Overall responses}

In the second blast test, due to a smaller applied load, the specimen survived the column loss event with a maximum MJD of $58 \mathrm{~mm}$ and a residual deformation of $45 \mathrm{~mm}$ as shown in Fig. 8.10(a). Based on logarithmic decrement technique, damping ratio of the vibration stage was computed to be $5 \%$, which was identical to that from SD-2 of Yu et al. (2014). Similar to the first test, there was a peak in accelerations during the initial duration of the blast event (Fig. 8.10(b)). Afterwards, there was no other acceleration peak showing that failure had not occurred. Damages and crack patterns after the blast test are shown in Fig. 8.11. At the beam regions near the end joints, there were several top-surface cracks propagating from the beam-column interface to the curtailment point at Section 2. At two sides of the middle joint (Section 4), bottom-surface cracks were also observed. However, there was no sign of critical failures such as concrete crushing or rebar fracturing. Besides, the side columns showed no obvious cracks or damages due to either the blast effect or the subsequent free-fall event. Under the small 
applied load of $20 \mathrm{kN}$, damages and cracks observed from CD2-F/20/M showed reasonable agreement with those from the corresponding free-fall test FD1/F/20. Readings from the pressure gauges showed similar values with the first blast test since both tests shared the same charge weight of $M=0.4 \mathrm{~kg}$ PETN explosive. The two blast tests also had similar damages of the middle column caused by the contact detonation (Fig. 8.12). The bottom end of the middle column was severely damaged after the blast, sharpened as a cone shape. The length of the damaged part was nearly 1.2 times the column size. Moreover, hairline cracks were found along the middle column due to shock wave propagation.

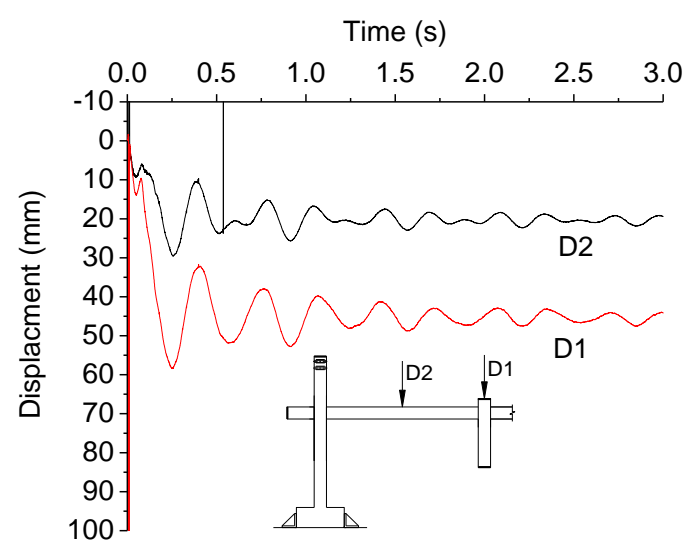

a) Displacement time-history

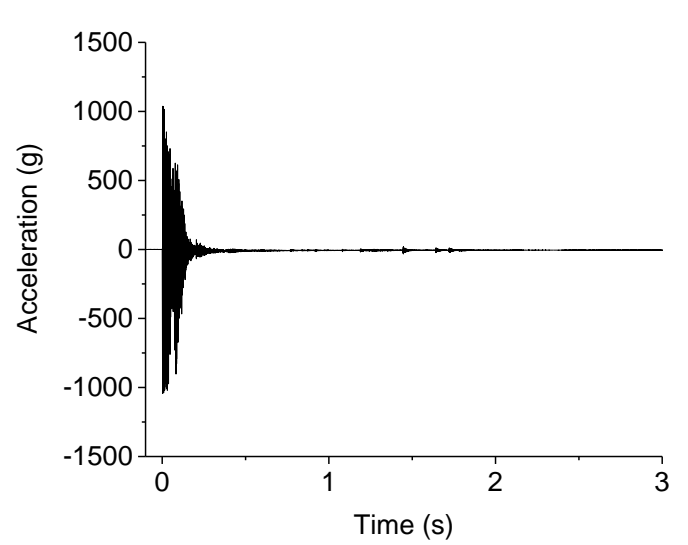

b) Acceleration time-history

Fig. 8.10 Response of the middle joint (CD2-F/20/M)

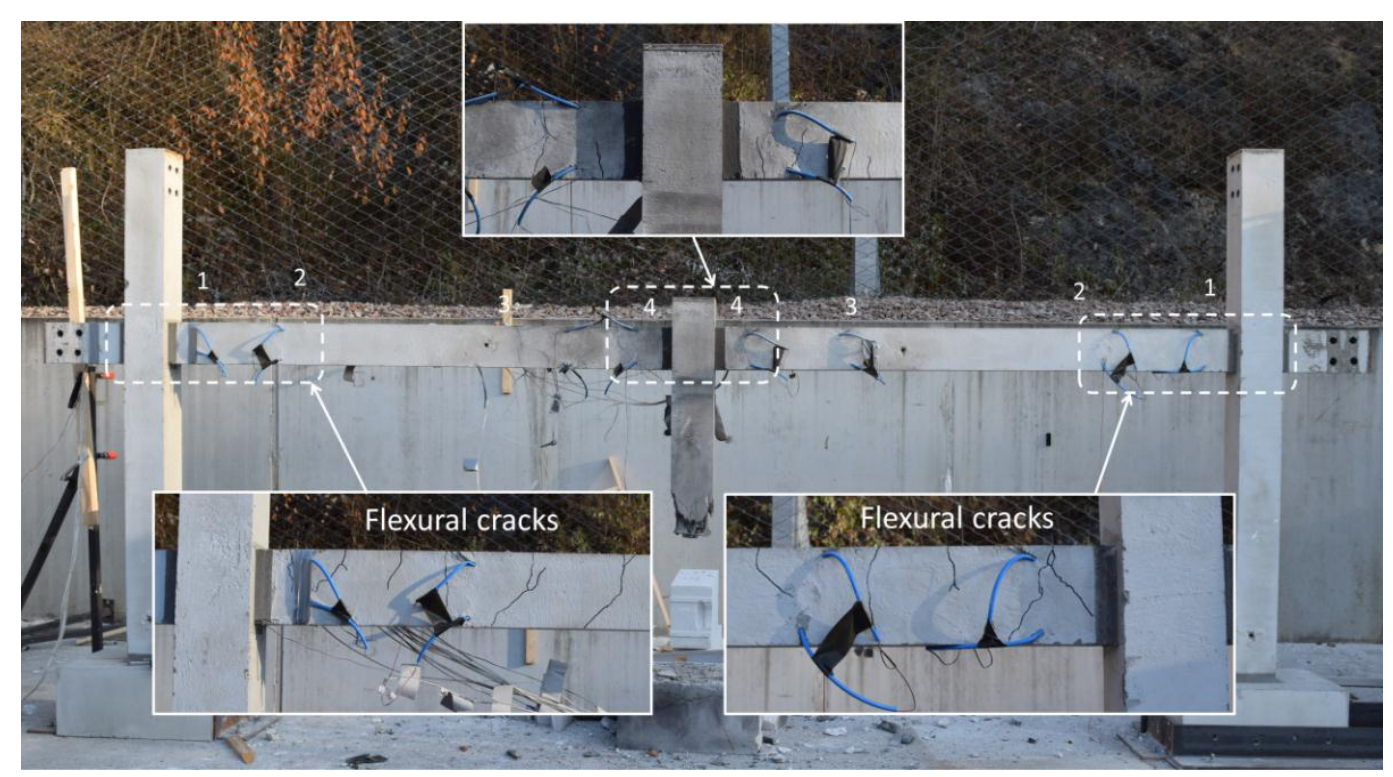

Fig. 8.11 Damages and failure modes of CD2-F/20/M 


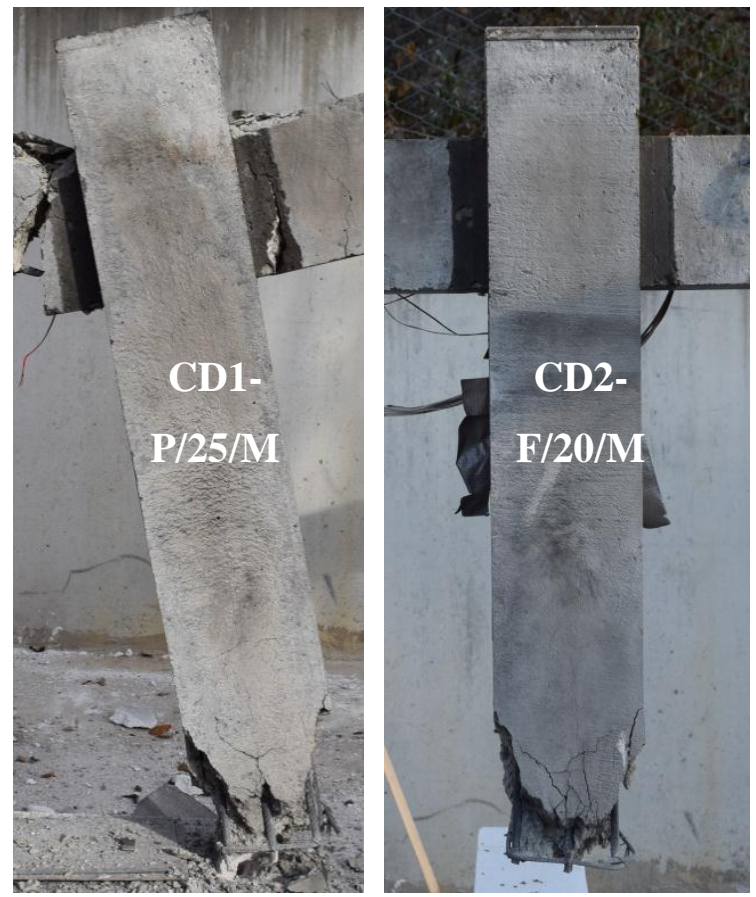

Fig. 8.12 Damages of middle column

\section{Horizontal reaction and material strain}

Total horizontal reaction from one side of the specimen (including the upper and the lower horizontal supports) is presented in Fig. 8.13. Similar to the first blast test (Fig. 8.6), reading distortions were observed at the beginning due to shock wave propagation. After the blast duration, the horizontal reaction was in compression at the early stage and then changed to tension at $270 \mathrm{~ms}$. That is to say, although the MJD had not yet reached one beam depth, total horizontal reaction from the upper and the lower horizontal supports had already changed to tension. This was the main difference in response between the frame structures in Series III (blast tests) and those in Series I (free-fall tests). Based on the reaction forces and displacements measured at the side columns, stiffness of the upper and the lower horizontal restraints was equal to $6000 \mathrm{kN} / \mathrm{m}$. 


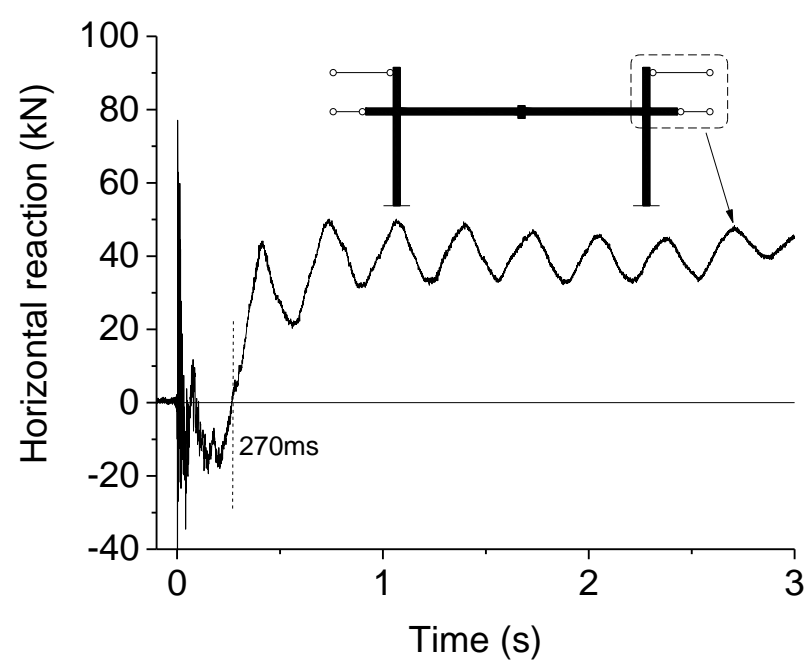

Fig. 8.13 Horizontal reaction

Readings from the concrete strain gauge near the middle joint (CS1) is shown in Fig. 8.14(a). At the early stage of first $20 \mathrm{~ms}$, there was a positive value showing tension of concrete at beam top surface due to uplift movement of the middle joint. Afterwards, the concrete strain became negative and reached a maximum value of $1.75 \mathrm{~mm} / \mathrm{m}$ (or 0.00175 ), which was smaller than the maximum compressive strain of concrete material. As a result, there was no concrete crushing occurring at the middle joint region. Fig. 8.14(b) presents the time-histories of the top rebar strains of the double-span beam. It shows that top rebars at Sections 1 and 2 (T1 and T2) had already exceeded yielding, illustrating the propagation of top-surface cracks within these two sections (Fig. 8.11).

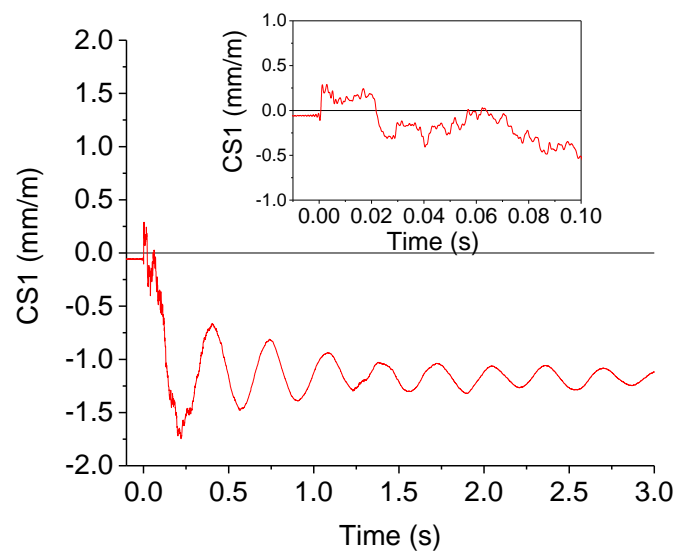

a) Concrete strains

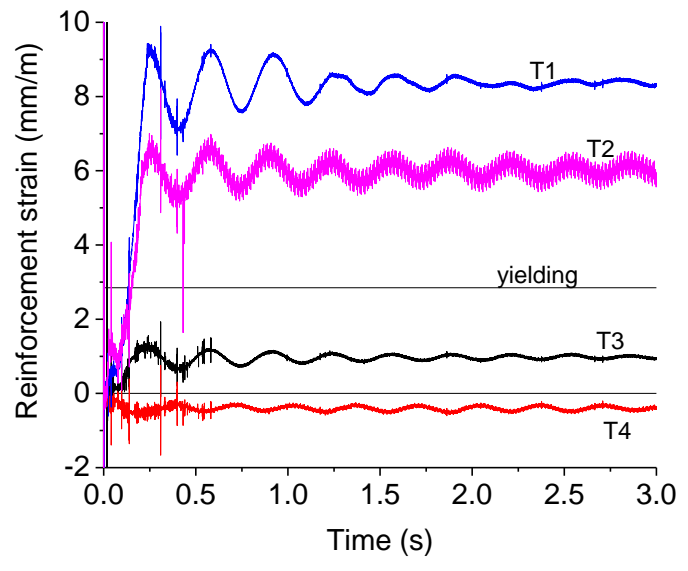

b) Reinforcement strains

Fig. 8.14 Concrete and reinforcement strains 


\subsection{Comparisons between free-fall and blast-induced dynamic tests}

By comparing the results from the blast tests to those from the corresponding freefall tests, the reduction in structural capacity due to blast effects is highlighted. The maximum deformations from the blast tests are also compared with those predicted using Izzuddin's method to verify the usefulness of this simplified approach in the context of blast-induced scenarios.

\section{FD1-F/20 and CD2-F/20/M}

Both the free-fall (Table 6.1) and the blast-induced (Fig. 8.10(a)) dynamic tests only responded with small deformations under the same applied load of $20 \mathrm{kN}$. However, the maximum deformation from the blast test $(58 \mathrm{~mm})$ was twice that of the free-fall test $(25.9 \mathrm{~mm})$. Moreover, beam top rebars in the blast test had yielded at both Sections 1 and 2 (Fig. 8.14(b)), whereas in the free-fall test, only the top rebars at Section 1 had almost reached the yield value (Fig. 6.7(a)). Regarding Izzuddin's method, it still provided conservative predictions compared to the actual blast test (Fig. 8.15(a)). That is to say, this approach could provide remarkably conservative predictions even under the blast-induced condition, provided that the structure only responds within flexure/CAA mechanism. This conclusion agrees well with those obtained from the free-fall tests of Series I and II.

\section{FD3-P/25 and CD1-P/25/M}

While the free-fall test of FD3-P/25 only showed small deformations with the maximum MJD of $52.4 \mathrm{~mm}$ (Table 6.1) and no major damages had occurred (Fig. 6.6(f)), the blast test of CD1-P/25/M caused a complete collapse with fracturing of top and bottom reinforcing bars (Fig. 8.3). Compared to the prediction from Izzuddin's method (Fig. 8.15(b)), the applied load of $25 \mathrm{kN}$ was still smaller than the maximum dynamic capacity of the partially-restrained structure. That is to say, while Izzuddin's method give conservative predictions for the free-fall tests of FD3$\mathrm{P} / 25$, it overestimated the result of $\mathrm{CD} 1-\mathrm{P} / 25 / \mathrm{M}$ under $\mathrm{CA}$ stage caused by a detonation. 


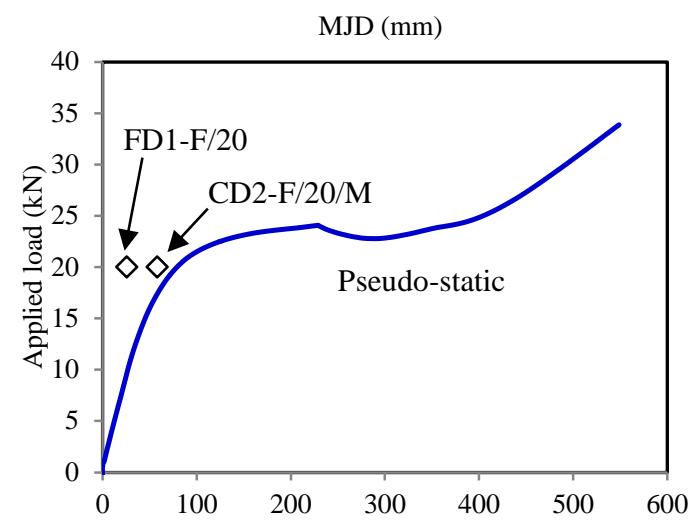

a) Full-restraint

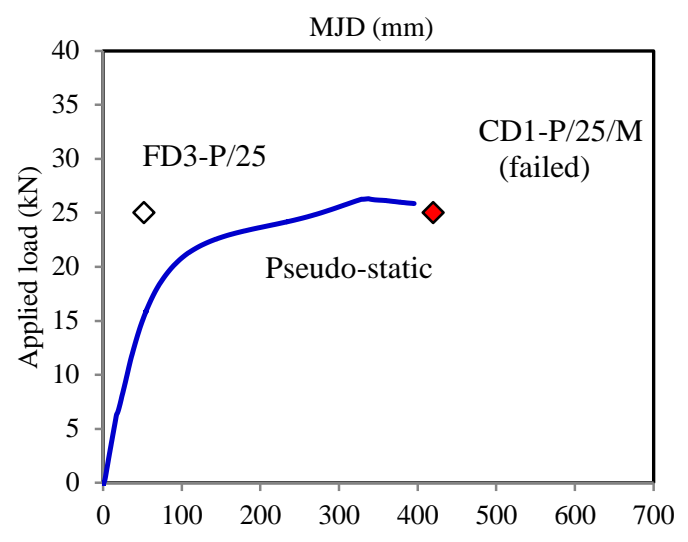

b) Partial-restraint

Fig. 8.15 Validations of Izzuddin's method with blast tests

\subsection{Numerical validations for the blast tests}

\subsubsection{Verification of blast pressure using CONWEP}

Due to the complexity of structural response when blast effects are involved, only 3D physics-based numerical models are suitable for simulating the progressive collapse blast-induced tests (Pham et al. 2016). In this chapter, numerical models in Section 6.4.2 for full- and partial-restraint frames are modified including the extended parts of both the side and the middle columns (Fig. 8.16). The concrete footing at the end of the side column is also considered. The footing is modelled with pinned supports at the locations where it was bolted to the concrete ground. The $0.4 \mathrm{~kg}$ PETN charge weight is converted into an ideal TNT explosive with a weight of $0.508 \mathrm{~kg}$ to provide equivalent weight pressure (Krauthammer 2008). Both the spherical free air-burst and the spherical air-burst with ground reflection scenarios for blast pressure are considered. It is shown that the latter can generate a second peak of positive pressure, but overestimates total incident impulse compared to the actual tests (Fig. 8.17(a)). This is because, in the CONWEP model for blast pressure, the ground is considered to be perfectly rigid and reflects all the shock wave originated from the charge. However, in the actual tests, the concrete ground below the specimens could partially absorb some energy from the shock wave. On the other hand, although the spherical free air-burst option cannot provide the second peak, it gives better agreement with the measured blast pressure in terms of 
total impulse (Fig. 8.17(b)). It also shows that the use of free air-burst explosive for validation of blast-induced tests on sub-assemblages presented in Section 3.2.7 is reasonable. Therefore, this blast-pressure scenario is employed in the FEM models to simulate the blast tests of Series III. The distribution of incident pressure on the bottom surface of beam and the side surface of column is shown in Fig. 8.18(a) and (b), respectively. Blast pressure arrives at the beam soffit much earlier than at the column side with a much larger magnitude. However, its distribution along the double-span beam is not uniform due to the position of the beam with respect to the charge. On the other hand, blast distribution on the column side is much more uniform in terms of magnitude and duration.

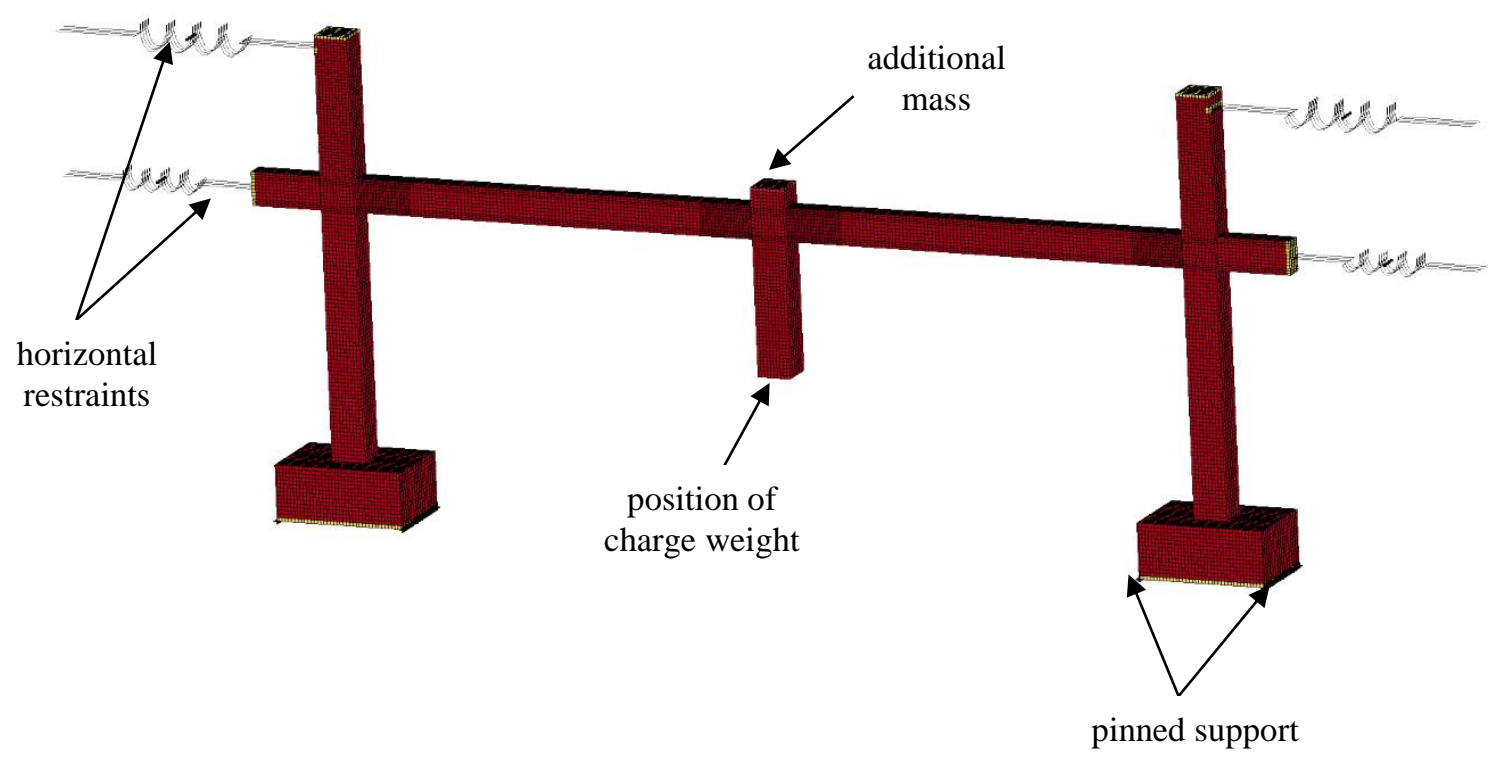

Fig. 8.16 FEM model for blast test validation (full-restraint)

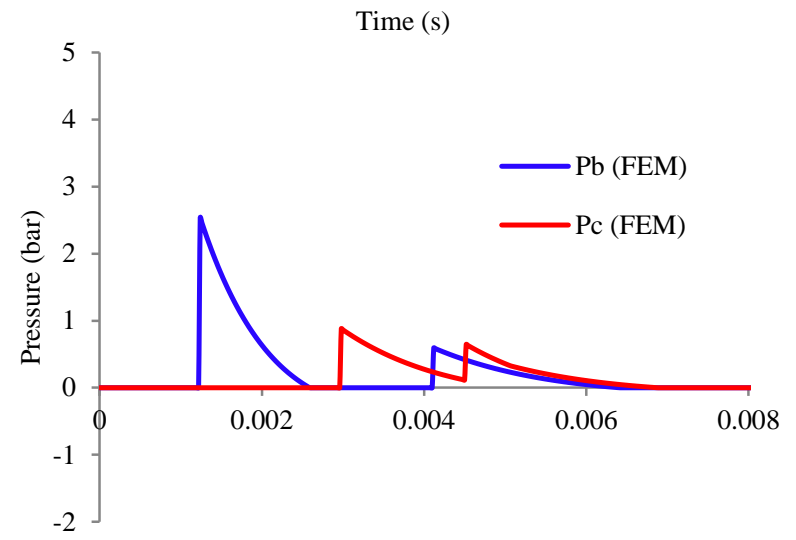

a) FEM results using air-burst with ground reflection scenario 


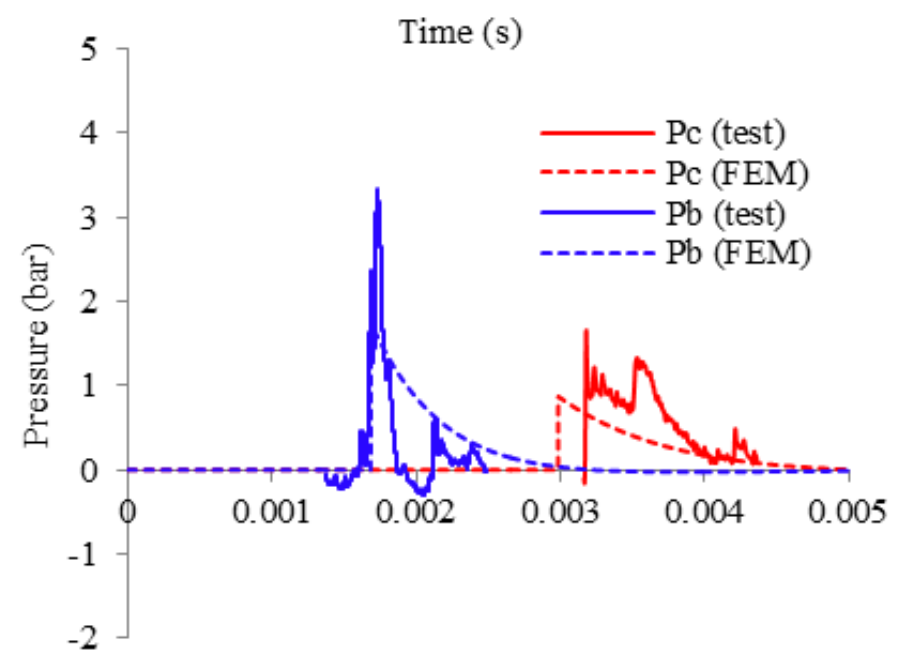

b) FEM results using free air-burst scenario

Fig. 8.17 Validation of blast incident pressure using CONWEP data (Pc for column pressure and $\mathrm{Pb}$ for beam pressure)

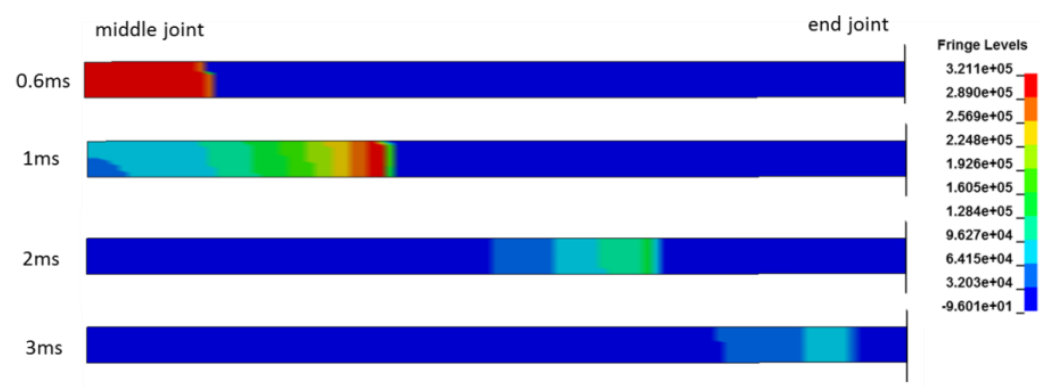

a) On beam soffit

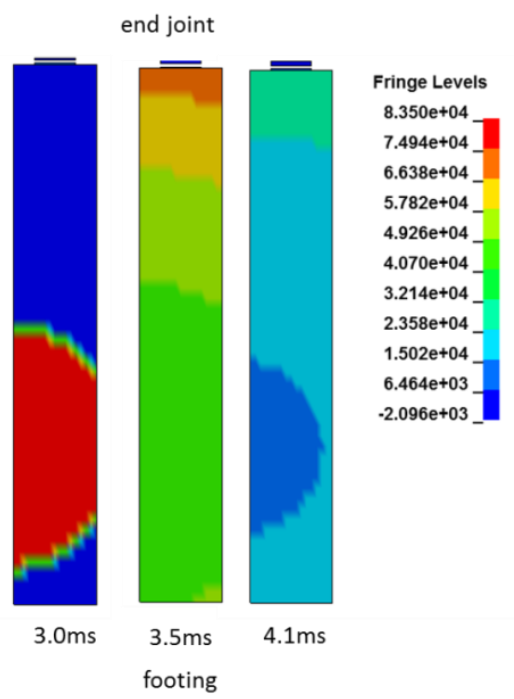

b) On side column

Fig. 8.18 Distribution of incident pressure $\left(\mathrm{N} / \mathrm{m}^{2}\right)$ 


\subsubsection{Validation results}

Parameters of the blast simulations used in this part (damping ratio, strain rate effect, etc.) apply the same criteria as those from the blast-test simulations in Chapter 3 for sub-assemblages. Two analyses are conducted to replicate the two blast tests of Series III. Gravitational action from the imposed load is immediately applied after the specimen reaches the maximum uplift due to the blast effect. The numerical results are presented in Fig. 8.19. In general, the responses of MJD in both tests are well captured in the FEM models.

Damages and failure mode in the model for CD1-P/25/M (Fig. 8.20) follow those observed in the test, including fracture of beam rebars, excessive inward movement and damages of the right-side end joint, and concrete damages at the bottom end of the partially-restrained side column. For CD2-F/20/M model, damage patterns of the simulation are presented in Fig. 8.21, showing relatively good agreement with the cracking zones in the actual test (Fig. 8.11 and Fig. 8.12). Damages of the middle column under contact detonation are similar to the actual test, showing the length of the damaged part of more than one column size.

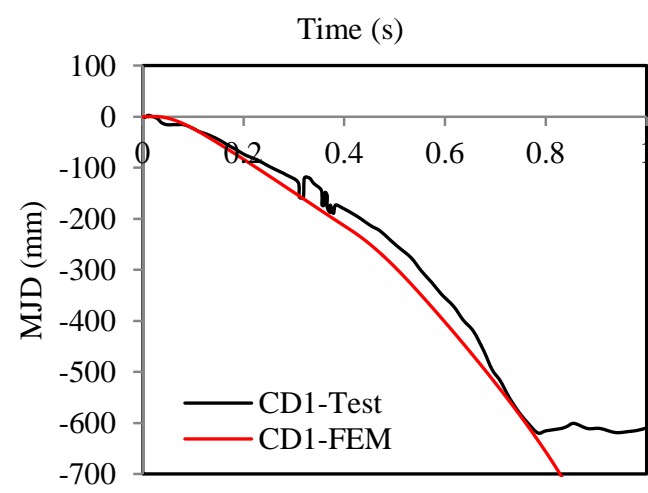

a) $\mathrm{CD} 1-\mathrm{P} / 25 / \mathrm{M}$

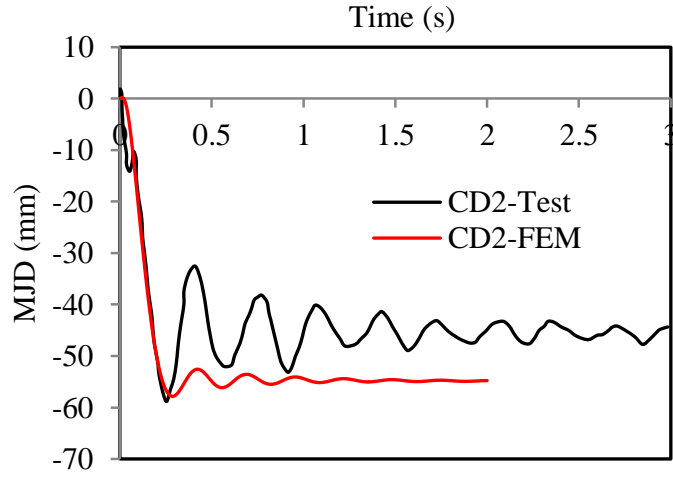

b) $\mathrm{CD} 2-\mathrm{F} / 20 / \mathrm{M}$

Fig. 8.19 Validation results for blast tests 


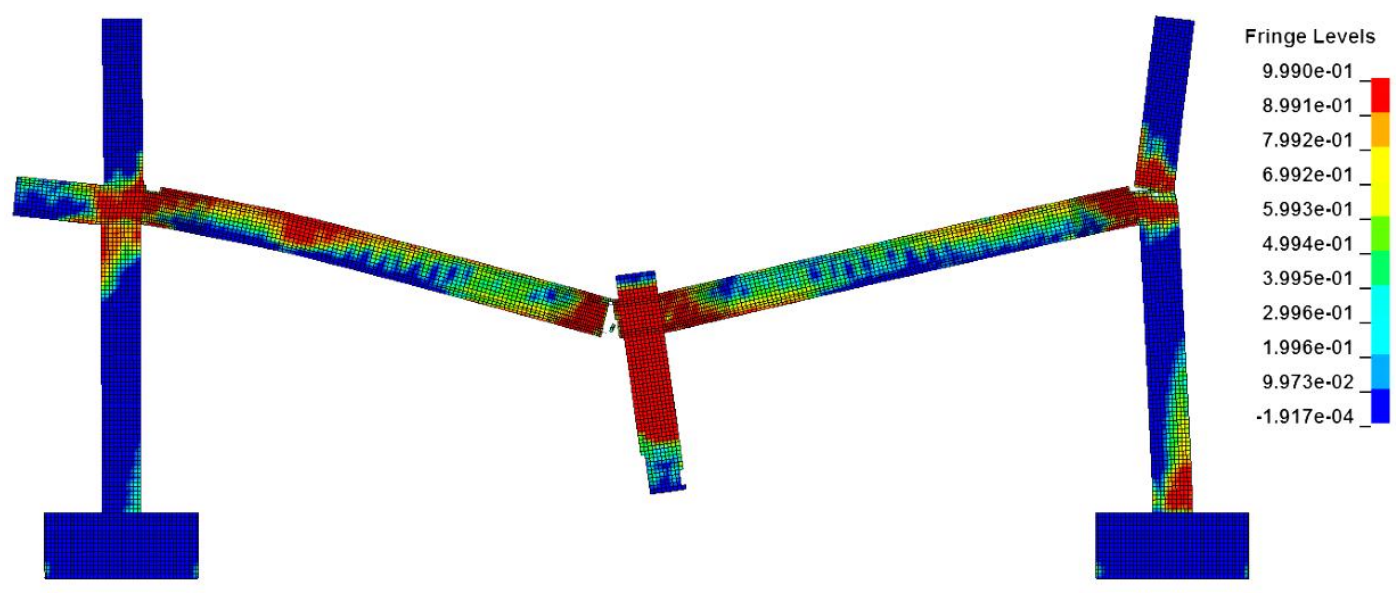

Fig. 8.20 Damages and failure modes of CD1 model

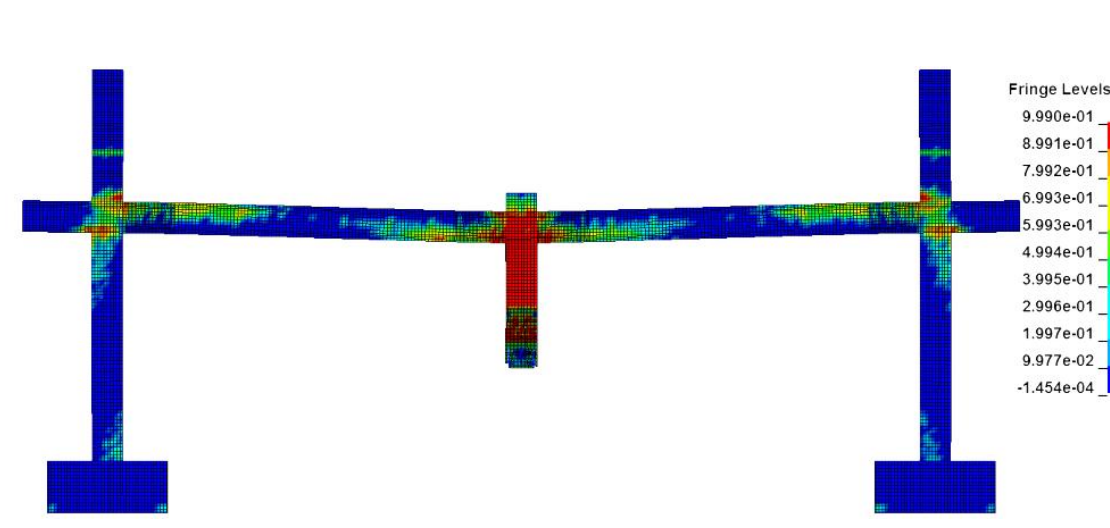

a) Overall specimen

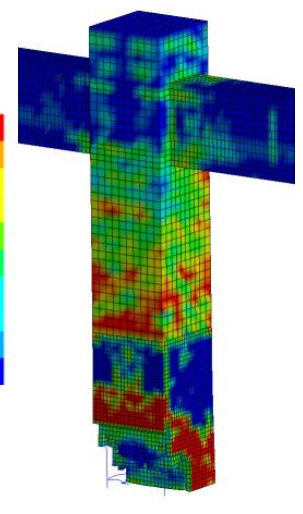

b) Middle joint

Fig. 8.21 Damage patterns of CD2 model

\subsection{Conclusions on experimental and numerical results of Series III}

Expanding the experimental progressive collapse studies on 2D frames under quasistatic (Lim et al. 2015) and free-fall dynamic (Chapter 6) conditions, contact detonation tests from Series III were carried out on two specimens applying different boundary conditions and imposed loads. However, the charge weights used in the two tests were similar. The following conclusions are obtained:

(1) Compared to the free-fall tests generated at laboratory environment, the blastinduced tests properly simulated the actual condition of a progressive collapse event triggered by a contact detonation. The duration of the blast was extremely short, in the order of milliseconds. Therefore, the targeted column could be considered to be destroyed immediately. Initial blast effect on the remaining 
structures which was not quantitatively investigated in previous blast-test studies, i.e. uplift of the double-span beam and blast pressure on beam and column, were well captured. Effects of the initial blast event were also quantitatively highlighted as CD1-P/25/M completely collapsed (FD3-P/25 only had small deformations), and CD2-F/20/M sustained twice the maximum MJD of FD1-F/20.

(2) The strain rates of materials during the blast duration could reach hundred /s, much larger than those collected from the free-fall dynamic tests. However, due to the short duration of the blast, enhancement on material strength could only marginally increase the blast resistance capacity, rather than the progressive collapse resistance capacity. After the blast had totally ceased and during the gravitational movement stage, the DIFs for yield and ultimate strengths of reinforcement were up to 1.21 and 1.08 , respectively, which were similar to those recorded from the free-fall tests of Series I and II.

(3) Since the initial detonation did not directly destroy the frame specimens (due to a small charge), damage pattern and failure modes caused by the sudden column removal in the blast tests reasonably mirrored those observed in the quasi-static and the free-fall dynamic tests. Nevertheless, if a relatively bigger charge weight had been used, it could have severely damaged the double-span beam or even the side columns. As a result, these surrounding members could have immediately failed within the blast duration, violating the assumption of single column removal.

(4) When the specimen only sustained small deformations, i.e. its behaviour was controlled by flexure/CAA (CD2-F/20/M), Izzuddin's method still provided conservative prediction of maximum deformation compared to the actual blast test. However, when the applied load was high and as the behaviour went into CA stage (CD1-P/25/M), this method overestimated the capacity of the structure and therefore could lead to unsafe predictions. Thus, in a blast- 
induced progressive collapse event, Izzuddin's approach should be used with caution when predicting ultimate capacities due to mobilisation of CA.

(5) Due to the complexity of blast effect, detailed FEM models are employed to simulate the blast test results. The use of free air-burst scenario from CONWEP data is a convenient way to reproduce the blast effect in terms of peak positive pressure and impulse. The FEM models show good results compared with actual tests for both structural response and damage patterns. Further numerical studies can then be employed to investigate the response of 2D RC frames under various conditions of blast parameters, applied load, or boundary stiffness. 


\section{CHAPTER 9 EVALUATION OF TENSILE MEMBRANE ACTION IN RC BEAM-SLAB STRUCTURES UNDER COLUMN REMOVAL SCENARIOS}

\subsection{Introduction}

Behaviours of RC beam-column sub-assemblages and frames have been extensively investigated and discussed in Chapters 3 to 8 of this thesis, both experimentally and numerically. The contribution of CA in beams to resist progressive collapse has been quantitatively studied by proposing simplified engineering models for both CL (Section 4.3) and UDL (Section 7.3) configurations. In this chapter, an investigation on the effects of TMA in beam-slab structures under different boundary and loading conditions, as well as under different column loss scenarios, is carried out to investigate the contribution of this mechanism.

Concerning effect of boundary conditions on structural resistance against progressive collapse, mobilisation of CA in beams under CL condition is noticeably influenced by restraint stiffness as shown in the static tests as well as from numerical investigations ( $\mathrm{Yu}$ and Tan 2013a, Lim et al. 2015, Pham et al. 2016). However, such similar studies on TMA in slabs are limited. Besides, existing experimental work on beam-slab systems (Qian and Li 2012a, Qian et al. 2014, Lu et al. 2016, Ren et al. 2016) prefers CL configuration to UDL method due to its simplicity, although UDL is a more practical action in slab design. In an RC building, current studies consider the loss of a corner column as the most critical scenario of progressive collapse. As a result, CA and TMA are neglected in this situation (Qian and Li 2012a). However, comprehensive investigations on column loss locations are required to confirm this conclusion, especially when UDL is applied. Regarding simplified methods for TMA prediction, although there are some analytical approaches evaluating TMA on RC slab-only structures under large deformations (Park 1964, Bailey 2001), there is no practical method considering TMA in a combined beam-slab system. 
To investigate the above-mentioned technical issues, simulations based on detailed FEM models are employed in this chapter and validated by a quasi-static test series on 3D beam-slab structures (Qian et al. 2014). Comparisons of structural responses subject to either CL or UDL conditions and sensitivity of TMA to horizontal and rotational restraints are then carried out. Thereafter, the effects of different columnloss locations on progressive collapse resistance are discussed to identify the most critical scenario for analysis and design. A simplified analytical model is then proposed to predict the response of RC beam-slab structures under an internalcolumn removal scenario taking TMA into account. Last but not least, blast effects from a contact detonation scenario on structural capacity of beam-slab systems is numerically investigated employing the blast-loading simulation which is verified in Chapter 3 for sub-assemblages and in Chapter 8 for beam-column frames. The purpose is to address the limitation of Izzuddin's method for dynamic assessment of beam-slab structures when an actual explosive threat is involved. This issue is also mentioned in Chapter 8 regarding beam-column structural responses.

\subsection{Numerical models of beam-only and beam-slab structures under progressive collapse}

\subsubsection{Quasi-static tests on RC structures under internal-column removal scenario}

To investigate the beam-slab effect against progressive collapse, Qian et al. (2014) conducted a series of quarter-scaled quasi-static tests on beam-only (beam-column sub-assemblage) and beam-slab systems under an internal-column loss scenario using CL method. The tests included 2D beam-only specimens (P1 and P2), 3D beam-only specimens ( $\mathrm{T} 1$ and $\mathrm{T} 2$ ), and 3D beam-slab specimens ( $\mathrm{S} 1$ and $\mathrm{S} 2$ ). While the 2D and the 3D beam-only specimens had double-span beams with a middle joint and a column stub at each beam end, the beam-slab systems included a 2x2-span panel with internal and edge beams plus slab extensions equal to a quarter of the span. In this chapter, numerical models are developed and validated by one 2D beam-only test (P2), one 3D beam-only test (T2), and one beam-slab test (S2) (Pham et al. 2017). All these specimens had the same centre-to-centre span of $1.5 \mathrm{~m}$ 
and the same beam section of $140 \times 80 \mathrm{~mm}^{2}$. All the beams had continuous longitudinal reinforcement (top and bottom rebars). Bottom reinforcement of the slab was continuously arranged on the entire specimen. On the other hand, top reinforcement was provided at the edge regions only. Fig. 9.1(a) and (b) illustrate the respective test setup of the 3D beam-only and the beam-slab specimens. Boundary conditions of the specimens in the static tests were ensured by connecting concrete column stubs to steel circular hollow sections, which in turn were fixed to the strong floor. Removal of the middle column was simulated by gradually increasing the displacement of the middle joint using a hydraulic jack.

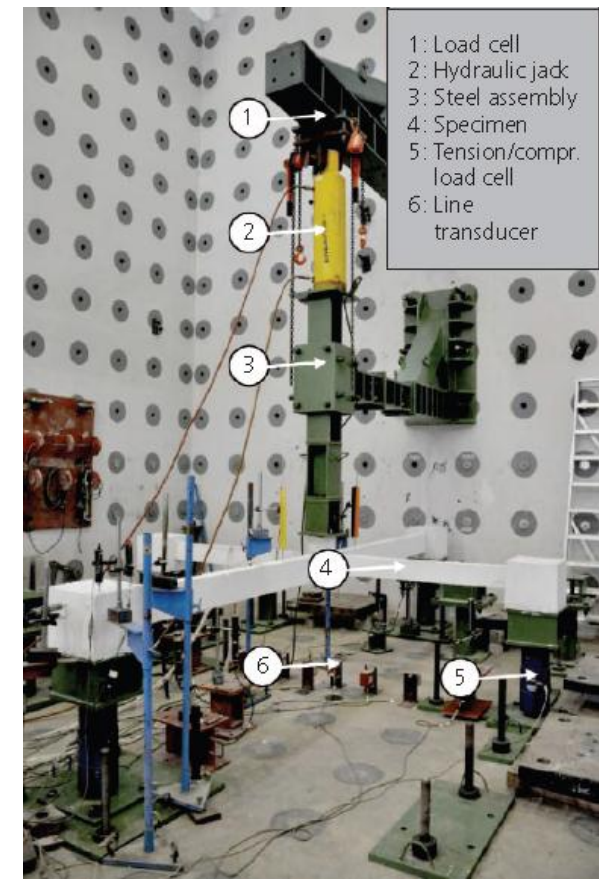

a) 3D beam-only

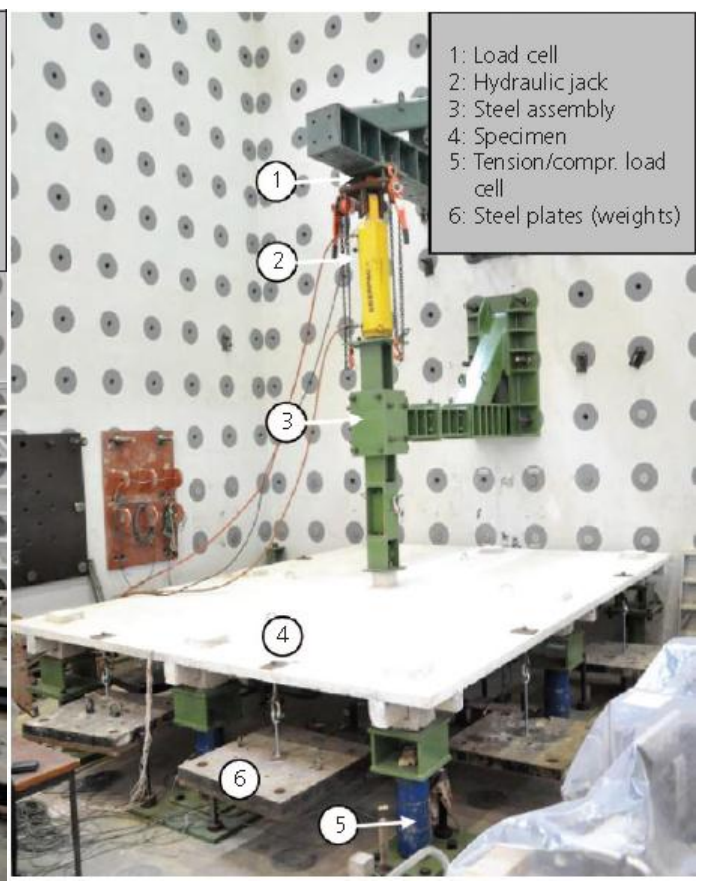

b) $3 \mathrm{D}$ beam-slab

Fig. 9.1 Test setup (Qian et al. 2014)

\subsubsection{Numerical model}

Similar to the FEM models for sub-assemblages under static and blast conditions presented in Chapter 3 (Pham et al. 2016), LS-Dyna (Hallquist 2007) is used to simulate the RC tests of P2, T2 and S2 (Pham et al. 2017). Concrete is simulated using 8-node solid elements with reduced integration scheme. Reinforcing bars are explicitly modelled by the 2-node Hughes-Liu beam element with 2x2 Gauss quadrature integration. Continuous surface cap model MAT_159 is employed to 
simulate the behaviour of concrete material implementing element erosion with the maximum principal strain value of 0.1 . Two mesh sizes are applied for solid elements of beams, including $10 \mathrm{~mm}$ for joint regions and $20 \mathrm{~mm}$ for the other noncritical regions. Only one mesh size of 20x20x6 mm is used for the concrete slab. All rebar elements have a mesh size of $20 \mathrm{~mm}$ length. Full models of the beam-only tests are developed, whereas only a quarter model of the beam-slab test is simulated. The Contact_1D function of LS-Dyna (Hallquist 2007) is employed for simulating bond-slip behaviour between rebars and concrete material in the beams at the beam-column joint regions applying the modelling procedure presented in Chapter 3. For the reinforcement in slabs, since mild-steel bars with high ductility were used in the tests (Qian et al. 2014), fracture of this reinforcement occurred much later than the high yield deformed bars used in the beams. However, to simplify the modelling and to save computational time, perfect bonding is assumed between mild steel reinforcement and concrete slabs.

\subsubsection{Boundary condition}

In the actual tests (Qian et al. 2014), horizontal forces in the steel supports connected to the column stubs were not measured; instead, LVDTs were placed to measure horizontal movements of the supports (Fig. 9.2(a)). The actual lateral movements of column stubs are extrapolated from LVDT readings and are used to compare with numerical predictions. For simplicity, it is assumed that the connections between the column stubs and the steel supports were rigid so that the beam-column joint was rotationally restrained. Besides, horizontal restraint of the joint at each beam end is simulated by two horizontal springs connected to the centre of the column stub. The stiffness of the horizontal springs is based on the stiffness of the steel supports, which was roughly equal to $50,000 \mathrm{kN} / \mathrm{m}$. The spring is assumed to behave elastically in both the beam-only (2D and 3D) and the 3D beam-slab models. Fig. 9.2 (b) shows the simplified support in numerical simulations and Fig. 9.3 shows three FEM models used in the validations with test results. 


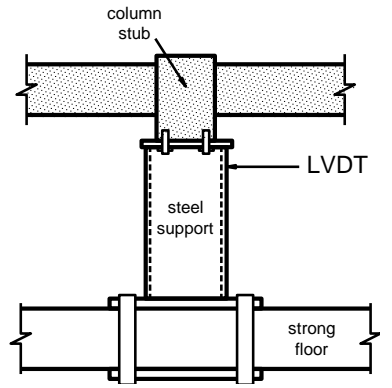

a) LVDT at support

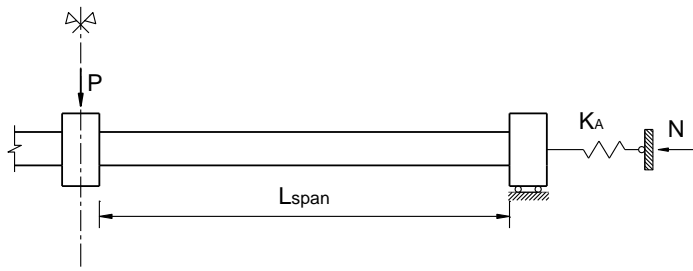

b) Simplified supports at beam's end

Fig. 9.2 Horizontal support of the system

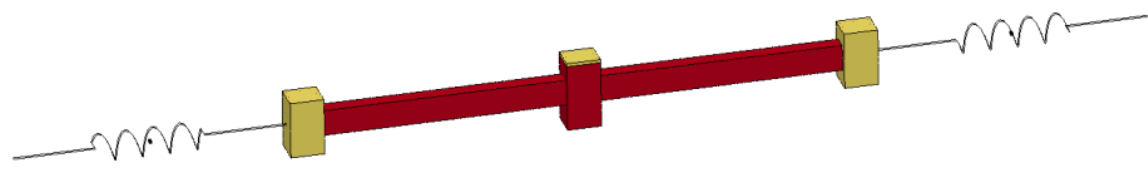

(a) 2D beam-only model

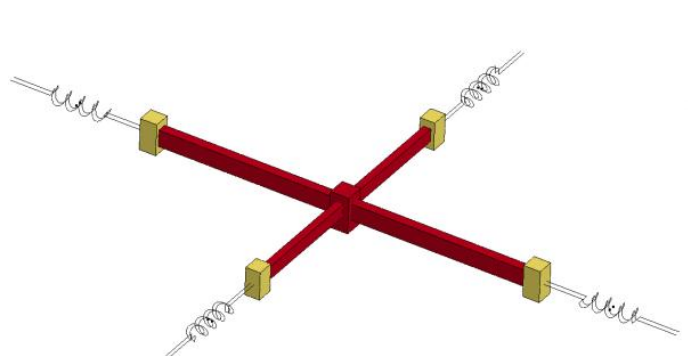

a) $3 \mathrm{D}$ beam-only model

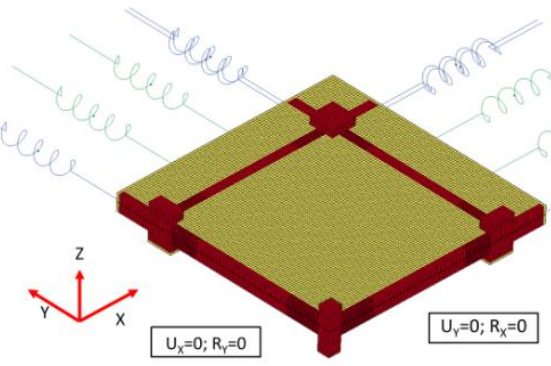

b) 3D beam-slab model

Fig. 9.3 FEM models

\subsubsection{Validation results for beam-only tests}

Comparisons of applied load versus vertical deflection between numerical and test results are presented in Fig. 9.4 and they generally show good agreement. Similar to other CL static tests from the literature, structural responses of the beam-only systems from both the actual tests (Qian et al. 2014) and the simulations (Pham et al. 2017) exhibit the mobilisation of both CAA and CA. In terms of horizontal movement of the edge joints, numerical predictions provide a reasonable trend compared to actual test results, which verifies the modelling of boundary restraint conditions. Both the FEM and the test results show an outward movement of the edge joints at the beginning stage due to CAA, and an inward displacement at the later part due to $\mathrm{CA}$. The numerical simulations also match well with the test results in terms of sequence of failure modes, which includes the fracture of bottom rebars at the middle joint followed by the fracture of top rebars at the end joint. Failure 
modes and damage patterns of FEM models are shown in Fig. 9.5 for beam-only models, similar to those observed from testing (Qian et al. 2014).

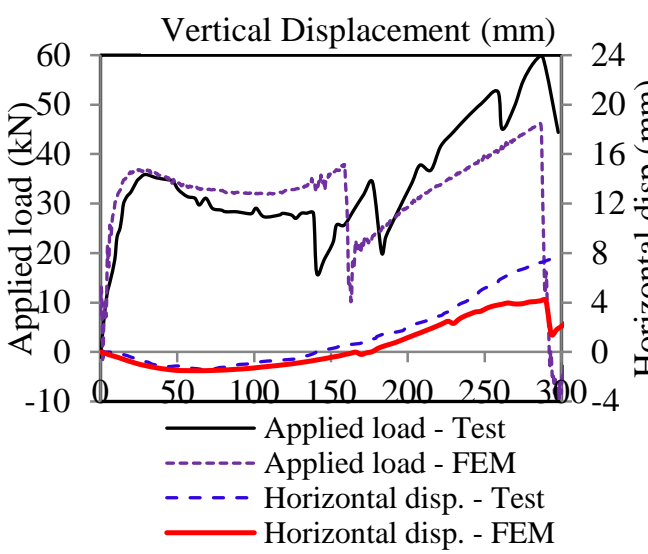

a) P2

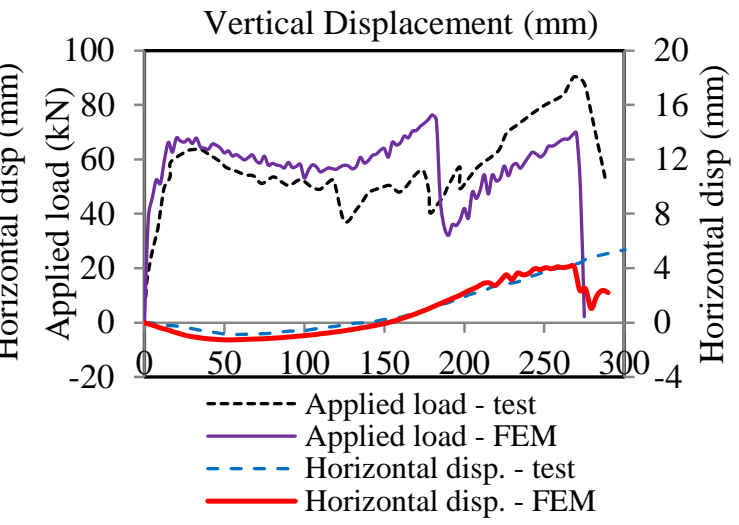

b) $\mathrm{T} 2$

Fig. 9.4 Test validation results of beam-only structures

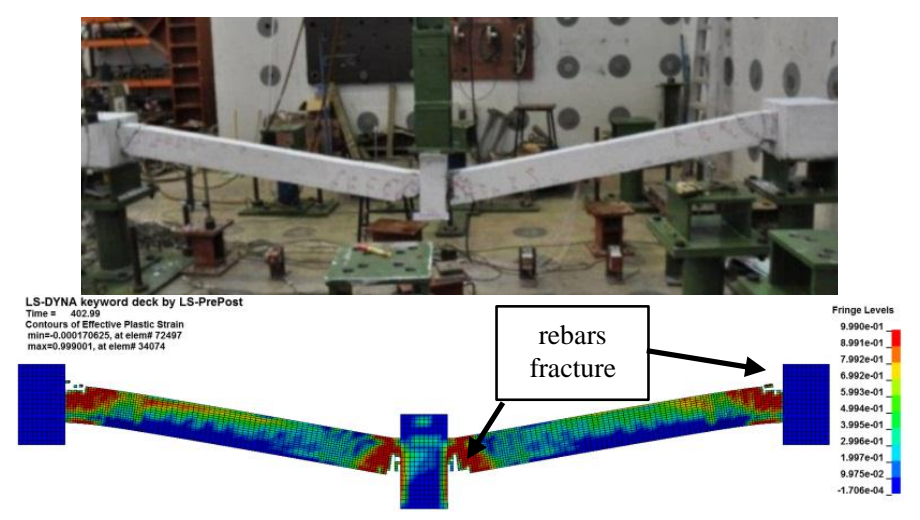

a) 2D beam-only

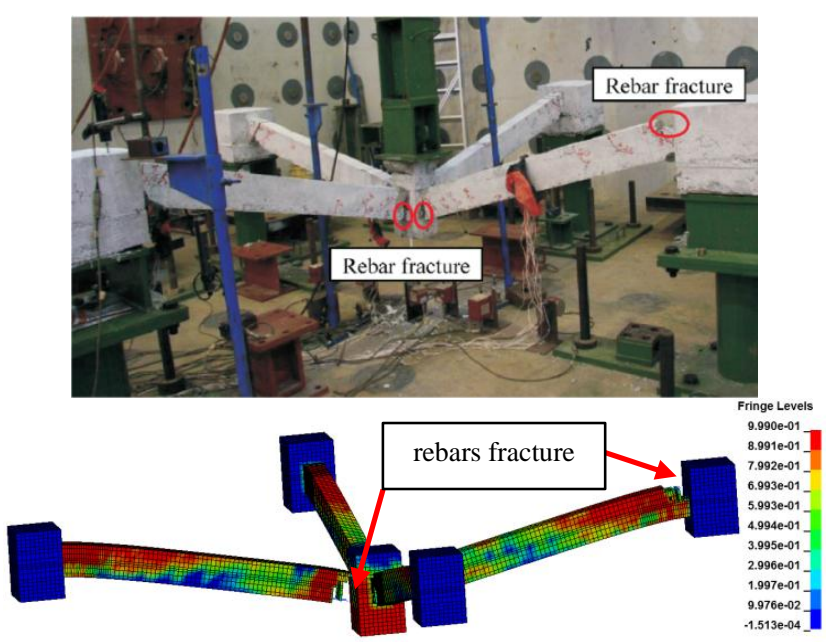

b) $3 \mathrm{D}$ beam-only

Fig. 9.5 Damage patterns of beam-only structures 


\subsubsection{Validation results for beam-slab test}

Numerical predictions of S2 achieve good agreement with test results in terms of load-carrying capacity (Fig. 9.6). Considering damage patterns and failure modes, the FEM simulation also provides good matching with actual responses (Fig. 9.7). At large deformation stage, the formations of a tensile net at the centre and a peripheral compressive ring surrounding the tensile region are well replicated in the one-quarter numerical model. The model also successfully simulates the failure modes that occurred in the tests, i.e. punching shear failure around the middle column and fracture of internal-beam bottom rebars near the middle column. That is to say, the developed FEM model can simulate well the behaviour of 3D RC beamslab systems.

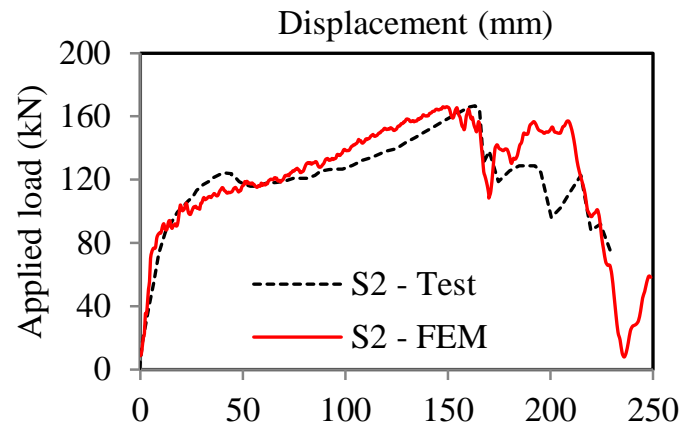

Fig. 9.6 Test validation results of S2
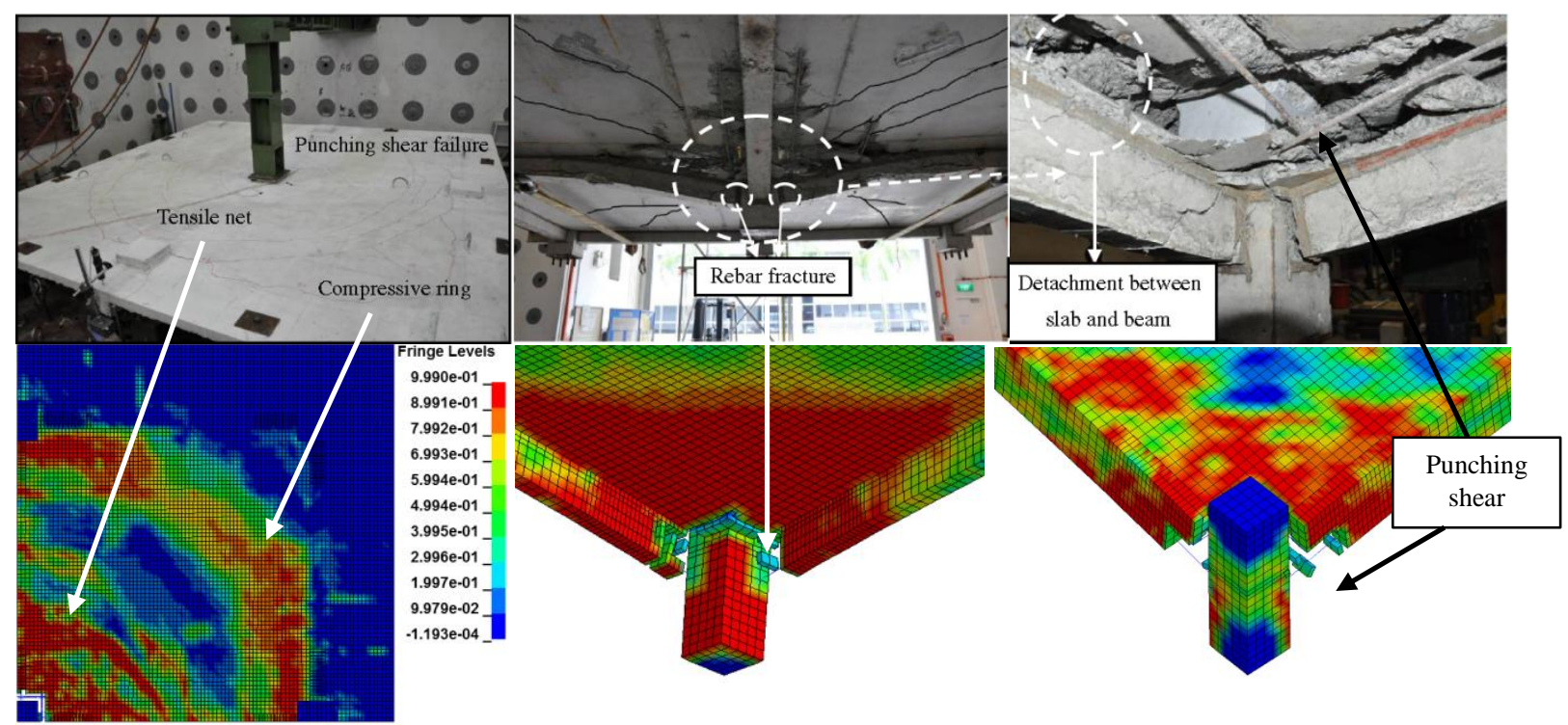

Fig. 9.7 Damage patterns of S2 


\subsection{Investigations of structural responses comparing between $\mathrm{CL}$ and UDL conditions}

Having validated the FEM models for $2 \mathrm{D}$ and $3 \mathrm{D}$ tests, they are then used to investigate the differences in structural behaviour subject to either CL or UDL conditions. The study includes two levels of structural system, viz. slab-only and beam-slab models.

\subsubsection{Proposed loading method representing UDL condition}

The validated FEM models are analysed under the UDL condition using a displacement-controlled method. However, if the actual distributed loads are applied onto the slab, then the FEM analysis is load-controlled. When its structural capacity reaches the peak and starts decreasing, the analysis may not capture the snap-through phase. Therefore, instead of using distributed loads, a 16-point loading method is applied for the slab-only and the beam-slab models (Fig. 9.8(a)). In this method, loads from the sixteen points acting on the slab are directed by a downward movement of a master point on top, which is displacement-controlled. Numerical results show that the discrepancies in terms of structural response within the initial stage (up to the first peak of compressive arch/membrane action) between the force-controlled UDL and the equivalent 16-point methods are negligible (Fig. 9.9). Moreover, this 16-point displacement-controlled procedure can still capture the correct behaviour after the load-carrying capacity attains the peak value and decreases afterwards. Besides, compared to the 12-point loading system normally used for slab tests (Foster et al. 2004, Pham and Tan 2013, 2015, Qian et al. 2016b), the proposed loading system ensures symmetry in two directions. Hence, only a quarter-type model is needed for simulations (Fig. 9.8(b)). 


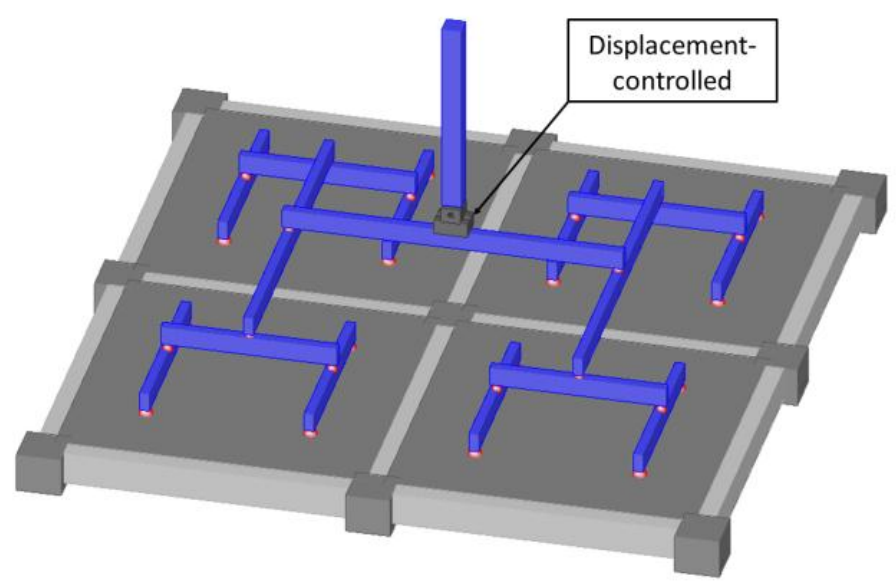

a) Full model

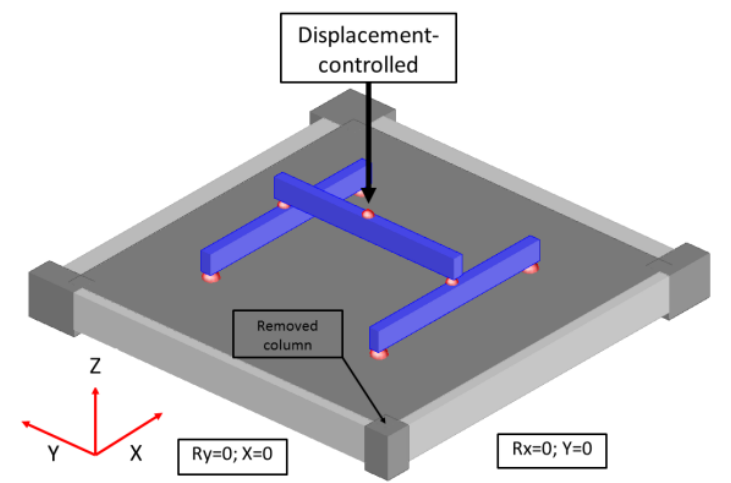

b) Quarter model

Fig. 9.8 Equivalent UDL by multiple-point loading systems

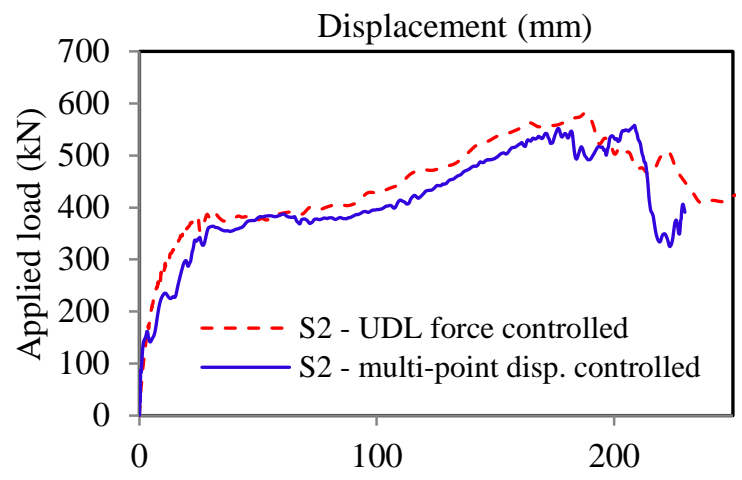

Fig. 9.9 Comparisons between true UDL force-controlled and equivalent multiplepoint displacement-controlled methods 


\subsubsection{Slab-only structure}

To compare the behaviour between CL and UDL conditions for the 3D slab-only structure, a FEM model is constructed similar to S2 model, except that all internal beams are removed. Hence, the RC slab is considered as the only isolated element within the region of the removed column. A comparison of structural responses between the two loading cases (CL and UDL) is presented in Fig. 9.10. Basically, the response of the CL case has a much smaller central deflection than the UDL case. From the initial stage to the first peak, the capacity in the UDL case is approximately four times that in the CL case. This relationship can be explained by employing the yield-line theory at small deformations. At the yielding state of reinforcement in the slab, it is assumed that the yield-line pattern under the UDL condition includes four triangular segments, whereas under the CL condition the slab shows a circular pattern of yield-line (Fig. 9.11). According to Park and Gamble (2000), for a square slab under fixed boundary condition and with equal reinforcement in orthogonal directions, total applied load on the slab at yielding for the $\mathrm{CL}\left(P_{c l}^{\text {slab }}\right)$ and the UDL $\left(P_{u d l}^{\text {slab }}\right)$ conditions can be calculated by using Eqs. (9.1) and (9.2), respectively. It is shown from these two equations that $P_{u d l}^{\text {slab }} \approx$ $3.82 P_{c l}^{\text {slab }}$, which explains why the slab resistance in the UDL case is nearly four times that in the CL case. Nonetheless, if the slab has an aspect ratio larger than one or had different rebar arrangements in two orthogonal directions, then this value will be different.

$$
\begin{aligned}
& P_{c l}^{\text {slab }}=2 \pi\left(m^{\prime}{ }_{s}+m_{s}\right)=6.28\left(m^{\prime}{ }_{s}+m_{s}\right) \\
& P_{u d l}^{\text {slab }}=w_{u} l^{2}=24\left(m_{s}^{\prime}+m_{s}\right)
\end{aligned}
$$

where $m_{s}^{\prime}$ and $m_{s}$ are the negative and the positive moment capacities per unit width of the slab, respectively; $w_{u}$ and $l$ are the maximum distributed load and the clear span of the slab, respectively; $m^{\prime}$ and $m_{s}$ can be simply calculated based on Eq. (9.3) (Park and Gamble 2000). 
$m_{s}=A_{s} f_{y}\left(d_{s}-\frac{0.59 A_{s} f_{y}}{f_{c k}}\right)$

where $A_{s}$ and $f_{y}$ are the reinforcement area per unit length and the yield strength of tensile rebars, respectively; $d_{s}$ is the effective depth of the slab and $f_{c k}$ is concrete cylinder strength.

After the first stage of flexural and CMA in the slabs, TMA is more efficiently mobilised in the UDL case whereas development of TMA is limited in the CL case due to potential premature punching shear failure. Concerning failure modes, the governing damage of the CL condition is punching shear around the removed column location (Fig. 9.12(a)). Since the structure under the CL case behaves like a flat slab, punching shear limits the maximum displacement which leads to less effective mobilisation of TMA. On the other hand, the slab under UDL sustains greater deflection, leading to a higher capacity at the final stage compared to the first peak of CMA. Failure modes of the UDL case include occurrence of full-depth cracks and fracture of rebars near the slab edges (Fig. 9.12(b)).

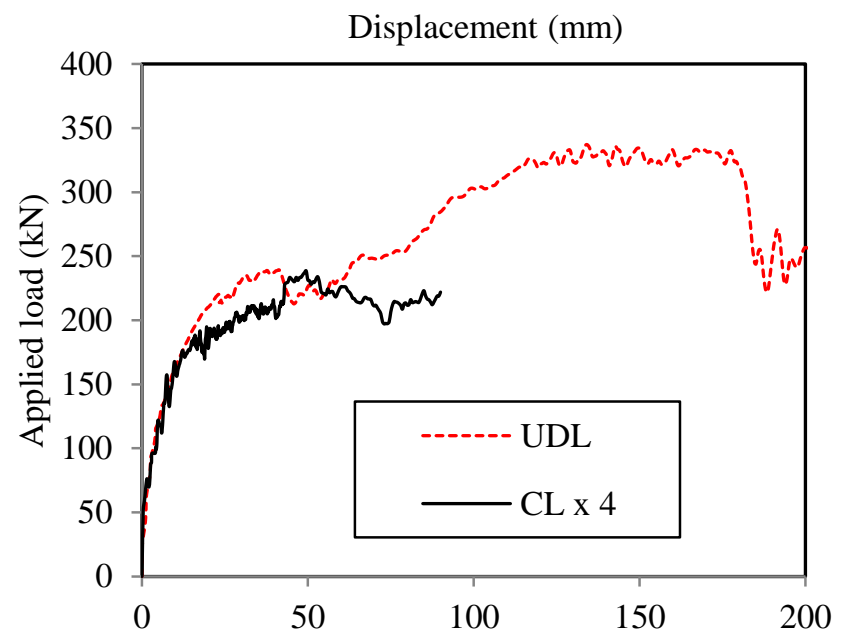

Fig. 9.10 Responses of slab-only structures 


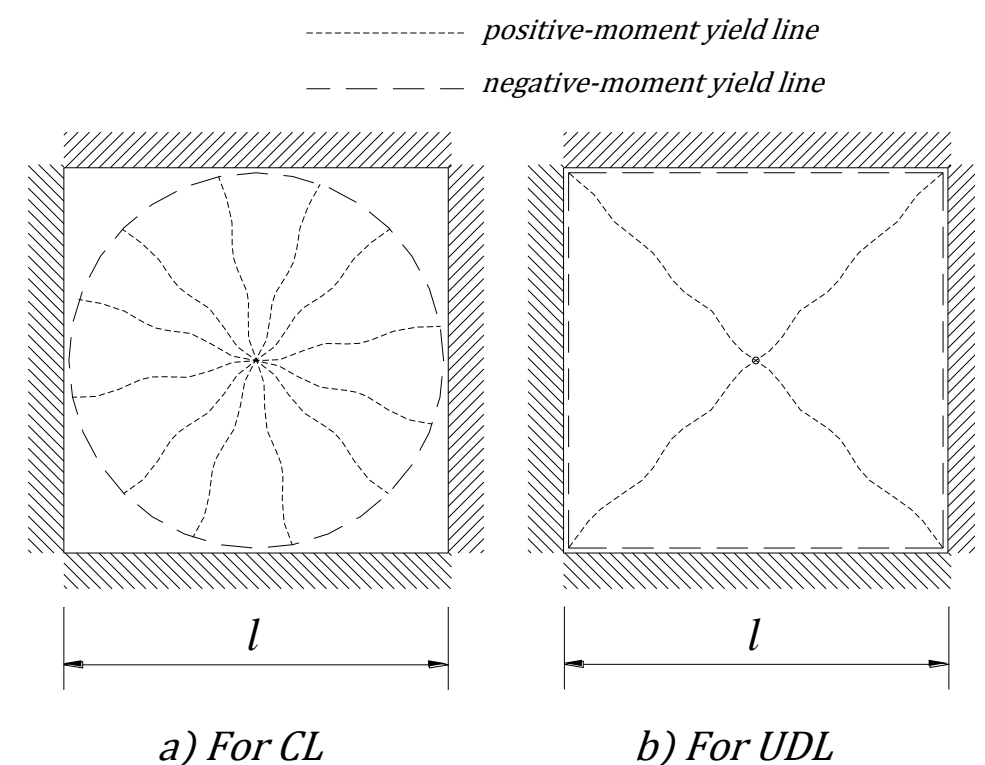

Fig. 9.11 Yield-line patterns of slab-only structures

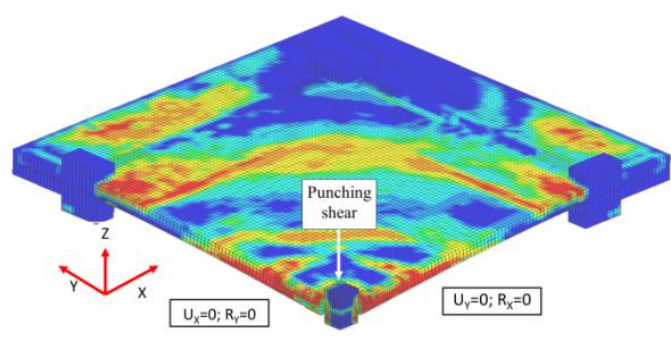

a) $\mathrm{CL}$

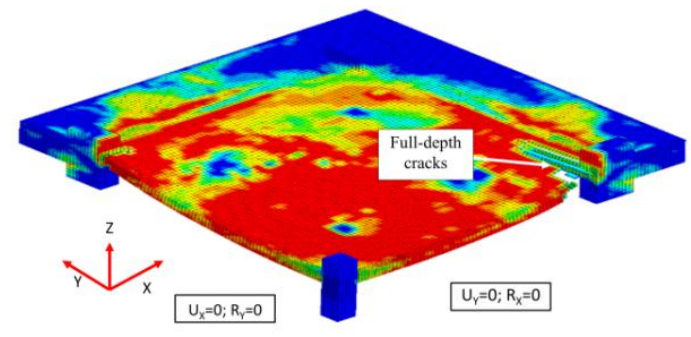

b) UDL

Fig. 9.12 Failure modes of slab-only structures

Fig. 9.13 shows the yielding spread of reinforcement in the slab at a vertical deflection of $55 \mathrm{~mm}$, or one slab thickness. For the CL case, the top rebars have yielded locally within one-quarter of the slab edges. But for the UDL case, most of the top rebars along the slab edges have already yielded at this state. Similarly for the bottom-rebar layer in the slab, yielding in the CL case only concentrates around the central column, whereas yielding in the UDL case develops along the slab diagonal and extends to nearly one-quarter of the area. That is to say, for the same central deflection, both the top and the bottom reinforcing bars are stressed more fully in the UDL case compared to the CL case with concentrated regions of rebar yielding. 


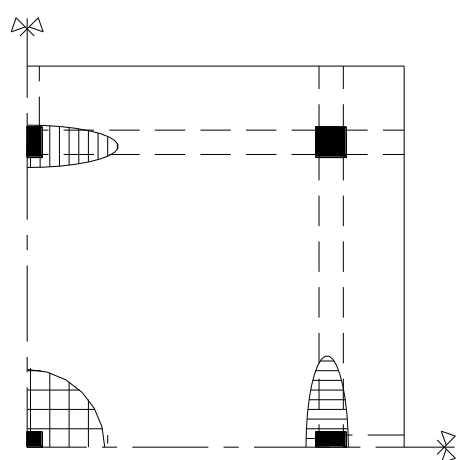

a) $C L$

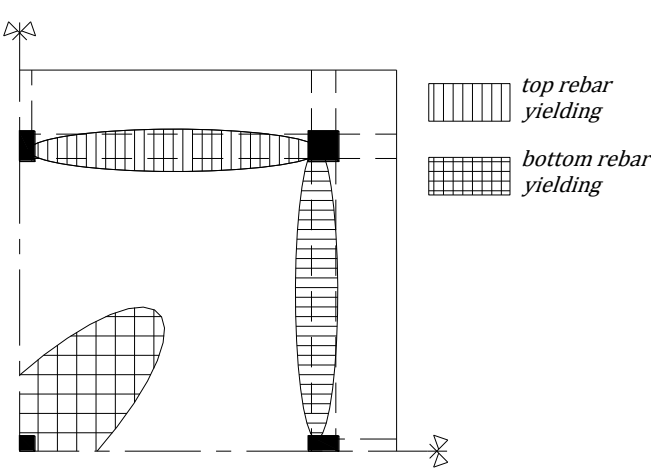

b) $U D L$

Fig. 9.13 Yielding of slab-only rebars at $55 \mathrm{~mm}$ deflection $\left(1.0 d_{\text {slab }}\right)$

\subsubsection{Beam-slab structure}

The second level of comparison between CL and UDL cases involves the full 3D beam-slab system (S2 model). Numerical results show that, the load-carrying capacity in the UDL case is nearly three times that in the CL case as shown in Fig. 9.14. It is assumed that at yielding state (small deformations), structural capacity of the beam-slab system is equal to the sum of yielding capacities from the beam-only and the slab-only structures. From Chapter 7, it is shown that structural capacity based on plastic hinge theory for UDL case is twice that for CL case in beam-only systems. For the slab-only system, the ratio between UDL and CL conditions is nearly four (Section 9.3.2). In this particular RC specimen of $\mathrm{S} 2$, the capacities of the 3D beam-only structure (internal beams) based on plastic-hinge theory under UDL and CL conditions are $96 \mathrm{kN}$ and $48 \mathrm{kN}$, respectively, whereas the capacities of the slab-only structure ( $\mathrm{S} 2$ without internal beams) based on yield-line theory under UDL and CL conditions (Eqs. (9.1) and (9.2)) are $85 \mathrm{kN}$ and $23 \mathrm{kN}$, respectively. Therefore, when combining the beam-only and the slab-only capacities together, the yielding capacity of the beam-slab system under UDL condition $(181 \mathrm{kN})$ is approximately 2.6 times that under CL condition $(71 \mathrm{kN})$. That is to say, structural capacity of a beam-slab system can be computed by separately considering the capacities contributed by beam-only and slab-only parts. Failure modes of the beam-slab structure at the final stage are shown in Fig. 9.15 and are further discussed in later part. 


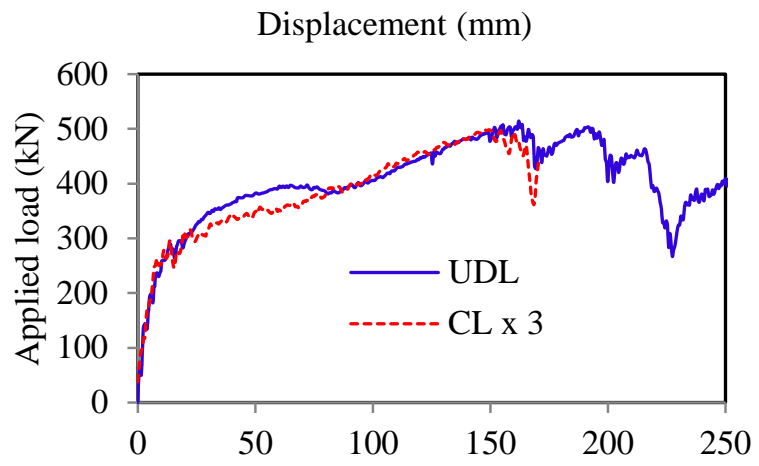

Fig. 9.14 Responses of beam-slab structures

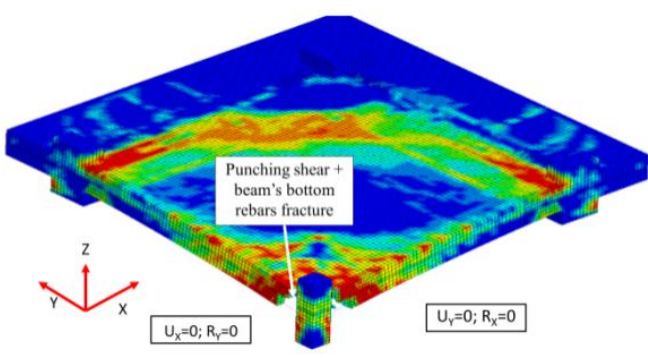

a) $\mathrm{CL}$

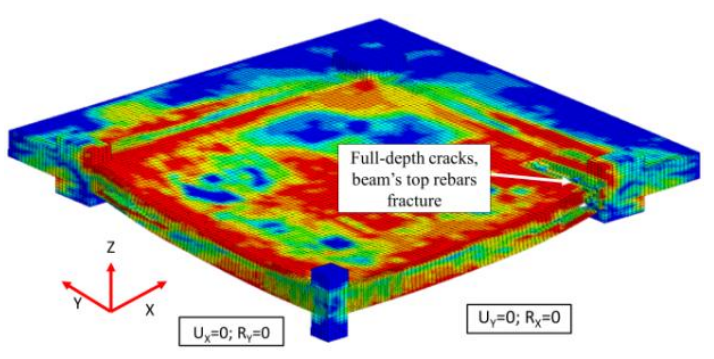

b) UDL

Fig. 9.15 Failure modes of beam-slab structures

\subsubsection{Discussions on the combined effects of beams and slabs under both CL and UDL conditions}

Under CL condition, responses of the two modelling levels from Sections 9.3.2 to 9.3.3 are shown in Fig. 9.16(a) together with the results from the 3D beam-only structure for comparison. For the beam-slab model, not only does its structural capacity increase compared to the slab-only model, the maximum deflection also increases because punching shear occurs later due to shear strength contribution from internal beams. Besides punching shear failure, the final peak of the beam-slab model is denoted by the fracture of bottom rebars in internal beams. In other words, the failure mode of the beam-slab system at the final stage under CL condition is a culmination of failure modes from both the beam-only and the slab-only structures (Fig. 9.15(a)). Moreover, the deformation corresponding to fracture of beam bottom rebars is also consistent between the beam-slab and the beam-only structures (Fig. 9.16(a)). This observation agrees well with the experimental study on beam-slab 
structures under edge column removal subjected to CL condition conducted by $\mathrm{Lu}$ et al. (2016).

Similar to the CL case, FEM results from all the three structural levels (3D beamonly, slab-only, and beam-slab) under UDL condition are shown in Fig. 9.16(b). In terms of failure mode, the beam-slab model fails when full-depth cracks in the slab occur near the edge beams and top rebars of internal beams fracture near the edge columns (Fig. 9.15(b)). From Chapter 7, it is concluded that the final failure of beam-only structures under UDL condition is denoted by top rebar fracture at the end joint. Moreover, from previous beam-slab UDL tests (Pham and Tan 2013, 2015, Qian et al. 2016b), fracture of beam top rebars near the slab edges can be conservatively considered as the failure state of the system. That is to say, failure mode of the beam-slab system under UDL is also a combination of failure modes from both the beam-only and the slab-only models. TMA, which is effectively mobilised in the slab-only system, is also well developed in the beam-slab model.

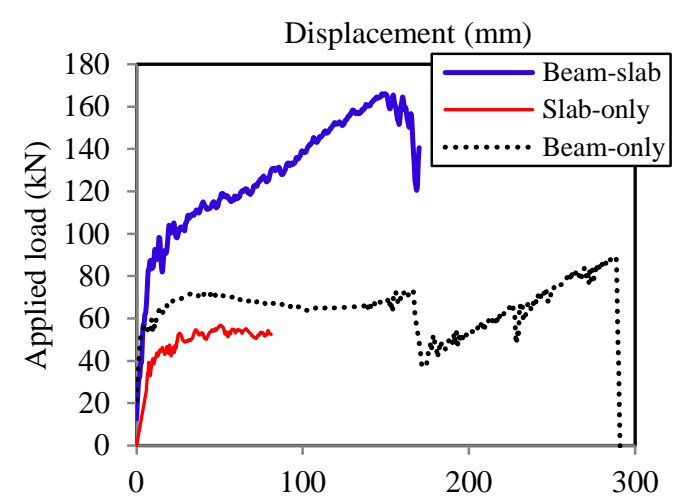

a) $\mathrm{CL}$

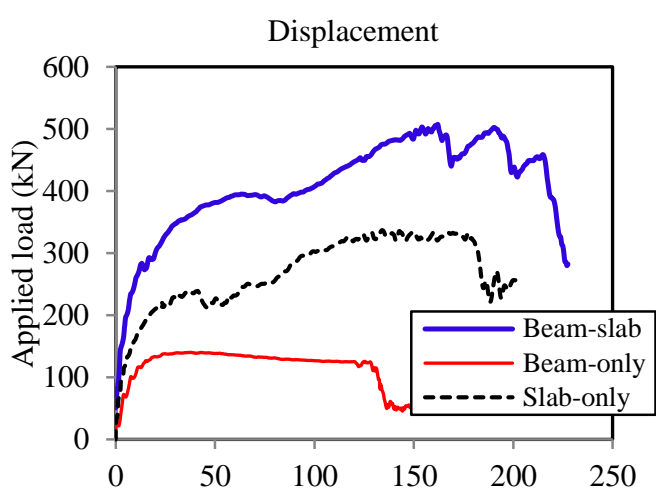

b) UDL

Fig. 9.16 Effects of beam and slab

\subsection{Effect of boundary stiffness on mobilisation of TMA}

\subsubsection{Horizontal restraint}

To study the sensitivity of TMA to horizontal restraint conditions, the beam-slab model of S2 is analysed with several values of horizontal spring stiffness, including zero stiffness, perfectly rigid, and 50,000 kN/m (semi-rigid) using both CL and UDL conditions. Besides the horizontal springs connected to column stubs, 
additional horizontal springs are added to the slab extension part, representing horizontal restraints from adjacent spans (Fig. 9.17(a)). Values of total applied load versus central displacement are presented in Fig. 9.18. For the first peak of CMA, applied load from the rigid-restraint case is larger than that from the zero-restraint case by $17 \%$ and $25 \%$ with regard to CL and UDL conditions, respectively. However, there is almost no difference in the load-carrying capacity for the last peak before the models fail under both CL and UDL cases. Suffice to say, the stiffness of horizontal restraint has some effect on CMA, but little or no effect on TMA. In fact, the formation of the peripheral compressive ring in some way provides sufficient restraint for the mobilisation of tensile membrane forces at the centre.

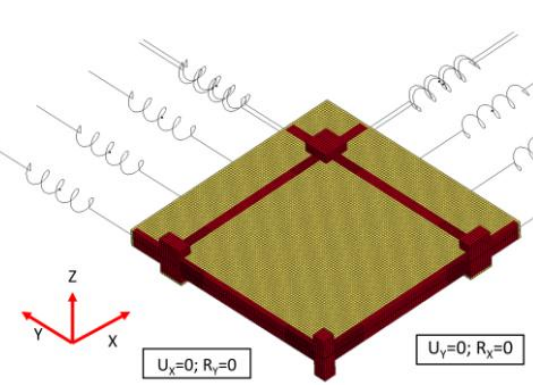

a) S2 with additional springs

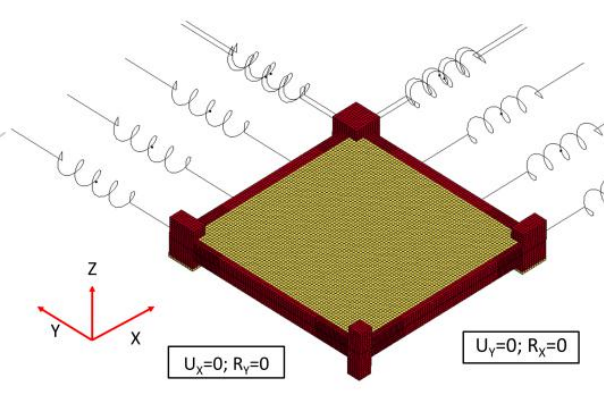

b) S2 without slab extension

Fig. 9.17 S2 models for restraint stiffness studies

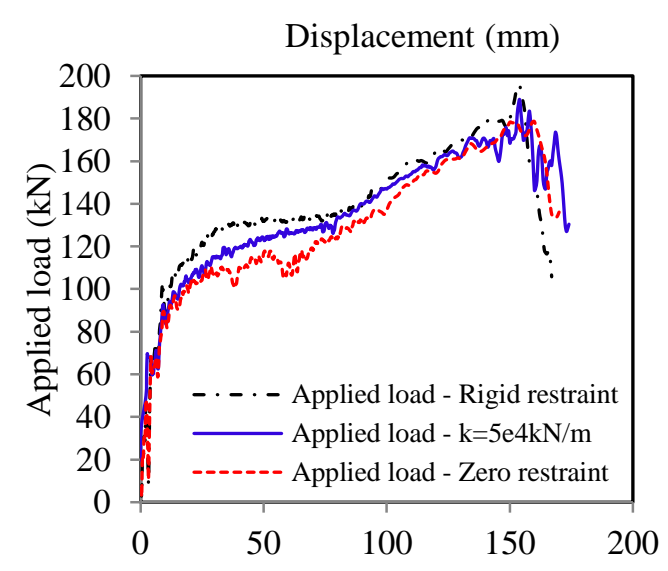

a) $\mathrm{CL}$

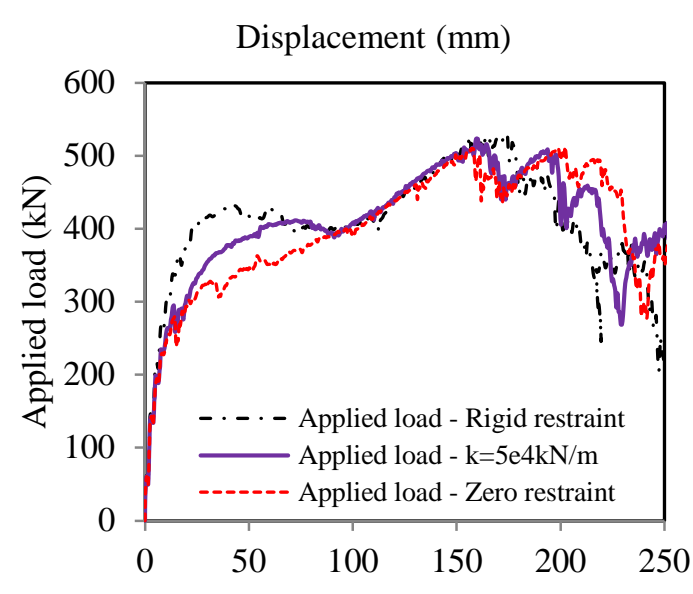

b) UDL

Fig. 9.18 Effects of horizontal restraint stiffness on beam-slab structures 


\subsubsection{Rotational restraint}

To investigate the effect of rotational restraint at the slab edges, the extended part of the slab in the beam-slab model is deleted to reduce rotational stiffness at the slab perimeter (Fig. 9.17(b)). Both CL and UDL cases are considered. Results from the modified model are compared with those from the original model which includes the slab extension (Fig. 9.19). It is shown that under the CL condition, there is almost little difference in terms of structural response between the two models. Nonetheless, for the UDL case, the overall load-carrying capacity of the modified model (without slab extension) is smaller than that of the original model (with slab extension) for both the first peak of CMA and the later part of TMA. Additionally, TMA in the model without slab extension is developed later compared to the original model. This observation agrees well with Section 9.3.2 since stresses of top reinforcement at the slab edges under the UDL case can reach yielding faster and more uniformly than those under the CL case. Hence, it is concluded that beam-slab models under UDL are influenced by rotational stiffness of slab edges whereas models under CL are less affected by this factor.

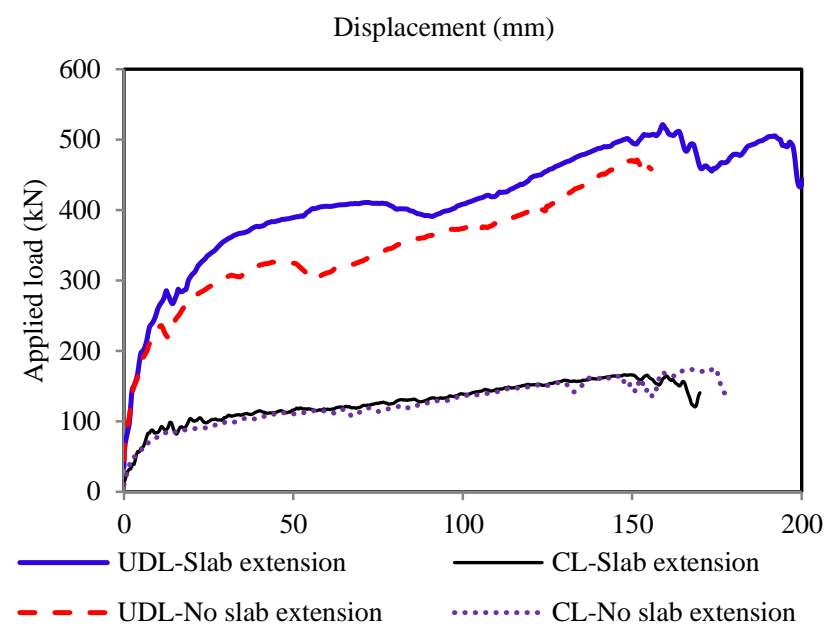

Fig. 9.19 Effects of rotational restraint on beam-slab structures

\subsection{Effect of location of removed column on structural response}

To date, most studies comment that the removal of a corner column is the most critical scenario for progressive collapse due to a lack of restraints from two 
adjacent sides of the removed column. Besides, penultimate columns which are next to the corner column are considered as the second most critical scenario for progressive collapse analysis. Experimental studies (Pham and Tan 2013, 2015) show that the removal of a penultimate column leads to large inward movements of adjacent columns, which in turn may precipitate collapse. However, there is no reported experimental work for beam-slab structures that systematically consider all the possible scenarios of missing column. In this section, several scenarios of column loss (Fig. 9.20) are numerically investigated using the validated beam-slab models. Distributed loads are applied to the slab area that is disturbed by the column removal (DOD 2013), the so-called affected area (Fig. 9.20). Five scenarios are considered including internal-column, corner-column, edge-column, penultimate-internal (PI) and penultimate-external (PE) column removal cases. The internal-column case is used as the baseline for comparison since its structural capacity is the greatest. The relationship between displacement of the joint above the removed column and structural capacity per unit area from the affected slab is used as a comparison basis among the five different scenarios of column removal as shown in Fig. 9.20. 
뜬

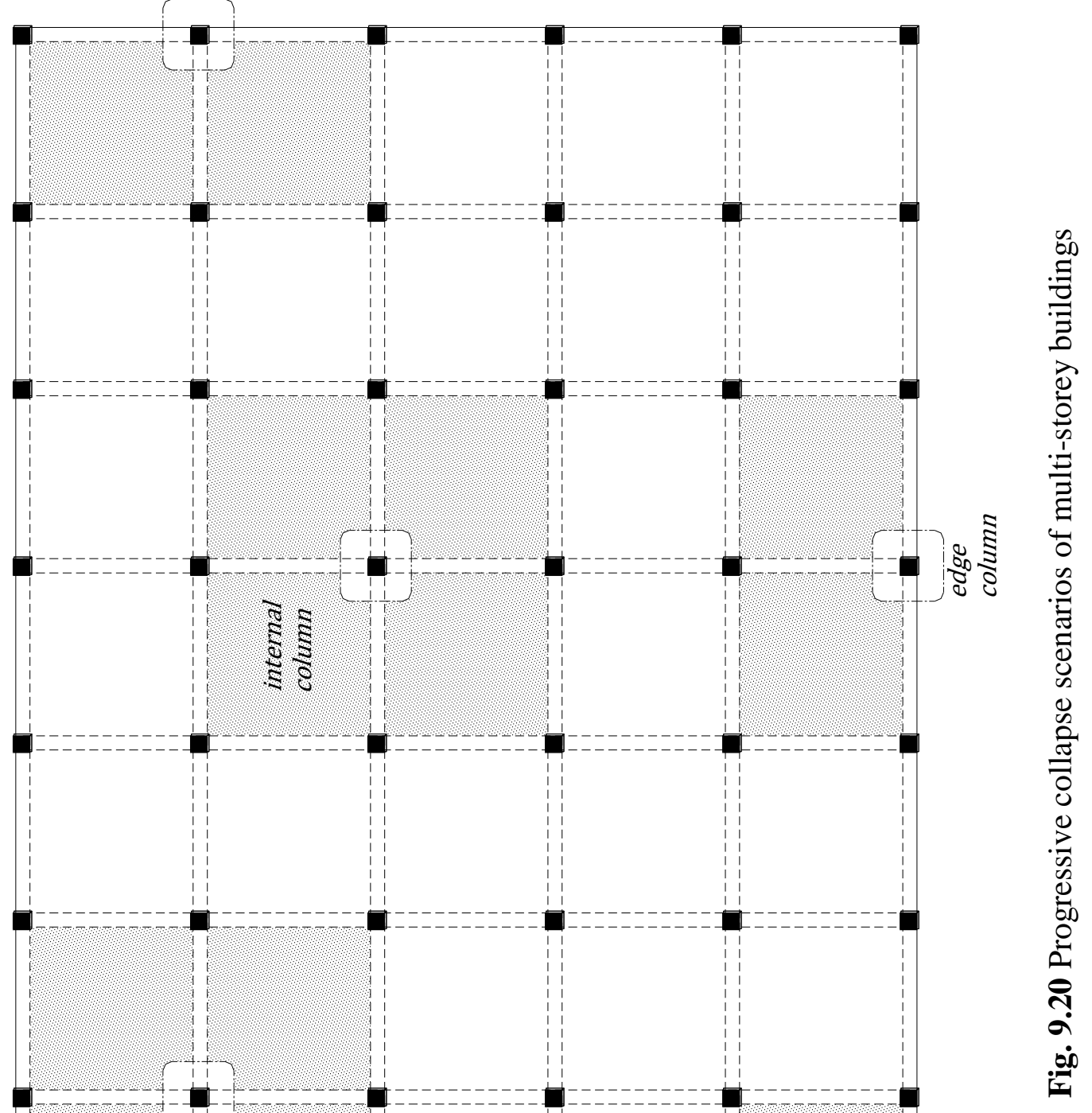

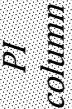

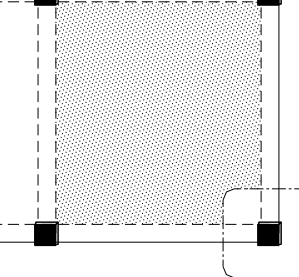

ฮั 


\subsubsection{Corner-column removal}

The comparison results in Fig. 9.21 show that the corner-column case is not the one with the smallest structural capacity. In fact, the response of the corner-column scenario is comparable with the internal-column and the edge-column cases. Although the effect of CMA on the corner-column scenario is unnoticeable due to a lack of horizontal restraints, the final capacity and the maximum central deflection are comparable to internal- and edge-column cases. Indeed, it is the PI- and the PEcolumn cases that have the lowest structural capacities. Therefore, it is concluded that the corner-column loss is not necessarily the most critical scenario. This is explained through the yield-line formation of slab top and bottom rebars at a deflection of one slab thickness for the internal- and the corner-column cases, shown in Fig. 9.22. The formation of positive- and negative-moment yield lines is also illustrated. For the internal-column case, bottom-rebar yielding zone concentrates near the slab centre and along the slab diagonal, whereas for the corner-column case, yielding develops towards the edge columns besides the diagonal region. It is shown that the two edge beams DA and DC (Fig. 9.22(b)) can provide noticeable strength (based on their stiffness) to the slab area so that additional positive yield lines ( $\mathrm{AO}$ and $\mathrm{CO}$ ) can form and therefore increase the capacity of the structure.

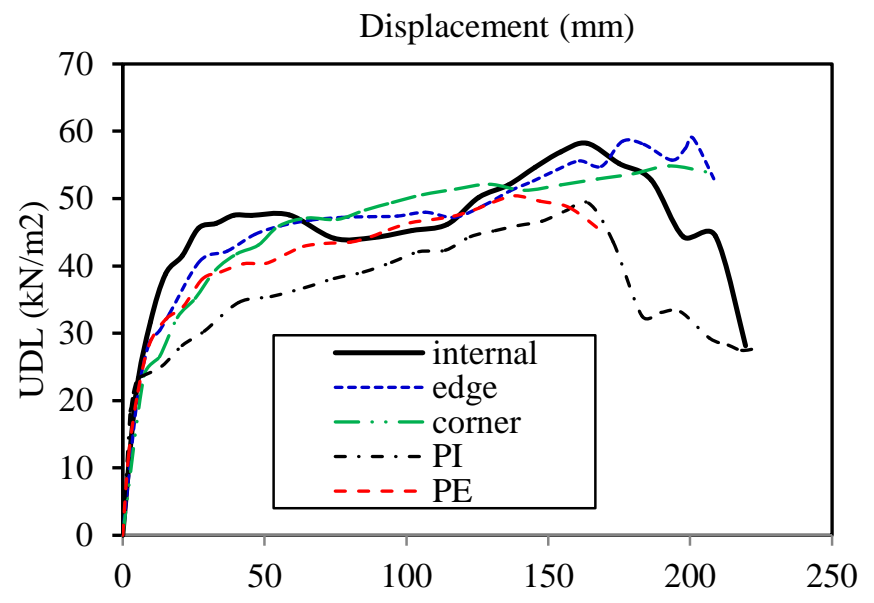

Fig. 9.21 Structural responses from different column loss scenarios 


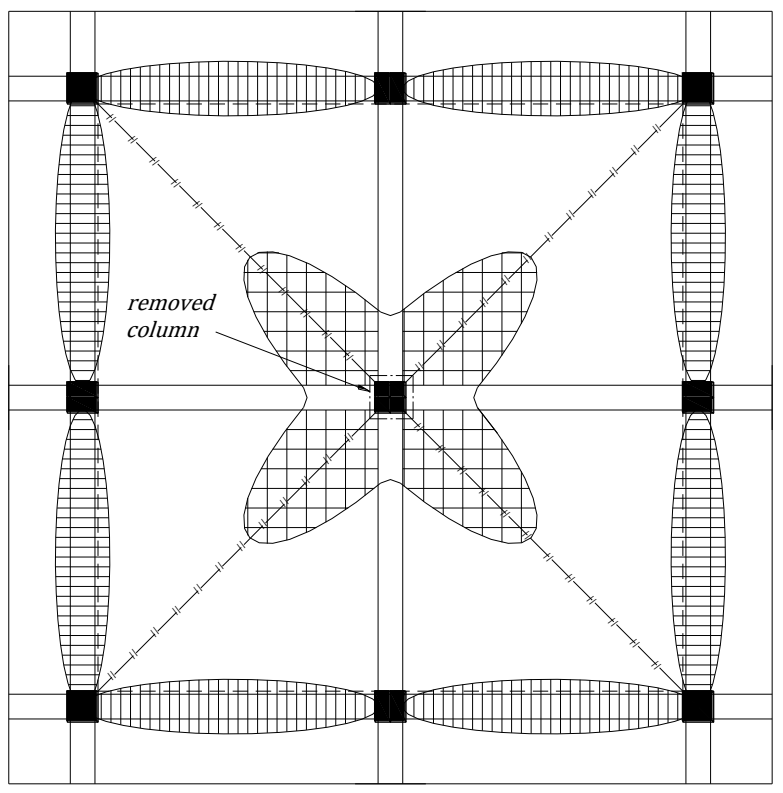

a) Internal-column case
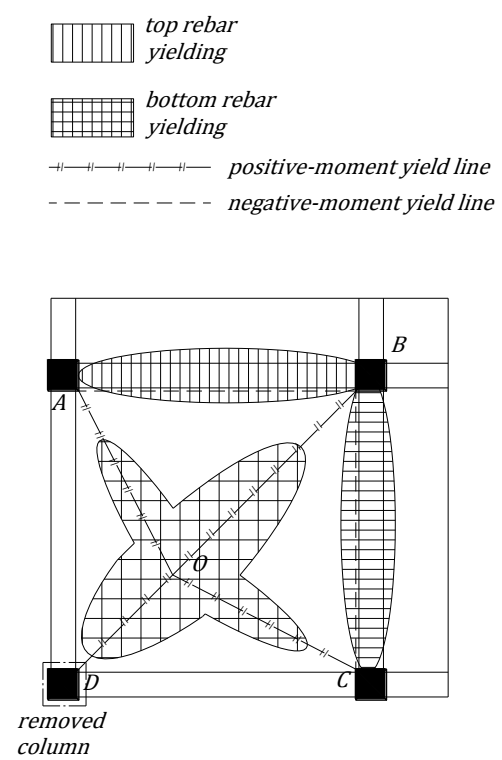

b) Corner-column case

Fig. 9.22 Rebar yielding at $55 \mathrm{~mm}$ deflection of internal- and corner-column cases

Regarding the development of TMA, in the internal-column case, it is ensured by the formation of a peripheral compressive ring which relies on surrounding columns (Fig. 9.23(a)). On the other hand, for the corner-column case, the presence of edge beams DA and DC at discontinuous sides helps to reduce the slab deformation at these edges. As a result, a peripheral compressive ring can still be formed (Fig. 9.23(b)) and limited TMA can be mobilised. In summary, it is concluded that although having two unrestrained sides DA and DC, the corner-column scenario can still develop sufficient load-carrying capacity and ductility compared to the internalor the edge-column scenarios if the boundary beams at discontinuous edges have sufficient stiffness and strength relative to those of the slab. 


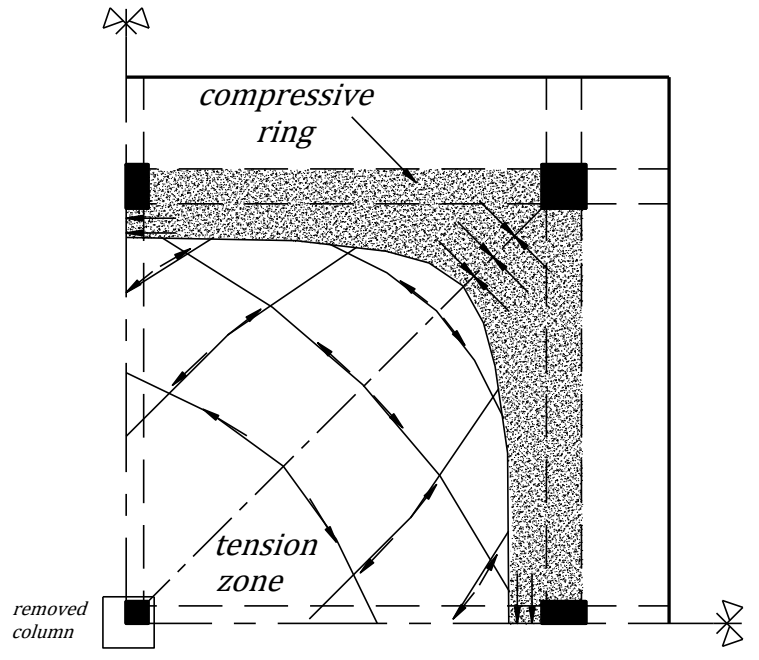

a) internal-column

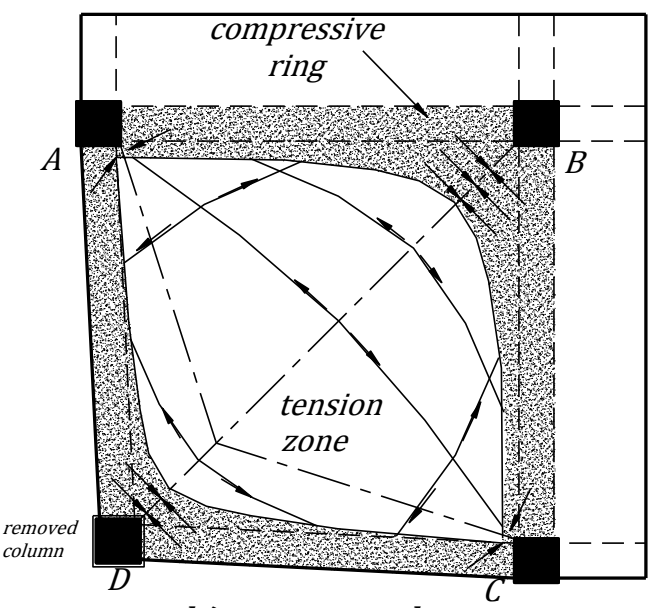

b) corner-column

Fig. 9.23 TMA in slabs

\subsubsection{Penultimate-column removals}

From Fig. 9.21, ultimate capacities of the PI- and the PE-column cases are the smallest among all the five scenarios. Besides, structural capacity of the PE-column case is slightly greater than that of the PI-column case, although the load from the PI-column case can be redistributed to a larger area (Fig. 9.20). These observations can be explained by the presence of some discontinuous slab edges in the PI- or the PE-column cases which provide hogging-moment resistance. Since the boundary beams at these discontinuous sides do not have relatively good rotational restraint compared to those from the continuous sides, slab hogging-moment resistances at these regions are affected. Fig. 9.24 shows the yielding zones of top- and bottomlayer rebars at a deflection of one slab thickness for the PI- and the PE-column cases. The yielding of top reinforcement at discontinuous edges is rather limited compared to that at continuous edges and mostly concentrates at the areas above the peripheral columns. Therefore, hogging moment resistance as well as tensile membrane effect from the slab top reinforcement cannot be effectively mobilised at those edges. This phenomenon does not exist in the internal- and the corner-column cases (Fig. 9.22). Besides, in the PI-column model, one-half of hogging-moment edges is discontinuous, whereas in the PE-column model it is only one quarter. As a 
result, structural resistance per unit area from the PE-column model is generally greater than that from the PI-column model.

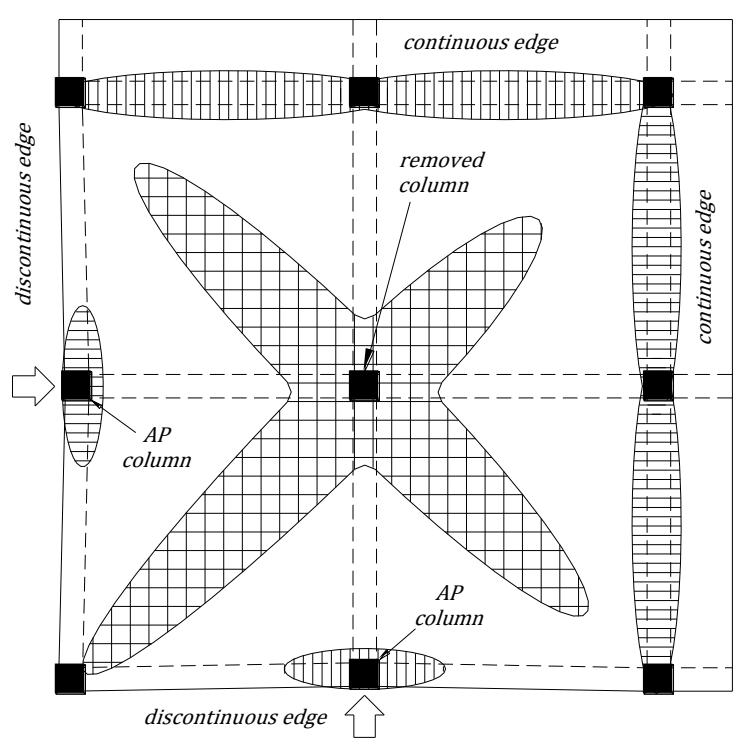

a) PI-column

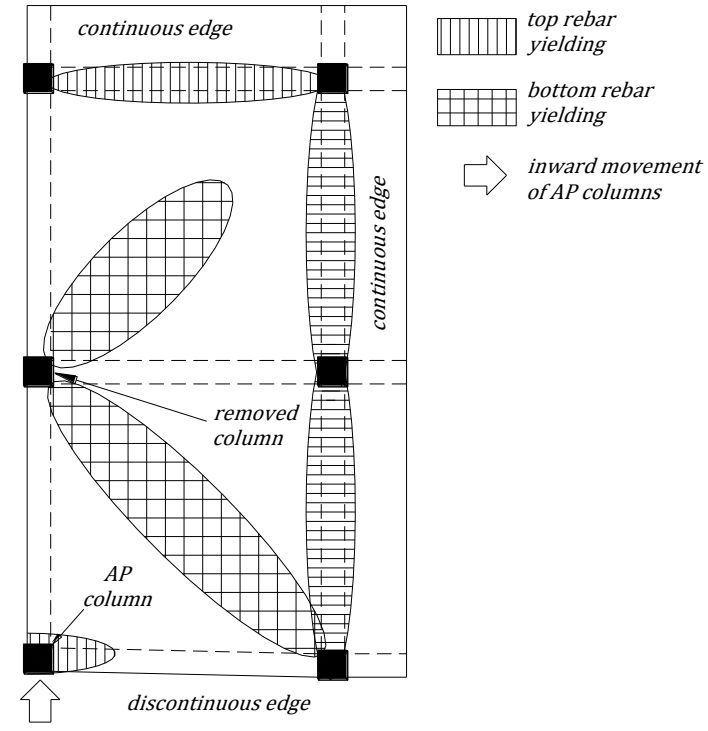

b) PE-column

Fig. 9.24 Rebar yielding at $55 \mathrm{~mm}$ deflection of PI- and PE-column cases

In terms of maximum vertical deflections above the missing column, values from the PI- and the PE-column cases are comparable with those from the other column loss scenarios (Fig. 9.21). However, to obtain this order of vertical deflections, the peripheral columns which are next to the removed column, so-called the affected peripheral (AP) columns, will experience excessive inward horizontal displacement. Again, this phenomenon does not occur under the edge- and the corner-column scenarios. Fig. 9.24 shows the positions of the AP columns as well as their inward movements in the respective PE- and PI-column cases. At a vertical deflection of $160 \mathrm{~mm}$ (Fig. 9.21), at which both the PE- and the PI-column cases almost attain their ultimate resistance, inward movements of the AP columns from the PE- and the PI-column cases are 17 and $11 \mathrm{~mm}$, respectively. With such large horizontal deflections, the AP columns may fail prematurely. In summary, the PE- and the PIcolumn models analysed in this study are more vulnerable compared to the other cases such as the corner- or the edge-column scenarios due to smaller structural capacity and the vulnerability of AP columns against pulling-in forces. Comparing between the PE- and the PI-column cases, although the PE-column case has a 
greater structural response, it also generates a larger movement for its AP column, which in turn makes it the most critical to progressive collapse. In fact, the beamslab tests for PE-column removal from Pham and Tan (2015) and Qian et al. (2016b) showed limited mobilisation of TMA.

\subsection{Simplified analytical model for structural response of RC beam- slab systems}

\subsubsection{Assumptions}

Based on the observations and findings from Sections 9.3 to 9.5 as well as to evaluate the structural capacity of RC beam-slab systems under the scenario of internal-column removal, a simplified analytical procedure is proposed in this part. The general response of a symmetric beam-slab structure, whether under CL or UDL condition, is simplified into a polyline curve OAB as shown in Fig. 9.25. It represents the relationship between total applied load $P$ and central deflection $d$ of the middle joint above the removed column. For the CL case, $P$ is the applied load at the position of the removed column. For the UDL case, $P$ is equal to the applied pressure multiplied by the area of the affected region $\left(q L_{x} L_{y}\right)$. Line OA represents the initial stage when the structural response changes from elastic to yielding while line $\mathrm{AB}$ describes the latter part of response when the structure is under plastic behaviour with the enhancement of TMA in the slab. Neglecting the beneficial effect of horizontal restraints, neither CAA in the internal beams nor CMA in the slabs is considered for OA. The contribution of CA in the internal beams can be computed using the simplified semi-analytical model presented in Section 4.3. Nonetheless, to be conservative, this contribution is not considered in this model and only flexural capacity of internal beams is used. It is also assumed that the final peak of response for the beam-slab system (point B) is governed by rebar fracture in the internal beams as observed in Section 9.3.4 and in recent beam-slab tests (Pham and Tan 2013, Qian et al. 2014, Pham and Tan 2015, Lu et al. 2016, Qian et al. 2016b). As the model is developed for the internal-column removal case, appropriate rotational restraints along the slab boundaries are assumed. In other words, development of TMA is not affected by the rotational stiffness of the slab 
edges. In Fig. 9.25, $L_{n}^{x}$ and $L_{n}^{y}$ are the clear spans of the original internal beams (before the targeted column is removed) in x- and y-directions. The double-span beam-only (sub-assemblage) structures in the $\mathrm{x}$ - and the $\mathrm{y}$-directions of the system are denoted as Beam $\mathrm{X}$ and Beam Y, respectively, while $L_{x}$ and $L_{y}$ are the clear spans of the affected slab area, respectively, and $n$ is equal to $L_{x} / L_{y}$ (slab aspect ratio) with $L_{x}$ as the larger dimension.
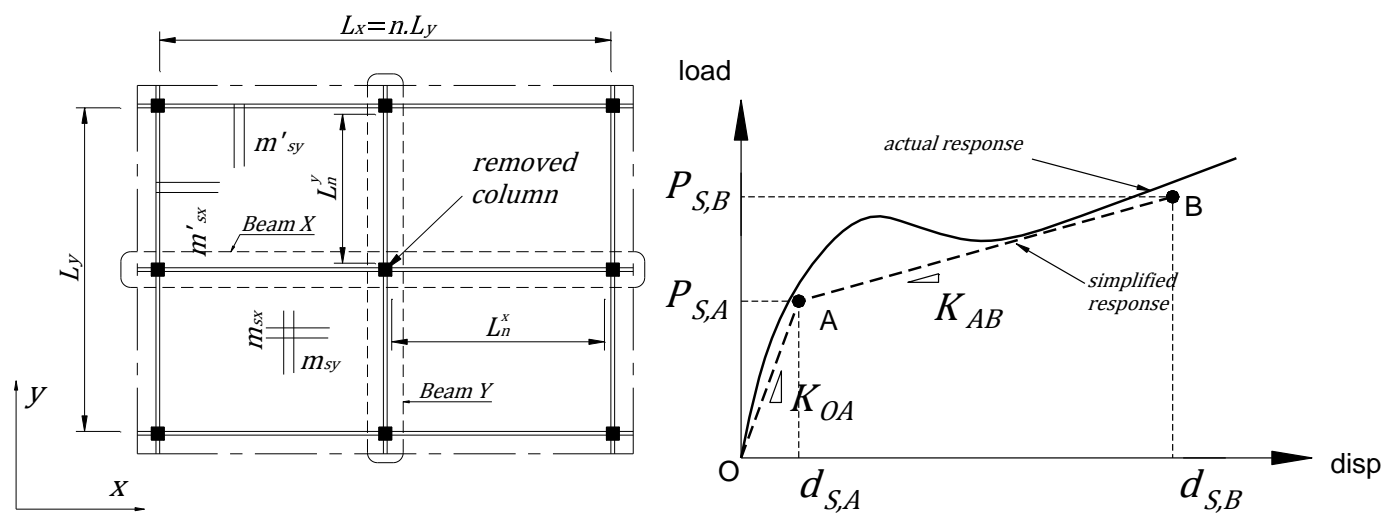

Fig. 9.25 Simplified response of RC beam-slab systems

\subsubsection{Load capacity at point A}

For the CL case, it is assumed that an elliptical yield-line pattern is formed in the slab together with the formation of plastic hinges in the internal beams near the end joints and the middle joints (Fig. 9.26(a)). Such a yield-line pattern is confirmed by the slab tests S1 and S2 from Qian et al. (2014). Based on plastic theory for the internal beams (Section 4.3.1) and yield-line theory for the slab (Park and Gamble 2000), $P_{S, A}$ is calculated as follows:

$$
P_{S, A}=\frac{2\left(M_{b, x}+M^{\prime}{ }_{b, x}\right)}{L_{n}^{x}}+\frac{2\left(M_{b, y}+M^{\prime}{ }_{b, y}\right)}{L_{n}^{y}}+\pi\left(\sqrt{m_{s x} m_{s y}}+\sqrt{m_{s x}^{\prime} m_{s y}^{\prime}}\right)
$$

where $M_{b, x}$ and $M_{b, x}^{\prime}$ are the positive and the negative bending capacities of the internal beams in the X-direction (Beam X), $M_{b, y}$ and $M_{b, y}^{\prime}$ are the positive and the negative bending capacities of the internal beams in the y-direction (Beam Y). These beam bending capacities are computed based on Eq. (4.4). The terms $m_{s x}$ and $m_{s x}^{\prime}$ are the positive and the negative bending capacities per unit width of the 
slab in the x-direction, while $m_{s y}$ and $m_{s y}^{\prime}$ are the positive and the negative bending capacities per unit width of the slab in the y-direction. These slab bending capacities are calculated based on Eq. (9.3).

For the UDL case, a four-segment yield-line pattern is assumed for the slab area, whereas plastic hinges in the internal beams are assumed to occur at intersections of the beams with the slab yield lines (Fig. 9.26(b)). Such formations of plastic hinges and yield lines have been confirmed in the isolated beam-slab tests of Pham and Tan (2013). Eq. (9.5) is then employed to calculate total structural capacity $P_{A}$.

$$
\begin{aligned}
P_{S, A}=q L_{x} L_{y}= & \frac{12 L_{x}}{L_{y}\left[3 L_{x}-L_{y}\right]}\left[2\left(L_{y} m_{s x}^{\prime}+L_{x} m_{s y}^{\prime}+M_{b, x}^{\prime}+M_{b, y}^{\prime}\right)\right. \\
& \left.+2\left(L_{y} m_{s x}+L_{x} m_{s y}+M_{b, x}+M_{b, y}\right)\right]
\end{aligned}
$$

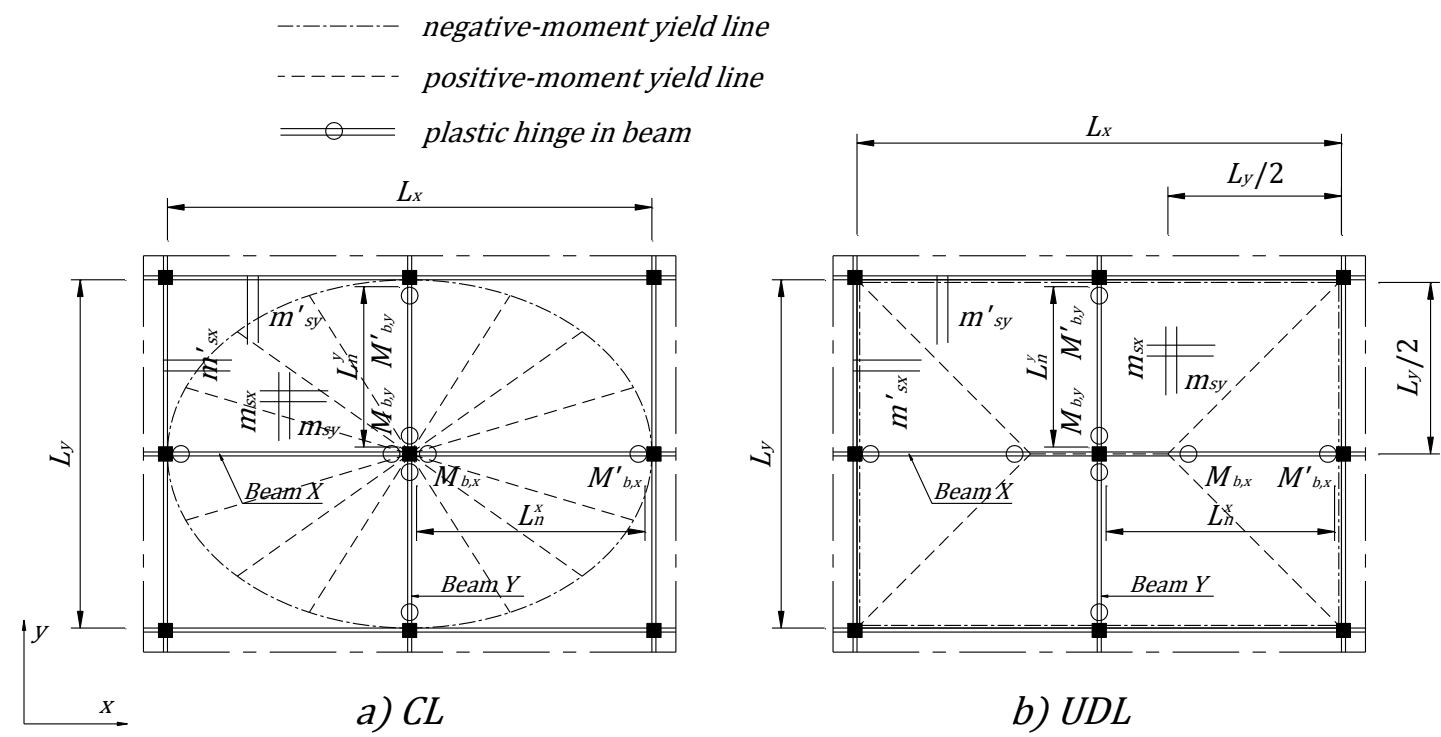

Fig. 9.26 Assumed yield-line patterns

\subsubsection{Central deflection at point $\mathrm{A}\left(d_{S, A}\right)$}

The term $d_{S, A}$ is equal to $P_{A} / K_{O A}$ where $K_{O A}$ is the stiffness of the system (gradient of OA in Fig. 9.25) and is equal to the sum of structural stiffness of Beam X, Beam Y, and the slab-only part (neglecting internal beams), as shown in Eq. (9.6).

$$
K_{O A}=K_{\text {beam }}^{x}+K_{\text {beam }}^{y}+K_{\text {slab }}
$$


For the CL case, it is assumed that plastic hinges are formed at both end joints of Beam $\mathrm{X}$ and Beam $\mathrm{Y}$, and the sub-assemblages are free to rotate at these joints. Therefore, the stiffness values of Beam X and Beam Y under the CL condition are defined by Eqs. (9.7) and (9.8), respectively.

$$
\begin{aligned}
& K_{\text {beam }}^{x}=\frac{48\left(0.5 E I_{x}\right)}{\left(2 L_{n}^{x}\right)^{3}} \\
& K_{\text {beam }}^{y}=\frac{48\left(0.5 E I_{y}\right)}{\left(2 L_{n}^{y}\right)^{3}}
\end{aligned}
$$

where $I_{x}$ and $I_{y}$ are the moments of inertial of concrete sections from Beam X and Beam Y, respectively. $E$ is the elastic modulus of concrete. Factor 0.5 is to consider cracked sections.

For the UDL case, total applied loads on Beam $\mathrm{X}\left(P_{x}\right)$ and Beam Y $\left(P_{y}\right)$ are calculated based on the distribution of uniform load $q$ on these two sub-assemblages (Fig. 9.27), i.e. triangular shape for shorter beam and trapezoidal shape for longer span. The total loads transferring on Beam X and Beam Y are computed as shown in Eqs. (9.9) and (9.10).

$$
\begin{aligned}
& P_{x}=\frac{q L_{y}{ }^{2}}{4}(2 n-1)=\frac{P_{S, A}(2 n-1)}{4 n} \\
& P_{y}=\frac{q L_{y}{ }^{2}}{4}=\frac{P_{S, A}}{4 n}
\end{aligned}
$$

where $P_{S, A}=q L_{x} L_{y}=q \cdot n L_{y}{ }^{2}$

It is assumed that $P_{x}$ and $P_{y}$ are equally distributed along Beam $\mathrm{X}$ and Beam $\mathrm{Y}$, respectively. The applied loads per unit length of these two sub-assemblages are $Q_{x}$ and $Q_{y}$ as shown in Fig. 9.27. $Q_{x}$ and $Q_{y}$ are related to $P_{x}$ and $P_{y}$ by Eqs. (9.11) and (9.12), respectively. 
$P_{x}=\left(2 L_{n}^{x}\right) Q_{x}$

$P_{y}=\left(2 L_{n}^{y}\right) Q_{y}$

Therefore, assuming the formation of plastic hinges at the end joints of the doublespan beams, the stiffness values of Beam $\mathrm{X}$ and Beam $\mathrm{Y}$ with respect to $P_{S, A}$ under UDL condition are defined as follows:

$$
\begin{aligned}
K_{\text {beam }}^{x}=\frac{P_{S, A}}{d_{A}} & =P_{S, A} \times \frac{384\left(0.5 E I_{x}\right)}{5\left(2 L_{n}^{x}\right)^{4} \cdot Q_{x}}=P_{S, A} \times \frac{384\left(0.5 E I_{x}\right)}{5\left(2 L_{n}^{x}\right)^{3} \cdot P_{x}} \\
& =\frac{4 n}{2 n-1} \times \frac{384\left(0.5 E I_{x}\right)}{5\left(2 L_{n}^{x}\right)^{3}} \\
K_{\text {beam }}^{y}=\frac{P_{S, A}}{d_{A}} & =P_{S, A} \times \frac{384\left(0.5 E I_{y}\right)}{5\left(2 L_{n}^{y}\right)^{4} \cdot Q_{y}}=P_{S, A} \times \frac{384\left(0.5 E I_{y}\right)}{5\left(2 L_{n}^{y}\right)^{3} \cdot P_{y}} \\
& =4 n \frac{384\left(0.5 E I_{y}\right)}{5\left(2 L_{n}^{y}\right)^{3}}
\end{aligned}
$$

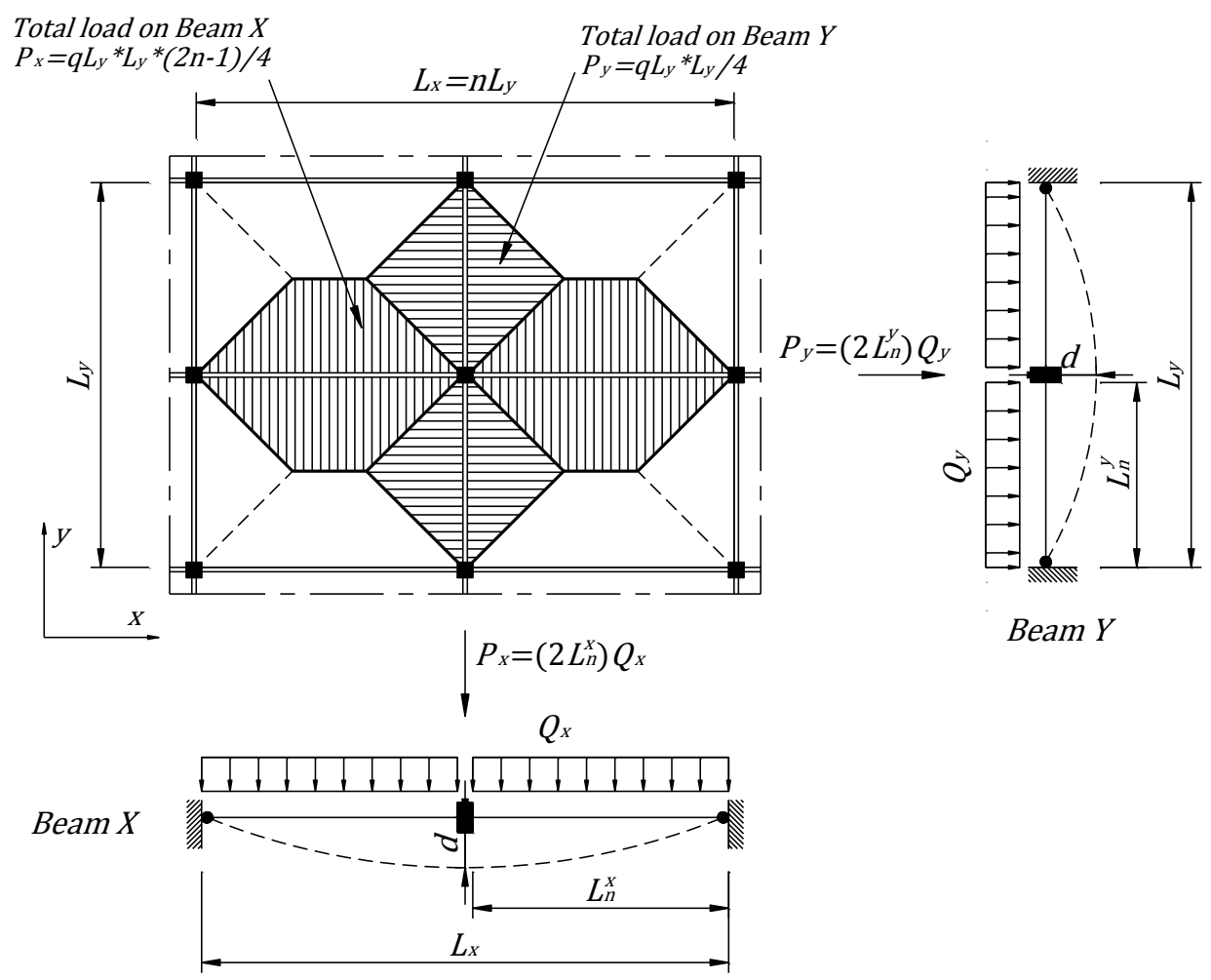

Fig. 9.27 Distribution of load to internal beams under UDL case 
To calculate the stiffness of the slab-only part, elastic theory on plates is applied (Timoshenko et al. 1959). Hence, $K_{\text {slab }}$ is defined by Eqs. (9.15) and (9.16) for the CL and the UDL cases, respectively.

$K_{\text {slab }}=\frac{0.5 D}{\alpha L_{y}^{2}}$

$K_{\text {slab }}=\frac{n q L_{y}{ }^{2}}{d}=n L_{y}{ }^{2} \frac{0.5 D}{\alpha L_{y}{ }^{4}}$

where:

$D=\frac{E \cdot t_{s}{ }^{3}}{12\left(1-v^{2}\right)}$

$t_{s}$ is the slab thickness, $v$ is the Poisson's factor of concrete, $\alpha$ is the dimensionless factor depending on slab aspect ratio $(n)$ and the type of applied load. Table 9.1 presents different values of $\alpha$ corresponding to $n$ (Timoshenko et al. 1959). In Eqs. (9.13) to (9.16), factor 0.5 is used to consider stiffness reduction due to cracked sections at yielding stage.

Table 9.1 $\alpha$ factor (Timoshenko et al. 1959)

\begin{tabular}{|c|c|c|c|c|c|c|c|c|c|}
\hline$n$ & 1.0 & 1.1 & 1.2 & 1.4 & 1.6 & 1.8 & 2.0 & 3.0 & $\infty$ \\
\hline $\begin{array}{c}\alpha \\
(\mathrm{CL} \text { case })\end{array}$ & 0.01160 & 0.01265 & 0.01353 & 0.01484 & 0.01570 & 0.01620 & 0.01651 & 0.01690 & 0.01695 \\
\hline $\begin{array}{c}\alpha \\
(\text { UDL case })\end{array}$ & 0.00406 & 0.00485 & 0.00564 & 0.00705 & 0.00830 & 0.00931 & 0.01013 & 0.01223 & 0.01302 \\
\hline
\end{tabular}

\subsubsection{Gradient of line $\mathrm{AB}\left(K_{A B}\right)$}

The enhancement of structural capacity for line AB in Fig. 9.25 is contributed by TMA developed in the slab. During this stage, it is assumed that the slab top rebars at negative-moment yield-line regions have reached the yield strength and contribute for all of the tensile effects, whereas the tensile contribution of the slab bottom rebars at these areas are conservatively neglected. The elliptical and the four-segment yield-line patterns are still valid for the CL and the UDL cases, 
respectively (Fig. 9.28). The gradient of $\mathrm{AB}$ is computed based on the increment of tensile forces along the negative-moment yield lines with respect to an increment of deformation at the middle of the slab. At a central deflection $d$ and corresponding applied load $P$ (for both the CL and the UDL cases), a deflection increment $\delta d$ will cause a load increment $\delta P$. Considering a small length $\delta i$ along the negativemoment yield line with a tensile force per unit width $F_{a, i}$, it will contribute an increment of vertical applied load $\delta P_{i}$ to $\delta P$, defined by Eq. (9.18). $F_{a, i}$ is computed from yielding forces of top rebars from both directions at $\delta i$ (Fig. 9.29), as shown in Eq. (9.19). Hence, the total increase of load $\delta P$ contributed by all the slab top-rebar yielding forces along the negative-moment yield line is $\sum \delta P_{i}$. Thereafter, $K_{A B}$ can be computed as $\delta P / \delta d$. Based on equilibrium of forces, the gradient of $\mathrm{AB}$ is calculated following Eqs. (9.20) and (9.21) for CL and UDL conditions, respectively.

$\delta P_{i}=F_{a, i} \delta i \frac{\delta d}{L_{i}}$

$F_{a, i}=F_{a}^{y} \cos ^{2} \alpha_{i}+F_{a}^{x} \sin ^{2} \alpha_{i}$

where $F_{a}^{x}$ and $F_{a}^{y}$ are the yield forces per unit width of the slab top rebars along the $\mathrm{x}$ - and the y-directions, respectively. $L_{i}$ is defined as shown in Fig. 9.28.

$$
\begin{aligned}
& K_{A B}=\frac{\delta P}{\delta d}=\frac{\sum \delta P_{i}}{\delta d}=\oint_{\text {yield-line }}^{\text {elliptical }} \frac{F_{a, i}}{L_{i}} \delta i \\
& K_{A B}=\frac{\delta P}{\delta d}=\frac{\sum \delta P_{i}}{\delta d}=\frac{\sum \delta q_{i} L_{x} L_{y}}{\delta d}=\oint_{y \text { ield-line }}^{\text {rectangular }} \frac{F_{a, i}}{L_{i}} \delta i
\end{aligned}
$$




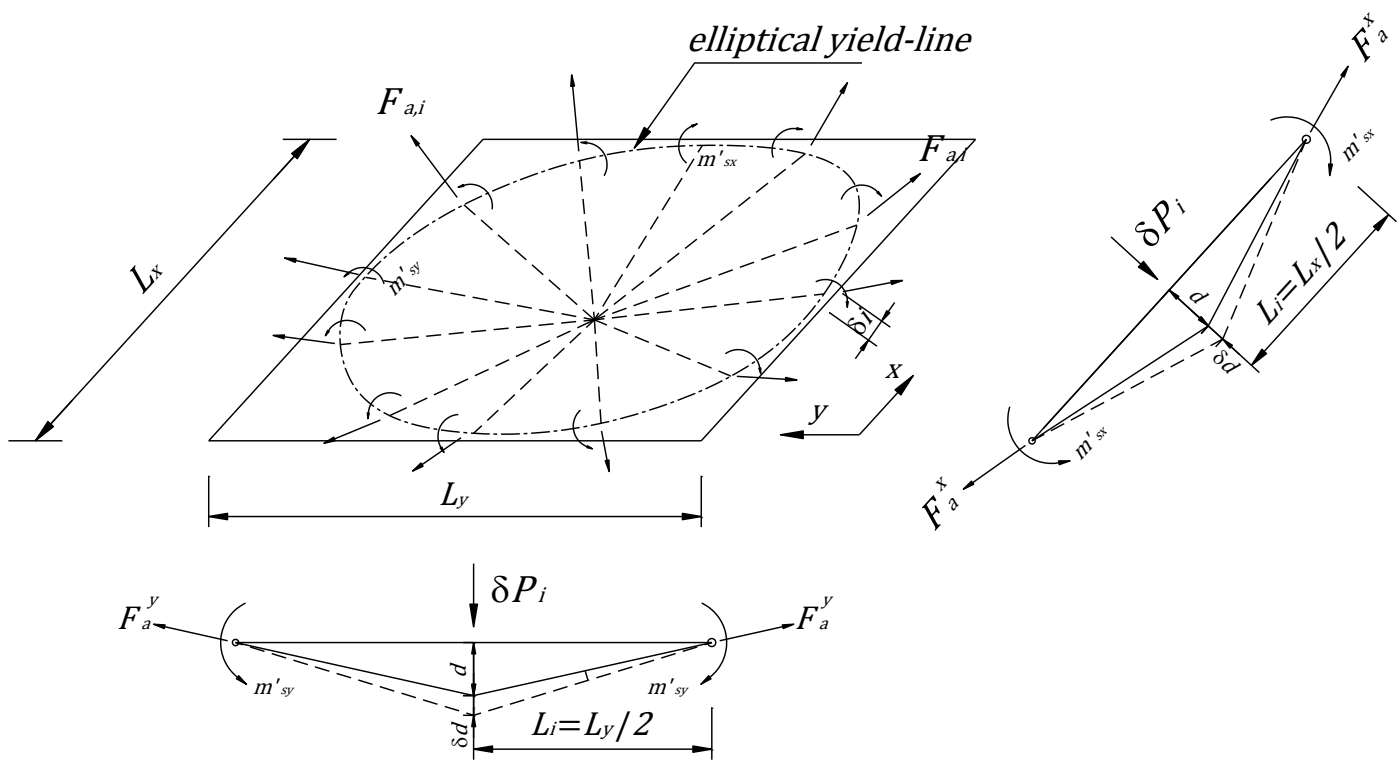

a) $\mathrm{CL}$

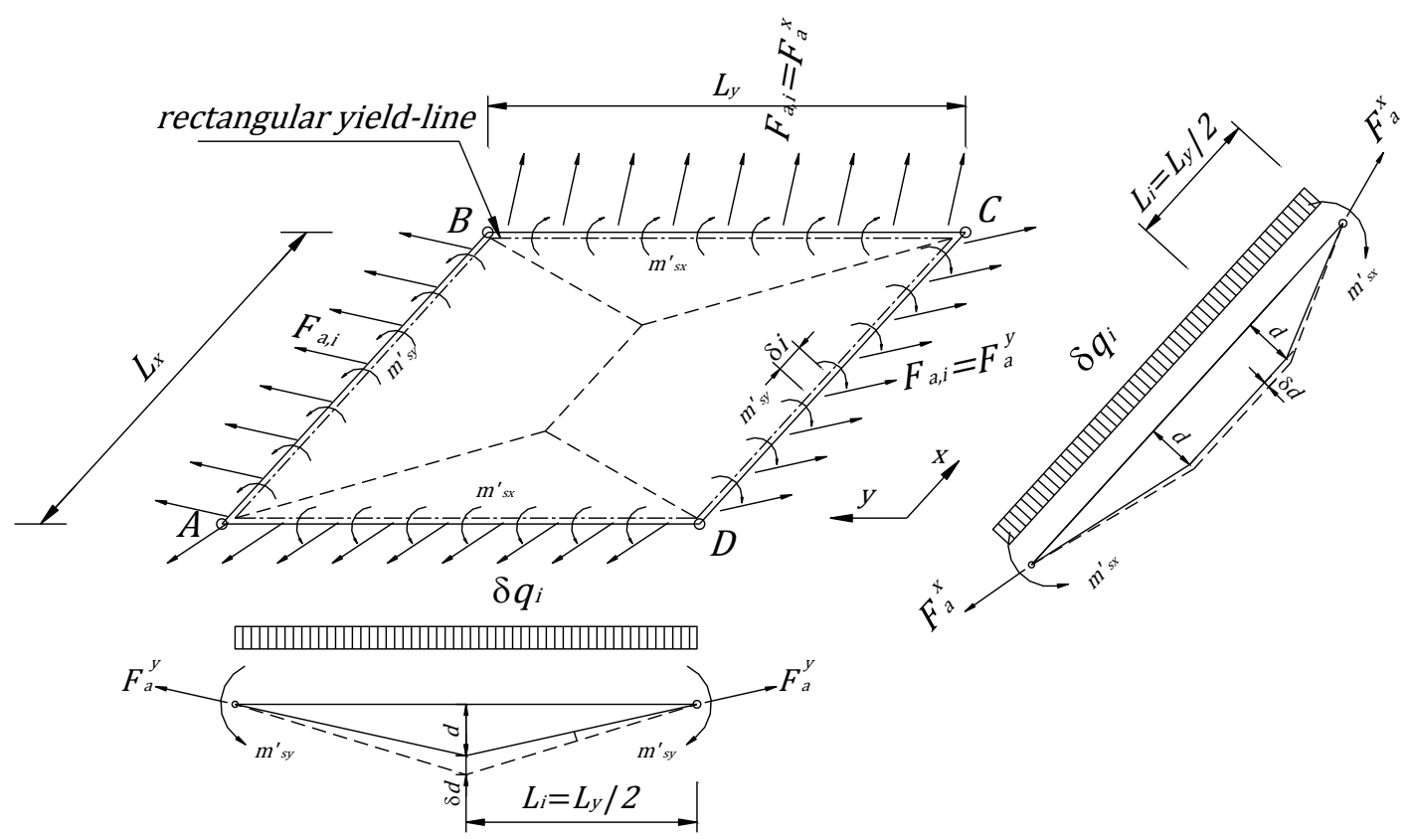

b) UDL

Fig. 9.28 Yielding forces of top rebars at negative yield lines 


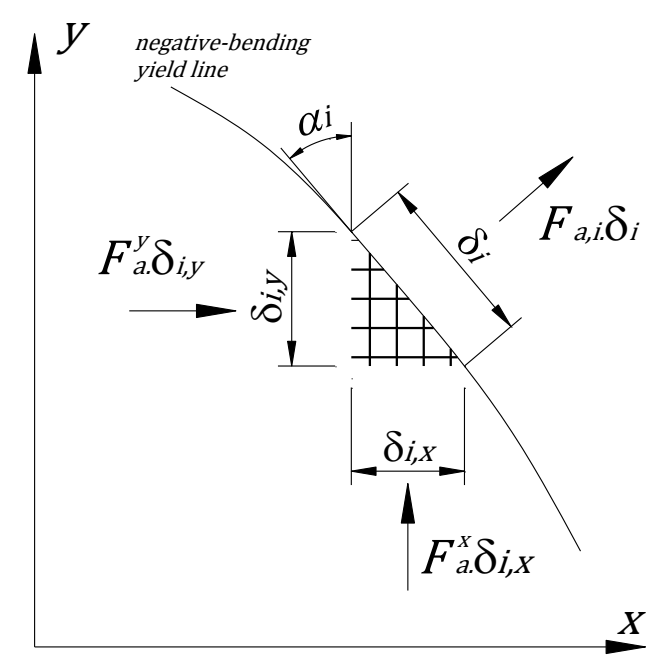

Fig. 9.29 Contribution of top-rebar yielding forces on $F_{a, i}$

\section{For CL condition:}

Considering an ideal scenario of isotropically-reinforced square slab, i.e. $L_{x}=L_{y}$ and $F_{a}^{x}=F_{a}^{y}$, as shown in Fig. 9.30, the negative-moment yield line will have a circular-fan shape (similar to specimen S2 from Qian et al. (2014)). At an arbitrary location of $\delta i$ along this yield line, $L_{i}$ is always equal to $L_{y} / 2$. Therefore, from Eqs. (9.18) and (9.19), $\delta P_{i}$ can be computed as follows:

$\delta P_{i}=\left(F_{a}^{y} \cos ^{2} \alpha_{i}+F_{a}^{x} \sin ^{2} \alpha_{i}\right) \delta i \frac{\delta d}{\frac{L_{y}}{2}}=\frac{2 F_{a}^{y} \delta i}{L_{y}} \delta d$

Applying Eq. (9.22) into Eq. (9.20), $K_{A B}$ is calculated as:

$K_{A B}=\frac{\sum \delta P_{i}}{\delta d}=\oint_{\text {yield-line }}^{\text {circular }} \frac{2 F_{a}^{y}}{L_{y}} \delta i=\frac{2 F_{a}^{y}}{L_{y}} \pi L_{y}=2 \pi F_{a}^{y}$

To apply the calculation of $K_{A B}$ from the isotropically-reinforced square slab to a normal orthotropically-reinforced rectangular slab, a method presented in Park and Gamble (2000) is employed. This method converts an orthotropic slab with elliptical yield line to an equivalent (affine) isotropic slab with circular yield line as shown in Fig. 9.31. A factor $\mu$ is defined as $F_{a}^{x} / F_{a}^{y}$. The equivalent slab has the $\mathrm{x}-$ 
direction span of $L_{x}^{\prime}=L_{x} / \sqrt{\mu}$, and the y-direction span of $L_{y}$ (unchanged). The yield force per unit width of the new slab is $F_{a}^{y}$ for both directions (isotropically reinforced). Besides, the applied load increment of the equivalent slab is $\delta P^{\prime}{ }_{i}=$ $\delta P_{i} / \sqrt{\mu}$. Eq. (9.23) is then applied for the circular-fan yield line of the equivalent isotropic slab as follows:

$\frac{\sum \delta P_{i}^{\prime}}{\delta d}=\frac{\sum \delta P_{i} / \sqrt{\mu}}{\delta d}=2 \pi F_{a}^{y}$

Therefore, $K_{A B}$ of the actual othortropic slab is calculated as:

$K_{A B}=\frac{\sum \delta P_{i}}{\delta d}=2 \pi F_{a}^{y} \sqrt{\mu}=2 \pi F_{a}^{y} \sqrt{F_{a}^{x} / F_{a}^{y}}=2 \pi \sqrt{F_{a}^{x} \cdot F_{a}^{y}}$

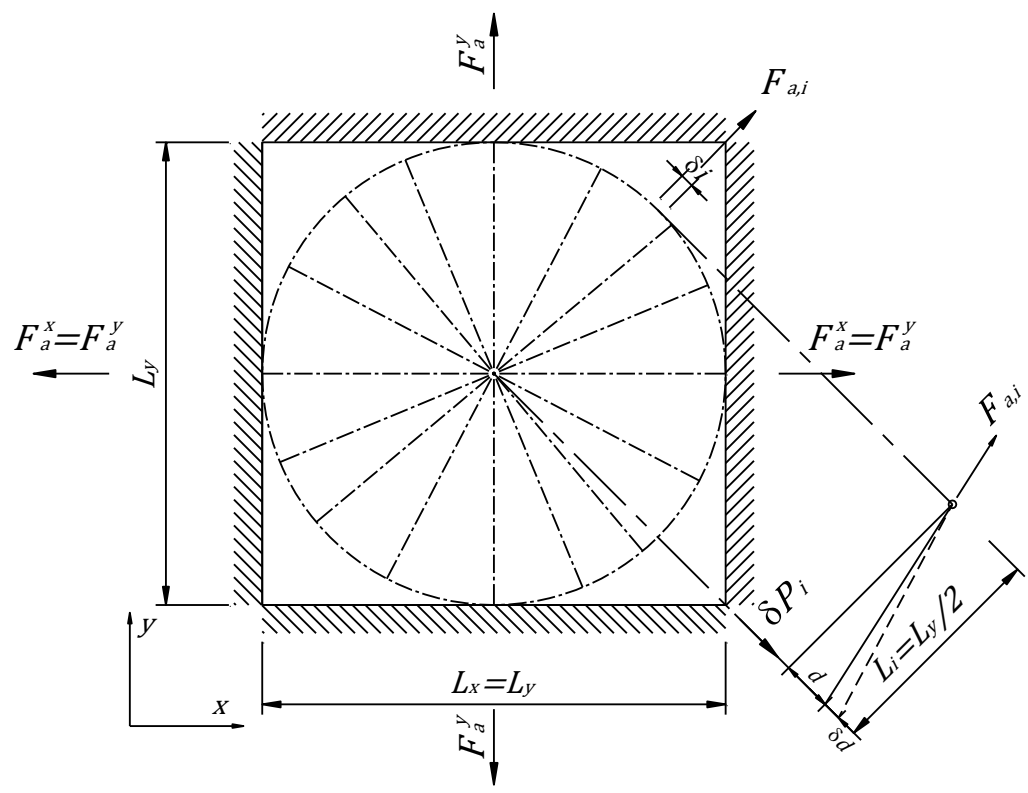

Fig. 9.30 Ideal scenario of isotropically-reinforced square slab 


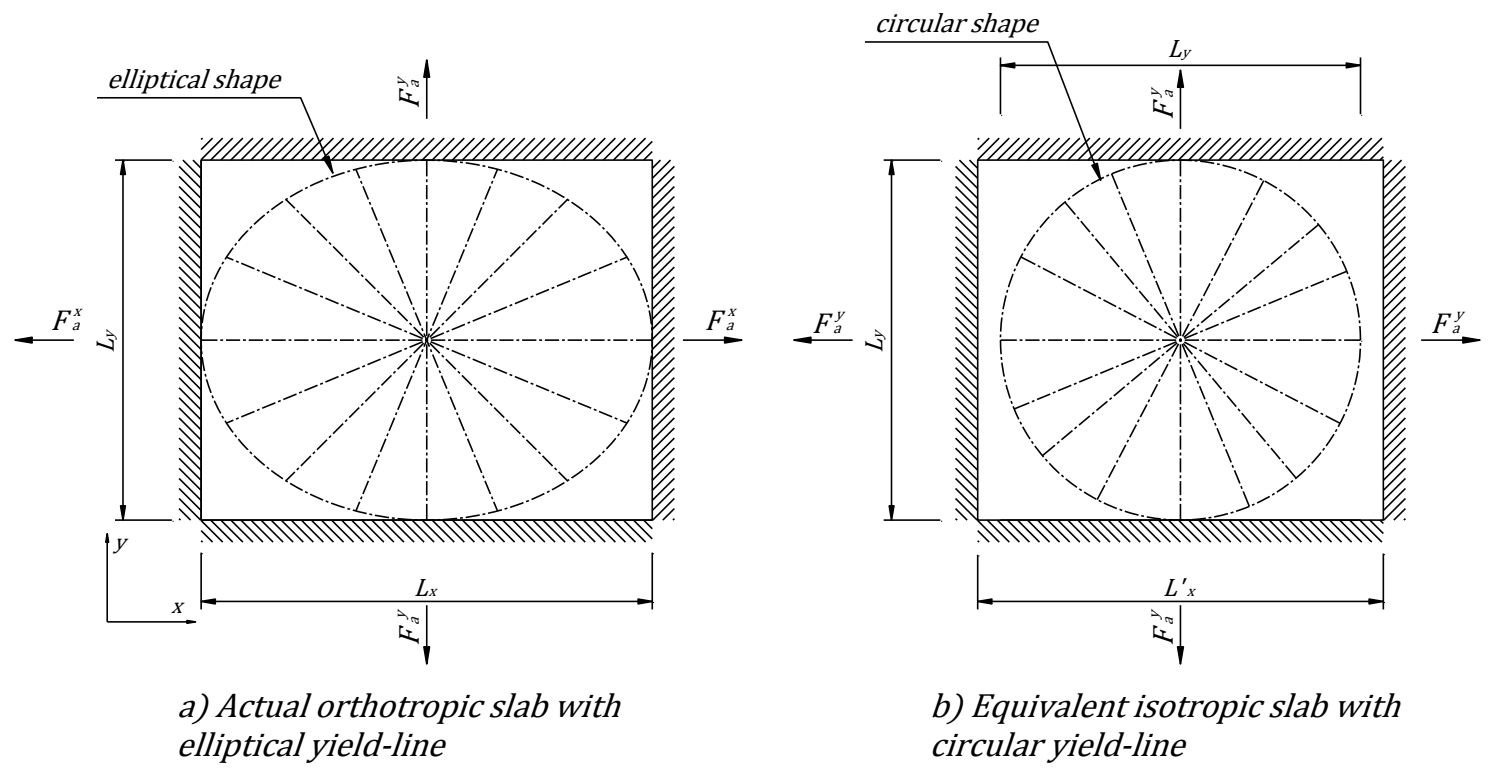

Fig. 9.31 Conversion of actual orthotropic slab into isotropic slab

\section{For UDL condition:}

Based on the four-segment yield-line patterns, $\sum \delta P_{i}$ is calculated along the four edges of the rectangular slab, i.e. $\mathrm{AB}$ and $\mathrm{CD}$ in the $\mathrm{x}$-direction and $\mathrm{AD}$ and $\mathrm{BC}$ in the y-direction (Fig. 9.32). Due to symmetry, the contributions of $A B$ and $C D$ to $\sum \delta P_{i}$ are equal, and similarly for $\mathrm{AD}$ and $\mathrm{BC}$. Along the $\mathrm{x}$-direction yield lines $\mathrm{AB}$ and $\mathrm{DC}, F_{a, i}$ is equal to $F_{a}^{y}$, whereas along the y-direction yield lines $\mathrm{AD}$ and $\mathrm{BC}$, $F_{a, i}$ is equal to $F_{a}^{x}$. However, at any location of $\delta i, L_{i}$ is always equal to $L_{y} / 2$. Therefore, substituting for Eq. (9.21), $K_{A B}$ for the UDL condition is calculated as below:

$$
\begin{aligned}
K_{A B}=\frac{\sum \delta P_{i}}{\delta d} & =2 \int_{B}^{A} \frac{F_{a}^{y}}{\frac{L_{y}}{2}} \delta i+2 \int_{D}^{A} \frac{F_{a}^{x}}{\frac{L_{y}}{2}} \delta i=\frac{4 F_{a}^{y}}{L_{y}} L_{x}+\frac{4 F_{a}^{x}}{L_{y}} L_{y} \\
& =4 F_{a}^{y} \frac{L_{x}}{L_{y}}+4 F_{a}^{x}
\end{aligned}
$$




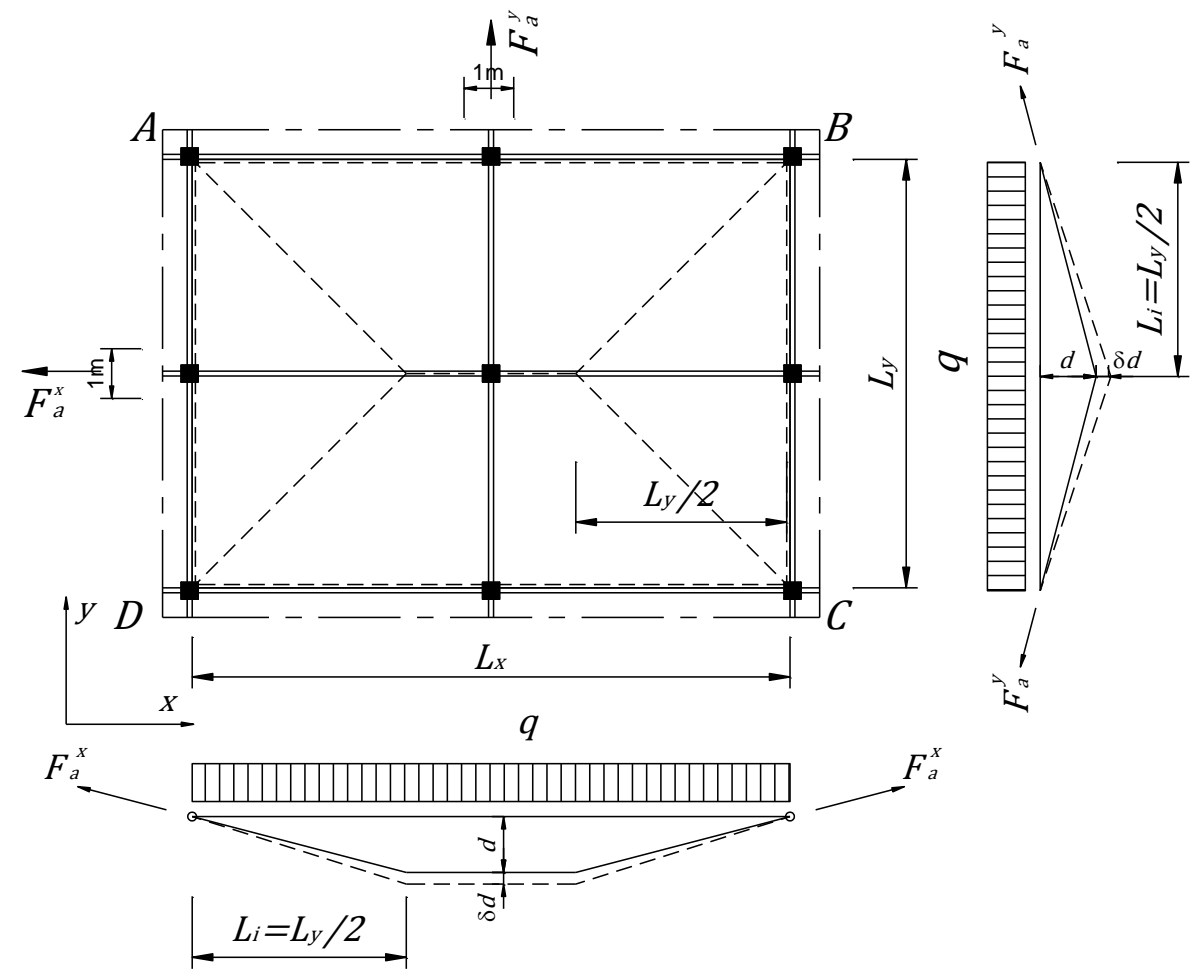

Fig. 9.32 Distribution of slab top-rebar yielding force (UDL)

\subsubsection{Maximum deflection at point $B$ of the beam-slab model}

The term $d_{S, B}$ of the beam-slab model in Fig. 9.25 is determined according to the fracture of rebars within the internal beams, whether the fracture stems from the bottom rebars near the removed column (CL case) or from the top rebars near the end joints (UDL case). Assuming the deflections of beams at rebar fracturing state are similar between the beam-only and the beam-slab systems, the simplified CL sub-assemblage model from Section 4.3 and the simplified UDL sub-assemblage model in Section 7.3 are employed to define the displacement where top or bottom rebars fracture in Beam $X$ and Beam $Y$ (Fig. 7.13). In these models, $d_{D}$ and $d_{D^{\prime}}$ represent the deflections corresponding to bottom rebar fracture near the middle joint in CL response and top rebar fracture near the end joint in UDL response, respectively. For the beam-slab system under CL case, $d_{D}$ from the CL subassemblage model is defined for both Beam X and Beam Y. Therefore, $d_{S, B}$ of the beam-slab model is equal to the smaller value of $d_{D}$. For the beam-slab system under UDL case, deformation when top rebars at the end joint fracture in Beam X 
or Beam Y $\left(d_{D^{\prime}}\right)$ is computed using the UDL sub-assemblage model (Fig. 7.13). Similar to the CL case, $d_{S, B}$ of the beam-slab model is the smaller value of $d_{D \prime}$.

In summary, to predict structural response of a symmetric beam-slab system under internal-column removal, the following steps are conducted:

- Define $P_{S, A}$ based on plastic-hinge and yield-line theories, following Eqs. (9.4) and (9.5) for CL and UDL cases, respectively.

- Define $d_{S, A}$ based on $P_{S, A}$ and the stiffness $K_{O A}$. The term $K_{O A}$ is computed following Eqs. (9.7), (9.8), and (9.15) for CL case, and Eqs. (9.13), (9.14), and (9.16) for UDL case.

- Define gradient $K_{A B}$ of line $\mathrm{AB}$ based on yielding forces of slab top rebars along the negative-moment yield lines. $K_{A B}$ is calculated following Eqs. (9.25) and (9.26) for CL and UDL cases, respectively.

- $\quad$ Define $d_{S, B}$ based on rebar fractures of internal beams (Fig. 7.13).

After defining the simplified static response (Fig. 9.25) of the beam-slab structure, the maximum dynamic load can be computed based on strain energy (Fig. 9.33), as shown in Eq. (9.27).

$P_{d y n}=\left[P_{S, A} d_{S, A}+\left(P_{S, B}+P_{S, A}\right)\left(d_{S, B}-d_{S, A}\right)\right] / 2 d_{S, B}$ 


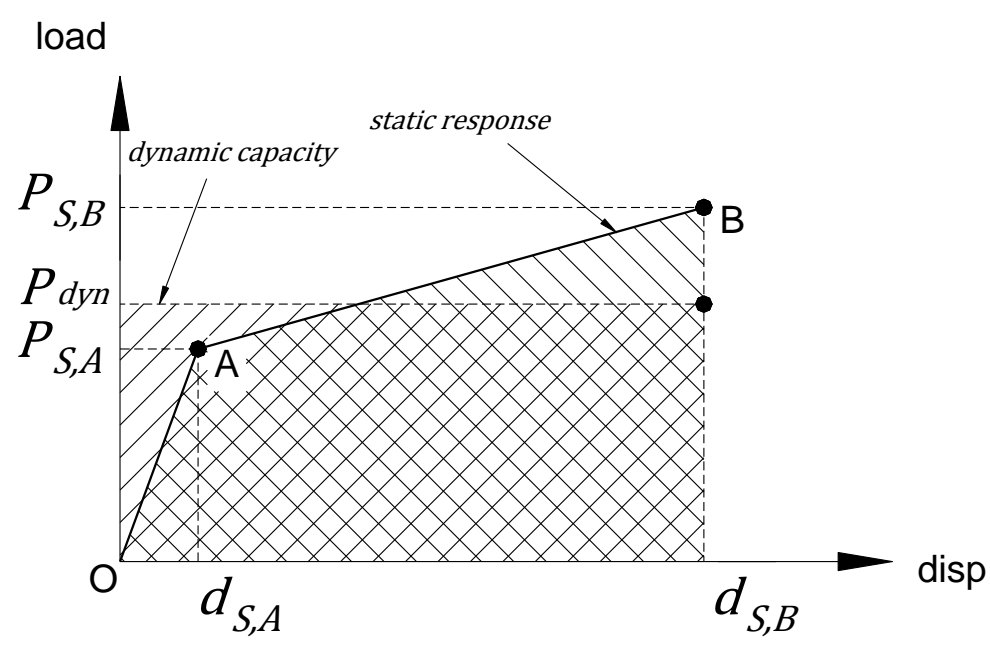

Fig. 9.33 Dynamic capacity based on the simplified model

\subsubsection{Validations of the simplified model for beam-slab structures}

To validate the conservatism of the proposed model, results from published beamslab tests under both CL and UDL conditions are employed. From Table 2.2 in the literature review chapter, it is shown that only two series of beam-slab tests under internal-column removal subjected to CL were conducted, i.e. Qian et al. (2014) and Ren et al. (2016). However, the latter only studied one-way behaviour of the beamslab structures. Therefore, only two slab tests S1 and S2 from Qian et al. (2014) are used to verify the proposed model under the CL case. For the UDL condition, there is no existing beam-slab test under internal-column removal available to date. Therefore, to validate the analytical model under the UDL case, beam-slab tests from Pham and Tan (2013) are used. In this test series, six specimens of 2-by-2span isolated slabs were conducted representing the removal of a penultimateinternal column. Although they were different from the case of an internal-column loss due to a lack of slab extensions at four sides, the first four specimens (PI-1 to PI-4) are still used to validate the beam-slab model as there was no major torsional failure of edge beams from these tests. The last two specimens, PI-5 and PI-6, experienced severe torsional failures of the boundary beams and hence are not used for validation since the assumption of appropriate rotational restraints is violated.

Validation results for the CL and the UDL tests are shown in Fig. 9.34. For the case of CL condition (Fig. 9.34(a) and (b)), the proposed model provides conservative 
predictions compared to the actual tests for both the yielding and the TMA parts. The reasons are probably due to the neglect of CAA and CA in internal beams, as well as the neglect of tensile contribution from bottom rebars in slabs at the negative-bending yield lines. For the UDL case (Fig. 9.34(c) to (f)), predictions from the model have good agreement with test results, even though the actual tests had partially-rigid rotational restraints of the slab edges. If the specimens from Pham and Tan (2013) had been provided with slab extensions to represent the actual internal-column removal scenario, the test results could have increased. In summary, the proposed model is shown to provide conservative predictions for static structural capacities of beam-slab systems under the internal-column loss event.

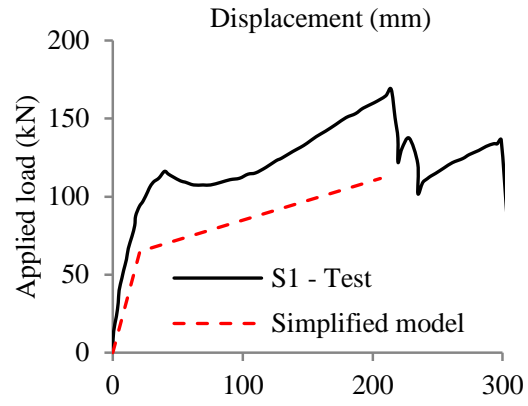

a)

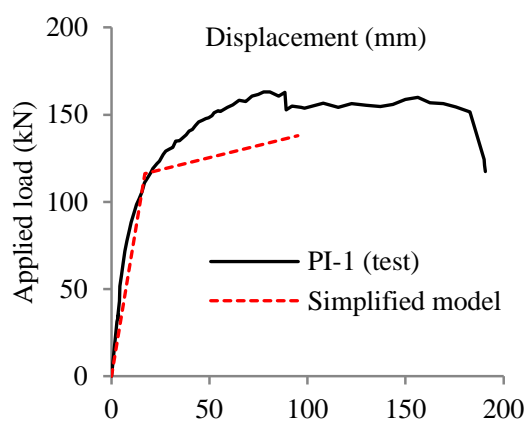

c)

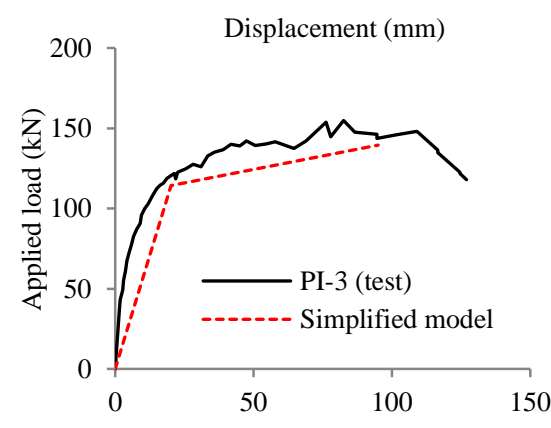

e)

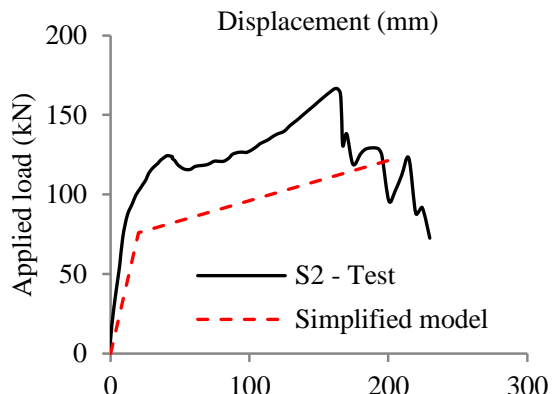

b)

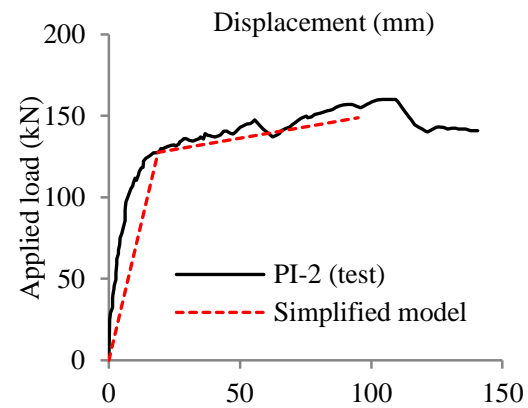

d)

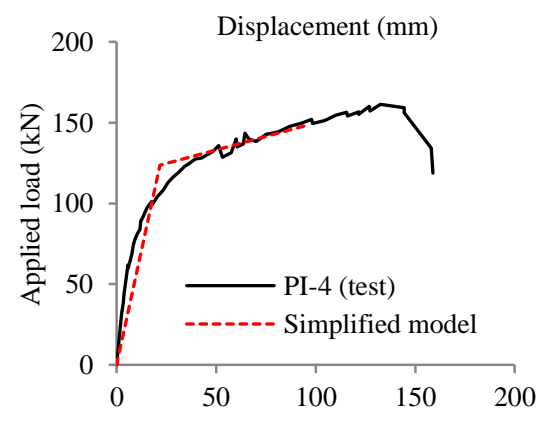

f)

Fig. 9.34 Model validations 


\subsection{Effect of contact detonation on 3D beam-slab structures}

The simplified analytical method presented in Section 9.6, together with the use of dynamic assessment based on strain energy (Fig. 9.33) proposed by Izzuddin et al. (2008), provide a convenient tool to engineers for designing beam-slab structures under progressive collapse threats. During design procedure, the maximum dynamic load computed from Eq. (9.27) can be used to compare with the partial safety factor for load defined from guidelines for progressive collapse, such as the combination values of 1.2 for dead load and 0.5 for live load prescribed in DOD (2013). However, as observed in the actual blast tests of Series III presented in Chapter 8, damages from an initial event of terrorist attacks involving contact detonation can significantly reduce overall structural responses of the surviving structures after the targeted column has been completely removed. If the structure is applied with the imposed load close to the maximum dynamic value based on tensile mechanisms such as CA or TMA, effects from a contact detonation, even with a small amount of charge weight, can easily cause the structure to collapse (CD1-P/25/M).

In an actual building, besides the beam-column frames, presence of the slab will trap the blast-induced pressure and cause more damages to the structure. According to Guideline for Enhancing Building Security in Singapore (MHA 2010), the possible threats created by an improvised explosive device can be up to $20 \mathrm{~kg}$ TNT (for commercial/office areas) whereas those created by a vehicle-borne improvised explosive device can be up to $500 \mathrm{~kg}$ TNT (for parking areas). Therefore, studies on beam-slab structures under progressive collapse triggered by actual explosive attacks are of great importance. However, the conduct of such experimental work is greatly constrained by costs and safety. On the other hand, numerical studies on beam-slab structural responses induced by blast are tremendously time-consuming due to complexities of blast effect simulations, as well as a variety of blast parameters (explosive type, charge weight, stand-off distance, etc.) that need to be considered.

To investigate the effect of blast loads on the beam-slab system compared to the predicted dynamic capacity based on static response, an exploratory study is conducted using the FEM beam-slab model of specimen S2 from Qian et al. (2014) 
which is developed and validated in Section 9.2. Izzuddin's method is used to convert the actual static response of S2 under CL condition (Qian et al. 2014) into a pseudo-static response. As a result, the maximum progressive collapse load of S2 acting on the middle column is $126 \mathrm{kN}$. A series of numerical dynamic analyses which employs a contact-detonation event is carried out. The explosive charge is located at the middle column of the model and is $750 \mathrm{~mm}$ below the bottom surface of the slab. The Load_blast_enhanced function integrated into LS-Dyna is employed to calculate the blast pressure and to assign this effect to the bottom surfaces of the structure. From the actual blast-test results of Series III (Chapter 8), it is shown that the spherical free air-burst scenario can be conveniently used to replicate the effect of contact detonation to the surviving members in a blastinduced single column removal event.

To have consistency with quasi-static behaviour, CL condition is employed in the blast simulations with the imposed mass at the top of the middle column equal to $100 \mathrm{kN}$ which is $79 \%$ of the predicted dynamic capacity based on Izzuddin's method. Several charge weights are used including 1, 2 and $4 \mathrm{~kg}$ of equivalent TNT. Additionally, one free-fall dynamic analysis (without blast) is also conducted as a baseline for comparison. At the beginning of the analysis, the explosive is activated and only lasts for a few milliseconds. Under the blast pressure, the slab system starts deforming upwards. At the moment when it attains the maximum uplift deflection, gravity load and imposed mass are applied. If the damages from the blast event are not severe, the structure starts oscillating and gradually obtains its equilibrium position. Otherwise, the beam-slab system will collapse due to strength reduction caused by the blast effect.

Numerical results for central displacement time-histories are presented in Fig. 9.35. It clearly shows a remarkable increase in deformation between the two cases, i.e. with and without blast effect. The structure can only survive the sudden column loss event if the charge weight is not greater than $1 \mathrm{~kg}$ of TNT. For 2 and $4 \mathrm{~kg}$ of TNT, the beam-slab system fails to resist the given applied load and totally collapses. Fig. 9.36 describes the responses and damages of the model under blast effect at the 
moment when the slab attains its maximum uplift movement. As observed, the structure attacked by $4 \mathrm{~kg}$ of TNT suffers the most severe damages for both the slab and the internal beams in the vicinity of the middle column. On the other hand, the structure with 1 or $2 \mathrm{~kg}$ of TNT only sustains minor or moderate damages caused by the blast loads. From the numerical results, it shows that application of the ALP method using the assumption of single column removal is not necessarily safe if the actual incident involves contact detonation, and especially if the structure is loaded close to its maximum dynamic capacity calculated based on tensile mechanisms. To precisely predict the actual maximum dynamic load of the S2 model with regard to a specific charge weight, as well as to find a certain blast-loading scenario in which the assumption of single column removal is no longer valid, comprehensive numerical work will have to be conducted. Several blast parameters (charge weight, stand-off distance) should be considered. Nonetheless, due to computational constraint, such objective is outside the scope of this study.

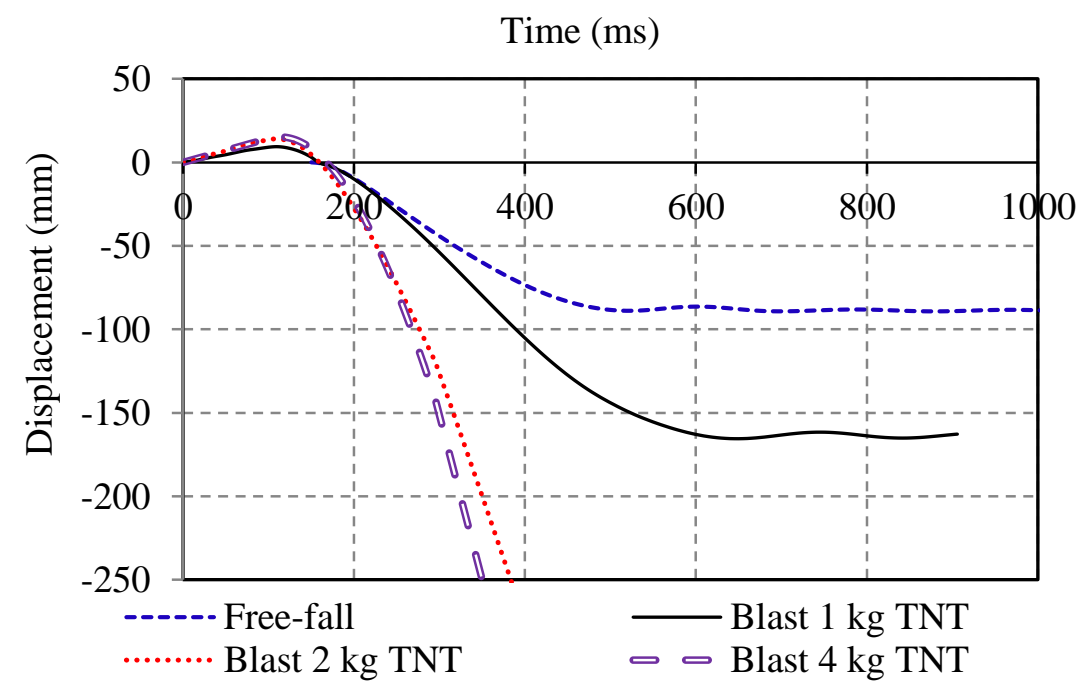

Fig. 9.35 Dynamic responses of the slab structure 

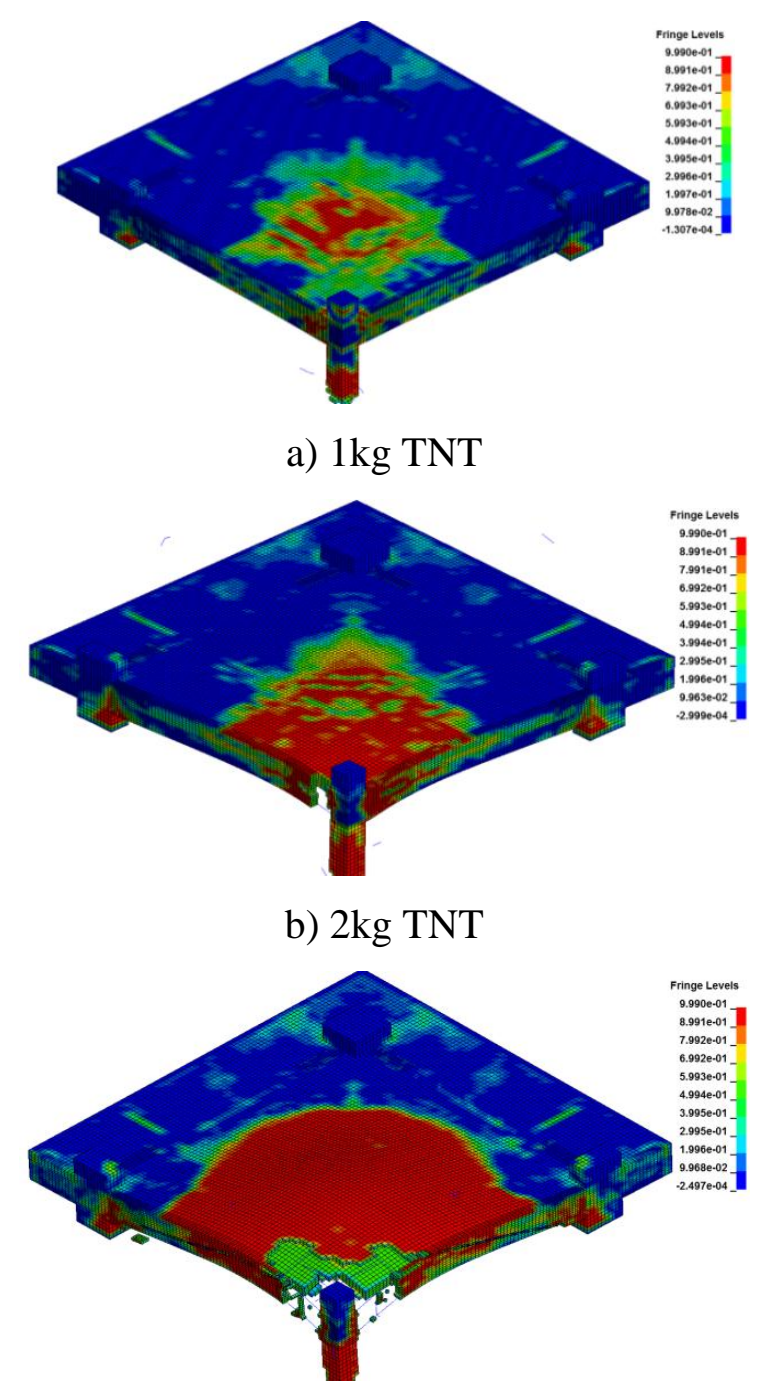

c) $4 \mathrm{~kg} \mathrm{TNT}$

Fig. 9.36 Effects of blast loading at maximum uplift deformation

\subsection{Summary}

To supplement the studies on beam-column structures presented in the previous chapters, in this chapter, an investigation on beam-slab systems under progressive collapse is conducted focusing on the contribution of TMA provided by slab reinforcement. The following conclusions are drawn:

(1) For beam-slab behaviour under single column removal, fracture of reinforcement in internal beams can be used as an indicator of load-carrying capacity. The combined effects of beams and slabs can be considered by separately taking into account the behaviour of the beam-only and the slab-only 
structures under both types of loading method. While CMA is influenced by the stiffness of horizontal restraints, TMA is little affected by this parameter. This finding is observed in both CL and UDL configurations. On the other hand, the response of the beam-slab structure under UDL condition is dependent on rotational restraint of the slab edges.

(2) Under UDL condition, among different scenarios of column loss, the corner column case is not necessarily the most critical. If the boundary beams along the slab's discontinuous edges are strong and stiff enough, the slab can develop limited TMA even under unrestrained conditions from two sides of the removed column. Instead, the scenarios involving penultimate columns can be more critical due to the weak rotational restraint of discontinuous edges which affects the stress development of the slab's top reinforcement at these areas. Moreover, excessive inward movement of affected penultimate columns next to the removed column makes the penultimate-column scenarios more critical.

(3) To evaluate the static structural capacity of RC beam-slab systems under internal-column removal scenario, a simplified analytical procedure is proposed. Both CL and UDL methods, as well as enhancement from TMA to structural resistance, are considered. It is conservatively assumed that only the slab top reinforcement aTlong negative-moment yield lines contributes to TMA. Validations with actual test results show that the simplified beam-slab model provides conservative predictions for the case of appropriate rotational restraint and can even give good agreement with test results that had imperfect rotational restraint.

(4) Although it is convenient to apply the immaculate column removal scenario to study the structural responses of beam-slab systems under static environment, its threat-independent characteristics become less conservative if a contact detonation threat is involved. When a beam-slab structure is applied with imposed loads close to the maximum dynamic capacity calculated based on TMA, even a not-so-large contact detonation charge can easily cause a 
complete collapse. In such events, Izzuddin's method is not recommended for progressive collapse design. To investigate the actual blast scenario that limits the application of the single column removal approach, it is necessary to employ extensive high-fidelity analyses using advanced FEM tools. However, this is outside the scope of the thesis. 


\section{CHAPTER 10 CONCLUSIONS AND FUTURE WORK}

\subsection{Conclusions}

Although progressive collapse is a realistic dynamic event which involves several complicated phenomena, the most commonly-used approach to study this behaviour is through nonlinear static analysis with consideration of dynamic effects. The recommended method in DOD (2013) to mitigate progressive collapse, viz. the alternate load path approach, is based on the assumption of sudden removal of one vertical supporting component. It is important to ascertain the conservatism of this approach, based on nonlinear static analysis with dynamic enhancement factor for loads. In this thesis, numerical and experimental studies were conducted to investigate structural responses of RC substructures against the column loss event under different loading rates, loading configurations, and structural types (beamcolumn and beam-slab structures). Three loading rates were considered including quasi-static, free-fall dynamic, and ultra-fast blast-induced environments. Simultaneously, two loading configurations were investigated i.e. CL and UDL conditions. The objectives of the research are summarised below:

- To extensively investigate nonlinear static behaviours of 2D beam-column and 3D beam-slab systems under progressive collapse subjected to two types of loading configuration, CL and UDL. Upper-bound tensile mechanisms, i.e. CA in beams and TMA in slabs, were identified. Based on the observations from numerical and experimental work, simplified engineering models were proposed to predict quasi-static behaviour of these RC structures under both loading configurations. These models can serve as simple and convenient tools to practical design.

- To compare and contrast the behaviour and failure modes observed in the quasistatic tests with the free-fall dynamic tests on 2D beam-column structures. Both CL and UDL configurations were employed in Series I and II, respectively, for the dynamic tests. By doing so, it clearly establishes the applicability and conservatism of nonlinear static analysis compared to the actual dynamic 
responses. Besides, Izzuddin's method for dynamic assessment was also verified experimentally.

- To investigate the effects of blast on the behaviour of 2D frames in a contactdetonation progressive collapse event. By studying the blast effect and strength reduction, limitations of the single column removal scenario and the dynamic assessment method proposed by Izzuddin were addressed.

Detailed results and findings from the research work are listed as follows under separate headings:

\section{Numerical and semi-analytical approaches for beam-column sub-assemblages under quasi-static environments}

To develop robust numerical analyses for assessing $\mathrm{RC}$ sub-assemblage responses under single column removal scenario, FEM models are developed in Chapter 3 using LS-Dyna software:

(1) The continuous surface cap model is employed to simulate concrete material due to its ability of capturing post-peak softening, shear dilation, confinement effect, etc. Besides, element erosion is also employed using maximum principal strain criterion. It is shown that FEM predictions are significantly dependent on mesh size and erosion limit. In this study, the bond-slip behaviour between concrete and reinforcement is explicitly considered using the Contact_1D function of LS-Dyna, validated by a component pull-out test series. The blast effects on structures are simulated using the Load_blast_enhanced keyword in LS-Dyna, which is based on data from CONWEP (Hyde 1991). The developed models successfully replicate structural response as well as failure modes from both quasi-static and blast-induced tests on sub-assemblages.

(2) The subsequent parametric studies on quasi-static behaviour of RC subassemblages show that span-depth ratio and horizontal restraint stiffness greatly affect the mobilisation of $\mathrm{CA}$ in axially-restrained structures, not only before 
but also after the fracture of bottom rebars. If the span-depth ratio is less than 7 , CA may not be developed due to brittle failure after the peak of flexure/CAA behaviour. On the other hand, if the normalised horizontal stiffness $k_{h o r}$ is too small (Eq. (4.29)), development of CA after the bottom rebars have fractured is rather limited due to excessive inward movement of the end joint. Other factors such as concrete strength, connection gap, rotational restraint stiffness, may have significant influence on the mobilisation of CAA, but they have less contribution on CA.

(3) Based on extensive data from FEM analyses as well as test results from published literature, a semi-analytical model is proposed in Chapter 4 (Section 4.3) to predict the structural static response of an RC sub-assemblage under progressive collapse. The model accounts for horizontal stiffness of the boundary, as well as actual capacity of the restraint. Besides, the fracture of the top and bottom rebars is reasonably predicted by the proposed model. At the instants of rebar fracturing, plastic hinges are assumed to be fully developed at either the middle or the end joint. The simplified model provides a quick and convenient tool to structural engineers for preliminary sizing of members.

\section{Dynamic behaviour of RC frames under progressive collapse applying CL condition (Series I)}

To confirm structural behaviour and failure modes of RC beam-column structures obtained from nonlinear quasi-static responses, dynamic tests of Series I were conducted using a quick-release device to simulate sudden removal of a supporting column:

(1) Under CL condition, the development of CA after the bottom rebars had fractured, which had not been confirmed in any previous dynamic studies, was clearly observed. It showed that CA relying only on top reinforcement could still provide adequate ALP to prevent collapse. Failure modes, deflection profile, as well as behaviour at structural and cross-sectional levels of the freefall tests, agreed closely with observations from the corresponding quasi-static 
tests. Moreover, the responses of reaction forces versus MJD achieved from the free-fall dynamic tests also followed those in the static environment. Suffice to say, the sudden removal of a column will not change the failure modes observed in the static environment. It confirms the applicability and usefulness of nonlinear static tests.

(2) Dynamic effects of the sudden column loss event such as DLIFs for reaction forces, DIFs for material strength and stiffness, were clearly observed. It was shown that the strain rate effects created during the column removal incident were beneficial to the development of flexural/CAA strength. On the other hand, structural capacity during the CA stage was less influenced by strain rate effects. As a result, the simplified framework proposed by Izzuddin et al. (2008) is conservative during the CAA stage as it neglects the contribution of damping and strain rate enhancement. However, during the CA stage, the maximum structural capacity predicted by this method should not be exceeded.

(3) To simulate the beam-column frame responses under dynamic environment, 3D physics-based modelling can be applied. Despite good predictions in terms of both structural behaviour and failure modes, this rigorous analysing method is very time-consuming. On the other hand, the use of equivalent SDOF models employing the semi-analytical model for static response is shown to be not only conservative but also cost-and-time effective for investigating maximum displacement under various conditions of applied load, release time, or damping ratio.

Behaviour and failure modes of RC beam-column structures subjected to UDL condition under both quasi-static and dynamic environments (Series II)

Although the behaviour of RC beam-column structures under progressive collapse has been studied extensively in previous research work, there is no quasi-static test applying UDL condition. Therefore, the static and dynamic frame tests subjected to UDL in Series II are needed: 
(1) Compared to the behaviour under CL condition which has been widely investigated, structural response under UDL configuration was different in terms of load-carrying capacity, sequence of failure, deformation profile, as well as maximum deflection. The fracture of bottom rebars, which was observed in all the reported quasi-static tests as well as in the free-fall tests of Series I, did not occur in any of the Series II tests. Instead, fracture of top rebars at the end joint could be considered as the failure criterion of the structure subjected to UDL. Besides, mobilisation of CA in the UDL tests was rather limited compared to the corresponding CL tests due to smaller deflections in the UDL tests, showing that such upper-bound mechanism in progressive collapse resistance has less influence on structures under UDL condition.

(2) A simplified response of beam-column structures subjected to UDL is proposed (Fig. 7.13) based on the semi-analytical model under CL condition developed in Section 4.3. The predictions are conservative, compared to the actual test results of FR-U specimen.

(3) Similar to the free-fall tests under CL condition (Series I), the free-fall tests under UDL condition of Series II agreed well with the corresponding UDL quasi-static tests in terms of failure modes and structural response. Therefore, it again verifies the use of nonlinear static analysis incorporating Izzuddin's method for analysing RC frames under missing column scenario.

\section{Effects of contact detonation in structural behaviour under progressive collapse} (Series III)

Blast effect on surviving structural members in a blast-induced progressive collapse event is of great importance as it shows the limitations of both the single column removal scenario and the simplified dynamic assessment method proposed by Izzuddin. Compared to the laboratory free-fall tests (Series I), the contact detonation tests conducted at site (Series III) were closer to the actual scenario of a terrorist attack: 
(1) Under the detonation of the explosive charge, the support at the middle column was immediately eliminated within few milliseconds, much faster than the laboratory tests. Moreover, effects of blast pressure on the remaining beamcolumn frame were elucidated via structural uplift and pressure time-histories recorded at the beam soffit and the side column. The reduction of structural resistance due to the blast event was obvious when comparing the behaviour and failure modes of the blast tests to those of the corresponding free-fall tests.

(2) Although the strain rate recorded during the blast duration was very high (up to hundred /s), its enhancement on progressive collapse resistance was rather limited as its duration was very short. After the blast effect had ceased, DIFs for material strength observed in the blast tests were not noticeably larger than those from the free-fall tests.

(3) Applying $0.4 \mathrm{~kg}$ PETN contact charge, Izzuddin's method can still provide conservative predictions for flexure/CAA response (CD2-F/20/M). Nonetheless, it overestimates the response under CA stage of CD1-P/25/M. Therefore, this approach should be applied with caution when predicting structural capacity based on CA in a blast-induced progressive collapse event.

\section{Numerical and analytical investigation of tensile membrane action in beam-slab} systems

Since slab members can significantly contribute to the structural capacity of an RC system against progressive collapse, their behaviour should not be omitted in the analysis procedure. Moreover, similar to CA, development of TMA under large deformations is a promising mechanism for preventing disproportionate collapse:

(1) The numerical investigation on beam-slab structures shows that fracture of rebars in internal beams can be considered as indicator of final structural capacity of the whole system. It should be noted that TMA in slabs is less influenced by horizontal restraint stiffness for both the CL and the UDL 
conditions. Nevertheless, this mechanism is affected by rotational restraint of the slab edges if UDL configuration is employed.

(2) An extensive numerical study on the locations of removed column is carried out under UDL condition, showing that the loss of a corner column is not necessarily the most vulnerable scenario. Limited TMA in the slab can still be mobilised if the beams at discontinuous edges are relatively stiff. In fact, due to the weak rotational restraint of the discontinuous slab edges, as well as the inward movement of affected edge columns, the removal of penultimate columns can be more critical to progressive collapse.

(3) The analytical model for internal-column loss scenarios proposed in Section 9.6 can consider mobilisation of TMA in the slabs. Load-bearing capacity of this mechanism is conservatively assumed to rely on yielding forces of slab top reinforcement along the negative-bending yield lines. The approach can be used for either the CL or the UDL conditions. The failure criteria of the 3D structure are based on rebar fracturing of internal beams, which can be conveniently computed using either the semi-analytical model in Section 4.3 (for CL case) or the simplified model in Section 7.3 (for UDL case). This method provides a convenient tool to engineers to assessing vulnerability of beam-slab structures under progressive collapse events.

\subsection{Future work}

Experimental studies on structural behaviour of beam-column frames under UDL condition, as well as under non-symmetric design condition

The semi-analytical model proposed in Chapter 4 is based on numerical and experimental studies under symmetric condition (Fig. 4.2). Some simplified adaptations are made to consider non-symmetric structures (Section 4.5.2). Nonetheless, actual experimental studies applying unequal spans or different sectional designs at the two sides of the double-span beam need to be conducted to verify the model. 
Although the quasi-static tests in Series II indicated differences in responses between the two loading configurations (CL and UDL), there is a need to extend the experimental work on RC beam-column structures under UDL condition covering various ranges of geometry and reinforcement properties and verifying the simplified response proposed in Section 7.3. Parameters which are important to CA development such as span-depth ratio, horizontal stiffness, reinforcement ratio, should also be considered.

\section{Structural responses of beam-slab systems under actual dynamic conditions}

The blast-induced dynamic tests presented in Chapter 8 provide valuable data on the behaviour of 2D RC frames under actual progressive collapse events triggered by explosives. However, dynamic tests should be conducted for 3D beam-slab structure. Both free-fall dynamic and blast-induced scenarios are required to investigate the dynamic behaviour of the substructure, and to evaluate the blast effect on surviving members. Under blast pressure from a contact detonation, uplift movement of the beam-slab structure can be much more obvious compared to the 2D frame system due to larger areas of blast exposures. As a result, the reduction in structural capacity against progressive collapse may become more critical, as shown in the numerical study in Section 9.7.

\section{Semi-empirical approach for progressive collapse assessment of $\mathrm{RC}$ frames}

Blast pressure generated from an explosive not only affects the general behaviour of structures but also causes localised damages. Simplified analytical methods for blast resistance, such as the SDOF method employed in UFC 3-340-02, can only be applied for simple problems in which only one major mechanism dominates the overall response of the structure. In more complicated conditions, such as an initial blast attack triggering a single column loss event which inflicts both local and global damages to the specimen, high-fidelity physics-based modelling techniques can provide more accurate results. The use of these advanced methods, however, requires intensive studies and can be daunting to structural engineers.

Alternatively, based on the detailed numerical models validated by test results in Chapter 8, parametric studies for different blast parameters can be performed. From 
numerical data, semi-empirical approaches can be proposed for different design conditions. Such approaches are similar to the pressure-impulse methods for blast resistance of structural members (Shi et al. 2008, Krauthammer et al. 2009). Thus, a semi-empirical numerical-based method for assessing the blast-induced progressive collapse resistance of $2 \mathrm{D}$ frames is a promising direction due to high costs associated with actual blast tests or complicated physics-based modelling.

The objective of this approach is to assess the applicability of the single-column removal scenario for RC frame structures under progressive collapse induced by contact detonation. This simplified method may require iso-damage curves to differentiate failure modes of the structure. The approach can also provide semiempirical equations to define the application limit of the single-column removal scenario based on parameters from geometries and materials. 



\section{REFERENCES}

American Society of Civil Engineers (ASCE) (2010). "Minimum design loads for buildings and other structures", ASCE 7-10. American Society of Civil Engineers, Reston, VA

Department of Defense (2008). "Structures to resist the effects of accidental explosions", UFC 3-340-02. Washington, D.C

Department of Defense (2013). "Design of buildings to resist progressive collapse", Unified Facilities Criteria (UFC) 4-023-03. Washington, D.C

Bailey, C., White, D. and Moore, D. (2000). "The tensile membrane action of unrestrained composite slabs simulated under fire conditions." Engineering Structures 22(12): 1583-1595.

Bailey, C. G. (2001). "Membrane action of unrestrained lightly reinforced concrete slabs at large displacements." Engineering Structures 23(5): 470-483.

Baker, W. E. (1973). Explosion in air, University of Texas press.

Bao, Y., Kunnath, S., El-Tawil, S. and Lew, H. (2008). "Macromodel-Based Simulation of Progressive Collapse: RC Frame Structures." Journal of Structural Engineering 134(7): 1079-1091.

Bao, Y., Lew, H. S. and Kunnath, S. K. (2014). "Modeling of Reinforced Concrete Assemblies under Column-Removal Scenario." Journal of Structural Engineering 140(1): 04013026.

Biggs, J. M. (1964). Introduction to structural dynamics, McGraw-Hill College.

Brannon, R. M. and Leelavanichkul, S. (2009). Survey of Four Damage Models for Concrete, Sandia National Laboratories. 
Brown, D. (2012). "Tracker video analysis and modeling tool." from www.cabrillo.edu/ dbrown/tracker/.

European Committee for Standardization (2004). "Eurocode 2: Design of concrete structures - General rules and rules for buildings", 1992-1-1: 2004. CEN, Brussels

Choi, H. and Kim, J. (2011). "Progressive collapse-resisting capacity of RC beamcolumn sub-assemblage." Magazine of concrete research 63(4): 297-310.

CEB-FIP Model Code (2010). "Design of concrete structures", Fédération Internationale du Béton fib/International Federation for Structural Concrete

Eligehausen, R., Popov, E. P. and Bertero, V. V. (1983). Local Bond Stress-Slip Relationships of Deformed Bars Under Generalized Excitations. Report UCB/EERC-83/23. Berkeley, EERC, Univeristy of California.

Ellingwood, B. R. and Leyendecker, E. (1978). "Approaches for design against progressive collapse." Journal of the Structural Division 104(3): 413-423.

FarhangVesali, N., Valipour, H., Samali, B. and Foster, S. (2013). "Development of arching action in longitudinally-restrained reinforced concrete beams." Construction and Building Materials 47: 7-19.

Foster, S., Bailey, C., Burgess, I. and Plank, R. (2004). "Experimental behaviour of concrete floor slabs at large displacements." Engineering Structures 26(9): 12311247.

General Services Administration (2003). "Progressive collapse analysis and design guidelines for new federal office buildings and major modernization projects", Washington, D.C 
Habibullah, A. and Wilson, E. (2005). SAP 2000 "Static and Dynamic Finite Element Analysis of Structures". Computers and Structures Inc. Berkeley, California.

Hallquist, J. O. (2007). "LS-DYNA Keyword User's Manual Version 971." Livermore Software Technology Corporation.

Hawkins, N. M., Lin, I. J. and Jeang, F. L. (1982). Local bond strength of concrete for cyclic reversed loadings. Bond in Concrete, Paisley College of Technology, Scotland, Applied Science Publishers, London, UK.

Hyde, D. (1991). "ConWep, conventional weapons effects program." US Army Engineer Waterways Experiment Station, USA.

American Concrete Institute (2011). "Building Code Requirements for Structural Concrete and Commentary", ACI 318-11.

British Standard Institution (1997). "Structural use of concrete", BS 8110:1997.

Izzuddin, B. and Elghazouli, A. (2004). "Failure of Lightly Reinforced Concrete Members under Fire. I: Analytical Modeling." Journal of Structural Engineering 130(1): 3-17.

Izzuddin, B., Vlassis, A., Elghazouli, A. and Nethercot, D. (2008). "Progressive collapse of multi-storey buildings due to sudden column loss-Part I: Simplified assessment framework." Engineering Structures 30(5): 1308-1318.

Izzuddin, B. and Nethercot, D. (2009). Design-Oriented Approaches for Progressive Collapse Assessment: Load-Factor vs. Ductility-Centred Methods. Structures Congress, American Society of Civil Engineers. 
Krauthammer, T. (2003). AISC research on structural steel to resist blast and progressive collapse. Proceedings of AISC Steel Building Symposium: Blast and Progressive Collapse Resistance, New York City, NY.

Krauthammer, T. (2008). Modern protective structures, CRC Press.

Krauthammer, T., Astarlioglu, S. and Blasko, J. R. (2009). Load-Impulse Diagrams for Protected Facility Assessment. Structures Congress 2009@ sDon’t Mess with Structural Engineers: Expanding Our Role, ASCE.

Li, Y., Lu, X., Guan, H. and Ye, L. (2014). "Progressive Collapse Resistance Demand of Reinforced Concrete Frames under Catenary Mechanism." $\underline{\text { ACI }}$ Structural Journal 111(5).

Lim, N. S., Lee, C. K. and Tan, K. H. (2015). Experimental studies on 2-D RC frame with middle column removed under progressive collapse. Proceedings of fib symposium 2015. Copenhagen, Denmark.

Lim, N. S. (2017). Systematic study on reinforced concrete structures under progressive collapse. PhD Thesis, Nanyang Technological University, Singapore.

Liu, C., Fung, T. C. and Tan, K. H. (2015). "Dynamic performance of flush endplate beam-column connections and design applications in progressive collapse." Journal of Structural Engineering 142(1): 04015074.

Lu, X., Lin, K., Li, Y., Guan, H., Ren, P. and Zhou, Y. (2016). "Experimental investigation of RC beam-slab substructures against progressive collapse subject to an edge-column-removal scenario." $\quad$ Engineering $\quad$ Structures http://dx.doi.org/10.1016/j.engstruct.2016.07.039.

Luccioni, B. and Aráoz, G. (2011). Erosion criteria for frictional materials under blast load, Mecánica Computacional 30 
Malvar, L. J. (1998). "Review of static and dynamic properties of steel reinforcing bars." ACI Materials Journal 95(5).

MHA (2010). Guideline for Enhancing Building Security in Singapore. S. Ministry of Home Affair (MHA). Singapore.

Murray, Y. D. (2007). Users manual for LS-DYNA concrete material model 159.

Namyo Salim Lim (2014). Quasi-static tests of reinforced concrete frames under progressive collapse - Test report, Nanyang Technological University.

Orton, S., Jirsa, J. O. and Bayrak, O. (2009). "Carbon Fiber-Reinforced Polymer for Continuity in Existing Reinforced Concrete Buildings Vulnerable to Collapse." $\underline{\mathrm{ACI}}$ Structural Journal 106(5).

Orton, S. L. and Kirby, J. E. (2013). "Dynamic response of a RC frame under column removal." Journal of Performance of Constructed Facilities 28(4): 04014010 .

Park, R. (1964). "Tensile membrane behaviour of uniformly loaded rectangular reinforced concrete slabs with fully restrained edges." Magazine of Concrete Research 16(46): 39-44.

Park, R. and Paulay, T. (1975). Reinforced concrete structures, John Wiley \& Sons.

Park, R. and Gamble, W. L. (2000). Reinforced concrete slabs, John Wiley \& Sons.

Paulay, T. and Priestley, M. (1992). Seismic design of reinforced concrete and masonry buildings, Wiley, New York.

Pham, A. T., Tan, K. H. and Yu, J. (2016). "Numerical investigations on static and dynamic responses of reinforced concrete sub-assemblages under progressive collapse." Structures

\section{http://dx.doi.org/10.1016/j.engstruct.2016.07.042.}


Pham, A. T., Lim, N. S. and Tan, K. H. (2017). "Investigations of tensile membrane action in beam-slab systems under progressive collapse subject to different loading configurations and boundary conditions." Engineering Structures 150: 520-536.

Pham, A. T. and Tan, K. H. (2017a). "Experimental study on dynamic responses of reinforced concrete frames under sudden column removal applying concentrated loading." Engineering Structures 139: 31-45.

Pham, A. T. and Tan, K. H. (2017b). "Static and dynamic responses of reinforced concrete structures under sudden column removal scenario subjected to distributed loading." Journal of Structural Engineering (submitted).

Pham, A. T. and Tan, K. H. (2017c). "A simplified model of catenary action in reinforced concrete frames under axially restrained conditions." Magazine of Concrete Research http://dx.doi.org/10.1680/jmacr.17.00009.

Pham, X. D. and Tan, K. H. (2013). "Experimental study of beam-slab substructures subjected to a penultimate-internal column loss." Engineering Structures 55: 2-15.

Pham, X. D., Tan, K. H. and Jun, Y. (2015). "A simplified approach to assess progressive collapse resistance of reinforced concrete framed structures." Engineering Structures 101: 45-57.

Pham, X. D. and Tan, K. H. (2015). "Experimental Response of Beam-Slab Substructures Subject to Penultimate-External Column Removal." Journal of Structural Engineering 141(7).

Qian, K. and Li, B. (2012a). "Slab effects on response of reinforced concrete substructures after loss of corner column." ACI Structural Journal 109(6). 
Qian, K. and Li, B. (2012b). "Dynamic performance of RC beam-column substructures under the scenario of the loss of a corner column-Experimental results." Engineering Structures 42: 154-167.

Qian, K. and Li, B. (2012c). "Performance of three-dimensional reinforced concrete beam-column substructures under loss of a corner column scenario." Journal of Structural Engineering 139(4): 584-594.

Qian, K. and Li, B. (2014). "Dynamic disproportionate collapse in flat-slab structures." Journal of Performance of Constructed Facilities 29(5): B4014005.

Qian, K., Li, B. and Ma, J. X. (2014). "Load-carrying mechanism to resist progressive collapse of RC buildings." Journal of Structural Engineering 141(2): 04014107.

Qian, K., Li, B. and Tian, Y. (2016a). "Recent Progress in Understanding of Load Resisting Mechanisms for Mitigating Progressive Collapse." $\underline{\text { ACI Special }}$ Publication 309(SP-309: Structural Integrity and Resilience).

Qian, K., Li, B. and Zhang, Z. (2016b). "Influence of Multicolumn Removal on the Behavior of RC Floors." Journal of Structural Engineering 142(5): 04016006 04016001-04016013.

Ren, P., Li, Y., Lu, X., Guan, H. and Zhou, Y. (2016). "Experimental investigation of progressive collapse resistance of one-way reinforced concrete beam-slab substructures under a middle-column-removal scenario." Engineering Structures 118: $28-40$.

Sadek, F., Main, J. A., Lew, H. S. and Bao, Y. (2011). "Testing and Analysis of Steel and Concrete Beam-Column Assemblies under a Column Removal Scenario." Journal of Structural Engineering 137(9): 881-892. 
Sagiroglu, S. (2012). Analytical and experimental evaluation of progressive collapse resistance of reinforced concrete structures, NORTHEASTERN UNIVERSITY.

Sasani, M., Bazan, M. and Sagiroglu, S. (2007). "Experimental and analytical progressive collapse evaluation of actual reinforced concrete structure." $\underline{\mathrm{ACI}}$ Structural Journal 104(6).

Sasani, M. (2008). "Response of a reinforced concrete infilled-frame structure to removal of two adjacent columns." Engineering Structures 30(9): 2478-2491.

Sasani, M. and Kropelnicki, J. (2008). "Progressive collapse analysis of an RC structure." The Structural Design of Tall and Special Buildings 17(4): 757-771.

Sasani, M. and Sagiroglu, S. (2010). "Gravity Load Redistribution and Progressive Collapse Resistance of 20-Story Reinforced Concrete Structure following Loss of Interior Column." ACI Structural Journal 107(6).

Sasani, M., Kazemi, A., Sagiroglu, S. and Forest, S. (2011). "Progressive collapse resistance of an actual 11-story structure subjected to severe initial damage." Journal of Structural Engineering 137(9): 893-902.

Shi, Y., Hao, H. and Li, Z. X. (2008). "Numerical derivation of pressure-impulse diagrams for prediction of RC column damage to blast loads." International Journal of Impact Engineering 35(11): 1213-1227.

Shi, Y., Li, Z. X. and Hao, H. (2009). "Bond slip modelling and its effect on numerical analysis of blast-induced responses of RC columns." Structural Engineering and Mechanics 32(2): 251-267.

Shi, Y., Li, Z. X. and Hao, H. (2010). "A new method for progressive collapse analysis of RC frames under blast loading." Engineering Structures 32(6): 16911703. 
Shima, H., Chou, L. and Okamura, H. (1987). "Micro and macro model for bond behavior in RC." Journal of the Faculty of Engineering, The University of Tokyo(B) 39(2): 133-194.

European Committee for Standardization (2006). "Eurocode 1: Actions on structures-General actions-Accidental actions", EN 1991-1-7: 2006. CEN, Brussels

Stevens, D., Marchand, K. and McKay, A. (2009). Revision of the tie force and alternate path approaches in the DoD progressive collapse design requirements. Structures Congress 2009 Proceedings, ASCE, Austin, Texas.

Stinger, S. M. and Orton, S. L. (2013). "Experimental evaluation of disproportionate collapse resistance in reinforced concrete frames." $\underline{\text { ACI Structural }}$ journal 110(3): 521.

Su, Y., Tian, Y. and Song, X. (2009). "Progressive Collapse Resistance of AxiallyRestrained Frame Beams." ACI Structural Journal 106(5).

Tan, K. H. and Yang, B. (2012). "Behaviour of Different Types of Steel Connections in Steel Frames against Progressive Collapse." Advanced Materials Research 374: 1330-1341.

Tang, E. K. and Hao, H. (2010). "Numerical simulation of a cable-stayed bridge response to blast loads, Part I: Model development and response calculations." Engineering Structures 32(10): 3180-3192.

Tian, Y. and Su, Y. (2011). "Dynamic response of reinforced concrete beams following instantaneous removal of a bearing column." International Journal of Concrete Structures and Materials 5(1): 19-28.

Timoshenko, S., Woinowsky-Krieger, S. and Woinowsky-Krieger, S. (1959). Theory of plates and shells, McGraw-hill New York. 
Technical Manual TM5-855-1 (1986). "Fundamentals of protective design for conventional weapons", Department of the Army, Washington D.C, 1986

Technical Manual TM5-1300 (1990). "Structures to Resist the Effects of Accidental Explosions", Departments of the Army, Washington D.C, November 1990

Tsai, M.-H. (2010). "An analytical methodology for the dynamic amplification factor in progressive collapse evaluation of building structures." Mechanics Research Communications 37(1): 61-66.

Ueda, T., Lin, I. and Hawkins, N. M. (1986). "Beam Bar Anchorage in Exterior Beam-Column Connections." ACI Journal, Proceedings 83(3): 412-422.

Valipour, H., FarhangVesali, N. and Foster, S. (2013). "A generic model for investigation of arching action in reinforced concrete members." Construction and Building Materials 38: 742-750.

Valipour, H. R. and Foster, S. J. (2010). "Finite element modelling of reinforced concrete framed structures including catenary action." Computers \& structures 88(9): 529-538.

Viawanthanatepa, S., Popov, E. P. and Bertero, V. V. (1979). Effects of Generalized Loadings on Bond of Reinforcing Bars Embedded in Confined Concrete Blocks. Report UCB/EERC-79/22. R. UCB/EERC-79/22. Berkeley, EERC, University of California. Report UCB/EERC-79/22.

Wu, K. C., Li, B. and Tsai, K. C. (2011). "Residual axial compression capacity of localized blast-damaged RC columns." International Journal of Impact Engineering 38(1): $29-40$.

Wu, Y., Crawford, J. E. and Magallanes, J. M. (2012). Performance of LS-DYNA concrete constitutive models. 12th International LS-DYNA Users Conference. 
Yi, W. J., He, Q. F., Xiao, Y. and Kunnath, S. K. (2008). "Experimental study on progressive collapse-resistant behavior of reinforced concrete frame structures." ACI Structural Journal 105(4).

Yu, J. and Tan, K. H. (2010). Progressive collapse resistance of RC beam-column sub. Proceedings of the third International Conference on Design and Analysis of Protective Structures 2010 : advances in protective technology, 10th-12th May, Singapore.

Yu, J. (2012). Structural behavior of reinforced concrete frames subjected to progressive collapse. Thesis, Nanyang Technological University, Singapore.

Yu, J. and Tan, K. H. (2013a). "Experimental and numerical investigation on progressive collapse resistance of reinforced concrete beam column subassemblages." Engineering Structures 55: 90-106.

Yu, J. and Tan, K. H. (2013b). "Structural Behavior of RC Beam-Column Subassemblages under a Middle Column Removal Scenario." Journal of Structural Engineering 139(2): 233-250.

Yu, J., Rinder, T., Stolz, A., Tan, K. H. and Riedel, W. (2014). "Dynamic Progressive Collapse of an RC Assemblage Induced by Contact Detonation." Journal of Structural Engineering 140(6).

Yu, J. and Tan, K. H. (2014). "Analytical model for the capacity of compressive arch action of reinforced concrete sub-assemblages." Magazine of concrete research 66(3): 109-126. 